\title{
Systematic Control of Aged Skeletal Muscle Following High- Intensity Stretch-Shortening Contraction Exercise Training: Epigenomic Regulation and Signaling Factors Underpinning Adaptation
}

Marshall Alan Naimo Jr.

West Virginia University, manaimo@mix.wvu.edu

Follow this and additional works at: https://researchrepository.wvu.edu/etd

Part of the Other Medicine and Health Sciences Commons, Sports Sciences Commons, and the Translational Medical Research Commons

\section{Recommended Citation}

Naimo, Marshall Alan Jr., "Systematic Control of Aged Skeletal Muscle Following High-Intensity StretchShortening Contraction Exercise Training: Epigenomic Regulation and Signaling Factors Underpinning Adaptation" (2019). Graduate Theses, Dissertations, and Problem Reports. 4094.

https://researchrepository.wvu.edu/etd/4094

This Dissertation is protected by copyright and/or related rights. It has been brought to you by the The Research Repository @ WVU with permission from the rights-holder(s). You are free to use this Dissertation in any way that is permitted by the copyright and related rights legislation that applies to your use. For other uses you must obtain permission from the rights-holder(s) directly, unless additional rights are indicated by a Creative Commons license in the record and/ or on the work itself. This Dissertation has been accepted for inclusion in WVU Graduate Theses, Dissertations, and Problem Reports collection by an authorized administrator of The Research Repository @ WVU.

For more information, please contact researchrepository@mail.wvu.edu. 
SYSTEMATIC CONTROL OF AGED SKELETAL MUSCLE FOLLOWING HIGH-INTENSITY
STRETCH-SHORTENING CONTRACTION EXERCISE TRAINING:
EPIGENOMIC REGULATION AND SIGNALING FACTORS UNDERPINNING ADAPTATION

MARSHALL ALAN NAIMO, JR., M.S.

A Dissertation submitted to the

School of Medicine at West Virginia University

in partial fulfillment of the

requirements for the degree of

Doctor of Philosophy in Exercise Physiology

Stephen E. Alway, Ph.D., Committee Chair

Brent A. Baker, Ph.D., Dissertation Mentor

John M. Hollander, Ph.D.

Emidio E. Pistilli, Ph.D.

David P. Siderovski, Ph.D.

Michael L. Kashon, Ph.D.

Division of Exercise Physiology

Morgantown, West Virginia

May 2019

Keywords: Adaptation, exercise training, stretch-shortening contractions, Fisher 344 x Brown Norway rats, growth and remodeling, aged skeletal muscle, epigenomics

Copyright 2019, Marshall A. Naimo 


\section{ABSTRACT}

\section{Systematic Control of Aged Skeletal Muscle Following High-Intensity Stretch-Shortening Contraction Exercise Training: Epigenomic Regulation and Signaling Factors Underpinning Muscle Adaptation}

\section{Marshall A. Naimo}

Sarcopenia, the age-related decline in skeletal muscle mass, results in a loss of strength and functional capacity, which subsequently increases the risk of disease, disability, frailty, and all-cause mortality. Exercise is known to be an efficacious paradigm for improving health and attenuating or preventing many chronic diseases. For the previous two decades, our laboratory has established an in vivo rodent dynamometer model to explore the effects of various skeletal muscle training paradigms following stretch-shortening contractions (SSCs). The responses to high-intensity resistance-type exercise training (RTET) using this physiological model ranges from adaptation, characterized by enhanced skeletal muscle performance along with increased muscle mass, to maladaptation, defined as an absence or diminishment of skeletal muscle performance and no improvements in muscle mass. Utilizing a non-injurious SSC protocol, training-induced adaptation occurs in young rodents; this response is altered with age in which old rodents undergo maladaptation when exposed to this same chronic loading protocol. Additionally, with respect to chronologically advancing age, our previous work indicates an altered adaptive phenotype following SSC RTET occurs prior to complete biological development of the rodent - six months of age in the adult rat, which we believe may indicate the onset of a loss in homeostatic control leading to an age-specific biological departure from the adaptive response. However, recently we have shown that modifying the frequency of RTET from three to two days per week in older rodents (e.g. 30-31 months of age) attenuates age-dependent maladaptation and restores muscle quality to a younger phenotype.

Despite the therapeutic potential of RTET, a fundamental basis for evidence-based exercise prescription is still largely undetermined because the molecular, cellular and integrated physiological pathways involved in exercise-induced muscle adaptation are not fully understood. Aging in-and-of-itself is a biological process associated with an altered phenotype, and emerging evidence suggests these changes are possibly linked to epigenomic processes. Excitingly, recent research has shown that exercise can influence changes in DNA methylation in skeletal muscle. However, it is currently unknown how exactly DNA methylation may be influencing the adaptation of skeletal muscle to high-intensity SSC RTET, which could be an important mechanism underlying the responsivity of the muscle to training in the context of aging. Traditionally, the term muscle memory has been defined as describing the capability of skeletal muscle to respond more quickly to an applied stimulus that has been encountered previously in spite of periods of inactivity. Recently, emerging evidence has pointed to the existence of a cellular foundation of skeletal muscle memory. Because environmental stimuli and stressors lead to modifications in gene expression, epigenetics/epigenomics are highly likely to form the underlying basis for this cellular memory. However, despite this collective knowledge, to date no studies have determined whether or not changes via DNA methylation that occur as a result of exposure to an adaptive exercise stimulus has a lasting influence on the adaptability of skeletal muscle upon reintroduction to the same stimulus at a later life. In order to examine these unresolved issues, the purpose of this dissertation followed three specific aims: 1) To determine the effects of aging and a reduced training frequency on the activation of molecular signaling pathways associated with the adaptation of skeletal muscle following one month of high-intensity SSC RTET in old rats; 2) to investigate whether DNA methylation influences the molecular signaling activity and adaptability of skeletal muscle following one month of highintensity SSC RTET, and whether reducing the training frequency modifies the methylation profile of skeletal muscle in response to the training stimulus; 3) to examine if introducing high-intensity SSC RTET at an earlier relative age promotes changes in molecular signaling and DNA methylation that positively influences the ability 
of skeletal muscle to adapt upon re-exposure to the same paradigm at a later age previously shown to have the inability to go through the full adaptive response .

The hypotheses for this research were that the ability of aged muscle to adapt to high-intensity SSC RTET would be compromised when exposed to an inappropriate stimulus (i.e., maladaptive) as a consequence of a dysregulated molecular signaling response which would be observable in distinct pathways crucial in muscle homeostasis and remodeling; furthermore, these potential age-related dysregulated events in gene activity would occur as a consequence to altered DNA methylation. Moreover, older animals exposed to a reduced frequency of high-intensity SSC RTET would respond favorably to the training stimulus and in an appropriate manner (i.e., adaptation) and would have a resemblance more like young rats in terms of the molecular signaling pathway and DNA methylation responses compared to age-matched counterparts exposed to a higher frequency that induces maladaptation. Additionally, training rodents at a younger relative age compared to where age-dependent maladaptation occurs would attenuate DNA methylation and therefore positively augment the adaptability of muscle to respond favorably to chronic SSC RTET at a later age following detraining. The results from this study could be vital in understanding the underlying performance, physiological, molecular, and environmental factors influencing the capability of aged skeletal muscle to undergo adaptation in response to RTET; and, thus have important ramifications in the attenuation and/or reversal of sarcopenia. Additionally, we sought to determine the therapeutic efficacy of a training-retraining paradigm using our in vivo highintensity RTET paradigm by investigating whether or not training at an earlier age is able to prevent functional and physiological decrements of skeletal muscle during the later stages of life. 


\section{DEDICATION}

To the memory of my aunt, Diana O. Naimo (July 15, 1944 - August 13, 2015) who taught me many things about life and gave me so much support and encouragement during the pursuit of my education and very badly wanted to be there when I finished my degree; you may not be there for it physically, but you will certainly be there in spirit.

To my Mom, Dad, sister, Aunt Laura, Uncle Alan, Aunt Deb, cousins, and to all my late grandparents for all the love, care, support, encouragement, and influence they provided me during this endeavor that has helped me grow into the person that I am today; I am eternally grateful.

To my close friends, particularly my first-year classmates that became like my second family away from home, for always being there when I needed their help most, for making the journey fun, and for keeping me from going crazy by helping me maintain a work/life balance.

To my other friends and fellow members of the Morgantown Mountain Goats Disc Golf Club, WVU Disc Golf Club, and men's softball teams for helping me find a competitive outlet in sports while being fortunate to be a part of something with a true purpose and a sense of camaraderie.

To all the owners, staff, and regulars at Mario’s Fishbowl, past and present, for all the cold beers, delicious food, and great times you have provided me following a tough exam or a long day in lab. All the memories, laughter, and friendships forged here go beyond anything I would have ever expected from a watering hole and there is no other place that could ever replace it.

Last but not least, to the great state of West Virginia, for your stunning and endless beauty, Appalachian soul, and the people living here that welcomed this Florida boy with open arms and made me feel like I had been here my whole life. Words cannot describe everything this state has given me since I came here. If the time ever comes where I have to leave this amazing place, may these country roads eventually take me back home to the place where I belong. 


\section{ACKOWLEDGEMENTS}

First and foremost, to Mom, Dad, my sister Elise, and Aunt Laura, that have been there for me this whole time while pursuing my dream of getting a Ph.D.; thank you for everything that you have ever done for me. Without your support and encouragement, I would not be in this position today and I am forever grateful of that. To the rest of my extended family as well as my closest friends from Florida, for being highly supportive, especially in times of need, along with providing me with the necessary breaks from the hardships of lab; some of my favorite memories have taken place with all of you at Shadow Lake in Vermont or on the disc golf courses and beaches in Florida. To my fellow classmates, particularly Emily, Ray, Kayla, Adam, Brenen, Peter, Evan, Dudley, and Dylan, who made the transition moving up here go so much better than I could have ever imagined. We all became very close and they were there for me every step of the way during the first couple years here when a lot of things in my personal life were changing, and for that I will always be so appreciative.

To my lab at NIOSH, starting with my mentor Dr. Baker. I knew within that first week of rotating that this was the right fit for me, and nothing has ever happened to make me think otherwise since. I have learned so much during my time here under your guidance, and you have always been there for me for advice whether it was a personal matter or professional. I have always enjoyed all of our conversations about exercise training and sports! James Ensey, our lab technician, for answering what may have literally been thousands of questions I had about techniques and bench science, as well as for always being willing to provide a helping hand without hesitation; and for all our conversations about Game of Thrones! Dr. Erik Rader, for being the one responsible for introducing me to Dr. Baker and the lab at NIOSH; I enjoy your dry humor and way of thinking on science, both of which have been very helpful and enjoyable. Dr. Bob Chetlin, for being the first one to show me around the lab during my rotation; I always enjoyed talking hockey with you and hope things are well in Erie.

To Dr. Alway, for being willing to be my committee chair and for seeing it through to the end despite the move to Tennessee. Your helpfulness and wisdom you have provided throughout the years has gone a long way in aiding me get to where I am at today and I wish you nothing but the best in Memphis. To Dr. Pistilli, for being the first faculty member I came in to contact with about WVU and allowing me to come in and gain valuable research experience within the first week of moving up here. You were the one who gave me my initiation in to the world of basic science research, and I really enjoyed the time spent in your lab during my first ever rotation all those years ago as I got my feet wet learning bench science. To Dr. Hollander, for your guidance as program director of Exercise Physiology as well as your valuable feedback on experiments from my dissertation as a member on my committee. To Dr. Siderovski and Dr. Kashon as my outside committee members; not only have you provided helpful guidance over the years, but your kind personalities and sense of humor always made it enjoyable to chat with both of you whenever I stopped by your offices.

To my Thesis mentor at Florida State University, Dr. Lynn Panton, for helping me get through my first experiences of designing and running a research project; I would not where I am at today without this prior experience I gained while in your lab. Also a big thanks to Dr. Panton for giving me guidance on what it would take to get a Ph.D. and for writing me all those letters of recommendation. Big thanks to Kyle Mandler, who is probably the person most responsible for why I ended up applying to and subsequently deciding to come to WVU. I was fortunate to get to know you during our time at FSU when you were applying to doctorate programs across the country, and it was during that time you planted the seed for me to apply to the program here a couple years later. I will always remember how much you were willing to help me out before I even got to Morgantown by giving me all the ins and outs of the program and for scouting out an apartment for me I found on Craigslist because I was 1,500 miles away in Florida. Also, our whitewater trips that you took me on over the years are still some of my favorite memories of West Virginia to this day!

To the Damron and Barbier labs, for giving me several good whitewater and outdoor adventures all over the state the last few years! I would rank a lot of those experiences up there as my best memories during my time here and because of them I have gotten to see some of the most remote and beautiful parts of West Virginia. Finally, to all other staff, both at NIOSH and at WVU, past and present, not mentioned by name above, for all the help, guidance, and resources you have given me over the years to make the completion of this dissertation possible. 


\section{TABLE OF CONTENTS}

ABSTRACT

DEDICATION

ACKNOWLEDGEMENTS N V

LIST OF ABBREVIATIONS Ix

$\begin{array}{ll}\text { I. INTRODUCTION } & 1\end{array}$

Purpose 2

Specific Aims $\quad 2$

Research Hypotheses $\quad 2$

Expected Outcomes 3

References

II. REVIEW OF LITERATURE $\quad 6$

Aging, Sarcopenia, and Resistance Exercise Training as a Therapeutic Aid 6

Adaptation versus Maladaptation with RTET 9

Reduced Frequency and Muscle Adaptation with Advanced Age 12

The Interaction between Epigenetics/Epigenomics, Aging, and Exercise 15

Effects of DNA Methylation and Exercise 16

Muscle Memory and DNA Methylation as a Cellular Basis for its Existence 18

The Application of a Training-Retraining Paradigm towards Muscle Memory 22

Conclusions and Purpose of the Dissertation 24

$\begin{array}{ll}\text { References } & 27\end{array}$

III. ENHANCEMENT OF SKELETAL MUSCLE IN AGED RATS FOLLOWING HIGH-INTENSITY STRECH-SHORTENING CONTRACTION TRAINING 39

$\begin{array}{ll}\text { Abstract } & 40\end{array}$

$\begin{array}{ll}\text { Introduction } & 42\end{array}$

Materials \& Methods $\quad 43$

$\begin{array}{ll}\text { Results } & 46\end{array}$

$\begin{array}{ll}\text { Discussion } & 49\end{array}$

$\begin{array}{ll}\text { References } & 52\end{array}$ 
Figure Legends

Figures

IV. REDUCED FREQUENCY OF RESISTANCE-TYPE EXERCISE TRAINING PROMOTES ADAPTATION OF THE AGED SKELETAL MUSCLE MICROENVIRONMENT 67

Abstract 70

Introduction 71

Materials \& Methods 72

Results 75

Discussion 78

References 82

Tables 87

Figure Legends 95

Figures 97

V. REDUCING THE FREQUENCY OF RESISTANCE-TYPE EXERCISE TRAINING POSITIVELY MODIFIES APOPTOSIS SIGNALING, DNA DAMAGE RESPONSE, AND MYONUCLEI MORPHOLOGY IN AGED SKELETAL MUSCLE

Abstract

Introduction 119

Materials \& Methods 122

Results 127

Discussion 131

References 142

Tables 154

Figure Legends 176

Figures 180

VI. THE THERAPEUTIC EFFECTS OF A RESISTANCE-TYPE EXERCISE TRAINING-RETRAINING PARADIGM ON TRANSCRIPTION FACTOR REGULATION AND MUSCLE ADAPTATION WITH AGING 201 Abstract 202 
$\begin{array}{ll}\text { Introduction } & 204\end{array}$

Materials \& Methods $\quad 207$

$\begin{array}{ll}\text { Results } & 211\end{array}$

$\begin{array}{ll}\text { Discussion } & 214\end{array}$

$\begin{array}{ll}\text { References } & 222\end{array}$

$\begin{array}{ll}\text { Tables } & 228\end{array}$

$\begin{array}{ll}\text { Figure Legends } & 238\end{array}$

$\begin{array}{ll}\text { Figures } & 241\end{array}$

$\begin{array}{ll}\text { VII. DISCUSSION } & 251\end{array}$

$\begin{array}{ll}\text { Future Directions } & 260\end{array}$

$\begin{array}{ll}\text { Conclusions } & 265\end{array}$

$\begin{array}{ll}\text { References } & 267\end{array}$

APPENDIX A: RESISTANCE TRAINING FREQUENCY CONFERS GREATER MUSCLE QUALITY IN AGED INDIVIDUALS: A BRIEF NHANES REPORT 


\section{LIST OF ABBREVIATIONS}

1RM: One-repetition maximum

4-HNE: 4-hydroxynonenal

8-OHdG: 8-hydroxydeoxyguanosine

AAALAC: Association for Assessment and Accreditation of Laboratory Animal Care

ACTB: Actin beta

AKT: Protein kinase B

AMPK: AMP-activated protein kinase

ATF3: Activating transcription factor 3

B2M: Beta-2 microglobulin

BAX: Bcl2-associated X protein

BCL2: B-cell lymphoma 2

BCL2A1: B-cell lymphoma 2-related protein A1

BER: Base Excision Repair

CNF: Central nucleated fiber

CpG: cytosine-guanosine base pairing

CSA: Cross sectional area

DAPI: 4', 6-diamidino-2-phenylindole

DHFR: Chromosome dihydrofolate reductase

DSB: Double-strand break

EB1: Microtubule associated protein RP/EB family member 1

ERK: Extracellular signal-regulated kinase

ELISA: Enzyme-linked immunosorbent assay

EWGSOP: European Working Group on Sarcopenia in Older People

F344xBN: Fischer 344 x Brown Norway

FADD: Fas-associated via death domain

FN14: Fibroblast growth factor-inducible 14

GADD45: Growth arrest and DNA damage

GSK3: Glycogen synthase kinase 3

HPRT1: Hypoxanthine phosphoribosyltransferase 1

HR: Homologous recombination

ILK: Integrin linked kinase

IPA: Ingenuity Pathway Analysis

LDHA: Lactate dehydrogenase 1

MAPK: Mitogen-activated protein kinases

MDA: Malondialdehyde

MEF2A: Myocyte enhancer factor 2AMMR: Mismatch Repair

MPG: N-methylpurine-DNA glycosylase

MQ: Muscle quality

mTOR: Mammalian target of rapamycin

MUTYH: MutY homolog

MYOD1: Myogenic differentiation protein 1

MYF5: Myogenic factor 5

NDUFC2: NADH dehydrogenase [ubiquinone] 1 subunit C2

NER: Nucleotide Excision Repair

NF- $\kappa$ B: Nuclear factor kappa-light-chain-enhancer of activated B cells

NHANES: National Health and Nutrition Examination Survey

NHEJ: Non-homologous end joining

NLC: Non-loaded control 
NR4A3: Nuclear receptor subfamily 4 group A member 3

O2x: Old rats SSC-loaded two days per week

O3x: Old rats SSC-loaded three days per week

$\mathrm{OD}_{405}$ : Optical density at $405 \mathrm{~nm}$

OGG1: 8-oxoguanine DNA glycosylase-1

p53: Tumor protein p53

PGC-1 $\alpha$ : Peroxisome proliferator-activated receptor gamma, coactivator $1 \alpha$

PDK4: Pyruvate dehydrogenase kinase 4

PI3K: Phosphoinositide 3-kinase

PPAR- $\delta$ : Peroxisome proliferator-activated receptor $\delta$

qRT-PCR: Quantitative real-time polymerase chain reaction

ROS: Reactive oxygen species

RPLP1: Ribosomal protein, large, P1

RTET: Resistance-type exercise training

RUNX1: Runt related transcription factor 1

SA: Synergistic ablation

SDEG: Significantly differentially expressed gene

SHOT: Cytoplasmic factors short stop, isoform a

SSC: Stretch-shortening contraction

TA: Tibialis Anterior

TDG: Thymine-DNA glycosylase

TF: Transcription factor

TFAM: Transcription factor A, mitochondrial

TGF- $\beta$ : Transforming growth factor beta

THADA: Thyroid adenoma associated

TNF- $\alpha$ : Tumor necrosis factor alpha

TNFR1: Tumor necrosis factor receptor 1

TRAF3: TNF receptor associated factor 3

TUNEL: Terminal deoxynucleotidyl transferase dUTP nick end labeling

TWEAK: Tumor necrosis factor-like weak inducer of apoptosis

TRT: Training-retraining

Y2x: Young rats SSC-loaded two days per week

Y3x: Young rats SSC-loaded three days per week

YAP: Yes-associated protein 


\section{CHAPTER I}

\section{INTRODUCTION}

Aging can be described as the gradual changes in the molecular physiology of cells leading to declines in normal tissue function(s) (1). Sarcopenia, the age-related decline in skeletal muscle mass, results in a loss of strength and functional capacity, which subsequently increases the risk of disease, disability, frailty, and allcause mortality $(3,4,7,12,13)$. Exercise is known to be an efficacious paradigm for improving health and attenuating or preventing many chronic diseases. For the previous two decades, our laboratory has established an in vivo rodent dynamometer model to explore the effects of various skeletal muscle training paradigms following stretch-shortening contractions (SSCs) $(6,8)$. The responses to high-intensity resistance-type exercise training (RTET) using this physiological model ranges from adaptation, characterized by enhanced skeletal muscle performance along with increased muscle mass, to maladaptation, defined as an absence or diminishment of skeletal muscle performance and no improvements in muscle mass. Utilizing a non-injurious SSC protocol, training-induced adaptation occurs in young rodents; this response is altered with age in which old rodents undergo maladaptation when exposed to this same chronic loading protocol (5). Additionally, with respect to chronologically advancing age, our previous work indicates an altered adaptive phenotype following SSC RTET occurs prior to complete biological development of the rodent - six months of age in the adult rat, which we believe may indicate the onset of a loss in homeostatic control leading to an age-specific biological departure from the adaptive response. However, recently we have shown that modifying the frequency of RTET from three to two days per week in older rodents (e.g. 30-31 months of age) attenuates age-dependent maladaptation and restores muscle quality to a younger phenotype (11). Collectively, the implications of these findings in older rats trained at two or three days per week demonstrates that the adaptive capacity of skeletal muscle in response to high-intensity SSC RTET is age-specific and dependent upon how components of the exercise prescription are structured. Additionally, with age individuals are increasingly sensitive to the exercise stimulus presented to them and are progressively more susceptible to the untoward effects of an inappropriate exposure; and, appropriately modified exercise prescription is a critical component that enables adaptation to occur.

Despite the therapeutic potential of RTET, a fundamental basis for evidence-based exercise prescription is still largely undetermined because the molecular, cellular and integrated physiological pathways involved in exercise-induced muscle adaptation are not fully understood. Aging in-and-of-itself is a biological process that is associated with an altered phenotype, and emerging evidence suggests these changes are possibly linked to epigenomic processes. Excitingly, recent research has shown that exercise can influence changes in DNA methylation in skeletal muscle $(2,9,10)$. However, it is currently unknown how exactly DNA methylation may 
be influencing the adaptation of skeletal muscle to high-intensity SSC RTET, which could be an important mechanism underlying the responsivity of the muscle to training in the context of aging. Traditionally, the term muscle memory has been defined as describing the capability of skeletal muscle to respond more quickly to an applied stimulus that has been encountered previously in spite of periods of inactivity. Recently, emerging evidence has pointed to the existence of a cellular foundation of skeletal muscle memory. Because environmental stimuli and stressors lead to modifications in gene expression, epigenetics/epigenomics are highly likely to form the underlying basis for this cellular memory. However, despite this collective knowledge, to date no studies have determined whether or not changes via DNA methylation that occur as a result of exposure to an adaptive exercise stimulus has a lasting influence on the adaptability of skeletal muscle upon reintroduction to the same stimulus at a later life.

\section{Purpose}

Therefore, the purpose of this dissertation was to use a valid and reliable in vivo rodent model of highintensity SSC RTET in order to fill in the gaps in our knowledge about the underlying molecular signaling pathways and epigenetic/epigenomic processes (i.e., DNA methylation) mediating aged-related muscle adaptation in response to a both a reduced frequency training and a training-retraining exercise paradigm. The purpose of this dissertation followed three specific aims.

\section{Specific Aims}

Specific Aim 1: To determine the effects of aging and a reduced training frequency using high-intensity SSC loading two or three days per week on the activation of molecular signaling pathways associated with the maintenance and adaptation of skeletal muscle following one month of chronic high-intensity SSC RTET in old rats.

Specific Aim 2: To investigate whether DNA methylation influences the molecular signaling activity and adaptability of skeletal muscle following one month of high-intensity SSC RTET, and whether reducing the training frequency modifies the methylation profile of skeletal muscle in response to the training stimulus

Specific Aim 3: To examine if introducing chronic high-intensity SSC RTET at an earlier relative age promotes changes in molecular signaling and DNA methylation that positively influences the ability of skeletal muscle to adapt upon re-exposure to the same paradigm at a later age previously shown to have the inability to go through the full adaptive response (i.e., skeletal muscle memory).

\section{Research Hypotheses}


Hypothesis 1: Older rats exposed to a reduced frequency of high-intensity SSC RTET would undergo adaptation and have a distinct molecular signature in comparison to old rats trained at a higher frequency and more closely resemble young rats in terms of the signaling pathway and DNA methylation responses

Hypothesis 2: The ability of aged muscle to adapt to high-intensity SSC RTET would be compromised when exposed to an inappropriate stimulus (i.e., maladaptive) as a consequence of a dysregulated molecular signaling response which would be observable in distinct pathways crucial in the muscle adaptive response; furthermore, these potential age-related dysregulated events in gene activity would occur as a consequence to altered DNA methylation.

Hypothesis 3: Training rodents at a younger relative age compared to where age-dependent maladaptation occurs (i.e., three months old) would attenuate DNA methylation and therefore positively augment the adaptability of muscle upon reintroduction to the same stimulus at adulthood (i.e., six months of age).

\section{Expected Outcomes:}

The results from this study would be vital in understanding the underlying performance, physiological, molecular, and environmental factors influencing the capability of aged skeletal muscle to undergo adaptation in response to RTET; and, thus have important ramifications in the attenuation and/or reversal of sarcopenia. Additionally, we sought to determine the therapeutic efficacy of a training-retraining paradigm using our in vivo high-intensity RTET paradigm by investigating whether or not training at an earlier age is able to prevent functional and physiological decrements of skeletal muscle during the later stages of life. 


\section{REFERENCES}

1. Akbari M, and Krokan HE. Cytotoxicity and mutagenicity of endogenous DNA base lesions as potential cause of human aging. Mech Ageing Dev 129: 353-365, 2008.

2. Barres R, Yan J, Egan B, Treebak JT, Rasmussen M, Fritz T, Caidahl K, Krook A, O'Gorman DJ, and Zierath JR. Acute exercise remodels promoter methylation in human skeletal muscle. Cell Metab 15: 405-411, 2012.

3. Bruyère O, Beaudart C, Ethgen O, Reginster JY, and Locquet M. The health economics burden of sarcopenia: a systematic review. Maturitas 119: 61-69, 2019.

4. Cruz-Jentoft AJ, Bahat G, Bauer J, Boirie Y, Bruyere O, Cederholm T, Cooper C, Landi F, Rolland Y, Sayer AA, Schneider SM, Sieber CC, Topinkova E, Vandewoude M, Visser M, Zamboni M, Writing Group for the European Working Group on Sarcopenia in Older P, and the Extended Group for E. Sarcopenia: revised European consensus on definition and diagnosis. Age Ageing 48: 16-31, 2019.

5. Cutlip RG, Baker BA, Geronilla KB, Mercer RR, Kashon ML, Miller GR, Murlasits Z, and Alway SE. Chronic exposure to stretch-shortening contractions results in skeletal muscle adaptation in young rats and maladaptation in old rats. Appl Physiol Nutr Metab 31: 573-587, 2006.

6. Cutlip RG, Stauber WT, Willison RH, McIntosh TA, and Means KH. Dynamometer for rat plantar flexor muscles in vivo. Med Biol Eng Comput 35: 540-543, 1997.

7. Evans WJ. What is sarcopenia? J Gerontol A Biol Sci Med Sci 50 Spec No: 5-8, 1995.

8. Geronilla KB, Miller GR, Mowrey KF, Wu JZ, Kashon ML, Brumbaugh K, Reynolds J, Hubbs A, and Cutlip RG. Dynamic force responses of skeletal muscle during stretch-shortening cycles. Eur J Appl Physiol 90: 144-153, 2003.

9. Kanzleiter T, Jahnert M, Schulze G, Selbig J, Hallahan N, Schwenk RW, and Schurmann A. Exercise training alters DNA methylation patterns in genes related to muscle growth and differentiation in mice. Am J Physiol Endocrinol Metab 308: E912-920, 2015.

10. Nitert MD, Dayeh T, Volkov P, Elgzyri T, Hall E, Nilsson E, Yang BT, Lang S, Parikh H, Wessman Y, Weishaupt H, Attema J, Abels M, Wierup N, Almgren P, Jansson PA, Ronn T, Hansson O, Eriksson KF, Groop L, and Ling C. Impact of an exercise intervention on DNA methylation in skeletal muscle from first-degree relatives of patients with type 2 diabetes. Diabetes 61: 3322-3332, 2012. 


\section{Rader EP, Naimo MA, Layner KN, Triscuit AM, Chetlin RD, Ensey J, and Baker BA.}

Enhancement of skeletal muscle in aged rats following high-intensity stretch-shortening contraction training. Rejuvenation Res 20: 93-102, 2017.

12. Seals DR, Justice JN, and LaRocca TJ. Physiological geroscience: targeting function to increase healthspan and achieve optimal longevity. J Physiol 594: 2001-2024, 2016.

13. Seals DR, and Melov S. Translational geroscience: emphasizing function to achieve optimal longevity. Aging (Albany NY) 6: 718-730, 2014. 


\section{CHAPTER II}

\section{REVIEW OF LITERATURE}

\section{Aging, Sarcopenia, and Resistance Exercise Training as a Therapeutic Aid}

Overall, aging is definitively one of the most concerning issues facing the world, particularly in the developed nations. These concerns are notably urgent in today's society due to the fact that the world's population is aging; by 2050, at least $25 \%$ of the population will be over the age of 65 , and that number could be as high as 40\% in certain places (103). Moreover, the global population above the ages of 65, 85 and 100 years is predicted to increase by 188, 551, and 1004\%, respectively (73). Additionally, it is now estimated that $20 \%$ of the current U.S. labor force is comprised of workers over the age of 55, and as early as 2020 that number could be as high as $25 \%(1,5)$. In the United States alone, the number of adults aged 65 and over is predicted to double during this period, and the number of people 80 years of age and older will increase by at least three fold $(81,103)$. Moreover, Europe already has more individuals over the age of 60 in comparison to those under 15, and this is expected to happen in North America by 2030 (54). Not only are people living longer, but these same individuals have different expectations about aging than previous generations, creating a much higher demand for not only extending lifespan, but an increased desire to live with optimal longevity, which may be regarded as healthspan (104). Thus, it is imperative to investigate and evaluate evidence-based interventions that have immediate translational impact to help counteract and/or attenuate the negative effects of aging across all demographics.

Aging is a multifactorial process that can be influenced by a variety of factors, such as genetics, nutrition, hormones, neurodegeneration, and lifestyle influences (4, 39, 130). Even though aging in itself is not considered a disease (91), concurrent with the aging process is an increased susceptibility to various acute and chronic disease states, which is associated with the progressive decline in biomechanical and physiological function that potentially leads to dysfunction, disability, loss of independence, and increased susceptibility to death (57). With advanced age, there are typically reductions in physical activity, specifically load-dependent movements, that contribute to declines in motor function and the metabolic quality of skeletal muscle, as well as loss of bone and muscle mass $(39,125)$.

One of the tissues in the body most affected by aging is skeletal muscle, which comprises more than $40 \%$ of body mass $(4,73)$. Skeletal muscle is critical for locomotion, the storage of nutrients, and in the regulation of metabolism (73). Additionally, skeletal muscle serves as an endocrine organ by secreting growth factors and myokines upon stimulation by contractile activity (10). A principal issue associated with aging is sarcopenia, which traditionally has been defined as the age-related loss of skeletal muscle mass (39). Sarcopenia 
is associated with an increased risk of adverse outcomes such as disability, frailty, cachexia, metabolic syndromes, poor quality of life and premature death $(73,111,128)$. During the aging process, there is evidence demonstrating age-related reductions in muscle mass and function (39). For instance, MRI imaging indicates age-related declines in skeletal muscle size starting around the age of 25 that continues to progress thereafter (69). Aging, and sarcopenia in particular, is associated with significant healthcare and socio-economic costs (10, $17,54,79,104,109)$. However, in more recent years, it has become increasingly recognized through scientific evidence that the characterization of sarcopenia extends beyond the loss of muscle mass.

Of note, the European Working Group on Sarcopenia in Older People (EWGSOP) extended the definition of sarcopenia as having the presence of both low muscle mass and low muscle function in the form of either strength or physical performance (32). More recently, the EWGSOP came out with an updated definition characterizing sarcopenia indicating that a loss or decline of skeletal muscle strength is the most important feature of the definition because strength is a more accurate predictor of adverse outcomes than muscle mass and is also the most reliable measure of the functional capabilities of the tissue (33). The authors further characterized sarcopenia by declaring the diagnosis could be confirmed by either low muscle mass or low muscle quality (i.e., muscle strength or power per unit of muscle mass) (MQ), and that decrements in physical performance in addition to low muscle strength and mass/quality should be further categorized as a severe form of sarcopenia (33). In particular, MQ has been demonstrated by our laboratory, as well as others, to be a particularly sensitive indicator of age-related negative outcomes, and arguably more functionally important than the actual amount of muscle mass for describing alterations in muscle performance as a consequence of sarcopenia (7, 43, 72, 89).

Recently, age-related physical changes have been suggested to be modifiable by increased physical activity (31). Furthermore, regular physical activity, more so than any other lifestyle or behavioral strategy (e.g., pharmacological interventions, diet, ergogenic aids, etc.), has the most convincing evidence for the preservation of function with aging (103). In fact, several pharmacological agents are currently undergoing testing and development that were designed in a way to attempt to simulate the positive effects of physical exercise on molecular signaling pathways involved in the physiological response to exercise. However, this approach has several potential concerns, such as possibly having negative interactions with multiple organ systems simultaneously $(10,103)$. Additionally, several reports have demonstrated the benefits of regularly-structured physical exercise as an effective, therapeutic approach to mitigate sarcopenic outcomes (e.g., loss of function, loss of muscle quality, etc.) with aging $(13,34,71,86,89,119,127,128,130)$. Specifically, evidence indicates that chronic high-intensity mechanical loading (i.e., resistance-type exercise training) has been shown to be the most highly effective tool in order to attenuate the effects of sarcopenia $(13,50,60,89,128)$, thus making it a potentially effective form of "physiotherapy" to counteract age-related decrements in muscle structure and 
function (23). This is due to its proven effects in preventing the losses in muscle performance, muscle mass, and motor units commonly associated with general aging and/or age-related inactivity via improvements in muscle capillarization and cardiorespiratory fitness, positive effects on lipid profiles, and its ability to maximize motor unit recruitment; and, subsequently increase the number and specific type of muscle fibers capable of being activated during training (i.e., type II fibers) (44, 50, 59, 85, 86, 101, 124). Additionally, previous work in our laboratory as well as others has provided supporting evidence that older individuals have the ability to safely tolerate high-intensity physical exercise without limitations and in the absence of any apparent physiological, histological, or cytological indices of damage or injury $(7,24,29,34,50)$.

Previously, studies have shown that one of the defining characteristics of exercise is its ability to initiate a complex assortment of highly coordinated molecular and cellular signaling events and networks, as well as transcriptional regulators, which has a profound impact on almost every single human organ system and tissue in the body (10). Upon exposure to an exercise bout, skeletal muscle responds in a coordinated fashion through initiating various signaling events that drive changes in gene expression of intracellular processes, such as metabolism, alterations in hormone signaling, defense mechanisms against oxidative stress, DNA damage and repair, protein turnover, and muscle remodeling $(20,82,90)$. In addition, repeated exposures have been previously suggested to lead to a "hormesis" effect in skeletal muscle (47, 62). Given that the implementation of high-intensity mechanical loading may be the preeminent means for attenuating the negative consequences of age-associated sarcopenia $(7,10,41,95)$, and considering that habitual or chronic activity can result in an integrated adaptive response in skeletal muscle, it is plausible that changes in functional performance, physiology, and cellular morphology should be corroborated by a molecular signature that is continuously being modified to maintain and establish new levels of homeostasis following repetitive loading (84). Thus, day-today stimuli prompt changes in local as well as global gene and protein expression, which impacts micro- and macro-cellular organization and structure, ultimately resulting in an integrated physiological and functional adaptation/maladaptation in skeletal muscle and the surrounding soft tissue.

Interestingly, gene expression, protein expression, and cell signaling events that have been investigated with respect to muscle growth and atrophy have been suggested to be the result of distinct molecular signaling pathways and/or mechanisms, which have been the topics of extensive review (14, 61, 76, 83, 93, 110). Additionally, several months of resistance training has been suggested to alter the aging phenotype by modifying the transcriptome and molecular networks in humans $(75,82)$. In this regard, resistance exercise is known to upregulate the gene expression of various myosin heavy chains as well as the myogenic regulatory factors, which included myogenic determination protein 1 (MYOD1), myogenin, myogenic factor 5 (MYF5), and myogenic factor 6, leading to increased protein synthesis and, ultimately, muscle hypertrophy (55). Thus, exercise variables that are prescribed in an appropriate manner for aged individuals could potentially be used in 
a therapeutic manner by improving the systemic environment and promote muscle growth via restoration of the remodeling capacity of skeletal muscle. Moreover, as mentioned previously, these myogenic events are occurring independent of the putative muscle injury response, since our laboratory $(7,34)$ as well as others $(24$, 29) have previously demonstrated that high-intensity physical exercise does not display evidence for any indices of damage or injury, along with a subsequent requisite inflammatory, degenerative, and regenerative processes. In an important recent review article by Miranda Grounds (48), she elegantly explains that evidence in the literature is indicative that various properties of new muscle formation (e.g., central nuclei, split myofibers, etc.) are not exclusive to regenerative events.

The age-related decline in muscle performance and muscle mass has in part been associated with detrimental effects in several key molecular and cellular processes at baseline (26, 27, 87). However, several investigators have found ways to modulate and/or restore some of the dysregulated signaling events in old skeletal muscle and reverse the aging phenotype. For example, Conboy et al. (26) demonstrated that forcible activation of Notch signaling in aged skeletal muscle restored the regenerative potential in old mice. Yousef et al. (129) were able to demonstrate through pharmacological inhibition of the pathway in old mice that they could rejuvenate skeletal muscle myogenesis. Kovacheva et al. (65) showed that testosterone supplementation in a mouse model protected against age-related declines in muscle mass, satellite cell proliferation and differentiation. With this in mind, the following sections will outline and characterize previous work conducted by our laboratory using resistance-type exercise training (RTET) as a paradigm for investigating age-related differences in the responsivity of the muscle to adapt properly in response to this type of training.

\section{Adaptation versus Maladaptation with RTET}

Both the acute and chronic effects that result from RTET are dependent on the biomechanical loading signature (i.e., intensity, duration/duty cycle, frequency, number of repetitions, velocity, and range of motion) (6). The responses to RTET can either result in adaptation, characterized by enhanced skeletal muscle performance along with increased muscle mass, to maladaptation, defined as an absence or diminishment of skeletal muscle performance and no improvements in muscle mass (34). Exercise training involving the use of stretch-shortening contractions (SSCs), is a common type of contraction inherent in RTET in which the muscle is activated prior to and during the initial stretch and subsequent shortening, which is in contrast to isolated isometric, concentric, or eccentric only movements $(88,89)$; SSCs have been particularly valuable in elucidating the benefits of exercise training. For nearly two decades, our laboratory has utilized an in vivo rodent dynamometer to investigate the underlying mechanics of muscle adaptation to RTET using SSCs (7, 35). This methodology allows for precise control of the various components of the exercise prescription and is minimally invasive, meaning it does not compromise the physiological response to training; moreover, it allows 
for examination of the adaptive responses of muscle following in vivo mechanical loading (6). Since highintensity RTET has been demonstrated to have the greatest potential for producing gains in muscle hypertrophy and performance due to the maximization of motor unit recruitment and the subsequent number of muscle fibers that are capable of being activated during training (101), the goal of using our in vivo animal model was to establish a model capable of testing the most "extreme" form of this type of training in order to examine the capabilities of young and old muscle to respond to true high-intensity exercise.

Utilizing a non-injurious paradigm of SSCs, in which young and old rodents were exposed to a protocol consisting of maximal intensity training for eight sets of ten moderate-velocity SSCs (80 total repetitions), three times per week for a duration of 4.5 weeks, previous data from our laboratory supports a divergence in the response of skeletal muscle to RTET in young versus aged rodents $(6,7,9,34,86,89)$. Specifically, young rats (3 months old) responded with a training-induced adaptation in the tibialis anterior (TA) muscle as reflected by a roughly 15-20\% increase in muscle wet-weight and approximately $20 \%$ increase in isometric and dynamic performance (34). This is in line with other studies showing the beneficial effects of higher frequencies on muscle adaptation in younger individuals; for instance, McLester and colleagues (74) compared the effects of training one and three days per week with equal volumes of RET in experienced subjects; each subject trained using a variety of upper and lower body exercises for 12 weeks. In the first group, three sets per exercise were performed in one day during the week; in the second group, subjects did one set on three separate days. The results showed that individuals who trained for one day during the week produced only about $62 \%$ of the strength gains observed in the individuals who trained for three days during the week.

However, the adaptive response is altered with age; specifically, several studies done in our laboratory $(6,8,9,34,86,89)$ have demonstrated that aged rodents (30 months old) undergo a maladaptive response when they are exposed to a general, chronic loading protocol specifically designed to induce adaptation in their younger counterparts, as reflected by no changes in muscle mass and an approximately 20-30\% decrease in muscle performance via static and dynamic force output. Moreover, histological analysis using hematoxylin \& eosin staining showed little to no signs of cell swelling, the presence of cellular infiltrates, or degenerative fibers in both the young and old rats. These results indicate that younger muscle can be exposed to highintensity SSC training at higher frequencies in a safe and effective manner that promotes muscle adaptation and performance gains. Meanwhile, in aged muscle, the lack of degenerative fibers and swelling suggests that the maladaptive response observed in aged muscle is not due to an injury response, and that they are still capable of being trained at higher intensities without compromising the muscle fibers. However, the caveat is that titration of the exercise prescription by potentially reducing the frequency of exercise may be necessary to allow old muscle to adapt properly. Moreover, with respect to advancing age, a more recent study from our laboratory (86) indicated that there is an altered phenotype that occurs prior to the complete biological development of 
rodents - six months of age in the adult Fisher 344 x Brown Norway (F344xBN) rat, which is highlighted by impairments in the adaptive capacity due to an attenuation of performance enhancement along with decreased muscle quality following one month of high-intensity RTET. Therefore, this study along with other recent literature $(28,92)$ suggests that age-related declines may potentially occur much sooner than what would be expected, potentially before any overt symptoms of the onset of chronic disease, which indicates that testing intermediate age groups is critical $(86,106)$.

As mentioned in the previous section, age-related declines in muscle performance and mass has been partly associated with the negative effects in several key molecular and cellular processes at baseline (26, 27, 87); however, it is clear from our previous work that this also true following inappropriately prescribed mechanical loading $(87,89)$. One potential reason for an impairment in the ability of aged muscle to respond appropriately to this general, adaptive SSC training protocol may be due to higher baseline levels of oxidative stress and inflammation within the muscle. Specifically, a recent study by Rader et al. (87) showed that in old rats, pathways related to oxidative stress, such as the growth arrest and DNA damage (GADD45) signaling, were high at baseline and remained unaltered following an SSC-injury protocol, whereas young rats had a heightened GADD45 response immediately following the injury exposure out to ten days, before decreasing back to lower levels as seen prior to the injury stimulus (87). Specific to an RTET stimulus, a couple studies by Ryan et al. $(97,98)$ demonstrated that old rats have increased levels of hydrogen peroxide and other markers of oxidative stress in the absence of a heightened antioxidant response. Collectively, aged muscles have higher levels of oxidants, oxidative stress, and oxidative damage following SSC training compared to their younger counterparts $(97,98)$. However, old rats that were supplemented with vitamins $E$ and $C$ had an attenuation of SSC-induced oxidative stress and significantly increased positive work following one month of training (98).

More recently, our laboratory examined whether or not altering other variables of the biomechanical loading signature (i.e., contraction mode and repetition number) could induce a similar adaptive response in aged muscle by training rats either at four sets of one maximally activating isometric contraction or dynamic training at four sets of 10 repetitions (40 total SSCs); both groups trained three days per week for one month (88). Young rats had gains in performance and increased muscle mass regardless of the training protocol examined. On the other hand, there were no changes in adaptive outcomes in old rats exposed to four maximal isometric contractions three days per week. Old rats exposed to 40 SSCs three days per week responded by having gains in muscle mass and decreased protein expression of tumor necrosis factor alpha (TNF- $\alpha$ ) levels; however, muscle quality was unaltered, which was divergent from the results seen when modifying the training frequency. Overall, the conclusions from this study were that high-intensity RTET at a moderate repetition number is limited in its ability to improve muscle quality, and demonstrated that the responsiveness of muscle to adapt is sensitive to various component(s) of the exercise prescription that are being modified (88). 
Collectively, these studies are suggestive that the adaptive capacity of skeletal muscle in response to high-intensity SSC RTET is age-specific and dependent upon specific variables of the exercise prescription, which has the capability of affecting the organism on a systems level (86, 89). Despite previous findings supporting the notion that RTET can reduce the negative effects of aging through improvements in strength, mass, neuromuscular function, vascular function, and metabolic function (56, 71, 72, 86), it is still unclear why aged muscle cannot adapt to the same loading paradigm as young muscle. However, there is evidence to suggest that although aged muscle has an impaired remodeling capacity, altering variables of the biomechanical loading signature (e.g., intensity, frequency) can improve the adaptive capacity of the muscle and attenuate the negative effects of sarcopenia (3); the following section will specifically address the potential role of a reduced frequency of training as a modulator of adaptation in aged skeletal muscle.

\section{Reduced Frequency and Muscle Adaptation with Advanced Age}

As a result of the findings pertaining to maladaptation in old rodents exposed to high-intensity SSC RTET three days per week, our laboratory examined the effects of reducing the training frequency as a means for positively influencing the adaptive response in aged muscle. In this study, young and old rats were exposed to the exact same 80 repetition SSC RTET program done previously, but at a reduced training frequency of two days per week (89). Our data clearly showed that the frequency of training is an important factor comprising the SSC exposure that results in muscle adaptation (Figure 1). Specifically, young rats presented the ability to adapt to multiple SSC paradigms by having comparable adaptation at both frequencies. However, in old rats that were trained three days per week, maladaptation occurred in which muscle mass was unchanged and peak force decreased following one month of training; this was the same response that had been shown in previous results $(9,34)$. Additionally, oxidative stress, as indicated by lipid peroxidation levels, remained high, whereas muscle quality was low. However, rats trained at a reduced frequency of two days per week resulted in muscle adaptation at old age; decreasing the frequency of training led to gains in muscle performance and muscle mass; importantly, MQ was revitalized to values similar to younger rodents. Moreover, in contrast to old rats that trained three days per week, there was a significant decrease in lipid peroxidation levels only in old rats trained two days per week, with levels that were indistinguishable from young rats. 

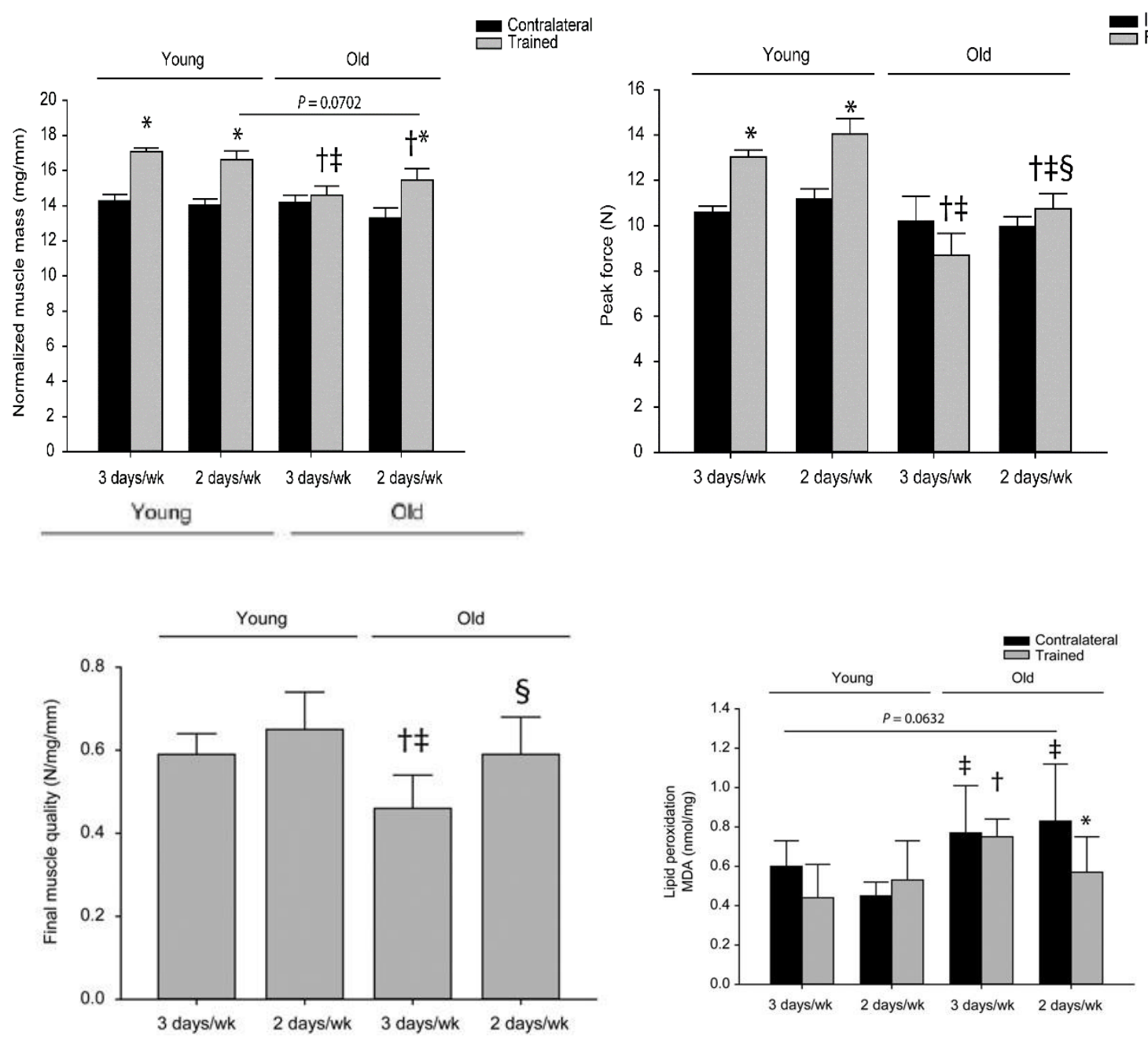

Figure 1. Normalized muscle mass (top left graph), peak force (top right graph), muscle quality (bottom left graph) and lipid peroxidation levels (bottom right graph) for young and old rats exposed to two different resistance-type training protocols. Protocols highlighted in green represent an optimal adaptive response and are age-specific. Values are mean \pm SD. *Different from contralateral (non-exposed) value (left graph) or different from initial value (right graph); †Different from value for young rats exposed three days per week; $\ddagger$ Different from value for young rats exposed two days per week; $\S$ Different from value for old rats exposed three days per week, $\mathrm{p}<0.05$. Adapted from Rader et al. (89).

Therefore, the findings of this study demonstrated that a reduced training frequency results in muscle adaptation with old age, which indicates that providing additional recovery time between exercise bouts allowed for a more favorable redox environment of the muscle, and subsequently provides an environment that promotes muscle adaptation $(85,89)$. Additionally, and perhaps most importantly, the results shown in this study by our group are supportive of other research findings demonstrating the restoration of functional, physiological, and molecular outcomes in aged human skeletal muscle in response to adaptive RTET. For instance, Melov et al. (75) found that following six months of training in humans there was an enhancement in the transcriptome profile of genes associated with mitochondrial function, thus providing evidence that resistance exercise can partially reverse mitochondrial impairment seen in older adults. Stec and colleagues (114) demonstrated that 
resistance training augmented rRNA production, enhanced ribosomal biogenesis, and led to muscle hypertrophy in older adults.

Our results are also in agreement with other studies in humans looking at the effects of a reduced frequency of exercise on muscle adaptation in older adults. For example, a meta-analysis by Borde et al. (15) using data from 25 studies found that a training frequency of two days per week at a relatively high training intensity of 70-79\% of one-repetition maximum (1RM) is most optimal for increasing muscle strength in healthy old adults. Moreover, our results indicating positive effects for two day per week high-intensity RTET in older rodent maximally-activate skeletal muscle are in line with a recent paper by Stec et al. (115), who found that two days per week high-intensity training (denoted "HH” in their study) was beneficial for increasing muscle strength and performance compared to three day (analogous to old rats that trained three days per week in our previous research) or one day per week high-intensity training. It should be noted the authors found that one additional day of low-intensity training interspersed between two days of high-intensity training may elicit further benefits that may potentially maximize training benefits; this conclusion was based on the significantly greater thigh muscle mass compared to the other interventions, including the two day per week high-intensity only training. This would indicate that there are potential additional adaptive benefits when incorporating an extra day of low intensity exercise to a training program that is absent with only two days of high intensity training represented in our current study. However, the authors from Stec et al. stated in the discussion that “... the benefits of the $\mathrm{HH}$ prescription could not be overlooked, as meaningful gains in muscle performance were noted, and 60\% responded with TMM hypertrophy. It could be argued that - for many older adults - HH offers a reasonable balance of efficacy and practicality, requiring only $2 \mathrm{~d} /$ week.” Along with several other studies that have shown successful adaptive outcomes in muscle with similar two day per week high-intensity RTET programs (50-52, 60, 75, 123), we believe the findings from our study are very complementary and support previous findings of the benefits of an “optimized” frequency of training in older adults.

Furthermore, our findings in old rats that trained at a reduced frequency can be used as fundamental/basic science evidence that supports recent literature in humans with both resistance training (42) as well as cardiorespiratory exercise $(45,46,118)$ of the benefits of a "minimal-dose approach" for exercise in older adults. Specifically, Fisher et al. (42) explained eloquently in their mini-review that a minimal dose approach of two day/week exercise for less than $60 \mathrm{~min}$, on par with our prescription, has many physiological and psychological benefits in older adults based on their examination of past studies detailed in their review. In fact, the authors pointed out that the minimal-dose approach is based on the premise that the subjects in these studies achieved these benefits only at moderate to high intensities (50-90\% 1RM). The physiological benefits, of which it should be noted are found similarly in studies using three day per week training as well, included outcomes such as improvements in muscle mass, muscle strength, functional capacity, reduced lower back pain 
and lower blood pressure. There are additional psychological benefits to a lower frequency of training that fall into the "minimal-dose approach" that the authors discussed. First, the reduced time to exercise is appealing with this type of approach because lack of time is often cited as arguably the most common reason people do not adhere to an exercise program $(120,126)$. Secondly, previous research has suggested that too much exercise can result in overreaching or overtraining, which not only has physiological effects (e.g., decreased immune function, increased catabolic hormone production, etc.) but also negative psychological disturbances on mood and self-esteem that are even more sensitive in older individuals (53); thus, the authors encourage training that avoids reaching this state. The next section will discuss epigenetic/epigenomic regulation, particularly DNA methylation, and its influences on aging as well as the adaptations to exercise, both acute and chronic.

\section{The Interaction between Epigenetics/Epigenomics, Aging, and Exercise}

One of the most defining characteristics of skeletal muscle is its remarkable plasticity to various forms of stimuli. Skeletal muscle is dynamically regulated by changes in physical demands and environmental challenges (19). Epigenetics, which literally means “above genetics,” is defined as changes in gene expression due to structural modifications, which are potentially reversible, occur as a result of external or environmental factors, and transpire independently of alterations to the actual genetic code itself (2, 22, 90). Each cell type in the body owns some type of distinctive epigenetic regulatory network, which is defined as the epigenome (90). Epigenomic processes are delineated as reversible marks that modulate chromatin structure, and comprise a macromolecular structure that contains unmodified as well as chemically modified nucleic acids and amino acids found in the nucleus (18).

Double-helix strands of DNA are packaged tightly inside the nucleus of each cell using proteins called histones; these proteins act as little spools around which segments of DNA are coiled, forming nucleosomes (i.e., "beads-on-a-string”). Threads of nucleosomes are then woven in to a ropelike structure referred to as chromatin. Collectively, access to genes by transcription factors, RNA polymerase, etc. are controlled mainly through DNA methylation and histone modifications on chromatin. Given the various mechanisms implicated in the epigenome of cells, DNA methylation, described as the attachment of a methyl group to the 5' position of a cytosine within DNA, is considered to be the most robust form of epigenomic regulation (25, 38). When methyl groups are located within the promoter region of genes, this typically leads to transcriptional repression due to the mobilization of methyl cytosine-guanine base pairing (CpG) binding domain proteins, which in turn recruit repressor complexes. These repressor complexes contain histone deacetylases, which further contributes to chromatin remodeling and alters gene expression by keeping the DNA in a closed state (i.e., heterochromatic), thereby acting as a blockade to transcription factors and RNA polymerase which leaves them unable to access the DNA $(21,66,90)$. Although there are a few exceptions, DNA methylation occurring at the promoter or 
enhancer regions of DNA almost always results in a suppression of gene expression. Thus, while excluding other variables, the higher the level of methylation, the lower the amount of gene expression.

Aging in and of itself is a biological process associated with an altered phenotype, and emerging evidence suggests that these changes are possibly linked to epigenetic/epigenomic changes (117). In fact, evidence indicates that the epigenome is continuously maturating with time over the course of a person's lifespan and that the changes that occur with aging appear progressively over time as a result of their interaction with the environment (117). Although other factors also account for age-associated changes in gene expression, a large proportion of these alterations may be linked to epigenetic/epigenomic mechanisms. Thus, epigenetic/epigenomic modifications of gene expression due to genetic and environmental factors results in agedependent patterns of gene expression. In post-mitotic tissues such as skeletal muscle, evidence seems to indicate that DNA methylation, in particular, is actively occurring as a result of an individual's interaction with their environment $(38,66,90,117)$. Additionally, histone modification by DNA methylation can lead to chromatin undergoing nuclear compartmentalization, possibly effecting the structure and functional features of myonuclei (12). The next section will look more in depth at the epigenomic process of DNA methylation in skeletal muscle and how exercise can influence its effects.

\section{Effects of DNA Methylation and Exercise}

Excitingly, recent research has shown that exposure to exercise can influence changes in DNA methylation in skeletal muscle. For instance, acute exposures to varying intensities of aerobic exercise have resulted in altered methylation as well as expression levels of genes related to muscle metabolism, mitochondrial biogenesis, and muscle growth in both human and rodent skeletal muscle tissue $(11,63,64,67)$. For instance, King-Himmelreich et al. (64) found that methylation of both gene and protein expression of AMPactivated protein kinase (AMPK) $\alpha 2$ was reduced in the skeletal muscle of mice following one hour of treadmill exercise. Barrès et al. (11) performed a global CpG methylation analysis from human skeletal muscle biopsies at baseline and 20 minutes after acute endurance exercise and found that overall methylation significantly decreased following the acute exercise bout. Additionally, the authors did a promoter-specific analysis of methylation and found significantly decreased methylation at the promoters of the metabolic genes peroxisome proliferator-activated receptor gamma, coactivator $1 \alpha$ (PGC-1 $\alpha)$, transcription factor A, mitochondrial (TFAM), peroxisome proliferator-activated receptor $\delta$ (PPAR- $\delta$ ), pyruvate dehydrogenase kinase 4 (PDK4) and citrate synthase following acute endurance exercise. These results mostly held true in a high-intensity bout of 80\% aerobic capacity in a separate cohort of eight young healthy sedentary men, in which PGC-1 $\alpha$, TFAM, and PDK4 had significantly decreased methylation following the acute exposure; PPAR- $\delta$ was not significantly demethylated immediately following the exercise but methylation was decreased three hours after. PGC-1 $\alpha$, 
PDK4, and PPAR- $\delta$ methylation was similarly decreased in mouse skeletal muscle 45 minutes following ex vivo muscular contractions. In another acute study, Lane and colleagues (67) utilized skeletal muscle biopsies of highly trained cyclists and found changes in DNA methylation in skeletal muscle at the promotor of cytochrome c oxidase subunit IV isoform 1 , fatty acid binding protein 3 , and PPAR- $\delta$ two hours following a high-intensity exercise exposure. Finally, Pattamaprapanont et al. (80) performed an electrical pulse stimulation protocol on mouse skeletal muscle C2C12 cells and found rapid demethylation at the promoter of the nuclear receptor subfamily 4 group A member 3 (NR4A3) 60 minutes after stimulation. Prior to the electrical stimulation, the authors had shown NR4A3 to be robustly upregulated (26-fold increase) following acute endurance exercise in skeletal muscle from young healthy adults.

In regards to chronic exercise training, while not explored as in-depth as with acute bouts of exercise, past findings from others suggest that changes in methylation are associated with alterations in expression of genes related to muscle adaptation $(66,78)$. Nitert et al. (78) sought to look at the impact of a six month aerobic exercise intervention on DNA methylation in human skeletal muscle in males with or without a past family history of type 2 diabetes mellitus. What they found was regardless of past family history, six months of mainly endurance exercise resulted in significant reductions in promoter DNA methylation of genes with known functions in both skeletal muscle and type 2 diabetes mellitus, including thyroid adenoma associated (THADA), myocyte enhancer factor 2A (MEF2A), runt related transcription factor 1 (RUNX1), and NADH dehydrogenase [ubiquinone] 1 subunit C2 (NDUFC2). In addition, the gene expression levels of THADA, MEF2A, RUNX1, and NDUFC2 were all negatively correlated with higher amounts of DNA methylation.

Kanzleiter and colleagues (63) had nine-week old male mice perform five days of endurance treadmill exercise training for four weeks. An initial bisulfite sequencing analysis was performed and it was found that compared to sedentary controls, 2,762 genes displayed differentially methylated CpGs in their promoter regions. Further analysis of gene expression data revealed that about 200 out of the 2,762 genes had negative correlations between CpG methylation status and gene expression; most of these genes were related to muscle growth, differentiation, and metabolism. Amongst the genes that were further highlighted by showing significantly reduced levels of methylation and higher gene expression in exercised individuals compared to no effects in sedentary counterparts included: the muscle growth/differentiation factors myogenin plexin A2 and insulin-like growth factor-binding protein-4; the muscle innervation factor docking protein-7; the angiogenesis factor CDP-diacylglycerol synthase; the glucose metabolism factor glutamine fructose-6-phosphate transaminase-2; the mitochondrial lipid metabolism factors START domain containing 10 and sodiumdependent phosphate transporter 1; and calcium-binding protein-39, which is associated with both glucose and mitochondrial lipid metabolism via its involvement in the activation of the AMPK pathway (63). 
Nguyen et al. (77) used a mouse model of severe dyslipidemic mice that lacked low-density lipoprotein receptor and had them go through three months of endurance exercise using a voluntary wheel; they found that one of the most important antioxidant enzymes, glutathione peroxidase 1, had significant alterations in gene expression that was accompanied by significant decreases in DNA methylation following the training protocol. Finally, Lindholm and colleagues (70) conducted a three month one-legged exercise training study in young adult humans; in this study, the non-exercise leg served as an inter-individual control. Training consisted of one-legged knee extension endurance training on a cycle four times per week; two unilateral knee extension performance tests were implemented on a modified cycle prior to and following the three month training period. Skeletal muscle biopsies were taken at rest, before and after training in both legs. The authors explained that their main findings were endurance training induced significant changes in methylation (primarily at enhancer regions) at approximately 5,000 sites across the genome, along with significant differential expression of roughly 4,000 genes. Genes with increased levels of methylation were mainly associated with structural remodeling of muscle and glucose metabolism, whereas genes with decreased methylation were mainly associated with inflammatory processes and transcriptional regulation. Genes related to muscle biology with increased methylation included myogenic regulatory factors and myocyte enhancer factors. Collectively, the authors suggested their findings demonstrated that exercise leads to epigenetic/epigenomic modifications in the form of DNA methylation and these changes are associated with changes in gene expression as well as performance (70). With these findings in mind, the next section will focus on DNA methylation as an underlying basis for the existence of a cellular form of muscle memory.

\section{Muscle Memory and DNA Methylation as a Cellular Basis for its Existence}

In general, the term muscle memory has been applied in the strength and conditioning, sport, and exercise domains as a general term describing the capability of skeletal muscle to respond more quickly to an applied stimulus that someone has been exposed to previously; hence, making skeletal muscle more responsive and enhancing its ability to rebuild mass and strength in spite of periods of inactivity $(49,106)$. There is scientific evidence supporting the existence of this phenomenon in the human performance literature. For example, Staron et al. (112) took a subset of six women who had participated in a previous 20 week resistance study in which they trained two days per week (113), and had them go through a 30-32 week detraining phase, then subsequently retrained them for six weeks. Their results indicated a significant retention of strength and muscle cross sectional area (CSA) during retraining following the long detraining phase; specifically, retraining for six weeks resulted in significant increases in the CSAs of type IIa and IIab/IIb fibers compared with detrained values along with a rapid return of strength values to their previously trained state. Thus, the authors concluded that the results demonstrated a significant retention of strength and muscle CSA following long 
periods of inactivity, thus supporting previous empirical observations that previously trained individuals who detrain are capable of returning to form quickly (112).

More recently, a well-designed study performed by Taffe and colleagues (116) was conducted where 13 men and women (healthy and no resistance training done within the past year) performed 24 weeks of resistance training, followed by 24 weeks of detraining, and then subsequently 12 weeks of retraining. Training was done two days per week using various upper and lower body exercises, including the chest press and leg press. Significant increases in bone-free lean mass was found during the initial 24 week training period before dropping back to levels near baseline during the 24 week detraining period. However, the 12 week retraining period resulted in a significant increase in bone-free lean mass levels close to what was seen during the initial 24 week training period. Similar results were seen in 1RM muscle strength measures for all six exercises (chest press, seated row, biceps curl, leg press, leg extension, and leg curl). In addition, the authors found significant alterations in muscle attenuation as measured in Hounsfield units, a noninvasive measure of muscle density that correlates with intramuscular lipid content (i.e., lower values reflect higher intramuscular lipid content) for the quadriceps and hamstring muscles following detraining and subsequent retraining (116). Specifically, 12 weeks of retraining resulted in significantly higher quadriceps and hamstring Hounsfield units compared to detraining, and was similar to values seen following the initial 24 week training period. Collectively, the results of this study demonstrated that resuming resistance training following a detraining period attenuated intramuscular fat content, improved muscle mass, and led to increased strength in older healthy adults. A very recent study by Sakugawa et al. (100) found similar results in elderly men and women who completed 12 weeks of training, 16 weeks of detraining, and then 8 weeks of retraining; these individuals were able to recover maximum strength gains, rate of torque development, and functional capacity to levels similar to after the initial training period and before the detraining phase. Finally, Henwood and Taffe (58) also demonstrated that older adults were able to recover muscle power and strength during a 12 week retraining period that was preceded by a 24 week training and 24 week detraining period.

Historically, the term muscle memory has almost exclusively been applied in the context of motor learning and neurological adaptations to training $(37,96)$. In a very elegant, recently published review paper by Sharples et al. (106), the authors promoted the idea of a "skeletal muscle memory" in the context of epigenetic regulation by defining it as "the capacity of skeletal muscle to respond differently to environmental stimuli in an adaptive or maladaptive manner if the stimuli have been previously encountered.” In other words, this term refers to the ability of the tissue to retain prior environmental stimuli, such as adaptation to exercise training, so that there is a change in the responsiveness of the muscle when this same stimulus is reintroduced at a later time period. Under these circumstances, the muscle tissue could have the potential to retain cellular/molecular information after encountering an environmental stimulus in a manner that "primes" the muscle upon which 
when it is re-exposed to the same stimuli it can bring about subsequent adaptation or maladaptation; this can be described as a "muscle epi-memory" (106). Thus, if the environment of the muscle is anabolic, causing growth synthesis pathways to respond in a manner that leads to positive alterations in epigenetic/epigenomic

modifications, the muscle will retain these modifications and affect the control of gene activity in a manner that will allow the muscle to be more likely to respond to this same stimulus at later life by maintaining or perhaps even increasing muscle mass across the lifespan of the organism (106).

It is known that different cell types have unique functions despite containing the same DNA, and that these unique functions are attributed to the proteins expressed in each cell type, which is determined early on in development (38). The relatively permanent protein production patterns of cells throughout an organism’s lifespan is the underlying basis for a general cellular memory (38). Cellular memory has been suggested to be regulated by epigenetic/epigenomic mechanisms that allows for diverse physiological roles in different cell types without changing the actual DNA itself $(38,90)$. Intriguingly, previous evidence $(40,90)$ suggests that DNA methylation, specifically, is the driving force behind an intrinsic cellular memory through the regulation of global patterns of gene expression that controls cellular function (38). Moreover, the fact that methylation is known to be a highly dynamic process that is reversible and occurs quickly in response to environmental signals (90) lends support to this concept as well.

As previously mentioned, Sharples et al. (106) have defined muscle memory as the responsiveness of muscle to various environmental stimuli and results in either adaptation or maladaptation, provided that the organism has been previously exposed to the stimulus. Thus, since environmental stimuli and stressors lead to modifications in gene expression, epigenetics/epigenomics are highly likely to form the underlying foundation for skeletal muscle memory $(90,106)$. Previous research has shown that myocytes exposed to early-life TNF- $\alpha$ induced acute inflammatory stress had an increased vulnerability to impaired differentiation when exposed to the same stress stimulus in later proliferative life; additionally, these cells retained elevated levels of DNA methylation for MYOD1 through multiple passages (105). Recent data by Sousa-Victor and colleagues (108) have shown that the loss of the ability of aging muscle satellite cells to maintain a normal quiescent state during homeostasis is caused by a loss of downregulation of $\mathrm{p} 16$, and that this is epigenetically regulated by the polycomb protein 1/H2A ubiquitination. These findings suggest that stable epigenetic/epigenomic mechanisms, such as DNA methylation and histone modifications, can be retained by muscle cells, which may affect them at the physiological level at later life (106).

Recently, evidence has emerged giving credence to the existence of a cellular form of muscle memory. This concept was first reported by Bruusgaard et al. (16), in which the researchers employed a synergistic ablation (SA) model and then used a live in vivo imaging technique in order to track and quantify myonuclei during an overload period followed by surgical denervation. Two weeks of overload with SA resulted in close 
to even gains in both myonuclei and muscle CSA (37\% vs. 35\%, respectively). However, when surgical denervation was introduced following the initial overload period, CSA significantly and rapidly declined by approximately $40 \%$ as a result; conversely, the total number of myonuclei held steady with a very slight, nonsignificant decrease in number despite the denervation period. Additionally, in a separate experiment male Wistar rats that were overloaded and compared to a control group not overloaded (i.e., no SA) before both groups underwent denervation, the results showed the group that had undergone a two week period of overload had a significantly higher number of nuclei $(\sim 30 \%)$ and muscle CSA ( 33\%) compared to the muscles of the control group with no prior exposure of overload. These results indicate that the addition of nuclei seems to be long lasting and persists even through a period where the muscle is denervated. The authors proposed a model on the relationship between myonuclei number and muscle hypertrophy in which increases in myonuclei number precedes muscle mass gains, with a subsequent detraining period leading to muscle atrophy but little to no loss of myonuclei; subsequently, a previous exposure to muscle hypertrophy (e.g., SA, mechanical overload, etc.), would bring about a more rapid gain in muscle mass to prior levels compared to those that did not have this previous experience, thus forming the underlying basis for a cellular muscle memory.

As a follow up to this study by Egner et al. (37), mice that were administered testosterone for 14 days showed robust increases in myonuclei as well as muscle mass. The authors were able to illustrate that not only were these nuclei retained following the removal of the drug despite the fact that muscle mass went back down to baseline levels, but that a subset of mice re-exposed to a physical loading stimulus exhibited significant increases in muscle mass relative to control mice. The authors concluded that although muscle hypertrophy can be reversed due to an atrophic stimulus (e.g., inactivity), a previous anabolic event left a lasting imprint on the muscle fiber in the form of increased myonuclei, and suggested that because myonuclei increase as a result of RTET, it may benefit younger individuals to perform this type of exercise modality in order to gain benefits prior to advanced age (37). However, in a very recent study by Dungan et al. (36), these researchers trained adult female mice for eight weeks using a progressive weighted wheel running model, and they found results in direct contrast to this previous study. Specifically, the authors demonstrated that the plantaris muscle was in an untrained state as reflected by decreased muscle mass; and, more importantly, the authors showed less myonuclei following detraining, which is in disagreement with previous studies mentioned (16, 37).As mentioned by the authors in their discussion, one of the main reasons for the conflict in findings could potentially be the model; these authors used a volitional, non-surgical, physiological model of exercise training, which is fully reversible due to the non-invasiveness as well as the avoidance of removing muscles or causing denervation compared to the SA model employed by the earlier studies. However, other differences exist as well between the two studies that confound the interpretation of the findings between the two groups of studies; Dungan et al. (36) used C57 black 6 mice ( $\geq$ four months of age prior to training) and examined the plantaris 
muscles, whereas Bruusgaard’s and Egner's groups $(16,37)$ used Naval Medical Research Institute mice and all their findings were reported using the EDL; it should also be noted that while bodyweights were similar between these different groups, the latter studies did not report the age of the mice. Most importantly, all of these studies did not incorporate a true detraining phase followed by a retraining period to simulate the previous work mentioned earlier done in human exercise training studies $(58,100,112,116)$, further limiting the translation of the results for application towards the concept of muscle memory within the context of exercise training; this will be the focus of the final section of this review.

\section{The Application of a Training-Retraining Paradigm towards Muscle Memory}

Research by the same group of investigators who wrote the initial review proposing the notion of a skeletal muscle memory (106) have started to unravel the basis of this within the context of a training-retraining (TRT) paradigm in the human literature. The first study to examine this research question was by Seaborne et al. (102), who attempted to characterize a TRT paradigm by means of a genome-wide methylation analysis in human skeletal muscle using eight previously untrained young male adults. For this study, participants completed an acute bout of resistance exercise, followed by seven weeks (three days per week) of RTET, then a subsequent seven week detraining period, and finally a seven week (three days per week) retraining phase. The main finding of this study was there were 18,816 hypomethylated CpG sites following the retraining period, which was higher than at any of the other time points measured (i.e., baseline, acute, and the initial loading phases). Of particular note, the results showed a significant enhancement of hypomethylation within the PI3KAKT pathway, a well-known regulator of muscle adaptation in response to RTET, when comparisons were made between baseline versus initial loading as well as the detrained versus retrained states. In addition, a group of almost 9,000 CpG sites that became hypomethylated following the initial training period maintained their reduced methylation through the detraining period when muscle mass had returned to baseline values; intriguingly, it was found that an even further enhancement of the hypomethylation at these same CpG sites occurred following the seven weeks of retraining. The authors were also able to demonstrate a relationship between decreased methylation and enhanced expression of a subset of genes at various parts of the acute loading, training, and retraining phases that have been previously implicated in the regulation of skeletal muscle. Overall, their findings provided the first scientific evidence for the existence of an epigenetic memory in skeletal muscle in response to exercise training.

As a follow up, Turner, Seaborne, and Sharples (122) performed a complex analysis using bioinformatics in order to examine whether or not large scale alterations in gene expression, due to alterations of DNA methylation in response to acute and chronic RTET, were present in the transcriptome. In order to do this, the researchers took publicly available transcriptome data from previous acute and chronic resistance 
exercise studies, combined them, and then directly compared with their previous results of genome-wide DNA methylation in human skeletal muscle following acute resistance exercise, as well as after the chronic RTET, detraining, and retraining paradigm mentioned earlier (102). They did their analysis using Venn diagram followed by subsequent bioinformatics using the Kyoto Encyclopedia of Genes and Genomes pathway analysis tool. Their analysis revealed 2,018 genes that were upregulated and 430 genes downregulated; nearly 600 out of the 2,018 genes that were upregulated were also found to have decreased methylation, while close to 100 out of the 430 genes were shown to have increased methylation following training. Collectively, through subsequent bioinformatics using KEGG pathway analysis, the combined methylation and transcriptome data implicated important roles of genes regulated epigenetically through DNA methylation with well-known processes involved in the adaptive response to RTET, such as extracellular matrix remodeling, mechanotransduction, and transforming growth factor-beta signaling.

Another recent and complementary study was conducted by Sailani et al. (99) that examined in older, healthy aged men the effects of lifelong physical activity (defined as having exercised on average three or more times per week throughout the majority of their lives) compared to sedentary controls on global DNA methylation status. The authors discovered a differential response in the DNA methylation profile in the lifelong physically active group in comparison to sedentary age-matched counterparts in which over 700 genes had significantly lower methylation at the promoter region; these included various genes involved in critical skeletal muscle regulatory processes such as glycogen metabolism, glycolysis, muscular contraction, myogenesis, and oxidative stress. Fascinatingly, these results complement on an epigenetic/epigenomic level previous research showing the beneficial effects of lifelong physical activity on morphological and physiological outcomes in aged skeletal muscle (30, 103, 104, 107, 121, 130).

Finally, there has been one study to date that has looked at the effects of a true TRT paradigm in an animal model of RTET. Lee and colleagues (68) used female Sprague-Dawley rats and had them perform a volitional weight-loaded ladder climbing exercise two times per week for eight weeks. Rats were randomly assigned to one of four different experimental groups: a non-exercised control group; a "training” group that performed RTET for eight weeks at 36-44 weeks of age; a "pre-training/detraining” group that trained for eight weeks at 8-16 weeks of age; and a "retraining" group that exercised at 8-16 weeks of age, and then again at 3644 weeks of age following a 20 week deloading phase. The results demonstrated a significantly higher number of myonuclei in all trained groups compared to the control, with no differences amongst the other three groups. However, retraining resulted in significantly higher relative muscle mass compared to the training only group. Additionally, it was found that retraining resulted in enhanced mitochondrial biogenesis as mitochondrial content and DNA copy number were significantly enhanced compared to all other groups; similar results were found for gene expression in the important mitochondrial genes PGC-1 $\alpha$, mitofusin-2, mitochondrial fission 1 , 
and dynamin-related protein 1. Following separate experiments using myonuclear-enriched myotubes, they concluded the beneficial effects seen in mitochondria were due to the increased number of myonuclei that resulted with training. Collectively, the authors inferred that an increased number of myonuclei from RTET is a potential underlying cause of enhanced muscle hypertrophy and mitochondrial biogenesis, and that exposing muscles to RTET at a younger age could potentially have benefits in improving the metabolic phenotype of skeletal muscle at later life (68).

\section{Conclusions and Purpose of the Dissertation}

In conclusion, while aged skeletal muscle has impairments in the capacity to respond favorably to generally-prescribed RTET, evidence from our laboratory as well as others provides clear evidence that the tissue still retains the capacity to undergo adaptation, both physiologically as well as in the molecular environment, as long as the stimulus is prescribed in a specific manner. While recognizing that it is not the sole component involved, there is a body of research that supports the notion a reduced frequency of exercise training is a highly influential factor that can be utilized in the titration of the exercise prescription in order to make aged muscle adapt properly to RTET. However, the integrated physiological pathways involved in exercise-induced muscle remodeling, and whether or not manipulating the frequency or other variables of exercise can be used to change the underlying signaling response of the muscle, particularly in a high-intensity SSC in vivo animal model of RTET, are not fully understood.

However, identifying the epigenetic/epigenomic mechanisms influencing the molecular signaling responses to SSC RTET are just as crucial in elucidating the determinants that promote age-specific adaptive or maladaptive outcomes. Epigenomic processes have a clearly defined role in the progression of aging; in particular, DNA methylation seems to be one of the chief mechanisms driving age-related changes both at baseline as well as in response to either acute or chronic exercise. Research has demonstrated that aerobic exercise and/or muscular contractions, both acute bouts and chronic training, can influence the epigenetic/epigenomic host environment through changes in DNA methylation levels across multiple tissues $(11,63,66,94)$. Therefore, if DNA methylation is the underlying basis for epigenetic/epigenomic regulation of cellular memory (38), then approaches aimed at reducing global levels of methylation in an attempt to restore or rejuvenate the developmental and/or differentiation potential of somatic cells should be beneficial for improving age-related negative health outcomes. However, studies examining how DNA methylation changes in response to SSC RTET intended to promote muscle adaptation, and how this response is altered with aging, is currently unknown. Intriguingly, these studies have suggested that not only is there a link between epigenetic/epigenomic regulation and gene expression, but that this may be a critical component impacting the age-specific ability of skeletal muscle to undergo adaptation in response to RTET. For this reason, further investigating the influences 
of DNA methylation upon the adaptive or maladaptive response of skeletal muscle in the context of aging is imperative for future research questions on how we can identify and possibly mitigate the onset of maladaptation so that aging can be positively impacted by the usage of SSC RTET as a form of physiotherapy for attenuating and/or preventing age-related sarcopenia.

Despite the therapeutic potential and supporting evidence for exercise, its application in positively influencing the total health of an individual across the lifespan is still not fully resolved. The term muscle memory has been one of the most commonly used terms discussed amongst wellness, therapists, and strength and conditioning practitioners, and refers to the capability of a person to more quickly or superiorly respond to a specific exercise stimulus that has previously been encountered in comparison to a naïve one (e.g., improvement in muscle mass, strength, etc.). The existence of muscle memory in research has been demonstrated nominally in the literature through findings that skeletal muscle responds differently when training ceases and then starts again (i.e., retraining), particularly with SSC RTET $(106,112,116)$. However, until recently, the underlying basis for muscle memory had largely been based on motor learning and the neurological adaptations to exercise training. Yet, there seems to be a growing body of evidence that points to a molecular/cellular basis for the concept of muscle memory.

Despite this collective knowledge and suggested underlying basis for the involvement of epigenetic/epigenomic regulation in the capability of skeletal muscle to adapt to exercise, no studies to date have determined whether or not alterations in DNA methylation that occur as a result of exposure to an adaptive or maladaptive exercise stimulus has a lasting influence on the epigenetic/epigenomic responsivity and adaptability of skeletal muscle upon reintroduction to the same stimulus at a later life. Based on the beneficial potential suggested by high-intensity SSC RTET for counteracting age-related declines in muscle mass and function, investigating the TRT paradigm could have important implications for furthering our understanding of muscle adaptation. Moreover, showing positive effects in later life from SSC RTET at an earlier age has enormous therapeutic ramifications in preventing and/or decreasing the susceptibility to diseases directly associated with aging, such as sarcopenia, as well as other age-related diseases (e.g., heart disease, diabetes, cancer, etc.), ultimately resulting in increased lifespan and healthspan (i.e., quality of life) in our increasingly growing aging population.

Therefore, the purpose of this dissertation was to use a valid and reliable in vivo rodent model of highintensity SSC RTET in order to fill in the gaps in our knowledge about the underlying molecular signaling pathways and epigenomic processes (i.e., DNA methylation) mediating aged-related skeletal muscle adaptation in response to both a reduced frequency training and a training-retraining exercise paradigm. The results from this study will be vital in better understanding the underlying performance, physiological, molecular, and environmental factors influencing the capability of aged skeletal muscle to undergo adaptation with training, 
which has important implications in the attenuation and/or reversal of the negative consequences of sarcopenia. Additionally, we will determine the therapeutic efficacy of a RTET TRT paradigm starting at an earlier relative age on the prevention of impairments of skeletal muscle during a later period of life. 


\section{REFERENCES}

1. In: Musculoskeletal Disorders and the Workplace: Low Back and Upper Extremities. Washington (DC): 2001.

2. Alabert C, and Groth A. Chromatin replication and epigenome maintenance. Nat Rev Mol Cell Biol 13: 153-167, 2012.

3. Alway SE. Inflammation and oxidative stress limit adaptation to stretch-shortening contractions in aging. Exerc Sport Sci Rev 45: 194, 2017.

4. Argiles JM, Busquets S, Stemmler B, and Lopez-Soriano FJ. Cachexia and sarcopenia: mechanisms and potential targets for intervention. Curr Opin Pharmacol 22: 100-106, 2015.

5. Baker BA. An old problem: aging and skeletal-muscle-strain injury. J Sport Rehabil 26: 180-188, 2017.

6. Baker BA, and Cutlip RG. The application of high-intensity muscular contractions for maximal training gains: the impact of age on the continuum of muscle injury, maladaptation and adaptation. In: Strength Training: Types and Principles, edited by Kai JT. New York: Nova Science Publishers, 2010, p. 119-148.

7. Baker BA, and Cutlip RG. Skeletal muscle injury versus adaptation with aging: novel insights on perplexing paradigms. Exerc Sport Sci Rev 38: 10-16, 2010.

8. Baker BA, Hollander MS, Kashon ML, and Cutlip RG. Effects of glutathione depletion and age on skeletal muscle performance and morphology following chronic stretch-shortening contraction exposure. Eur $J$ Appl Physiol 108: 619-630, 2010.

9. Baker BA, Hollander MS, Mercer RR, Kashon ML, and Cutlip RG. Adaptive stretch-shortening contractions: diminished regenerative capacity with aging. Appl Physiol Nutr Metab 33: 1181-1191, 2008.

10. Bamman MM, Cooper DM, Booth FW, Chin ER, Neufer PD, Trappe S, Lightfoot JT, Kraus WE, and Joyner MJ. Exercise biology and medicine: innovative research to improve global health. Mayo Clin Proc 89: 148-153, 2014.

11. Barres R, Yan J, Egan B, Treebak JT, Rasmussen M, Fritz T, Caidahl K, Krook A, O'Gorman DJ, and Zierath JR. Acute exercise remodels promoter methylation in human skeletal muscle. Cell Metab 15: 405-411, 2012. 
12. Bartova E, Krejci J, Harnicarova A, Galiova G, and Kozubek S. Histone modifications and nuclear architecture: a review. J Histochem Cytochem 56: 711-721, 2008.

13. Bechshøft RL, Malmgaard-Clausen NM, Gliese B, Beyer N, Mackey AL, Andersen JL, Kjær M, and Holm L. Improved skeletal muscle mass and strength after heavy strength training in very old individuals. Exp Gerontol 92: 96-105, 2017.

14. Bolster DR, Kimball SR, and Jefferson LS. Translational control mechanisms modulate skeletal muscle gene expression during hypertrophy. Exerc Sport Sci Rev 31: 111-116, 2003.

15. Borde R, Hortobagyi T, and Granacher $\mathbf{U}$. Dose-response relationships of resistance training in healthy old adults: a systematic review and meta-analysis. Sports Med 45: 1693-1720, 2015.

16. Bruusgaard JC, Johansen IB, Egner IM, Rana ZA, and Gundersen K. Myonuclei acquired by overload exercise precede hypertrophy and are not lost on detraining. Proc Natl Acad Sci U S A 107: 1511115116, 2010.

17. Bruyère O, Beaudart $\mathbf{C}$, Ethgen $\mathbf{O}$, Reginster JY, and Locquet M. The health economics burden of sarcopenia: a systematic review. Maturitas 119: 61-69, 2019.

18. Bultmann S, and Stricker SH. Entering the post-epigenomic age: back to epigenetics. Open Biol 8: 2018.

19. Caldow MK, Thomas EE, Dale MJ, Tomkinson GR, Buckley JD, and Cameron-Smith D. Early myogenic responses to acute exercise before and after resistance training in young men. Physiol Rep 3: 2015.

20. Camera DM, Smiles WJ, and Hawley JA. Exercise-induced skeletal muscle signaling pathways and human athletic performance. Free Radic Biol Med 2016.

21. Cedar H, and Bergman Y. Linking DNA methylation and histone modification: patterns and paradigms. Nat Rev Genet 10: 295-304, 2009.

\section{Cheedipudi S, Puri D, Saleh A, Gala HP, Rumman M, Pillai MS, Sreenivas P, Arora R,} Sellathurai J, Schroder HD, Mishra RK, and Dhawan J. A fine balance: epigenetic control of cellular quiescence by the tumor suppressor PRDM2/RIZ at a bivalent domain in the cyclin a gene. Nucleic Acids Res 43: 6236-6256, 2015. 
23. Cholewa J, Guimaraes-Ferreira L, da Silva Teixeira T, Naimo MA, Zhi X, de Sa RB, Lodetti A, Cardozo MQ, and Zanchi NE. Basic models modeling resistance training: an update for basic scientists interested in study skeletal muscle hypertrophy. J Cell Physiol 229: 1148-1156, 2014.

24. Cisterna B, Giagnacovo M, Costanzo M, Fattoretti P, Zancanaro C, Pellicciari C, and Malatesta M. Adapted physical exercise enhances activation and differentiation potential of satellite cells in the skeletal muscle of old mice. J Anat 228: 771-783, 2016.

25. Clark SJ, Statham A, Stirzaker C, Molloy PL, and Frommer M. DNA methylation: bisulphite modification and analysis. Nat Protoc 1: 2353-2364, 2006.

26. Conboy IM, Conboy MJ, Smythe GM, and Rando TA. Notch-mediated restoration of regenerative potential to aged muscle. Science 302: 1575-1577, 2003.

27. Conboy IM, Conboy MJ, Wagers AJ, Girma ER, Weissman IL, and Rando TA. Rejuvenation of aged progenitor cells by exposure to a young systemic environment. Nature 433: 760-764, 2005.

28. Cooper R, Bann D, Wloch EG, Adams JE, and Kuh D. "Skeletal muscle function deficit" in a nationally representative British birth cohort in early old age. J Gerontol A Biol Sci Med Sci 70: 604-607, 2015.

29. Crameri RM, Aagaard P, Qvortrup K, Langberg H, Olesen J, and Kjaer M. Myofibre damage in human skeletal muscle: effects of electrical stimulation versus voluntary contraction. J Physiol 583: 365-380, 2007.

30. Crane JD, Macneil LG, and Tarnopolsky MA. Long-term aerobic exercise is associated with greater muscle strength throughout the life span. J Gerontol A Biol Sci Med Sci 68: 631-638, 2013.

31. Crawford JO, Graveling RA, Cowie HA, and Dixon K. The health safety and health promotion needs of older workers. Occup Med (Lond) 60: 184-192, 2010.

32. Cruz-Jentoft AJ, Baeyens JP, Bauer JM, Boirie Y, Cederholm T, Landi F, Martin FC, Michel JP, Rolland Y, Schneider SM, Topinkova E, Vandewoude M, Zamboni M, and European Working Group on Sarcopenia in Older P. Sarcopenia: European consensus on definition and diagnosis: report of the European Working Group on Sarcopenia in Older People. Age Ageing 39: 412-423, 2010.

33. Cruz-Jentoft AJ, Bahat G, Bauer J, Boirie Y, Bruyere O, Cederholm T, Cooper C, Landi F, Rolland Y, Sayer AA, Schneider SM, Sieber CC, Topinkova E, Vandewoude M, Visser M, Zamboni M, 
Writing Group for the European Working Group on Sarcopenia in Older P, and the Extended Group for

E. Sarcopenia: revised European consensus on definition and diagnosis. Age Ageing 48: 16-31, 2019.

34. Cutlip RG, Baker BA, Geronilla KB, Mercer RR, Kashon ML, Miller GR, Murlasits Z, and Alway

SE. Chronic exposure to stretch-shortening contractions results in skeletal muscle adaptation in young rats and maladaptation in old rats. Appl Physiol Nutr Metab 31: 573-587, 2006.

35. Cutlip RG, Stauber WT, Willison RH, McIntosh TA, and Means KH. Dynamometer for rat plantar flexor muscles in vivo. Med Biol Eng Comput 35: 540-543, 1997.

36. Dungan CM, Murach KA, Frick KK, Jones SR, Crow SE, Englund DA, Vechetti IJ, Jr., Figueiredo VC, Levitan BM, Satin J, McCarthy JJ, and Peterson CA. Elevated myonuclear density during skeletal muscle hypertrophy in response to training is reversed during detraining. Am J Physiol Cell Physiol 2019.

37. Egner IM, Bruusgaard JC, Eftestol E, and Gundersen K. A cellular memory mechanism aids overload hypertrophy in muscle long after an episodic exposure to anabolic steroids. J Physiol 591: 6221-6230, 2013.

38. Eilertsen KJ, Power RA, Harkins LL, and Misica P. Targeting cellular memory to reprogram the epigenome, restore potential, and improve somatic cell nuclear transfer. Anim Reprod Sci 98: 129-146, 2007.

39. Evans WJ. What is sarcopenia? J Gerontol A Biol Sci Med Sci 50 Spec No: 5-8, 1995.

40. Feng YQ, Desprat R, Fu H, Olivier E, Lin CM, Lobell A, Gowda SN, Aladjem MI, and Bouhassira EE. DNA methylation supports intrinsic epigenetic memory in mammalian cells. PLoS Genet 2: e65, 2006.

41. Fiatarone MA, Marks EC, Ryan ND, Meredith CN, Lipsitz LA, and Evans WJ. High-intensity strength training in nonagenarians. Effects on skeletal muscle. JAMA 263: 3029-3034, 1990.

42. Fisher JP, Steele J, Gentil P, Giessing J, and Westcott WL. A minimal dose approach to resistance training for the older adult; the prophylactic for aging. Exp Gerontol 99: 80-86, 2017.

43. Fragala MS, Kenny AM, and Kuchel GA. Muscle quality in aging: a multi-dimensional approach to muscle functioning with applications for treatment. Sports Med 45: 641-658, 2015.

44. Frontera WR, Meredith CN, O'Reilly KP, Knuttgen HG, and Evans WJ. Strength conditioning in older men: skeletal muscle hypertrophy and improved function. 64: 1038-1044, 1988. 
45. Gillen JB, and Gibala MJ. Interval training: a time-efficient exercise strategy to improve cardiometabolic health. Appl Physiol Nutr Metab 43: iii-iv, 2018.

46. Gillen JB, Percival ME, Skelly LE, Martin BJ, Tan RB, Tarnopolsky MA, and Gibala MJ. Three minutes of all-out intermittent exercise per week increases skeletal muscle oxidative capacity and improves cardiometabolic health. PLoS One 9: e111489, 2014.

47. Goto S, and Radak Z. Hormetic effects of reactive oxygen species by exercise: a view from animal studies for successful aging in human. Dose Response 8: 68-72, 2009.

48. Grounds MD. The need to more precisely define aspects of skeletal muscle regeneration. Int J Biochem Cell Biol 56: 56-65, 2014.

49. Gundersen K. Muscle memory and a new cellular model for muscle atrophy and hypertrophy. $J$ Exp Biol 219: 235-242, 2016.

50. Hagerman FC, Walsh SJ, Staron RS, Hikida RS, Gilders RM, Murray TF, Toma K, and Ragg KE. Effects of high-intensity resistance training on untrained older men. I. Strength, cardiovascular, and metabolic responses. J Gerontol A Biol Sci Med Sci 55: B336-346, 2000.

51. Hakkinen K, Kraemer WJ, Newton RU, and Alen M. Changes in electromyographic activity, muscle fibre and force production characteristics during heavy resistance/power strength training in middle-aged and older men and women. Acta Physiol Scand 171: 51-62, 2001.

52. Hakkinen K, Pakarinen A, Kraemer WJ, Hakkinen A, Valkeinen H, and Alen M. Selective muscle hypertrophy, changes in EMG and force, and serum hormones during strength training in older women. J Appl Physiol (1985) 91: 569-580, 2001.

53. Halson SL, and Jeukendrup AE. Does overtraining exist? An analysis of overreaching and overtraining research. Sports Med 34: 967-981, 2004.

54. Harper S. Economic and social implications of aging societies. Science 346: 587-591, 2014.

55. Harridge SD. Plasticity of human skeletal muscle: gene expression to in vivo function. Exp Physiol 92: 783-797, 2007.

56. Hartman MJ, Fields DA, Byrne NM, and Hunter GR. Resistance training improves metabolic economy during functional tasks in older adults. J Strength Cond Res 21: 91-95, 2007. 
57. Hayflick L. Biological aging is no longer an unsolved problem. Ann N Y Acad Sci 1100: 1-13, 2007.

58. Henwood TR, and Taaffe DR. Detraining and retraining in older adults following long-term muscle power or muscle strength specific training. J Gerontol A Biol Sci Med Sci 63: 751-758, 2008.

59. Hepple RT, Mackinnon SL, Thomas SG, Goodman JM, and Plyley MJ. Quantitating the capillary supply and the response to resistance training in older men. Pflügers Archiv - European Journal of Physiology 433: 238-244, 1997.

60. Hikida RS, Staron RS, Hagerman FC, Walsh S, Kaiser E, Shell S, and Hervey S. Effects of highintensity resistance training on untrained older men. II. Muscle fiber characteristics and nucleo-cytoplasmic relationships. J Gerontol A Biol Sci Med Sci 55: B347-354, 2000.

61. Jackman RW, and Kandarian SC. The molecular basis of skeletal muscle atrophy. Am J Physiol Cell Physiol 287: C834-843, 2004.

62. Jargin SV. Hormetic use of stress in gerontological interventions requires a cautious approach. Biogerontology 17: 417-420, 2016.

63. Kanzleiter T, Jahnert M, Schulze G, Selbig J, Hallahan N, Schwenk RW, and Schurmann A. Exercise training alters DNA methylation patterns in genes related to muscle growth and differentiation in mice. Am J Physiol Endocrinol Metab 308: E912-920, 2015.

64. King-Himmelreich TS, Schramm S, Wolters MC, Schmetzer J, Moser CV, Knothe C, Resch E, Peil J, Geisslinger G, and Niederberger E. The impact of endurance exercise on global and AMPK genespecific DNA methylation. Biochem Biophys Res Commun 474: 284-290, 2016.

65. Kovacheva EL, Hikim AP, Shen R, Sinha I, and Sinha-Hikim I. Testosterone supplementation reverses sarcopenia in aging through regulation of myostatin, c-Jun NH2-terminal kinase, Notch, and Akt signaling pathways. Endocrinology 151: 628-638, 2010.

66. Laker RC, and Ryall JG. DNA Methylation in skeletal muscle stem cell specification, proliferation, and differentiation. Stem Cells Int 2016: 5725927, 2016.

67. Lane SC, Camera DM, Lassiter DG, Areta JL, Bird SR, Yeo WK, Jeacocke NA, Krook A, Zierath JR, Burke LM, and Hawley JA. Effects of sleeping with reduced carbohydrate availability on acute training responses. J Appl Physiol (1985) 119: 643-655, 2015. 
68. Lee H, Kim K, Kim B, Shin J, Rajan S, Wu J, Chen X, Brown MD, Lee S, and Park JY. A cellular mechanism of muscle memory facilitates mitochondrial remodelling following resistance training. $J$ Physiol 596: 4413-4426, 2018.

69. Lexell J. Human aging, muscle mass, and fiber type composition. J Gerontol A Biol Sci Med Sci 50 Spec No: 11-16, 1995.

\section{Lindholm ME, Marabita F, Gomez-Cabrero D, Rundqvist H, Ekstrom TJ, Tegner J, and}

Sundberg CJ. An integrative analysis reveals coordinated reprogramming of the epigenome and the transcriptome in human skeletal muscle after training. Epigenetics 9: 1557-1569, 2014.

71. Malatesta M, Fattoretti P, Giagnacovo M, Pellicciari C, and Zancanaro C. Physical training modulates structural and functional features of cell nuclei in type II myofibers of old mice. Rejuvenation Res 14: 543-552, 2011.

72. McGregor RA, Cameron-Smith D, and Poppitt SD. It is not just muscle mass: a review of muscle quality, composition and metabolism during ageing as determinants of muscle function and mobility in later life. Longev Healthspan 3: 9, 2014.

73. McLeod M, Breen L, Hamilton DL, and Philp A. Live strong and prosper: the importance of skeletal muscle strength for healthy ageing. Biogerontology 17: 497-510, 2016.

74. McLester JR, Bishop E, and Guilliams ME. Comparison of 1 day and 3 days per week of equalvolume resistance training in experienced subjects. 14: 273-281, 2000.

75. Melov S, Tarnopolsky MA, Beckman K, Felkey K, and Hubbard A. Resistance exercise reverses aging in human skeletal muscle. PLoS One 2: e465, 2007.

76. Morton JP, Kayani AC, McArdle A, and Drust B. The exercise-induced stress response of skeletal muscle, with specific emphasis on humans. Sports Med 39: 643-662, 2009.

77. Nguyen A, Duquette N, Mamarbachi M, and Thorin E. Epigenetic regulatory effect of exercise on glutathione peroxidase 1 expression in the skeletal muscle of severely dyslipidemic mice. PLoS One 11: e0151526, 2016.

78. Nitert MD, Dayeh T, Volkov P, Elgzyri T, Hall E, Nilsson E, Yang BT, Lang S, Parikh H, Wessman Y, Weishaupt H, Attema J, Abels M, Wierup N, Almgren P, Jansson PA, Ronn T, Hansson O, 
Eriksson KF, Groop L, and Ling C. Impact of an exercise intervention on DNA methylation in skeletal muscle from first-degree relatives of patients with type 2 diabetes. Diabetes 61: 3322-3332, 2012.

79. Norman K, and Otten L. Financial impact of sarcopenia or low muscle mass - a short review. Clin Nutr 2018.

80. Pattamaprapanont P, Garde C, Fabre O, and Barres R. Muscle contraction induces acute hydroxymethylation of the exercise-responsive gene Nr4a3. Front Endocrinol (Lausanne) 7: 165, 2016.

81. Petsko GA. A seat at the table. Genome Biol 9: 113, 2008.

82. Phillips BE, Williams JP, Gustafsson T, Bouchard C, Rankinen T, Knudsen S, Smith K, Timmons JA, and Atherton PJ. Molecular networks of human muscle adaptation to exercise and age. PLoS Genet 9: e1003389, 2013.

83. Philp A, Hamilton DL, and Baar K. Signals mediating skeletal muscle remodeling by resistance exercise: PI3-kinase independent activation of mTORC1. J Appl Physiol (1985) 110: 561-568, 2011.

84. Radak Z, Chung HY, Koltai E, Taylor AW, and Goto S. Exercise, oxidative stress and hormesis. Ageing Res Rev 7: 34-42, 2008.

85. Rader EP, and Baker BA. Inflammaging and the age-specific responsiveness to stretch-shortening contractions. Exerc Sport Sci Rev 45: 195-200, 2017.

86. Rader EP, Layner K, Triscuit AM, Chetlin RD, Ensey J, and Baker BA. Age-dependent muscle adaptation after chronic stretch-shortening contractions in rats. Aging Dis 7: 1-13, 2016.

87. Rader EP, Layner KN, Triscuit AM, Kashon ML, Gu JK, Ensey J, and Baker BA. Desensitized morphological and cytokine response after stretch-shortening muscle contractions as a feature of aging in rats. Exp Gerontol 72: 138-149, 2015.

88. Rader EP, Naimo MA, Ensey J, and Baker BA. High-intensity stretch-shortening contraction training modifies responsivity of skeletal muscle in old male rats. Exp Gerontol 104: 118-126, 2018.

\section{Rader EP, Naimo MA, Layner KN, Triscuit AM, Chetlin RD, Ensey J, and Baker BA.}

Enhancement of skeletal muscle in aged rats following high-intensity stretch-shortening contraction training. Rejuvenation Res 20: 93-102, 2017. 
90. Rasmussen M, Zierath JR, and Barres R. Dynamic epigenetic responses to muscle contraction. Drug Discov Today 19: 1010-1014, 2014.

91. Rattan SI. Aging is not a disease: implications for intervention. Aging Dis 5: 196-202, 2014.

92. Roberts MD, Kerksick CM, Dalbo VJ, Hassell SE, Tucker PS, and Brown R. Molecular attributes of human skeletal muscle at rest and after unaccustomed exercise: an age comparison. J Strength Cond Res 24: 1161-1168, 2010.

93. Röckl KS, Witczak CA, and Goodyear LJ. Signaling mechanisms in skeletal muscle: acute responses and chronic adaptations to exercise. IUBMB Life 60: 145-153, 2008.

\section{Ronn T, Volkov P, Davegardh C, Dayeh T, Hall E, Olsson AH, Nilsson E, Tornberg A, Dekker} Nitert M, Eriksson KF, Jones HA, Groop L, and Ling C. A six months exercise intervention influences the genome-wide DNA methylation pattern in human adipose tissue. PLoS Genet 9: e1003572, 2013.

95. Roth SM, Ferrell RF, and Hurley BF. Strength training for the prevention and treatment of sarcopenia. J Nutr Health Aging 4: 143-155, 2000.

96. Rutherford OM, and Jones DA. The role of learning and coordination in strength training. Eur J Appl Physiol Occup Physiol 55: 100-105, 1986.

97. Ryan MJ, Dudash HJ, Docherty M, Geronilla KB, Baker BA, Haff GG, Cutlip RG, and Alway SE. Aging-dependent regulation of antioxidant enzymes and redox status in chronically loaded rat dorsiflexor muscles. J Gerontol A Biol Sci Med Sci 63: 1015-1026, 2008.

98. Ryan MJ, Dudash HJ, Docherty M, Geronilla KB, Baker BA, Haff GG, Cutlip RG, and Alway SE. Vitamin E and C supplementation reduces oxidative stress, improves antioxidant enzymes and positive muscle work in chronically loaded muscles of aged rats. Exp Gerontol 45: 882-895, 2010.

\section{Sailani MR, Halling JF, Moller HD, Lee H, Plomgaard P, Pilegaard H, Snyder MP, and}

Regenberg B. Lifelong physical activity is associated with promoter hypomethylation of genes involved in metabolism, myogenesis, contractile properties and oxidative stress resistance in aged human skeletal muscle. Sci Rep 9: 3272, 2019.

100. Sakugawa RL, Moura BM, Orssatto L, Bezerra ES, Cadore EL, and Diefenthaeler F. Effects of resistance training, detraining, and retraining on strength and functional capacity in elderly. Aging Clin Exp Res 31: 31-39, 2019. 
101. Schoenfeld BJ, Wilson JM, Lowery RP, and Krieger JW. Muscular adaptations in low- versus highload resistance training: a meta-analysis. Eur J Sport Sci 16: 1-10, 2016.

102. Seaborne RA, Strauss J, Cocks M, Shepherd S, O'Brien TD, van Someren KA, Bell PG, Murgatroyd C, Morton JP, Stewart CE, and Sharples AP. Human skeletal muscle possesses an epigenetic memory of hypertrophy. Sci Rep 8: 1898, 2018.

103. Seals DR, Justice JN, and LaRocca TJ. Physiological geroscience: targeting function to increase healthspan and achieve optimal longevity. J Physiol 594: 2001-2024, 2016.

104. Seals DR, and Melov S. Translational geroscience: emphasizing function to achieve optimal longevity. Aging (Albany NY) 6: 718-730, 2014.

105. Sharples AP, Polydorou I, Hughes DC, Owens DJ, Hughes TM, and Stewart CE. Skeletal muscle cells possess a 'memory' of acute early life TNF-alpha exposure: role of epigenetic adaptation. Biogerontology 17: 603-617, 2016.

106. Sharples AP, Stewart CE, and Seaborne RA. Does skeletal muscle have an 'epi'-memory? The role of epigenetics in nutritional programming, metabolic disease, aging and exercise. Aging Cell 15: 603-616, 2016.

107. Shibata S, Fujimoto N, Hastings JL, Carrick-Ranson G, Bhella PS, Hearon CM, Jr., and Levine BD. The effect of lifelong exercise frequency on arterial stiffness. J Physiol 596: 2783-2795, 2018.

108. Sousa-Victor P, Gutarra S, Garcia-Prat L, Rodriguez-Ubreva J, Ortet L, Ruiz-Bonilla V, Jardi M, Ballestar E, Gonzalez S, Serrano AL, Perdiguero E, and Munoz-Canoves P. Geriatric muscle stem cells switch reversible quiescence into senescence. Nature 506: 316-321, 2014.

109. Sousa AS, Guerra RS, Fonseca I, Pichel F, Ferreira S, and Amaral TF. Financial impact of sarcopenia on hospitalization costs. Eur J Clin Nutr 70: 1046-1051, 2016.

110. Spiering BA, Kraemer WJ, Anderson JM, Armstrong LE, Nindl BC, Volek JS, and Maresh CM. Resistance exercise biology: manipulation of resistance exercise programme variables determines the responses of cellular and molecular signalling pathways. Sports Med 38: 527-540, 2008.

111. Srikanthan P, and Karlamangla AS. Muscle mass index as a predictor of longevity in older adults. Am J Med 127: 547-553, 2014. 
112. Staron RS, Leonardi MJ, Karapondo DL, Malicky ES, Falkel JE, Hagerman FC, and Hikida RS. Strength and skeletal muscle adaptations in heavy-resistance-trained women after detraining and retraining. $J$ Appl Physiol (1985) 70: 631-640, 1991.

\section{Staron RS, Malicky ES, Leonardi MJ, Falkel JE, Hagerman FC, and Dudley GA. Muscle} hypertrophy and fast fiber type conversions in heavy resistance-trained women. Eur J Appl Physiol Occup Physiol 60: 71-79, 1990.

114. Stec MJ, Kelly NA, Many GM, Windham ST, Tuggle SC, and Bamman MM. Ribosome biogenesis may augment resistance training-induced myofiber hypertrophy and is required for myotube growth in vitro. Am J Physiol Endocrinol Metab 310: E652-E661, 2016.

\section{Stec MJ, Thalacker-Mercer A, Mayhew DL, Kelly NA, Tuggle SC, Merritt EK, Brown CJ,} Windham ST, Dell'Italia LJ, Bickel CS, Roberts BM, Vaughn KM, Isakova-Donahue I, Many GM, and Bamman MM. Randomized, four-arm, dose-response clinical trial to optimize resistance exercise training for older adults with age-related muscle atrophy. Exp Gerontol 99: 98-109, 2017.

116. Taaffe DR, Henwood TR, Nalls MA, Walker DG, Lang TF, and Harris TB. Alterations in muscle attenuation following detraining and retraining in resistance-trained older adults. Gerontology 55: 217-223, 2009.

117. Tan Q, Christiansen L, von Bornemann Hjelmborg J, and Christensen K. Twin methodology in epigenetic studies. J Exp Biol 218: 134-139, 2015.

118. Tan R, Nederveen JP, Gillen JB, Joanisse S, Parise G, Tarnopolsky MA, and Gibala MJ. Skeletal muscle fiber-type-specific changes in markers of capillary and mitochondrial content after low-volume interval training in overweight women. Physiol Rep 6: 2018.

119. Tanaka H, and Seals DR. Endurance exercise performance in Masters athletes: age-associated changes and underlying physiological mechanisms. J Physiol 586: 55-63, 2008.

120. Trost SG, Owen N, Bauman AE, Sallis JF, and Brown W. Correlates of adults' participation in physical activity: review and update. Med Sci Sports Exerc 34: 1996-2001, 2002.

121. Tucker LA. Physical activity and telomere length in U.S. men and women: an NHANES investigation. Prev Med 100: 145-151, 2017. 
122. Turner DC, Seaborne RA, and Sharples AP. Comparative transcriptome and methylome analysis in human skeletal muscle anabolism, hypertrophy and epigenetic memory. Sci Rep 9: 4251, 2019.

123. Turpela M, Hakkinen K, Haff GG, and Walker S. Effects of different strength training frequencies on maximum strength, body composition and functional capacity in healthy older individuals. Exp Gerontol 98: 13-21, 2017.

124. Vaczi M, Nagy SA, Koszegi T, Ambrus M, Bogner P, Perlaki G, Orsi G, Toth K, and Hortobagyi T. Mechanical, hormonal, and hypertrophic adaptations to 10 weeks of eccentric and stretch-shortening cycle exercise training in old males. Exp Gerontol 58: 69-77, 2014.

125. Welle S. Cellular and molecular basis of age-related sarcopenia. Can J Appl Physiol 27: 19-41, 2002.

126. Winett RA, Williams DM, and Davy BM. Initiating and maintaining resistance training in older adults: a social cognitive theory-based approach. Br J Sports Med 43: 114-119, 2009.

127. Yarasheski KE. Managing sarcopenia with progressive resistance exercise training. J Nutr Health Aging 6: 349-356, 2002.

128. Yoo SZ, No MH, Heo JW, Park DH, Kang JH, Kim SH, and Kwak HB. Role of exercise in agerelated sarcopenia. J Exerc Rehabil 14: 551-558, 2018.

129. Yousef H, Conboy MJ, Morgenthaler A, Schlesinger C, Bugaj L, Paliwal P, Greer C, Conboy IM, and Schaffer D. Systemic attenuation of the TGF-beta pathway by a single drug simultaneously rejuvenates hippocampal neurogenesis and myogenesis in the same old mammal. Oncotarget 6: 11959-11978, 2015.

130. Zampieri S, Pietrangelo L, Loefler S, Fruhmann H, Vogelauer M, Burggraf S, Pond A, GrimStieger M, Cvecka J, Sedliak M, Tirpakova V, Mayr W, Sarabon N, Rossini K, Barberi L, De Rossi M, Romanello V, Boncompagni S, Musaro A, Sandri M, Protasi F, Carraro U, and Kern H. Lifelong physical exercise delays age-associated skeletal muscle decline. J Gerontol A Biol Sci Med Sci 70: 163-173, 2015. 


\section{CHAPTER III}

ENHANCEMENT OF SKELETAL MUSCLE IN AGED RATS FOLLOWING HIGH-INTENSITY STRECHSHORTENING CONTRACTION TRAINING

Erik P. Rader ${ }^{1 *}$, Marshall A. Naimo ${ }^{1,2}$, Kayla N. Layner ${ }^{1}$, Alyssa M. Triscuit ${ }^{1}$, Robert D. Chetlin ${ }^{1,2,3}$, James Ensey $^{1}$, and Brent A. Baker ${ }^{1}$

${ }^{1}$ Centers for Disease Control and Prevention, National Institute for Occupational Safety and Health, Morgantown, West Virginia 26505. ²West Virginia School of Medicine, Division of Exercise Physiology, Morgantown, West Virginia. ${ }^{3}$ Mercyhurst University, Department of Sports Medicine, Erie, Pennsylvania 16546.

*Address for correspondence and reprints:

Erik P. Rader, PhD

MS L3014, 1095 Willowdale Rd.

Morgantown, WV, 26505

WLZ4@cdc.gov

Office: 304-285-6242, Fax: 304-285-5708

Keywords: dorsiflexor muscles, dynamometer, Fisher 344XBrown Norway rats, repetitive exposure, oxidative stress

Running title: Skeletal muscle enhancement in aged rats

\section{Received: February 19, 2016}

Accepted: July 5, 2016 


\section{ABSTRACT}

Exercise is the most accessible, efficacious, and multifactorial intervention to improve health and treat chronic disease. High-intensity resistance exercise, in particular, also maximizes skeletal muscle size and strength outcomes crucial at advanced age. However, such training is capable of inducing muscle maladaptation when misapplied at old age. Therefore, characterization of parameters (e.g. mode and frequency) which foster adaptation is an active research area. To address this issue, we utilized a rodent model that allowed training at maximal intensity in terms of muscle activation and tested the hypothesis that muscles of old rats adapt to stretch-shortening contraction training, provided the training frequency is sufficiently low. At termination of training, normalized muscle mass (i.e. muscle mass divided by tibia length) and muscle quality (isometric force divided by normalized muscle mass) were determined. For young rats, normalized muscle mass increased by $\sim 20 \%$ regardless of training frequency. No difference was also observed for muscle quality values after 2 days vs 3 days per week training ( $0.65 \pm 0.09 \mathrm{~N} / \mathrm{mg} / \mathrm{mm}$ vs $0.59 \pm 0.05 \mathrm{~N} / \mathrm{mg} / \mathrm{mm}$, respectively). For old rats following 3 days per week training, normalized muscle mass was unaltered and muscle quality was 30\% lower than young levels. Following 2 days per week training at old age, normalized muscle mass increased by $17 \%$ and muscle quality was restored to young levels. To investigate this enhanced response, oxidative stress was assessed by lipid peroxidation quantification. For young rats, lipid peroxidation levels were unaltered by training. With aging, baseline levels of lipid peroxidation increased by 1.5-fold. For old rats, only 2 days per week training decreased lipid peroxidation to levels indistinguishable from young values. These results imply

appropriately scheduled high-intensity stretch-shortening contraction training at old age is capable of restoring muscle to a younger phenotype in terms of lipid peroxidation levels and muscle quality. 


\section{PUBLICATIONS DISCLAIMER:}

"The findings and conclusions in this report are those of the author(s) and do not necessarily represent the views of the National Institute for Occupational Safety and Health." 


\section{INTRODUCTION}

Exercise is an effective intervention for improving overall health and alleviating chronic diseases ranging from cancer to psychiatric, neurological, metabolic, cardiovascular, musculoskeletal, and pulmonary diseases $(20,21,25,27,28)$. Strenuous physical activity in the form of exercise is as efficacious as many commonly prescribed drug treatments for heart disease and diabetes and is more beneficial than drug intervention in rehabilitation from stroke (25). Evidence for the pluripotency of exercise led the American Medical Association and the American College of Sports Medicine to launch the Exercise is Medicine initiative in 2007 - a now global initiative to translate scientifically proven health benefits of physical exercise into the healthcare system (20).

Among the various forms of exercise ranging from aerobic to anaerobic, resistance exercise in particular is promoted for increasing skeletal muscle size and strength for those with deficiencies in these attributes such as the elderly (28). However, the training parameters for intensity, mode, and frequency of resistance exercise to prescribe is an active area of research. Exposure to sessions of muscular activity that are extreme in terms of volume (i.e. product of intensity and repetition number) result in maladaptation - especially at advanced age.(6, $11,22)$. Concerns about high-intensity training ( $\geq 70-85 \%$ of one repetition maximum) at old age has led some researchers to investigate lower intensity training alternatives $(26,38)$. Reports utilizing experimental rodent models has been especially informative in regards to negative outcomes of some high-intensity resistance-type training regimens because of the ability to precisely control intensity and mode. In several such studies, muscles of rats underwent maximal intensity training in terms of muscle activation 3 days per week for 1 month (2, 9, 33). In these studies, the mode utilized was stretch-shortening contractions (SSCs), a common form of contraction mode utilized by all mammals during resistance-type exercise in which muscle is activated prior to and during the initial stretch and subsequent shortening; as opposed to isolated eccentric, isometric, or shortening contractions. For young rats, this training induced gains of $\sim 20 \%$ in muscle mass and performance. In contrast, for old rats, the exposure typically induced marginal muscle mass gains $(<10 \%)$ and decreased performance output $(2,9,33)$. Such findings indicated that high-intensity SSC training has the potential to not only be ineffective at old age but also detrimental if inappropriately utilized.

Although resistance exercise programs performed at a range of intensities can produce substantial muscle hypertrophy and strength gains, high-intensity resistance training demonstrates the most potential for maximizing these outcomes presumably because of the maximization of motor unit recruitment and, consequently, the number of muscle fibers activated in the training (35). Therefore, the need to characterize training parameters (e.g. mode and frequency) which are compatible with muscle adaptation from high-intensity training at old age is of great importance. The present study was undertaken for SSC training at maximal muscle activation (i.e. high-intensity training) to determine whether decreasing the frequency of training, and thereby increasing recovery time between training sessions, would be sufficient to induce an adaptive response.

Specifically, the hypothesis tested was that decreasing the frequency of training from 3 to 2 days per week 
would improve the adaptive outcome for old rats. For assessing adaptation/maladaptation, muscle mass (normalized to tibia length), isometric force, muscle quality (i.e. isometric force divided by normalized muscle mass), dynamic peak force, negative work, and positive work were analyzed. Quantitative morphology for tissue percentage of normal muscle fibers, degenerative muscle fibers, non-cellular interstitium, and cellular interstitium was assessed by a standardized stereological method. Lipid peroxidation was also quantified as an indicator of oxidative stress because of findings in previous reports indicating oxidative stress as a factor in SSC-induced adaptation/maladaptation $(33,34)$. In these studies, aging was accompanied by an increase in baseline levels of lipid peroxidation in skeletal muscle and a reduction in these levels induced by Vitamin E and C supplementation improved the response to SSC training $(33,34)$. The measurement of lipid peroxidation in the present study enabled the assessment of whether changes in lipid peroxidation levels accompany differential muscle performance outcomes when training frequency is altered. The findings demonstrate the efficacy of age-appropriate high-intensity SSC training - training with potential to reduce lipid peroxidation levels, offset sarcopenia, and promote healthy aging.

\section{MATERIALS \& METHODS}

\section{Animals}

Male Fischer Brown Norway hybrid rats (F344 X BN) at 3 and 30 months of age were obtained from the National Institute of Aging colony and housed in an Association for Assessment and Accreditation of Laboratory Animal Care-accredited animal quarters. All animal procedures were approved by the Animal Care and Use Committee at the National Institute for Occupational Safety and Health in Morgantown, WV.

\section{High-intensity SSC training}

The 2 and 3 days per week high-intensity SSC training exposures were identical with the exception of frequency of exposure with the 2 days per week training administered Mondays and Thursdays while the 3 days per week training was administered Mondays, Wednesdays, and Fridays. Both exposures were administered over 4.5 weeks. Each exposure was based on a previously described procedure in which rats were exposed to 80 SSCs (9). Each rat was anesthetized with isoflurane gas and placed in dorsal recumbency on a heated table with the left knee secured in flexion at $90^{\circ}$. The left foot was secured to a fixture containing a load cell. Platinum electrodes were placed subcutaneously in the region of the common peroneal nerve for activation of the dorsiflexor muscles. Muscle stimulation was set at parameters for maximal contraction; 4-V magnitude, 0.2-ms pulse duration, and 120-Hz frequency (12).

Both static and dynamic performance was assessed prior to exposure to the 80 SSC protocol. Static performance was assessed by exposing the muscles to a single maximal isometric tetanic contraction for $300 \mathrm{~ms}$ with the ankle at $90^{\circ}$. After a 2-minute rest, dynamic performance was assessed by exposure to a single SSC test consisting of an isometric contraction for $300 \mathrm{~ms}$ at an ankle angle of $70^{\circ}$, then rotating the ankle through the 
entire physiologic range from $70^{\circ}$ to $140^{\circ}$ at $500^{\circ}$ per second, then returning to $70^{\circ}$ at $500^{\circ}$ per second, and lastly continuing activation for an additional $300 \mathrm{~ms}$. Peak force was determined by assessing the maximum force during the stretch phase. Negative and positive work referred to the work required to stretch the muscle and the work done by the muscle during the shortening phase, respectively.

The 80 SSC protocol was administered 2 minutes following the single SSC test. The protocol consisted of 8 sets of SSCs (2-minute intervals between sets) and 10 SSCs per set (2-second intervals between SSCs) with the interval durations chosen so as to be comparable to those typically suggested for resistance exercise training (7). For each SSC, while the muscles were maximally activated, the ankle was set to $90^{\circ}$ for 100 ms, rotated to $140^{\circ}$ at $60^{\circ} / \mathrm{s}$, returned to $90^{\circ}$ at $60^{\circ}$ s, and deactivated $300 \mathrm{~ms}$ later. The ankle range of $90^{\circ}$ (angle associated with maximum isometric force) to $140^{\circ}$ maximized the force output during each training SSC while decreasing the factor of fatigue which would have been a greater factor had the complete physiologic range $\left(70^{\circ}\right.$ to $\left.140^{\circ}\right)$ been utilized (10). The velocity of $60^{\circ}$ per second was chosen to promote performance adaptation and hypertrophy rather than injury (9). Muscle inflammation and degeneration are not overt for young and old rats in the days to weeks following the SSC exposure utilized in the present study (2, 3, 9). The performance measures for sessions during the first and last week of the SSC exposures were averaged to determine initial and final values, respectively. At 24 hours after the final SSC exposure, the tibialis anterior (TA) muscle was surgically removed, weighed, and the tibia length recorded. This time period was chosen to be beyond the acute effects of the first several hours post-training yet be within the time period conducive for detecting any overt muscle degeneration resulting from the last training session. The mid-belly of the TA muscle was covered with tissue freezing media (Tissue-Tek, 4583 O.C.T. Compound, Sakura Finetek) and frozen in cold isopentane (-80 ${ }^{\circ} \mathrm{C}$ ) for quantitative morphology. A portion of the remaining TA tissue was allocated for lipid peroxidation analysis. Normalized muscle mass was determined by dividing the muscle mass by tibia length. Muscle quality was quantified as maximum isometric tetanic force for the final week of training divided by normalized muscle mass.

\section{Quantitative morphology}

The mid-belly of each TA muscle was cryosectioned at $12 \mu \mathrm{m}$ thickness and then hematoxylin and eosin stained. A standardized stereological method was used for quantitative morphology $(4,5)$. At two regions, $1 \mathrm{~mm}$ to the right of the section midline and $1 \mathrm{~mm}$ to the left of the section midline, stereological analysis was performed at 5 equally spaced sites across the muscle section. At each site, points of a 121-point 11-line overlay graticule (0.04 $\mathrm{mm}^{2}$ square with 100 divisions) were evaluated at 40X magnification. Therefore, a total of 1210 points were analyzed per section since 10 total fields were evaluated. Each point was identified as overlaying a normal muscle fiber, degenerative muscle fiber, cellular interstitium, or non-cellular interstitium (Figure 1). Degenerative muscle fibers were considered to have 1) loss of contact with surrounding fibers, 2) interdigitation of the sarcolemma by cellular infiltrates, and 3) internalization of cellular infiltrates (5). If a muscle fiber did not 
have these characteristics, the fiber was considered normal. Cellular interstitium were counted when points overlaid nuclei in between muscle fibers. Points that overlaid interstitial regions without nuclei were counted as non-cellular interstitium. Percent of muscle tissue comprised of normal muscle fibers, degenerative muscle fibers, centrally nucleated muscle fibers, cellular interstitium, or non-cellular interstitium were calculated as the percentage of points which overlaid each type of tissue relative to the total number of points.

\section{INSERT FIGURE 1 HERE}

\section{Immunofluorescence}

Frozen TA muscle sections (cryosectioned at $12 \mu \mathrm{m}$ thickness) were stained for 4-hydroxynonenal (4HNE; Calbiochem; no. 393206; at 1:250) to determine lipid peroxidation distribution and $\beta$-dystroglycan (Santa Cruz Biotechnology; sc-33701; at 1:100) to outline the sarcolemma for muscle fiber size measurements. First, sections were fixed in methanol $\left(-20^{\circ} \mathrm{C}\right)$ for 10 minutes and then acetone $\left(-20^{\circ} \mathrm{C}\right)$ for 10 minutes. Sections were washed with PBS and then blocked with 10\% goat serum at room temperature for 1 hour. Primary was applied overnight at $4^{\circ} \mathrm{C}$. After three washes with PBS (5 minutes each), secondary antibodies (goat anti-rabbit IgG Alexa Fluor 488 and goat anti-mouse IgG1 Alexa Fluor 594) were applied for 1 hour. The investigator was blinded to sample identification and for each muscle section, 10 images were captured at site locations determined in the same manner as described for quantitative morphology. Images were then analyzed utilizing ImageJ (version 1.46, National institutes of Health, USA). Each muscle fiber (208 \pm 21 fibers per section) was traced to determine minimum feret diameter, a measure of muscle fiber size that is resistant to variations in the orientation of muscle fibers during sectioning. For analysis of 4-HNE, threshold was set (based on images of secondary only stained sections) and percent area of signal within each muscle fiber measured.

\section{Lipid peroxidation levels}

Total malondialdehyde (MDA) content was assessed using a commercially available kit (Bioxytech MDA-586, Oxis International, Inc., Beverly Hills, CA, USA). TA muscle tissue was homogenized in PBS containing butylated hydroxytoluene $(5 \mathrm{mM})$. The homogenate was spun at $2000 \mathrm{~g}$ at $4{ }^{\circ} \mathrm{C}$ and the supernatant used for the total protein quantification and the total MDA assay per manufacturer's instructions. Protein quantification was performed using a standard colorimetric bicinchoninic acid (BCA) protein assay (Pierce, Rockford, IL, USA). MDA levels were normalized by amount of muscle protein for each sample.

\section{Statistical Analysis}

When assumptions of normality and equal variance were justified, data were analyzed using ANOVA (JMP version 11, SAS Institute, Inc., Cary, NC) with animal represented as a random factor to account for repeated measures within animal when appropriate. Post hoc comparisons were performed using Fisher's least 
significant difference method. Regarding data for percent of tissue comprised of degenerative muscle fibers, normality and equal variance could not be assumed so non-parametric tests (using SigmaPlot version 12.5, Systat Software, Inc., San Jose, CA) were utilized - comparisons for groups of old vs young rats were analyzed by the Mann-Whitney Rank Sum Test and Kruskal-Wallis Analysis of Variance on Ranks when appropriate while comparisons for trained vs contralateral muscles within each experimental group were analyzed by Wilcoxon Signed Ranks to account for repeated measures. Chi-square analysis (SigmaPlot version 12.5) was utilized to determine training-induced differences in the absolute frequency distributions of minimum feret diameter. Pearson product correlations (SigmaPlot version 12.5) were performed between SSC-induced alterations in MDA levels vs. muscle mass/performance outcomes. All data are expressed as means \pm SD. $P<$ 0.05 was considered statistically significant.

\section{RESULTS}

\section{Muscle mass and performance}

For young rats, muscle mass and strength gains were largely independent of whether they were subjected to 2 or 3 days per week high-intensity SSC training. Specifically, such training induced increases in peak force and the capacity for negative work (Figure 2A and Figure 2B). Positive work capacity and maximum isometric force was unaltered by training regardless of frequency (Figure 2C and 3A). Relative to contralateral control muscles, muscle mass increases of $19 \pm 4 \%$ and $20 \pm 4 \%$ were observed for muscles of young rats exposed to 2 vs. 3 days per week training, respectively (Figure 3B). To determine whether muscle mass changes were reflected at the muscle fiber level, minimum feret diameter was measured for individual muscle fibers (Table S1). The muscle mass increases for the young rats were accompanied by shifts in muscle fiber size distribution (Figures 4A and 4B). No difference in muscle quality, a measure of maximum isometric force normalized by muscle size, was observed between the training protocols (Figure 2C). Therefore, muscles of young rats displayed the ability to adapt in distinct performance measures following multiple SSC regimes by demonstrating comparable adaptation to both training frequencies tested.

For old rats, SSC training frequency resulted in distinct outcomes. Following 3 days per week training, the capacity for positive work diminished relative to initial values while peak force and negative work were unaltered compared with initial values (Figures 2A, 2B, and 2C). Muscle mass was unchanged when considered in comparison with contralateral control muscle mass data (Figures 3B). Muscle fiber size distribution also remained unchanged by 3 days per week training (Figure 4C). Final muscle quality values were depressed relative to those of young rats (Figure 3C). In contrast, following 2 days per week training, positive work capacity was maintained, muscle mass increased by $17 \pm 8 \%$ relative to contralateral control values $(P<$ 0.0001), and muscle fiber size distribution shifted to larger values (Figure 2C, 3B, and 4D). Furthermore, training 2 days per week restored muscle quality to levels indistinguishable from young levels. The implication 
was that at old age, decreasing frequency of high-intensity SSC training enabled muscle performance and muscle mass gains accompanied by revitalization of muscle quality.

\section{INSERT FIGURES 2-4 HERE}

\section{Quantitative morphology}

Analysis by quantitative morphology demonstrated an age-related decrease in tissue composed of normal muscle fibers (Table 1). Besides the difference between young and old contralateral control muscles following 2 days per week training $(P=0.039)$, a main effect for age was observed by ANOVA $(P=0.01)$. This decrease with age was due to an increase in the percentage of tissue other than that of normal muscle fibers which consists of degenerative muscle fibers, cellular interstitium, and non-cellular interstitium. When considering these tissue constituents separately, comparisons among individual groups did not reach significance but trends for the main effect of age were observed by ANOVA for increases in non-cellular interstitium $(P=0.057)$ and cellular interstitium $(P=0.09)$ with aging. The effect of age could not be assessed by ANOVA for degenerative muscle fibers since normality and equal variance could not be assumed for that data. However, when degenerative fiber data for contralateral muscles were pooled within age groups and analyzed by Mann-Whitney Rank Sum Test, muscles of old rats were comprised of a greater percentage of degenerative muscle fibers, $0.4 \pm 0.5 \%$, relative to young rats, $0.2 \pm 0.5 \%(P=0.001)$. Both the 2 and 3 days per week training had no significant effect for young and old rats (Table 1 and Figure 5). The lack of SSCinduced histological alterations demonstrated that the factors responsible for the distinct functional outcomes following the 2 vs. 3 days per week SSC training for the old rats were not apparent at the level of quantitative morphology. Rather, in agreement with multiple reports, this suggested that a more subtle process must be involved $(2,9)$.

INSERT TABLE 1 HERE

INSERT FIGURE 5 HERE

\section{Lipid peroxidation}

To investigate the possibility whether alterations in lipid peroxidation, an indicator of oxidative stress, correlate with the differential response in 2 days per week exposed old rats, lipid peroxidation assays were performed. Lipid peroxidation levels rather than spatial distribution of lipid peroxidation within muscle fibers appeared to be dependent on training frequency for old rats. Spatial distribution was investigated by immunofluorescence staining for 4-HNE in transverse sections of muscles. For all conditions (i.e. non-trained, trained 2 days per week, or trained 3 days per week) lipid peroxidation was apparent both at the sarcolemma and within muscle fibers of old rats (Figure 6). Interestingly for young rats, training either 2 or 3 days per week 
altered the distribution within muscles. While cytoplasmic staining of 4-HNE was present in all conditions, sarcolemma staining of HNE was modest in contralateral muscles of young rats whereas such staining was substantial in trained muscles (Figure 6). These observations were consistent with quantification of muscle fiber percent area positive for 4-HNE staining; $33 \pm 20 \%$ and $44 \pm 39 \%$ for contralateral and exposed muscles of young rats trained 3 days per week, $49 \pm 29 \%$ and $68 \pm 28 \%$ for contralateral and exposed muscles of young rats trained 2 days per week, $47 \pm 39 \%$ and $36 \pm 22 \%$ for contralateral and exposed muscles of old rats trained 3 days per week, and $61 \pm 29 \%$ and $50 \pm 21 \%$ for contralateral and exposed muscles of old rats trained 2 days per week ( $N=4$ to 5 per group). While no significant differences were observed between any of these groups when considered individually, an ANOVA demonstrated a significant interaction $(P=0.02)$ between age and limb (i.e. right contralateral muscle vs left trained muscle) independent of training frequency. Therefore, when data for 2 and 3 days per week trained groups were pooled, the percentage of each muscle fiber positive for HNE staining in trained muscles of young rats, $56 \pm 34 \%$, was increased relative to that of contralateral muscles, $41 \pm$ 25\% $(P=0.048)$. This dispersal of lipid peroxidation was in agreement with the observation of the addition of greater sarcolemma staining of 4-HNE upon training in the young rats indicating a substantial level of lipid peroxidation at the sarcolemma accompanies adaptation. The percentage area of 4-HNE staining was unaltered for old rats even upon pooling of the data for 2 and 3 days per week training. This was not surprising since sarcolemma lipid peroxidation was already overtly present in the non-trained condition and remained posttraining.

\section{INSERT FIGURE 6 HERE}

To determine whether lipid peroxidation levels differ with training frequency in muscle tissue, MDA levels were quantified. For contralateral control muscles, MDA levels were 1.5-fold greater for old rats relative to those of young rats (Figure 7). Exposure to 3 days per week of SSCs did not alter MDA levels. In contrast, 2 days per week of SSC training decreased MDA levels for muscles of old rats to levels of young rats (Figure 7). For old rats, Pearson product correlation analysis resulted in a negative correlation coefficient $(r=-0.719, P=$ 0.03) between SSC-induced alterations in MDA levels vs muscle mass (i.e. percentage difference in MDA levels relative to contralateral control levels vs percentage difference in muscle mass relative to contralateral control values). A trend for a negative correlation was noted between SSC-induced changes in MDA levels vs positive work (i.e. percentage difference in MDA levels relative to contralateral control levels vs percentage change in positive work relative to initial values; $r=-0.579, P=0.10$ ). A negative correlation trend was also observed between MDA levels of SSC trained muscles vs final muscle quality for those muscles $(r=-0.641, P$ =0.06). Overall, this data indicated that returning lipid peroxidation levels to young levels was inversely associated with improved skeletal muscle size, function, and quality concomitant with the 2 days per week training. 


\section{INSERT FIGURE 7 HERE}

\section{DISCUSSION}

Sarcopenia consists of muscle mass decreases of 1-2\% per year beyond 50 years of age so that 30\% of muscle mass and strength is lost by the eighth decade of life (18). Concomitant with this decline is increased incidence of falls, insulin resistance, mitochondrial impairment, chronic heart failure, and bone density loss - all conditions which high-intensity resistance exercise demonstrates potential to help prevent, delay, or improve (1, 23, 30). Unfortunately with aging, adaptation to various high-intensity resistance exercise programs becomes limited as well $(14,32,36)$. Therefore, systematic research is necessary to identify the key exercise parameters to ensure adaptation to high-intensity training at old age. Utilizing an experimental rodent model in the present study, allowed us to precisely test whether at advanced age, muscle adaptation can occur following training at the highest intensity possible (i.e. maximal muscle activation) for chronic SSC exposure. We demonstrate successful skeletal muscle enhancement following this high-intensity SSC training when frequency of training is optimized at old age. Following 3 days per week training, positive work capacity diminished, muscle mass was unaltered, and muscle quality was low while 2 days per week training preserved positive work capacity, induced muscle mass gains and restored muscle quality values to values comparable to those of young rats. The restoration of final muscle quality to young levels is impressive given the $20 \%$ decrement in non-trained muscle quality values with aging (old vs. young; $0.55 \pm 0.13$ vs. $0.69 \pm 0.04 \mathrm{~N} / \mathrm{mg} / \mathrm{mm}, P=0.027$ ) observed for Fisher Brown Norway hybrid rats reported on in a previous study (3). Overall, such an improvement to chronic exposure of maximal intensity contractions demonstrates that high-intensity SSC training is capable of rejuvenating skeletal muscle at advanced age given training frequency is modulated appropriately.

The muscles of young rats adapted to the SSC training with a 20\% increase in muscle mass and peak force capacity regardless of whether frequency of training was 2 or 3 days per week. Research regarding human subjects indicate that muscles of young individuals can also adapt similarly in terms of muscle size and strength to two distinct exercise modes, conventional resistance training vs plyometric training (39). Two reports regarding human subjects suggest that a high responsiveness to exercise is present at such young ages as adolescence and the transition from adolescence to adulthood.(13, 29) Compared with adults, exercise-induced growth hormone response (13) and gains in explosive power (29) were superior in young subjects. Likewise, for rats exposed to 1 month of SSCs 3 days per week, muscles of young rats (3 months old) increase isometric and dynamic force output by $20 \%$ to $30 \%$ whereas at adulthood (6 months), no such force gains are realized (31). Overall, these robust responses to a wide range of exposures at young age are consistent with an exceptional adaptive capacity at this stage of life.

Substantial muscle fiber size gains have been realized in older men and women at such low training frequencies as 1 to 2 days per week $(15,16,23)$. Indeed for the elderly, low volume of training has been 
previously recommended based on the observation of compromised recovery following contractions with aging.(17, 19) In a recent report focused on exercise mode, 60-70 year old males gained muscle size and strength following a regimen consisting of SSCs for 10-weeks (37). The frequency of training was 2 to 3 days per week such that the exposure for the first week was 2 days per week, the exposure for the second and third weeks were 3 days per week, and then this exposure sequence was repeated for the remaining 7 weeks. Overall, the findings suggest that aging is accompanied by an increased sensitivity to training frequency.

For muscles of young rats, maintenance of relatively low levels of lipid peroxidation was presumably conducive for adaptation. However, a training-induced alteration in the spatial distribution of lipid peroxidation towards increased lipid peroxidation at the sarcolemma was observed by 4-HNE staining. This indicated that an increased dispersal of lipid peroxidation (rather than increased tissue levels) accompanies adaptation. With aging, substantial lipid peroxidation at the sarcolemma was present even in non-trained muscles. In this scenario, this may have been the result of excessive lipid peroxidation since high tissue levels of lipid peroxidation were observed. Such age-related increases in lipid peroxidation levels have been observed previously.(33, 34) The unique finding of the present investigation was the lowered lipid peroxidation levels for old rats exclusively following the adaptive 2 days per week training. This implies that when baseline lipid peroxidation levels exceed a certain threshold as in the case of old rats of the present study, a decreased training frequency is necessary to allow training to diminish such a heightened state of oxidative stress and permit muscle adaptation. A negative correlation between lipid peroxidation status and SSC-induced performance is consistent with previous investigations in two studies by Ryan et al.(33, 34) In these reports, 3 days per week SSC training was accompanied by decreased lipid peroxidation levels and a lack of maladaptive performance. Ryan et al. described how the lack of maladaptive performance in these two studies was atypical and attributed this to differences in individual animal cohorts obtained from the National Institute of Aging, a possibility because of the tendency for increased variation observed with increased age of organisms/subjects.(8, 9, 24, 33, 34) Nevertheless, the general finding from these studies was that low lipid peroxidation levels accompany a beneficial functional response. Future study is required to confirm whether the lowered lipid peroxidation levels induced by 2 days per week training are indeed indicative of a role for overall oxidative stress in the outcome to high-intensity SSC training. Such a role for oxidative stress in adaptation/maladaptation is consistent with the finding that antioxidant supplementation with Vitamin E and Vitamin C increases oxidant buffering capacity and improves adaptation to SSC training for old rats.(34) Provided the lipid peroxidation data are representative of oxidative stress, the present study indicates that increasing recovery time between training sessions at advanced age decreases the redox environment to low levels, an environment important for maintaining performance during individual contractions as well as diminishing risk of other age-related oxidative stress induced impairments (34). Therefore, such a modulation of high-intensity resistance exercise has the potential to revitalize skeletal muscles at old age and improve quality of life at advanced age. 


\section{ACKNOWLEDGEMENTS}

This study was supported by Internal NIOSH funds.

\section{AUTHOR DISCLOSURE STATEMENT}

No conflicting financial interests exist. 


\section{REFERENCES}

1. Alves JP, Nunes RB, Stefani GP, and Dal Lago P. Resistance training improves hemodynamic function, collagen deposition and inflammatory profiles: experimental model of heart failure. PLoS One 9: e110317, 2014.

2. Baker BA, Hollander MS, Kashon ML, and Cutlip RG. Effects of glutathione depletion and age on skeletal muscle performance and morphology following chronic stretch-shortening contraction exposure. Eur $J$ Appl Physiol 108: 619-630, 2010.

3. Baker BA, Hollander MS, Mercer RR, Kashon ML, and Cutlip RG. Adaptive stretch-shortening contractions: diminished regenerative capacity with aging. Appl Physiol Nutr Metab 33: 1181-1191, 2008.

4. Baker BA, Mercer RR, Geronilla KB, Kashon ML, Miller GR, and Cutlip RG. Impact of repetition number on muscle performance and histological response. Med Sci Sports Exerc 39: 1275-1281, 2007.

5. Baker BA, Mercer RR, Geronilla KB, Kashon ML, Miller GR, and Cutlip RG. Stereological analysis of muscle morphology following exposure to repetitive stretch-shortening cycles in a rat model. Appl Physiol Nutr Metab 31: 167-179, 2006.

6. Barbe MF, Gallagher S, Massicotte VS, Tytell M, Popoff SN, and Barr-Gillespie AE. The interaction of force and repetition on musculoskeletal and neural tissue responses and sensorimotor behavior in a rat model of work-related musculoskeletal disorders. BMC Musculoskelet Disord 14: 303, 2013.

7. Borde R, Hortobagyi T, and Granacher U. Dose-response relationships of resistance training in healthy old adults: a systematic review and meta-analysis. Sports Med 45: 1693-1720, 2015.

8. Cutlip RG, Baker BA, Geronilla KB, Kashon ML, and Wu JZ. The influence of velocity of stretchshortening contractions on muscle performance during chronic exposure: age effects. Appl Physiol Nutr Metab 32: 443-453, 2007.

9. Cutlip RG, Baker BA, Geronilla KB, Mercer RR, Kashon ML, Miller GR, Murlasits Z, and Alway SE. Chronic exposure to stretch-shortening contractions results in skeletal muscle adaptation in young rats and maladaptation in old rats. Appl Physiol Nutr Metab 31: 573-587, 2006.

10. Cutlip RG, Geronilla KB, Baker BA, Kashon ML, Miller GR, and Schopper AW. Impact of muscle length during stretch-shortening contractions on real-time and temporal muscle performance measures in rats in vivo. J Appl Physiol (1985) 96: 507-516, 2004. 
11. Fry AC, Kraemer WJ, van Borselen F, Lynch JM, Marsit JL, Roy EP, Triplett NT, and Knuttgen HG. Performance decrements with high-intensity resistance exercise overtraining. Med Sci Sports Exerc 26: 1165-1173, 1994.

12. Geronilla KB, Miller GR, Mowrey KF, Wu JZ, Kashon ML, Brumbaugh K, Reynolds J, Hubbs A, and Cutlip RG. Dynamic force responses of skeletal muscle during stretch-shortening cycles. Eur J Appl Physiol 90: 144-153, 2003.

13. Gilbert KL, Stokes KA, Hall GM, and Thompson D. Growth hormone responses to 3 different exercise bouts in 18- to 25- and 40- to 50-year-old men. Appl Physiol Nutr Metab 33: 706-712, 2008.

14. Greig CA, Gray C, Rankin D, Young A, Mann V, Noble B, and Atherton PJ. Blunting of adaptive responses to resistance exercise training in women over 75y. Exp Gerontol 46: 884-890, 2011.

\section{Hakkinen K, Newton RU, Gordon SE, McCormick M, Volek JS, Nindl BC, Gotshalk LA,} Campbell WW, Evans WJ, Hakkinen A, Humphries BJ, and Kraemer WJ. Changes in muscle morphology, electromyographic activity, and force production characteristics during progressive strength training in young and older men. J Gerontol A Biol Sci Med Sci 53: B415-423, 1998.

16. Hakkinen K, Pakarinen A, Kraemer WJ, Hakkinen A, Valkeinen H, and Alen M. Selective muscle hypertrophy, changes in EMG and force, and serum hormones during strength training in older women. $J$ Appl Physiol (1985) 91: 569-580, 2001.

17. Hunter GR, McCarthy JP, and Bamman MM. Effects of resistance training on older adults. Sports Med 34: 329-348, 2004.

18. Kim TN, and Choi KM. Sarcopenia: definition, epidemiology, and pathophysiology. Journal of bone metabolism 20: 1-10, 2013.

19. Kosek DJ, Kim JS, Petrella JK, Cross JM, and Bamman MM. Efficacy of 3 days/wk resistance training on myofiber hypertrophy and myogenic mechanisms in young vs. older adults. J Appl Physiol (1985) 101: 531-544, 2006.

20. Lobelo F, Stoutenberg M, and Hutber A. The Exercise is Medicine Global Health Initiative: a 2014 update. Br J Sports Med 48: 1627-1633, 2014.

21. Lucas SJ, Cotter JD, Brassard P, and Bailey DM. High-intensity interval exercise and cerebrovascular health: curiosity, cause, and consequence. J Cereb Blood Flow Metab 35: 902-911, 2015. 
22. Massicotte VS, Frara N, Harris MY, Amin M, Wade CK, Popoff SN, and Barbe MF. Prolonged performance of a high repetition low force task induces bone adaptation in young adult rats, but loss in mature rats. Exp Gerontol 72: 204-217, 2015.

23. Melov S, Tarnopolsky MA, Beckman K, Felkey K, and Hubbard A. Resistance exercise reverses aging in human skeletal muscle. PLoS One 2: e465, 2007.

24. Murlasits Z, Cutlip RG, Geronilla KB, Rao KM, Wonderlin WF, and Alway SE. Resistance training increases heat shock protein levels in skeletal muscle of young and old rats. Exp Gerontol 41: 398-406, 2006.

25. Naci H, and Ioannidis JP. Comparative effectiveness of exercise and drug interventions on mortality outcomes: metaepidemiological study. BMJ 347: f5577, 2013.

26. Nicholson VP, McKean MR, and Burkett BJ. Low-load high-repetition resistance training improves strength and gait speed in middle-aged and older adults. J Sci Med Sport 18: 596-600, 2015.

27. Oliveira PF, Gadelha AB, Gauche R, Paiva FM, Bottaro M, Vianna LC, and Lima RM. Resistance training improves isokinetic strength and metabolic syndrome-related phenotypes in postmenopausal women. Clin Interv Aging 10: 1299-1304, 2015.

28. Pedersen BK, and Saltin B. Exercise as medicine - evidence for prescribing exercise as therapy in 26 different chronic diseases. Scand J Med Sci Sports 25 Suppl 3: 1-72, 2015.

29. Pesta D, Thaler A, Hoppel F, Macek C, Schocke M, and Burtscher M. Effects of a 10-week conventional strength training program on lower leg muscle performance in adolescent boys compared to adults. The Journal of sports medicine and physical fitness 54: 147-153, 2014.

30. Phillips MD, Flynn MG, McFarlin BK, Stewart LK, and Timmerman KL. Resistance training at eight-repetition maximum reduces the inflammatory milieu in elderly women. Med Sci Sports Exerc 42: 314325, 2010.

31. Rader EP, Layner KN, Triscuit AM, Chetlin RD, Ensey J, and Baker BA. Age-dependent muscle adaptation after chronic stretch-shortening contractions in rats. Aging Dis 2015.

32. Raue U, Slivka D, Minchev K, and Trappe S. Improvements in whole muscle and myocellular function are limited with high-intensity resistance training in octogenarian women. J Appl Physiol (1985) 106: 1611-1617, 2009. 
33. Ryan MJ, Dudash HJ, Docherty M, Geronilla KB, Baker BA, Haff GG, Cutlip RG, and Alway SE. Aging-dependent regulation of antioxidant enzymes and redox status in chronically loaded rat dorsiflexor muscles. J Gerontol A Biol Sci Med Sci 63: 1015-1026, 2008.

34. Ryan MJ, Dudash HJ, Docherty M, Geronilla KB, Baker BA, Haff GG, Cutlip RG, and Alway SE. Vitamin E and C supplementation reduces oxidative stress, improves antioxidant enzymes and positive muscle work in chronically loaded muscles of aged rats. Exp Gerontol 45: 882-895, 2010.

35. Schoenfeld BJ, Wilson JM, Lowery RP, and Krieger JW. Muscular adaptations in low- versus highload resistance training: a meta-analysis. Eur J Sport Sci 16: 1-10, 2016.

36. Slivka D, Raue U, Hollon C, Minchev K, and Trappe S. Single muscle fiber adaptations to resistance training in old (>80 yr) men: evidence for limited skeletal muscle plasticity. American journal of physiology Regulatory, integrative and comparative physiology 295: R273-280, 2008.

\section{Vaczi M, Nagy SA, Koszegi T, Ambrus M, Bogner P, Perlaki G, Orsi G, Toth K, and Hortobagyi}

T. Mechanical, hormonal, and hypertrophic adaptations to 10 weeks of eccentric and stretch-shortening cycle exercise training in old males. Exp Gerontol 58: 69-77, 2014.

38. Van Roie E, Delecluse C, Coudyzer W, Boonen S, and Bautmans I. Strength training at high versus low external resistance in older adults: effects on muscle volume, muscle strength, and force-velocity characteristics. Exp Gerontol 48: 1351-1361, 2013.

39. Vissing K, Brink M, Lonbro S, Sorensen H, Overgaard K, Danborg K, Mortensen J, Elstrom O, Rosenhoj N, Ringgaard S, Andersen JL, and Aagaard P. Muscle adaptations to plyometric vs. resistance training in untrained young men. J Strength Cond Res 22: 1799-1810, 2008. 
Table 1. Quantitative morphology for SSC trained and contralateral control muscles of young and old rats.

\begin{tabular}{|c|c|c|c|c|c|c|c|c|}
\hline & \multicolumn{4}{|c|}{ Young } & \multicolumn{4}{|c|}{ Old } \\
\hline & \multicolumn{2}{|c|}{3 days/wk } & \multicolumn{2}{|c|}{2 days/wk } & \multicolumn{2}{|c|}{3 days/wk } & \multicolumn{2}{|c|}{2 days/wk } \\
\hline & Contralateral & Trained & Contralateral & Trained & $\begin{array}{c}\text { Contralater } \\
\text { al }\end{array}$ & Trained & $\begin{array}{c}\text { Contralater } \\
\text { al }\end{array}$ & Trained \\
\hline $\begin{array}{l}\text { Normal muscle } \\
\text { fibers } \\
\text { (\% of tissue) }\end{array}$ & $93.4 \pm 0.7$ & $\begin{array}{c}92.9 \pm \\
1.1\end{array}$ & $95.4 \pm 1.0$ & $94.6 \pm 1.2$ & $92.7 \pm 0.4$ & $91.3 \pm 1.6 \neq$ & $92.4 \pm 0.8 \neq$ & $91.8 \pm 0.7 \ddagger$ \\
\hline $\begin{array}{l}\text { Degenerative } \\
\text { muscle fibers } \\
\text { (\% of tissue) }\end{array}$ & $0.0 \pm 0.0$ & $0.6 \pm 0.3$ & $0.0 \pm 0.0$ & $0.0 \pm 0.0$ & $0.5 \pm 0.4$ & $0.1 \pm 0.0$ & $0.4 \pm 0.1$ & $0.2 \pm 0.1$ \\
\hline $\begin{array}{l}\text { Cellular } \\
\text { interstitium } \\
\text { (\% of tissue) }\end{array}$ & $1.7 \pm 0.3$ & $1.6 \pm 0.2$ & $1.7 \pm 0.2$ & $1.3 \pm 0.2$ & $2.1 \pm 0.2$ & $2.3 \pm 0.6$ & $1.4 \pm 0.2$ & $1.9 \pm 0.3$ \\
\hline $\begin{array}{l}\text { Non-cellular } \\
\text { interstitium } \\
\text { (\% of tissue) }\end{array}$ & $4.9 \pm 0.9$ & $5.0 \pm 1.2$ & $2.9 \pm 0.9$ & $4.1 \pm 1.0$ & $4.7 \pm 0.8$ & $6.3 \pm 1.1$ & $5.8 \pm 0.8$ & $6.3 \pm 0.8$ \\
\hline
\end{tabular}

Sample sizes were $N=4$ to 5 per group. Values are means \pm S.E.M. $\neq$ Different from young 2 days/wk value, $P$ $<0.05$. 
Table S1. Percentage of muscle fibers designated by minimum feret diameter (increments of $10 \mu \mathrm{m}$ ).

\begin{tabular}{|c|c|c|c|}
\hline & $\begin{array}{l}\text { Minimum feret } \\
\text { diameter }(\mu \mathrm{m})\end{array}$ & $\begin{array}{c}\text { Contralateral - number of } \\
\text { muscle fibers (\%) }\end{array}$ & $\begin{array}{c}\text { Trained - number of } \\
\text { muscle fibers }(\%)\end{array}$ \\
\hline \multicolumn{4}{|l|}{ Young - 3 days/week } \\
\hline & 10 & $0.0 \pm 0.0$ & $0.2 \pm 0.4$ \\
\hline & 20 & $0.2 \pm 0.5$ & $0.2 \pm 0.3$ \\
\hline & 30 & $3.7 \pm 2.6$ & $1.2 \pm 1.2$ \\
\hline & 40 & $27.5 \pm 2.6$ & $15.5 \pm 5.6$ \\
\hline & 50 & $26.9 \pm 2.8$ & $33.9 \pm 3.5$ \\
\hline & 60 & $20.6 \pm 2.4$ & $25.0 \pm 4.7$ \\
\hline & 70 & $14.6 \pm 3.6$ & $15.0 \pm 2.4$ \\
\hline & 80 & $5.2 \pm 1.8$ & $6.3 \pm 2.6$ \\
\hline & 90 & $1.1 \pm 1.0$ & $1.9 \pm 2.1$ \\
\hline & $\geq 100$ & $0.1 \pm 0.2$ & $0.7 \pm 1.1$ \\
\hline \multicolumn{4}{|l|}{ Young - 2 days/week } \\
\hline & 10 & $0.0 \pm 0.0$ & $0.0 \pm 0.0$ \\
\hline & 20 & $0.1 \pm 0.2$ & $0.2 \pm 0.3$ \\
\hline & 30 & $2.0 \pm 1.2$ & $1.1 \pm 0.7$ \\
\hline & 40 & $22.3 \pm 6.8$ & $11.2 \pm 5.7$ \\
\hline & 50 & $31.1 \pm 4.4$ & $32.3 \pm 3.7$ \\
\hline & 60 & $23.2 \pm 3.5$ & $26.3 \pm 3.7$ \\
\hline & 70 & $12.0 \pm 4.2$ & $18.0 \pm 2.1$ \\
\hline & 80 & $5.9 \pm 2.1$ & $6.6 \pm 4.1$ \\
\hline & 90 & $2.1 \pm 2.6$ & $3.2 \pm 2.0$ \\
\hline & $\geq 100$ & $1.3 \pm 2.4$ & $1.2 \pm 1.6$ \\
\hline \multicolumn{4}{|l|}{ Old -3 days/week } \\
\hline & 10 & $0.0 \pm 0.0$ & $0.0 \pm 0.0$ \\
\hline & 20 & $0.7 \pm 0.9$ & $0.6 \pm 0.5$ \\
\hline & 30 & $6.3 \pm 2.7$ & $5.4 \pm 2.6$ \\
\hline & 40 & $19.0 \pm 6.2$ & $17.8 \pm 6.1$ \\
\hline & 50 & $30.5 \pm 0.7$ & $29.6 \pm 8.5$ \\
\hline & 60 & $21.5 \pm 2.0$ & $23.1 \pm 6.5$ \\
\hline & 70 & $11.8 \pm 3.2$ & $13.8 \pm 2.4$ \\
\hline & 80 & $5.7 \pm 2.3$ & $5.9 \pm 2.4$ \\
\hline & 90 & $3.1 \pm 1.6$ & $2.6 \pm 1.7$ \\
\hline & $\geq 100$ & $1.4 \pm 1.1$ & $1.3 \pm 1.4$ \\
\hline \multicolumn{4}{|l|}{ Old - 2 days/week } \\
\hline & 10 & $0.1 \pm 0.2$ & $0.3 \pm 0.6$ \\
\hline & 20 & $0.5 \pm 0.7$ & $0.7 \pm 1.3$ \\
\hline & 30 & $6.8 \pm 0.8$ & $2.7 \pm 3.9$ \\
\hline & 40 & $17.2 \pm 4.2$ & $13.2 \pm 4.1$ \\
\hline & 50 & $28.9 \pm 2.6$ & $27.9 \pm 4.3$ \\
\hline & 60 & $22.8 \pm 3.1$ & $29.1 \pm 7.6$ \\
\hline & 70 & $12.2 \pm 3.9$ & $13.4 \pm 3.1$ \\
\hline & 80 & $7.0 \pm 2.7$ & $7.3 \pm 2.0$ \\
\hline & 90 & $3.1 \pm 1.2$ & $2.9 \pm 2.2$ \\
\hline & $\geq 100$ & $1.3 \pm 0.8$ & $2.4 \pm 2.6$ \\
\hline
\end{tabular}

Data were obtained from $N=4$ to 5 animals per group. Values are means \pm SD. 


\section{FIGURE LEGENDS}

Figure 1. Quantitative morphology was assessed by a standardized stereological method. Representative image for how stereological analysis was performed. At each site, points (i.e. nodes where lines intersect) of a 121point graticule overlaid a field at 40X magnification. Each point was identified as overlaying a normal muscle fiber (NMF), degenerative muscle fiber (DMF), cellular interstitium (CI), or non-cellular interstitium (NCI). Examples for each of these designations are labeled (arrows). Of the total 121 points for this image, the following were the designations: 105 NMF, 6 DM, 7 NCI, and 3 CI. Scale bar $=100 \mu \mathrm{m}$.

Figure 2. Effect of altering training frequency on dynamic performance. Initial and final values of (A) peak force, (B) negative work (i.e. work required to stretch muscle), and (C) positive work (i.e. work done by the muscle during shortening) were assessed. For young rats (final vs initial values), both 2 and 3 days per week training increased peak force and 2 days per week training increased negative work capacity (a trend was observed for 3 days per week training). For old rats, 3 days per week training induced a decrease in positive work capacity relative to initial values. This decrease was absent with 2 days per week training. The 2 days per week training also increased final peak force values relative to final values for 3 days per week training. Sample sizes were $N=4$ to 8 per group. Values are means \pm SD. *Different from initial value; $†$ Different from value for young rats exposed 3 days per week; $\ddagger$ Different from value for young rats exposed 2 days per week; $\S$ Different from value for old rats exposed 3 days per week, $P<0.05$.

Figure 3. Decreasing the frequency of training improved muscle mass and static performance for old rats. (A) Maximum isometric forces were assessed for the initial and final weeks of training. (B) TA muscle mass was normalized by tibia length. (C) Final maximum isometric forces were divided by normalized muscle mass of exposed muscles to determine muscle quality values. Sample sizes were $N=4$ to 8 per group. Values are means \pm SD. *Different from non-exposed value (i.e. contralateral muscle in context of muscle mass or initial data in context of maximum isometric force and muscle quality); †Different from value for young rats exposed 3 days per week; $\ddagger$ Different from value for young rats exposed 2 days per week; §Different from value for old rats exposed 3 days per week, $P<0.05$.

Figure 4. Decreasing SSC frequency enabled training induced shifts to larger muscle fibers for muscles of old rats. Frequency distributions of fiber minimum feret diameters of individual muscle fibers are presented as percentage of total fibers measured for each experimental group. Therefore, within each experimental group, the number of muscle fibers of a specific size were summed across all the muscles and then expressed as a percentage of total fibers counted for all of the muscles ( $N=4$ to 5 rats per group). Chi-square analysis was performed to determine alterations in distribution with training. All the groups with the exception of 3 days per week trained old rats demonstrated a significant difference in distribution. 
Figure 5. Transverse sections of contralateral and exposed muscles following SSC training stained with hematoxylin and eosin for young and old rats. Scale bar $=100 \mu \mathrm{m}$.

Figure 6. Immunofluorescence staining for the lipid peroxidation marker 4-HNE in contralateral and trained muscles for young and old rats. Scale bar $=50 \mu \mathrm{m}$.

Figure 7. Oxidative stress levels as determined by lipid peroxidation marker MDA, was elevated in muscles of old rats and restored to young levels exclusively by SSC training 2 days per week. MDA measures were normalized to total protein content. Lipid peroxidation was elevated by aging as evident by increased levels in the contralateral muscles of old rats trained 2 and 3 days per week relative to those of the 2 times per week trained young rats. A trend for greater age-related lipid peroxidation was observed between contralateral control muscles of 2 days per week trained old rats and 3 days per week trained young rats. The only difference observed between trained muscles and contralateral muscles were for old rats trained 2 days per week. Sample sizes were $N=4$ to 5 per group. Values are means \pm SD. *Different from value for contralateral muscles; $\nmid$ Different from value for young rats exposed 3 days per week; $\ddagger$ Different from value for young rats exposed 2 days per week, $P<0.05$. 
FIGURE 1

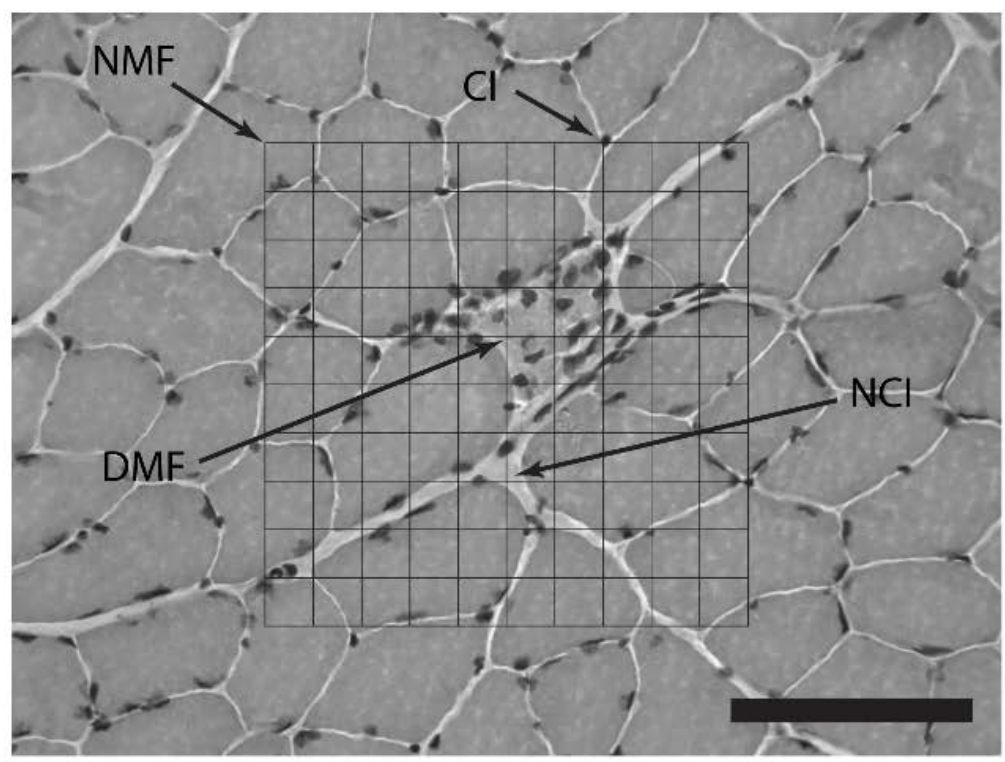


FIGURE 2

A

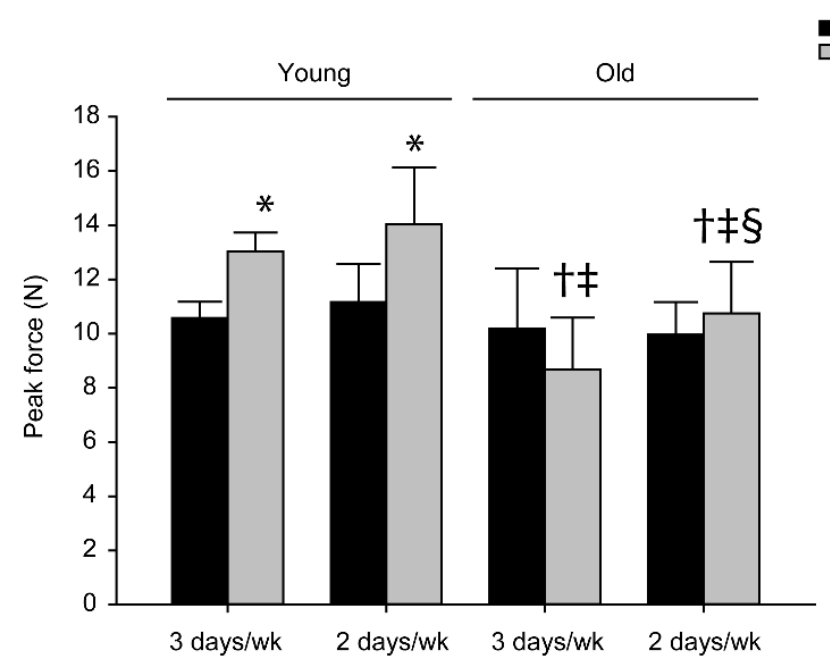

B

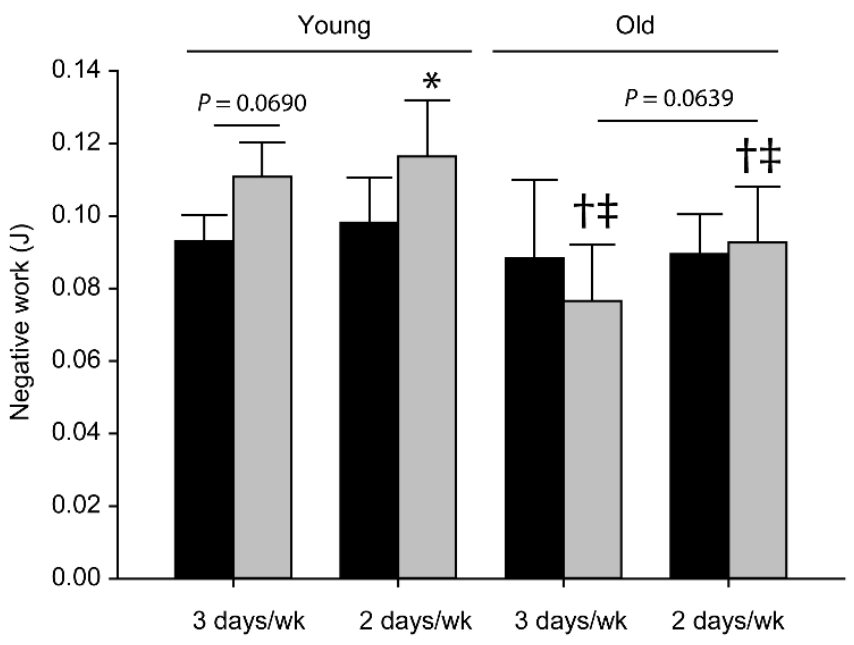

C

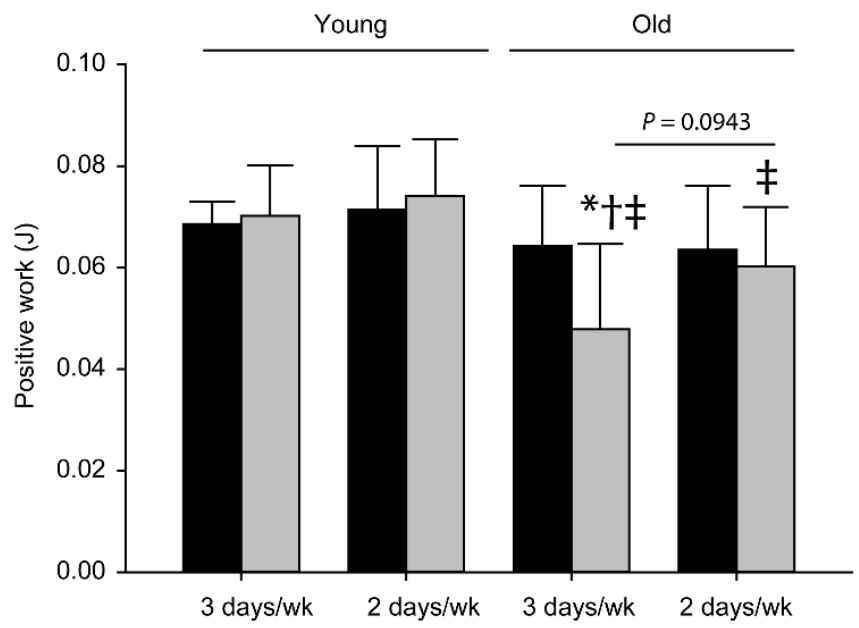


FIGURE 3

A

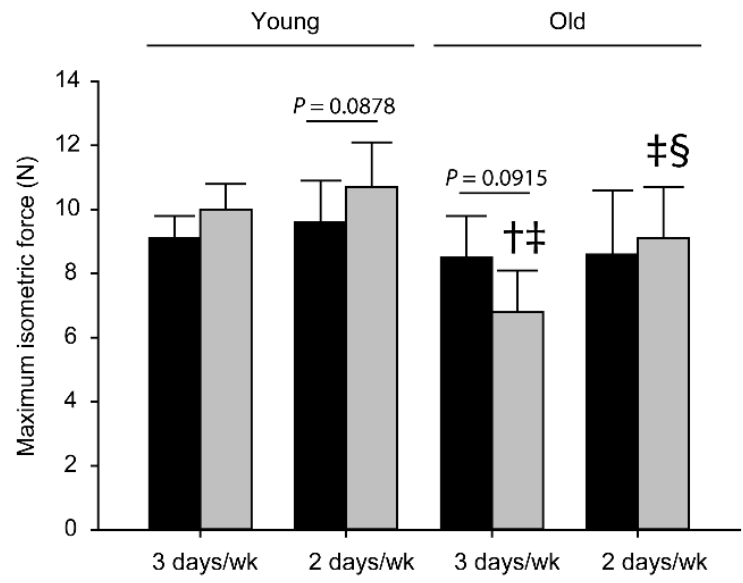

B

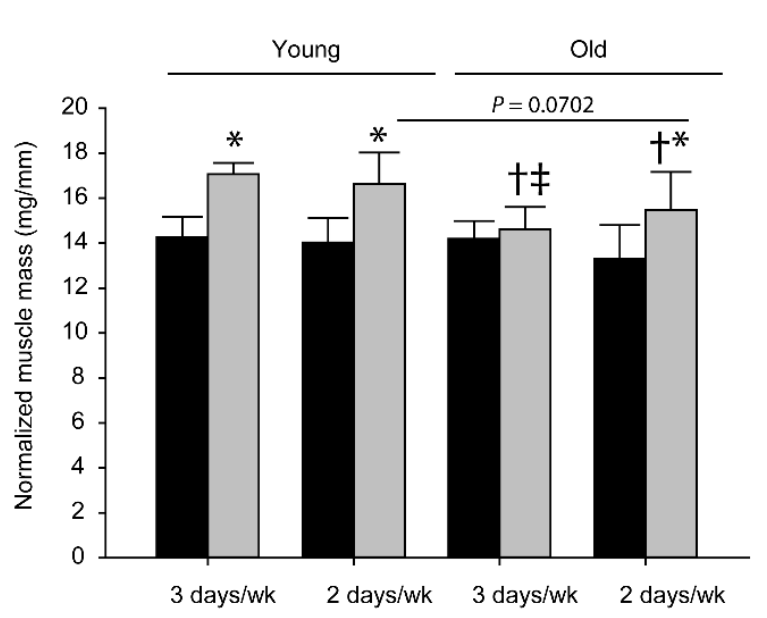

C

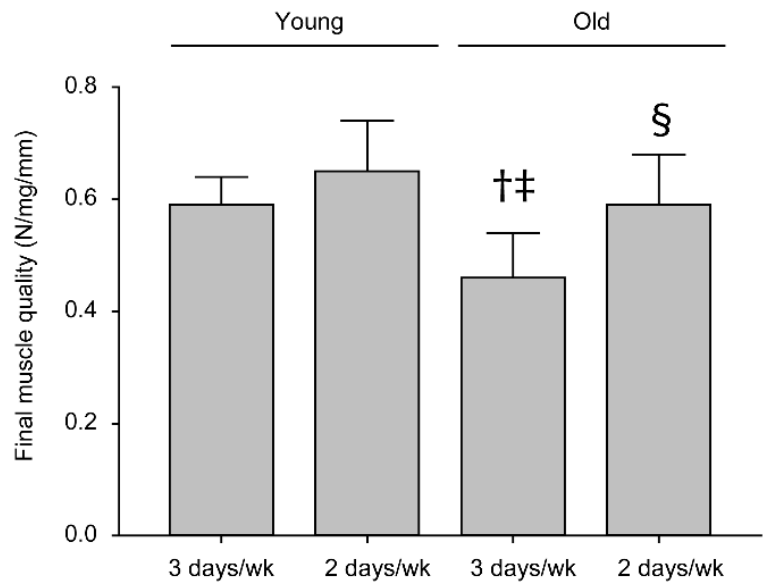




\section{FIGURE 4}

$$
\begin{aligned}
& \text { Contralateral } \\
& \text { Trained }
\end{aligned}
$$

A

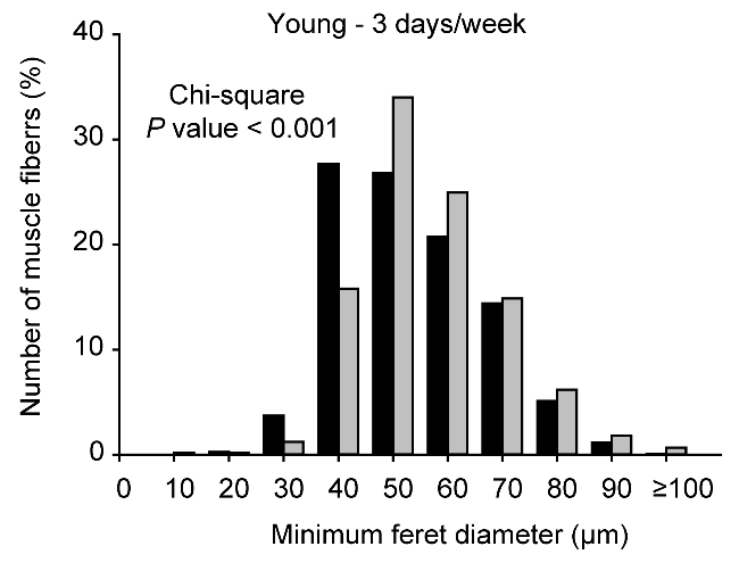

C

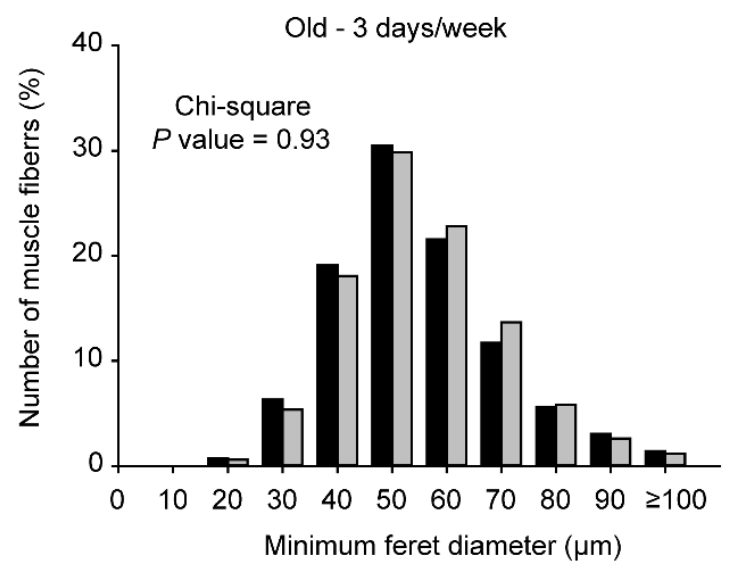

B

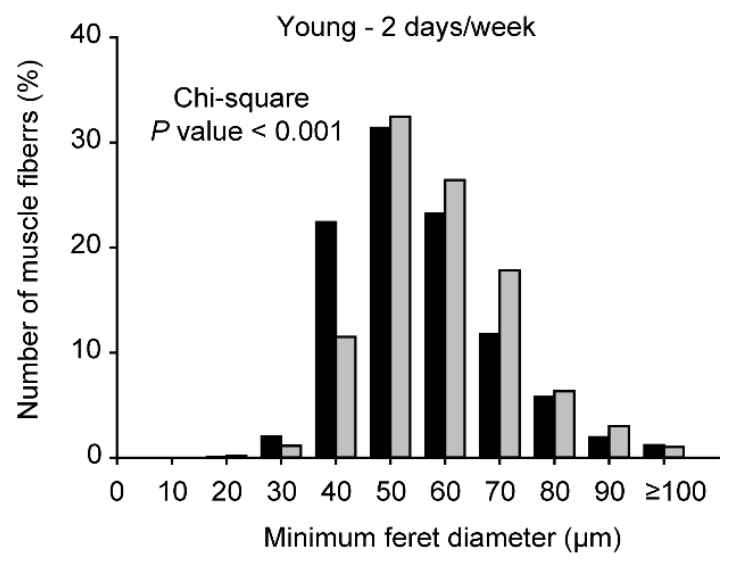

D

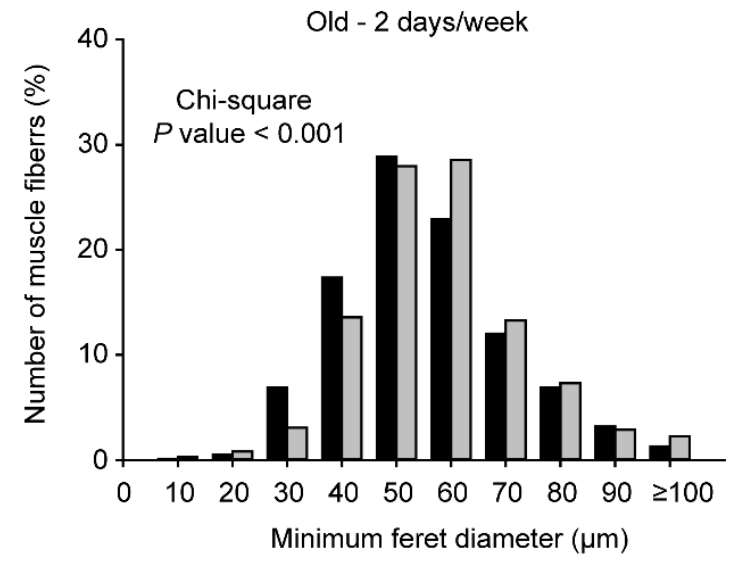


FIGURE 5
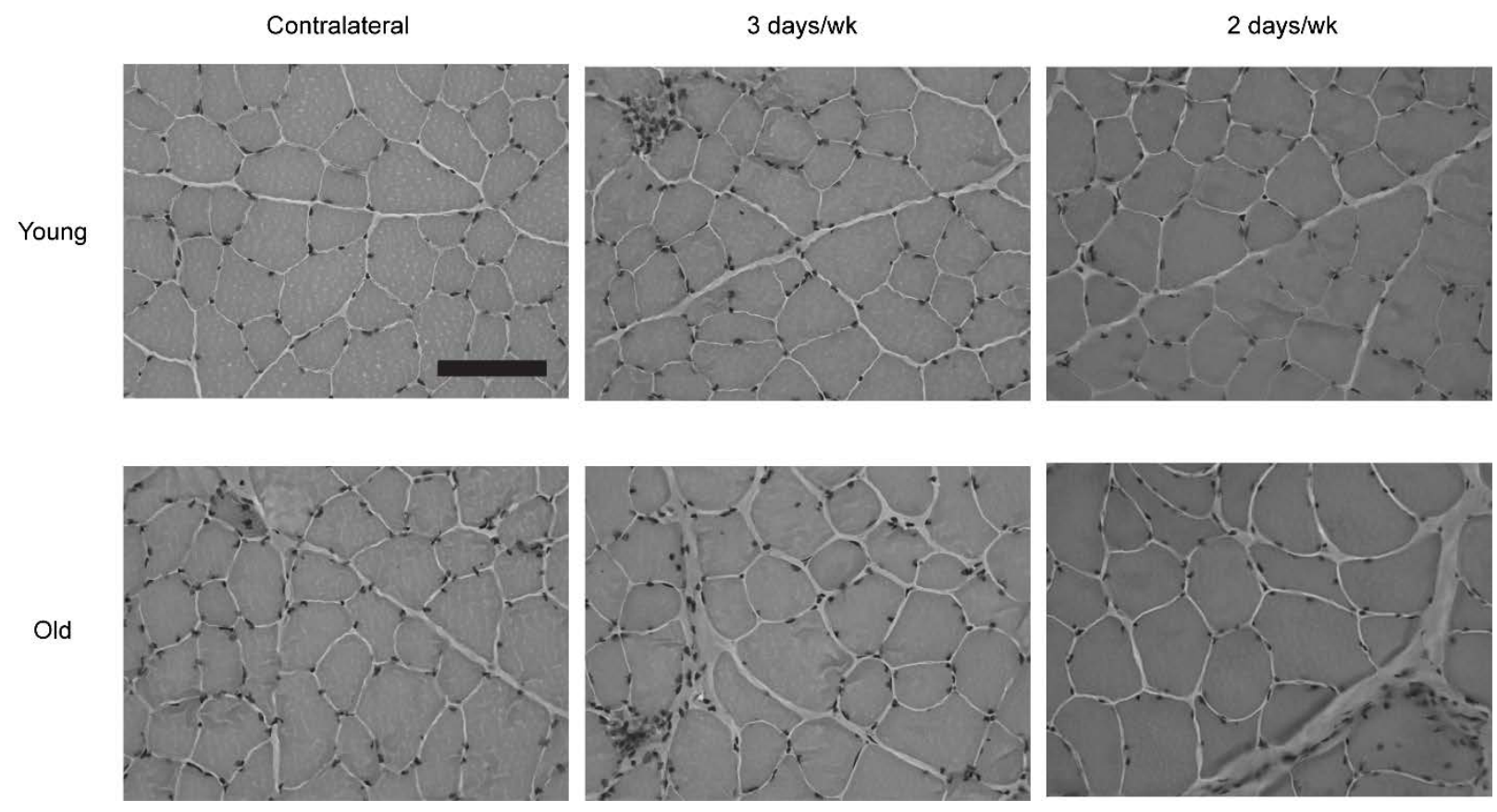
FIGURE 6

Contralateral

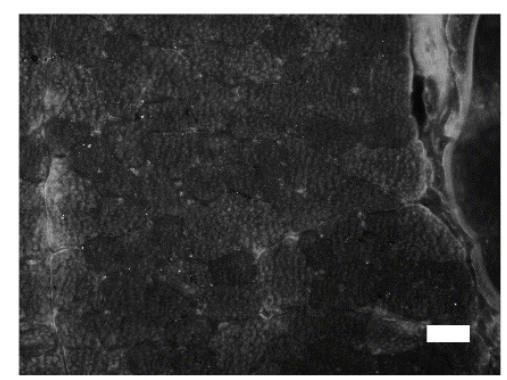

Old

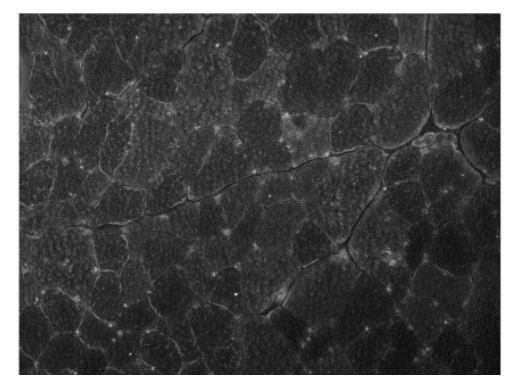

3 days/wk
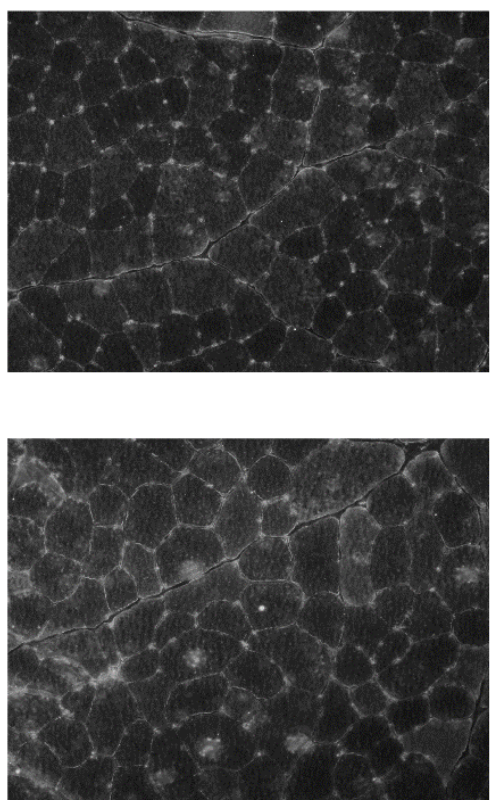

2 days/wk
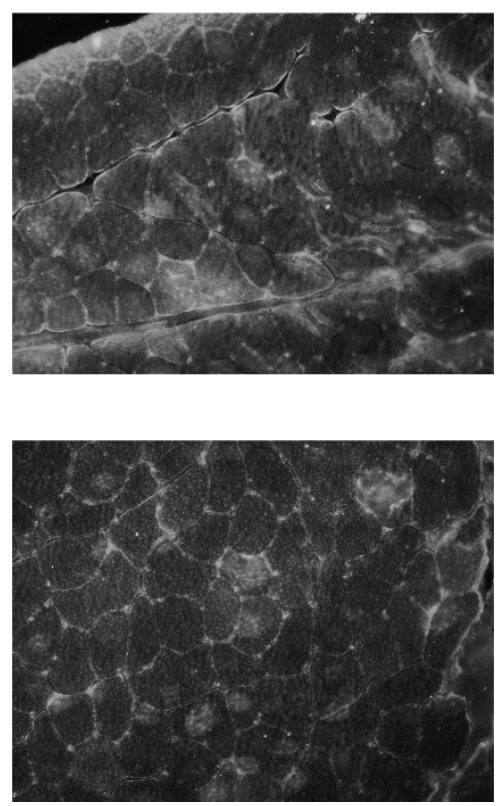
FIGURE 7

Contralateral

Trained

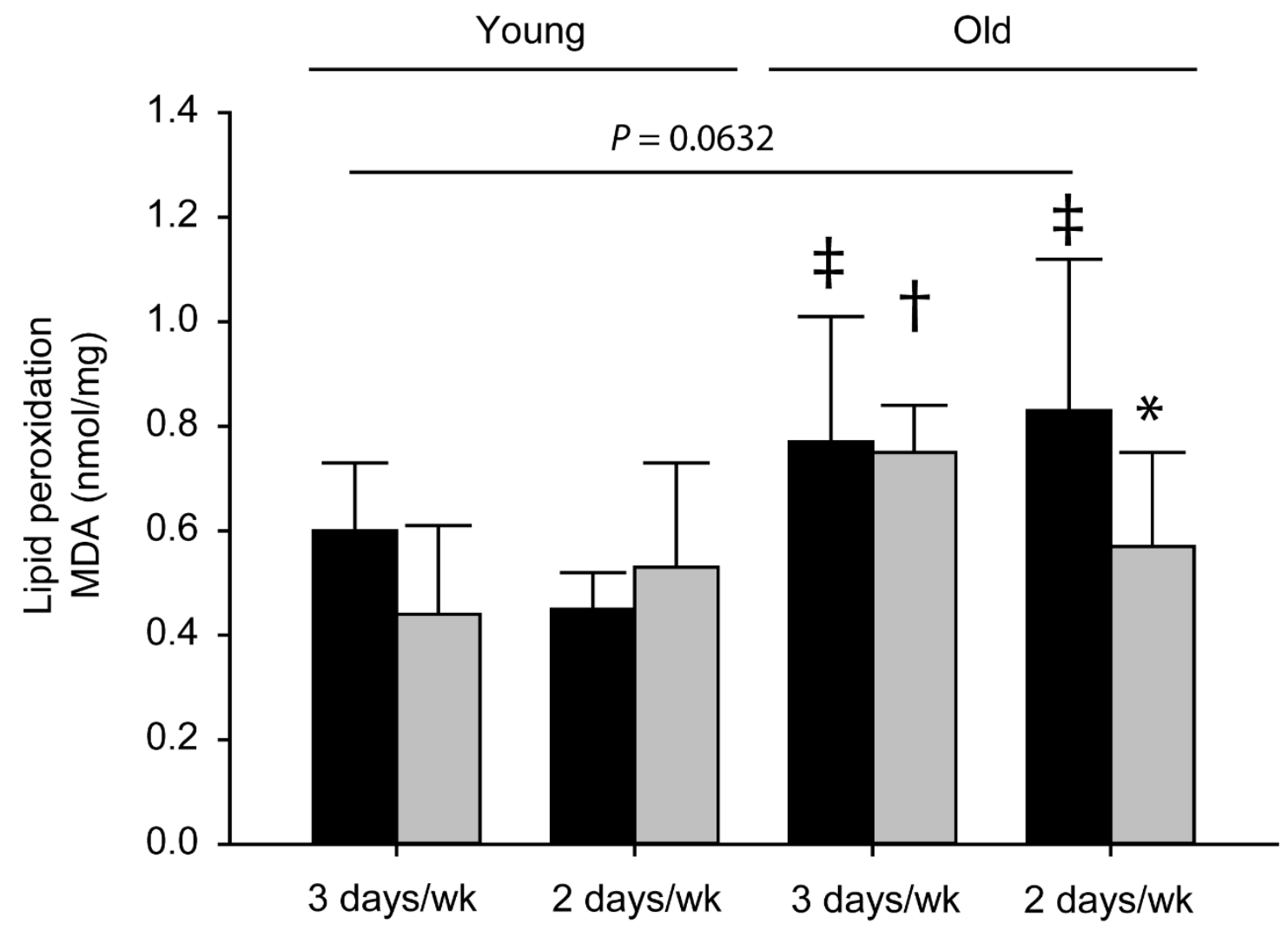




\section{CHAPTER IV}

REDUCED FREQUENCY OF RESISTANCE-TYPE EXERCISE TRAINING PROMOTES ADAPTATION

OF THE AGED SKELETAL MUSCLE MICROENVIRONMENT

Authors: Marshall A. Naimo ${ }^{1,2}$, Erik P. Rader ${ }^{1}$, James Ensey ${ }^{1}$, Michael L. Kashon ${ }^{1}$, Brent A. Baker ${ }^{1}$

\section{Author Contributions:}

M.A. Naimo, E.P. Rader, J. Ensey, M.L. Kashon, and B.A. Baker designed the study; M.A. Naimo, E.P. Rader, J. Ensey, and B.A. Baker conducted the study; M.A. Naimo and B.A. Baker collected the data; M.A. Naimo, M.L. Kashon, and B.A. Baker analyzed the data; M.A. Naimo and B.A. Baker interpreted the data; M.A. Naimo and B.A. Baker drafted the manuscript; M.A. Naimo, E.P. Rader, and B.A. Baker revised the manuscript content; M.A. Naimo, E.P. Rader, J. Ensey, M.L. Kashon, and B.A. Baker approved the final version of the manuscript; and B.A. Baker was responsible for the integrity of the data analysis.

Affiliations: ${ }^{1}$ Centers for Disease Control and Prevention, National Institute for Occupational Safety and Health, Health Effects Laboratory Division, Morgantown, West Virginia, USA; 'West Virginia University, School of Medicine, Division of Exercise Physiology, Morgantown, West Virginia, USA

Running Head: Growth and Remodeling Responses to Stretch-Shortening Contraction Training

Address for correspondence: Brent A. Baker, Centers for Disease Control and Prevention, National Institute for Occupational Safety and Health, Health Effects Laboratory Division, Morgantown, West Virginia, USA 1095 Willowdale Road, MS-L3027, Morgantown, WV 26505-2888,

Email: bwb3@cdc.gov 


\section{NEW \& NOTEWORTHY}

Decreasing resistance-type exercise training frequency in old rodents led to adaptation through enhancements in performance, fiber areas, and myonuclear domains. Modifying frequency influenced the molecular environment through improvements in PI3K-AKT pathway-specific expression and bioinformatics indicating increased protein synthesis. Reducing training frequency may be appropriate in older individuals who respond unfavorably to higher frequencies (i.e., maladaptation); overall, modifying the parameters of the exercise prescription can affect the cellular environment, ultimately leading to adaptive or maladaptive outcomes.

Keywords: Stretch-shortening contractions, Fisher 344 x Brown Norway rats, growth and remodeling, central nuclei, rejuvenation 


\section{PUBLICATIONS DISCLAIMER:}

"The findings and conclusions in this report are those of the author(s) and do not necessarily represent the official position of the National Institute for Occupational Safety and Health, Centers for Disease Control and Prevention.” 


\section{ABSTRACT}

The purpose of this study was to characterize the growth and remodeling molecular signaling response in aged skeletal muscle following one month of “resistance-type exercise” training. Male Fischer344x Brown Norway hybrid rats aged 3 (young) and 30 months (old) underwent stretch-shortening contraction (SSC) loading two or three days per week; muscles were removed 72 hours post-training. Young rats SSC-loaded three (Y3x) or two days per week (Y2x) adapted via increased work performance. Old rats SSC-loaded three days per week (O3x) maladapted via decreased negative work; however, old rats SSC-loaded two days per week (O2x) adapted through improved negative and positive work. Y3x, Y2x, and O2x, but not O3x, displayed hypertrophy via larger fiber area and myonuclear domains. Y3x, Y2x, and O2x differentially expressed 19, 30, and 8 PI3K-AKT genes, respectively, whereas O3x only expressed two. Bioinformatics analysis revealed that rats in the adapting groups presented growth and remodeling processes (i.e. increased protein synthesis), whereas O3x demonstrated inflammatory signaling. In conclusion, reducing SSC-loading frequency in aged rodents positively rejuvenates the molecular signaling microenvironment, promoting muscle adaptation. 


\section{INTRODUCTION}

Significant issues associated with aging, such as frailty and cachexia, are related to sarcopenia, typically defined as the age-related loss of skeletal muscle mass with concomitant weakness $(19,36)$. Thus, strategies aiming to improve muscle performance and muscle mass in older individuals should be pursued in order to improve functional capacity and decrease musculoskeletal disorders. Recently, age-related physical changes have been suggested to be modifiable by increased physical activity (14). Evidence for the pluripotent effects of exercise led both the American Medical Association and the American College of Sports Medicine to launch an initiative in 2007 called "Exercise is Medicine,” a global initiative to translationally incorporate scientifically supported health benefits of physical exercise into our healthcare system (9). Despite the therapeutic potential and supporting evidence, how age-specific resistance exercise positively influences the total health of an individual across the lifespan is equivocal. Furthermore, the integrated physiological pathways involved in exercise-induced muscle adaptation are not fully understood.

The responses to “resistance-type exercise” training (RTET) can range from adaptation, defined as gains in muscle mass and performance, to maladaptation, characterized by an absence of muscle mass gains and diminished performance (16). Exercise training involving the use of stretch-shortening contractions (SSCs), a common type of contraction in which the muscle is activated prior to and during the initial stretch and subsequent shortening (36), has been particularly beneficial in elucidating the benefits of exercise training. For nearly two decades, our laboratory has utilized an in vivo rodent dynamometer to investigate the underlying mechanics of muscle adaptation to $\operatorname{RTET}(4,17)$; this methodology allows for precise control of the biomechanical loading signature (i.e., frequency) and is minimally invasive. The combination of this methodology with the use of SSCs, as opposed to isometric or lengthening only contractions, makes it a physiologically representative model for the longitudinal study of muscle adaptation to RTET. Using a noninjurious paradigm of SSCs previously designed by our laboratory (8 sets of 10 SSCs, three days per week for 4.5 weeks) RTET-induced adaptation occurs in young rodents (16); however, this response is altered with age. Specifically, results demonstrated that aged rodents undergo a maladaptive response when exposed to this protocol $(5,6,16,35,36,41)$. Similarly, other studies in older humans have also found a reduced capacity to adapt to resistance exercise training $(37-39,44)$. Moreover, a recent NHANES analysis showed statistical associations between sufficient levels of resistance training and greater muscle quality with select age groups at early old age but not at later old age (30). These results, along with the studies mentioned above, indicate that as one gets older, appropriate age-specific exercise prescription may be a critical component that enables skeletal muscle adaptation in response to RTET.

In recent work by our laboratory, we investigated whether modulating the exercise prescription by reducing the training frequency from three to two days per week resulted in decreased oxidative stress and enhanced muscle quality in older rodents to similar levels to young rats that adapted (36). These results indicate that additional recovery between training bouts may be beneficial for developing a favorable redox environment 
and subsequently promoting muscle adaptation (34). Protein kinase B (AKT) is a serine/threonine kinase that is a widely accepted key regulator of muscle growth (21). Activation of AKT has several downstream

physiological outcomes, including roles in metabolism, protein synthesis, apoptosis, the regulation of transcription factors, and involvement in the cell cycle. Mechanical loading has been shown to cause increased activation of AKT independently of growth factors or nutrient stimulation through multiple mechanosensory molecules (26, 27). This suggests that SSC-loading may directly modify AKT signaling during active RTET, possibly leading to an adaptive or maladaptive fate. Despite the well-known benefits of PI3K-AKT signaling on the hypertrophic response to resistance exercise, no studies have examined the PI3K-AKT pathway response and determined the distinct molecular signature occurring with aging while connecting it to adaptive outcomes (i.e., force output, changes in muscle fiber area), particularly in a precisely controlled in vivo physiologically representative model of resistance exercise. Additionally, it is not known whether or not the PI3K-AKT pathway response is altered when parameters of the exercise prescription are manipulated in a manner that causes adaptation or maladaptation to occur. Therefore, the purpose of this study was to define and extend our previous findings by implementing an integrated systems biology approach using representative PI3K-AKT pathway gene expression and bioinformatics via ingenuity pathway analysis (IPA), in addition to biochemical, morphological, and physiological muscle measures in addition to muscle performance. Our hypothesis was that young rats would respond at the performance, physiological and molecular level in an adaptive manner regardless of whether or not training frequency was reduced, whereas old rats trained at a higher frequency would have a blunted response indicative of maladaptation. Additionally, we hypothesized that old rats trained at a reduced frequency would have a molecular growth and remodeling response that more closely resemble younger rats that adapt following SSC-training.

\section{MATERIALS AND METHODS}

\section{Animals}

Young (3 mo.) and old (30 mo.) male Fischer 344 x Brown Norway (F344xBN) rats were obtained from the National Institute of Aging colony and housed in an Association for Assessment and Accreditation of Laboratory Animal Care International-accredited animal quarters. All animal procedures were approved by the Animal Care and Use Committee at the National Institute for Occupational Safety and Health in Morgantown, WV and were in compliance with the NIH guide for the care and use of animals in research.

\section{RTET Paradigm}

F344xBN male rats were randomly assigned to a high-intensity SSC RTET protocol for 4.5 weeks in one of the following four groups: 1) Young rats SSC-loaded three days per week (Y3x; n=10); 2) Young rats SSC-loaded two days per week (Y2x; n=10); 3) Old rats SSC-loaded three days per week (O3x; n=10); 4) Old rats SSC-loaded two days per week $(\mathrm{O} 2 \mathrm{x} ; \mathrm{n}=8)$. Due to limited tissue availability, experiments that yielded the 
results done in Figures 2-4 were done on an subset of animals within each condition (n=4-6 per group) rather than the entire experimental cohort. The loading paradigms were identical with the exception of the training frequency. For the two days per week training groups, training was conducted on Mondays and Thursdays, with 72 hours between training bouts. For the three days per week training group, training was carried out on Mondays, Wednesdays, and Fridays, with 48 hours rest between training sessions. The training protocol was based on a previously validated, SSC RTET protocol $(16,17,36)$. Briefly, rats were anesthetized with isoflurane gas and placed in dorsal recumbency on a heated table with the left knee secured in flexion at $90^{\circ}$ and the left foot secured in a load cell. Platinum electrodes were then placed subcutaneously for activation of the dorsiflexor muscles. Muscle stimulation parameters (4-V magnitude, 0.2-ms pulse duration, and 120-Hz frequency) were based on previous studies of this model that found these settings to be optimal for producing supramaximal force outputs of the TA muscle in male rats $(16,17)$. Training consisted of 80 total SSCs per session, which comprised eight sets of 10 repetitions, with 2-minute rest intervals between sets. For every SSC, the muscles were maximally activated, with the ankle angle set to $90^{\circ}$ of flexion for $100 \mathrm{~ms}$, rotated out to $140^{\circ}$ of flexion at a movement velocity of $60^{\circ}$ s, then returned back to $90^{\circ}$ flexion at $60 \%$ s. Using this protocol, agespecific performance adaptation occurs with a concomitant absence of overt muscle inflammation and degeneration in the days and weeks following training $(5,6,16,36)$.

During each training session, muscle performance was assessed during the first SSC repetition of the first training set. Both the work required to stretch the muscle (i.e., negative work) and the work performed by the muscle during the shortening phase (i.e., positive work) were measured and recorded using MatLab software (Version 9.3; MathWorks ${ }^{\circledR}$, Natick, MA, USA). Seventy two hours after the final SSC-loading protocol, rats were euthanized and the tibialis anterior (TA) muscle was surgically removed, weighed, and recorded as the raw muscle mass (i.e., wet-weight) in grams. Seventy two hours was chosen because this would be beyond the acute effects of first several hours post-training, but within the timeline where any overt signs of muscle degeneration as a consequence to the last training session would occur (36). The mid-belly of the TA was coated with tissue freezing media (Tissue-Tek, 4583 O.C.T Compound; Sakura Finetek, Torrance, CA, USA) and frozen in isopentane cooled to the temperature of liquid nitrogen then stored at $-80^{\circ} \mathrm{C}$ for quantitative morphology, gene expression and protein analysis.

\section{Muscle Fiber Area, Myonuclei Number, and Myonuclear Domain}

Frozen TA muscle sections (12 $\mu$ m thickness) were previously stained for 4-hydroxynonenal (4-HNE; no. 393206; at 1:250; Calbiochem, La Jolla, CA, USA), $\beta$-dystroglycan (sc-33701; at 1:100; Santa Cruz Biotechnology, Dallas, TX, USA) in order to outline the muscle sarcolemma, and 4',6-diamidino-2phenylindole (DAPI) (P36931; Thermo Fisher Scientific, Rockville, IL, USA) stain for myonuclei and representative images were reported elsewhere (36). The midpoint of the TA muscle was identified and nonoverlapping images were captured at 40x magnification at the site of most area in both the lateral and medial 
regions of the TA muscle section. A standardized stereological method was used for quantitative morphology (7). Briefly, at two regions ( $1 \mathrm{~mm}$ to the right and $1 \mathrm{~mm}$ to the left of the section midline) the analysis was performed at five equally spaced sites across the muscle section. At each site, points of a 121-point, 11-line overlay graticule $\left(0.04 \mathrm{~mm}^{2}\right.$ square boundary with 100 divisions) was placed at the center of each image and was used to count various features, which included muscle fibers and myonuclei. A total of 1,210 points were analyzed per section since 10 total fields (i.e., images) were evaluated.

The investigator was blinded to sample identity and samples were analyzed utilizing Image J (Version 1.48; NIH, Bethesda, MD, USA). Each muscle fiber (208 \pm 21 fibers per section) was traced to determine muscle fiber area. Myonuclei were manually counted within the sarcoplasm regions of muscle sections. Myonuclei per unit of muscle cross-sectional area (nuclei per $\mathrm{mm}^{2}$ ) was determined by dividing the number of myonuclei by the total muscle fiber area sampled. The percentage of central nucleated fibers (CNFs) was quantified by dividing the number of muscle fibers that presented with central nuclei by the total number of muscle fibers sampled. Myonuclear domain (MND), typically defined as the volume unit of which the transcriptional activity level of a muscle fiber is under the direct control of a single myonucleus (15), was quantified as the ratio of the muscle fiber area relative to the number of myonuclei for each myofiber.

\section{Gene Expression Analysis}

Muscle tissue stored at $-80^{\circ} \mathrm{C}$ was retrieved and then a $50 \mathrm{mg}$ portion was homogenized using a MiniBeadbeater 8 (Biospec Products, Bartlesville, OK, USA) with $1 \mathrm{ml}$ TRIzol and $1.0 \mathrm{~mm}$ zirconia beads (Catalog \#22079110zx; Biospec). RNA was subsequently isolated using the RNAqueous ${ }^{\mathrm{TM}}$ Phenol-free total RNA Isolation Kit (Cat\# AM1912; Thermo Fisher Scientific) following kit instructions. Next, cDNA was synthesized using the RT ${ }^{2}$ First Strand Kit (Catalog\# 330401; Qiagen). Samples were then analyzed using the Rat Skeletal Muscle PI3K-AKT RT² Profiler PCR Array (Catalog\# PARN-058Z; Qiagen) and RT² SYBR Green Mastermix (Catalog\# 330523; Qiagen) per manufacturer’s instructions with use of an ABI PRISM 7500 instrument (Applied Biosystems, Foster City, CA, USA). The RT² Profiler PCR Array was able to simultaneously assay 84 genes related to the PI3K-AKT signaling pathway (Table 1). Fold changes and $P$ values were determined from comparisons of $\Delta \mathrm{C}_{\mathrm{t}}$ values between SSC-loaded and non-loaded control (NLC) muscles (i.e., the contralateral limb that was not subjected to RTET) and analyzed through the GeneGlobe Data Analysis Center (Qiagen). Bioinformatic analysis was performed using IPA (Ingenuity Systems, www.ingenuity.com), which allowed for functional annotation of differentially expressed genes. All IPA biological functions except cancer specific, reproductive system, and psychological functions were evaluated.

INSERT TABLE 1 HERE 
RNA was isolated following the protocol outlined in the previous section on gene expression. Total DNA was isolated using the DNeasy ${ }^{\circledR}$ Blood \& Tissue Kit (Qiagen) according to kit instructions. Both RNA and DNA concentrations were obtained using a spectrophotometer (ND-1000; NanoDrop Technologies Inc., Wilmington, DE, USA). Cytoplasmic protein isolation extracts were obtained from the TA muscles using a method described by Siu et al. (43). Total protein contents of the cytoplasmic protein extracts were quantified and reported as protein concentration ( $\mu$ g protein/mg tissue - [protein]) in triplicate using the Pierce ${ }^{\mathrm{TM}}$ BCA Protein Assay Kit (Thermo Scientific). Additionally, values for the [RNA] to [DNA] ratio (i.e., assumed to be a surrogate measure of global transcriptional efficiency) and the [protein] to [RNA] ratio (i.e., assumed to be a surrogate measure of global translational efficiency) were analyzed (40).

\section{Statistical Analysis}

Negative work, positive work, myonuclei count, muscle RNA, muscle DNA, muscle protein, muscle transcriptional efficiency, and muscle translational efficiency were all analyzed using a mixed model analysis of variance (ANOVA) with the variable animal identification as a random factor to account for repeated measures (JMP version 11; SAS Institute, Inc., Cary, NC, USA). PI3K-AKT PCR data was analyzed using unpaired student's t-test in the GeneGlobe Data Analysis Center (Qiagen) online platform. Chi-square analysis (SigmaPlot version 12.5; Systat Software, Inc., San Jose, CA, USA) was utilized to determine training-induced differences in the absolute frequency distributions of muscle fiber area and MND. All data are expressed as means \pm SE, with $p<0.05$ being considered statistically significant.

\section{RESULTS}

\section{Dorsiflexor Muscle Performance}

Negative work performed on the first SSC repetition of the first training set at initial and final is shown in Figure 1. In response to dorsiflexion SSC-loading, Y3x and Y2x adapted to SSC-training as indicated by significantly higher negative work following training (387 \pm 15 to $484 \pm 26 \mathrm{~N}$ and $395 \pm 20$ to $489 \pm 30 \mathrm{~N}$, respectively; $\mathrm{p}<0.01$ ), whereas O3x had significantly decreased negative work ( $343 \pm 20$ to $277 \pm 29 \mathrm{~N} ; \mathrm{p}<0.05$ ). Meanwhile, O2x had a trend for increased negative work following training (328 \pm 7 to $392 \pm 31 \mathrm{~N}$; $\mathrm{p}=0.07$ ). For positive work, Y3x had a trend for a higher initial value relative to O3x (296 \pm 20 vs. $240 \pm 21 \mathrm{~N}$; p = 0.08),

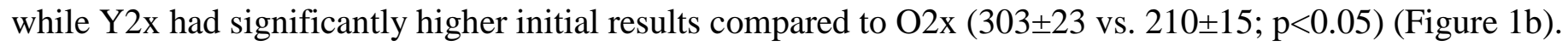
Following training, only O3x had significantly decreased positive work (240 \pm 21 to $174 \pm 24 \mathrm{~N}$; $\mathrm{p}<0.05$ ). Further, Y3x (317 $\pm 26 \mathrm{~N} ; \mathrm{p}<0.0001)$ and Y2x (323 $\pm 22 \mathrm{~N} ; \mathrm{p}<0.0001)$, as well as O2x $(241 \pm 23 \mathrm{~N} ; \mathrm{p}<0.05)$, exhibited significantly higher positive work than O3x (174 $\pm 24 \mathrm{~N})$. 


\section{Muscle Mass, Fiber Area, Myonuclei Count, Central Nuclei, and MND}

No differences were found between young and old NLC raw muscle mass (585 \pm 13 vs. $572 \pm 24$ mg; $\mathrm{p}>0.05)$. All four groups had significantly increased raw muscle mass following the SSC-loading training period

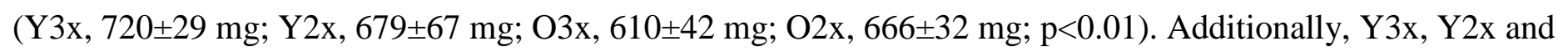
$\mathrm{O} 2 \mathrm{x}$ had significantly higher muscle mass compared to O3x following training $(\mathrm{p}<0.05)$. Frequency distributions for muscle fiber areas are shown in Figure 2. Following training, chi-square analysis revealed that muscle fiber area distributions there were significantly increased fiber areas for $\mathrm{Y} 3 \mathrm{x}, \mathrm{Y} 2 \mathrm{x}$ and $\mathrm{O} 2 \mathrm{x}$ relative to NLC muscle $(p<0.001)$ (Figure 2a-2b, 2d), whereas no changes were found in O3x $(p>0.05)$ (Figure 2c).

\section{INSERT FIGURE 2 HERE}

The total number of myonuclei and the percentage CNF results are shown in Table 2. Old NLC muscle had a significantly higher myonuclei count relative to young NLC muscle $(\mathrm{p}<0.05)$; there were no effects of three or two day per week loading on myonuclei counts in any group. Similar to myonuclei count, the percentage of CNFs were significantly higher in old compared to young NLC muscle $(\mathrm{p}<0.05)$. Following SSCloading, the percentage of CNF was significantly lower in O3x compared to old NLC muscle $(\mathrm{p}<0.05)$. Both young and old rats SSC-loaded two days per week had significantly higher percentage of CNFs relative to agematched counterparts ( $\mathrm{p}<0.05)$. MND was calculated by dividing muscle fiber area by the number of myonuclei for that myofiber (Figure 3). When looking at each SSC-loaded group relative to age-matched NLC muscle, all groups except for O3x had a significant increase in their respective MNDs following training, particularly for O2x $(p<0.001)$ (Figures 3a-3d).

\section{INSERT TABLE 2 HERE}

INSERT FIGURE 3 HERE

\section{PI3K-AKT Gene Expression and Bioinformatics Analysis}

The significantly differentially expressed genes (SDEGs) in Rat Skeletal Muscle PI3K-AKT RT ${ }^{2}$ Profiler PCR Array are shown in Table 3 and Figure 4 and are defined as having a p-value less than 0.05. Y3x and Y2x had 19 and 30 SDEGs, respectively, in the PI3K-AKT signaling pathway. In contrast, O3x only had 2 SDEGs; however, O2x had 8 SDEGs for the PI3K-AKT signaling pathway, a four-fold increase. Intriguingly, integrin beta 1 (Itgb1), a key regulator of mechanotransduction signaling events in skeletal muscle in response to exercise (10), was significantly upregulated in both Y3x and Y2x, as well as in O2x (1.53 fold vs. 1.95 fold vs. 2.34 fold), whereas there was only a trend for upregulation in O3x. Additionally, Fos and Jun, two important regulators of satellite cell activation (2), were significantly upregulated in O2x (Fos, 6.3 fold; Jun, 1.6 fold). 
INSERT TABLE 3 HERE

INSERT FIGURE 4 HERE

Bioinformatics analysis via IPA revealed several differential patterns when comparisons were made between the adapting groups versus the O3x maladapted group (Table 4). For the top canonical pathways, Y3x, Y2x and O2x had processes mainly associated with growth and remodeling events in muscle (e.g., ERK/MAPK signaling, mTOR signaling, HGF signaling, IGF-1 signaling), while old rats loaded three days per week had processes mainly related to inflammatory signaling (e.g., IL17A signaling, T-cell signaling). When evaluating the top networks, Y3x and Y2x had networks related to growth and remodeling processes such as cellular movement, cell death and survival, etc. After the networks in the adapted groups were merged in order to generate top merged-network functions, the processes were reflective of events associated with growth and/or the stress response to exercise (e.g., apoptosis, carbohydrate metabolism, cell cycle, and protein synthesis). O3x had a lone represented top network, and because of this, merged-network function analysis was unable to be performed. Meanwhile, O2x had two top networks and the top merged network functions was similar to both young groups, with processes related to the growth response to exercise (e.g., protein expression, proliferation of fibroblasts, angiogenesis).

\section{INSERT TABLE 4 HERE}

\section{Muscle RNA, DNA and Protein}

Figure 5 displays skeletal muscle concentrations for RNA, DNA, and protein. There were no age or treatment effects for any group on muscle [RNA] ( $>>0.05)$ (Figure 5a). For muscle [DNA], old NLC muscle had significantly higher concentrations compared to young NLC muscle $(\mathrm{p}<0.05)$ (Figure 5b); additionally, Y3x and O2x had significantly increased muscle [DNA] compared to age-matched NLC muscle $(\mathrm{p}<0.05)$. Moreover, O2x had significantly higher muscle [DNA] compared to Y2x $(\mathrm{p}<0.01)$. For muscle [protein], old NLC’s muscle [protein] was not different compared to young NLC muscle (Figure 5c). For Y3x, there was a significant increase in [protein] compared to Y2x $(\mathrm{p}<0.001)$ and O3x $(\mathrm{p}<0.01)$. O2x significantly increased muscle [protein] compared to old NLC muscle following training $(\mathrm{p}<0.01)$. Strikingly, O2x also had significantly higher [protein] compared with O3x $(\mathrm{p}<0.01)$ and Y2x $(\mathrm{p}<0.001)$. While there were no effects for surrogate muscle transcriptional efficiency index (Figure 5d), there was a significantly higher surrogate muscle translational efficiency index in young compared to old NLC muscle $(p<0.01)$. There were no effects of SSCloading in the old rats ( $\mathrm{p}>0.05)$. 


\section{DISCUSSION}

The main findings from this study are: 1) There is a distinct molecular signature following RTET utilizing SSCs in old rats that accompanies adaptive and maladaptive outcomes, and that these responses appear to be connected to the PI3K-AKT signaling pathway, with SDEGs involved in muscle growth and remodeling present in both Y3x and Y2x, as well as O2x; 2) effects on gene expression in O2x had a positive influence on downstream outcomes including central nucleation and increased MND; and 3) this work advances recent work from our laboratory demonstrating the efficacy of a reduced-frequency exercise paradigm aiding the adaptability of aged muscle in response to SSC-training, as reflected by positive changes in muscle performance and muscle mass.

To determine if muscles are capable of adapting to chronic RTET at an old age, we exposed muscles of young and old rats to 80 SSCs, two days per week, (72 hours between training bouts). These protocols were altered from our originally published protocol (80 SSCs, three days per week; 48 hours between training bouts) in which young rats adapted and old rats maladapted (16). Similar to the response to 80 SSCs, three days per week exposure, the modified exposure protocols induced muscle mass increases for Y3x, Y2x, and O2x. For young rats, the modified exposure protocol yielded similar gains in performance and muscle mass, indicating that muscles of young rats have the ability to adapt following a variety of SSC paradigms.

In the present study, O2x was superior over O3x as indicated by increases in muscle performance as well as improvements in muscle fiber area and MND that were similar to younger rodents. Our results indicating positive effects for two day per week high-intensity RTET in older muscle are in line with a recent paper by Stec et al. (45), who found that two days per week high-intensity training was beneficial for increasing muscle strength and performance compared to three day (analogous to O3x) or one day per week high-intensity training. Moreover, it should be noted the authors found that one additional day of low-intensity training interspersed between two days of high-intensity training may elicit further benefits; this conclusion was based on the significantly greater thigh muscle mass compared to the other interventions, including the two day per week high-intensity only training. This would indicate that there are potential additional adaptive benefits when incorporating an extra day of low intensity exercise to a training program that is absent with only two days of high intensity training; because we did not directly examine this paradigm in the present study, we cannot directly compare $\mathrm{O} 2 \mathrm{x}$ or O3x to this paradigm and confirm the conclusions shown from the Stec et al. report, although it would certainly warrant examination in future research in order to confirm and elucidate the underlying integrated physiological processes supporting these findings. Indeed, with appreciation for the current molecular data described herein (i.e., $\mathrm{RT}^{2}$ profiler array gene expression, IPA, central nucleated fibers, and myonuclear domains) as well as the positive results, namely that of muscle performance and muscle mass, that are complementary to the Stec et al. investigation as well as other studies in humans (22-25, 29), our study provides support at a fundamental level that a reduced frequency of high-intensity RTET can be highly beneficial for aged muscle, particularly those who do not respond favorably when compared with higher 
frequencies of training. Additionally, the O2x paradigm in our study also falls in line with recent support for a “minimal-dose approach” for RTET of two days per week of resistance exercise for less than 60 minutes that was based on previous research showing various physiological and psychological benefits to this type of training in older adults (20), or simply desire to achieve the benefits of RTET at a reduced time commitment.

The present data demonstrates that $\mathrm{O} 2 \mathrm{x}$ were able to undergo adaptation concomitant with an enhanced activation of the PI3K-AKT pathway. Specifically, Fos and Jun, key regulators of satellite cell activation and migration involved during the myogenic process, were upregulated only in the O2x group. Fos and Jun dimerize together and form the transcription factor complex AP-1 $(1,12)$, and have been demonstrated to play a crucial role in the regulation of satellite cells during both myogenesis and skeletal muscle regeneration (1) as well as being involved in the control of muscle adaptation in response to exercise $(12,28,31)$. Intriguingly, the top canonical pathway in the IPA analysis for O2x was ERK/MAPK signaling. Thus, exercise variables that are prescribed in an appropriate manner for aged individuals, such as reducing the frequency, may provide evidence for an augmented systemic environment in aged skeletal muscle. However, these findings should be viewed within the perspective that we did not use traditional markers of satellite cell abundance, location, or function, such as Pax7 or MyoD; because of the role of Fos and Jun in many other regulatory processes besides satellite cells, this response will have to be assessed in future studies to confirm these findings.

Itgb1, a molecule crucial for mechanotransduction signaling of AKT, independent of growth factor or nutrient factors (26), was upregulated only in Y3x, Y2x and O2x (Table 3). In response to mechanical activation, Itgb1 signals to integrin linked kinase (ILK), leading to the subsequent activation of the mammalian target of rapamycin (mTOR); mTOR subsequently increases protein synthesis, ribosomal biogenesis, and eventual muscle growth $(11,32,46)$. Moreover, through canonical PI3K signaling, ILK works in concert with pyruvate dehydrogenase kinase 1 to stimulate Akt, eventually leading to increased protein synthesis and expression of genes related to growth and remodeling in skeletal muscle (11). Interestingly, the IPA analysis revealed that adapted groups have higher activation states of pathways related to PI3K canonical signaling, such as ERK/MAPK, HGF, IGF-1, and mTOR (Table 4). Thus, the results of the present study indicate that RTET that promotes adaptation (i.e., Y3x, Y2x, and O2x) enhances mechanotransduction signaling through Itgb1, subsequently increasing muscle protein content and skeletal muscle fiber area.

The PI3K-AKT signaling pathway has been shown to stimulate and control the activity of multiple functional processes, including protein synthesis, glucose uptake, glycogen synthesis, cell cycle progression, cell growth, and an inhibition of apoptosis (21). Therefore, modulation of genes and proteins within this pathway have a crucial role in integrating both anabolic and catabolic signals, ultimately leading to changes in myofiber area and overall muscle mass. In the present study, we have shown that appropriate, age-specific RTET (i.e., Y3x, Y2x and O2x) leads to increased expression of PI3K-AKT genes as reflected by the RT ${ }^{2}$ Profiler $^{\mathrm{TM}}$ Arrays and bioinformatics analysis via IPA, whereas a dysregulated response occurs in O3x. This is an important distinction because these observations suggest that SSC-loading, when prescribed in the 
appropriate manner, directly modifies local PI3K-AKT activity within skeletal muscle, which improves cellular metabolism and leads to an adaptive fate. Therefore, reducing the frequency of RTET, thereby allowing additional recovery time to initiate muscle repair and remodeling processes, provides an optimal environment for aged muscle to adapt properly and aids in restoring muscle performance, physiology as well as the microenvironment, subsequently improving soft-tissue quality and functional outcomes with advanced age.

While we believe the results from the present study provide valuable information about optimizing the spacing of exercise in an old versus young state, there are limitations to the current study. First, our study was limited to two age groups, which consisted of young (3 mo.) and old (30 mo.) that were SSC-loaded three or two days per week. Recent literature suggests that age-related declines may potentially occur much sooner than what would be expected, potentially before any overt symptoms of the onset of chronic disease $(13,40)$, which indicates that testing intermediate age groups is critical $(35,42)$. Moreover, our findings of no differences in muscle mass wet-weight between young and old NLC may also be due to a lack of intermediate age groups; 3 month old rats (comparable with 16 to 20 year old humans) are at late adolescence/young adulthood, before they reach their adult weight. Previous research from our laboratory has demonstrated that 6 to 9 month old F344xBN rats, but not 3 month old rats, have significantly less NLC muscle relative to 26 to 33 month old rodents $(18,35)$. Emphasizing our previous work $(6,16,36)$, the basis for choosing 3 month old rats was to select a population that had the highest potential in terms of adaptability to high-intensity SSC RTET as well as serving as a representative age group for a young occupational demographic. Importantly, our findings of significantly enhanced muscle performance and muscle fiber areas (Figures 1 \& 2) still provide a fundamental basis for the use of appropriately prescribed RTET in preventing the onset of age-associated sarcopenia and/or dynapenia.

Next, the study was limited by investigating only rats, and not humans. Although using the F344xBN rats allowed for finding important molecular findings that are driving muscle adaptation (e.g., gene expression of signaling pathways, etc.), caution should be used in directly translating the findings to humans. However, it is noteworthy that in a recent review by Ballak et al. (8), after an extensive review of the literature, found that the model used in the current study, the F344xBN, most closely resembled the aging of muscle in men more so than any other animal model they examined.

Another limitation to the present study was we did not look at fiber type distribution. In a previous study by our laboratory (35), we showed that in younger rodents subjected to three days per week chronic SSC RTET that there was a significant shift in fiber type distribution from type IIb to type IIx fibers, whereas old rats that maladapted had a blunted fiber type shift response. Overall, in younger rodents, the high-intensity training of the current model that is intended to target type II fibers resulted in a more oxidative phenotype, explaining the ability to recover from fatigue. This suggests that the shift in fiber type distribution could be playing a role in the adaptive or maladaptive response to SSC training. While not looked at in the present study, a future research question is what the fiber type distribution would look like in O2x following chronic RTET. We would 
speculate based on the current data and past research by our laboratory $(35,36)$ that this group would have fiber type distribution shift that looks more similar to young rats that adapted to the three days per week loading paradigm; this will need to be confirmed in future studies. Finally, although it was outside the scope of this study, only signaling events associated with growth and remodeling were investigated, while excluding pathways involved in the stress response to exercise that also underlies muscle adaptation, such as apoptosis, reaction oxygen species metabolism, and autophagy $(3,33)$. Of note, IPA analysis (Table 4) indicated that cell death and survival was a highly expressed functional outcome in the adaptive groups; thus, investigating the stress response involvement following modification of variables involving the RTET prescription with aging are warranted in future research.

In conclusion, we provide key insights into the growth and remodeling signaling response in skeletal muscle to appropriately prescribed, age-specific RTET involving PI3K-AKT pathway expression, which partially underlies training adaptation with aging. Aged skeletal muscle demonstrates an impairment in the ability to adapt properly to chronic SSC RTET when conducted at a higher frequency; however, when reducing the frequency of exercise, old muscle can adapt through enhanced muscle performance and increased muscle fiber areas. Additionally, these features are supported by a concomitant improvement in the growth and remodeling molecular environment, specifically the PI3K-AKT pathway that promotes central nucleation and MND remodeling, and partially explains the underlying basis for adaptive outcomes in aged rodents following RTET. Thus, the findings in the present study support the notion that age-appropriate exercise prescription, in this case reducing the frequency of training in aged rodents, reverses aged-associated negative alterations in key pathways involved in growth and remodeling of skeletal muscle, and rejuvenates the systemic environment in a favorable manner that results in muscle adaptation. Future studies should focus on revealing additional molecular influences (e.g. gene signaling, DNA methylation, etc.,) that affect muscle adaptation occurring with RTET and how they are influenced by titrating the parameters of the exercise prescription. 


\section{REFERENCES}

1. Alli NS, Yang EC, Miyake T, Aziz A, Collins-Hooper H, Patel K, and McDermott JC. Signaldependent fra-2 regulation in skeletal muscle reserve and satellite cells. Cell Death Dis 4: e692, 2013.

2. Almada AE, and Wagers AJ. Molecular circuitry of stem cell fate in skeletal muscle regeneration, ageing and disease. Nat Rev Mol Cell Biol 17: 267-279, 2016.

3. Alway SE. Inflammation and oxidative stress limit adaptation to stretch-shortening contractions in aging. Exerc Sport Sci Rev 45: 194, 2017.

4. Baker BA, and Cutlip RG. Skeletal muscle injury versus adaptation with aging: novel insights on perplexing paradigms. Exerc Sport Sci Rev 38: 10-16, 2010.

5. Baker BA, Hollander MS, Kashon ML, and Cutlip RG. Effects of glutathione depletion and age on skeletal muscle performance and morphology following chronic stretch-shortening contraction exposure. Eur $J$ Appl Physiol 108: 619-630, 2010.

6. Baker BA, Hollander MS, Mercer RR, Kashon ML, and Cutlip RG. Adaptive stretch-shortening contractions: diminished regenerative capacity with aging. Appl Physiol Nutr Metab 33: 1181-1191, 2008.

\section{Baker BA, Mercer RR, Geronilla KB, Kashon ML, Miller GR, and Cutlip RG. Stereological} analysis of muscle morphology following exposure to repetitive stretch-shortening cycles in a rat model. Appl Physiol Nutr Metab 31: 167-179, 2006.

8. Ballak SB, Degens H, de Haan A, and Jaspers RT. Aging related changes in determinants of muscle force generating capacity: a comparison of muscle aging in men and male rodents. Ageing Res Rev 14: 43-55, 2014.

9. Bamman MM, Cooper DM, Booth FW, Chin ER, Neufer PD, Trappe S, Lightfoot JT, Kraus WE, and Joyner MJ. Exercise biology and medicine: innovative research to improve global health. Mayo Clin Proc 89: 148-153, 2014.

10. Boppart MD, Burkin DJ, and Kaufman SJ. Alpha7beta1-integrin regulates mechanotransduction and prevents skeletal muscle injury. Am J Physiol Cell Physiol 290: C1660-1665, 2006.

11. Burkholder TJ. Mechanotransduction in skeletal muscle. Front Biosci 12: 174-191, 2007.

12. Caldow MK, Steinberg GR, and Cameron-Smith D. Impact of SOCS3 overexpression on human skeletal muscle development in vitro. Cytokine 55: 104-109, 2011. 
13. Cooper R, Bann D, Wloch EG, Adams JE, and Kuh D. "Skeletal muscle function deficit" in a nationally representative British birth cohort in early old age. J Gerontol A Biol Sci Med Sci 70: 604-607, 2015.

14. Crawford JO, Graveling RA, Cowie HA, and Dixon K. The health safety and health promotion needs of older workers. Occup Med (Lond) 60: 184-192, 2010.

15. Cristea A, Qaisar R, Edlund PK, Lindblad J, Bengtsson E, and Larsson L. Effects of aging and gender on the spatial organization of nuclei in single human skeletal muscle cells. Aging Cell 9: 685-697, 2010.

16. Cutlip RG, Baker BA, Geronilla KB, Mercer RR, Kashon ML, Miller GR, Murlasits Z, and Alway SE. Chronic exposure to stretch-shortening contractions results in skeletal muscle adaptation in young rats and maladaptation in old rats. Appl Physiol Nutr Metab 31: 573-587, 2006.

17. Cutlip RG, Stauber WT, Willison RH, McIntosh TA, and Means KH. Dynamometer for rat plantar flexor muscles in vivo. Med Biol Eng Comput 35: 540-543, 1997.

18. Degens H, and Alway SE. Skeletal muscle function and hypertrophy are diminished in old age. Muscle Nerve 27: 339-347, 2003.

19. Evans WJ. What is sarcopenia? J Gerontol A Biol Sci Med Sci 50 Spec No: 5-8, 1995.

20. Fisher JP, Steele J, Gentil P, Giessing J, and Westcott WL. A minimal dose approach to resistance training for the older adult; the prophylactic for aging. Exp Gerontol 99: 80-86, 2017.

21. Frost RA, and Lang CH. Protein kinase B/Akt: a nexus of growth factor and cytokine signaling in determining muscle mass. J Appl Physiol (1985) 103: 378-387, 2007.

22. Hagerman FC, Walsh SJ, Staron RS, Hikida RS, Gilders RM, Murray TF, Toma K, and Ragg KE. Effects of high-intensity resistance training on untrained older men. I. Strength, cardiovascular, and metabolic responses. J Gerontol A Biol Sci Med Sci 55: B336-346, 2000.

23. Hakkinen K, Kraemer WJ, Newton RU, and Alen M. Changes in electromyographic activity, muscle fibre and force production characteristics during heavy resistance/power strength training in middle-aged and older men and women. Acta Physiol Scand 171: 51-62, 2001.

24. Hakkinen K, Pakarinen A, Kraemer WJ, Hakkinen A, Valkeinen H, and Alen M. Selective muscle hypertrophy, changes in EMG and force, and serum hormones during strength training in older women. $J$ Appl Physiol (1985) 91: 569-580, 2001. 
25. Hikida RS, Staron RS, Hagerman FC, Walsh S, Kaiser E, Shell S, and Hervey S. Effects of highintensity resistance training on untrained older men. II. Muscle fiber characteristics and nucleo-cytoplasmic relationships. J Gerontol A Biol Sci Med Sci 55: B347-354, 2000.

26. Hornberger TA, and Esser KA. Mechanotransduction and the regulation of protein synthesis in skeletal muscle. Proc Nutr Soc 63: 331-335, 2004.

\section{Hornberger TA, Stuppard R, Conley KE, Fedele MJ, Fiorotto ML, Chin ER, and Esser KA.}

Mechanical stimuli regulate rapamycin-sensitive signalling by a phosphoinositide 3-kinase-, protein kinase Band growth factor-independent mechanism. Biochem J 380: 795-804, 2004.

\section{LaFramboise WA, Jayaraman RC, Bombach KL, Ankrapp DP, Krill-Burger JM, Sciulli CM,} Petrosko P, and Wiseman RW. Acute molecular response of mouse hindlimb muscles to chronic stimulation. Am J Physiol Cell Physiol 297: C556-570, 2009.

29. Melov S, Tarnopolsky MA, Beckman K, Felkey K, and Hubbard A. Resistance exercise reverses aging in human skeletal muscle. PLoS One 2: e465, 2007.

30. Naimo MA, Gu JK, Lilly C, Kelley GA, and Baker BA. Resistance training confers greater muscle quality in aged individuals: A brief NHANES report. J Cachexia Sarcopenia Muscle - Clinical Reports In Press, 2018.

31. Nikolaidis MG, Papazisis KT, Kortsaris AH, and Mougios V. Exercise-induced changes in c-Fos protein levels in skeletal muscle of trained and untrained rats. Int J Sports Med 24: 96-100, 2003.

32. Philp A, Hamilton DL, and Baar K. Signals mediating skeletal muscle remodeling by resistance exercise: PI3-kinase independent activation of mTORC1. J Appl Physiol (1985) 110: 561-568, 2011.

33. Quadrilatero J, Alway SE, and Dupont-Versteegden EE. Skeletal muscle apoptotic response to physical activity: potential mechanisms for protection. Appl Physiol Nutr Metab 36: 608-617, 2011.

34. Rader EP, and Baker BA. Inflammaging and the age-specific responsiveness to stretch-shortening contractions. Exerc Sport Sci Rev 45: 195-200, 2017.

35. Rader EP, Layner K, Triscuit AM, Chetlin RD, Ensey J, and Baker BA. Age-dependent muscle adaptation after chronic stretch-shortening contractions in rats. Aging Dis 7: 1-13, 2016. 
36. Rader EP, Naimo MA, Layner KN, Triscuit AM, Chetlin RD, Ensey J, and Baker BA.

Enhancement of skeletal muscle in aged rats following high-intensity stretch-shortening contraction training. Rejuvenation Res 20: 93-102, 2017.

37. Raue U, Slivka D, Jemiolo B, Hollon C, and Trappe S. Proteolytic gene expression differs at rest and after resistance exercise between young and old women. J Gerontol A Biol Sci Med Sci 62: 1407-1412, 2007.

38. Raue U, Slivka D, Minchev K, and Trappe S. Improvements in whole muscle and myocellular function are limited with high-intensity resistance training in octogenarian women. J Appl Physiol (1985) 106: 1611-1617, 2009.

39. Raue U, Trappe TA, Estrem ST, Qian HR, Helvering LM, Smith RC, and Trappe S. Transcriptome signature of resistance exercise adaptations: mixed muscle and fiber type specific profiles in young and old adults. J Appl Physiol (1985) 112: 1625-1636, 2012.

40. Roberts MD, Kerksick CM, Dalbo VJ, Hassell SE, Tucker PS, and Brown R. Molecular attributes of human skeletal muscle at rest and after unaccustomed exercise: an age comparison. J Strength Cond Res 24: 1161-1168, 2010.

41. Ryan MJ, Dudash HJ, Docherty M, Geronilla KB, Baker BA, Haff GG, Cutlip RG, and Alway SE. Aging-dependent regulation of antioxidant enzymes and redox status in chronically loaded rat dorsiflexor muscles. J Gerontol A Biol Sci Med Sci 63: 1015-1026, 2008.

42. Sharples AP, Stewart CE, and Seaborne RA. Does skeletal muscle have an 'epi'-memory? The role of epigenetics in nutritional programming, metabolic disease, aging and exercise. Aging Cell 15: 603-616, 2016.

43. Siu PM, Bryner RW, Martyn JK, and Alway SE. Apoptotic adaptations from exercise training in skeletal and cardiac muscles. FASEB J 18: 1150-1152, 2004.

44. Slivka D, Raue U, Hollon C, Minchev K, and Trappe S. Single muscle fiber adaptations to resistance training in old (>80 yr) men: evidence for limited skeletal muscle plasticity. American journal of physiology Regulatory, integrative and comparative physiology 295: R273-280, 2008.

45. Stec MJ, Thalacker-Mercer A, Mayhew DL, Kelly NA, Tuggle SC, Merritt EK, Brown CJ, Windham ST, Dell'Italia LJ, Bickel CS, Roberts BM, Vaughn KM, Isakova-Donahue I, Many GM, and Bamman MM. Randomized, four-arm, dose-response clinical trial to optimize resistance exercise training for older adults with age-related muscle atrophy. Exp Gerontol 99: 98-109, 2017. 
46. Yamada AK, Verlengia R, and Bueno Junior CR. Mechanotransduction pathways in skeletal muscle hypertrophy. J Recept Signal Transduct Res 32: 42-44, 2012. 
Table 1. RT $^{2}$ Profiler PI3K-AKT PCR Array.

\begin{tabular}{|c|c|c|c|c|c|c|c|c|c|c|c|c|}
\hline & 1 & 2 & 3 & 4 & 5 & 6 & 7 & 8 & 9 & 10 & 11 & 12 \\
\hline A & Adar & Akt1 & Akt2 & Akt3 & Арс & Bad & Btk & Casp9 & Ccnd1 & Cd14 & Cdc42 & Cdkn1b \\
\hline B & Chuk & Csnk2a1 & Ctnnb1 & Eif2ak2 & Eif4b & Eif4e & Eif4ebp1 & Eif4g1 & Faslg & Fkbp1a & Fos & Foxg1 \\
\hline C & Foxo3 & Gja1 & Grb10 & Grb2 & Gsk3b & Hras & Hspb1 & Igf1 & Igf1r & Ilk & Irak1 & Irs1 \\
\hline D & Itgb1 & Jun & Kcnh8 & Map2k1 & Mapk1 & Mapk14 & Mapk3 & Mapk8 & Mtcp1 & Mtor & Myd88 & Nfkb1 \\
\hline E & Nfkbia & Pabpc1 & Pak1 & Pdgfra & Pdk1 & Pdk2 & Pdpk1 & Pik3ca & Pik3cg & Pik3r1 & Pik3r2 & Prkca \\
\hline $\mathrm{F}$ & Prkcb & Prkcz & Pten & Ptk2 & Ptpn11 & Rac1 & Raf1 & Rasa1 & Rbl2 & Rheb & Rhoa & Rps6ka1 \\
\hline G & Rps6kb1 & Shc1 & Sos1 & Srf & Tcl1a & Tirap & Tlr4 & Tollip & Tsc1 & Tsc2 & Wasl & Ywhah \\
\hline $\mathrm{H}$ & Actb & $\mathrm{B} 2 \mathrm{~m}$ & Hprt1 & Ldha & Rplp1 & RGDC & RTC & RTC & RTC & PPC & PPC & PPC \\
\hline
\end{tabular}


Table 2. Quantitative myonuclei count, myonuclear domain and central nuclei for SSC-loaded and non-loaded control muscles of young and old rats.

\begin{tabular}{cccccc} 
& Young & & \multicolumn{3}{c}{ Old } \\
Non-Loaded & 3 days/wk. Loaded & 2 days/wk. & Non-Loaded & 3 & 2 days/wk. \\
Control & & Loaded & Control & days/wk. & Loaded \\
& & & & Loaded &
\end{tabular}

Myonuclei Count (nuclei

per $\mathbf{m m}^{2}$ )

$1046 \pm 51$

$1031 \pm 55$

$1043 \pm 99$

$1223 \pm 50^{*}$

$1155 \pm 55 \quad 1105 \pm 26$

Central Nucleated Fibers

(\%)

Sample sizes were $N=4$ to 5 per group. Values are means \pm S.E.M. ${ }^{*}$ Different from value of the young nonloaded control muscle limb; ${ }^{\#}$ Different from value of the non-loaded control muscle within age; ${ }^{\dagger}$ Different from value of the trained limb for rats exposed three days per week within age; $p<0.05$. 
Table 3. Significantly differentially expressed genes (SDEGs) relevant to PI3K-AKT and muscle growth for the tibialis anterior muscle following dorsiflexion SSC-loading relative to non-loaded muscles.

\begin{tabular}{|c|c|c|c|c|c|c|c|c|c|c|c|}
\hline & & & & \multicolumn{2}{|c|}{ Young 3x/wk. } & \multicolumn{2}{|c|}{ Young 2x/wk. } & \multicolumn{2}{|c|}{ Old 3x/wk. } & \multicolumn{2}{|c|}{ Old 2x/wk. } \\
\hline Genes & Symbol & Description & RefSeq \# & FC & $P$ value & FC & $P$ value & $\mathrm{FC}$ & $P$ value & $\mathrm{FC}$ & $P$ value \\
\hline \multirow[t]{10}{*}{$\begin{array}{l}\text { AKT/PI3K } \\
\text { family } \\
\text { members \& } \\
\text { their regulators }\end{array}$} & Akt2 & $\begin{array}{l}\text { V-akt murine } \\
\text { thymoma viral } \\
\text { oncogene } \\
\text { homolog } 2\end{array}$ & $\begin{array}{l}\text { NM_0170 } \\
93\end{array}$ & $\downarrow 1.38$ & 0.0173 & $\downarrow 1.63$ & 0.0054 & $\downarrow 1.20$ & 0.1842 & $\downarrow 1.17$ & 0.8244 \\
\hline & Grb10 & $\begin{array}{l}\text { Growth factor } \\
\text { receptor bound } \\
\text { protein } 10 \\
\end{array}$ & $\begin{array}{l}\text { NM_0011 } \\
09093\end{array}$ & $\downarrow 1.31$ & 0.2144 & $\downarrow 1.40$ & 0.0426 & $\downarrow 1.22$ & 0.5728 & $\uparrow 1.22$ & 0.2888 \\
\hline & Grb2 & $\begin{array}{l}\text { Growth factor } \\
\text { receptor bound } \\
\text { protein } 2\end{array}$ & $\begin{array}{l}\text { NM_0308 } \\
46\end{array}$ & $\downarrow 1.17$ & 0.1810 & $\downarrow 1.17$ & 0.0819 & $\downarrow 1.05$ & 0.9621 & $\uparrow 1.11$ & 0.4180 \\
\hline & Hspb1 & $\begin{array}{l}\text { Heat shock } \\
\text { protein } 1\end{array}$ & $\begin{array}{l}\text { NM_0319 } \\
70\end{array}$ & $\uparrow 1.08$ & 0.6024 & $\uparrow 2.07$ & 0.0011 & $\downarrow 1.08$ & 0.4440 & $\uparrow 2.16$ & 0.0398 \\
\hline & Ilk & $\begin{array}{l}\text { Integrin-linked } \\
\text { kinase }\end{array}$ & $\begin{array}{l}\text { NM_1334 } \\
09\end{array}$ & $\downarrow 1.02$ & 0.8547 & $\uparrow 1.00$ & 0.9749 & $\uparrow 1.10$ & 0.4336 & $\uparrow 1.29$ & 0.0993 \\
\hline & Mtcp1 & $\begin{array}{l}\text { Mature T-cell } \\
\text { proliferation } 1 \\
\end{array}$ & $\begin{array}{l}\text { XM_0010 } \\
70795\end{array}$ & $\downarrow 1.59$ & 0.0358 & $\downarrow 1.50$ & 0.0308 & $\downarrow 1.42$ & 0.1733 & $\downarrow 1.14$ & 0.8505 \\
\hline & Pdk2 & $\begin{array}{l}\text { Pyruvate } \\
\text { dehydrogenase } \\
\text { kinase, isozyme } \\
2\end{array}$ & $\begin{array}{l}\text { NM_0308 } \\
72\end{array}$ & $\downarrow 1.45$ & 0.0046 & $\downarrow 1.55$ & 0.0010 & $\downarrow 1.31$ & 0.3230 & $\downarrow 1.09$ & 0.7266 \\
\hline & Pdpk1 & $\begin{array}{l}\text { 3- } \\
\text { phosphoinositid } \\
\text { e dependent } \\
\text { protein kinase-1 }\end{array}$ & $\begin{array}{l}\text { NM_0310 } \\
81\end{array}$ & $\downarrow 1.26$ & 0.0759 & $\downarrow 1.25$ & 0.0386 & $\downarrow 1.14$ & 0.3988 & $\uparrow 1.14$ & 0.3459 \\
\hline & Pi3k3r1 & $\begin{array}{l}\text { Phosphoinositid } \\
\text { e-3-kinase, } \\
\text { regulatory } \\
\text { subunit } 1 \\
\text { (alpha) }\end{array}$ & $\begin{array}{l}\text { NM_0130 } \\
05\end{array}$ & $\downarrow 1.43$ & 0.0565 & $\downarrow 1.58$ & 0.0391 & $\downarrow 1.42$ & 0.1040 & $\downarrow 1.24$ & 0.3978 \\
\hline & Prkcz & $\begin{array}{l}\text { Protein kinase } \\
\text { C, zeta }\end{array}$ & $\begin{array}{l}\text { NM_0225 } \\
07\end{array}$ & $\downarrow 2.05$ & 0.0084 & $\downarrow 1.98$ & 0.0017 & $\downarrow 1.33$ & 0.4962 & $\downarrow 1.56$ & 0.2448 \\
\hline \multirow{2}{*}{$\begin{array}{l}\text { Force } \\
\text { transmission \& } \\
\text { mechanotrans- } \\
\text { duction }\end{array}$} & Itgb1 & Integrin, beta 1 & $\begin{array}{l}\text { NM_0170 } \\
22\end{array}$ & $\uparrow 1.53$ & 0.0123 & $\uparrow 1.95$ & $<0.0001$ & $\uparrow 1.70$ & 0.0775 & $\uparrow 2.34$ & 0.0072 \\
\hline & Ptk2 & $\begin{array}{l}\text { PTK2 protein } \\
\text { tyrosine kinase } \\
2 \text { (also known } \\
\text { as focal } \\
\text { adhesion } \\
\text { kinase) }\end{array}$ & $\begin{array}{l}\text { NM_0130 } \\
81\end{array}$ & $\downarrow 1.35$ & 0.0258 & $\downarrow 1.43$ & 0.0106 & $\downarrow 1.15$ & 0.6602 & $\downarrow 1.03$ & 0.7613 \\
\hline \multirow[t]{7}{*}{$\begin{array}{l}\text { IGF-1 signaling } \\
\text { pathway }\end{array}$} & Csnk2a1 & $\begin{array}{l}\text { Casein kinase 2, } \\
\text { alpha } 1 \\
\text { polypeptide }\end{array}$ & $\begin{array}{l}\text { NM_0538 } \\
24\end{array}$ & $\downarrow 1.20$ & 0.2549 & $\downarrow 1.46$ & 0.0570 & $\downarrow 1.13$ & 0.8687 & $\downarrow 1.03$ & 0.7479 \\
\hline & Fos & $\begin{array}{l}\text { FBJ } \\
\text { osteosarcoma } \\
\text { oncogene } \\
\end{array}$ & $\begin{array}{l}\text { NM_0221 } \\
97\end{array}$ & $\uparrow 1.47$ & 0.4709 & $\uparrow 1.27$ & 0.2837 & $\uparrow 2.57$ & 0.0458 & $\uparrow 6.25$ & 0.0168 \\
\hline & Hras & $\begin{array}{l}\text { Harvey rat } \\
\text { sarcoma virus } \\
\text { oncogene }\end{array}$ & $\begin{array}{l}\text { NM_0010 } \\
98241\end{array}$ & $\downarrow 1.46$ & 0.0195 & $\downarrow 1.21$ & 0.1173 & $\downarrow 1.29$ & 0.1931 & $\uparrow 1.09$ & 0.4611 \\
\hline & Irs1 & $\begin{array}{l}\text { Insulin receptor } \\
\text { substrate } 1\end{array}$ & $\begin{array}{l}\text { NM_0129 } \\
69\end{array}$ & $\uparrow 1.02$ & 0.9168 & $\downarrow 1.11$ & 0.5557 & $\downarrow 1.03$ & 0.9305 & $\uparrow 1.53$ & 0.0777 \\
\hline & Jun & Jun oncogene & $\begin{array}{l}\text { NM_0218 } \\
35\end{array}$ & $\uparrow 1.16$ & 0.2424 & $\downarrow 2.36$ & 0.6896 & $\downarrow 1.23$ & 0.0683 & $\uparrow 1.57$ & 0.0241 \\
\hline & Map2k1 & $\begin{array}{l}\text { Mitogen } \\
\text { activated } \\
\text { protein kinase 1 } \\
\end{array}$ & $\begin{array}{l}\text { NM_0316 } \\
43\end{array}$ & $\downarrow 1.27$ & 0.1934 & $\downarrow 1.27$ & 0.0253 & $\downarrow 1.14$ & 0.7504 & $\uparrow 1.01$ & 0.6985 \\
\hline & Mapk8 & $\begin{array}{l}\text { Mitogen- } \\
\text { activated } \\
\text { protein kinase } 8\end{array}$ & $\begin{array}{l}\text { NM_0538 } \\
29\end{array}$ & $\downarrow 1.17$ & 0.6829 & $\downarrow 1.77$ & 0.0022 & $\downarrow 1.09$ & 0.8617 & $\uparrow 1.13$ & 0.4917 \\
\hline
\end{tabular}




\begin{tabular}{|c|c|c|c|c|c|c|c|c|c|c|c|}
\hline & Raf1 & $\begin{array}{l}\text { V-raf-leukemia } \\
\text { viral oncogene } \\
1\end{array}$ & $\begin{array}{l}\text { NM_0126 } \\
39\end{array}$ & $\downarrow 1.21$ & 0.0614 & $\downarrow 1.21$ & 0.0745 & $\downarrow 1.08$ & 0.8582 & $\uparrow 1.21$ & 0.2527 \\
\hline & Sos1 & $\begin{array}{l}\text { Son of } \\
\text { sevenless } \\
\text { homolog } 1 \\
\text { (Drosophila) }\end{array}$ & $\begin{array}{l}\text { NM_0011 } \\
00716\end{array}$ & $\downarrow 1.24$ & 0.0111 & $\downarrow 1.23$ & 0.0636 & $\downarrow 1.13$ & 0.5640 & $\uparrow 1.11$ & 0.4472 \\
\hline & Ptpn11 & $\begin{array}{l}\text { Protein tyrosine } \\
\text { phosphatase, } \\
\text { non-receptor } \\
\text { type } 11\end{array}$ & $\begin{array}{l}\text { NM_0130 } \\
88\end{array}$ & $\downarrow 1.37$ & 0.0128 & $\downarrow 1.44$ & 0.0153 & $\downarrow 1.25$ & 0.4620 & $\uparrow 1.01$ & 0.6772 \\
\hline & Srf & $\begin{array}{l}\text { Serum response } \\
\text { factor (c-fos } \\
\text { serum response } \\
\text { element- } \\
\text { binding } \\
\text { transcription } \\
\text { factor) } \\
\end{array}$ & $\begin{array}{l}\text { NM_0011 } \\
09302\end{array}$ & $\downarrow 1.18$ & 0.0188 & $\downarrow 1.00$ & 0.9511 & $\downarrow 1.10$ & 0.8794 & $\uparrow 1.44$ & 0.0633 \\
\hline \multirow[t]{10}{*}{$\begin{array}{l}\text { mTOR } \\
\text { signaling } \\
\text { pathway }\end{array}$} & Eif4b & $\begin{array}{l}\text { Eukaryotic } \\
\text { translation } \\
\text { initiation factor } \\
\text { 4B } \\
\end{array}$ & $\begin{array}{l}\text { NM_0010 } \\
08324\end{array}$ & $\downarrow 1.25$ & 0.1695 & $\downarrow 1.47$ & 0.0076 & $\downarrow 1.21$ & 0.3759 & $\uparrow 1.00$ & 0.7125 \\
\hline & Eif4e & $\begin{array}{l}\text { Eukaryotic } \\
\text { translation } \\
\text { initiation factor } \\
4 \mathrm{E} \\
\end{array}$ & $\begin{array}{l}\text { NM_0539 } \\
74\end{array}$ & $\downarrow 1.20$ & 0.0839 & $\downarrow 1.23$ & 0.0050 & $\downarrow 1.07$ & 0.8048 & $\uparrow 1.11$ & 0.4189 \\
\hline & Eif4ebp1 & $\begin{array}{l}\text { Eukaryotic } \\
\text { translation } \\
\text { initiation factor } \\
4 \mathrm{E} \text { binding } \\
\text { protein } 1 \\
\end{array}$ & $\begin{array}{l}\text { NM_0538 } \\
57\end{array}$ & $\downarrow 1.47$ & 0.0225 & $\downarrow 1.29$ & 0.0069 & $\downarrow 1.16$ & 0.3081 & $\downarrow 1.06$ & 0.6880 \\
\hline & Eif4g1 & $\begin{array}{l}\text { Eukaryotic } \\
\text { translation } \\
\text { initiation factor } \\
4 \text { gamma, } 1 \\
\end{array}$ & $\begin{array}{l}\text { XM_0062 } \\
21191\end{array}$ & $\downarrow 1.40$ & 0.1074 & $\downarrow 1.52$ & 0.0145 & $\downarrow 1.33$ & 0.5250 & $\downarrow 1.13$ & 0.7690 \\
\hline & Fkbp1a & $\begin{array}{l}\text { FK506 binding } \\
\text { protein 1a }\end{array}$ & $\begin{array}{l}\text { NM_0131 } \\
02\end{array}$ & $\downarrow 1.19$ & 0.0471 & $\downarrow 1.09$ & 0.2031 & $\uparrow 1.01$ & 0.9536 & $\uparrow 1.10$ & 0.3988 \\
\hline & Mtor & $\begin{array}{l}\text { Mechanistic } \\
\text { target of } \\
\text { rapamycin } \\
\text { (serine/threonin } \\
\text { e kinase) }\end{array}$ & $\begin{array}{l}\text { NM_0199 } \\
06\end{array}$ & $\downarrow 1.19$ & 0.2779 & $\downarrow 1.27$ & 0.0329 & $\downarrow 1.07$ & 0.8875 & $\uparrow 1.15$ & 0.3924 \\
\hline & Rheb & $\begin{array}{l}\text { Ras homolog } \\
\text { enriched in } \\
\text { brain }\end{array}$ & $\begin{array}{l}\text { NM_0132 } \\
16\end{array}$ & $\uparrow 1.00$ & 0.9960 & $\uparrow 1.17$ & 0.0672 & $\downarrow 1.05$ & 0.9051 & $\uparrow 1.41$ & 0.0341 \\
\hline & Rps6kb1 & $\begin{array}{l}\text { Ribosomal } \\
\text { protein S6 } \\
\text { kinase, } \\
\text { polypeptide } 1\end{array}$ & $\begin{array}{l}\text { NM_0319 } \\
85\end{array}$ & $\downarrow 1.17$ & 0.0939 & $\downarrow 1.16$ & 0.2515 & $\downarrow 1.04$ & 0.9257 & $\uparrow 1.04$ & 0.5851 \\
\hline & Tsc1 & $\begin{array}{l}\text { Tuberous } \\
\text { sclerosis } 1\end{array}$ & $\begin{array}{l}\text { NM_0218 } \\
54\end{array}$ & $\downarrow 1.23$ & 0.2577 & $\downarrow 1.36$ & 0.0325 & $\downarrow 1.19$ & 0.6980 & $\uparrow 1.11$ & 0.4780 \\
\hline & Tsc2 & $\begin{array}{l}\text { Tuberous } \\
\text { sclerosis } 2 \\
\end{array}$ & $\begin{array}{l}\text { NM_0126 } \\
80\end{array}$ & $\downarrow 1.29$ & 0.0173 & $\downarrow 1.22$ & 0.0385 & $\downarrow 1.28$ & 0.1314 & $\downarrow 1.01$ & 0.9511 \\
\hline \multirow{3}{*}{$\begin{array}{l}\text { PI3K subunit } \\
\text { genes \& } \\
\text { regulation of } \\
\text { actin } \\
\text { organization/ } \\
\text { cell migration }\end{array}$} & Cdc42 & $\begin{array}{l}\text { Cell division } \\
\text { cycle } 42 \text { (GTP } \\
\text { binding protein) }\end{array}$ & $\begin{array}{l}\text { NM_1719 } \\
94\end{array}$ & $\downarrow 1.17$ & 0.0279 & $\downarrow 1.14$ & 0.0426 & $\downarrow 1.04$ & 0.7280 & $\uparrow 1.12$ & 0.2488 \\
\hline & Pak 1 & $\begin{array}{l}\text { P21 protein } \\
\text { (Cdc42/Rac)- } \\
\text { activated kinase } \\
1\end{array}$ & $\begin{array}{l}\text { NM_0171 } \\
98\end{array}$ & $\uparrow 1.03$ & 0.7088 & $\uparrow 1.39$ & 0.0603 & $\uparrow 1.22$ & 0.4118 & $\uparrow 1.42$ & 0.0022 \\
\hline & Rac1 & $\begin{array}{l}\text { Ras-related C3 } \\
\text { botulinum toxin } \\
\text { substrate } 1\end{array}$ & $\begin{array}{l}\text { NM_1343 } \\
66\end{array}$ & $\downarrow 1.24$ & 0.0142 & $\downarrow 1.19$ & 0.0171 & $\downarrow 1.12$ & 0.5877 & $\uparrow 1.06$ & 0.5195 \\
\hline
\end{tabular}




\begin{tabular}{|c|c|c|c|c|c|c|c|c|c|c|c|}
\hline & Wasl & $\begin{array}{l}\text { Wiskott- } \\
\text { Aldrich } \\
\text { syndrome-like }\end{array}$ & $\begin{array}{l}\text { NM_0011 } \\
10365\end{array}$ & $\downarrow 1.30$ & 0.0983 & $\downarrow 1.24$ & 0.1464 & $\downarrow 1.28$ & 0.2234 & $\downarrow 1.08$ & 0.8867 \\
\hline \multirow[t]{3}{*}{$\begin{array}{l}\text { Regulation of } \\
\text { eIF4e \& } \\
\text { p70s6K }\end{array}$} & Mapk1 & $\begin{array}{l}\text { Mitogen } \\
\text { activated } \\
\text { protein kinase } 1\end{array}$ & $\begin{array}{l}\text { NM_0538 } \\
42\end{array}$ & $\downarrow 1.15$ & 0.0309 & $\downarrow 1.23$ & 0.0019 & $\downarrow 1.03$ & 0.8166 & $\uparrow 1.04$ & 0.5864 \\
\hline & Mapk14 & $\begin{array}{l}\text { Mitogen } \\
\text { activated } \\
\text { protein kinase } \\
14\end{array}$ & $\begin{array}{l}\text { NM_0310 } \\
20\end{array}$ & $\downarrow 1.51$ & 0.0110 & $\downarrow 1.53$ & 0.0023 & $\downarrow 1.34$ & 0.2748 & $\downarrow 1.00$ & 0.7664 \\
\hline & Pabpc1 & $\begin{array}{l}\text { Poly(A) binding } \\
\text { protein, } \\
\text { cytoplasmic } 1\end{array}$ & $\begin{array}{l}\text { NM_1343 } \\
53\end{array}$ & $\uparrow 1.07$ & 0.5336 & $\uparrow 1.17$ & 0.1203 & $\uparrow 1.32$ & 0.0079 & $\uparrow 1.39$ & 0.0063 \\
\hline $\begin{array}{l}\text { Other PI3K- } \\
\text { AKT Signaling } \\
\text { Pathway Genes }\end{array}$ & Chuk & $\begin{array}{l}\text { Conserved } \\
\text { helix-loop-helix } \\
\text { ubiquitous } \\
\text { kinase }\end{array}$ & $\begin{array}{l}\text { NM_0011 } \\
07588\end{array}$ & $\downarrow 1.15$ & 0.1168 & $\downarrow 1.23$ & 0.0601 & $\downarrow 1.07$ & 0.8291 & $\uparrow 1.11$ & 0.4602 \\
\hline \multirow{5}{*}{$\begin{array}{l}\text { Inactivation of } \\
\text { Gsk3 \& } \\
\text { accumulation } \\
\text { of } \beta \text {-Catenin }\end{array}$} & Cd14 & CD14 molecule & $\begin{array}{l}\text { NM_0217 } \\
44\end{array}$ & $\uparrow 2.04$ & 0.4715 & $\uparrow 2.69$ & 0.2001 & $\uparrow 1.72$ & 0.0607 & $\uparrow 1.52$ & 0.1089 \\
\hline & Ctnnb1 & $\begin{array}{l}\text { Catenin } \\
\text { (cadherin } \\
\text { associated } \\
\text { protein), beta } 1\end{array}$ & $\begin{array}{l}\text { NM_0533 } \\
57\end{array}$ & $\downarrow 1.22$ & 0.1685 & $\downarrow 1.48$ & 0.0054 & $\uparrow 1.28$ & 0.7227 & $\downarrow 1.23$ & 0.6579 \\
\hline & Tirap & $\begin{array}{l}\text { Toll-interleukin } \\
1 \text { receptor } \\
\text { (TIR) domain- } \\
\text { containing } \\
\text { adaptor protein }\end{array}$ & $\begin{array}{l}\text { XM_0010 } \\
55833\end{array}$ & $\downarrow 1.29$ & 0.0327 & $\downarrow 1.49$ & 0.0211 & $\downarrow 1.26$ & 0.5200 & $\uparrow 1.05$ & 0.5846 \\
\hline & Tlr4 & $\begin{array}{l}\text { Toll-like } \\
\text { receptor } 4\end{array}$ & $\begin{array}{l}\text { NM_0191 } \\
78\end{array}$ & $\uparrow 1.35$ & 0.2286 & $\uparrow 1.37$ & 0.0756 & $\downarrow 1.27$ & 0.9067 & $\uparrow 1.56$ & 0.0771 \\
\hline & Tollip & $\begin{array}{l}\text { Toll interacting } \\
\text { protein }\end{array}$ & $\begin{array}{l}\text { NM_0011 } \\
09668\end{array}$ & $\downarrow 1.13$ & 0.6168 & $\downarrow 1.25$ & 0.0570 & $\downarrow 1.27$ & 0.6514 & $\downarrow 1.09$ & 0.8961 \\
\hline \multirow[t]{3}{*}{$\begin{array}{l}\text { PTEN } \\
\text { Dependent Cell } \\
\text { Cycle Arrest \& } \\
\text { Apoptosis }\end{array}$} & Cdkn1b & $\begin{array}{l}\text { Cyclin- } \\
\text { dependent } \\
\text { kinase inhibitor } \\
\text { 1B } \\
\end{array}$ & $\begin{array}{l}\text { NM_0317 } \\
62\end{array}$ & $\downarrow 1.28$ & 0.0570 & $\downarrow 1.42$ & 0.0127 & $\downarrow 1.07$ & 0.8595 & $\uparrow 1.04$ & 0.6191 \\
\hline & Foxo3 & $\begin{array}{l}\text { Forkhead box } \\
\text { O3 }\end{array}$ & $\begin{array}{l}\text { NM_0011 } \\
06395 \\
\end{array}$ & $\downarrow 1.35$ & 0.2981 & $\downarrow 1.39$ & 0.0261 & $\downarrow 1.18$ & 0.4787 & $\downarrow 1.01$ & 0.7489 \\
\hline & Rbl2 & $\begin{array}{l}\text { Retinoblastoma } \\
\text {-like } 2\end{array}$ & $\begin{array}{l}\text { NM_0310 } \\
94\end{array}$ & $\downarrow 1.30$ & 0.0320 & $\downarrow 1.36$ & 0.0132 & $\downarrow 1.28$ & 0.1827 & $\uparrow 1.24$ & 0.7618 \\
\hline $\begin{array}{l}\text { BAD } \\
\text { Phosphorylatio } \\
\text { n \& Anti- } \\
\text { Apoptosis }\end{array}$ & Ywhah & $\begin{array}{l}\text { Tyrosine 3- } \\
\text { monooxygenase } \\
\text { /tryptophan 5- } \\
\text { monooxygenase } \\
\text { activation } \\
\text { protein, eta } \\
\text { polypeptide }\end{array}$ & $\begin{array}{l}\text { NM_0130 } \\
52\end{array}$ & $\uparrow 1.07$ & 0.5539 & $\uparrow 1.30$ & 0.0137 & $\uparrow 1.19$ & 0.2628 & $\uparrow 1.61$ & 0.0473 \\
\hline
\end{tabular}

SDEGs surpassed 1.1-fold change with a $P$ value $<0.05$ have bold text. Italicized text $=$ trend $(0.05<P<0.10)$ for increased/decreased expression. Sample sizes were $N=5$ to 6 per group. FC $=$ fold change 
Table 4. Ingenuity Pathway Analysis (IPA) of gene expression results from the PI3K-AKT RT ${ }^{2}$ Profiler Pathway Array for the tibialis anterior muscle following dorsiflexion SSC-loading relative to non-loaded control muscles; the top 5 results from each category were included in the table.

\begin{tabular}{|c|c|c|c|c|c|c|}
\hline Condition & Analyses & \multicolumn{5}{|c|}{ 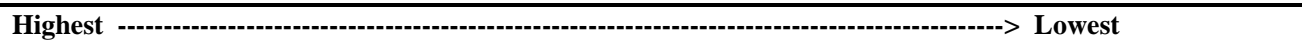 } \\
\hline \multirow{3}{*}{$\begin{array}{l}\text { Young ( } 3 \text { mo.) } \\
\text { SSC-loaded } 3 \\
\text { days per week }\end{array}$} & $\begin{array}{c}\text { Top Canonical } \\
\text { Pathways (p-value) }\end{array}$ & $\begin{array}{l}\text { HGF Signaling } \\
(6.90 E-17)\end{array}$ & $\begin{array}{l}\text { IGF-1 Signaling } \\
\quad(6.91 E-15)\end{array}$ & $\begin{array}{c}\text { Fc Episolon RI } \\
\text { Signaling } \\
(1.67 E-14)\end{array}$ & $\begin{array}{c}\text { PTEN Signaling } \\
\text { (3.47E-14) }\end{array}$ & $\begin{array}{c}\text { PI3K/AKT Signaling } \\
(4.87 E-14)\end{array}$ \\
\hline & $\begin{array}{l}\text { Top Networks } \\
\text { (Score) }\end{array}$ & $\begin{array}{l}\text { Cell Death \& } \\
\text { Survival, Cellular } \\
\text { Development, } \\
\text { Dermatological } \\
\text { Diseases and } \\
\text { Conditions } \\
\text { (22) }\end{array}$ & $\begin{array}{l}\text { Cell Death \& } \\
\text { Survival, Embryonic } \\
\text { Development, } \\
\text { Organismal } \\
\text { Development } \\
\text { (6) }\end{array}$ & $\begin{array}{c}\text { Cell-to-Cell } \\
\text { Signaling \& } \\
\text { Interaction, Nervous } \\
\text { System Development } \\
\text { and Function, } \\
\text { Carbohydrate } \\
\text { Metabolism } \\
\text { (6) }\end{array}$ & $\begin{array}{c}\text { Cell Death \& } \\
\text { Survival, Cell } \\
\text { Morphology, } \\
\text { Cellular Assembly } \\
\text { and Organization } \\
\text { (6) }\end{array}$ & $N / A$ \\
\hline & $\begin{array}{c}\text { Top Merged Network } \\
\text { Functions } \\
\text { (Bias-corrected } \\
\text { activation z-score) }\end{array}$ & $\begin{array}{c}\text { Cell Death \& } \\
\text { Survival (Apoptosis } \\
\text { of Kidney Cell } \\
\text { Lines) } \\
\text { (2.419) }\end{array}$ & $\begin{array}{c}\text { Cell Death \& } \\
\text { Survival (Cell Death } \\
\text { of Immune Cells) } \\
(-2.010)\end{array}$ & $\begin{array}{c}\text { Carbohydrate } \\
\text { Metabolism (Uptake } \\
\text { of Monosaccharide) } \\
(-1.916)\end{array}$ & $\begin{array}{c}\text { Cellular } \\
\text { Development, } \\
\text { Cellular Growth \& } \\
\text { Proliferation, } \\
\text { Embryonic } \\
\text { Development } \\
\text { (Proliferation of } \\
\text { Embryonic Cell } \\
\text { Lines) } \\
(-1.736) \\
\end{array}$ & $\begin{array}{c}\text { Cell Cycle, Gene } \\
\text { Expression (Binding } \\
\text { of Protein Binding } \\
\text { Site) } \\
(-1.729)\end{array}$ \\
\hline
\end{tabular}

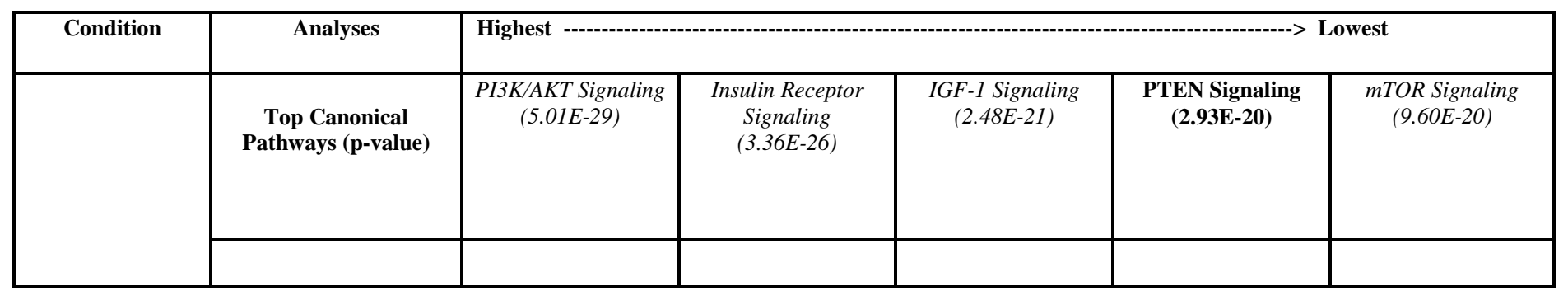




\begin{tabular}{|c|c|c|c|c|c|c|}
\hline \multirow[t]{2}{*}{$\begin{array}{l}\text { Young ( } 3 \text { mo.) } \\
\text { SSC-loaded } 2 \\
\text { days per week }\end{array}$} & $\begin{array}{l}\text { Top Networks } \\
\text { (Score) }\end{array}$ & $\begin{array}{l}\text { Protein Synthesis, } \\
\text { Cell Morphology, } \\
\text { Cellular Function } \\
\text { and Maintenance } \\
\text { (25) }\end{array}$ & $\begin{array}{c}\text { Cell Cycle, } \\
\text { Connective Tissue } \\
\text { Development and } \\
\text { Function, Cardiac } \\
\text { Proliferation } \\
\text { (12) }\end{array}$ & $\begin{array}{c}\text { Developmental } \\
\text { Disorder, } \\
\text { Neurological } \\
\text { Disease, Connective } \\
\text { Tissue Disorders } \\
\text { (10) }\end{array}$ & \begin{tabular}{|} 
Cellular Movement, \\
Cell Death and \\
Survival, Skeletal \\
and Muscular System \\
Development and \\
Function \\
(7)
\end{tabular} & $\begin{array}{c}\text { Cellular } \\
\text { Development, Cell- } \\
\text { To-Cell Signaling and } \\
\text { Interaction, Cellular } \\
\text { Assembly and } \\
\text { Organization } \\
\text { (3) }\end{array}$ \\
\hline & $\begin{array}{c}\text { Top Merged Network } \\
\text { Functions } \\
\text { (Bias-corrected } \\
\text { activation z-score) }\end{array}$ & $\begin{array}{c}\text { Cellular } \\
\text { Development, } \\
\text { Cellular Growth \& } \\
\text { Proliferation } \\
\text { (Proliferation of } \\
\text { Stem Cells) } \\
(2.246)\end{array}$ & $\begin{array}{c}\text { Carbohydrate } \\
\text { Metabolism (Uptake } \\
\text { of Monosaccharide) } \\
(-2.138)\end{array}$ & $\begin{array}{c}\text { Cell Death \& } \\
\text { Survival } \\
\text { (Apoptosis of } \\
\text { Leukocyte Cell } \\
\text { Lines) } \\
(-2.119)\end{array}$ & $\begin{array}{c}\text { Cell Death \& } \\
\text { Survival (Cell Death } \\
\text { of Immune Cells) } \\
(-2.007)\end{array}$ & $\begin{array}{c}\text { Cellular } \\
\text { Development, } \\
\text { Cellular Growth \& } \\
\text { Proliferation, } \\
\text { Hematological } \\
\text { System Development } \\
\text { \& Function, } \\
\text { Inflammatory } \\
\text { Response, Lymphoid } \\
\text { Tissue Structure \& } \\
\text { Development } \\
\text { (Proliferation of } \\
\text { Phagocytes) } \\
\text { (1.953) } \\
\end{array}$ \\
\hline
\end{tabular}

\begin{tabular}{|c|c|c|c|c|c|c|}
\hline Condition & Analyses & Highest ----------- & 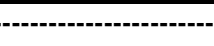 & 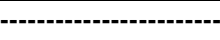 & 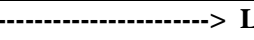 & \\
\hline \multirow{3}{*}{$\begin{array}{r}\text { Old (30 mo.) } \\
\text { SSC-loaded } 3 \\
\text { days per week }\end{array}$} & $\begin{array}{c}\text { Canonical Pathways } \\
\text { (p-value) }\end{array}$ & $\begin{array}{c}\text { IL-17A Signaling in } \\
\text { Gastric Cells } \\
(2.40 E-03)\end{array}$ & $\begin{array}{c}\text { Antiproliferative } \\
\text { Role of TOB in T } \\
\text { Cell Signaling } \\
\text { (2.49E-03) }\end{array}$ & $\begin{array}{l}\text { TNFR2 Signaling } \\
\quad(2.78 \mathrm{E}-03)\end{array}$ & $\begin{array}{c}L-17 A \text { Signaling in } \\
\text { Fibroblasts } \\
(3.36 E-03)\end{array}$ & $\begin{array}{l}\text { April Mediated } \\
\text { Signaling } \\
(3.64 \mathrm{E}-03)\end{array}$ \\
\hline & $\begin{array}{l}\text { Top Network } \\
\text { (Score) }\end{array}$ & $\begin{array}{c}\text { Protein Synthesis, } \\
\text { Cell Death and } \\
\text { Survival, RNA } \\
\text { Post- } \\
\text { Transcriptional } \\
\text { Modification } \\
\text { (6) }\end{array}$ & & & & \\
\hline & $\begin{array}{l}\text { Top Merged Network } \\
\text { Functions } \\
\text { (z-score) }\end{array}$ & N/A & N/A & N/A & N/A & N/A \\
\hline
\end{tabular}




\begin{tabular}{|c|c|c|c|c|c|c|}
\hline Condition & Analyses & \multicolumn{5}{|c|}{ 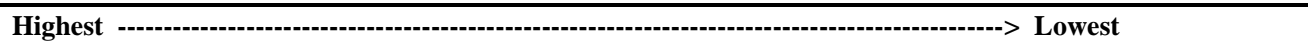 } \\
\hline \multirow{3}{*}{$\begin{array}{r}\text { Old ( } 30 \text { mo.) } \\
\text { SSC-loaded } 2 \\
\text { days per week }\end{array}$} & $\begin{array}{c}\text { Canonical Pathways } \\
\text { (p-value) }\end{array}$ & $\begin{array}{c}\text { ERK/MAPK } \\
\text { Signaling } \\
\text { (3.02E-09) }\end{array}$ & $\begin{array}{c}\text { HGF Signaling } \\
(4.19 E-08)\end{array}$ & $\begin{array}{l}\text { Cdc42 Signaling } \\
\quad(2.72 \mathrm{E}-07)\end{array}$ & $\begin{array}{l}\text { TNFR1 Signaling } \\
\text { (6.78E-07) }\end{array}$ & $\begin{array}{c}\text { Signaling by Rho } \\
\text { Family GTPases } \\
(1.05 E-06)\end{array}$ \\
\hline & $\begin{array}{l}\text { Top Networks } \\
\text { (Score) }\end{array}$ & $\begin{array}{c}\text { Cellular } \\
\text { Development, Cell } \\
\text { Death and Survival, } \\
\text { Skeletal and } \\
\text { Muscular System } \\
\text { Development and } \\
\text { Function } \\
\text { (10) }\end{array}$ & $\begin{array}{c}\text { Behavior, Cell } \\
\text { Death and Survival, } \\
\text { Cellular } \\
\text { Compromise } \\
\text { (10) }\end{array}$ & & & \\
\hline & $\begin{array}{c}\text { Top Merged Network } \\
\text { Functions } \\
\text { (Bias-corrected } \\
\text { activation z-score) }\end{array}$ & $\begin{array}{l}\text { Protein Synthesis } \\
\text { (Expression of } \\
\text { Protein) } \\
\text { (1.569) }\end{array}$ & $\begin{array}{c}\text { Cellular } \\
\text { Development, } \\
\text { Cellular Growth \& } \\
\text { Proliferation, } \\
\text { Connective Tissue } \\
\text { Development } \\
\text { (Cell Proliferation } \\
\text { of Fibroblasts) } \\
\text { (1.257) }\end{array}$ & $\begin{array}{c}\text { Cardiovascular } \\
\text { System } \\
\text { Development \& } \\
\text { Function, } \\
\text { Organismal } \\
\text { Development } \\
\text { (Angiogenesis) } \\
\text { (1.193) }\end{array}$ & $\begin{array}{c}\text { Cellular } \\
\text { Development, } \\
\text { Cellular Growth \& } \\
\text { Proliferation, } \\
\text { Connective Tissue } \\
\text { Development \& } \\
\text { Function } \\
\text { (Proliferation of } \\
\text { Fibroblast Cell } \\
\text { Lines) } \\
(\mathbf{0 . 6 8 7 )}\end{array}$ & \begin{tabular}{|c|} 
Cellular \\
Development, \\
Cellular Growth \& \\
Proliferation, Hair \\
and Skin \\
Development \& \\
Function, Organ \\
Development, Tissue \\
Development \\
(Proliferation of \\
Epidermal Cell \\
Lines) \\
(0.639) \\
\end{tabular} \\
\hline
\end{tabular}

The top canonical pathways and top networks were color highlighted based upon the percentage of genes associated with the pathway array that were up- or downregulated, while the top merged network functions were text highlighted based on z-score (i.e., activation state); bold text = increased activation, italicized text = decreased activation, standard text = unchanged. All IPA functions with the exception of cancer specific, reproductive system, and psychological functions were considered for this table. Sample sizes were $N=5$ to 6 per group. 


\section{FIGURE LEGENDS}

Figure 1. Reduced frequency of SSC “resistance-type exercise” training improves muscle performance of aged rats. During each training session, TA muscle performance was assessed during the first SSC repetition of the first training set. Both the work required to stretch the muscle (i.e., negative work) and the work performed by the muscle during the shortening phase (i.e., positive work) were measured and recorded values of A) dorsiflexion dynamic performance in terms of negative work performed on the first repetition of the first training set of each training session; B) dorsiflexion dynamic performance in terms of positive work performed on the first repetition of the first training set of each training session. For each group, the work performed was averaged based on recorded force values during the first week (initial) and last week of training (final). Sample sizes were $N=8$ to 10 per group. * Different from initial; ${ }^{*}$ Different from initial for old rats trained 2 days per week; ${ }^{\dagger}$ Different from final for old rats trained 3 days per week; $\ddagger$ Different from final for old rats trained 2 days per week, $\mathrm{p}<0.05$. Data presented as means \pm SEM. SSC $=$ stretch-shortening contraction; $\mathrm{TA}=$ tibialis anterior.

Figure 2. Reduced frequency of SSC “resistance-type exercise” training increases TA muscle mass size of aged rats. Frozen TA sections stained for 4-HNE and $\beta$-dystroglycan were manually traced to determine muscle fiber area. Frequency distributions for muscle fiber areas are presented as a percentage of the total myofibers measured for each group; A) young rats trained 3 days per week; B) young rats trained 2 days per week; C) old rats trained 3 days per week; D) old rats trained 2 days per week. Sample sizes were $N=4$ to 5 per group. Analyses for were done comparing SSC-loaded muscle following “resistance-type exercise” training with agematched non-loaded control TA muscle. All groups with the exception of old rats trained 3 days per week demonstrated a significant shift in distribution towards a greater fiber area following training, $\mathrm{p}<0.05$. SSC $=$ stretch-shortening contraction; TA = tibialis anterior; 4-HNE = 4-hydroxynonenal.

Figure 3. Reduced frequency of SSC “resistance-type exercise” training enhances increases the myonuclear domains of aged rats. Myonuclear domains were calculated as the ratio of the muscle fiber areas relative to the number of myonuclei per myofiber and was quantified by dividing the number of myonuclei by the total muscle fiber area sampled (nuclei per $\mathrm{mm}^{2}$ ). Frequency distributions for myonuclear domains are presented as a percentage of the total myonuclear domains measured for each group; A) young rats trained 3 days per week; B) young rats trained 2 days per week; C) old rats trained 3 days per week; D) old rats trained 2 days per week. Sample sizes were $N=4$ to 5 per group. Analyses were done comparing loaded muscle following SSC training with age-matched non-loaded control muscle. All groups with the exception of old rats trained 3 days per week demonstrated a significant shift in distribution following training, $\mathrm{p}<0.05$. Note in particular the pronounced 
shift towards a higher myonuclear domain in the old rats trained 2 days per week. SSC = stretch-shortening contraction; $\mathrm{TA}=$ tibialis anterior.

Figure 4. Reduced frequency of SSC “resistance-type exercise” training increases PI3K-AKT signaling in aged rats. The volcano plot graphs of the PI3K-AKT PCR array. These graphs show the log2 of the fold change for each gene's expression between SSC-loaded and non-loaded control TA muscle for each group. Fold changes and $P$ values were determined from comparisons of $\Delta \mathrm{C}_{\mathrm{t}}$ values between SSC-loaded and non-loaded control TA muscles analyzed through the GeneGlobe Data Analysis Center (Qiagen). A) Young rats trained 3 days per week; B) young rats trained 2 days per week; C) old rats trained 3 days per week; D) old rats trained 2 days per week. Sample sizes were $N=5$ to 6 per group. The vertical solid line indicates fold changes of 0 . Vertical dotted lines indicate that the fold change in gene expression threshold is 1.1. The horizontal solid line indicates that the P-value of the $t$ test threshold is 0.05 . Genes that were significantly differentially expressed are labeled and colored according to whether or not they were up- or downregulated. SSC = stretch-shortening contraction; PI3K = Phosphoinositide 3-kinase; AKT = Protein kinase B; TA = tibialis anterior.

Figure 5. Reduced frequency of SSC “resistance-type exercise” training augments muscle DNA, protein and translational efficiency in aged rats. Comparison of the molecular attributes between young and old rodent skeletal muscle for A) muscle RNA; B) muscle DNA; C) muscle protein; D) transcription efficiency index (RNA:DNA); E) translation efficiency index (protein:RNA). Both RNA and DNA concentrations were obtained using a spectrophotometer (ND-1000; NanoDrop Technologies Inc., Wilmington, DE, USA). Total protein contents of the cytoplasmic protein extracts were quantified and reported as protein concentration $(\mu \mathrm{g}$ protein/mg tissue - [protein]). The transcription efficiency index and translation efficiency index are surrogate measures of global transcriptional and translational efficiency and have been used previously (Roberts et al., 2010). ${ }^{\#}$ Different from non-loaded control within age; ${ }^{\dagger}$ Different from treatment within age; ${ }^{\$}$ Different from treatment by age. SSC = stretch-shortening contraction; $\mathrm{TA}=$ tibialis anterior. 
FIGURE 1

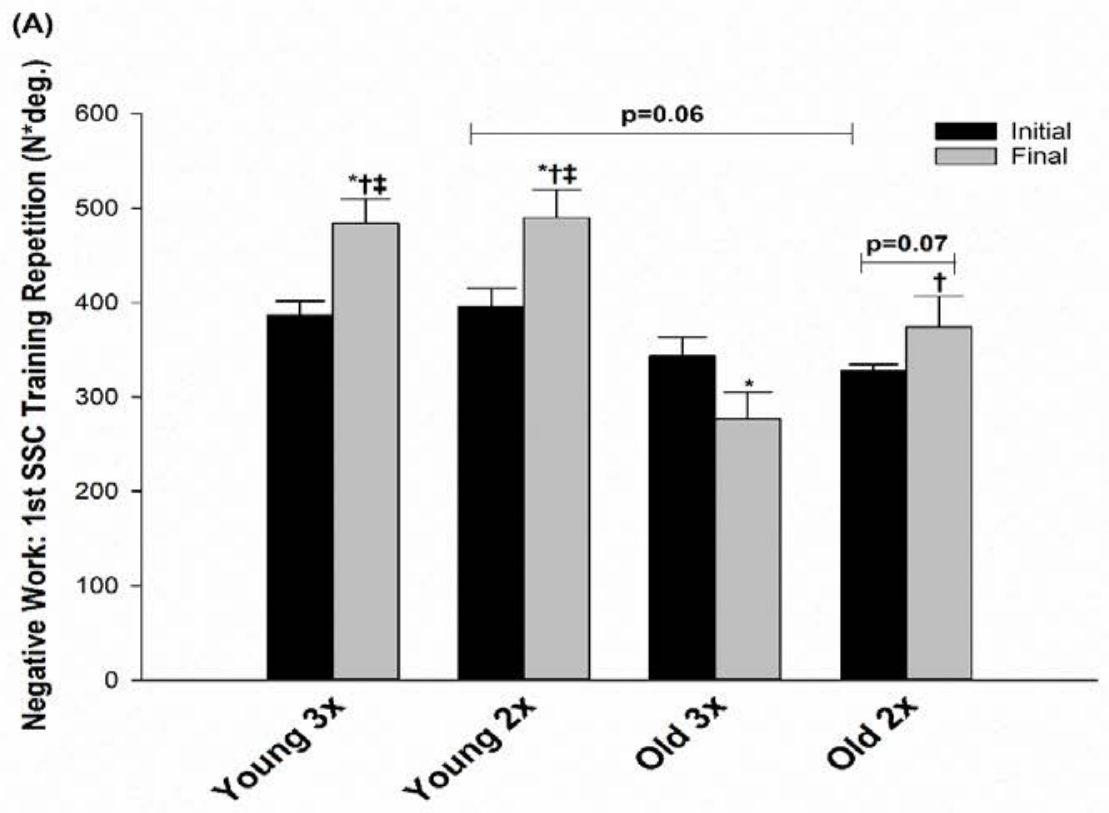


(B)

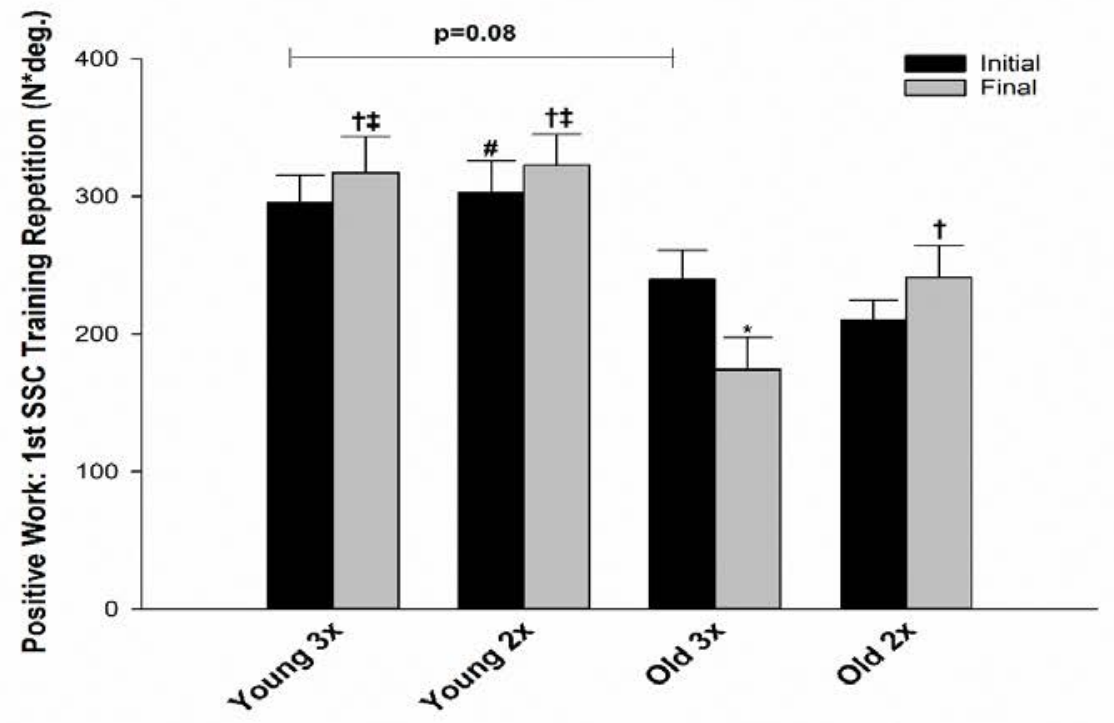


FIGURE 2

(A)

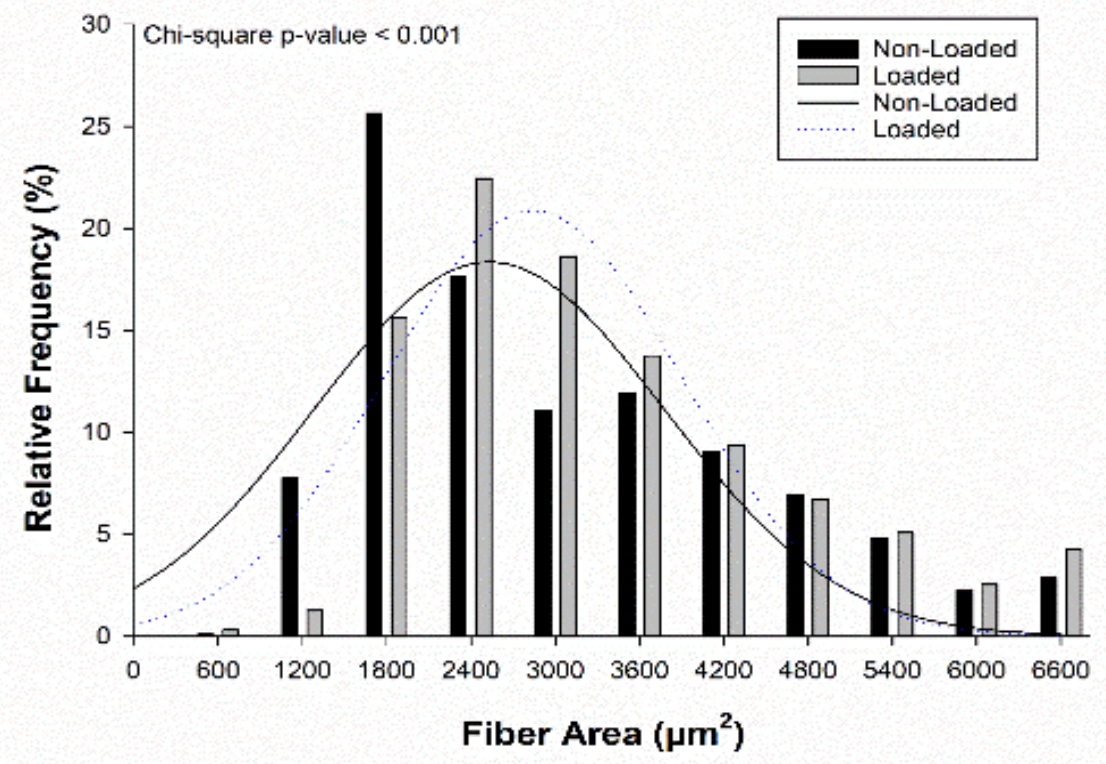


(B)

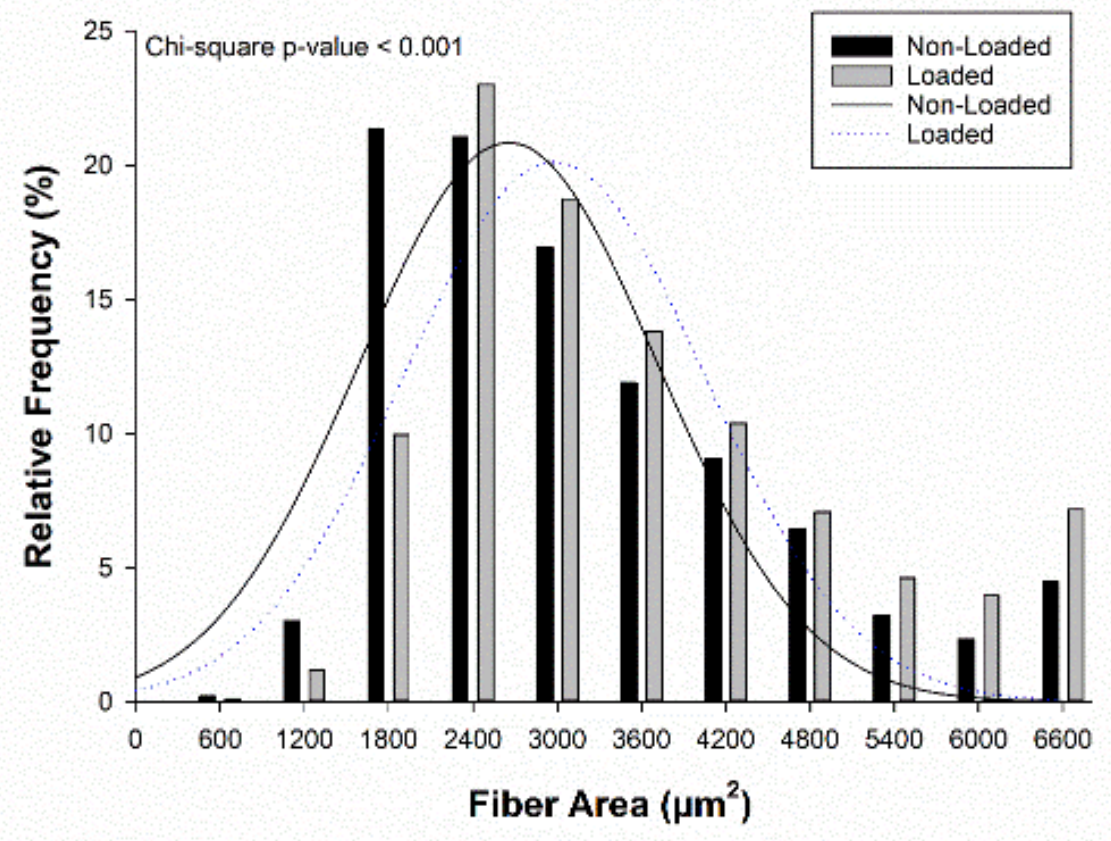


(C)

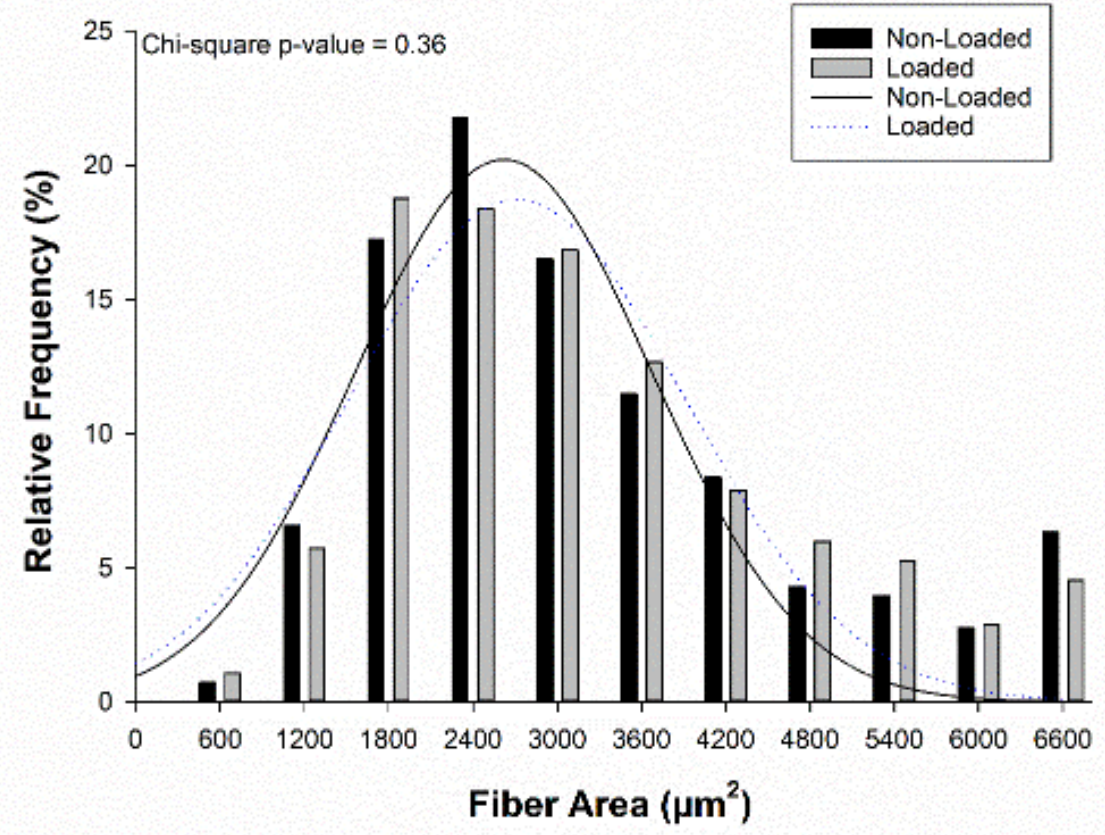


(D)

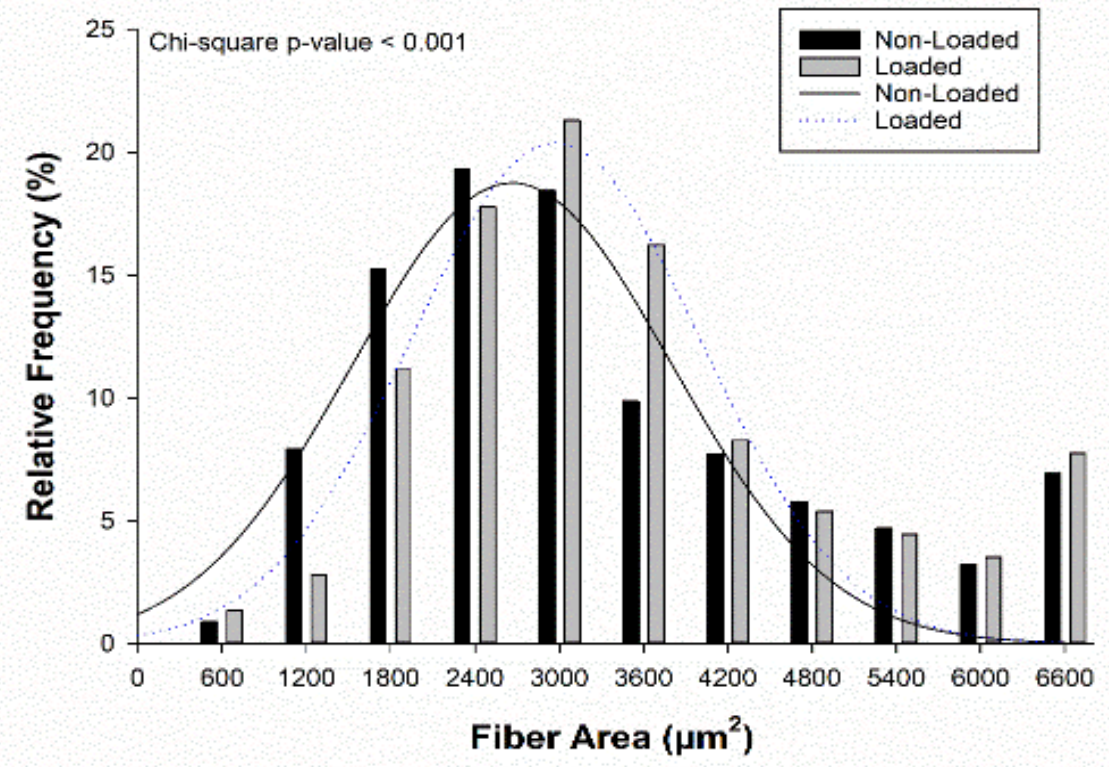




\section{FIGURE 3}

(A)

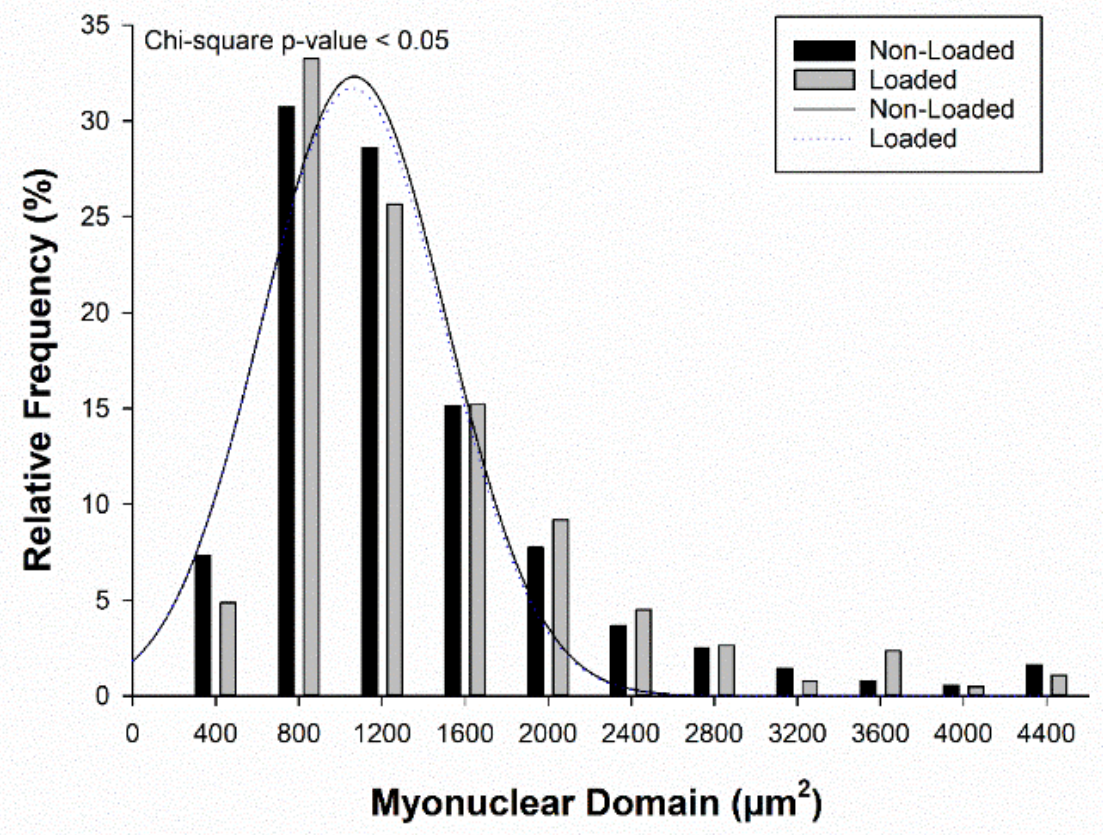


(B)

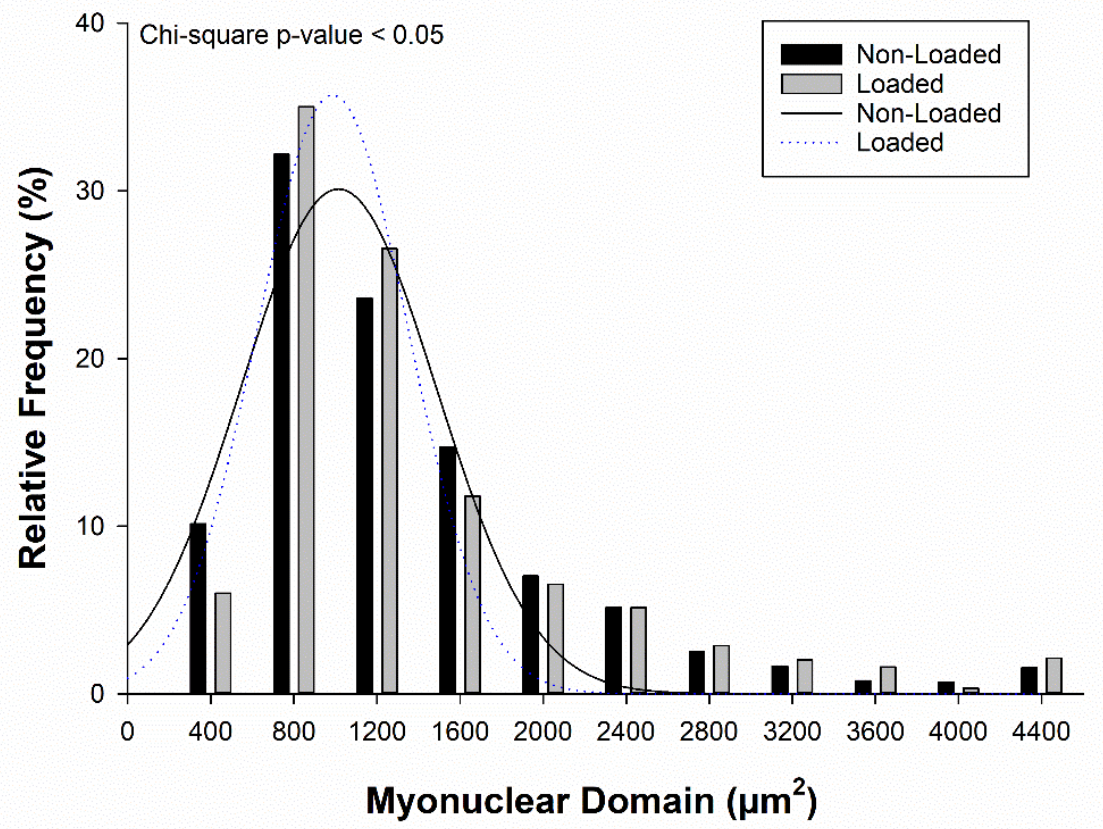


(C)

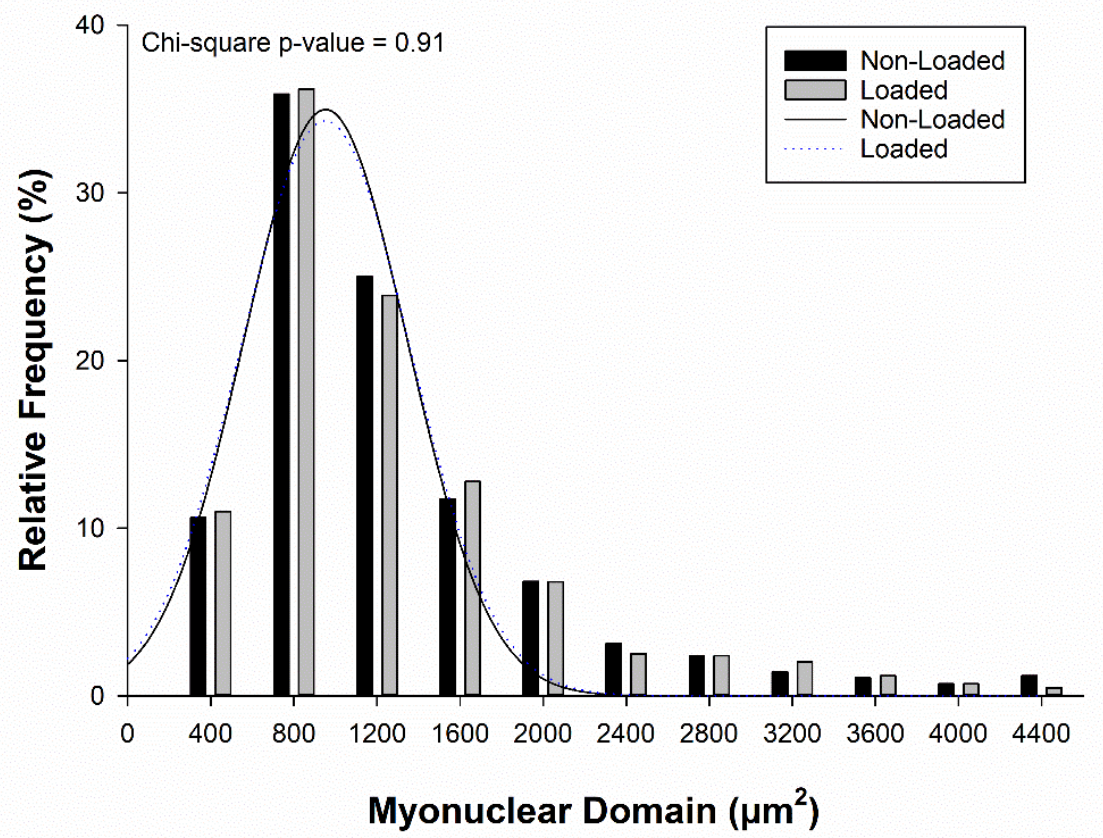


(D)

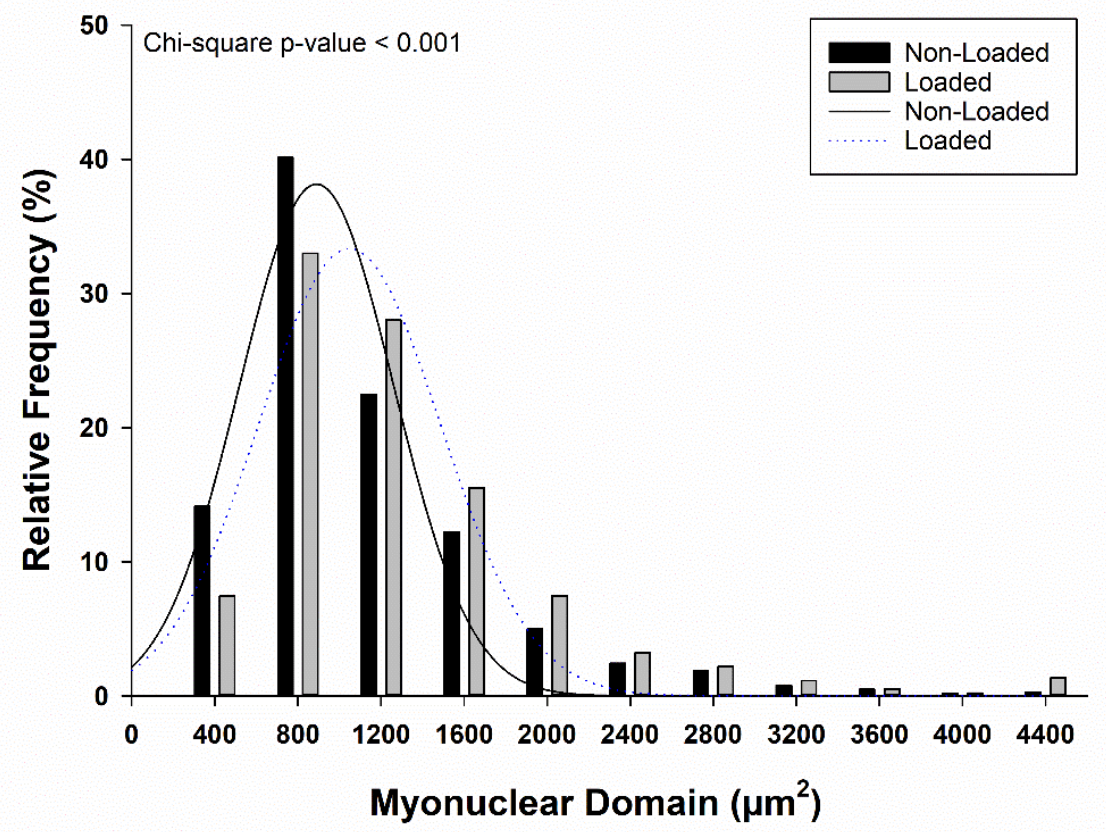




\section{FIGURE 4}

(A)

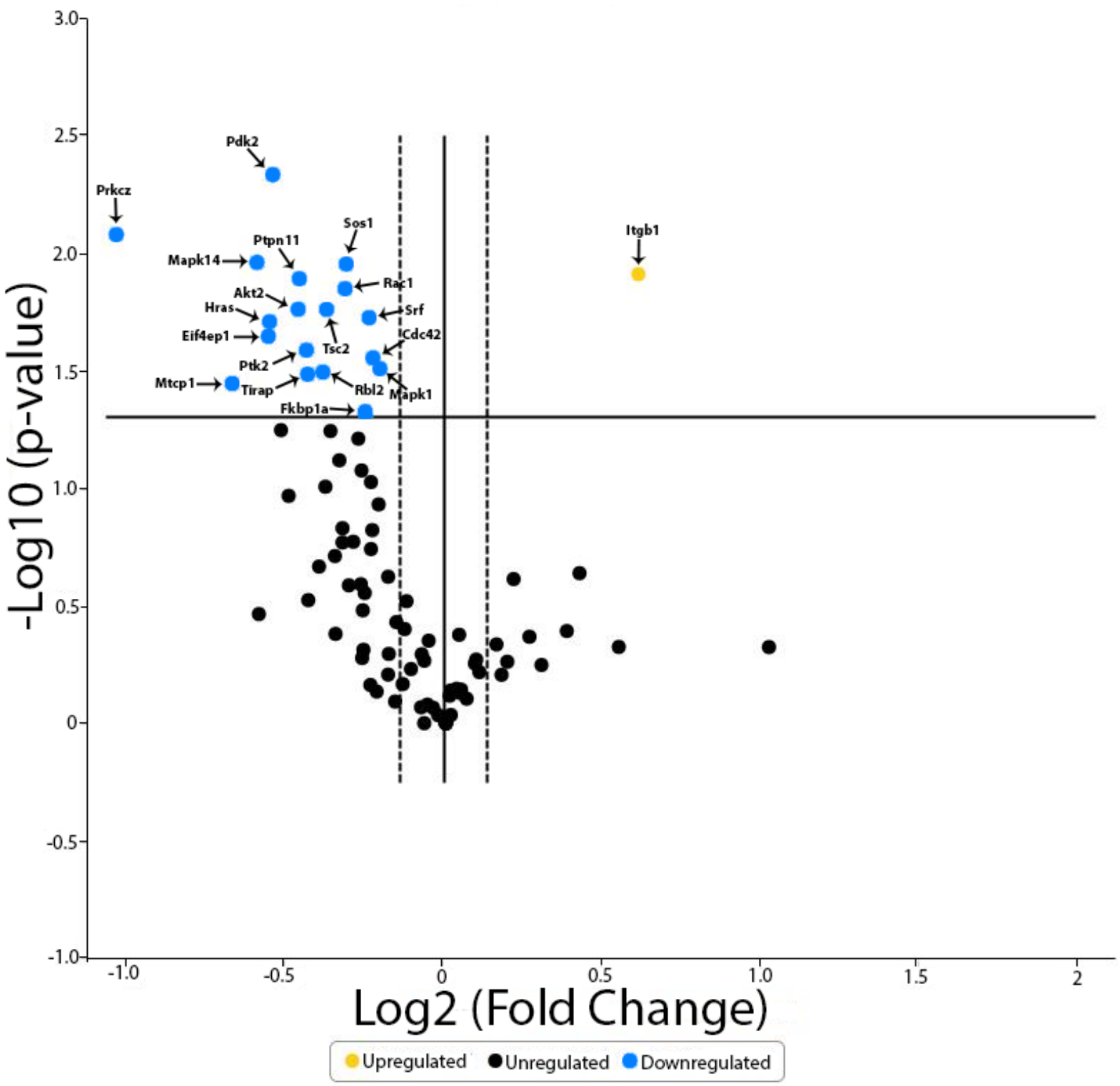




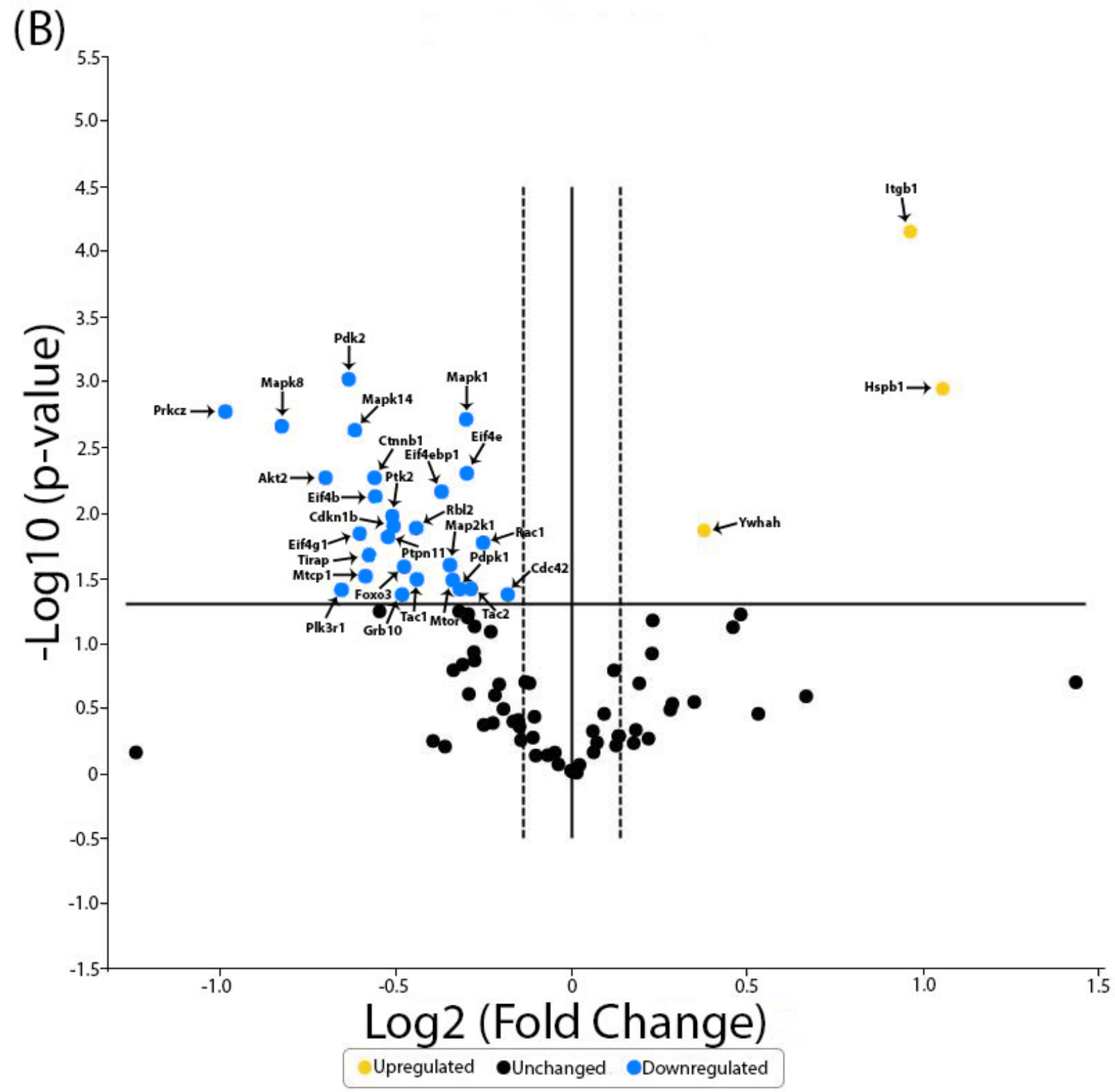


(C)

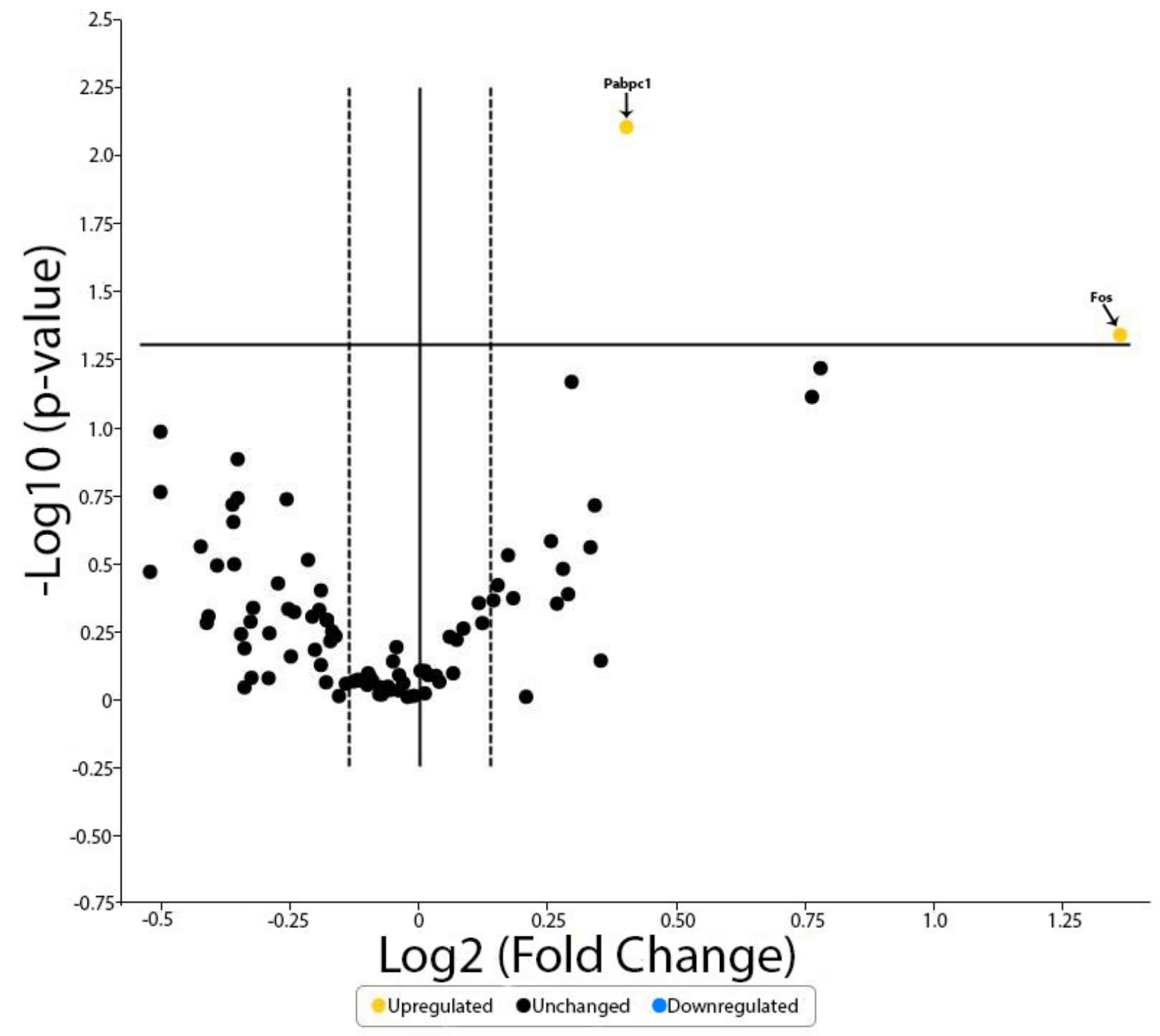




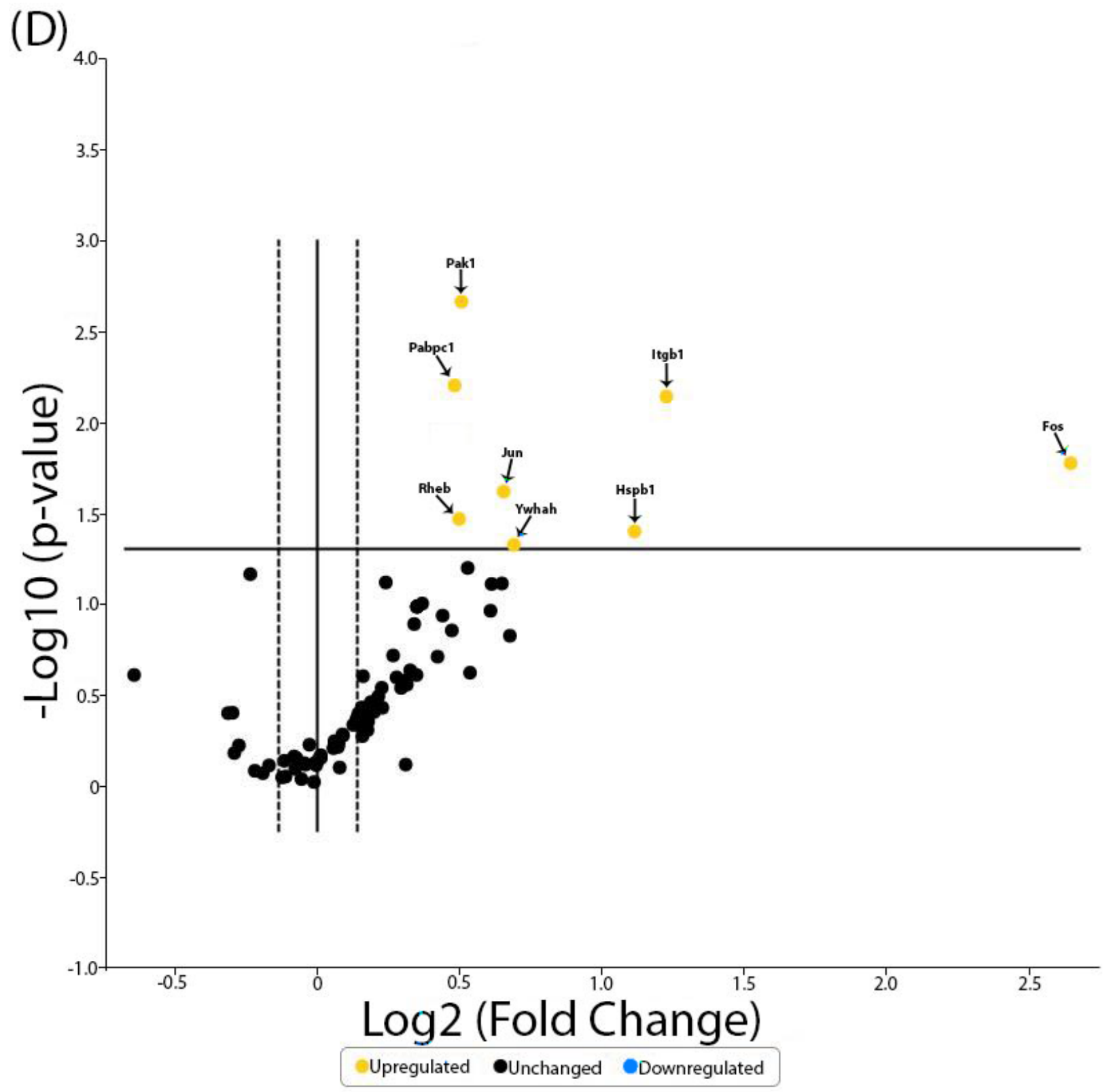


FIGURE 5

(A)

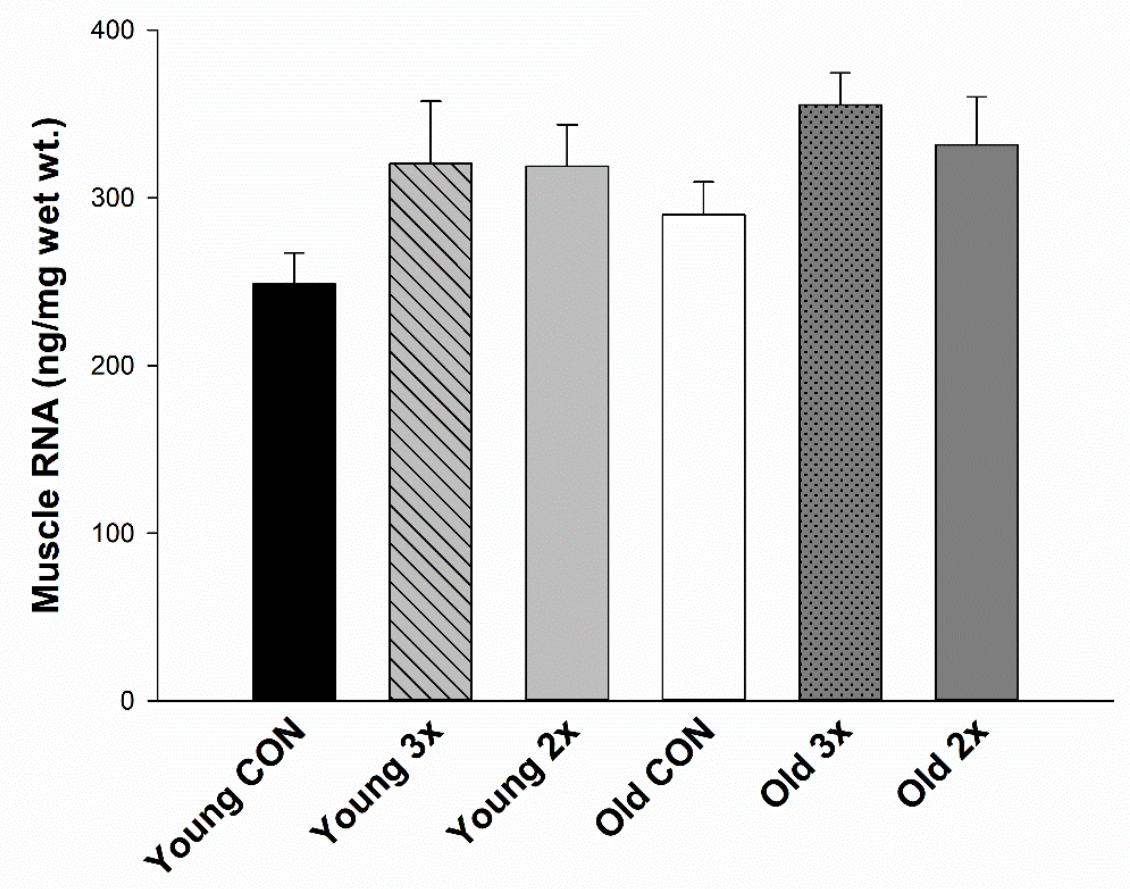


(B)

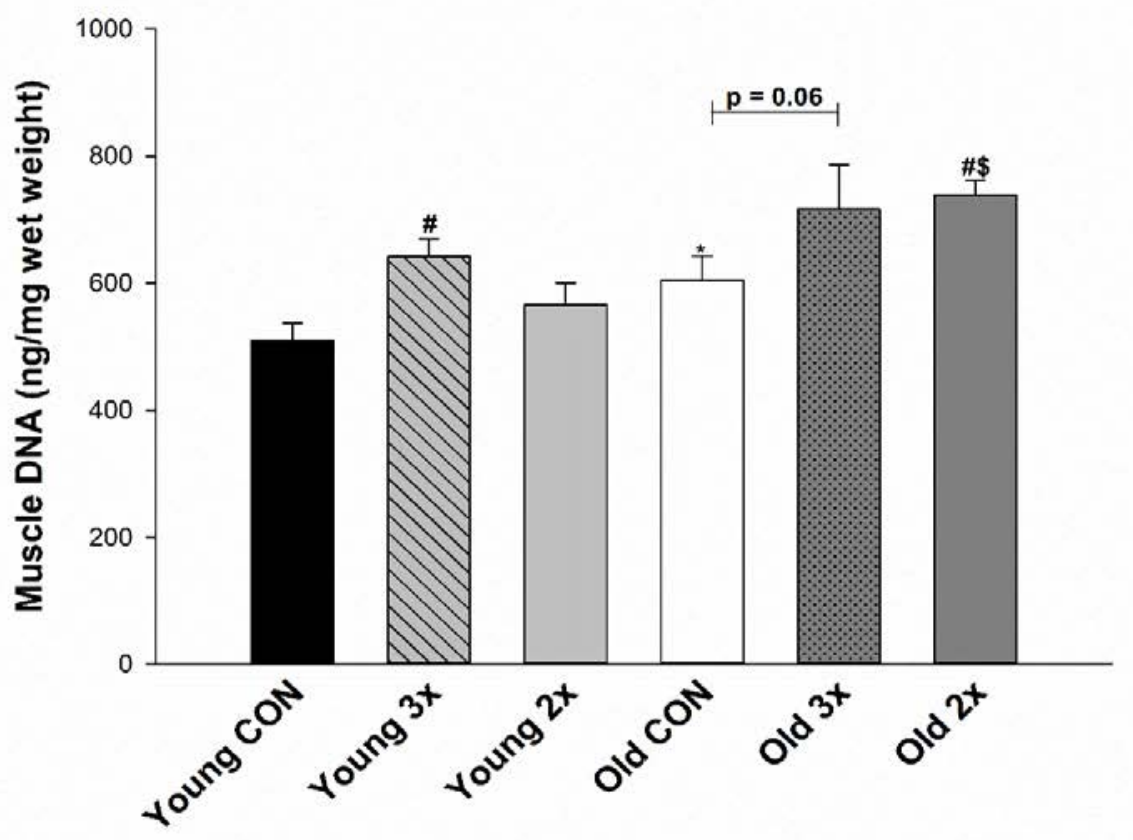


(C)

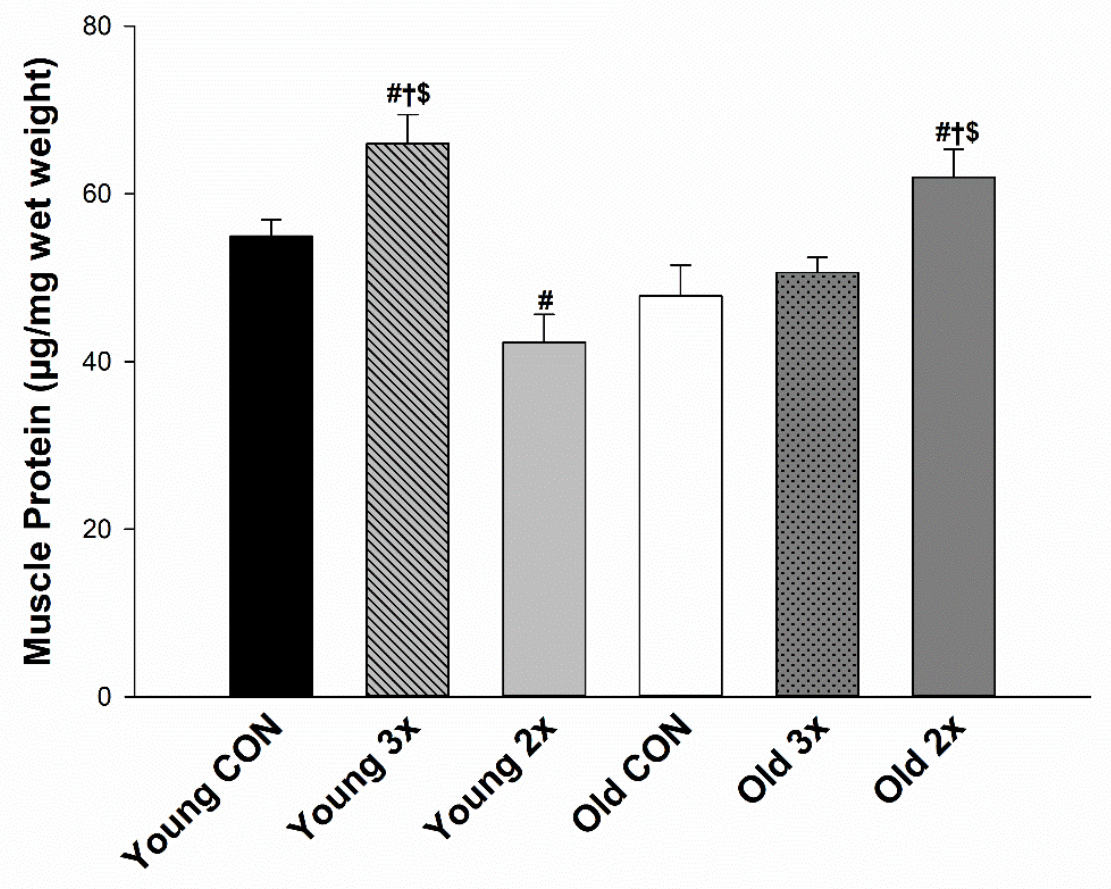


(D)

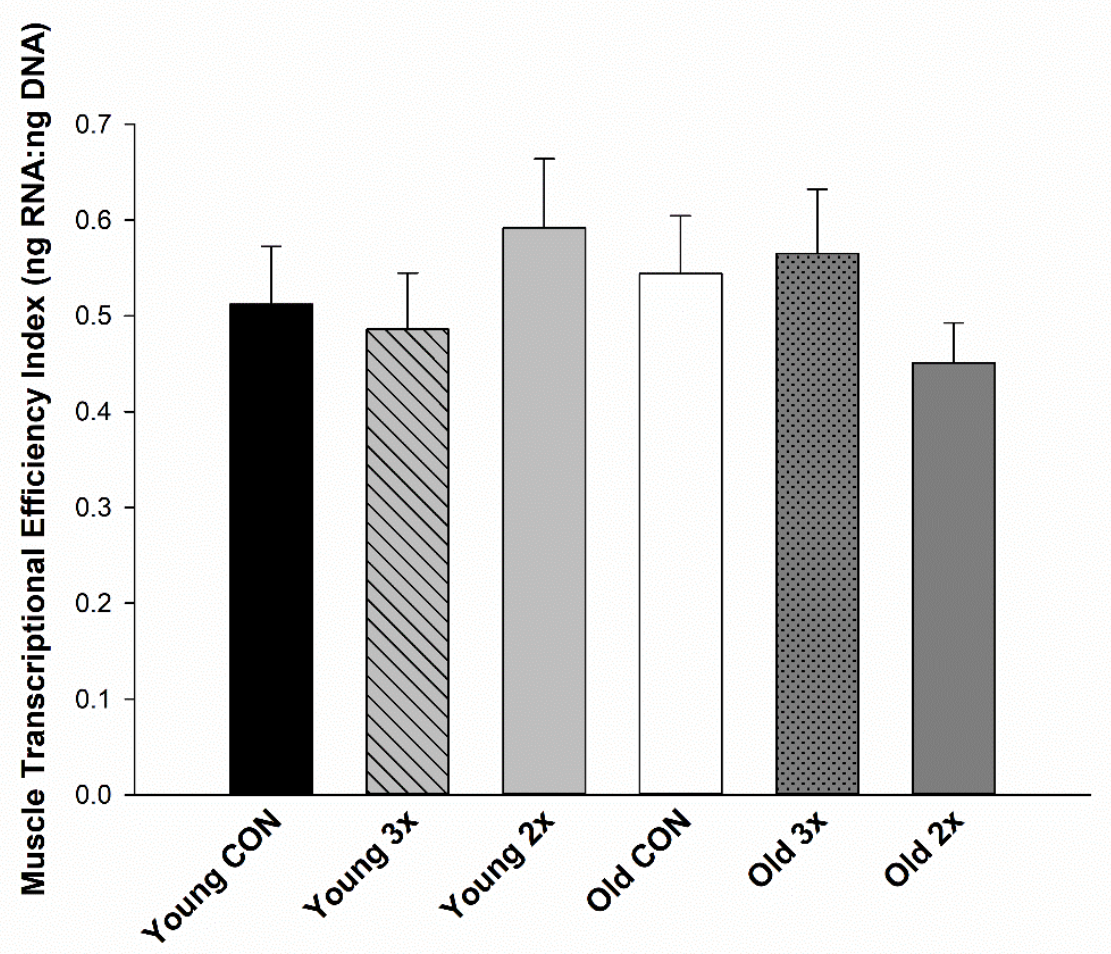




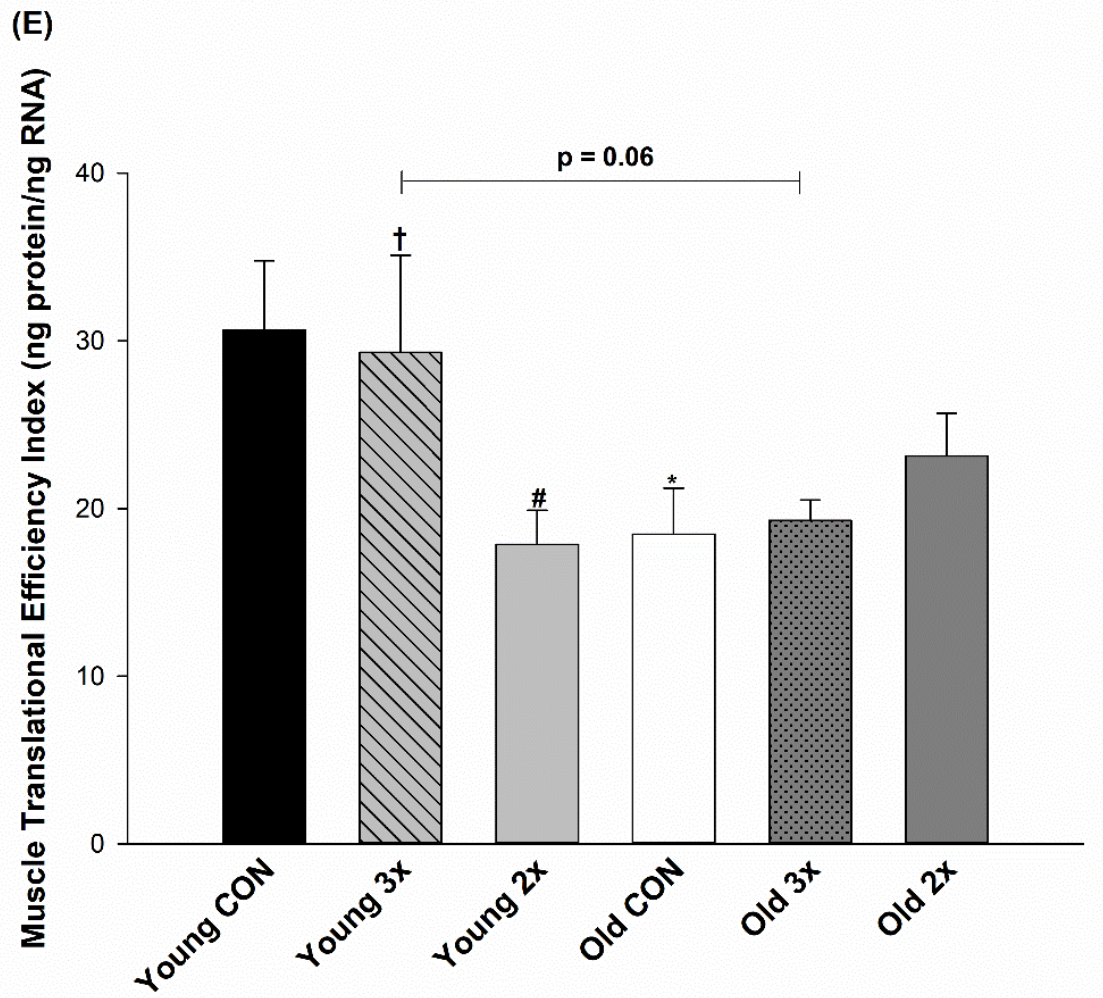




\section{CHAPTER V}

REDUCING THE FREQUENCY OF RESISTANCE-TYPE EXERCISE TRAINING POSITIVELY MODIFIES APOPTOSIS SIGNALING, DNA DAMAGE RESPONSE, AND MYONUCLEI MORPHOLOGY IN AGED SKELETAL MUSCLE

Authors: Marshall A. Naimo ${ }^{1,2}$, Erik P. Rader ${ }^{1}$, James Ensey ${ }^{1}$, Michael L. Kashon ${ }^{1}$, Brent A. Baker ${ }^{1}$

Affiliations: ${ }^{1}$ Centers for Disease Control and Prevention, National Institute for Occupational Safety and Health, Health Effects Laboratory Division, Morgantown, West Virginia, USA; ${ }^{2}$ West Virginia University, School of Medicine, Division of Exercise Physiology, Morgantown, West Virginia, USA

Running Head: Exercise Stress Response to Stretch-Shortening Contraction Training

Keywords: Stretch-shortening contractions, Fisher 344 x Brown Norway rats, muscle homeostasis, aging, rejuvenation, microenvironment 


\section{ABSTRACT}

Previously, our laboratory has shown that modifying the frequency of resistance-type exercise training (RTET) using stretch-shortening contractions (SSCs) from three to two days per week attenuates age-dependent maladaptation and restores muscle quality to a younger phenotype. The evidence suggests a link between stressinduced pathway responses as well as mechanical perturbations with nuclei morphology and epigenomics, but the precise mechanisms and the effects of these skeletal muscle regulatory responses on myonuclei with aging and RTET is unknown. Therefore, the purpose of this study was to utilize a pathway-specific approach to investigate if a differential response for apoptosis and DNA repair to a reduced training frequency was present after one month of high-intensity SSC RTET, specifically for gene expression and methylation status as well as alterations in myonuclei morphology. Tibialis anterior (TA) muscles of young (three months old) and old (30 months old) male Fischer x Brown Norway rats exposed to 80 SSCs for two or three days per week for one month were harvested 72 hours post-training. Gene expression and methylation were quantified via Qiagen ${ }^{\circledR}$ pathway gene expression and methylation arrays. Frozen TA tissue cross-sections were stained for $\beta$ dystroglycan and DAPI to perform myonuclei morphology via manual tracings using Image J. Cell death and 8hydroxydeoxyguanosine levels were quantified using enzyme-linked immunosorbent assays. Young rats adapted to training three days per week (Y3x) and two days per week (Y2x) and had 21 and seven apoptotic significantly differentially expressed genes (SDEGs) $(\mathrm{p}<0.05)$, respectively. Old rats maladapted to three days per week (O3x) training and only had increased expression of one anti-apoptotic gene (B-cell lymphoma 2related protein A1); however, muscles from old rats that trained two days per week (O2x) had eight apoptotic SDEGs. DNA methylation was significantly increased $(\mathrm{p}<0.05)$ in SSC trained relative to non-loaded control (NLC) muscles only in O3x (2.2 \pm 0.5 vs. $0.9 \pm 0.2 \%)$, and was significantly higher compared to O2x (0.7 $\pm 0.1 \%)$. For the DNA repair pathway, O3x had the most SDEGs with 37, but 36 of them were downregulated, despite having significantly lower levels of methylation compared to old NLC ( $0.7 \pm 0.1 \%$ vs. $2.8 \pm 0.4 \%)$. In contrast, O2x had 10 SDEGs that were all upregulated, indicating enhanced DNA repair signaling. Finally, chi-square analysis for $\mathrm{Y} 3 \mathrm{x}, \mathrm{Y} 2 \mathrm{x}$, and $\mathrm{O} 2 \mathrm{x}$ revealed significant decreases $(\mathrm{p}<0.05)$ in myonuclei size (measured by minimum feret diameter) along with unaltered circularity values ( $\mathrm{p}>0.05)$; in contrast, O3x had no changes in myonuclei minimum feret diameter and significantly decreased $(\mathrm{p}<0.05)$ circularity. In conclusion, reduced SSC training frequency positively influences aged muscle by decreasing methylation of apoptotic genes, thereby increasing gene expression, concomitant with positive outcomes on DNA repair transcription, myonuclei size, and myonuclei morphology, thus aiding in promoting muscle adaptation following highintensity SSC RTET. 


\section{Publications Disclaimers:}

"The findings and conclusions in this report are those of the author(s) and do not necessarily represent the official position of the National Institute for Occupational Safety and Health, Centers for Disease Control and Prevention." 


\section{INTRODUCTION}

Exercise is known to be an efficacious model for improving health and attenuating or preventing many chronic diseases (96). For the previous two decades, our laboratory has established a non-injurious, minimally invasive in vivo rodent dynamometer model of resistance-type exercise training (RTET) in order to explore the effects of various skeletal muscle training paradigms using stretch-shortening contractions (SSCs) (29); SSCs are characterized by having an isometric preactivation phase prior to initiation of the movement followed by subsequent lengthening and shortening contractions $(60,107)$. Having a model that utilizes these type of movements is physiologically relevant because SSCs are inherent in activities of daily living as well as the majority of exercise movements (e.g., walking, sprinting, jumping, resistance training, plyometrics), which is in contrast to sequestered isometric, concentric, or eccentric only contractions $(85,107)$. The responses to RTET using this physiological model range from adaptation, characterized by enhanced skeletal muscle performance along with increased muscle mass, to maladaptation, defined as an absence or diminishment of skeletal muscle performance and no improvements in muscle mass.(28, 86). Specifically, in response to 80 SSC (eight sets of 10 repetitions, two minutes rest between sets) maximal intensity training for one month, training-induced adaptation occurs in young rodents characterized by increased muscle performance and mass; this response is altered with age, in which old rodents undergo maladaptation when exposed to this same general, chronic loading protocol (28).

One potential important reason for why aged muscle is unable to adapt to the same loading protocol in comparison to younger counterparts implied from previous research is high chronic levels of inflammatory signaling and oxidative stress present in the skeletal muscle tissue $(5,54,82)$. Moreover, oxidative stress and inflammatory signaling can potentially become exacerbated in response to increased mechanical loading, which can subsequently lead to an attenuation in parameters of muscle adaptation, such as force output (82, 92). For example, a pair of studies by Ryan et al. $(92,93)$ have shown that not only does aged skeletal muscle present with higher levels of oxidants, oxidative stress, and oxidative damage at baseline, but that these responses were significantly heightened compared to young rats following SSC RTET.

However, there have been potential solutions put forth aimed at combating these age-specific negative stress responses to RTET. For instance, old rodents given Vitamin E and C supplementation had significantly decreased baseline and post-SSC RTET levels of oxidative stress (indicated by the ratio of glutathione to oxidized glutathione) while simultaneously leading to significantly increased positive work capacity (93). Another study by Takahashi et al. (104) gave rodents epigallocatechin-3-gallate and found positive modifications aged skeletal muscle mass and autophagy signaling in response to 14 days of reloading immediately following 14 days of hindlimb-suspension. Moreover, several studies looking at supplementing aged rodents with resveratrol, a naturally occurring compound found in plants known to have powerful 
antioxidant and antiaging properties (52) following muscle disuse atrophy via hindlimb suspension have found that it led to declines in oxidative stress (e.g., hydrogen peroxide, lipid peroxidation) $(17,51,52)$, enhancements in antioxidant defense enzymes (52), improvements in force output (52), enhanced plantaris muscle mass/type II muscle cross-sectional area (CSA) (17), improved fast-twitch fiber contractile function (51), and increased osteoblast bone formation (32). Moreover, resveratrol was demonstrated in older adult humans to increase muscle strength, power, along with various measures of mitochondrial density following 12 weeks of RTET (6). Finally, Haramizu et al. (47) examined the effects of resveratrol in vitro using mouse C2C12 myoblasts and found it significantly increased antioxidant production and protected against oxidative stress through reductions in the apoptotic index along with activity levels of caspase 9, caspase 8, and caspase 3.

Despite these positive effects with supplementation, recent evidence has emerged indicating that modifying parameters of the exercise prescription (e.g., frequency, repetition number) may also be effective in improving the oxidative stress and adaptive responsivity of skeletal muscle without the requirement for supplementation. Intriguingly, modifying the frequency of RTET from three to two days per week or by altering the total amount of repetitions to a modest number in old rats not only attenuated some components of maladaptation, such as enhanced muscle mass and MQ, but that it also led to significantly lower levels of oxidative stress (i.e., lipid peroxidation) and inflammatory signaling via protein levels of TNF- $\alpha$ that were indistinguishable from younger muscle $(85,86)$. Therefore, these results demonstrated that not only modifying training frequency or repetition number may be beneficial by providing additional recovery and/or reducing the overall work demand, but that high-intensity SSC RTET prescribed in an appropriate manner can lead to positive changes in the overall muscle redox environment, decrease the susceptibility of aged muscle to the negative effects of chronic inflammatory signaling, and induce adaptation. Additionally, these studies suggest that the adaptive capacity of skeletal muscle in response to high-intensity SSC RTET is age-specific and dependent upon precise variables of the exercise prescription, which have the capability of affecting the organism on a systems level $(83,86)$. Despite the therapeutic potential demonstrated previously of RTET in the treatment of sarcopenia (13), a fundamental basis for evidence-based exercise prescription is still largely undetermined because the integrated physiological pathways involved in the exercise-induced stress response leading to muscle adaptation or maladaptation (e.g., apoptosis, DNA repair) are not fully understood.

Aging in and of itself is a biological process associated with an altered phenotype, and emerging evidence suggests that these effects are possibly linked to epigenetic/epigenomic regulation, which are changes in gene expression (and subsequently the phenotype) as a result of factors other than modifications in the actual DNA itself that are attributed to external/environmental influences (105). Given the various mechanisms implicated in the epigenome of cells, DNA methylation, the process of attaching a methyl group to the 5' position of a cytosine within DNA, is considered the most robust form of epigenomic regulation (25, 33). 
Methyl groups attached within the promoter region of genes typically leads to transcriptional repression due to the mobilization of methyl cytosine-guanine base pairing $(\mathrm{CpG})$ binding domain proteins, which subsequently recruit repressor complexes. These repressor complexes, which contain histone deacetylases, directly contribute to chromatin remodeling and changes in gene expression by keeping DNA in a closed state (i.e., heterochromatic), thereby acting as a blockade to transcription factors and RNA polymerase and leaving them unable to access the DNA $(22,61,87)$. Although a few exceptions have been noted, DNA methylation occurring at the promoter or enhancer regions of DNA almost always results in an attenuation of gene expression; therefore, while excluding other variables, the higher the amount of methylation present, the lower the gene expression that will be transcribed.

Excitingly, recent research has shown that exercise can influence changes in DNA methylation in skeletal muscle (61). For instance, acute exposures to varying intensities of aerobic exercise have resulted in altered methylation levels as well as expression of genes related to muscle metabolism, mitochondrial biogenesis, and muscle growth in both human and rodent skeletal muscle tissue $(14,55,57,62)$. In regards to chronic exercise, while not explored as in-depth as acute bouts of exercise, past findings from others imply that changes in methylation are associated with alterations in expression of genes related to muscle adaptation (61, 74). For instance, Nitert et al. (74) sought to look at the impact of a six month aerobic exercise intervention on DNA methylation in human skeletal muscle in males with or without a past family history of type 2 diabetes mellitus and found that six months of mainly endurance exercise resulted in significant reductions in promoter DNA methylation of genes with known functions in both muscle and type 2 diabetes mellitus. Kanzleiter and colleagues (55) had nine-week old male mice perform five days of endurance treadmill exercise training for four weeks. An initial bisulfite sequencing analysis performed found that compared to sedentary controls, exercised mice had 2,762 genes that displayed differentially methylated CpGs in their promoter regions. Further analysis of gene expression data revealed that approximately 200 out of the 2,762 genes had negative correlations between $\mathrm{CpG}$ methylation status and gene expression, with the majority of these genes being related to muscle growth, differentiation, and metabolism.

Nguyen et al. (73) used a mouse model of severe dyslipidemic mice lacking low-density lipoprotein receptor and had them go through three months of endurance exercise using a voluntary wheel. The authors found that one of the most important antioxidant enzymes, glutathione peroxidase 1, had significant alterations in gene expression accompanied by significant decreases in DNA methylation following the training protocol. Finally, Lindholm and colleagues (65) conducted a three month one-legged exercise training study in young adult humans. The authors stated their main findings were that endurance training induced significant changes in methylation at approximately 5,000 sites across the genome, along with significant differential expression of roughly 4,000 genes. Collectively, these studies suggest that a physiological stimulus with well-known health 
benefits (i.e., exercise) leads to epigenomic modifications in the form of DNA methylation, and that these alterations are associated with changes in gene expression as well as performance (65). However, no studies have investigated how DNA methylation is altered with aging, and whether or not it may be influencing the adaptation of skeletal muscle in response to a physiological model of high-intensity SSC RTET, which could be an important mechanism underlying the responsivity to training in the context of aging. Additionally, although a relationship between chromatin state and nuclei morphology has been previously established $(58,103,108)$, it is unknown what (if any) effect there is on myonuclei morphology in response to chronic high-intensity SSC loading.

In order to examine these unresolved issues, the purpose of this study was: 1) To determine the effects of aging and a reduced training frequency using high-intensity SSC loading two or three days per week on the activation of molecular signaling pathways associated with the maintenance and adaptation of skeletal muscle (i.e., apoptosis, DNA damage and repair) following one month of high-intensity SSC RTET in old rats; and 2) to investigate whether DNA methylation is influencing the molecular signaling activity/adaptability of skeletal muscle following this training protocol, and whether or not reducing the training frequency modifies the methylation profile of skeletal muscle . Our hypothesis was that older animals exposed to a reduced frequency of high-intensity SSC RTET would respond favorably to the training stimulus and in an appropriate manner (i.e., adaptation) and would more closely resemble younger rats in terms of the underlying molecular signaling pathway, DNA methylation, and redox environment responses that would be in contrast to age-matched counterparts exposed to a higher frequency that induces maladaptation. Moreover, we also postulated that the ability of aged muscle to adapt to high-intensity SSC RTET would be compromised when exposed to an inappropriate (i.e., maladaptive) exercise stimulus as a consequence of a dysregulated molecular signaling response, which would be observable in distinct pathways crucial in muscle homeostasis and remodeling; furthermore, these potential age-related dysregulated events in gene activity would occur as a consequence to alterations in DNA methylation. .

\section{MATERIALS AND METHODS}

\section{Animals}

Young (3 mo.) and old (30 mo.) male Fischer x Brown Norway (F344xBN) rats were obtained from the National Institute of Aging colony and housed in an Association for Assessment and Accreditation of Laboratory Animal Care International-accredited animal quarters. All animal procedures were approved by the Animal Care and Use Committee at the National Institute for Occupational Safety and Health in Morgantown, WV and were in compliance with the NIH guide for the care and use of animals in research. 


\section{RTET Paradigm}

F344xBN male rats were randomly assigned to a high-intensity SSC RTET protocol for 4.5 weeks in one of the following four groups: 1) Young rats SSC-loaded three days per week (Y3x; n=10); 2) Young rats SSC-loaded two days per week (Y2x; n=10); 3) Old rats SSC-loaded three days per week (O3x; $n=10)$; 4) Old rats SSC-loaded two days per week $(\mathrm{O} 2 \mathrm{x} ; \mathrm{n}=9)$. The loading paradigms were identical with the exception of the training frequency. For the two day per week training groups, training occurred on Mondays and Thursdays, with 72 hours between training bouts; for the three day per week training groups, training was carried out on Mondays, Wednesdays, and Fridays, with 48 hours rest between training sessions. The training protocol was based on a previously validated, SSC RTET protocol $(28,29,86)$. Briefly, rats were anesthetized with 2-5\% isoflurane gas and placed in dorsal recumbency on a heated table with the left knee secured in flexion at $90^{\circ}$ and the left foot secured in a load cell. Platinum electrodes were placed subcutaneously for activation of the dorsiflexor muscles. Muscle stimulation parameters (4-V magnitude, 0.2-ms pulse duration, and 120-Hz frequency) were based on previous studies of this model that found these settings to be optimal for producing supramaximal force outputs of the tibialis anterior (TA) muscle in male rats $(28,29)$. Training consisted of 80 total SSCs per session, comprised of eight sets of 10 repetitions, with 2-minute rest intervals between sets. For every SSC, muscles were maximally activated, with the ankle angle set to $90^{\circ}$ of flexion for 100 ms, rotated out to $140^{\circ}$ of flexion at a movement velocity of $60^{\circ} / \mathrm{s}$, then returned back to $90^{\circ}$ at $60^{\circ} \mathrm{s}$. Using this protocol, agespecific performance adaptation has been shown to occur along with a concomitant absence of overt muscle inflammation and degeneration in the days and weeks following training $(9,10,28,86)$.

During each training session, dynamic muscle performance was assessed during the first SSC repetition of the first training set by measurement of peak force, which was recorded and quantified using MatLab software (Version 9.3; MathWorks ${ }^{\circledR}$, Natick, MA, USA). Seventy two hours after the final SSC-loading protocol, rats were euthanized and the TA muscle was surgically removed, weighed, and recorded as the absolute muscle mass (i.e., wet-weight) in grams. Seventy two hours was chosen because this is considered beyond the acute effects of the immediate post-training responses but within the timeline where any overt signs of muscle degeneration as a consequence to the last training session would occur (86). Dynamic MQ was derived from both performance and muscle mass values by taking the SSC peak force and dividing by the normalized (i.e., muscle mass relative to tibia length) muscle mass, which were previously reported elsewhere $(84,86)$. The mid-belly of the TA was coated with tissue freezing media (Tissue-Tek, 4583 O.C.T Compound; Sakura Finetek, Torrance, CA, USA) and frozen in isopentane cooled to the temperature of liquid nitrogen and stored at $-80^{\circ} \mathrm{C}$ prior to conducting quantitative morphology, gene expression, and protein analysis.

\section{Gene Expression Analysis}


Muscle tissue stored at $-80^{\circ} \mathrm{C}$ was retrieved and a $50 \mathrm{mg}$ portion was homogenized using a MiniBeadbeater 8 (Biospec Products, Bartlesville, OK, USA) with $1 \mathrm{ml}$ TRIzol and $1.0 \mathrm{~mm}$ zirconia beads (Catalog \#22079110zx; Biospec). RNA was subsequently isolated using the RNAqueous ${ }^{\mathrm{TM}}$ Phenol-free total RNA Isolation Kit (Cat\# AM1912; Life Technologies, Carlsbad, CA, USA) following kit instructions. RNA concentrations were obtained using a spectrophotometer (ND-1000; NanoDrop Technologies Inc., Wilmington, DE, USA). Next, cDNA was synthesized using the RT² First Strand Kit (Catalog\# 330401; Qiagen, Valencia, CA, USA). Samples were then analyzed using the Rat Skeletal Muscle Apoptosis RT ${ }^{2}$ Profiler PCR Array (Catalog\# PARN-012Z; Qiagen), the Rat Skeletal Muscle DNA Repair RT² Profiler PCR Array (Catalog\# PARN-012Z; Qiagen), and RT² SYBR Green Mastermix (Catalog\# 330523; Qiagen) per manufacturer’s instructions with use of an ABI PRISM 7500 instrument (Applied Biosystems, Foster City, CA, USA). The RT ${ }^{2}$ Profiler PCR Arrays was able to simultaneously assay 84 genes considered a part of the apoptosis and/or DNA repair signaling pathways (Tables $1 \& 2$ ). Fold changes and $P$ values were determined from comparisons of $\Delta \mathrm{C}_{\mathrm{t}}$ values between SSC-loaded and non-loaded control (NLC) muscles (i.e., the contralateral limb not subjected to RTET) and were analyzed through the GeneGlobe Data Analysis Center (Qiagen). Five housekeeping genes [Actin beta (ACTB), beta-2 microglobulin (B2M), hypoxanthine phosphoribosyltransferase 1 (HPRT1), lactate dehydrogenase 1 (LDHA), and ribosomal protein, large, P1 (RPLP1)] were included on the array. The genes HPRT1 and LDHA were chosen as reference genes for normalization of values generated through GeneGlobe for the apoptosis arrays because they displayed the most stable results; HPRT1 and RPLP1 were chosen as reference genes for normalization of values for the DNA repair arrays for the same reason. Bioinformatic analysis was performed using IPA (Ingenuity Systems, www.ingenuity.com), which allowed for functional annotation of differentially expressed genes. All IPA biological functions except cancer specific, reproductive system, and psychological functions were evaluated.

\section{INSERT TABLE 1 HERE}

INSERT TABLE 2 HERE

\section{DNA Methylation Analysis}

Total DNA was isolated using the DNeasy ${ }^{\circledR}$ Blood \& Tissue Kit (Qiagen) according to kit instructions. DNA extracts were prepared for methylation analysis following restriction digestions using the EpiTect Methyl II DNA Restriction Kit provided by Qiagen. Digested DNA samples were subsequently investigated using the Rat Apoptosis EpiTect Methyl II Signature PCR Array (catalog no. EARN-121Z; Qiagen) and the Rat DNA Repair EpiTect Methyl II Signature PCR Array (catalog no. EARN-421Z; Qiagen), which profiled the promoter-specific methylation status of a panel of 22 genes related to these pathways (Tables 3-4). After the 
digestion, the remaining DNA in each individual enzyme was quantified using quantitative real-time polymerase chain reaction (qRT-PCR). The qRT-PCR arrays were performed in an ABI PRISM 7500 instrument (Applied Biosystems). In a similar manner to previous studies $(56,113)$, data was analyzed using the EpiTect Methyl II PCR Array system, which provided an integrated Microsoft Excel-based template that automatically performed all $\Delta \mathrm{Ct}$ based calculations from the raw $\mathrm{Ct}$ values in order to calculate the percentage of DNA that was methylated and unmethylated for each gene on the array. The method employed by the Epitect Methyl II PCR System was based on the detection of remaining input DNA following cleavage with a methylation-sensitive and/or methylation-dependent restriction enzymes, which digested unmethylated and methylated DNA, respectively $(56,71,75,113)$. The relative fractions of methylated and unmethylated DNA for each gene of interest on the arrays were determined by comparing the amount in each digest with a "mock digest” (i.e., no enzymes added) using the $\Delta \mathrm{Ct}$ method (113).

\section{INSERT TABLE 3 HERE}

INSERT TABLE 4 HERE

\section{ELISA levels of 8-hydroxydeoxyguanosine (8-OHdG) and Apoptotic Cell Death}

Total DNA was isolated using the DNeasy ${ }^{\circledR}$ Blood \& Tissue Kit (Qiagen) according to kit instructions. DNA concentrations were obtained using a spectrophotometer (ND-1000; NanoDrop Technologies Inc.). Oxidized DNA in samples was determined using an 8-OHdG sandwich ELISA, the OxiSelect ${ }^{\mathrm{TM}}$ Oxidative DNA Damage ELISA Kit (Cell Biolabs, Inc., San Diego, CA, USA), according to manufacturer's instructions. Samples were read at an absorbance of $450 \mathrm{~nm}$ using a 96-well plate reader (Epoch Microplate Spectrophotometer, BioTek Instruments, Inc., Winooski, VT, USA) and subsequently normalized to their respective DNA concentrations and expressed as nanograms per milliliter. All measurements were performed in duplicate. Cytoplasmic protein isolation extracts were obtained from the TA muscles using a method described by Siu et al. (98). Briefly, total protein contents of the cytoplasmic protein extracts were quantified and reported as a protein concentration ( $\mu \mathrm{g}$ protein/mg tissue - [protein]) in triplicate using the Pierce ${ }^{\mathrm{TM}}$ BCA Protein Assay Kit (Thermo Scientific, Rockford, IL, USA). Cytoplasmic protein extracts were used to quantify DNA fragmentation using the Cell Death Detection ELISA assay kit (Roche Diagnostics, Mannheim, Germany) to quantitatively detect levels of cytosolic histone-associated DNA fragmentation, in accordance to manufacturer's instructions. The extracted cytoplasmic fractions of TA muscles were used as an antigen source in a sandwich ELISA with a primary anti-histone mouse monoclonal antibody coated to the microtiter plate and a second antiDNA mouse monoclonal antibody coupled to peroxidase. The amount of peroxidase retained in the immunocomplex was determined photometrically by incubating with 2, 2'-azino-di-[3-ethylbenzthiazoline 
sulfonate] as a substrate for $10 \mathrm{~min}$ at $20^{\circ} \mathrm{C}$ (98). Samples were read at an absorbance of $405 \mathrm{~nm}$ using a 96 -well plate reader (Epoch Microplate Spectrophotometer, BioTek Instruments, Inc.). All measurements were performed in duplicate. The resulting optical density at $405 \mathrm{~nm}\left(\mathrm{OD}_{405}\right)$ reading was normalized to the total amount of protein in the sample and reported as an apoptotic index ( $\mathrm{OD}_{405} / \mathrm{mg}$ protein) to indicate the level of cytosolic mono-and oligonucleosomes present in the samples (78).

\section{Myonuclei Morphology}

Frozen TA muscle sections (12 $\mu$ m thickness) were previously stained for 4-hydroxynonenal (4-HNE; no. 393206; at 1:250; Calbiochem, La Jolla, CA, USA), $\beta$-dystroglycan (sc-33701; at 1:100; Santa Cruz Biotechnology, Dallas, TX, USA) in order to outline the muscle sarcolemma, and DAPI (P36931; Thermo Fisher Scientific, Rockville, IL, USA) to stain for myonuclei; representative images were reported elsewhere (86). The midpoint of the TA muscle was identified and non-overlapping images were captured at 40x magnification at the site of the most area in both the lateral and medial regions of the TA section. A standardized stereological method was used for quantitative morphology (11). Briefly, at two regions (1 mm to the right and $1 \mathrm{~mm}$ to the left of the section midline) the analysis was performed at five equally spaced sites across the muscle section. At each site, points of a 121-point, 11-line overlay graticule $\left(0.04 \mathrm{~mm}^{2}\right.$ square boundary with 100 divisions) was placed at the center of each image and was used in identifying various myonuclei for inclusion in quantification of various features of morphology. A total of 1,210 points were analyzed per section since 10 total fields (i.e., images) were evaluated.

Using images from these slides, preliminary data via total particle counting and analysis using ImageJ (version 1.48, National Institutes of Health, USA) indicated that both training age and frequency results in differences amongst rodents for total nuclei related morphology (data not shown). Because satellite cells and myonuclei are well documented to be a primary component responsible for gains in muscle mass in response to RTET, we did an analysis specifically for myonuclei (defined as nuclei that lied on or within the basal lamina of the muscle fibers). Using the ImageJ software, the investigator was blinded to animal identity and samples were analyzed via tracings of myonuclei in order to determine indices of size and shape characteristics of myonuclei, which included minimum feret diameter and circularity. Minimum feret diameter, defined as the closest possible distance between two parallel tangents of an object (i.e., the myonucleus), was chosen over other size variables (e.g., perimeter) because, in comparison to other morphometric parameters, it would attain the size least influenced by sample sectioning and processing $(31,88)$. Circularity was calculated by the following equation: circularity $=4 \Pi\left(\mathrm{area} /\right.$ perimeter $\left.^{2}\right)$; a circularity value of 1.0 indicates a perfect circle. As the value approaches 0.0 , it is indicative of an increasingly elongated polygon. 


\section{Statistical Analysis}

Peak force (means and relative), MQ, DNA methylation, cell death (apoptotic index), and 8-OHdG concentrations were all analyzed using a mixed model analysis of variance (ANOVA) with the variable animal identification as a random factor to account for repeated measures (JMP version 13; SAS Institute, Inc., Cary, NC, USA). Apoptosis and DNA repair PCR array data were analyzed using unpaired student's t-test in the GeneGlobe Data Analysis Center (Qiagen) online platform. Chi-square analysis (SigmaPlot version 12.5; Systat Software, Inc., San Jose, CA, USA) was utilized to determine training-induced differences in the absolute frequency distributions of myonuclei minimum feret diameter and circularity. All data with the exception of myonuclei minimum feret diameter and circularity, which were presented as frequency distributions, are expressed as means $\pm \mathrm{SE}$, with $p<0.05$ being considered statistically significant.

\section{RESULTS}

\section{Dorsiflexor Muscle Performance}

Muscle performance was measured via peak force performed on the first SSC repetition of the first training set during the initial and final week of training; this data is shown in Figure 1. In response to dorsiflexion SSC-loading, Y3x and Y2x had significantly higher peak force following training, whereas O3x had significantly decreased peak force at final compared to initial $(\mathrm{p}<0.05)$ (Figure 1a). Meanwhile, O2x significantly increased peak force following training; moreover, peak force was also higher in $\mathrm{O} 2 \mathrm{x}$ at final in

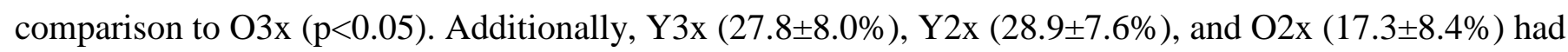

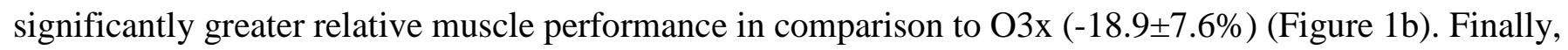
calculated MQ was significantly higher in both young groups $(\mathrm{p}<0.05)$ and exhibited a strong trend being increased in $\mathrm{O} 2 \mathrm{x}(\mathrm{p}=0.05)$ when compared to O3x (Figure 1c).

\section{INSERT FIGURE 1 HERE}

\section{Apoptosis DNA Methylation, Gene Expression, and Bioinformatics Analysis}

Apoptosis pathway methylation profiles from the EpiTect Methyl II PCR arrays are shown in Figure 2. When looking collectively at the entire pathway, there was no effect of age at baseline in DNA methylation between young and old NLC, nor was there any effect of training on methylation levels in the young groups ( $\mathrm{p}>0.05)$. However, following one month of high-intensity SSC RTET, there was a significant increase in methylation in O3x compared to old NLC and O2x (Figure 2). On an individual gene level, no genes were identified that had either significantly higher levels of methylation with concomitant downregulation of gene 
expression or significantly lower levels of methylation with simultaneous upregulation of gene expression from the pathway array.

\section{INSERT FIGURE 2 HERE}

The significantly differentially expressed genes (SDEGs) in the Rat Skeletal Muscle Apoptosis RT ${ }^{2}$ Profiler PCR Array are shown in Table 5 and Figure 3. The SDEGs were defined as having a fold change value of being up- or down-regulated by 1.1 fold or more along with a p-value of less than 0.05 . Y3x and Y2x had 21 and seven SDEGs, respectively, in the apoptosis signaling pathway; all genes were upregulated in Y2x while every gene except one was upregulated in Y3x. In contrast, O3x only had one SDEG, which notably, was an upregulation of B-cell lymphoma 2-related protein A1 (BCL2A1), a well-known anti-apoptotic molecule that can reduce the release of cytochrome c from mitochondria and block the activation of pro-apoptotic caspases (68). In contrast, O2x had eight SDEGs; all but one gene was downregulated, but three of the six downregulated molecules have been directly implicated and/or associated with anti-apoptotic processes [baculoviral IAP repeat-containing 3 (BIRC3), myeloid cell leukemia sequence 1 (MCL1), and receptor-interacting serinethreonine kinase 2 (RIPK)]. Interestingly, the pro-apoptotic molecule TNF receptor associated factor 3 (TRAF3), a key regulator of lymphotoxin-beta (LTB) receptor complex, which induces Nuclear factor kappalight-chain-enhancer of activated B cells (NF-kB) activation and cell death initiated by LTB ligation (114), was significantly upregulated in both Y3x and Y2x, as well as in O2x (2.36 fold vs. 1.95 fold vs. 1.63 fold), but not in O3x.

\section{INSERT TABLE 5 HERE}

\section{INSERT FIGURE 3 HERE}

Bioinformatics analysis of the top canonical pathways in IPA revealed similar pro-apoptotic signaling mechanisms in both young groups as well as in $\mathrm{O} 2 \mathrm{x}$ (Table 6), such as death receptor signaling, tumor necrosis factor receptor 1 (TNFR1) signaling, tumor protein p53 (p53) signaling, GADD45 signaling, and interferon signaling. In contrast, the list of top canonical pathways for O3x mostly contained processes related to cell survival and autoimmunity/inflammation (e.g., docosahexanoic acid signaling, B cell receptor signaling, etc.). Besides the general classification differences, two noteworthy observations stood out in the three adapting groups (Y3x, Y2x, O2x) in comparison to O3x: 1) Apoptosis signaling was ranked as either the first or second canonical pathway in the analysis, whereas it was third in O3x; 2) while being notably absent in O3x, the other three groups had tumor necrosis factor-like weak inducer of apoptosis (TWEAK) signaling in their individual analyses, including being the top ranked canonical pathway in O2x. TWEAK signaling has been implicated 
previously in a positive manner on promoting muscle repair in response to acute injury through its influential effects on muscle cells $(1,40)$, and a previous study by Walton et al. (110) showed an upregulation of the TWEAK receptor fibroblast growth factor-inducible 14 (FN14) following 12 weeks of endurance cycling training. Collectively their data suggested that macrophages are positively involved in the muscle adaptation response to endurance training, with modifications to TWEAK/FN14 signaling being one highly influential factor (110).

\section{INSERT TABLE 6 HERE}

\section{DNA Repair DNA Methylation, Gene Expression, and Bioinformatics Analysis}

The DNA repair pathway methylation profile from the EpiTect Methyl II PCR array is shown in Figure 4. When looking collectively at the entire pathway, there was no effect of age at baseline in levels of DNA methylation between young and old NLC, nor was there any effect of training on methylation levels in the young groups ( $\mathrm{p}>0.05)$. However, following one month of high-intensity SSC RTET, DNA methylation was significantly lower in O3x in comparison with old NLC and exhibited a trend ( $\mathrm{p}=0.07)$ for being less than O2x; O2x was not significantly different from old NLC nor either young group. On an individual gene level, there were no genes that had either significantly higher levels of methylation with concomitant downregulation of gene expression nor significantly lower levels of methylation with simultaneous upregulation of gene expression from the pathway array.

\section{INSERT FIGURE 4 HERE}

The SDEGs in the Rat Skeletal Muscle DNA Repair RT² Profiler PCR Array are shown in Table 7 and Figure 5. Y3x and Y2x had two (both upregulated) and 16 (two upregulated and 14 downregulated) SDEGs, respectively, in the DNA repair signaling pathway. Meanwhile, O3x had 37 SDEGs, all but one of which were downregulated in the SSC-loaded compared to NLC muscle. In comparison, O2x had 10 SDEGs, all of which were upregulated in the SSC-loaded compared to NLC muscle. There were a few notable genes that were differentially upregulated in the adapting groups; first, MutY homolog (MUTYH) was the only gene upregulated in both young groups. MUTYH is a DNA glycosylase strongly implicated as an important regulator of DNA repair by excising adenine base lesions from DNA located opposite of 8-OHdG during replication, thereby attenuating mutations caused by oxidative stress $(3,41)$. In O2x, thymine-DNA glycosylase (TDG) was one of the SDEGs upregulated following training. TDG has been previously shown to selectively excise 5formylcytosine and 5-carboxylcytosine, two oxidized byproducts of 5-methylcytosine in the activated cytosine 
demethylation pathway, thus implicating this molecule in epigenetic/epigenomic regulation (31). In contrast, O3x had a significant downregulation of another DNA glycosylase gene, N-methylpurine-DNA glycosylase (MPG), who functions as an initiator of base excision repair (BER) of DNA adducts produced as byproducts of lipid peroxidation, and whose inefficient turnover rate has been associated with delays in DNA repair, subsequently leading to genomic instability (115).

\section{INSERT TABLE 7 HERE}

INSERT FIGURE 5 HERE

Bioinformatics of the DNA repair pathway using IPA is shown in Table 8. (Table 8). For the top canonical pathways, Y3x had no available relevant functions that showed up in the analysis, likely due to the small number of SDEGs in this group, so therefore no top canonical pathways existed. The other three groups had top canonical pathways almost exclusively related to DNA repair, such as the nucleotide excision repair (NER) pathway, the BER pathway, and double-strand break (DSB) repair by homologous recombination (HR). Interestingly, Y2x and O2x, but not O3x, had DNA DSB repair by non-homologous end joining (NHEJ) listed; NHEJ works by directly rejoining DNA, rather than utilizing sister chromatids or homologous chromosomes as a template for DNA resynthesis and rejoining (16). NHEJ, rather than HR, is the predominate pathway used in many stages of the cell cycle, particularly early (i.e., G0 \& G1) (102). For the physiological systems and development section, O3x, in the context of a downregulation of DNA repair gene expression, top functions included “Organismal Survival,” “Organismal Development,” and “Connective Tissue Development and Function.” In contrast, O2x, within the context of an upregulation of DNA repair gene expression, top functions included “Nervous System Development and Function” and “Tissue Morphology.”

\section{INSERT TABLE 8 HERE}

\section{Cell Death and 8-OHdG ELISAs}

Cell Death ELISA values are shown as an apoptotic index (OD $405 / \mathrm{mg}$ protein) to indicate levels of cytosolic mono-and oligonucleosomes present in the samples (Figure 6). There were no age effects shown when looking at young and old NLC, as well as no treatment effects for both Y3x and Y2x (p>0.05). However, O3x had a significantly higher apoptotic index, and thus greater cell death, compared to both old NLC and O2x $(\mathrm{p}<0.05)$. Meanwhile, O2x showed no effect of training on the apoptotic index in comparison to old NLC and Y2x, meaning they had levels of cell death similar to younger rats. Oxidative stress, as measured by 8-OHdG concentrations (ng/ml), are shown in Figure 7. Similar to previous investigations by our laboratory (92, 93), old 
NLC muscle had significantly higher 8-OHdG compared to young NLC muscle $(\mathrm{p}<0.001)$. In both young groups, there was no effect of SSC RTET on 8-OHdG concentrations compared to young NLC nor each other. O3x had significantly lower levels of 8-OHdG compared to old NLC and O2x ( $<<0.05)$. In contrast, O2x had significantly higher concentrations of 8-OHdG compared to O3x and Y2x $(\mathrm{p}<0.05)$; additionally, a strong trend was noted $(\mathrm{p}=0.05)$ for higher 8-OHdG levels in comparison to old NLC.

INSERT FIGURE 6 HERE

INSERT FIGURE 7 HERE

\section{Myonuclei Morphology}

Frequency distributions for myonuclei minimum feret diameter are shown in Figure 8. Following training, chi-square analysis revealed significant shifts in distribution to the left (i.e., decreased) minimum feret diameters in Y3x, Y2x and O2x relative to NLC ( $<<0.001)$ (Figure 8a-8b, 8d), whereas no changes were found in O3x ( $>$ >0.05) (Figure 8c); this indicates that myonuclei got smaller as a result of one month of high-intensity SSC RTET that resulted in adaptation. Frequency distributions for myonuclei circularity are displayed in Figure 9, which was calculated based on the area and perimeter (see Materials and Methods Section). Interestingly, O3x was the only group that showed a significant decrease in circularity (Figure 9c); chi-square analysis revealed significance in $\mathrm{Y3X}$ as well $(\mathrm{p}<0.05)$, but, as can be seen in the figure, this was due to increases in the peak of the distribution rather than by any shifts to the left or right, which was attributed to a higher number of values in the 0.90-0.99 bin in the trained limb of these animals in comparison to NLC (Figure 9a). Meanwhile, no changes were found in both $\mathrm{Y} 2 \mathrm{x}$ and $\mathrm{O} 2 \mathrm{x}(\mathrm{p}>0.05)$.

INSERT FIGURE 8 HERE

INSERT FIGURE 9 HERE

\section{DISCUSSION}

The main findings from this study are: 1) There is a distinct molecular signature following RTET utilizing SSCs in old rats that accompanies adaptive and maladaptive outcomes, and these responses appear to be connected to the apoptosis and DNA repair signaling pathways, with a distinct pattern of SDEGs involved in the skeletal muscle stress and muscle homeostasis response present in both Y3x, Y2x, and O2x compared to O3x; 2) DNA methylation seems to be a highly influential epigenomic process involved in the regulation of the aged muscle stress response to exercise, specifically in apoptosis; 3) effects on gene expression in O2x had a positive influence on downstream outcomes including myonuclei size and morphology; and 4) this work 
advances recent work from our laboratory demonstrating the efficacy of a reduced frequency RTET paradigm in promoting adaptation in aged skeletal muscle following high-intensity SSC-loading, as reflected by positive changes in muscle performance and MQ.

To determine if muscles are capable of adapting to high-intensity RTET at an old age, we exposed muscles of young and old rats to 80 SSCs, two days per week, ( 72 hours between training bouts), which altered from our originally published protocol (80 SSCs, three days per week; 48 hours between training bouts) in which young rats adapted and old rats maladapted (28). Similar to the response to 80 SSCs, three days per week exposure shown previously in young rats, $\mathrm{Y3x}$ as well as the modified exposure protocols ( $\mathrm{Y} 2 \mathrm{x}$ and $\mathrm{O} 2 \mathrm{x}$ ) induced enhancements in dynamic muscle performance and final dynamic MQ. For Y2x, the modified exposure protocol yielded similar gains in performance and MQ in comparison to Y3x, which implies that young rodent muscles are robust in their ability to adapt to multiple frequency paradigms utilizing a highintensity SSC RTET regime. In aged skeletal muscle, our results demonstrated that O2x was clearly superior over O3x in eliciting the adaptive response to high-intensity SSC RTET as indicated by increases in muscle performance as well as improvements in MQ more similar to younger rodents. Indeed, with appreciation for the current molecular data described herein (i.e., profiler array gene expression, IPA, cell death ELISA, myonuclei size/morphology), as well as that of other studies in human investigations (18, 42, 44, 45, 48, 69, 101), these results have provided evidence that high-intensity SSC RTET prescribed in the appropriate manner (e.g., a reduced training frequency) can be highly beneficial for aged skeletal muscle, particularly in individuals who do not respond favorably to higher frequencies of exercise. Additionally, it should be noted that the O2x paradigm in our study parallels recent support for a “minimal-dose approach” for RTET of two days per week resistance exercise for less than 60 minutes, which was based on previous evidence showing various physiological and psychological benefits to this specific type of training in older adults or in those whose goal is to achieve the benefits of RTET at a reduced time commitment (37).

Long-term adaptations to RTET occur as a result of an accumulative effect of repeated, acute increases in gene expression for exercise-induced pathways of growth, stress, homeostasis, and remodeling that follow each individual exercise stimulus $(19,26)$. The present data demonstrates that O2x were able to undergo adaptation concomitant with an enhanced activation of the apoptosis and DNA repair signaling pathways. Specifically, TRAF3 was only significantly upregulated in Y3x, Y2x and O2x (Table 5); TRAF3 is a key regulator of the LTB receptor complex responsible for inducing NF- $\kappa$ B activation and cell death that is initiated by LTB ligation (114). More specifically, TRAF3 is a cytoplasmic factor recruited by membrane-bound TNF binding to TNF receptor 2 and directly interacts with cluster of differentiation 40 and latent membrane protein 1 , and has also been implicated as an important molecule in the signaling mechanisms enhancing the activation and subsistence of T cells $(30,63)$. Because the communication pathways that TNF and TNFR superfamilies 
(such as NF- $\kappa \mathrm{B}$ ) are known to regulate, which are highly critical for various developmental, homeostatic, and stimulus-responsive (e.g., exercise) events (30) such as cellular differentiation, cell survival, and programmed cell death (i.e., apoptosis), the upregulation of TRAF3 in the adapting groups could potentially be an underlying mechanism associated with the positive responses of muscle to a reduced frequency of high-intensity RTET.

Another notable observation present in all three groups that adapted to the exercise training, but not in the maladaptive O3x, was the IPA top canonical pathway analysis showing TWEAK signaling being implicated in the response to high-intensity SSC RTET. Interestingly, TWEAK and its receptor FN14 have recently come to light as important characteristics of physiological and pathophysiological tissue remodeling and have been implicated previously in a positive manner on promoting muscle repair and regeneration in response to acute injury through its influential effects on myogenic cells $(1,20,21,40)$. Within this context, TWEAK/FN14

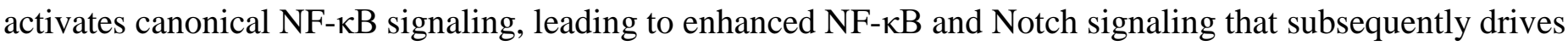
enhanced myogenic progenitor cell proliferation and increased myogenesis as well as angiogenesis $(1,21)$. TWEAK/FN14 signaling in an appropriate manner has been shown to contribute to normal tissue regeneration and repair, but within the context of a dysregulated signaling response, this pathway can lead to "progressive local tissue damage and maladaptive remodeling (20).” A recent study by Walton et al. (110) looked at whether or not increases in M2 macrophage content in response to exercise were correlated with increased muscle satellite cell content and transcriptional activation following 12 weeks of endurance cycling training in middle aged females. Following the training, participants had an enhanced upregulation of the TWEAK receptor FN14. Collectively, their overall data suggested that M2 macrophages are positively involved in the muscle adaptation response to endurance training, with modifications to TWEAK/FN14 signaling being one critically important factor (110). Thus, based on this information along with our IPA analysis showing enhanced TWEAK signaling activation in both young groups and $\mathrm{O} 2 \mathrm{x}$ within the context of muscle adaptation (via enhanced muscle performance and MQ), activation of the TWEAK/FN14 pathway may be an important mechanism by which aged muscle is able to go through remodeling in a positive manner and induce adaptation in response to an appropriate RTET stimulus. While not examined directly in the present study, future research is certainly warranted to examine if more direct evidence implicating TWEAK/FN14 with muscle adaptation within the context of high-intensity SSC RTET exists.

To our knowledge, this is the first study to demonstrate age and training specific differences in methylation of the apoptosis pathway following high-intensity SSC RTET. Specifically, O3x rats that maladapted exhibited higher levels of promoter specific DNA methylation that was concomitant with decreased apoptotic gene expression. Importantly, modifying the exposure in old rodents from three to two days per week resulted in a more favorable molecular environment compared to O3x via lower percentages of DNA promoter methylation along with increased apoptosis pathway gene expression. While increased apoptosis has previously 
been implicated as contributing to the negative effects of aging (64), a key part of apoptosis in general is that it leads to the quick and efficient elimination of dysfunctional cells while simultaneously avoiding the activation of inflammatory processes (35). Moreover, evidence has started to emerge indicating that processes that promote cell death (e.g., apoptosis, autophagy) is not always a negative event; in fact, a host of literature seems to indicate these processes may actually be critically important in the context of the normal physiological regulation of skeletal muscle $(8,23,30,38,39,77,82,104,112)$. For example, there is evidence indicating that in response to muscle wasting as a consequence of cancer cachexia, the muscle responds by activating pathways that positively alters the anabolic capacity in part due to increasing the activity of autophagy (77). It has also been shown that a requirement for myogenic differentiation (when satellite cells fuse with existing myofibers) is for apoptosis of M1 pro-inflammatory macrophages to occur followed by a subsequent replacement with M2 macrophages $(77,100)$.

Another example of the positive regulation of cells by apoptosis is in the case of senescence, which occurs when cells go into a state of irreversible growth arrest from the cell cycle. It is well known that senescent cells increase in frequency with age and are capable of contributing to age-related diseases of tissues (23, 38, 95). More specifically, an accumulation of senescent cells leads to high secretion of various cytokines, chemokines, and extracellular matrix-remodeling enzymes, which can have consequential effects on tumor production and tissue dysfunction; this state is referred to as the senescence-associated secretory phenotype (23). However, senescence, just like apoptosis, is not always a negative affair and can actually have positive effects on body function; during adulthood, short-lived acute senescent cells have multiple positive functions (e.g., tumor suppressants, aiding in wound repair, etc.); moreover, these senescent cells are capable of being cleared through apoptosis in vivo provided they are exposed to the appropriate stimulus (23). Conversely, as individuals age, senescent cells lose their capabilities for proper immune function, and as a byproduct of this they are less able to stabilize p53-mediated apoptotic signaling, and an accumulation of defective senescent cells increases the susceptibility to the assemblance of tumors and overall tissue dysfunction (23). It has previously been demonstrated that aging can result in an impairment of p53 signaling, which could be preventing cells that have severe amounts of damage from entering into apoptosis, thus contributing to the increased number of senescent and/or abnormal cell types with advanced age (23). Of note, reduced or a complete loss of expression of the tumor suppressor gene phosphatase and tensin homolog (PTEN) has been previously implicated in promoting cell senescence and inhibiting apoptosis (4); while there are several other factors also involved in determining whether or not a cell undergoes senescence or apoptosis, this is an interesting note because in a recently published study from our laboratory (71), our results showed that in young rats that adapted from the same one month high-intensity SSC RTET protocol as in the current study, IPA 
analysis of the PI3K-AKT pathway had an upregulation of PTEN signaling ranked as one of the top canonical pathways.

Of particular interest pertaining to our study, Sanders et al. (95) compared senescent and non-senescent high diploid fibroblasts and found that the senescent cells were more resistant to oxidative stress and apoptosis compared to the non-senescent cells. They found that the anti-apoptotic gene B-cell lymphoma 2 (BCL2) was upregulated in senescent fibroblasts while pro-apoptotic Bcl2-associated X protein (BAX) was downregulated. Interestingly, and in contrast to BAX, BCL2 was found to have a significant increase in the amount of repressive histone modifications (i.e., methylation) along with a simultaneous decrease in active histone modifications (i.e., acetylation). Given our results that O3x had significantly increased methylation following RTET and that the only gene that was found to be an SDEG in this group was another anti-apoptotic BCL-2 family member, BCL2A1, our results are in line with those found in the Saunders et al. study. In summary, these findings, combined with the well-known functions of various apoptotic molecules as essential regulators of a host of events related to development, homeostasis and "stimulus-driven events" within skeletal muscle (30), we propose that DNA methylation may be associated with a dysregulated apoptotic signaling cascade in O3x maladaptive muscle, which ultimately impairs the adaptive capacity of the muscle in response to an inappropriate high-intensity SSC RTET stimulus; our results illustrating significantly higher cell death in O3x relative to old NLC following training provide support for this statement. In O2x, increased apoptosis signaling is indicative that old skeletal muscle is capable of adapting to RTET in a manner that is more similar to young muscle by reducing the training frequency, which is supported by the findings that levels of cell death were not different from old NLC and indistinguishable from young rats that underwent the same SSC protocol (Figure 6).

DNA is continuously being challenged by various agents (e.g., metabolism, exercise, environmental exposures) that results in stress and damage, which includes any chemical alteration to the DNA and/or incorrect placement of nucleotides during replication. Cell cycle control, the regulation of cell death/senescence, and DNA damage signaling and repair are well-known to be critical in maintaining the genome integrity, thus decreasing the susceptibility to negative age-related effects. Because of the variety of damage-inducing agents that DNA is exposed to, the body has developed specialized repair mechanisms that can take on these different forms of damage. The three main types of repair are: direct reversal of damage, excision repair, and postreplication repair. These repair pathways are highly conserved and critically important for cell survival and maintaining the integrity of the genome because they prevent damaged DNA from being mistakenly copied via cellular replication, thereby preventing mutations that could lead to instability $(66,115)$. Evidence has emerged to show that with aging you have increased DNA damage and potential declines in DNA repair pathway activity (2, 46, 90, 109). Additionally, unrepaired/repeated oxidative lesions of DNA, and subsequently a decline of 
skeletal muscle function with increased age, may be linked to low expression of genes in the BER and NER repair pathways (106).

Collectively, old tissues accumulate functional declines as a result of constant mutations/epimutations that increase the amount of variability between cells in levels of transcriptional activity, which consequently transforms tissues into a medley of functional cells, lesions, and fibrotic tissue, ultimately leading to improper organ and/or tissue functioning (109). It has been shown that longer-lived species, such as the naked mole rat, have higher levels of expression of DNA repair genes, and it is known that DNA repair pathways are conserved and serve critical functions in cell survival and maintenance of the integrity of the genome (66); another study by Hyun et al. (50) demonstrated that long-lived, stress-resistant mutant C. Elegans have a significantly higher capacity for DNA repair compared to wild-type controls. Therefore, modulation of genes and proteins within this pathway have a crucial role in integrating both anabolic and catabolic signals, ultimately affecting changes in functional performance and muscle quality. In the present study, we have shown that appropriate, agespecific RTET for O2x caused increased expression of DNA repair genes as reflected by the $\mathrm{RT}^{2}$ Profiler $^{\mathrm{TM}}$ Arrays and bioinformatics analysis via IPA (Figure 10a), whereas a dysregulated response occurs in O3x via a downregulation of many genes in this pathway which is also demonstrated through IPA (Figure 10b). This is an important distinction because these observations suggest that SSC-loading, when prescribed in the appropriate manner such as reducing the frequency of high-intensity SSC RTET, directly modifies local DNA repair activity within skeletal muscle, which may be critically important in leading to an adaptive fate. Therefore, reducing the frequency of RTET, thereby allowing additional recovery time to initiate muscle repair and remodeling processes, provides an optimal environment for aged muscle to adapt properly and aids in restoring muscle performance, physiology (i.e., muscle fiber area and MQ data), and improves the microenvironment, ultimately enhancing tissue quality and functional outcomes with advanced age.

Despite the differential patterns of gene expression seen with age and training, DNA methylation levels did not correspond accordingly to these responses; O3x was the most prominent example of this, as they showed the least amount of methylation yet had a robust downregulation of DNA repair gene expression following one month of high-intensity SSC RTET. The only other study directly investigating DNA methylation levels and gene expression of DNA repair genes was conducted recently by $\mathrm{Hu}$ et al. (49) who found that workers exposed to hexavalent chromium had significantly higher levels of CpG methylation compared to control subjects not exposed to this substance, which contributed to differences seen in 8-OHdG levels. It is clear that environmental exposure to a chemical agent is not the same physiological stimulus as exercise training, yet this finding provides an additional exposure-response scenario in which an environmental stimulus has direct impact on the DNA damage and repair response. The results of the current study suggest that DNA methylation is not the primary epigenomic regulator of DNA repair pathway gene expression, and perhaps 
other regulatory mechanisms (i.e. chromatin morphology) may provide an active role in the control of DNA repair and/or perhaps another epigenomic process, such as acetylation, is more prominently involved. However, given the minimal evidence that is apparent for the involvement, let alone control, of DNA methylation as the primary regulator of DNA damage and repair response with respect to an exposure-response paradigm with aging, more research is needed.

Interestingly, despite enhanced activity of DNA repair gene expression in O2x that adapted and a suppression of this pathway in O3x that maladapted, the ELISA 8-OHdG results showed the opposite of what we expected (Figure 7). Specifically, O3x had significantly less 8-OHdG compared to both Old NLC and O2x; in contrast, O2x showed a strong trend for having elevated concentrations of 8-OHdG following training compared to old NLC. Both groups had significantly higher 8-OHdG compared to young trained-matched counterparts (i.e., O3x vs. Y3x, O2 vs. Y2x). These results were an unexpected finding given the outcomes seen in muscle adaptation as well as the upregulation of the DNA repair pathway in O2x. However, there is evidence in the literature that can potentially help explain these paradoxical findings. First, previous reports exist that have shown that longer-lived species actually have higher amounts of oxidative damage; for instance, reports have directly shown that the naked mole rat, the longest living rodent species known to the scientific community, produces significantly higher levels of reactive oxygen species (ROS) and DNA damage (approximately two to eight fold greater) at a young age when compared to physiologically aged-matched mice despite having a much longer overall lifespan (7, 94). Also, it has been demonstrated that long-lived birds, despite possessing higher basal metabolic rate and greater amounts of oxidative damage, live almost twice as long compared to similar-sized mammals $(7,90)$; additionally, long-lived bats and naked mole rats have been shown to produce greater amounts of protein carbonyls compared to shorter-lived rats and mice (90, 94). Finally, Sohal et al. (99) revealed longer-lived fruit flies have significantly more oxidative damage compared to shorter-lived bowflies. Therefore, these studies, at the very least, show that higher levels of DNA damage are not always detrimental to overall tissue function and lifespan under the right conditions, and perhaps even suggest that basal or appropriate levels of DNA damage are either neutral or are a positive influence in the adaptive morphology of tissue composition.

Secondly, evidence has emerged indicating that damage-induced oxidative stress molecules are required on some level as a stimulus in order for DNA repair to take place. For instance, the concept of hormesis relies on the idea that at least some DNA repair mechanisms require small amounts of a DNA damage signal from environmental exposures in order to become activated, which subsequently leads to increased resiliency in the organism following this stimulus $(53,81)$; in other words, damage is a necessity in order to activate repair mechanisms and protective cellular response pathways (e.g., apoptosis) (79). Other studies have shown that knockout mice who have a loss of activity of 8-oxoguanine DNA glycosylase-1 (OGG1), a DNA glycosylase 
enzyme whose primary functions are initiating BER by making incisions at 8-oxoguanine (8-oxoG) residues and excising lesions of damaged DNA during BER (76), results in an accumulation of excess 8-oxoG, yet not only did they show an increased resistance to oxidative stress and inflammatory processes, but also presented no evidence of having an altered phenotype or lifespan (59, 70). More recently, Hajas et al. (43) demonstrated that the presence of 8-oxoG, specifically its base, is a requirement for OGG1 interaction and subsequent stimulation of rac family small GTPase 1 (RAC1) dependent stimulation GDP for GTP conversion. This regulation of RAC1 by OGG1 subsequently causes increased levels of intracellular ROS, which the authors suggest is an important part of a physiological DNA damage and repair mechanism that prevents mutagenesis and helps maintain cellular homeostasis. In addition, previous research has demonstrated that in normal, healthy skeletal muscle, basal levels of ROS modulates force production and are vital for normal muscular contractions to occur (80). Also, ROS, along with growth factors and chemokines, are highly involved in the activation of pathways essential for muscle repair following injury $(80,106)$. Therefore, viewing these previous findings within the important context of our current results demonstrating adaptive outcomes in $\mathrm{O} 2 \mathrm{x}$ as opposed to maladaptation in O3x in response to RTET, we speculate that perhaps an appropriate exercise stimulus (e.g., O2x) induces a DNA damage signal as a prerequisite for aged skeletal muscle to have the ability to undergo an appropriate DNA repair response; and, subsequently actively facilitate adaptation. In contrast, when presented with an inappropriate exercise stimulus (e.g., O3x), the result is a blunted DNA damage response, which is subsequently accompanied by an attenuation of DNA repair signaling that does not allow for proper muscle remodeling and homeostasis to occur, thus leading to maladaptation. However, precisely how inappropriately prescribed exercise, such as a higher training frequency, leads to a blunted DNA damage and repair response is unclear and will have to be examined in future research.

As mentioned previously, in post-mitotic tissues such as skeletal muscle, evidence seems to indicate that DNA methylation, in particular, is actively occurring as a result of an individual's interaction with their environment (33, 61, 87, 105). Additionally, histone modifications through epigenetic/epigenomic mechanisms can lead to chromatin undergoing nuclear compartmentalization, possibly effecting structure and functional features of myonuclei (15). In these regards, perhaps the most fascinating result from our study was that there were characteristic differences in myonuclei size and morphology that occurred in response to high-intensity SSC RTET. Alterations in the frequency distributions for myonuclei size were noted in all adaptive states; myonuclei minimum feret decreased in all adaptive conditions (Y3x, Y2x, and O2x), whereas the O3x that maladapted were non-responsive. Strikingly, the myonuclei shape metric, circularity, decreased only in O3x, indicative of having more elongated nuclei. It is well known that myogenic cells are exposed continuously to varying levels of mechanical forces in the form of contractions, both during everyday movement and during resistance exercise. These mechanical cues elicit subsequent biological responses, which includes activating and 
modulating gene expression allowing cells the ability to adapt to their environment (58). Moreover, it has been suggested that aberrant nuclear morphology could be linked to various muscular dystrophies, such as EmeryDreifuss muscular dystrophy and cardiomyopathies (111). However, despite this information, there is a paucity of literature regarding changes in dynamics of myonuclei size and morphology, particularly when it comes to modifications following perturbations via exercise, both acute and chronic.

To our knowledge, there are only two studies to date that have attempted to characterize myonuclei size and/or morphology in response to exercise. Malatesta et al. (67) examined the effects of one month of treadmill running endurance exercise in old mice compared to an adult sedentary and old sedentary group. Using electron microscopy techniques, the authors revealed that this type of training modified structural and functional features of myonuclei in old mice that resembled the younger sedentary mice. Specifically, while myonuclear areas and the percentage of condensed chromatin were all lower in both old groups compared to young sedentary, the fibrillar center area and the nucleolar percentage of fibrillar centers were significantly higher in both adult sedentary and old trained compared to the old sedentary group. In a separate study by Cisterna and colleagues (24) using the same exercise paradigm for one month with the same strain of mice, the authors again used electron microscopy to characterize various parameters of myonuclei morphology. When researchers looked at electron micrograph images of myotubes, they found that the myonuclei were more round in the young sedentary and old trained groups, whereas in old sedentary they were irregularly shaped. Thus, the present study is the first to report training-induced alterations in myonuclei size and morphology in response to a resistance exercise training paradigm. We found training-induced alterations in myonuclei size and shape following one month of high-intensity SSC RTET in which Y3x, Y2x, and O2x had decreased size and maintained a more circular shape following training-induced adaptation, whereas O3x maintained their size and became more elongated (i.e., less circular) as a consequence to training that brought on maladaptation.

In response to mechanical loading, muscle cells respond by activating specific genes and signaling pathways which allows for adaptation to take place. The process of mechanotransduction is used to describe the collective cellular and molecular events involved in converting a mechanical stimulus into biochemical signals that subsequently lead to adaptive or maladaptive outcomes. There is support in the literature to indicate that alterations in cellular contractility can influence the myogenic phenotype, and that extracellular mechanical stimuli have the ability to modify cellular functions related to morphogenesis (91). Recently, evidence has emerged implicating the nucleus as a key “mechanosensor” that plays a critical role in mechanotransduction events of the cell, including in the direct response to mechanical forces as well as by integrating various mechanoresponsive signals $(36,91)$. One of the biggest advantages for the nucleus being involved in mechanotransduction is its ability to integrate force-transducing stimuli directly from the cytoskeleton, thereby increasing the speed at which the cell is able to integrate these signals and initiate signaling responses (72). 
Various mechanisms for how the nucleus enables regulation in response to mechanical perturbations includes nuclear protein unfolding in response to forces, which induces changes in chromatin conformation, alterations in gene location within the interior of the nucleus, modifications in nuclear volume, regulation of the nuclear pore complex, and adaptations in the composition of the nuclear membrane (36). Specific to myonuclei, a study by Wang et al. (111) provided results supporting a protective mechanism in which the cytoplasmic factors short stop, isoform a (SHOT) and microtubule associated protein RP/EB family member 1 (EB1) function together with nesprin (MSP300) in order to maintain myonuclei morphology. SHOT and EB1 helped stabilize dense nuclear-associated networks of microtubules that physically wrap around and protect the myonuclei, while nesprin enhanced their stretching capacity. Collectively, both these components form a protective shield aptly able to absorb various contractile strains on the cytoplasm, thereby maintaining appropriate myonuclei morphology in response to muscular contractions (111). Therefore, given our results of decreased circularity along with past findings on abnormal myonuclei morphology in response to mechanical forces, it is plausible that high-intensity SSC RTET leading to maladaptation may be a stimulus that induces negative alterations in these protective networks, subsequently altering morphology in a negative manner leading to an increased number of myonuclei with an aberrant/elongated shape.

Interestingly, relationships between cell size/shape with changes in nuclei shape, chromatin state, and cellular proliferation have been previously reported. Tajik et al. (103) showed a clear relationship between chromatin stretching and deformation with transcriptional regulation on chromosome dihydrofolate reductase (DHFR) at the interior of the nucleus. Specifically, the authors found that the upregulation of DHFR was directly related to the direction of the load and proportional to the degree of chromatin stretching. Moreover, they found endogenous contractions upregulated DHFR gene expression, whereas DHFR became downregulated in response to filamentous actin interference or by blocking actomyosin contractions. Stress angles, the specificity of ligand binding, and the stress amplitude/duration all had an impact on the degree of chromatin stretching, which subsequently affected DHFR gene expression. A recent study by Elosegui-Artola et al. (34) found that yes-associated protein (YAP) gene regulation was regulated through mechanical forces and successive changes in nuclear mechanics; specifically, an increase in force transmission caused nuclear flattening, followed by stretching of the nuclear pores, and finally an increase in the nuclear transport of YAP as a consequence of a decreased mechanical resistance to transport.

Finally, and most remarkably, Versaeval et al. (108) was able to establish a connection between cell shape and changes in nuclei morphology with cellular proliferation states in endothelial cells. Specifically, the authors were able to demonstrate that as cells became more elongated this caused a high amount of compressive forces to be placed laterally on the nuclei. As a result, nuclear volume decreased, which then caused the nuclei to elongate and become less circular, and this elongation of the nuclei subsequently led to increases in 
chromatin condensation (i.e. heterochromatic state) and ultimately a decrease in cell proliferation. While we did not examine the shape of the muscle cells directly, it is fascinating that there was a decrease in minimum feret diameter of the myonuclei of both young groups and O2x that adapted to our mechanical loading stimulus, whereas there was no change in O3x that maladapted. On the other hand, while both young groups as well as O2x were able to preserve circularity following one month of high-intensity SSC-RTET, O3x showed significantly decreased circularity, instead becoming more elongated, results that are directly in line with the Versaeval et al. findings. Overall, supporting various emerging evidence for the role of the nucleus in mediating mechanotransduction, chromatin condensation, and functional outcomes of cells, our data supports the premise that alterations in myonuclei morphology following exposure to either an appropriate (i.e., adaptive) or inappropriate (i.e., maladaptive) high-intensity RTET stimulus are directly influencing the myonuclei morphology; which, subsequently may impact molecular signaling events and is part of the overall biological response leading to either adaptation or maladaptation (Figure 11). While we believe the results from the present study provide valuable information about the titration of exercise frequency in an old versus young state, our study was limited to two age groups, consisting of 3 month old and 30 month old rodents; recent literature from our laboratory and others suggest age-related declines could potentially occur earlier than expected and before any overt symptoms of chronic disease $(27,83,89)$, which indicates that testing intermediate age groups is becoming increasingly more important $(83,97)$.

In conclusion, we provide key insights into the stress response and muscle homeostasis regulation of skeletal muscle to an appropriately prescribed, age-specific reduced frequency paradigm of high-intensity SSC RTET involving apoptosis and DNA repair pathway gene expression, which partially underlies training adaptation. Aged skeletal muscle demonstrates a deficiency in its ability to adapt properly to high-intensity SSC RTET when conducted at higher frequencies; however, when reducing the frequency of exercise, old muscle is able to adapt through augmented muscle performance and improved muscle quality. Moreover, for the first time we provide evidence that appropriately prescribed high-intensity SSC RTET induces positive modifications in myonuclei size and morphology, which we believe helps shape downstream events in transcriptional and epigenomic regulation. Therefore, the findings in the present study supports the concept that age-appropriate exercise prescription, in this case reducing the training frequency, reverses and/or attenuates aged-associated negative alterations in key pathways involved in the stress response of skeletal muscle, rejuvenates the systemic environment in a favorable manner that helps maintain muscle homeostasis, and eventually leads to adaptation. Future studies should focus on further characterizing how these molecular, epigenomic, and morphological variables are influenced by other components of the exercise prescription (e.g., repetition number, velocity, exercise selection, etc.) in order to fully characterize what encompasses an optimal RTET exercise prescription necessary for muscle adaptation to occur as one ages. 


\section{REFERENCES}

1. Akahori H, Karmali V, Polavarapu R, Lyle AN, Weiss D, Shin E, Husain A, Naqvi N, Van Dam R, Habib A, Choi CU, King AL, Pachura K, Taylor WR, Lefer DJ, and Finn AV. CD163 interacts with TWEAK to regulate tissue regeneration after ischaemic injury. Nat Commun 6: 7792, 2015.

2. Akbari M, and Krokan HE. Cytotoxicity and mutagenicity of endogenous DNA base lesions as potential cause of human aging. Mech Ageing Dev 129: 353-365, 2008.

3. Al-Tassan N, Chmiel NH, Maynard J, Fleming N, Livingston AL, Williams GT, Hodges AK, Davies DR, David SS, Sampson JR, and Cheadle JP. Inherited variants of MYH associated with somatic G:C-->T:A mutations in colorectal tumors. Nat Genet 30: 227-232, 2002.

4. Alimonti A, Nardella C, Chen Z, Clohessy JG, Carracedo A, Trotman LC, Cheng K, Varmeh S, Kozma SC, Thomas G, Rosivatz E, Woscholski R, Cognetti F, Scher HI, and Pandolfi PP. A novel type of cellular senescence that can be enhanced in mouse models and human tumor xenografts to suppress prostate tumorigenesis. J Clin Invest 120: 681-693, 2010.

5. Alway SE. Inflammation and oxidative stress limit adaptation to stretch-shortening contractions in aging. Exerc Sport Sci Rev 45: 194, 2017.

6. Alway SE, McCrory JL, Kearcher K, Vickers A, Frear B, Gilleland DL, Bonner DE, Thomas JM, Donley DA, Lively MW, and Mohamed JS. Resveratrol enhances exercise-induced cellular and functional adaptations of skeletal muscle in older men and women. J Gerontol A Biol Sci Med Sci 72: 1595-1606, 2017.

7. Andziak B, O'Connor TP, Qi W, DeWaal EM, Pierce A, Chaudhuri AR, Van Remmen H, and Buffenstein R. High oxidative damage levels in the longest-living rodent, the naked mole-rat. Aging Cell 5: 463-471, 2006.

8. Baker BA. An old problem: aging and skeletal-muscle-strain injury. J Sport Rehabil 26: 180-188, 2017.

9. Baker BA, Hollander MS, Kashon ML, and Cutlip RG. Effects of glutathione depletion and age on skeletal muscle performance and morphology following chronic stretch-shortening contraction exposure. Eur $J$ Appl Physiol 108: 619-630, 2010.

10. Baker BA, Hollander MS, Mercer RR, Kashon ML, and Cutlip RG. Adaptive stretch-shortening contractions: diminished regenerative capacity with aging. Appl Physiol Nutr Metab 33: 1181-1191, 2008. 
11. Baker BA, Mercer RR, Geronilla KB, Kashon ML, Miller GR, and Cutlip RG. Stereological analysis of muscle morphology following exposure to repetitive stretch-shortening cycles in a rat model. Appl Physiol Nutr Metab 31: 167-179, 2006.

12. Ballak SB, Degens H, de Haan A, and Jaspers RT. Aging related changes in determinants of muscle force generating capacity: a comparison of muscle aging in men and male rodents. Ageing Res Rev 14: 43-55, 2014.

13. Bamman MM, Cooper DM, Booth FW, Chin ER, Neufer PD, Trappe S, Lightfoot JT, Kraus WE, and Joyner MJ. Exercise biology and medicine: innovative research to improve global health. Mayo Clin Proc 89: 148-153, 2014.

\section{Barres R, Yan J, Egan B, Treebak JT, Rasmussen M, Fritz T, Caidahl K, Krook A, O'Gorman} DJ, and Zierath JR. Acute exercise remodels promoter methylation in human skeletal muscle. Cell Metab 15: 405-411, 2012.

15. Bartova E, Krejci J, Harnicarova A, Galiova G, and Kozubek S. Histone modifications and nuclear architecture: a review. J Histochem Cytochem 56: 711-721, 2008.

16. Beggs R, and Yang ES. Targeting DNA repair in precision medicine. In: Advances in Protein Chemistry and Structural Biology: DNA Repair, edited by Donev R. Cambridge, MA: Academic Press, 2019, p. 136-149.

17. Bennett BT, Mohamed JS, and Alway SE. Effects of resveratrol on the recovery of muscle mass following disuse in the plantaris muscle of aged rats. PLoS One 8: e83518, 2013.

18. Borde R, Hortobagyi T, and Granacher U. Dose-response relationships of resistance training in healthy old adults: a systematic review and meta-analysis. Sports Med 45: 1693-1720, 2015.

19. Burke LM, and Hawley JA. Swifter, higher, stronger: what's on the menu? Science 362: 781-787, 2018.

20. Burkly LC, and Dohi T. The TWEAK/Fn14 pathway in tissue remodeling: for better or for worse. Adv Exp Med Biol 691: 305-322, 2011.

21. Burkly LC, Michaelson JS, and Zheng TS. TWEAK/Fn14 pathway: an immunological switch for shaping tissue responses. Immunol Rev 244: 99-114, 2011. 
22. Cedar H, and Bergman Y. Linking DNA methylation and histone modification: patterns and paradigms. Nat Rev Genet 10: 295-304, 2009.

23. Childs BG, Baker DJ, Kirkland JL, Campisi J, and van Deursen JM. Senescence and apoptosis: dueling or complementary cell fates? EMBO Rep 15: 1139-1153, 2014.

\section{Cisterna B, Giagnacovo M, Costanzo M, Fattoretti P, Zancanaro C, Pellicciari C, and Malatesta}

M. Adapted physical exercise enhances activation and differentiation potential of satellite cells in the skeletal muscle of old mice. J Anat 228: 771-783, 2016.

25. Clark SJ, Statham A, Stirzaker C, Molloy PL, and Frommer M. DNA methylation: bisulphite modification and analysis. Nat Protoc 1: 2353-2364, 2006.

26. Coffey VG, and Hawley JA. The molecular bases of training adaptation. Sports Med 37: 737-763, 2007.

27. Cooper R, Bann D, Wloch EG, Adams JE, and Kuh D. "Skeletal muscle function deficit" in a nationally representative British birth cohort in early old age. J Gerontol A Biol Sci Med Sci 70: 604-607, 2015.

28. Cutlip RG, Baker BA, Geronilla KB, Mercer RR, Kashon ML, Miller GR, Murlasits Z, and Alway SE. Chronic exposure to stretch-shortening contractions results in skeletal muscle adaptation in young rats and maladaptation in old rats. Appl Physiol Nutr Metab 31: 573-587, 2006.

29. Cutlip RG, Stauber WT, Willison RH, McIntosh TA, and Means KH. Dynamometer for rat plantar flexor muscles in vivo. Med Biol Eng Comput 35: 540-543, 1997.

30. Dostert C, Grusdat M, Letellier E, and Brenner D. The TNF family of ligands and receptors: communication modules in the immune system and beyond. Physiol Rev 99: 115-160, 2019.

31. Dubache-Powell J. Quantitative determination of muscle fiber diameter (minimal Feret's diameter) and percentage of centralized nuclei. TREAT-NMD Neuromuscular Network 1: 2008.

\section{Durbin SM, Jackson JR, Ryan MJ, Gigliotti JC, Alway SE, and Tou JC. Resveratrol}

supplementation preserves long bone mass, microstructure, and strength in hindlimb-suspended old male rats. $J$ Bone Miner Metab 32: 38-47, 2014. 
33. Eilertsen KJ, Power RA, Harkins LL, and Misica P. Targeting cellular memory to reprogram the epigenome, restore potential, and improve somatic cell nuclear transfer. Anim Reprod Sci 98: 129-146, 2007.

34. Elosegui-Artola A, Andreu I, Beedle AEM, Lezamiz A, Uroz M, Kosmalska AJ, Oria R, Kechagia JZ, Rico-Lastres P, Le Roux AL, Shanahan CM, Trepat X, Navajas D, Garcia-Manyes S, and Roca-

Cusachs P. Force triggers YAP nuclear entry by regulating transport across nuclear pores. Cell 171: 1397-1410 e1314, 2017.

35. Erwig LP, and Henson PM. Clearance of apoptotic cells by phagocytes. Cell Death Differ 15: 243250, 2008.

36. Fedorchak GR, Kaminski A, and Lammerding J. Cellular mechanosensing: getting to the nucleus of it all. Prog Biophys Mol Biol 115: 76-92, 2014.

37. Fisher JP, Steele J, Gentil P, Giessing J, and Westcott WL. A minimal dose approach to resistance training for the older adult; the prophylactic for aging. Exp Gerontol 99: 80-86, 2017.

38. Garcia-Prat L, Martinez-Vicente M, Perdiguero E, Ortet L, Rodriguez-Ubreva J, Rebollo E, RuizBonilla V, Gutarra S, Ballestar E, Serrano AL, Sandri M, and Munoz-Canoves P. Autophagy maintains stemness by preventing senescence. Nature 529: 37-42, 2016.

39. Garcia-Prat L, Munoz-Canoves P, and Martinez-Vicente M. Dysfunctional autophagy is a driver of muscle stem cell functional decline with aging. Autophagy 12: 612-613, 2016.

40. Girgenrath M, Weng S, Kostek CA, Browning B, Wang M, Brown SA, Winkles JA, Michaelson JS, Allaire N, Schneider P, Scott ML, Hsu YM, Yagita H, Flavell RA, Miller JB, Burkly LC, and Zheng TS. TWEAK, via its receptor Fn14, is a novel regulator of mesenchymal progenitor cells and skeletal muscle regeneration. EMBO J 25: 5826-5839, 2006.

41. Gushima M, Hirahashi M, Matsumoto T, Fujita K, Fujisawa R, Mizumoto K, Nakabeppu Y, Iida M, Yao T, and Tsuneyoshi M. Altered expression of MUTYH and an increase in 8-hydroxydeoxyguanosine are early events in ulcerative colitis-associated carcinogenesis. J Pathol 219: 77-86, 2009.

42. Hagerman FC, Walsh SJ, Staron RS, Hikida RS, Gilders RM, Murray TF, Toma K, and Ragg KE. Effects of high-intensity resistance training on untrained older men. I. Strength, cardiovascular, and metabolic responses. J Gerontol A Biol Sci Med Sci 55: B336-346, 2000. 
43. Hajas G, Bacsi A, Aguilera-Aguirre L, Hegde ML, Tapas KH, Sur S, Radak Z, Ba X, and Boldogh

I. 8-Oxoguanine DNA glycosylase-1 links DNA repair to cellular signaling via the activation of the small GTPase Rac1. Free Radic Biol Med 61: 384-394, 2013.

44. Hakkinen K, Kraemer WJ, Newton RU, and Alen M. Changes in electromyographic activity, muscle fibre and force production characteristics during heavy resistance/power strength training in middle-aged and older men and women. Acta Physiol Scand 171: 51-62, 2001.

45. Hakkinen K, Pakarinen A, Kraemer WJ, Hakkinen A, Valkeinen H, and Alen M. Selective muscle hypertrophy, changes in EMG and force, and serum hormones during strength training in older women. $J$ Appl Physiol (1985) 91: 569-580, 2001.

46. Hanawalt PC. Emerging links between premature ageing and defective DNA repair. Mech Ageing Dev 129: 503-505, 2008.

47. Haramizu S, Asano S, Butler DC, Stanton DA, Hajira A, Mohamed JS, and Alway SE. Dietary resveratrol confers apoptotic resistance to oxidative stress in myoblasts. J Nutr Biochem 50: 103-115, 2017.

48. Hikida RS, Staron RS, Hagerman FC, Walsh S, Kaiser E, Shell S, and Hervey S. Effects of highintensity resistance training on untrained older men. II. Muscle fiber characteristics and nucleo-cytoplasmic relationships. J Gerontol A Biol Sci Med Sci 55: B347-354, 2000.

49. Hu G, Li P, Cui X, Li Y, Zhang J, Zhai X, Yu S, Tang S, Zhao Z, Wang J, and Jia G. Cr(VI)induced methylation and down-regulation of DNA repair genes and its association with markers of genetic damage in workers and 16HBE cells. Environ Pollut 238: 833-843, 2018.

50. Hyun M, Lee J, Lee K, May A, Bohr VA, and Ahn B. Longevity and resistance to stress correlate with DNA repair capacity in Caenorhabditis elegans. Nucleic Acids Res 36: 1380-1389, 2008.

51. Jackson JR, Ryan MJ, and Alway SE. Long-term supplementation with resveratrol alleviates oxidative stress but does not attenuate sarcopenia in aged mice. J Gerontol A Biol Sci Med Sci 66: 751-764, 2011.

52. Jackson JR, Ryan MJ, Hao Y, and Alway SE. Mediation of endogenous antioxidant enzymes and apoptotic signaling by resveratrol following muscle disuse in the gastrocnemius muscles of young and old rats. American journal of physiology Regulatory, integrative and comparative physiology 299: R1572-1581, 2010. 
53. Jargin SV. Hormetic use of stress in gerontological interventions requires a cautious approach. Biogerontology 17: 417-420, 2016.

54. Ji LL, and Kang C. Role of PGC-1alpha in sarcopenia: etiology and potential intervention - a minireview. Gerontology 61: 139-148, 2015.

55. Kanzleiter T, Jahnert M, Schulze G, Selbig J, Hallahan N, Schwenk RW, and Schurmann A.

Exercise training alters DNA methylation patterns in genes related to muscle growth and differentiation in mice. Am J Physiol Endocrinol Metab 308: E912-920, 2015.

56. Karatzas PS, Mantzaris GJ, Safioleas M, and Gazouli M. DNA methylation profile of genes involved in inflammation and autoimmunity in inflammatory bowel disease. Medicine (Baltimore) 93: e309, 2014.

57. King-Himmelreich TS, Schramm S, Wolters MC, Schmetzer J, Moser CV, Knothe C, Resch E, Peil J, Geisslinger G, and Niederberger E. The impact of endurance exercise on global and AMPK genespecific DNA methylation. Biochem Biophys Res Commun 474: 284-290, 2016.

58. Kirby TJ, and Lammerding J. Cell mechanotransduction: stretch to express. Nat Mater 15: 12271229, 2016.

59. Klungland A, Rosewell I, Hollenbach S, Larsen E, Daly G, Epe B, Seeberg E, Lindahl T, and Barnes DE. Accumulation of premutagenic DNA lesions in mice defective in removal of oxidative base damage. Proc Natl Acad Sci U S A 96: 13300-13305, 1999.

60. Komi PV. Stretch-shortening cycle: a powerful model to study normal and fatigued muscle. $J$ Biomech 33: 1197-1206, 2000.

61. Laker RC, and Ryall JG. DNA Methylation in skeletal muscle stem cell specification, proliferation, and differentiation. Stem Cells Int 2016: 5725927, 2016.

62. Lane SC, Camera DM, Lassiter DG, Areta JL, Bird SR, Yeo WK, Jeacocke NA, Krook A, Zierath JR, Burke LM, and Hawley JA. Effects of sleeping with reduced carbohydrate availability on acute training responses. J Appl Physiol (1985) 119: 643-655, 2015.

63. Lee NK, and Lee SY. Modulation of life and death by the tumor necrosis factor receptor-associated factors (TRAFs). J Biochem Mol Biol 35: 61-66, 2002. 
64. Leeuwenburgh C, Gurley CM, Strotman BA, and Dupont-Versteegden EE. Age-related differences in apoptosis with disuse atrophy in soleus muscle. American journal of physiology Regulatory, integrative and comparative physiology 288: R1288-1296, 2005.

65. Lindholm ME, Marabita F, Gomez-Cabrero D, Rundqvist H, Ekstrom TJ, Tegner J, and Sundberg CJ. An integrative analysis reveals coordinated reprogramming of the epigenome and the transcriptome in human skeletal muscle after training. Epigenetics 9: 1557-1569, 2014.

66. MacRae SL, Croken MM, Calder RB, Aliper A, Milholland B, White RR, Zhavoronkov A, Gladyshev VN, Seluanov A, Gorbunova V, Zhang ZD, and Vijg J. DNA repair in species with extreme lifespan differences. Aging (Albany NY) 7: 1171-1184, 2015.

67. Malatesta M, Fattoretti P, Giagnacovo M, Pellicciari C, and Zancanaro C. Physical training modulates structural and functional features of cell nuclei in type II myofibers of old mice. Rejuvenation Res 14: 543-552, 2011.

68. Medina-Echeverz J, Haile LA, Zhao F, Gamrekelashvili J, Ma C, Metais JY, Dunbar CE, Kapoor V, Manns MP, Korangy F, and Greten TF. IFN-gamma regulates survival and function of tumor-induced CD11b+ Gr-1high myeloid derived suppressor cells by modulating the anti-apoptotic molecule Bcl2a1. Eur $J$ Immunol 44: 2457-2467, 2014.

69. Melov S, Tarnopolsky MA, Beckman K, Felkey K, and Hubbard A. Resistance exercise reverses aging in human skeletal muscle. PLoS One 2: e465, 2007.

70. Minowa O, Arai T, Hirano M, Monden Y, Nakai S, Fukuda M, Itoh M, Takano H, Hippou Y, Aburatani H, Masumura K, Nohmi T, Nishimura S, and Noda T. Mmh/Ogg1 gene inactivation results in accumulation of 8-hydroxyguanine in mice. Proc Natl Acad Sci U S A 97: 4156-4161, 2000.

71. Naimo MA, Rader EP, Ensey J, Kashon ML, and Baker BA. Reduced frequency of resistance-type exercise training promotes adaptation of the aged skeletal muscle microenvironment. J Appl Physiol (1985) 2019.

72. Navarro AP, Collins MA, and Folker ES. The nucleus is a conserved mechanosensation and mechanoresponse organelle. Cytoskeleton (Hoboken) 73: 59-67, 2016. 
73. Nguyen A, Duquette N, Mamarbachi M, and Thorin E. Epigenetic regulatory effect of exercise on glutathione peroxidase 1 expression in the skeletal muscle of severely dyslipidemic mice. PLoS One 11: e0151526, 2016.

\section{Nitert MD, Dayeh T, Volkov P, Elgzyri T, Hall E, Nilsson E, Yang BT, Lang S, Parikh H,} Wessman Y, Weishaupt H, Attema J, Abels M, Wierup N, Almgren P, Jansson PA, Ronn T, Hansson O, Eriksson KF, Groop L, and Ling C. Impact of an exercise intervention on DNA methylation in skeletal muscle from first-degree relatives of patients with type 2 diabetes. Diabetes 61: 3322-3332, 2012.

75. Ordway JM, Bedell JA, Citek RW, Nunberg A, Garrido A, Kendall R, Stevens JR, Cao D, Doerge RW, Korshunova Y, Holemon H, McPherson JD, Lakey N, Leon J, Martienssen RA, and Jeddeloh JA. Comprehensive DNA methylation profiling in a human cancer genome identifies novel epigenetic targets. Carcinogenesis 27: 2409-2423, 2006.

76. Pan L, Zhu B, Hao W, Zeng X, Vlahopoulos SA, Hazra TK, Hegde ML, Radak Z, Bacsi A, Brasier AR, Ba X, and Boldogh I. Oxidized guanine base lesions function in 8-oxoguanine DNA glycosylase-1mediated epigenetic regulation of nuclear factor kappaB-driven gene expression. J Biol Chem 291: 2555325566, 2016.

77. Penna F, Ballaro R, Beltra M, De Lucia S, Garcia Castillo L, and Costelli P. The skeletal muscle as an active player against cancer cachexia. Front Physiol 10: 41, 2019.

78. Pistilli EE, Siu PM, and Alway SE. Molecular regulation of apoptosis in fast plantaris muscles of aged rats. J Gerontol A Biol Sci Med Sci 61: 245-255, 2006.

79. Pomatto LCD, Sun PY, and Davies KJA. To adapt or not to adapt: consequences of declining adaptive homeostasis and proteostasis with age. Mech Ageing Dev 177: 80-87, 2019.

80. Qaisar R, Bhaskaran S, and Van Remmen H. Muscle fiber type diversification during exercise and regeneration. Free Radic Biol Med 98: 56-67, 2016.

81. Radak Z, Chung HY, Koltai E, Taylor AW, and Goto S. Exercise, oxidative stress and hormesis. Ageing Res Rev 7: 34-42, 2008.

82. Rader EP, and Baker BA. Inflammaging and the age-specific responsiveness to stretch-shortening contractions. Exerc Sport Sci Rev 45: 195-200, 2017. 
83. Rader EP, Layner K, Triscuit AM, Chetlin RD, Ensey J, and Baker BA. Age-dependent muscle adaptation after chronic stretch-shortening contractions in rats. Aging Dis 7: 1-13, 2016.

84. Rader EP, Layner KN, Triscuit AM, Chetlin RD, Ensey J, and Baker BA. Age-dependent muscle adaptation after chronic stretch-shortening contractions in rats. Aging Dis 2015.

85. Rader EP, Naimo MA, Ensey J, and Baker BA. High-intensity stretch-shortening contraction training modifies responsivity of skeletal muscle in old male rats. Exp Gerontol 104: 118-126, 2018.

86. Rader EP, Naimo MA, Layner KN, Triscuit AM, Chetlin RD, Ensey J, and Baker BA.

Enhancement of skeletal muscle in aged rats following high-intensity stretch-shortening contraction training. Rejuvenation Res 20: 93-102, 2017.

87. Rasmussen M, Zierath JR, and Barres R. Dynamic epigenetic responses to muscle contraction. Drug Discov Today 19: 1010-1014, 2014.

88. Real-Martinez A, Brull A, Huerta J, Tarraso G, Lucia A, Martin MA, Arenas J, Andreu AL, Nogales-Gadea G, Vissing J, Krag TO, de Luna N, and Pinos T. Low survival rate and muscle fiberdependent aging effects in the McArdle disease mouse model. Sci Rep 9: 5116, 2019.

89. Roberts MD, Kerksick CM, Dalbo VJ, Hassell SE, Tucker PS, and Brown R. Molecular attributes of human skeletal muscle at rest and after unaccustomed exercise: an age comparison. J Strength Cond Res 24: 1161-1168, 2010.

90. Rodriguez KA, Wywial E, Perez VI, Lambert AJ, Edrey YH, Lewis KN, Grimes K, Lindsey ML, Brand MD, and Buffenstein R. Walking the oxidative stress tightrope: a perspective from the naked mole-rat, the longest-living rodent. Curr Pharm Des 17: 2290-2307, 2011.

91. Ron A. Mechano-molecular transduction: Putting the pieces together. Biophys Chem 241: 15-19, 2018.

92. Ryan MJ, Dudash HJ, Docherty M, Geronilla KB, Baker BA, Haff GG, Cutlip RG, and Alway SE. Aging-dependent regulation of antioxidant enzymes and redox status in chronically loaded rat dorsiflexor muscles. J Gerontol A Biol Sci Med Sci 63: 1015-1026, 2008.

93. Ryan MJ, Dudash HJ, Docherty M, Geronilla KB, Baker BA, Haff GG, Cutlip RG, and Alway SE. Vitamin E and C supplementation reduces oxidative stress, improves antioxidant enzymes and positive muscle work in chronically loaded muscles of aged rats. Exp Gerontol 45: 882-895, 2010. 
94. Saldmann F, Viltard M, Leroy C, and Friedlander G. The naked mole rat: a unique example of positive oxidative stress. Oxid Med Cell Longev 2019: 4502819, 2019.

95. Sanders YY, Liu H, Zhang X, Hecker L, Bernard K, Desai L, Liu G, and Thannickal VJ. Histone modifications in senescence-associated resistance to apoptosis by oxidative stress. Redox Biol 1: 8-16, 2013.

96. Seals DR, Justice JN, and LaRocca TJ. Physiological geroscience: targeting function to increase healthspan and achieve optimal longevity. J Physiol 594: 2001-2024, 2016.

97. Sharples AP, Stewart CE, and Seaborne RA. Does skeletal muscle have an 'epi'-memory? The role of epigenetics in nutritional programming, metabolic disease, aging and exercise. Aging Cell 15: 603-616, 2016.

98. Siu PM, Bryner RW, Martyn JK, and Alway SE. Apoptotic adaptations from exercise training in skeletal and cardiac muscles. FASEB J 18: 1150-1152, 2004.

99. Sohal RS, Sohal BH, and Orr WC. Mitochondrial superoxide and hydrogen peroxide generation, protein oxidative damage, and longevity in different species of flies. Free Radic Biol Med 19: 499-504, 1995.

100. St Pierre BA, and Tidball JG. Differential response of macrophage subpopulations to soleus muscle reloading after rat hindlimb suspension. J Appl Physiol (1985) 77: 290-297, 1994.

\section{Stec MJ, Thalacker-Mercer A, Mayhew DL, Kelly NA, Tuggle SC, Merritt EK, Brown CJ,} Windham ST, Dell'Italia LJ, Bickel CS, Roberts BM, Vaughn KM, Isakova-Donahue I, Many GM, and Bamman MM. Randomized, four-arm, dose-response clinical trial to optimize resistance exercise training for older adults with age-related muscle atrophy. Exp Gerontol 99: 98-109, 2017.

102. Stinson BM, Moreno AT, Walter JC, and Loparo JJ. Non-homologous end joining minimizes errors by coordinating DNA processing with ligation. 563197, 2019.

103. Tajik A, Zhang Y, Wei F, Sun J, Jia Q, Zhou W, Singh R, Khanna N, Belmont AS, and Wang N. Transcription upregulation via force-induced direct stretching of chromatin. Nat Mater 15: 1287-1296, 2016.

104. Takahashi H, Suzuki Y, Mohamed JS, Gotoh T, Pereira SL, and Alway SE. Epigallocatechin-3gallate increases autophagy signaling in resting and unloaded plantaris muscles but selectively suppresses autophagy protein abundance in reloaded muscles of aged rats. Exp Gerontol 92: 56-66, 2017. 
105. Tan Q, Christiansen L, von Bornemann Hjelmborg J, and Christensen K. Twin methodology in epigenetic studies. J Exp Biol 218: 134-139, 2015.

106. Trajano L, Trajano ETL, Silva M, Stumbo AC, Mencalha AL, and Fonseca ASD. Genomic stability and telomere regulation in skeletal muscle tissue. Biomed Pharmacother 98: 907-915, 2018.

107. Vaczi M, Nagy SA, Koszegi T, Ambrus M, Bogner P, Perlaki G, Orsi G, Toth K, and Hortobagyi T. Mechanical, hormonal, and hypertrophic adaptations to 10 weeks of eccentric and stretch-shortening cycle exercise training in old males. Exp Gerontol 58: 69-77, 2014.

108. Versaevel M, Grevesse T, and Gabriele S. Spatial coordination between cell and nuclear shape within micropatterned endothelial cells. Nat Commun 3: 671, 2012.

109. Vijg J. The role of DNA damage and repair in aging: new approaches to an old problem. Mech Ageing Dev 129: 498-502, 2008.

110. Walton RG, Kosmac K, Mula J, Fry CS, Peck BD, Groshong JS, Finlin BS, Zhu B, Kern PA, and Peterson CA. Human skeletal muscle macrophages increase following cycle training and are associated with adaptations that may facilitate growth. Sci Rep 9: 969, 2019.

111. Wang S, Reuveny A, and Volk T. Nesprin provides elastic properties to muscle nuclei by cooperating with spectraplakin and EB1. J Cell Biol 209: 529-538, 2015.

\section{White Z, Terrill J, White RB, McMahon C, Sheard P, Grounds MD, and Shavlakadze T.}

Voluntary resistance wheel exercise from mid-life prevents sarcopenia and increases markers of mitochondrial function and autophagy in muscles of old male and female C57BL/6J mice. Skelet Muscle 6: 45, 2016.

\section{Wichnieski C, Maheshwari K, Souza LC, Nieves F, Tartari T, Garlet GP, Carneiro E, Letra A,} and Silva RM. DNA methylation profiles of immune response-related genes in apical periodontitis. Int Endod $J$ 52: 5-12, 2019.

114. Wimmer N, Heigl U, Klingseisen L, Schneider-Brachert W, and Hehlgans T. Lymphotoxin-beta receptor signalling regulates cytokine expression via TRIM30alpha in a TRAF3-dependent manner. Mol Immunol 54: 40-47, 2013. 


\section{Woodrick J, Gupta S, Khatkar P, Sarangi S, Narasimhan G, Trehan A, Adhikari S, and Roy R.}

Slow repair of lipid peroxidation-induced DNA damage at p53 mutation hotspots in human cells caused by low turnover of a DNA glycosylase. Nucleic Acids Res 42: 9033-9046, 2014. 
Table 1. $\mathrm{RT}^{2}$ Profiler Apoptosis PCR Array.

$\begin{array}{ccccccccccccccc} & 1 & 2 & 3 & 4 & 5 & 6 & 7 & 8 & 9 & 10 & 11 & 12 \\ \text { A } & \text { Abl1 } & \text { Aifm1 } & \text { Akt1 } & \text { Anxa5 } & \text { Apaf1 } & \text { Api5 } & \text { Aven } & \text { Bad } & \text { Bag1 } & \text { Bak1 } & \text { Bax } & \text { Bcl10 } \\ \text { B } & \text { Bcl2 } & \text { Bcl2a1 } & \text { Bcl211 } & \text { Bcl2l11 } & \text { Bcl212 } & \text { Bid } & \text { Bik } & \text { Birc2 } & \text { Birc3 } & \text { Birc5 } & \text { Bnip2 } & \text { Bnip3 } \\ \text { C } & \text { Bok } & \text { Card10 } & \text { Casp1 } & \text { Casp12 } & \text { Casp14 } & \text { Casp2 } & \text { Casp3 } & \text { Casp4 } & \text { Casp6 } & \text { Casp7 } & \text { Casp8 } & \text { Casp8ap2 } \\ \text { D } & \text { Casp9 } & \text { Cd40 } & \text { Cd40lg } & \text { Cflar } & \text { Cidea } & \text { Cideb } & \text { Cycs } & \text { Dad1 } & \text { Dapk1 } & \text { Dffa } & \text { Dffb } & \text { Diablo } \\ \text { E } & \text { Fadd } & \text { Faim } & \text { Fas } & \text { Faslg } & \text { Gadd45a } & \text { Hrk } & \text { Il10 } & \text { Lta } & \text { Ltbr } & \text { Mapk1 } & \text { Mapk8ip1 } & \text { Mcl1 } \\ \text { F } & \text { Naip6 } & \text { Nfkb1 } & \text { Nol3 } & \text { Polb } & \text { Prdx2 } & \text { Prlr } & \text { Pycard } & \text { Ripk2 } & \text { Sphk2 } & \text { Tnf } & \text { Tnfrsf10b } & \text { Tnfrsf11b } \\ \text { G } & \text { Tnfrsf1a } & \text { Tnfrsf1b } & \text { Tnfsf10 } & \text { Tnfsf12 } & \text { Tp53 } & \text { Tp53bp2 } & \text { Tp63 } & \text { Tp73 } & \text { Tradd } & \text { Traf2 } & \text { Traf3 } & \text { Xiap } \\ \text { H } & \text { Actb } & \text { B2m } & \text { Hprt1 } & \text { Ldha } & \text { Rplp1 } & \text { RGDC } & \text { RTC } & \text { RTC } & \text { RTC } & \text { PPC } & \text { PPC } & \text { PPC }\end{array}$


Table 2. RT ${ }^{2}$ Profiler DNA Repair PCR Array.

$\begin{array}{cccccccccccccc} & 1 & 2 & 3 & 4 & 5 & 6 & 7 & 8 & 9 & 10 & 11 & 12 \\ \text { A } & \text { Alkbh2 } & \text { Alkbh3 } & \text { Apex1 } & \text { Atm } & \text { Atxn3 } & \text { Blm } & \text { Brca1 } & \text { Brca2 } & \text { Ccnh } & \text { Cdk7 } & \text { Dclre1a } & \text { Dclre1b } \\ \text { B } & \text { Ddb1 } & \text { Ddb2 } & \text { Dmc1 } & \text { Erc1 } & \text { Erc2 } & \text { Erc3 } & \text { Ercc4 } & \text { Ercc5 } & \text { Ercc6 } & \text { Ercc8 } & \text { Exo1 } & \text { Fen1 } \\ \text { C } & \text { Gen1 } & \text { Lig1 } & \text { Lig3 } & \text { Lig4 } & \text { Msh6 } & \text { Mgmt } & \text { Mlh1 } & \text { Mlh3 } & \text { Mpg } & \text { Mre11a } & \text { Msh2 } & \text { Msh3 } \\ \text { D } & \text { Msh5 } & \text { Mus81 } & \text { Mutyh } & \text { Neil1 } & \text { Neil2 } & \text { Nhej1 } & \text { Nthl1 } & \text { Ogg1 } & \text { Parp1 } & \text { Parp2 } & \text { Parp3 } & \text { Pms1 } \\ \text { E } & \text { Pms2 } & \text { Pnkp } & \text { Polb } & \text { Pold3 } & \text { Poll } & \text { Prkdc } & \text { Rad18 } & \text { Rad21 } & \text { Rad23a } & \text { Rad23b } & \text { Rad50 } & \text { Rad51 } \\ \text { F } & \text { Rad51c } & \text { Rad51d } & \text { Rad52 } & \text { Rad54l } & \text { Rad9b } & \text { Rfc1 } & \text { Rpa1 } & \text { Rpa3 } & \text { Slk } & \text { Smug1 } & \text { Tdg } & \text { Top3a } \\ \text { G } & \text { Top3b } & \text { Trex1 } & \text { Trex2 } & \text { Ung } & \text { Xab2 } & \text { Xpc } & \text { Xrcc1 } & \text { Xrcc2 } & \text { Xrcc4 } & \text { Xrcc5 } & \text { Xrcc6 } & \text { Xrcc6bp1 } \\ \text { H } & \text { Actb } & \text { B2m } & \text { Hprt1 } & \text { Ldha } & \text { Rplp1 } & \text { RGDC } & \text { RTC } & \text { RTC } & \text { RTC } & \text { PPC } & \text { PPC } & \text { PPC }\end{array}$


Table 3. Details of the Rat Apoptosis DNA Methylation PCR Array. All information on gene function(s) was obtained from Entrez Gene Summary and/or UniProtKB/Swiss-Prot.

\begin{tabular}{ccc}
\hline Genes & Symbol & Description \\
\hline Induction of Apoptosis & Bad & $\begin{array}{l}\text { BCL2-associated agonist of cell } \\
\text { death }\end{array}$
\end{tabular}

Bax

Bcl2-associated X protein

$\operatorname{Bcl} 2 \mathrm{l} 11$

Bclaf1

Bid

Bik

Bnip3l

Cideb

Cradd

Dapk1
BCL2-like 11 (apoptosis facilitator)

BCL2 associated transcription factor

BH3 interacting domain death agonist

BCL2-interacting killer (apoptosis-inducing)

BCL2/adenovirus E1B interacting protein 3-like

Cell death-inducing DFFA-like effector b

CASP2 and RIPK1 domain containing adaptor with death domain

Death associated protein kinase 1
Promotes cell death; positively regulates cell apoptosis by forming heterodimers with $\mathrm{Bcl}-\mathrm{xL}$ and $\mathrm{Bcl}-2$, reversing their death repressor activity Plays a role in the mitochondrial apoptotic process; forms a heterodimer with Bcl-2 and functions as an apoptotic activator; under stress conditions, undergoes a conformational change that causes translocation to the mitochondrion membrane, leading to release of cytochrome c that triggers apoptosis

Induces apoptosis and anoikis; been shown to interact with other members of the $\mathrm{Bcl}-2$ protein family and act as an apoptotic activator

Death-promoting transcriptional repressor; overexpression induces apoptosis

Mediator of mitochondrial damage induced by caspase 8; encodes a death agonist that heterodimerizes with either agonist Bax or antagonist $\mathrm{Bcl} 2$; triggers cytochrome $\mathrm{c}$ release Accelerates programmed cell death; shares a critical BH3 domain with other deathpromoting proteins that is required for its pro-apoptotic activity

Induces apoptosis; the protein directly targets mitochondria and causes apoptotic changes, including loss of membrane potential and the release of cytochrome $\mathrm{c}$ Activates apoptosis

Promotes apoptosis; apoptotic adaptor molecule specific for caspase-2 and FASL/TNF receptor-interacting protein Rip; In the presence of Rip and Tradd, recruits caspase- 2 to the Tnfr- 1 signaling complex

Positive mediator of gammainterferon-induced programmed cell death; involved in multiple cellular signaling pathways that triggers cell survival, apoptosis, and autophagy; regulates both type I apoptotic and type II autophagic cell death signals, depending on the cellular setting 
Dffa

Fadd

Gadd45a

Hrk

Tnfrsf21

Tp53

Anti-Apoptosis

Bcl2l1

Regulation of Apoptosis: Negative Regulation
DNA fragmentation factor, alpha subunit

Fas (TNFRSF6)-associated via death domain

Growth arrest and DNA-damageinducible, alpha

Harakiri, BCL2 interacting protein (contains only BH3 domain)

Tumor necrosis factor receptor superfamily, member 21

Tumor protein p53

Bcl2-like 1

Baculoviral IAP repeat-containing 2
Substrate for caspase 3 and triggers DNA fragmentation during apoptosis; Dff becomes activated when Dffa is cleaved by caspase 3; the cleaved fragments of Dffa dissociate from Dffb, the active component of Dff; Dffb has been found to trigger both DNA fragmentation and chromatin condensation during apoptosis Apoptotic adapter molecule that recruits caspase 8 or caspase 10 to the activated Fas or Tnfr-1 receptors. The resulting deathinducing signaling complex performs caspase 8 proteolytic cleavage; plays a role in the positive regulation of interferon signaling

Transcript levels are increased following stressful growth arrest conditions; responds to environmental stresses by mediating activation of the p38/JNK pathway; stimulates DNA excision repair in vitro and inhibits entry of cells into $S$ phase Promotes apoptosis by interacting with the apoptotic inhibitors Bcl-2 and Bcl-XL via its BH3 domain Promotes apoptosis possibly via a pathway that involves activation of NF- $\mathrm{BB}$; can also promote apoptosis mediated by Bax and by release of cytochrome c from the mitochondria into the cytoplasm; through its death domain, interacts with Tradd, which is known to mediate signal transduction of TNF receptors Induces growth arrest or apoptosis depending on the physiological circumstance and cell type; tumor suppressor; involved in cell cycle regulation as a trans-activator; apoptosis induction mediated either by stimulation of Bax and Fas antigen expression, or by repression of $\mathrm{Bcl} 2$ expression Potent inhibitor of cell death; inhibits activation of caspases; appears to regulate cell death by blocking voltage-dependent anion channel by binding and preventing release of Cyc1 from the mitochondrial membrane Multi-functional protein which not only regulates caspases and apoptosis, but also modulates inflammatory signaling/immunity, mitogenic kinase signaling, cell proliferation, cell invasion, and metastasis; member of a family of proteins that inhibit apoptosis by binding to Traf1 and Traf2 
Regulation of Apoptosis:

Positive Regulation

\section{Caspases \& Regulators}

Apaf1

Casp3

Casp9
Tumor necrosis factor receptor superfamily, member 10b

Apoptotic peptidase activating factor 1

Caspase 3

Caspase 9, apoptosis-related cysteine peptidase
Essential for ER stress-induced apoptosis; member of the TNFreceptor superfamily, contains an intracellular death domain Oligomeric Apaf1 mediates cytochrome c-dependent autocatalytic activation of procaspase 9 (Apaf3), leading to activation of caspase 3 and apoptosis

Plays central role in the execution-phase of cell apoptosis; involved in the activation cascade of caspases responsible for apoptosis execution; encoded protein cleaves and activates sterol regulatory binding proteins as well as caspases 6,7 , and 9 Involved in the activation cascade of caspases responsible for apoptosis execution; binding of caspase 9 to Apaf1 leads to activation of the protease which then cleaves and activates caspase 3 ; promotes DNA damageinduced apoptosis in a Abl1/cAbl-dependent manner 
Table 4. Details of the DNA Repair DNA Methylation PCR Array. All information on gene function(s) was obtained from Entrez Gene Summary and/or UniProtKB/Swiss-Prot.

\begin{tabular}{ccc}
\hline Genes & Symbol & Description \\
\hline Base Excision Repair (BER) & Apex1 & APEX nuclease (multifunctional \\
& & DNA repair enzyme) 1 \\
& & \\
& & \\
& & \\
& &
\end{tabular}

Lig3

Ung

Xrcc1

Nucleotide Excision Repair (NER)

Ccnh
Ligase III, DNA, ATP-dependent

Uracil-DNA glycosylase

$\mathrm{X}$-ray repair complementing defective repair in Chinese hamster cells 1

Rad23a

Rad23b
RAD23 homolog A (S. cerevisiae)
RAD23 homolog B (S. cerevisiae)
Multifunctional protein plays a central role in the cellular response to oxidative stress; two major activities are DNA repair and redox regulation of transcriptional factors; acts as a apurinic/apyrimidinic endodeoxyribonuclease in DNA BER pathway of DNA lesions induced by oxidative agents Member of DNA ligase family; isoform 3 functions as a heterodimer with Xrcc1 in the nucleus and can correct defective DNA strand-break repair and sister chromatid exchange; isoform 1 functions as a DNA ligase in mitochondrial baseexcision DNA repair One of several uracil-DNA glycosylases whose important function is to prevent mutagenesis by eliminating uracil residues from DNA molecules by cleaving the N-glycosylic bond and initiating the BER pathway Involved in DNA single-strand break repair by mediating the assembly of DNA break repair protein complexes; interacts with DNA ligase III, polymerase beta and poly (ADP-ribose) polymerase to participate in the BER pathway; negatively regulates $\mathrm{ADP}$-ribose levels by modulating ADP-

ribosyltransferase PARP1 activity Involved in cell cycle control and RNA transcription by RNA polymerase II; regulates CDK7, the catalytic subunit of the CDKactivating kinase enzymatic complex; participates in two different transcriptional regulation processes, indicating an important link between basal transcription control and the cell cycle system Multiubiquitin chain receptor involved in modulation of proteasomal degradation; plays important role in NER and in delivery of polyubiquitinated proteins to the proteasome; thought to be functionally equal to Rad23b in global genome NER by association with Xpc

Multiubiquitin chain receptor involved in modulation of proteasomal degradation; important in global genome NER 
$\mathrm{Xpc}$

Mismatch Repair (MMR) Repair
Xeroderma pigmentosum, complementation group C

by acting as a component of the XPC complex; shown to interact with, and elevate, the nucleotide excision activity of 3methyladenine-DNA glycosylase, suggesting a role in DNA damage recognition in BER

Involved in global genome NER by acting as damage sensing and DNA-binding factor component of the XPC complex; has a low DNA repair activity by itself, which is stimulated by Rad23a and Rad23b; the Xpc complex is proposed to represent the first factor bound at sites of DNA damage and together with other core recognition factors, Xpa, Rpa and TfIIh complex, is part of the pre-incision (or initial recognition) complex Heterodimerizes with Pms2 to form MutL alpha, a component of the post-replicative DNA MMR system; also involved in DNA damage signaling and can heterodimerize with DNA mismatch repair protein Mlh3 to form MutL gamma, which is involved in meiosis; introduces single-strand breaks near the mismatch thus generating new entry points for the exonuclease Exo1 to degrade the strain containing the mismatch

MutS homolog 2 (E. coli) Component of MMR; forms two different heterodimers: MutS alpha and MutS beta, which binds to DNA mismatches, thereby initiating DNA repair

PMS2 postmeiotic segregation Key component of MMR system that functions to correct DNA mismatches and small insertions/deletions that can occur during DNA replication and HR; introduces single-strand breaks near the mismatch, thus generating new entry points for exonuclease Exo1 to degrade the strand containing the mismatch

Polymerase (DNA-directed), delta 3 , accessory subunit

Brca1
Possesses both polymerase and 3'

to 5' exonuclease activity, plays a critical role in DNA replication and repair; stabilizes Pol-delta complex, plays a major role in Pol-delta stimulation by Pcna, thus is required for optimal Poldelta activity

Plays a major role in maintaining genomic stability; acts as a tumor suppressor; central role in DNA repair by facilitating cellular responses to DNA damage; also plays a role in transcription, repair of DSBs, and recombination; 
Brca2

Fen1

Mre11a

Prkdc

Rad50
Breast cancer 2

Flap structure-specific endonuclease 1

Protein kinase, DNA activated, catalytic polypeptide

RAD50 homolog (S. cerevisiae) homolog A (S. cerevisiae) required for normal cell cycle progression from $\mathrm{G} 2$ to mitosis Involved in DSB repair and/or $\mathrm{HR}$; involved in maintenance of genome stability; binds Rad51 and potentiates recombinational DNA repair by promoting assembly of Rad51 onto singlestranded DNA

Acts as a genome stabilization factor that prevents flaps from equilibrating into structures leading to duplications and deletions; removes 5 , overhanging flaps in DNA repair and processes the 5' ends of Okazaki fragments in lagging DNA strand synthesis; direct physical interaction between this protein and AP endonuclease 1 during long-patch BER provides coordinated loading of proteins onto the substrate, thus passing it from one enzyme to another; also involved in replication/repair of rDNA and in repairing mitochondrial DNA

MRE11 meiotic recombination 11

olved in $\mathrm{HR}$, telomere length maintenance, and DNA DSB repair; forms complex with Rad50 homolog, which is required for NHEJ of DNA ends and possesses increased single-stranded DNA endonuclease and 3' to 5' exonuclease activities; may be vital for DNA damage signaling via activation of Atm kinase Serine/threonine kinase that acts as a molecular sensor for DNA damage; encodes the protein for the catalytic subunit of DNAdependent protein kinase; involved in DNA NHEJ required for DSB repair and V (D) J recombination; required to protect and align broken ends of DNA; may act as a scaffold protein to aid localization of DNA repair proteins to the site of damage Component of the MRN complex, which plays a central role in DSB repair. The complex possesses single-strand endonuclease activity and double-strandspecific 3'-5' exonuclease activity, which are provided by Mre11; DNA recombination, maintenance of telomere integrity, and meiosis; may be required to bind DNA ends and hold them in close proximity; may also be required for DNA damage signaling via activation of the Atm kinase 
RAD51 homolog (RecA homolog, E. coli) (S. cerevisiae)

Rad51c

Xrcc2
Rad51 homolog c (S. cerevisiae)

$\mathrm{X}$-ray repair complementing defective repair in Chinese hamster cells 2
Plays important role in homologous strand exchange, a key step in DNA repair through HR; binds single and doublestranded DNA and exhibits DNAdependent ATPase activity; thought to play roles in homologous pairing and strand transfer of DNA; found to interact with Brca1 and Brca2, which may be important for the cellular response to DNA damage; catalyzes the recognition of homology and strand exchange between homologous DNA partners to form a joint molecule between a processed DNA break and the repair template; plays a role in regulating mitochondrial DNA copy number under conditions of oxidative stress in the presence of Rad51c and Xrcc3; involved in interstrand cross-link repair

Essential in HR repair pathway of double-stranded DNA breaks arising during DNA replication or induced DNA-damaging agents; important for Holliday junction resolution; has an early function in DNA repair in facilitating phosphorylation of the checkpoint kinase Chek2 and transduction of the damage signal, leading to cell cycle arrest and HR activation Involved in HR repair pathway of double-stranded DNA, repairs chromosomal fragmentation, translocations and deletions; encodes a member of the RecA/Rad51-related protein family that participates in HR to maintain chromosome stability and repair DNA damage; part of the Rad21 paralog protein complex Bcdx2, which acts in a Brca1-Brca2-dependent HR pathway. 
Other Genes Related to DNA Repair
Atm

Ataxia telangiectasia mutated homolog (human)
Serine/threonine protein kinase which acts as a DNA damage sensor by activating checkpoint signaling upon DSBs, apoptosis, and genotoxic stresses; important cell cycle checkpoint kinase that, along with Atr, is considered to be master controllers of cell cycle checkpoint signaling pathways required for a cell response to DNA damage and for genome stability; phosphorylates and functions as a regulator of a wide variety of downstream proteins, including Tp53 and Brca1 
Table 5. Significantly differentially expressed genes (SDEGs) relevant to the apoptosis pathway for the tibialis anterior muscle following dorsiflexion SSC-loading relative to non-loaded control muscles.

\begin{tabular}{|c|c|c|c|c|c|c|c|c|c|c|c|}
\hline & & & & \multicolumn{2}{|c|}{ Young 3x/wk. } & \multicolumn{2}{|c|}{ Young 2x/wk. } & \multicolumn{2}{|c|}{ Old 3x/wk. } & \multicolumn{2}{|c|}{ Old 2x/wk. } \\
\hline Genes & Symbol & Description & RefSeq \# & FC & $\begin{array}{c}P \\
\text { value }\end{array}$ & $\mathrm{FC}$ & $\begin{array}{c}P \\
\text { value }\end{array}$ & FC & $\begin{array}{c}P \\
\text { value }\end{array}$ & FC & $P$ value \\
\hline \multirow{2}{*}{$\begin{array}{l}\text { Induction of } \\
\text { Apoptosis: } \\
\text { Death } \\
\text { Domain } \\
\text { Receptors }\end{array}$} & Casp8ap2 & $\begin{array}{l}\text { Caspase } 8 \\
\text { associated protein } 2\end{array}$ & NM_001107921 & $\uparrow 1.39$ & 0.0686 & $\uparrow 1.04$ & 0.5665 & $\uparrow 1.22$ & 0.5101 & $\downarrow 1.02$ & 0.8577 \\
\hline & Fadd & $\begin{array}{l}\text { Fas (TNFRSF6)- } \\
\text { associated via death } \\
\text { domain }\end{array}$ & NM_152937 & $\uparrow 1.71$ & 0.0210 & $\uparrow 1.34$ & 0.0612 & $\uparrow 1.49$ & 0.6592 & $\downarrow 1.02$ & 0.7791 \\
\hline $\begin{array}{l}\text { Induction of } \\
\text { Apoptosis: } \\
\text { Extracellular } \\
\text { Apoptotic } \\
\text { Signals }\end{array}$ & Cflar & $\begin{array}{l}\text { CASP8 and FADD- } \\
\text { like apoptosis } \\
\text { regulator }\end{array}$ & NM_057138 & $\downarrow 1.16$ & 0.2223 & $\downarrow 1.23$ & 0.0941 & $\uparrow 1.04$ & 0.8794 & $\downarrow 1.19$ & 0.0046 \\
\hline \multirow{11}{*}{$\begin{array}{l}\text { Induction of } \\
\text { Apoptosis: } \\
\text { Other Pro- } \\
\text { Apoptotic } \\
\text { Genes }\end{array}$} & Bad & $\begin{array}{l}\text { BCL2-associated } \\
\text { agonist of cell death }\end{array}$ & NM_022698 & $\uparrow 1.58$ & 0.0051 & $\uparrow 1.00$ & 0.8339 & $\uparrow 1.83$ & 0.3226 & $\downarrow 1.02$ & 0.9104 \\
\hline & Bak1 & $\begin{array}{l}\text { BCL2- } \\
\text { antagonist/killer } 1\end{array}$ & NM_053812 & $\uparrow 1.50$ & 0.0548 & $\uparrow 1.31$ & 0.0367 & $\uparrow 1.77$ & 0.1996 & $\downarrow 1.02$ & 0.9576 \\
\hline & $\mathrm{Bax}$ & $\begin{array}{l}\text { Bcl2-associated X } \\
\text { protein }\end{array}$ & NM_017059 & $\uparrow 1.30$ & 0.0274 & $\uparrow 1.04$ & 0.6412 & $\uparrow 1.53$ & 0.3750 & $\downarrow 1.20$ & 0.1652 \\
\hline & Bcl10 & $\begin{array}{l}\text { B-cell } \\
\text { CLL/lymphoma } 10\end{array}$ & NM_031328 & $\uparrow 1.31$ & 0.0220 & $\uparrow 1.09$ & 0.5161 & $\uparrow 1.44$ & 0.3685 & $\downarrow 1.12$ & 0.1320 \\
\hline & $\mathrm{Bcl} 2 \mathrm{l} 11$ & $\begin{array}{l}\text { BCL2-like } 11 \\
\text { (apoptosis } \\
\text { facilitator) }\end{array}$ & NM_022612 & $\uparrow 1.20$ & 0.4687 & $\uparrow 1.40$ & 0.0298 & $\uparrow 1.11$ & 0.6739 & $\uparrow 1.00$ & 0.9251 \\
\hline & Bid & $\begin{array}{l}\text { BH3 interacting } \\
\text { domain death } \\
\text { agonist }\end{array}$ & NM_022684 & $\uparrow 2.46$ & 0.0004 & $\uparrow 1.67$ & 0.1324 & $\uparrow 1.41$ & 0.3001 & $\downarrow 1.28$ & 0.3197 \\
\hline & Bok & $\begin{array}{l}\text { BCL2-related } \\
\text { ovarian killer }\end{array}$ & NM_017312 & $\uparrow 2.15$ & 0.0159 & $\uparrow 1.37$ & 0.0177 & $\uparrow 1.30$ & 0.1324 & $\uparrow 1.29$ & 0.1138 \\
\hline & Gadd45a & $\begin{array}{l}\text { Growth arrest and } \\
\text { DNA-damage- } \\
\text { inducible, alpha }\end{array}$ & NM_024127 & $\uparrow 1.65$ & 0.0127 & $\uparrow 1.58$ & 0.0207 & $\uparrow 1.55$ & 0.6229 & $\downarrow 1.46$ & 0.2080 \\
\hline & Pycard & $\begin{array}{l}\text { PYD and CARD } \\
\text { domain containing }\end{array}$ & NM_172322 & $\uparrow 1.64$ & 0.0756 & $\uparrow 1.59$ & 0.2337 & $\uparrow 1.77$ & 0.1462 & $\downarrow 1.19$ & 0.2887 \\
\hline & Tp63 & Tumor protein p63 & NM_019221 & $\uparrow 1.93$ & 0.0001 & $\uparrow 1.57$ & 0.0073 & $\uparrow 1.19$ & 0.8005 & $\uparrow 1.13$ & 0.3090 \\
\hline & Traf3 & $\begin{array}{l}\text { Tnf receptor- } \\
\text { associated factor } 3\end{array}$ & NM_001108724 & $\uparrow 2.36$ & 0.0025 & $\uparrow 1.95$ & 0.0057 & $\uparrow 1.11$ & 0.6418 & $\uparrow 1.63$ & 0.0041 \\
\hline $\begin{array}{l}\text { Anti- } \\
\text { Apoptotic }\end{array}$ & Anxa5 & Annexin A5 & NM_013132 & $\uparrow 1.91$ & 0.0168 & $\uparrow 1.60$ & 0.1637 & $\uparrow 2.67$ & 0.1200 & $\uparrow 1.24$ & 0.1466 \\
\hline
\end{tabular}




\begin{tabular}{|c|c|c|c|c|c|c|c|c|c|c|c|}
\hline & Aven & $\begin{array}{l}\text { Apoptosis, caspase } \\
\text { activation inhibitor }\end{array}$ & NM_001107757 & $\uparrow 1.08$ & 0.6188 & $\downarrow 1.00$ & 0.9921 & $\uparrow 1.90$ & 0.0902 & $\uparrow 1.02$ & 0.8046 \\
\hline & $\mathrm{Bcl} 2$ & $\begin{array}{l}\text { B-cell } \\
\text { CLL/lymphoma } 2\end{array}$ & NM_016993 & $\uparrow 1.32$ & 0.0173 & $\uparrow 1.31$ & 0.0659 & $\uparrow 1.14$ & 0.9351 & $\downarrow 1.13$ & 0.3592 \\
\hline & Bcl2a1 & $\begin{array}{l}\text { B-cell } \\
\text { leukemia/lymphoma } \\
2 \text { related protein } \\
\text { A1d }\end{array}$ & NM_133416 & $\uparrow 2.81$ & 0.0522 & $\uparrow 2.42$ & 0.3267 & $\uparrow 3.07$ & 0.0308 & $\downarrow 1.19$ & 0.4663 \\
\hline & Birc3 & $\begin{array}{l}\text { Baculoviral IAP } \\
\text { repeat-containing } 3\end{array}$ & NM_023987 & $\uparrow 1.22$ & 0.3516 & $\downarrow 1.10$ & 0.6410 & $\uparrow 1.09$ & 0.8974 & $\downarrow 1.44$ & 0.0218 \\
\hline & Bnip2 & $\begin{array}{l}\text { Bcl2/adenovirus } \\
\text { E1B interacting } \\
\text { protein } 2\end{array}$ & NM_001106835 & $\downarrow 1.06$ & 0.7008 & $\uparrow 1.01$ & 0.8027 & $\downarrow 1.07$ & 0.9655 & $\downarrow 1.18$ & 0.0990 \\
\hline & Dad1 & $\begin{array}{l}\text { Defender against } \\
\text { cell death } 1\end{array}$ & NM_138910 & $\uparrow 1.13$ & 0.2784 & $\uparrow 1.23$ & 0.0503 & $\uparrow 1.14$ & 0.4290 & $\uparrow 1.00$ & 0.8963 \\
\hline & Faim & $\begin{array}{l}\text { Fas apoptotic } \\
\text { inhibitory molecule }\end{array}$ & NM_080895 & $\uparrow 1.24$ & 0.0933 & $\uparrow 1.40$ & 0.0610 & $\uparrow 1.78$ & 0.2940 & $\downarrow 1.12$ & 0.4811 \\
\hline & Mcl1 & $\begin{array}{l}\text { Myeloid cell } \\
\text { leukemia sequence } \\
1\end{array}$ & NM_021846 & $\uparrow 1.49$ & 0.0499 & $\uparrow 1.16$ & 0.2473 & $\uparrow 1.37$ & 0.3999 & $\downarrow 1.27$ & 0.0086 \\
\hline & Naip6 & $\begin{array}{l}\text { NLR family, } \\
\text { apoptosis inhibitory } \\
\text { protein } 6\end{array}$ & XM_006223993 & $\uparrow 1.70$ & 0.0999 & $\uparrow 1.63$ & 0.3292 & $\uparrow 1.55$ & 0.3668 & $\downarrow 1.02$ & 0.9041 \\
\hline & Nol3 & $\begin{array}{l}\text { Nucleolar protein } 3 \\
\text { (apoptosis repressor } \\
\text { with CARD } \\
\text { domain) }\end{array}$ & NM_053516 & $\uparrow 1.59$ & 0.0013 & $\uparrow 1.57$ & 0.0080 & $\uparrow 1.35$ & 0.2830 & $\uparrow 1.19$ & 0.1079 \\
\hline $\begin{array}{l}\text { Regulation } \\
\text { of Apoptosis: } \\
\text { Positive }\end{array}$ & Ripk2 & $\begin{array}{l}\text { Receptor-interacting } \\
\text { serine-threonine } \\
\text { kinase } 2\end{array}$ & NM_001191865 & $\uparrow 1.01$ & 0.9251 & $\uparrow 1.18$ & 0.2362 & $\uparrow 1.10$ & 0.6177 & $\downarrow 1.35$ & 0.0014 \\
\hline $\begin{array}{l}\text { Regulation } \\
\text { of Apoptosis }\end{array}$ & Tp53bp2 & $\begin{array}{l}\text { Tumor protein p53 } \\
\text { binding protein, } 2\end{array}$ & XM_223012 & $\uparrow 1.43$ & 0.0952 & $\uparrow 1.07$ & 0.4527 & $\downarrow 1.05$ & 0.9249 & $\downarrow 1.11$ & 0.3304 \\
\hline $\begin{array}{l}\text { Death } \\
\text { Domain }\end{array}$ & Dapk1 & $\begin{array}{l}\text { Death associated } \\
\text { protein kinase } 1\end{array}$ & NM_001107335 & $\downarrow 1.15$ & 0.7166 & $\downarrow 1.68$ & 0.0882 & $\downarrow 1.42$ & 0.3753 & $\downarrow 1.41$ & 0.2842 \\
\hline Receptors & Tnfrsf10b & $\begin{array}{l}\text { Tumor necrosis } \\
\text { factor receptor } \\
\text { superfamily, } \\
\text { member 10b }\end{array}$ & NM_001108873 & $\uparrow 1.56$ & 0.0993 & $\uparrow 1.64$ & 0.0803 & $\uparrow 1.34$ & 0.2085 & $\downarrow 1.32$ & 0.3248 \\
\hline & Tnfrsf11b & $\begin{array}{l}\text { Tumor necrosis } \\
\text { factor receptor } \\
\text { superfamily, } \\
\text { member 11b }\end{array}$ & NM_012870 & $\uparrow 3.09$ & 0.0141 & $\uparrow 1.23$ & 0.4504 & $\uparrow 1.33$ & 0.2808 & $\uparrow 1.30$ & 0.1345 \\
\hline & Tnfrsf1a & $\begin{array}{l}\text { Tumor necrosis } \\
\text { factor receptor } \\
\text { superfamily, } \\
\text { member 1a }\end{array}$ & NM_013091 & $\uparrow 1.48$ & 0.0328 & $\uparrow 1.22$ & 0.3073 & $\uparrow 1.19$ & 0.7155 & $\downarrow 1.05$ & 0.7968 \\
\hline
\end{tabular}




\begin{tabular}{|c|c|c|c|c|c|c|c|c|c|c|c|}
\hline & Tnfrsf1b & $\begin{array}{l}\text { Tumor necrosis } \\
\text { factor receptor } \\
\text { superfamily, } \\
\text { member 1b }\end{array}$ & NM_130426 & $\uparrow 1.81$ & 0.0306 & $\uparrow 1.20$ & 0.4457 & $\uparrow 1.38$ & 0.3536 & $\uparrow 1.06$ & 0.7545 \\
\hline & Tradd & $\begin{array}{l}\text { TNFRSF1A- } \\
\text { associated via death } \\
\text { domain }\end{array}$ & NM_001100480 & $\uparrow 1.84$ & 0.0383 & $\uparrow 1.27$ & 0.1893 & $\uparrow 1.15$ & 0.6397 & $\downarrow 1.35$ & 0.4974 \\
\hline \multirow{7}{*}{$\begin{array}{l}\text { Caspases \& } \\
\text { Regulators: } \\
\text { Caspases }\end{array}$} & Casp1 & Caspase 1 & NM_012762 & $\uparrow 1.22$ & 0.1183 & $\uparrow 1.08$ & 0.5417 & $\uparrow 1.17$ & 0.2744 & $\downarrow 1.22$ & 0.0072 \\
\hline & Casp12 & Caspase 12 & NM_130422 & $\uparrow 1.21$ & 0.1751 & $\downarrow 1.19$ & 0.5201 & $\uparrow 1.05$ & 0.8035 & $\downarrow 1.60$ & 0.0297 \\
\hline & Casp3 & Caspase 3 & NM_012922 & $\uparrow 2.09$ & 0.0070 & $\uparrow 1.47$ & 0.1607 & $\uparrow 1.43$ & 0.1515 & $\uparrow 1.23$ & 0.1200 \\
\hline & Casp4 & $\begin{array}{l}\text { Caspase } 4, \\
\text { apoptosis-related } \\
\text { cysteine peptidase }\end{array}$ & NM_053736 & $\uparrow 1.48$ & 0.0109 & $\uparrow 1.10$ & 0.2349 & $\uparrow 1.04$ & 0.8441 & $\downarrow 1.25$ & 0.0620 \\
\hline & Casp6 & Caspase 6 & NM_031775 & $\uparrow 1.40$ & 0.0581 & $\uparrow 1.08$ & 0.5570 & $\uparrow 1.15$ & 0.5092 & $\uparrow 1.13$ & 0.3866 \\
\hline & Casp7 & Caspase 7 & NM_022260 & $\downarrow 1.39$ & 0.0286 & $\downarrow 1.20$ & 0.1393 & $\downarrow 1.21$ & 0.0881 & $\downarrow 1.44$ & $<0.001$ \\
\hline & Casp9 & $\begin{array}{l}\text { Caspase 9, } \\
\text { apoptosis-related } \\
\text { cysteine peptidase }\end{array}$ & NM_031632 & $\uparrow 1.76$ & 0.0006 & $\uparrow 1.29$ & 0.1323 & $\uparrow 1.07$ & 0.8017 & $\downarrow 1.28$ & 0.2964 \\
\hline
\end{tabular}

Out of 84 mRNA transcripts in the apoptosis pathway array, a total of 29 were classified as SDEGs in one or more experimental groups, while another 11 had a trend for being an SDEG. All SDEGs that surpassed a 1.1fold change with a $P$ value $<0.05$ have bold text. Italicized text $=$ trend $(0.05<P<0.10)$ for increased/decreased expression. Sample sizes were $N=5$ to 6 per group. FC $=$ fold change. 
Table 6. Ingenuity Pathway Analysis (IPA) of gene expression results from the Apoptosis RT ${ }^{2}$ Profiler Pathway Array for the tibialis anterior muscle following dorsiflexion SSC-loading relative to non-loaded control muscles; the top 5 results from each category were included in the table.

\begin{tabular}{|c|c|c|c|c|c|c|}
\hline Condition & Analyses & \multicolumn{5}{|c|}{ 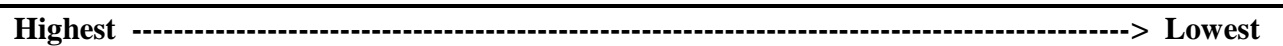 } \\
\hline \multirow{3}{*}{$\begin{array}{l}\text { Young ( } 3 \text { mo.) } \\
\text { SSC-loaded } 3 \\
\text { days per week }\end{array}$} & $\begin{array}{c}\text { Top Canonical } \\
\text { Pathways } \\
\text { (p-value) }\end{array}$ & $\begin{array}{c}\text { Apoptosis } \\
\text { Signaling } \\
(4.06 E-19)\end{array}$ & $\begin{array}{l}\text { Death Receptor } \\
\text { Signaling } \\
(1.20 \mathrm{E}-16)\end{array}$ & $\begin{array}{c}\text { TWEAK } \\
\text { Signaling } \\
(1.82 E-15)\end{array}$ & $\begin{array}{c}\text { TNFR1 Signaling } \\
(2.88 \mathrm{E}-14)\end{array}$ & $\begin{array}{c}\text { Tumoricidal } \\
\text { Function of Hepatic } \\
\text { Natural Killer Cells } \\
(6.35 E-14)\end{array}$ \\
\hline & $\begin{array}{l}\text { Top Networks } \\
\text { (Score) }\end{array}$ & $\begin{array}{c}\text { Cell Death \& } \\
\text { Survival, } \\
\text { Embryonic } \\
\text { Development, } \\
\text { Organismal Injury } \\
\text { \& Abnormalities } \\
\text { (37) }\end{array}$ & $\begin{array}{c}\text { Cellular Growth } \\
\text { and Proliferation, } \\
\text { Cellular } \\
\text { Movement, } \\
\text { Cellular } \\
\text { Development } \\
\text { (3) }\end{array}$ & N/A & N/A & N/A \\
\hline & $\begin{array}{c}\text { Physiological System } \\
\text { Development and } \\
\text { Function } \\
\text { (p-value range) }\end{array}$ & $\begin{array}{c}\text { Embryonic } \\
\text { Development } \\
(6.80 \mathrm{E}-05- \\
4.02 \mathrm{E}-24)\end{array}$ & $\begin{array}{c}\text { Hematological } \\
\text { System } \\
\text { Development and } \\
\text { Function } \\
(6.89 \mathrm{E}-05- \\
\text { 4.79E-13) }\end{array}$ & $\begin{array}{c}\text { Tissue } \\
\text { Morphology } \\
\text { (6.66E-05- } \\
\text { 4.79E-13) }\end{array}$ & $\begin{array}{c}\text { Cell-Mediated } \\
\text { Immune Response } \\
(1.69 \mathrm{E}-11-6.44 \mathrm{E}- \\
12)\end{array}$ & $\begin{array}{c}\text { Hematopoiesis } \\
(4.43 \mathrm{E}-05-7.10 \mathrm{E}- \\
13)\end{array}$ \\
\hline
\end{tabular}

\begin{tabular}{|c|c|c|c|c|c|c|}
\hline Condition & Analyses & \multicolumn{5}{|c|}{ Highest } \\
\hline \multirow{3}{*}{$\begin{array}{l}\text { Young (3 mo.) } \\
\text { SSC-loaded } 2 \\
\text { days per week }\end{array}$} & $\begin{array}{l}\text { Top Canonical } \\
\text { Pathways } \\
\text { (p-value) }\end{array}$ & $\begin{array}{l}\text { Apoptosis } \\
\text { Signaling } \\
(3.73 E-04)\end{array}$ & $\begin{array}{l}\text { p53 Signaling } \\
\text { (4.53E-04) }\end{array}$ & $\begin{array}{l}\text { GADD45 } \\
\text { Signaling } \\
(6.37 \mathrm{E}-03)\end{array}$ & $\begin{array}{l}\text { TWEAK Signaling } \\
(1.14 \mathrm{E}-02)\end{array}$ & $\begin{array}{l}\text { Interferon Signaling } \\
\quad(1.20 \mathrm{E}-02)\end{array}$ \\
\hline & $\begin{array}{l}\text { Top Networks } \\
\text { (Score) }\end{array}$ & $\begin{array}{l}\text { Dermatological } \\
\text { Diseases and } \\
\text { Conditions, } \\
\text { Developmental } \\
\text { Disorder, } \\
\text { Hereditary } \\
\text { Disorder (21) }\end{array}$ & N/A & N/A & N/A & N/A \\
\hline & $\begin{array}{c}\text { Physiological System } \\
\text { Development and } \\
\text { Function } \\
\text { (p-value range) }\end{array}$ & $\begin{array}{l}\text { Lymphoid Tissue } \\
\text { Structure and } \\
\text { Development } \\
(6.70 \mathrm{E}-3-2.81 \mathrm{E}- \\
06)\end{array}$ & $\begin{array}{c}\text { Organ } \\
\text { Morphology } \\
(6.70 \mathrm{E}-03- \\
2.81 \mathrm{E}-06)\end{array}$ & $\begin{array}{l}\text { Hematological } \\
\text { System } \\
\text { Development and } \\
\text { Function } \\
\text { (6.89E-03- } \\
\text { 5.35E-06) }\end{array}$ & $\begin{array}{c}\text { Immune Cell } \\
\text { Trafficking } \\
(2.35 \mathrm{E}-03-6.70 \mathrm{E}- \\
06)\end{array}$ & $\begin{array}{c}\text { Hematopoiesis } \\
(6.70 \mathrm{E}-03-1.82 \mathrm{E}- \\
05)\end{array}$ \\
\hline
\end{tabular}




\begin{tabular}{|c|c|c|c|c|c|c|}
\hline Condition & Analyses & \multicolumn{5}{|c|}{ 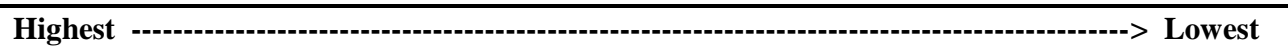 } \\
\hline \multirow{3}{*}{$\begin{array}{l}\text { Old (30 mo.) } \\
\text { SSC-loaded } 3 \\
\text { days per week }\end{array}$} & $\begin{array}{c}\text { Top Canonical } \\
\text { Pathways } \\
\text { (p-value) }\end{array}$ & $\begin{array}{l}\text { DHA Signaling } \\
(1.87 \mathrm{E}-03)\end{array}$ & $\begin{array}{c}\text { GM_CSF } \\
\text { Signaling } \\
(2.98 \mathrm{E}-03)\end{array}$ & $\begin{array}{c}\text { Apoptosis } \\
\text { Signaling } \\
(4.27 \mathrm{E}-03)\end{array}$ & $\begin{array}{l}\text { B Cell Receptor } \\
\text { Signaling } \\
(8.35 E-03)\end{array}$ & N/A \\
\hline & $\begin{array}{l}\text { Top Networks } \\
\text { (Score) }\end{array}$ & $\begin{array}{c}\text { Cell Death \& } \\
\text { Survival, } \\
\text { Hematological } \\
\text { System } \\
\text { Development \& } \\
\text { Function, Tissue } \\
\text { Morphology } \\
\text { (3) }\end{array}$ & N/A & N/A & N/A & N/A \\
\hline & $\begin{array}{c}\text { Physiological System } \\
\text { Development and } \\
\text { Function } \\
\text { (p-value range) }\end{array}$ & $\begin{array}{l}\text { Hematological } \\
\text { System } \\
\text { Development and } \\
\text { Function } \\
(3.40 \mathrm{E}-02- \\
1.25 \mathrm{E}-03)\end{array}$ & $\begin{array}{l}\text { Humoral Immune } \\
\text { Response } \\
(1.74 \mathrm{E}-02- \\
1.78 \mathrm{E}-03)\end{array}$ & $\begin{array}{c}\text { Tissue } \\
\text { Morphology } \\
(3.40 \mathrm{E}-02- \\
2.30 \mathrm{E}-03)\end{array}$ & $\begin{array}{c}\text { Cardiovascular } \\
\text { System } \\
\text { Development and } \\
\text { Function } \\
\text { (3.36E-03 - 3.36E- } \\
03)\end{array}$ & $\begin{array}{c}\text { Organismal } \\
\text { Development } \\
(4.85 \mathrm{E}-03-4.85 \mathrm{E}- \\
03)\end{array}$ \\
\hline
\end{tabular}

\begin{tabular}{|c|c|c|c|c|c|c|}
\hline Condition & Analyses & \multicolumn{5}{|c|}{ 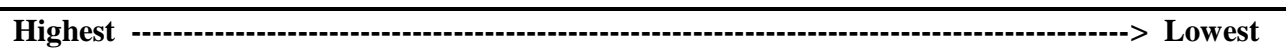 } \\
\hline \multirow{3}{*}{$\begin{array}{l}\text { Old (30 mo.) } \\
\text { SSC-loaded } 2 \\
\text { days per week }\end{array}$} & $\begin{array}{l}\text { Top Canonical } \\
\text { Pathways } \\
\text { (p-value) } \\
\text { (Overlap) } \\
\end{array}$ & $\begin{array}{c}\text { TWEAK } \\
\text { Signaling } \\
(2.21 E-07)\end{array}$ & $\begin{array}{c}\text { Apoptosis } \\
\text { Signaling } \\
(4.15 \mathrm{E}-06)\end{array}$ & $\begin{array}{c}\text { Death Receptor } \\
\text { Signaling } \\
(4.59 \mathrm{E}-06)\end{array}$ & $\begin{array}{c}\text { TNFR1 Signaling } \\
(1.50 \mathrm{E}-04)\end{array}$ & $\begin{array}{c}\text { PEDF Signaling } \\
(3.16 E-04)\end{array}$ \\
\hline & $\begin{array}{l}\text { Top Networks } \\
\text { (Score) }\end{array}$ & $\begin{array}{c}\text { Cell Death \& } \\
\text { Survival, } \\
\text { Gastrointestinal } \\
\text { Disease, Hepatic } \\
\text { System Disease } \\
\text { (24) }\end{array}$ & N/A & N/A & N/A & N/A \\
\hline & $\begin{array}{c}\text { Physiological System } \\
\text { Development and } \\
\text { Function } \\
\text { (p-value range) }\end{array}$ & $\begin{array}{c}\text { Embryonic } \\
\text { Development } \\
(4.85 \mathrm{E}-02- \\
5.16 \mathrm{E}-09)\end{array}$ & $\begin{array}{c}\text { Organismal } \\
\text { Functions } \\
(2.28 \mathrm{E}-02- \\
1.93 \mathrm{E}-06)\end{array}$ & $\begin{array}{c}\text { Tissue } \\
\text { Morphology } \\
(4.70 \mathrm{E}-02- \\
3.21 \mathrm{E}-06)\end{array}$ & $\begin{array}{c}\text { Hematological } \\
\text { System } \\
\text { Development and } \\
\text { Function } \\
(4.48 \mathrm{E}-02-3.21 \mathrm{E}- \\
06)\end{array}$ & $\begin{array}{l}\text { Digestive System } \\
\text { Development and } \\
\text { Function } \\
(4.77 \mathrm{E}-02-5.43 \mathrm{E}- \\
06)\end{array}$ \\
\hline
\end{tabular}

The top canonical pathways and top networks were noted based on the functional annotation of significantly differentially expressed genes from the apoptosis expression arrays. All IPA functions with the exception of cancer specific, reproductive system, psychological functions, and cell lines were considered for this table. Sample sizes were $\mathrm{N}=5$ to 6 per group. $\mathrm{N} / \mathrm{A}=$ Not available. SSC = stretch-shortening contraction; TWEAK = Tumor necrosis factor-like weak inducer of apoptosis; TNFR1: tumor necrosis factor receptor 1; p53 = tumor protein p53; GADD45 = growth arrest and DNA damage; DHA = docosahexanoic acid; PEDF = pigment epithelium-derived factor. 
Table 7. Significantly differentially expressed genes (SDEGs) relevant to the DNA repair pathway for the tibialis anterior muscle following dorsiflexion SSC-loading relative to non-loaded control muscles.

\begin{tabular}{|c|c|c|c|c|c|c|c|c|c|c|c|}
\hline & & & & Youn & $3 x /$ wk. & Your & $2 \mathrm{x} / \mathrm{wk}$ & & 3x/wk. & & 2x/wk. \\
\hline Genes & Symbol & Description & RefSeq \# & FC & $P$ value & FC & $P$ value & FC & $P$ value & FC & $P$ value \\
\hline \multirow[t]{10}{*}{$\begin{array}{l}\text { Base } \\
\text { Excision } \\
\text { Repair } \\
\text { (BER) }\end{array}$} & Apex1 & $\begin{array}{l}\text { APEX nuclease } \\
\text { (multifunctional } \\
\text { DNA repair } \\
\text { enzyme) } 1\end{array}$ & NM_024148 & $\downarrow 1.22$ & 0.5167 & $\downarrow 1.35$ & 0.1402 & $\downarrow 1.65$ & 0.0356 & $\uparrow 1.46$ & 0.1944 \\
\hline & Mpg & $\begin{array}{l}\text { N-methylpurine- } \\
\text { DNA } \\
\text { glycosylase }\end{array}$ & NM_012601 & $\uparrow 1.55$ & 0.2502 & $\downarrow 1.31$ & 0.1701 & $\downarrow 1.98$ & 0.0263 & $\uparrow 1.09$ & 0.5752 \\
\hline & Mutyh & $\begin{array}{l}\text { MutY homolog } \\
\text { (E. coli) }\end{array}$ & NM_133316 & $\uparrow 2.69$ & 0.0154 & $\uparrow 2.08$ & 0.0350 & $\uparrow 1.12$ & 0.6667 & $\uparrow 1.45$ & 0.0856 \\
\hline & Neil1 & $\begin{array}{l}\text { Nei } \\
\text { endonuclease } \\
\text { VIII-like } 1 \text { (E. } \\
\text { coli) }\end{array}$ & NM_001025754 & $\downarrow 1.20$ & 0.7257 & $\downarrow 2.12$ & 0.0097 & $\downarrow 2.37$ & 0.0005 & $\uparrow 1.18$ & 0.3847 \\
\hline & Nthl1 & $\begin{array}{l}\text { Nth } \\
\text { (endonuclease } \\
\text { III)-like } 1 \\
\text { (E.coli) }\end{array}$ & NM_001105728 & $\downarrow 1.01$ & 0.8492 & $\downarrow 1.16$ & 0.0880 & $\downarrow 1.35$ & 0.0936 & $\uparrow 1.21$ & 0.4098 \\
\hline & Parp1 & $\begin{array}{l}\text { Poly (ADP- } \\
\text { ribose) } \\
\text { polymerase } 1\end{array}$ & NM_013063 & $\downarrow 1.23$ & 0.3419 & $\downarrow 2.04$ & 0.0119 & $\downarrow 1.86$ & 0.0007 & $\uparrow 1.44$ & 0.2182 \\
\hline & Parp3 & $\begin{array}{l}\text { Poly (ADP- } \\
\text { ribose) } \\
\text { polymerase } \\
\text { family, member } \\
3\end{array}$ & NM_001008328 & $\downarrow 1.37$ & 0.3200 & $\downarrow 1.60$ & 0.0915 & $\downarrow 2.00$ & 0.0021 & $\uparrow 1.26$ & 0.9906 \\
\hline & Smug1 & $\begin{array}{l}\text { Single-strand- } \\
\text { selective } \\
\text { monofunctional } \\
\text { uracil-DNA } \\
\text { glycosylase } 1\end{array}$ & NM_177934 & $\uparrow 1.04$ & 0.9196 & $\downarrow 1.34$ & 0.3360 & $\downarrow 1.25$ & 0.3554 & $\uparrow 1.52$ & 0.0865 \\
\hline & Tdg & $\begin{array}{l}\text { Thymine-DNA } \\
\text { glycosylase }\end{array}$ & NM_053729 & $\downarrow 1.04$ & 0.9719 & $\downarrow 1.21$ & 0.3549 & $\downarrow 1.17$ & 0.3589 & $\uparrow 1.59$ & 0.0413 \\
\hline & Xrcc1 & $\begin{array}{l}\text { X-ray repair } \\
\text { complementing } \\
\text { defective repair } \\
\text { in Chinese } \\
\text { hamster cells } 1\end{array}$ & NM_053435 & $\downarrow 1.36$ & 0.2984 & $\downarrow 1.70$ & 0.1288 & $\downarrow 1.84$ & 0.0025 & $\uparrow 1.25$ & 0.5506 \\
\hline \multirow{2}{*}{$\begin{array}{l}\text { Nucleotide } \\
\text { Excision } \\
\text { Repair } \\
\text { (NER) }\end{array}$} & Atxn3 & Ataxin 3 & NM_021702 & $\uparrow 2.00$ & 0.0766 & $\downarrow 1.06$ & 0.7142 & $\downarrow 1.21$ & 0.1699 & $\uparrow 1.37$ & 0.0499 \\
\hline & Ccnh & Cyclin $\mathrm{H}$ & NM_052981 & $\downarrow 1.07$ & 0.4170 & $\downarrow 1.15$ & 0.0126 & $\downarrow 1.07$ & 0.3254 & $\uparrow 1.09$ & 0.4464 \\
\hline
\end{tabular}




\begin{tabular}{|c|c|c|c|c|c|c|c|c|c|c|}
\hline Cdk7 & $\begin{array}{l}\text { Cyclin- } \\
\text { dependent kinase } \\
7\end{array}$ & XM_008760700 & $\uparrow 1.02$ & 0.8950 & $\downarrow 1.19$ & 0.0889 & $\downarrow 1.27$ & 0.0657 & $\uparrow 1.12$ & 0.3404 \\
\hline Dclre1a & $\begin{array}{l}\text { DNA cross-link } \\
\text { repair 1A, PSO2 } \\
\text { homolog (S. } \\
\text { cerevisiae) }\end{array}$ & NM_001106201 & $\downarrow 1.20$ & 0.5232 & $\downarrow 1.25$ & 0.0359 & $\downarrow 1.43$ & 0.0427 & $\uparrow 1.13$ & 0.6431 \\
\hline Ddb1 & $\begin{array}{l}\text { Damage-specific } \\
\text { DNA binding } \\
\text { protein } 1\end{array}$ & NM_171995 & $\downarrow 1.12$ & 0.7910 & $\downarrow 1.46$ & 0.0579 & $\downarrow 1.85$ & 0.0019 & $\uparrow 1.91$ & 0.0411 \\
\hline Ddb2 & $\begin{array}{l}\text { Damage specific } \\
\text { DNA binding } \\
\text { protein } 2\end{array}$ & NM_001271346 & $\uparrow 1.06$ & 0.7229 & $\downarrow 1.88$ & 0.2714 & $\downarrow 1.55$ & 0.0115 & $\uparrow 1.16$ & 0.8714 \\
\hline Ercc1 & $\begin{array}{l}\text { Excision repair } \\
\text { cross- } \\
\text { complementing } \\
\text { rodent repair } \\
\text { deficiency, } \\
\text { complementation } \\
\text { group } 1\end{array}$ & NM_001106228 & $\downarrow 1.01$ & 0.8280 & $\downarrow 1.69$ & 0.0032 & $\downarrow 1.91$ & 0.0043 & $\uparrow 1.59$ & 0.0689 \\
\hline Ercc2 & $\begin{array}{l}\text { Excision repair } \\
\text { cross- } \\
\text { complementing } \\
\text { rodent repair } \\
\text { deficiency, } \\
\text { complementation } \\
\text { group } 2\end{array}$ & NM_001172809 & $\uparrow 1.05$ & 0.7647 & $\downarrow 1.73$ & 0.0437 & $\downarrow 1.45$ & 0.0136 & $\uparrow 1.68$ & 0.0472 \\
\hline Ercc3 & $\begin{array}{l}\text { Excision repair } \\
\text { cross- } \\
\text { complementing } \\
\text { rodent repair } \\
\text { deficiency, } \\
\text { complementation } \\
\text { group } 3\end{array}$ & NM_001031644 & $\uparrow 1.01$ & 0.8892 & $\downarrow 1.33$ & 0.0975 & $\downarrow 1.80$ & 0.0203 & $\uparrow 1.22$ & 0.2962 \\
\hline Ercc4 & $\begin{array}{l}\text { Excision repair } \\
\text { cross- } \\
\text { complementing } \\
\text { rodent repair } \\
\text { deficiency, } \\
\text { complementation } \\
\text { group } 4\end{array}$ & XM_003754645 & $\downarrow 1.22$ & 0.4480 & $\downarrow 1.54$ & 0.1869 & $\downarrow 2.14$ & 0.0007 & $\uparrow 1.39$ & 0.2594 \\
\hline Ercc5 & $\begin{array}{l}\text { Excision repair } \\
\text { cross- } \\
\text { complementing } \\
\text { rodent repair } \\
\text { deficiency, } \\
\text { complementation } \\
\text { group } 5\end{array}$ & NM_001106910 & $\downarrow 1.63$ & 0.3601 & $\downarrow 2.43$ & 0.0710 & $\downarrow 1.43$ & 0.0199 & $\uparrow 1.24$ & 0.2485 \\
\hline
\end{tabular}




\begin{tabular}{|c|c|c|c|c|c|c|c|c|c|c|c|}
\hline & Ercc6 & $\begin{array}{l}\text { Excision repair } \\
\text { cross- } \\
\text { complementing } \\
\text { rodent repair } \\
\text { deficiency, } \\
\text { complementation } \\
\text { group } 6\end{array}$ & NM_001107296 & $\downarrow 1.08$ & 0.9839 & $\downarrow 1.43$ & 0.0713 & $\downarrow 1.75$ & 0.0089 & $\uparrow 1.42$ & 0.3928 \\
\hline & Ercc8 & $\begin{array}{l}\text { Excision repair } \\
\text { cross- } \\
\text { complementing } \\
\text { rodent repair } \\
\text { deficiency, } \\
\text { complementation } \\
\text { group } 8\end{array}$ & NM_001107650 & $\downarrow 1.74$ & 0.6354 & $\downarrow 1.25$ & 0.2603 & $\downarrow 1.43$ & 0.0280 & $\uparrow 1.64$ & 0.0816 \\
\hline & Lig1 & $\begin{array}{l}\text { Ligase I, DNA, } \\
\text { ATP-dependent }\end{array}$ & NM_001024268 & $\downarrow 1.09$ & 0.7068 & $\downarrow 1.21$ & 0.0837 & $\downarrow 1.44$ & 0.0130 & $\uparrow 1.13$ & 0.6031 \\
\hline & Pnkp & $\begin{array}{l}\text { Polynucleotide } \\
\text { kinase 3'- } \\
\text { phosphatase }\end{array}$ & NM_001004259 & $\uparrow 1.22$ & 0.2283 & $\downarrow 1.16$ & 0.2170 & $\downarrow 1.50$ & 0.0398 & $\uparrow 1.33$ & 0.3004 \\
\hline & Poll & $\begin{array}{l}\text { Polymerase } \\
\text { (DNA directed), } \\
\text { lambda }\end{array}$ & NM_001014168 & $\downarrow 1.36$ & 0.3169 & $\downarrow 1.76$ & 0.0045 & $\downarrow 1.99$ & 0.0001 & $\uparrow 1.62$ & 0.3070 \\
\hline & Rad23a & $\begin{array}{l}\text { RAD23 homolog } \\
\text { A (S. cerevisiae) }\end{array}$ & NM_001013190 & $\downarrow 1.26$ & 0.5416 & $\downarrow 1.50$ & 0.1174 & $\downarrow 1.77$ & 0.0031 & $\uparrow 1.54$ & 0.2872 \\
\hline & Rad23b & $\begin{array}{l}\text { RAD23 homolog } \\
\text { B (S. cerevisiae) }\end{array}$ & NM_001025275 & $\downarrow 1.28$ & 0.3390 & $\uparrow 1.23$ & 0.5213 & $\downarrow 1.36$ & 0.0689 & $\uparrow 1.43$ & 0.1287 \\
\hline & Rpa1 & $\begin{array}{l}\text { Replication } \\
\text { protein A1 }\end{array}$ & NM_001047843 & $\downarrow 1.29$ & 0.3498 & $\downarrow 1.65$ & 0.0969 & $\downarrow 2.17$ & 0.0020 & $\uparrow 1.04$ & 0.7567 \\
\hline & Slk & $\begin{array}{l}\text { STE20-like } \\
\text { kinase (yeast) }\end{array}$ & NM_019349 & $\uparrow 1.07$ & 0.8095 & $\downarrow 1.01$ & 0.8951 & $\uparrow 1.03$ & 0.8254 & $\uparrow 1.32$ & 0.0447 \\
\hline & Xab2 & $\begin{array}{l}\text { XPA binding } \\
\text { protein } 2\end{array}$ & NM_139109 & $\downarrow 1.00$ & 0.8959 & $\downarrow 1.44$ & 0.0211 & $\downarrow 1.66$ & 0.0059 & $\uparrow 1.38$ & 0.3324 \\
\hline & Xpc & $\begin{array}{l}\text { Xeroderma } \\
\text { pigmentosum, } \\
\text { complementation } \\
\text { group C }\end{array}$ & NM_001107874 & $\downarrow 1.12$ & 0.7080 & $\downarrow 1.80$ & 0.0212 & $\downarrow 1.59$ & 0.0070 & $\uparrow 1.63$ & 0.0979 \\
\hline $\begin{array}{l}\text { Mismatch } \\
\text { Repair }\end{array}$ & Mlh1 & $\begin{array}{l}\text { MutL homolog } 1 \\
\text { (E. coli) }\end{array}$ & NM_031053 & $\downarrow 1.22$ & 0.5682 & $\downarrow 2.02$ & 0.0245 & $\downarrow 2.11$ & 0.0089 & $\uparrow 1.54$ & 0.2577 \\
\hline & Mlh3 & $\begin{array}{l}\text { MutL homolog } 3 \\
\text { (E. coli) }\end{array}$ & NM_001108043 & $\downarrow 1.11$ & 0.4529 & $\downarrow 1.84$ & 0.0344 & $\downarrow 2.07$ & $<0.0001$ & $\uparrow 1.07$ & 0.9973 \\
\hline & Msh3 & $\begin{array}{l}\text { MutS homolog } 3 \\
\text { (E. coli) }\end{array}$ & NM_001191957 & $\downarrow 1.32$ & 0.9533 & $\uparrow 1.04$ & 0.6611 & $\uparrow 1.19$ & 0.1943 & $\uparrow 1.33$ & 0.0117 \\
\hline & Msh6 & MutS homolog 6 & XM_006225670 & $\uparrow 1.07$ & 0.5933 & $\downarrow 1.15$ & 0.2824 & $\downarrow 1.21$ & 0.1398 & $\uparrow 1.28$ & 0.0350 \\
\hline
\end{tabular}




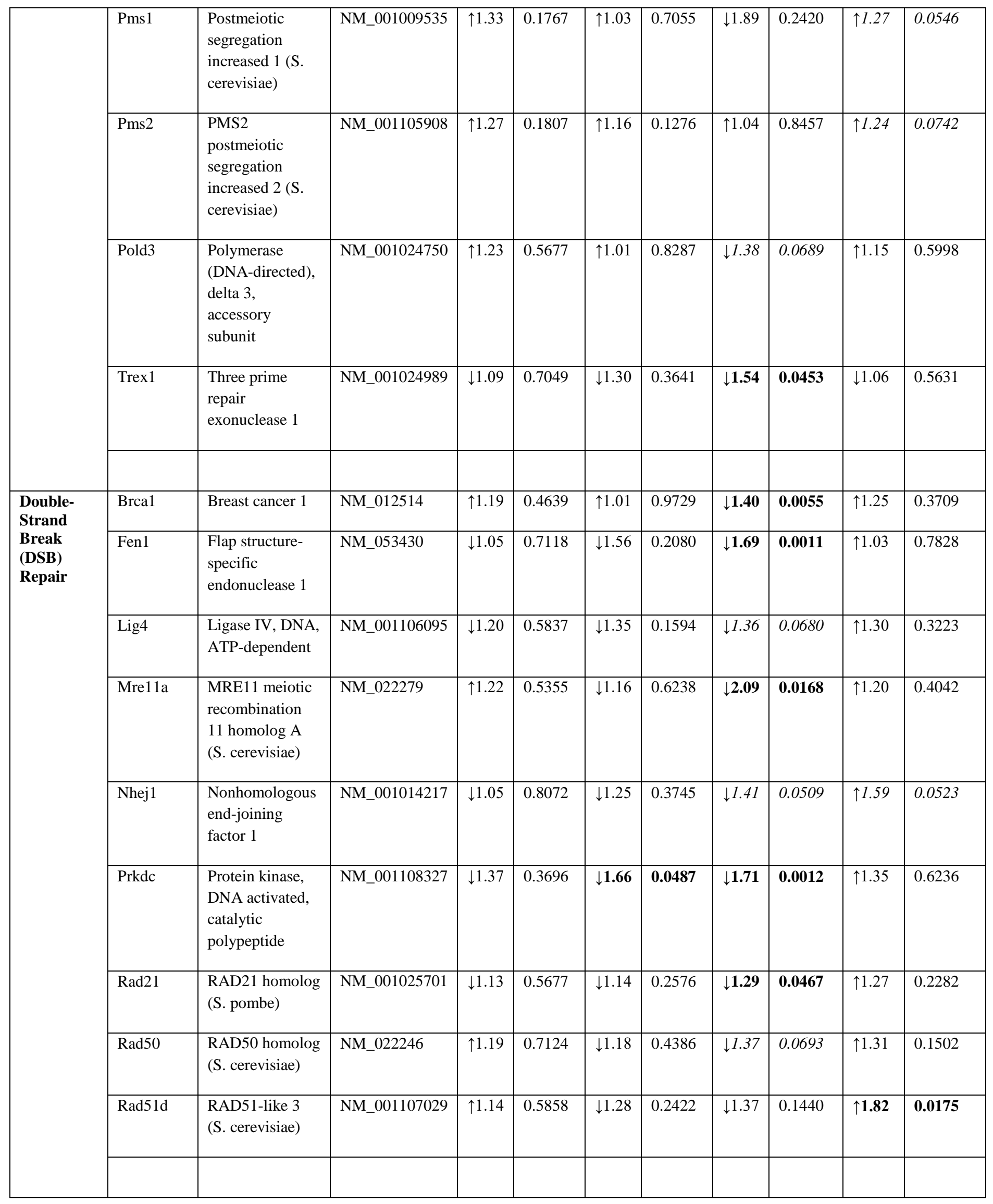




\begin{tabular}{|c|c|c|c|c|c|c|c|c|c|c|c|}
\hline \multirow[t]{9}{*}{$\begin{array}{l}\text { Other } \\
\text { DNA } \\
\text { Repair } \\
\text { Genes }\end{array}$} & Atm & $\begin{array}{l}\text { Ataxia } \\
\text { telangiectasia } \\
\text { mutated } \\
\text { homolog } \\
\text { (human) }\end{array}$ & NM_001106821 & $\uparrow 1.09$ & 0.6808 & $\downarrow 1.25$ & 0.2634 & $\downarrow 1.36$ & 0.1099 & $\uparrow 1.67$ & 0.0273 \\
\hline & Dclre1b & $\begin{array}{l}\text { DNA cross-link } \\
\text { repair 1B, PSO2 } \\
\text { homolog (S. } \\
\text { cerevisiae) }\end{array}$ & NM_001025687 & $\uparrow 1.05$ & 0.8761 & $\downarrow 1.54$ & 0.0378 & $\downarrow 1.46$ & 0.0487 & $\uparrow 1.34$ & 0.2414 \\
\hline & Gen1 & $\begin{array}{l}\text { Gen homolog } 1 \text {, } \\
\text { endonuclease } \\
\text { (Drosophila) }\end{array}$ & NM_001106717 & $\downarrow 1.03$ & 0.8349 & $\uparrow 1.08$ & 0.8920 & $\downarrow 1.23$ & 0.0715 & $\uparrow 1.30$ & 0.1414 \\
\hline & Mus81 & $\begin{array}{l}\text { MUS81 } \\
\text { endonuclease } \\
\text { homolog (S. } \\
\text { cerevisiae) }\end{array}$ & NM_001025645 & $\uparrow 1.09$ & 0.9689 & $\downarrow 1.42$ & 0.1293 & $\downarrow 2.25$ & 0.0006 & $\uparrow 1.79$ & 0.0388 \\
\hline & Rad9b & $\begin{array}{l}\text { RAD9 homolog } \\
\text { B (S. cerevisiae) }\end{array}$ & NM_001030042 & $\downarrow 1.31$ & 0.5407 & $\downarrow 1.26$ & 0.1286 & $\downarrow 1.63$ & 0.0352 & $\downarrow 1.14$ & 0.2862 \\
\hline & Rfc1 & $\begin{array}{l}\text { Replication } \\
\text { factor C } \\
\text { (activator 1) } 1\end{array}$ & NM_053547 & $\uparrow 1.07$ & 0.8671 & $\downarrow 1.42$ & 0.1376 & $\downarrow 1.35$ & 0.0319 & $\uparrow 1.37$ & 0.2760 \\
\hline & Top3a & $\begin{array}{l}\text { Topoisomerase } \\
\text { (DNA) III alpha }\end{array}$ & XM_006220998 & $\downarrow 1.06$ & 0.9537 & $\downarrow 1.42$ & 0.2418 & $\downarrow 1.61$ & 0.0261 & $\uparrow 1.32$ & 0.2589 \\
\hline & Top3b & $\begin{array}{l}\text { Topoisomerase } \\
\text { (DNA) III beta }\end{array}$ & NM_001105861 & $\downarrow 1.03$ & 0.9382 & $\downarrow 1.48$ & 0.0393 & $\uparrow 1.10$ & 0.4948 & $\uparrow 1.61$ & 0.0942 \\
\hline & Xrcc6bp1 & $\begin{array}{l}\text { XRCC6 binding } \\
\text { protein } 1\end{array}$ & NM_001134466 & $\uparrow 1.33$ & 0.0008 & $\uparrow 1.26$ & 0.0048 & $\uparrow 2.24$ & 0.0202 & $\uparrow 1.12$ & 0.2526 \\
\hline
\end{tabular}

Out of 84 mRNA transcripts in the DNA repair pathway array, a total of 47 were classified as SDEGs in one or more experimental groups, while another 11 had a trend for being an SDEG. All SDEGs that surpassed a 1.1fold change with a $P$ value $<0.05$ have bold text. Italicized text $=$ trend $(0.05<P<0.10)$ for increased/decreased expression. Sample sizes were $N=5$ to 6 per group. FC $=$ fold change. 
Table 8. Ingenuity Pathway Analysis (IPA) of gene expression results from the DNA Repair RT ${ }^{2}$ Profiler Pathway Array for the tibialis anterior muscle following dorsiflexion SSC-loading relative to non-loaded control muscles; the top 5 results from each category were included in the table.

\begin{tabular}{|c|c|c|c|c|c|c|}
\hline Condition & Analyses & \multicolumn{5}{|c|}{ Highest } \\
\hline \multirow{3}{*}{$\begin{array}{l}\text { Young ( } 3 \text { mo.) } \\
\text { SSC-loaded } 2 \\
\text { days per week }\end{array}$} & $\begin{array}{l}\text { Top Canonical } \\
\text { Pathways } \\
\text { (p-value) }\end{array}$ & $\begin{array}{l}\text { NER Pathway } \\
\text { (8.49E-09) }\end{array}$ & $\begin{array}{c}\text { DNA DSB Repair } \\
\text { by NHEJ } \\
(4.48 E-05)\end{array}$ & $\begin{array}{c}\text { Granzyme B } \\
\text { Signaling (5.90E- } \\
05)\end{array}$ & $\begin{array}{l}\text { Estrogen Receptor } \\
\text { Signaling } \\
(1.16 \mathrm{E}-04)\end{array}$ & $\begin{array}{l}\text { RAR Activation } \\
\quad(3.26 \mathrm{E}-04)\end{array}$ \\
\hline & $\begin{array}{l}\text { Top Networks } \\
\text { (Score) }\end{array}$ & $\begin{array}{l}\text { Cell Death and } \\
\text { Survival, } \\
\text { Organismal } \\
\text { Survival, DNA } \\
\text { Replication, } \\
\text { Recombination, } \\
\text { and Repair } \\
\text { (28) } \\
\end{array}$ & N/A & N/A & N/A & N/A \\
\hline & $\begin{array}{c}\text { Physiological System } \\
\text { Development and } \\
\text { Function } \\
\text { (p-value range) }\end{array}$ & $\begin{array}{c}\text { Organismal } \\
\text { Survival } \\
(6.92 \mathrm{E}-03- \\
5.41 \mathrm{E}-07)\end{array}$ & $\begin{array}{c}\text { Cell-Mediated } \\
\text { Immune Response } \\
(1.24 \mathrm{E}-02- \\
1.04 \mathrm{E}-05)\end{array}$ & $\begin{array}{c}\text { Connective } \\
\text { Tissue } \\
\text { Development and } \\
\text { Function } \\
(6.52 \mathrm{E}-03- \\
1.04 \mathrm{E}-05)\end{array}$ & $\begin{array}{c}\text { Embryonic } \\
\text { Development } \\
(1.43 \mathrm{E}-02-1.04 \mathrm{E}- \\
05)\end{array}$ & $\begin{array}{c}\text { Hematological } \\
\text { System } \\
\text { Development and } \\
\text { Function } \\
(1.43 \mathrm{E}-02-1.04 \mathrm{E}- \\
05)\end{array}$ \\
\hline
\end{tabular}

\begin{tabular}{|c|c|c|c|c|c|c|}
\hline Condition & Analyses & \multicolumn{5}{|c|}{ Highest -------------------------------------------------------------------------------------------------> Lowest } \\
\hline \multirow{4}{*}{$\begin{array}{l}\text { Old (30 mo.) } \\
\text { SSC-loaded } 3 \\
\text { days per week }\end{array}$} & $\begin{array}{c}\text { Top Canonical } \\
\text { Pathways } \\
\text { (p-value) }\end{array}$ & $\begin{array}{c}\text { NER Pathway } \\
\text { (3.31E-26) }\end{array}$ & $\begin{array}{c}\text { BER Pathway } \\
(1.34 \mathrm{E}-14)\end{array}$ & $\begin{array}{c}\text { DNA DSB } \\
\text { Repair by HR } \\
(6.66 \mathrm{E}-09)\end{array}$ & $\begin{array}{l}\text { Mismatch Repair } \\
\text { in Eukaryotes } \\
(1.21 E-08)\end{array}$ & $\begin{array}{c}\text { Role of BRCA1 in } \\
\text { DNA Damage } \\
\text { Response } \\
(2.21 \mathrm{E}-07)\end{array}$ \\
\hline & & & & & & \\
\hline & $\begin{array}{l}\text { Top Networks } \\
\text { (Score) }\end{array}$ & $\begin{array}{c}\text { DNA Replication, } \\
\text { Recombination, } \\
\text { and Repair, Cell } \\
\text { Morphology, } \\
\text { Cellular Function } \\
\text { and Maintenance } \\
\text { (68) }\end{array}$ & $\begin{array}{c}\text { Organ } \\
\text { Morphology, } \\
\text { Skeletal and } \\
\text { Muscular System } \\
\text { Development and } \\
\text { Function, Cell } \\
\text { Morphology } \\
(2) \\
\end{array}$ & N/A & N/A & N/A \\
\hline & $\begin{array}{l}\text { Physiological System } \\
\text { Development and } \\
\text { Function } \\
\text { (p-value range) } \\
\text { (\# molecules) }\end{array}$ & $\begin{array}{c}\text { Organismal } \\
\text { Survival } \\
(2.03 \mathrm{E}-04- \\
9.52 \mathrm{E}-14)\end{array}$ & $\begin{array}{c}\text { Organismal } \\
\text { Development } \\
(1.68 \mathrm{E}-03- \\
1.49 \mathrm{E}-07)\end{array}$ & $\begin{array}{c}\text { Connective } \\
\text { Tissue } \\
\text { Development and } \\
\text { Function } \\
(5.73 \mathrm{E}-05- \\
4.23 \mathrm{E}-07)\end{array}$ & $\begin{array}{l}\text { Digestive System } \\
\text { Development and } \\
\text { Function } \\
(1.68 \mathrm{E}-03-7.06 \mathrm{E}- \\
07)\end{array}$ & $\begin{array}{c}\text { Visual System } \\
\text { Development and } \\
\text { Function } \\
(2.74 \mathrm{E}-05-1.57 \mathrm{E}- \\
06)\end{array}$ \\
\hline
\end{tabular}




\begin{tabular}{|c|c|c|c|c|c|c|}
\hline Condition & Analyses & \multicolumn{5}{|c|}{ Highest } \\
\hline \multirow{3}{*}{$\begin{array}{l}\text { Old (30 mo.) } \\
\text { SSC-loaded } 2 \\
\text { days per week }\end{array}$} & $\begin{array}{l}\text { Top Canonical } \\
\text { Pathways } \\
\text { (p-value) }\end{array}$ & $\begin{array}{l}\text { Mismatch Repair } \\
\text { in Eukaryotes } \\
(2.22 \mathrm{E}-05)\end{array}$ & $\begin{array}{c}\text { Role of BRCA1 in } \\
\text { DNA Damage } \\
\text { Response (5.75E- } \\
04)\end{array}$ & $\begin{array}{l}\text { NER Pathway } \\
\text { (9.51E-04) }\end{array}$ & $\begin{array}{c}\text { DNA DSB Repair } \\
\text { by HR } \\
(6.34 \mathrm{E}-03)\end{array}$ & $\begin{array}{c}\text { DNA DSB Repair } \\
\text { by NHEJ } \\
(6.34 \mathrm{E}-03)\end{array}$ \\
\hline & $\begin{array}{l}\text { Top Networks } \\
\text { (Score) }\end{array}$ & $\begin{array}{l}\text { DNA Replication, } \\
\text { Recombination, } \\
\text { and Repair, Cell } \\
\text { Death and } \\
\text { Survival, Cell } \\
\text { Cycle } \\
(17)\end{array}$ & $\begin{array}{l}\text { DNA Replication, } \\
\text { Recombination, } \\
\text { and Repair, Cell } \\
\text { Cycle, Cancer } \\
\text { (3) }\end{array}$ & N/A & N/A & N/A \\
\hline & $\begin{array}{c}\text { Physiological System } \\
\text { Development and } \\
\text { Function } \\
\text { (p-value range) }\end{array}$ & $\begin{array}{c}\text { Organismal } \\
\text { Survival } \\
(4.27 \mathrm{E}-02- \\
1.01 \mathrm{E}-06)\end{array}$ & $\begin{array}{c}\text { Connective Tissue } \\
\text { Development and } \\
\text { Function } \\
(3.35 \mathrm{E}-02- \\
1.85 \mathrm{E}-06)\end{array}$ & $\begin{array}{l}\text { Nervous System } \\
\text { Development and } \\
\text { Function } \\
(4.14 \mathrm{E}-02- \\
\text { 4.54E-04) }\end{array}$ & \begin{tabular}{|c|} 
Tissue \\
Morphology \\
$(3.40 \mathrm{E}-02-4.54 \mathrm{E}-$ \\
$04)$
\end{tabular} & N/A \\
\hline
\end{tabular}

The top canonical pathways and top networks were noted based on the functional annotation of significantly differentially expressed genes from the apoptosis expression arrays. All IPA functions with the exception of cancer specific, reproductive system, psychological functions, and cell lines were considered for this table. Young rats SSC-loaded three days per week had no available relevant functions that came up in the analysis, so therefore there is no table displayed for that group. Sample sizes were $N=5$ to 6 per group. N/A= Not available. SSC = stretch-shortening contraction; NER = nucleotide excision repair; DSB = double-strand break; NHEJ = non-homologous end joining; RAR = retinoic acid receptor; $\mathrm{HR}$ = homologous recombination; BRCA1 $=$ Breast cancer 1 . 


\section{FIGURE LEGENDS}

Figure 1. Reduced frequency of SSC resistance-type exercise training improves muscle performance of aged rats. During each training session, TA muscle performance was assessed during the first SSC repetition of the first training set. The maximum force was measured and recorded for each group; A) Dorsiflexion dynamic performance in terms of peak force performed on the first repetition of the first training set of each training session; B) the relative changes in peak force performed on the first repetition of the first training set of each training session; C) final SSC peak force values were divided by normalized muscle mass of SSC-loaded muscles to determine muscle quality values. For each group, the peak force performed was averaged based on recorded force values during the first week (initial) and last week of training (final). Sample sizes were $N=9$ to 10 per group. ${ }^{*}$ Different from young initial; ${ }^{*}$ Different from initial value within each group; ${ }^{\dagger}$ Different from old rats trained 3 days per week; $\ddagger$ Different from old rats trained 2 days per week, $\mathrm{p}<0.05$. Data presented as means \pm SEM. SSC = stretch-shortening contraction; TA = tibialis anterior.

Figure 2. Reduced frequency of SSC resistance-type exercise training decreases apoptosis DNA methylation in the TA skeletal muscle of aged rodents. Epitect Methyl II PCR array looking at the promoter-specific methylation status of genes implicated as important regulators of the apoptosis pathway were calculated as a percentage for each gene and then collectively combined to quantify the overall DNA methylation status of the entire pathway for each group. Analyses were done comparing SSC-loaded muscle following one month of high-intensity SSC RTET with age-match NLC TA muscle. Sample sizes were $N=4$ to 6 per group. ${ }^{*}$ Different from NLC within age; "Different from treatment within age, $\mathrm{p}<0.05$. Data presented as means $\pm \mathrm{SEM}$. SSC = stretch-shortening contraction; TA = tibialis anterior; NLC = non-loaded control.

Figure 3. Reduced frequency of SSC resistance-type exercise training increases apoptosis signaling in the TA skeletal muscle of aged rats. The volcano plot graphs of the apoptosis PCR array. These graphs show the log2 of the fold change for each gene's expression between SSC-loaded and NLC muscle for each group. Fold changes and $P$ values were determined from comparisons of $\Delta \mathrm{C}_{\mathrm{t}}$ values between SSC-loaded and NLC TA muscles analyzed through the GeneGlobe Data Analysis Center (Qiagen). A) Young rats trained 3 days per week; B) young rats trained 2 days per week; C) old rats trained 3 days per week; D) old rats trained 2 days per week. Sample sizes were $N=5$ to 6 per group. The vertical solid line indicates fold changes of 0 . Vertical dotted lines indicate that the fold change in gene expression threshold is 1.1; the horizontal solid line indicates that the P-value of the $t$ test threshold is 0.05 . Genes that were significantly differentially expressed are labeled and colored according to whether or not they were up- or downregulated. SSC = stretch-shortening contraction; $\mathrm{TA}=$ tibialis anterior; NLC $=$ non-loaded control. 
Figure 4. Reduced frequency of SSC resistance-type exercise training has no effect on DNA methylation of the DNA repair pathway in the TA skeletal muscle of aged rats. Epitect Methyl II PCR array looking at the promoter-specific methylation status of genes implicated as important regulators of the DNA repair pathway were calculated as a percentage for each gene and then collectively combined to quantify the overall DNA methylation status of the entire pathway for each group. Analyses were done comparing SSC-loaded muscle following one month of high-intensity SSC RTET with age-match NLC TA muscle. Sample sizes were $N=4$ to 6 per group. " Different from NLC within age; ${ }^{*}$ Different from treatment within age, $\mathrm{p}<0.05$. Data presented as means \pm SEM. SSC = stretch-shortening contraction; TA = tibialis anterior; NLC = non-loaded control.

Figure 5. Reduced frequency of SSC resistance-type exercise training enhances DNA repair signaling in the TA skeletal muscle of aged rats. The volcano plot graphs of the DNA Repair PCR array. These graphs show the log2 of the fold change for each gene’s expression between SSC-loaded and NLC TA muscle for each group. Fold changes and $P$ values were determined from comparisons of $\Delta C_{t}$ values between SSC-loaded and NLC TA muscles analyzed through the GeneGlobe Data Analysis Center (Qiagen). A) Young rats trained 3 days per week; B) young rats trained 2 days per week; C) old rats trained 3 days per week; D) old rats trained 2 days per week. Sample sizes were $N=5$ to 6 per group. The vertical solid line indicates fold changes of 0 . Vertical dotted lines indicate that the fold change in gene expression threshold is 1.1; the horizontal solid line indicates that the P-value of the $t$ test threshold is 0.05 . Genes that were significantly differentially expressed are labeled and colored according to whether or not they were up- or downregulated. SSC = stretch-shortening contraction; $\mathrm{TA}=$ tibialis anterior; NLC $=$ non-loaded control.

Figure 6. Reduced frequency of SSC resistance-type exercise training protects against cell death. Comparison of the DNA fragmentation cell death via an ELISA between young and old rodent TA skeletal muscle. Total protein contents of the cytoplasmic protein extracts were quantified and reported as protein concentration ( $\mu g$ protein/mg tissue - [protein]) in triplicate using the Pierce ${ }^{\mathrm{TM}}$ BCA Protein Assay Kit (Thermo Scientific, Rockford, IL, USA). Cytoplasmic protein extracts were used to quantitatively detect the cytosolic histoneassociated DNA fragmentation. The resulting optical density at $405 \mathrm{~nm}$ was then normalized to the total amount of protein in the sample and the data was reported as an apoptotic index ( $\mathrm{OD}_{405} / \mathrm{mg}$ protein) to indicate the level of cytosolic mono-and oligonucleosomes present in the samples. All measurements were performed in duplicate. ${ }^{*}$ Different from NLC within age; ${ }^{\dagger}$ Different from treatment by age, $\mathrm{p}<0.05$. SSC $=$ stretch-shortening contraction; TA = tibialis anterior; $\mathrm{OD}_{405}=$ Optical density at $405 \mathrm{~nm}$; NLC = non-loaded control. 
Figure 7. Reduced frequency of SSC resistance-type exercise training elevates 8-OHdG. Oxidized DNA in samples was determined using an 8-OHdG sandwich ELISA. Samples were read and subsequently normalized to their respective DNA concentrations and expressed as nanograms per milliliter. All measurements were performed in duplicate. * Different from Young CON; ${ }^{*}$ Different from NLC within age; ${ }^{\dagger}$ Different from treatment within age; ${ }^{\ddagger}$ Different from treatment by age, $\mathrm{p}<0.05$. SSC = stretch-shortening contraction; 8-OHdG: 8-hydroxydeoxyguanosine; ELISA = enzyme-linked immunosorbent assay; TA = tibialis anterior; NLC = nonloaded control.

Figure 8. Adaptation from one month high-intensity SSC resistance-type exercise training results in altered myonuclei size as indicated by changes in minimum feret diameter. Frozen TA sections stained for 4-HNE and $\beta$-dystroglycan were manually traced to determine minimum feret diameter using Image J (Version 1.48; NIH, Bethesda, MD, USA). Frequency distributions for minimum feret diameter are presented as a percentage of the total myonuclei measured for each group. A) Young rats trained 3 days per week; B) young rats trained 2 days per week; C) old rats trained 3 days per week; D) old rats trained 2 days per week. Sample sizes were $N=4$ to 5 per group. Analyses for were done comparing SSC-loaded muscle following RTET with age-matched NLC TA muscle. The minimum feret diameter was quantified in ImageJ as the closest possible distance between two parallel tangents of a myonucleus. All groups with exception of old rats trained 3 days per week demonstrated a significant shift in distribution towards decreased minimum feret diameter following training, $\mathrm{p}<0.05$. SSC = stretch-shortening contraction; TA = tibialis anterior; 4-HNE = 4-hydroxynonenal; NLC = non-loaded control.

Figure 9. Maladaptation from one month high-intensity SSC resistance-type exercise training results in modified myonuclei morphology as indicated by changes in circularity. Frozen TA sections stained for 4-HNE and $\beta$-dystroglycan were manually traced to determine circularity using Image J (Version 1.48; NIH, Bethesda, MD, USA). Frequency distributions for circularity are presented as a percentage of the total myonuclei measured for each group. A) Young rats trained 3 days per week; B) young rats trained 2 days per week; C) old rats trained 3 days per week; D) old rats trained 2 days per week. Sample sizes were $N=4$ to 5 per group. Analyses were done comparing SSC-loaded muscle following RTET with age-matched NLC TA muscle. A circularity value of 1.0 indicates a perfect circle. As the value approaches 0.0 , it indicates an increasingly elongated polygon. Old rats trained 3 days per week were the only group that demonstrated a significant shift in distribution towards decreased circularity following training, $\mathrm{p}<0.05$. SSC $=$ stretch-shortening contraction; TA $=$ tibialis anterior; 4-HNE = 4-hydroxynonenal; NLC = non-loaded control. 
Figure 10. The merging of top network functions shows enhanced activation of DNA repair in old rats exposed to a reduced frequency of training. Through IPA analysis, the Top Networks were merged and the resulting Top Network Merged Functions is reported. This figure was generated through IPA using the path designer feature and displays the number one Top Network based on the functional annotation of key differentially expressed genes from the DNA Repair PCR Array for the following groups: A) Old rats trained three days per week; B) old rats trained two days per week. Yellow colored shapes indicates an upregulation of that molecule, whereas blue indicates a downregulation. For the lines, colors indicate predicted relationships based off the upregulation or downregulation of the genes in the dataset that are presented on the figure; yellow lines designate a predicted relationship leading to activation, whereas blue lines designate a predicted relationship leading to inhibition. Note that there is a collective downregulation of DNA repair genes and an overall predicted suppression of "Repair of DNA” in O3x, whereas O2x has a collective upregulation of DNA repair genes and an overall predicted activation of “Cell Survival.” From top to bottom, individual molecules have identified z-scores and p-values associated with them. IPA = Ingenuity Pathway Analysis.

Figure 11. Conceptual model for the relationship between myonuclei morphology, chromatin condensation, and transcriptional activation in aged skeletal muscle. Our data supports a fundamental model in which appropriately prescribed resistance-type exercise training (i.e., reduced frequency) allows aged myonuclei to maintain circularity, possibly reducing chromatin condensation, which would subsequently keep chromatin in a more open (euchromatic) state and lead to an increase in transcriptional activity, ultimately contributing to the positive results that come with muscle adaptation. In contrast, inappropriately prescribed exercise prescription (i.e., higher frequency) to resistance-type exercise training has a negative impact on aged myonuclei by causing them to become less circular (i.e., elongated), driving chromatin to become more condensed (heterochromatic) and leading to decreased transcriptional activation, consequently promoting maladaptation. 
FIGURE 1

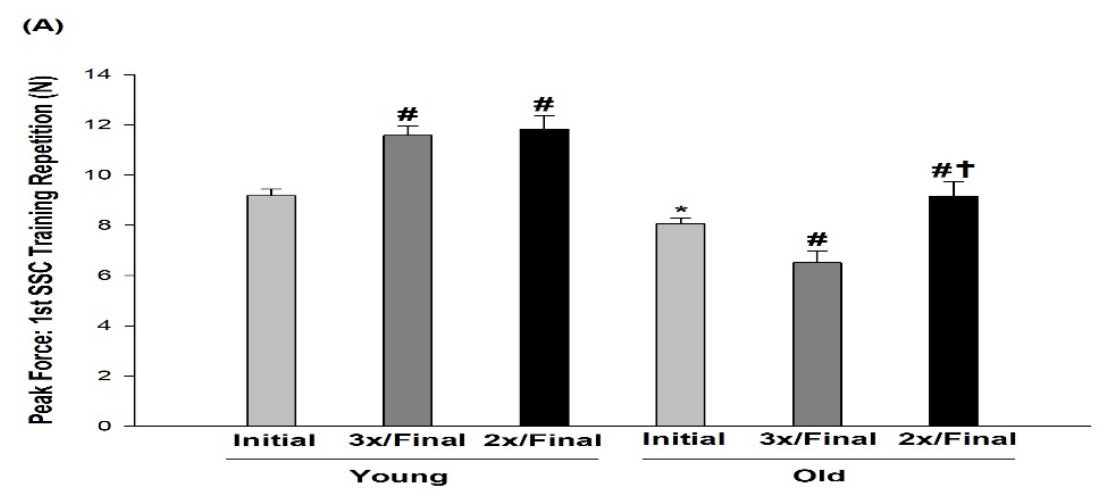

(B)

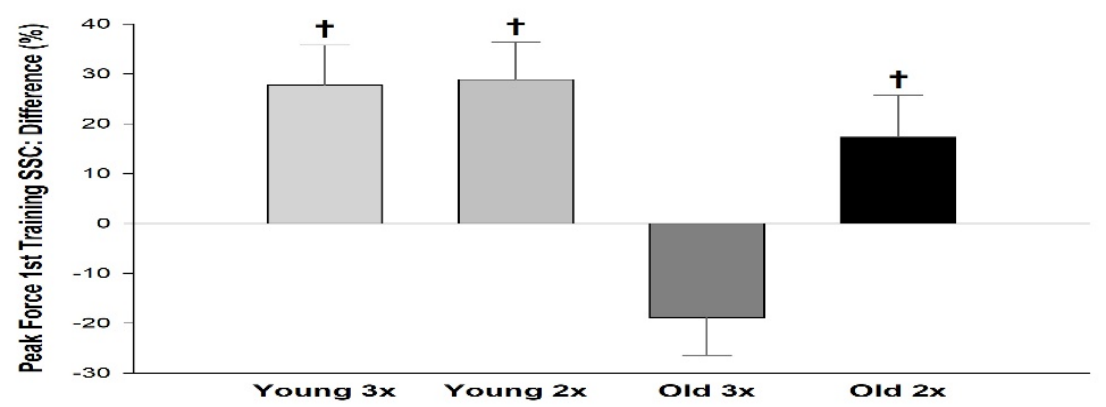


(C)

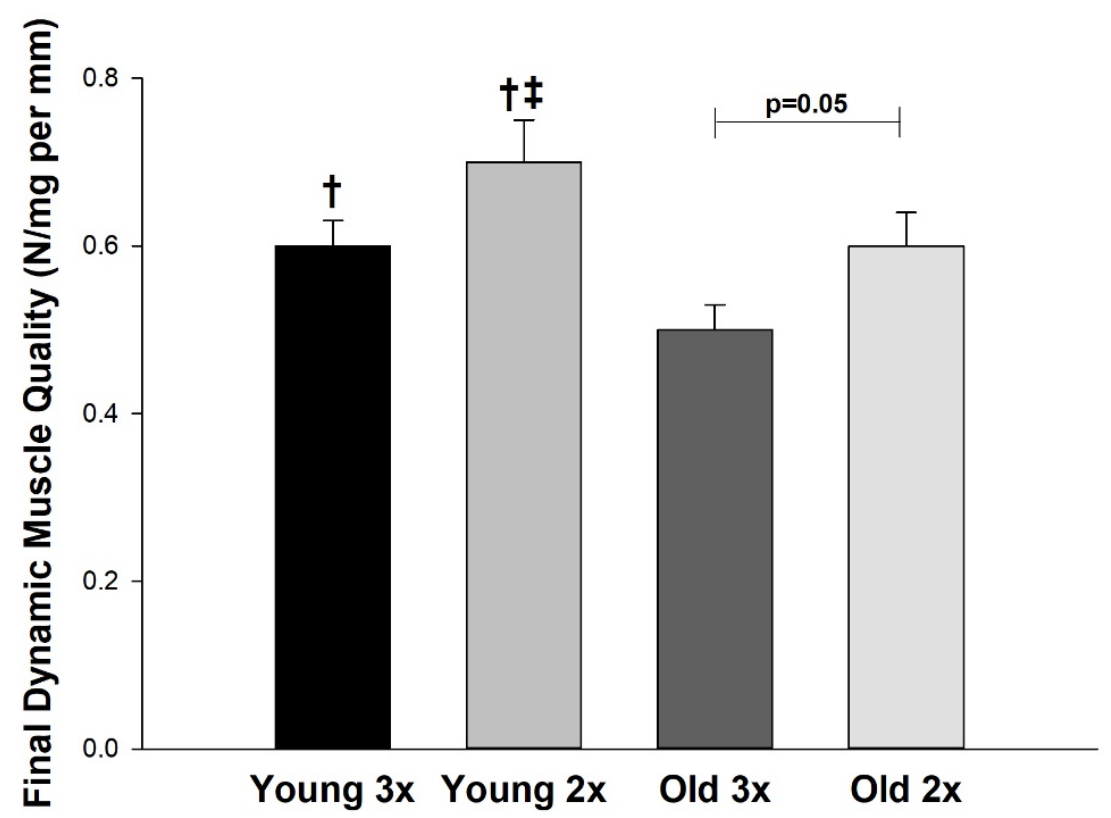


FIGURE 2

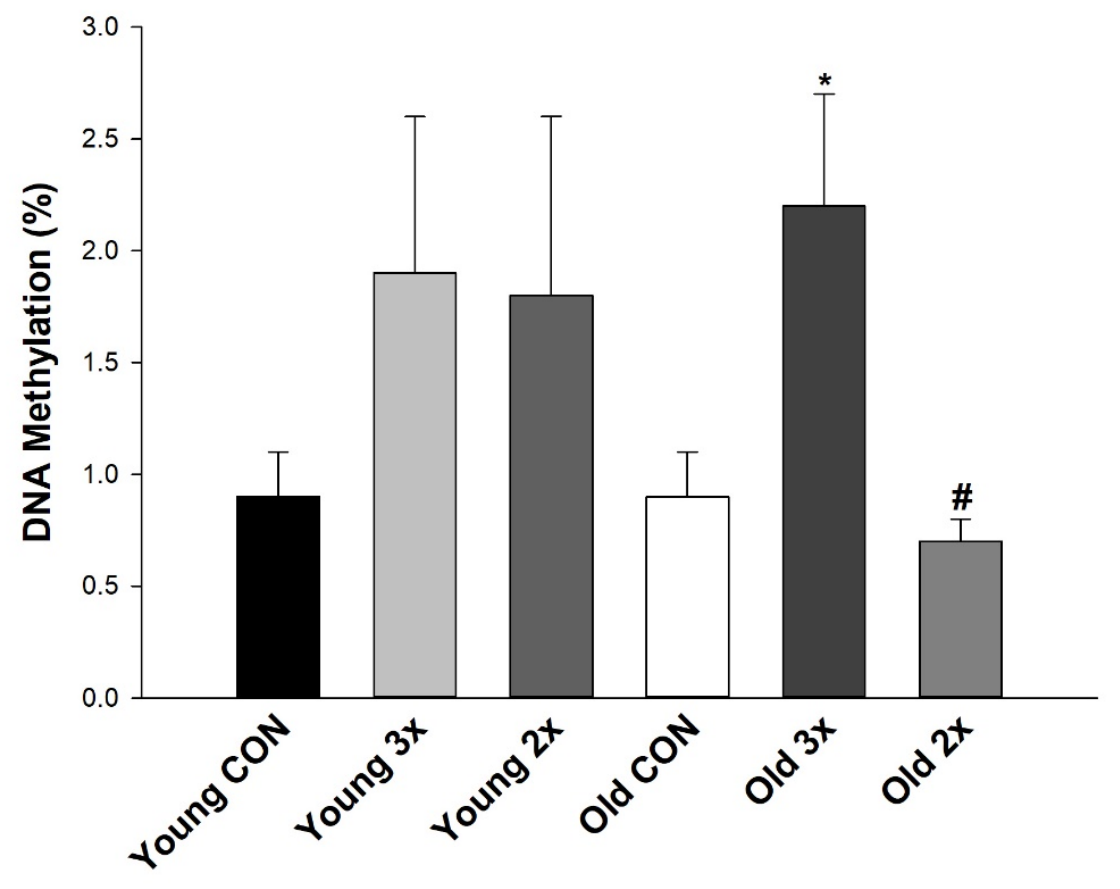




\section{FIGURE 3}

(A)

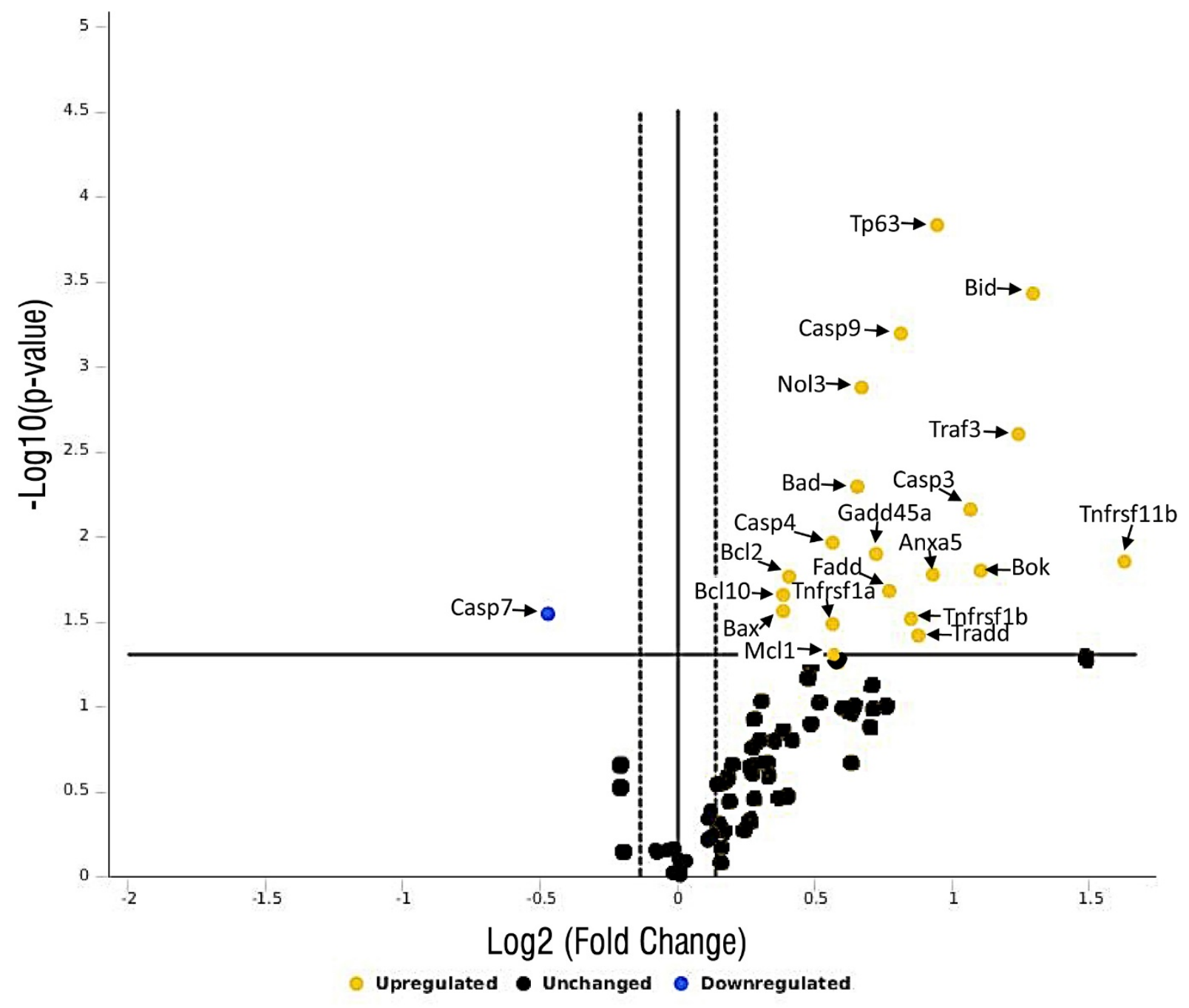


(B)

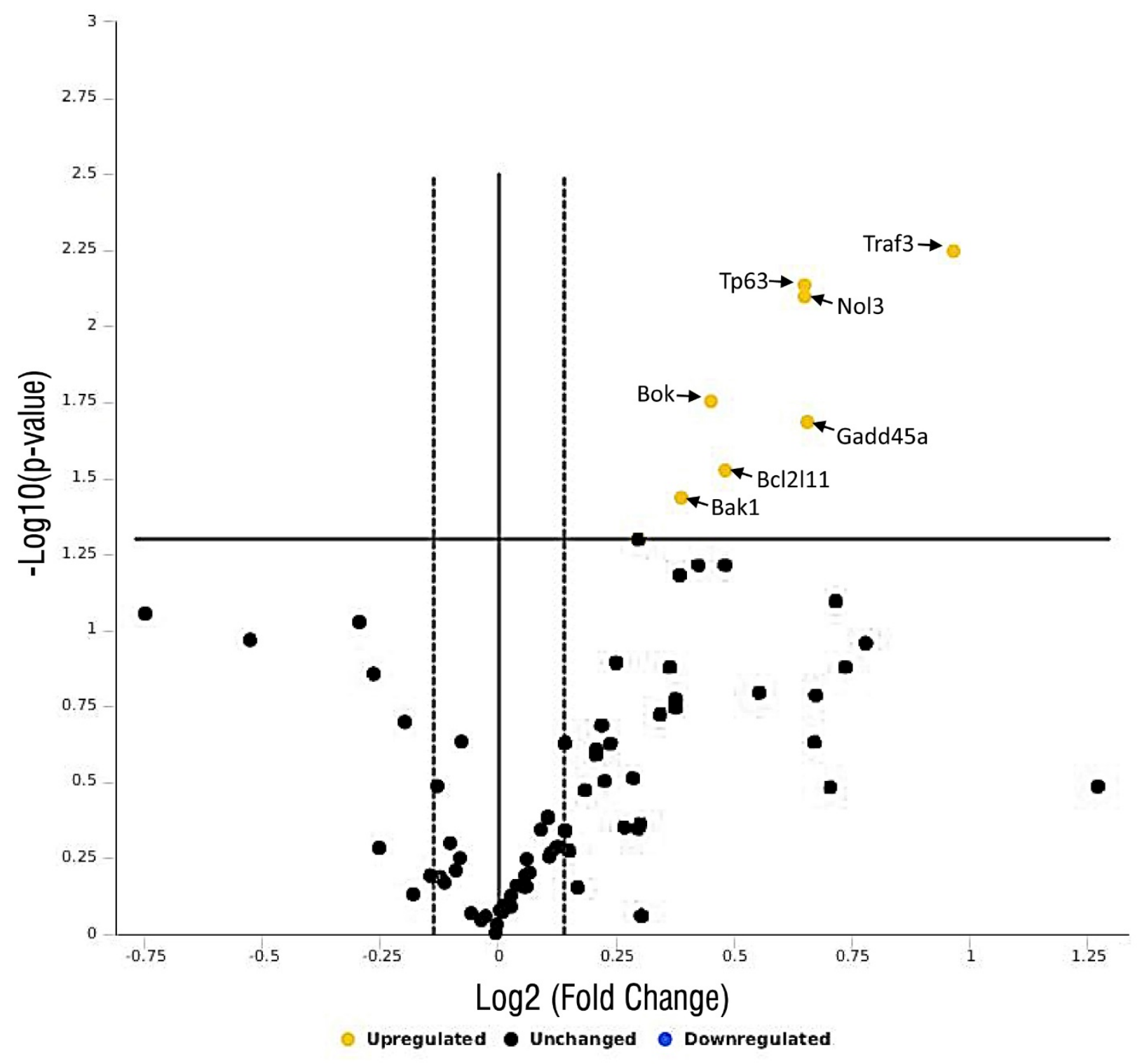


(C)

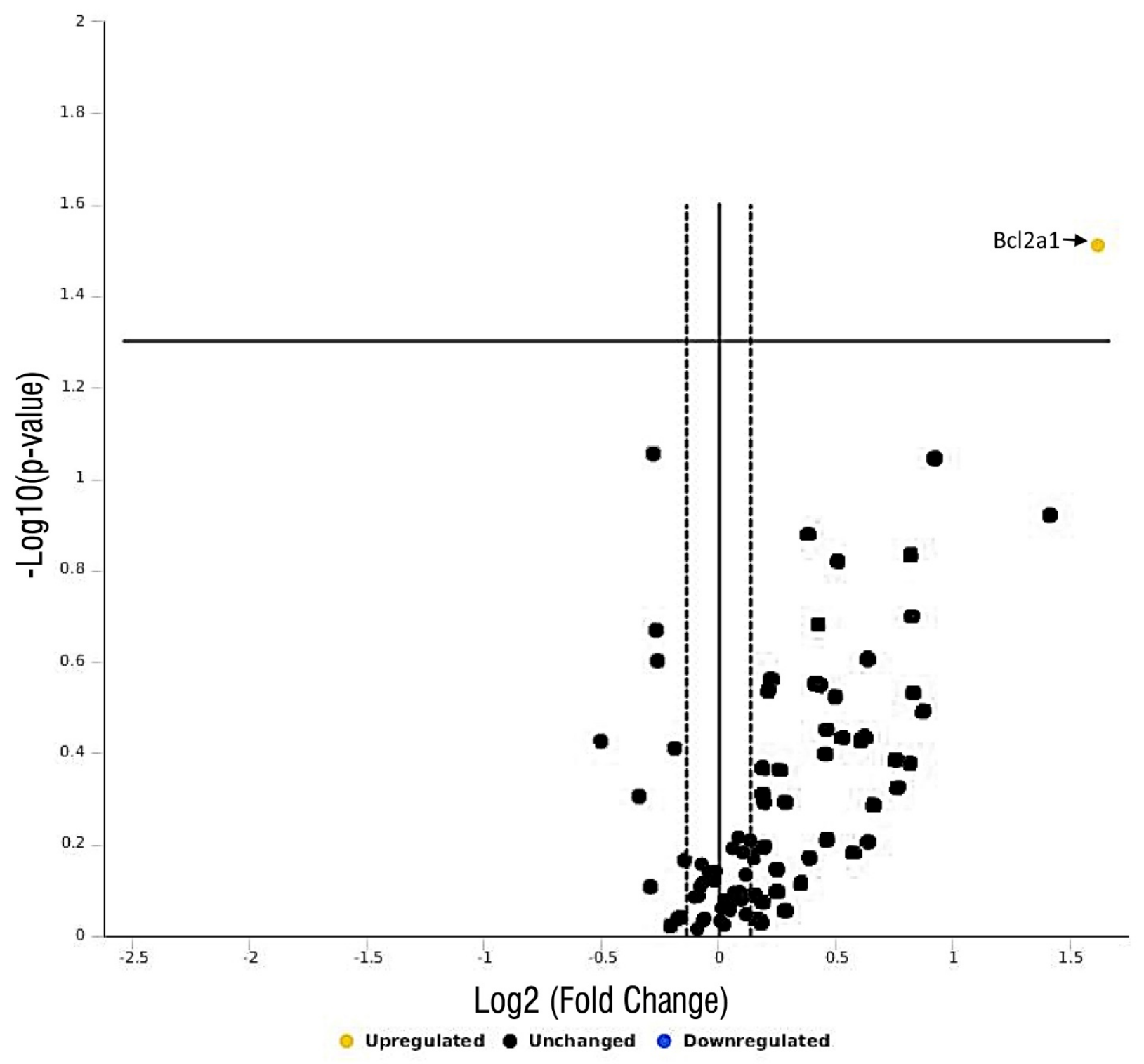


(D)

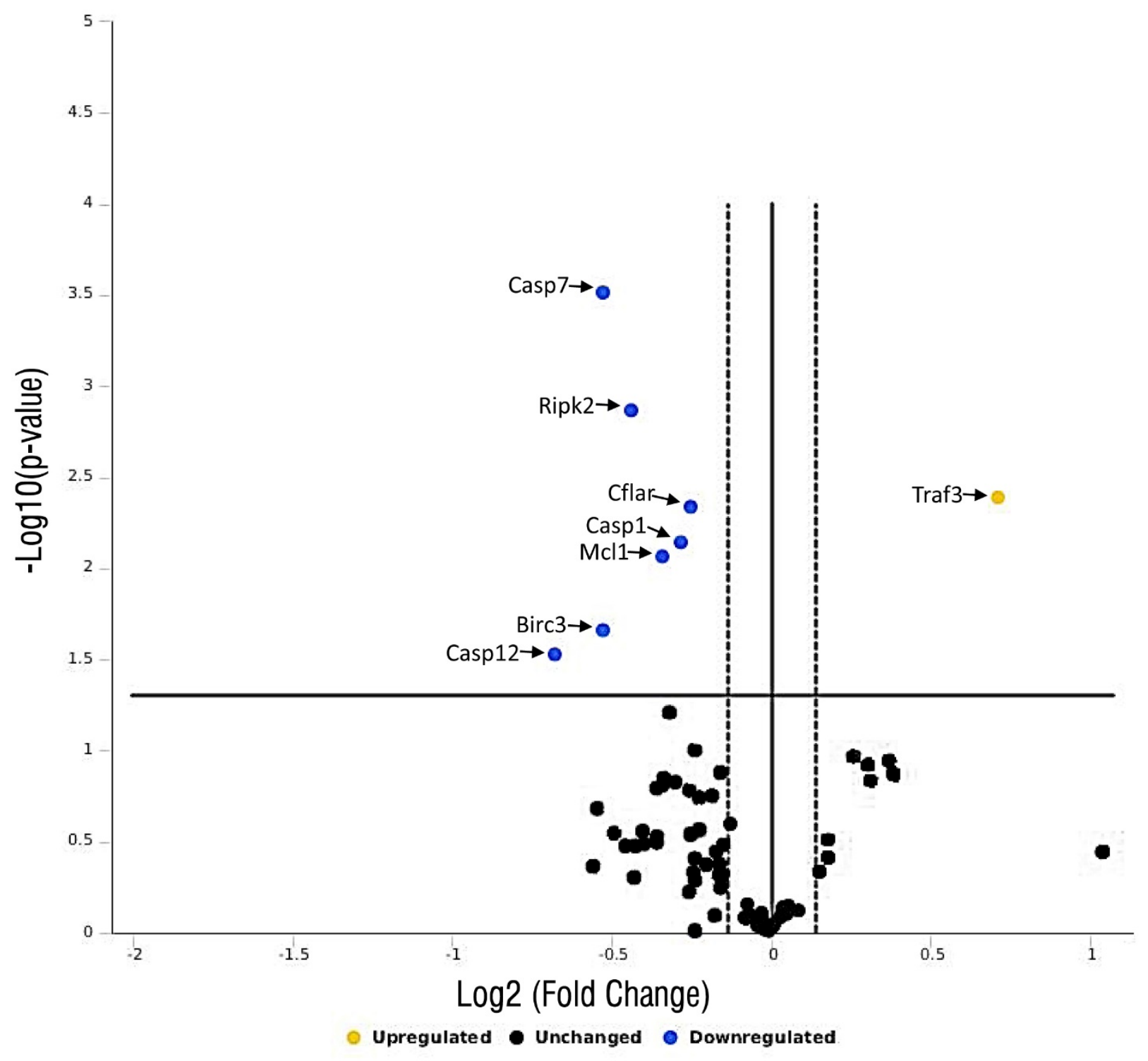


FIGURE 4

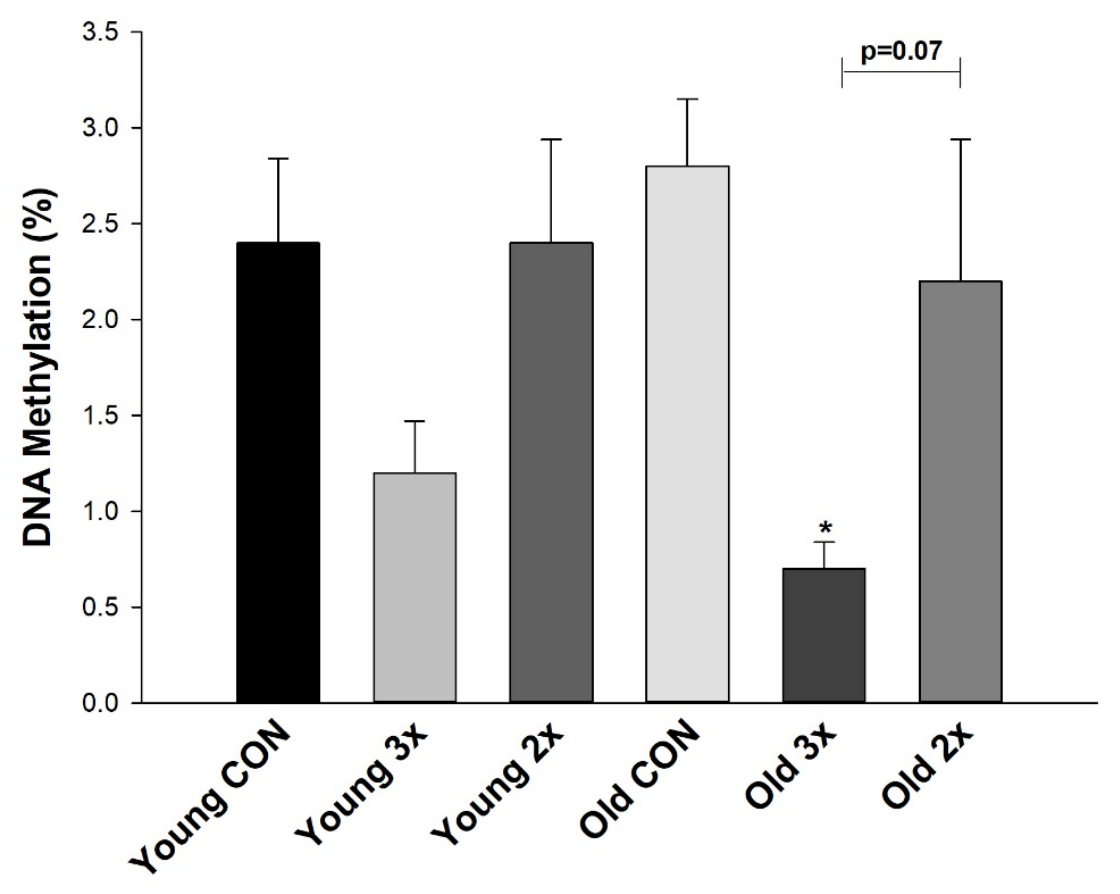


FIGURE 5

(A)

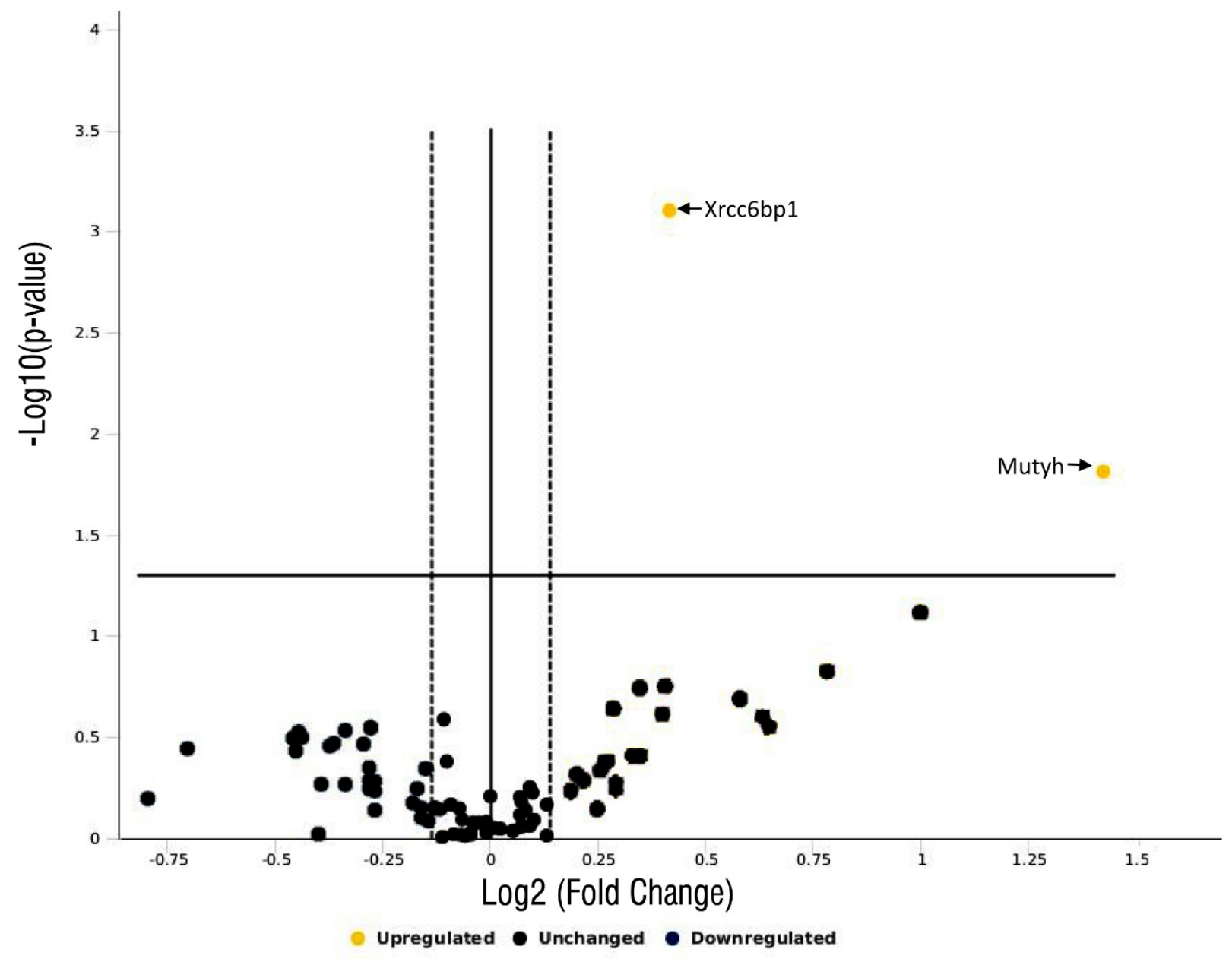


(B)

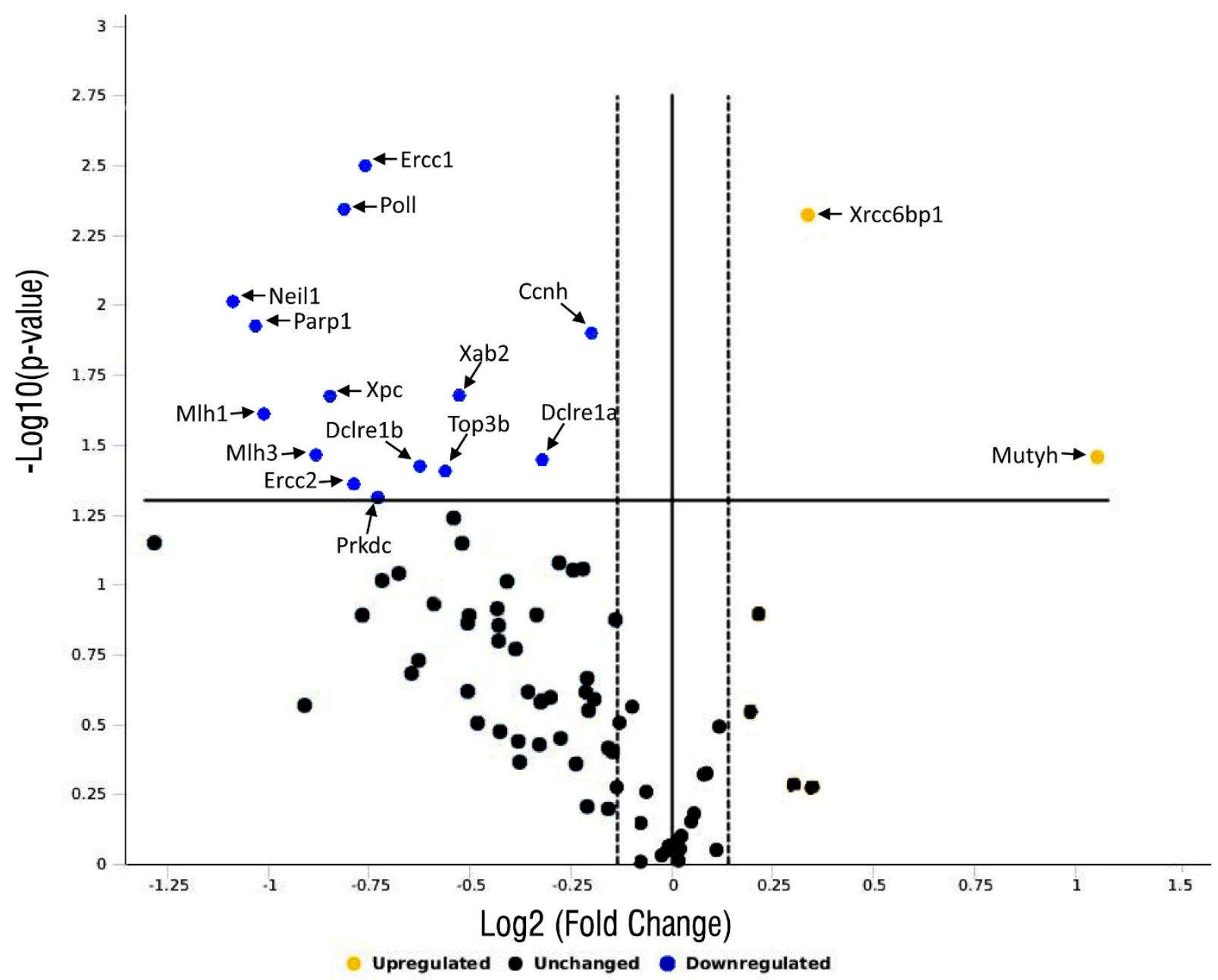


(C)

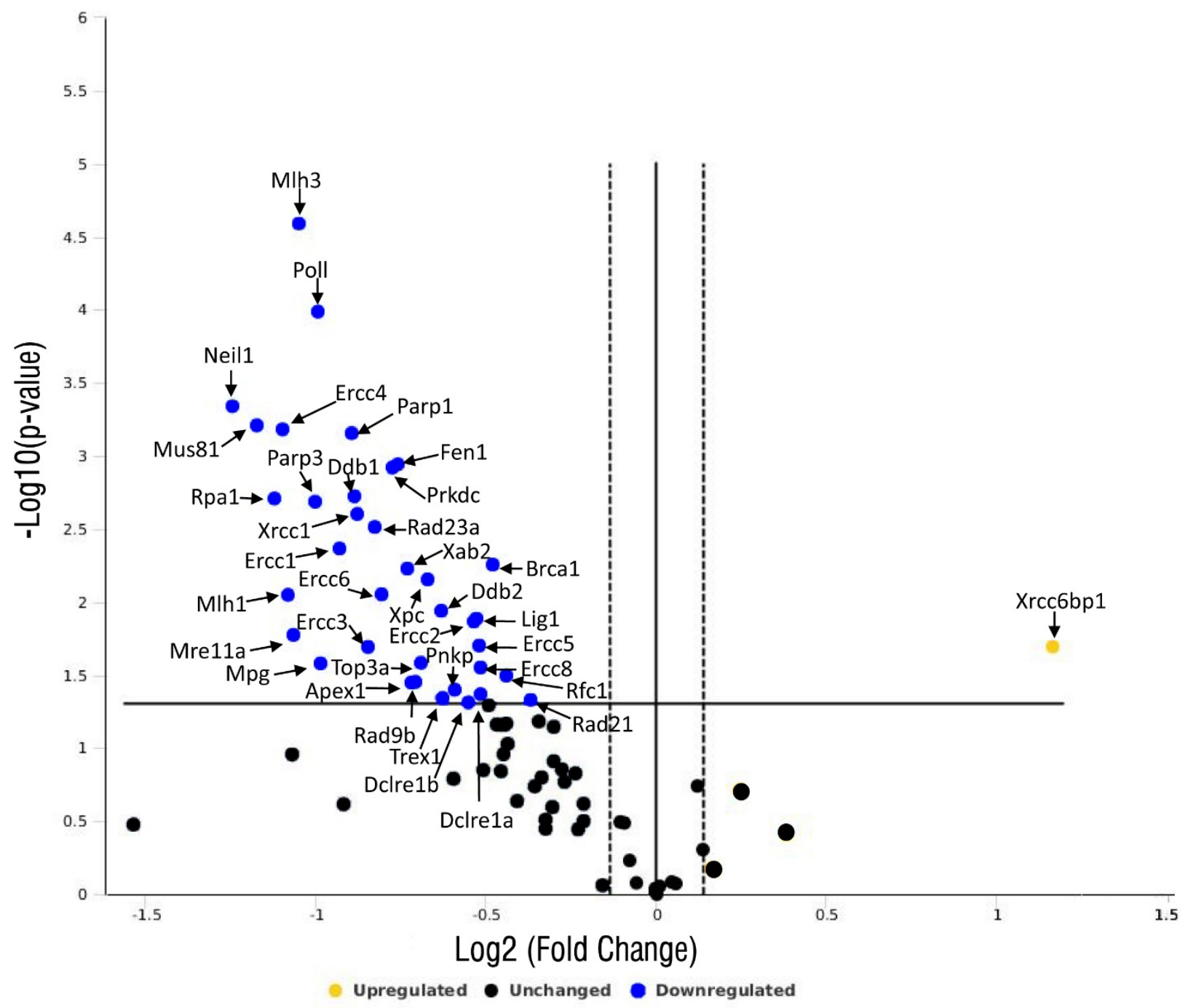


(D)

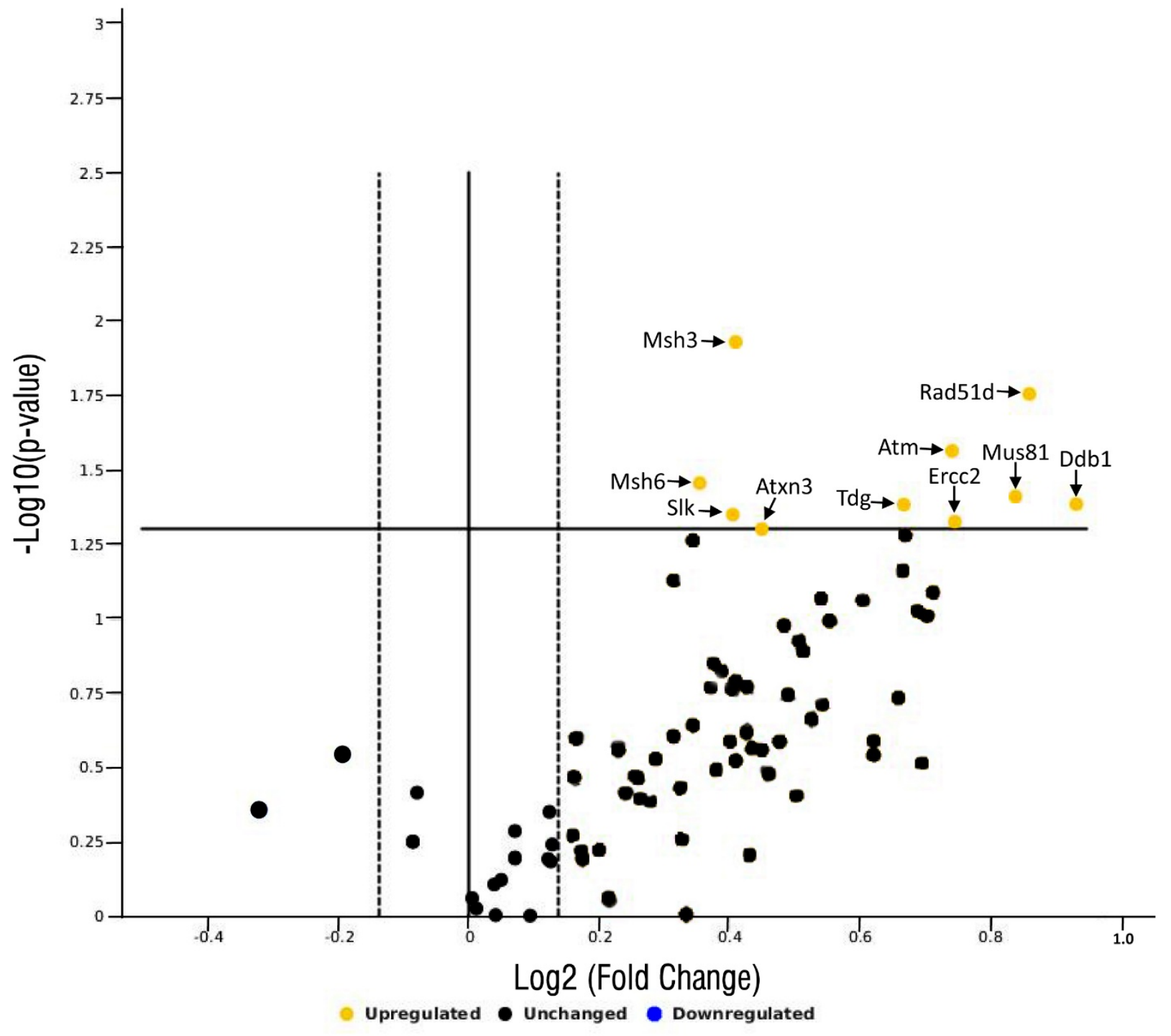


FIGURE 6

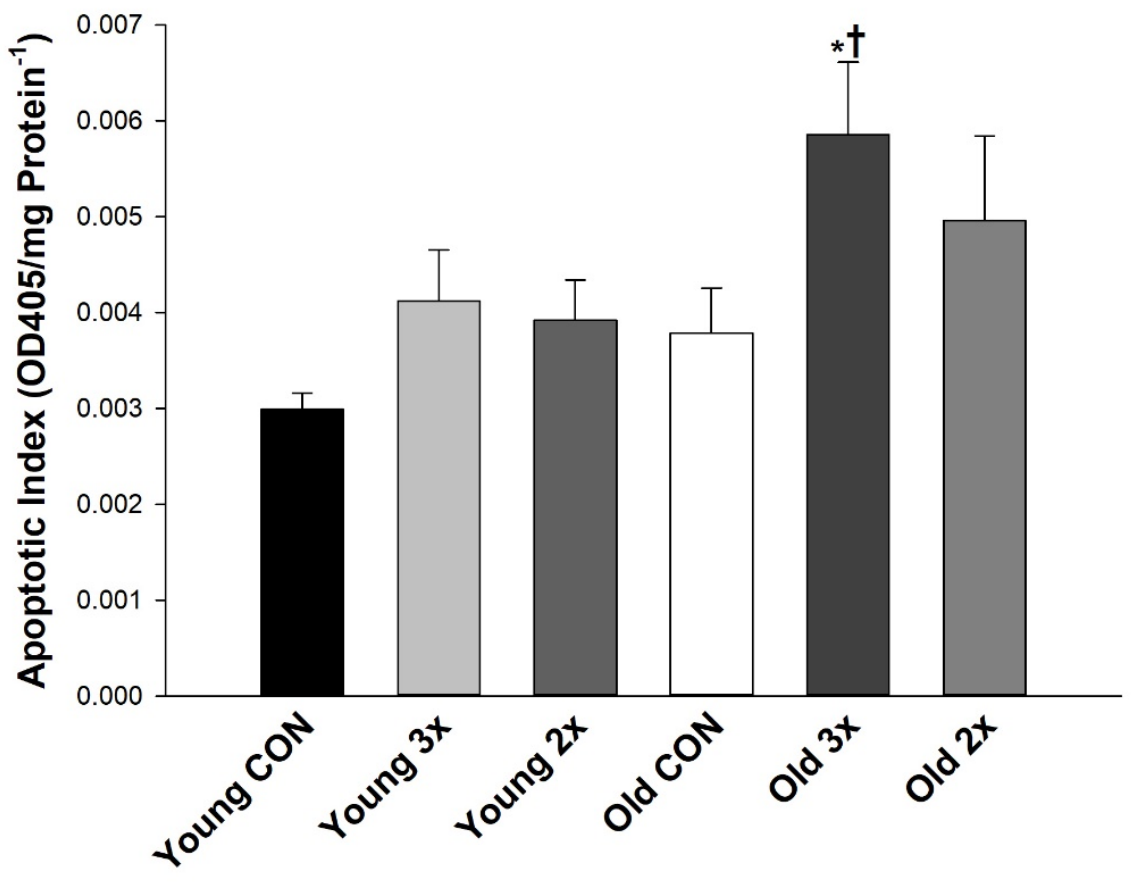


FIGURE 7

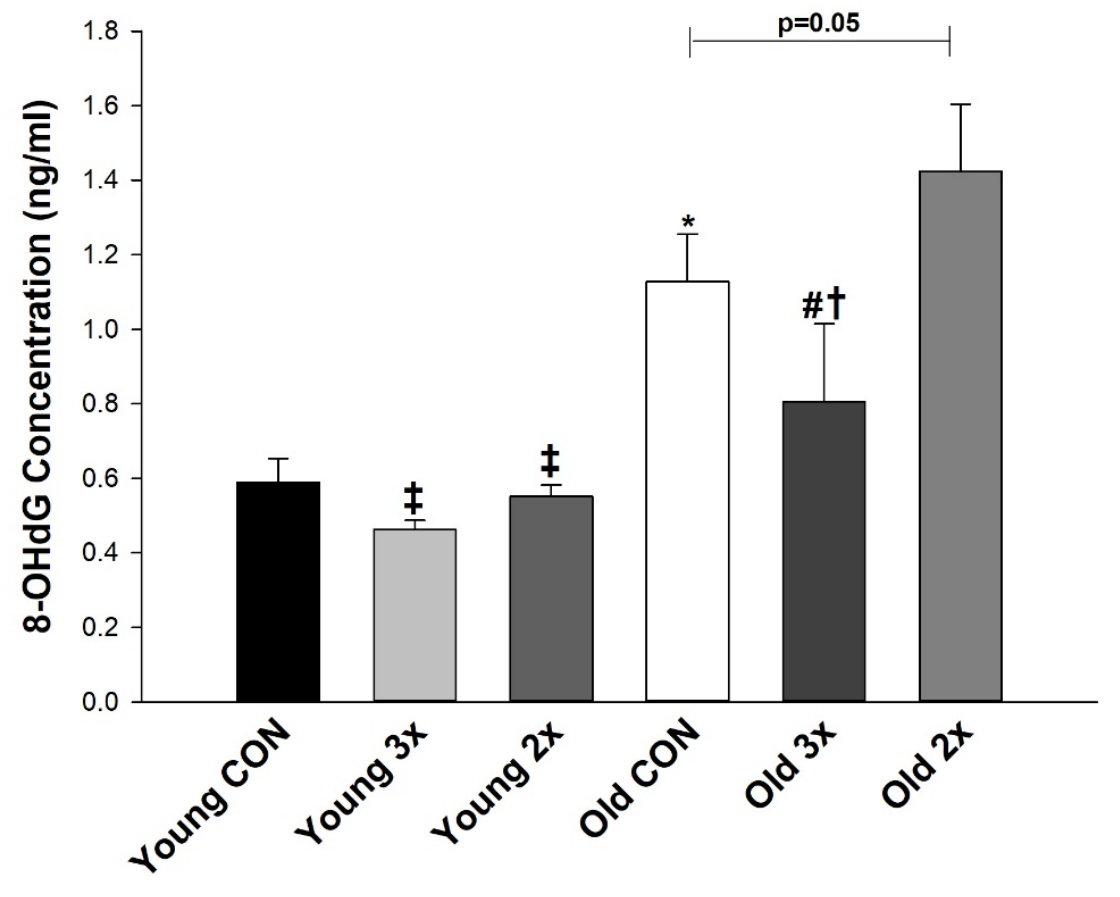




\section{FIGURE 8}
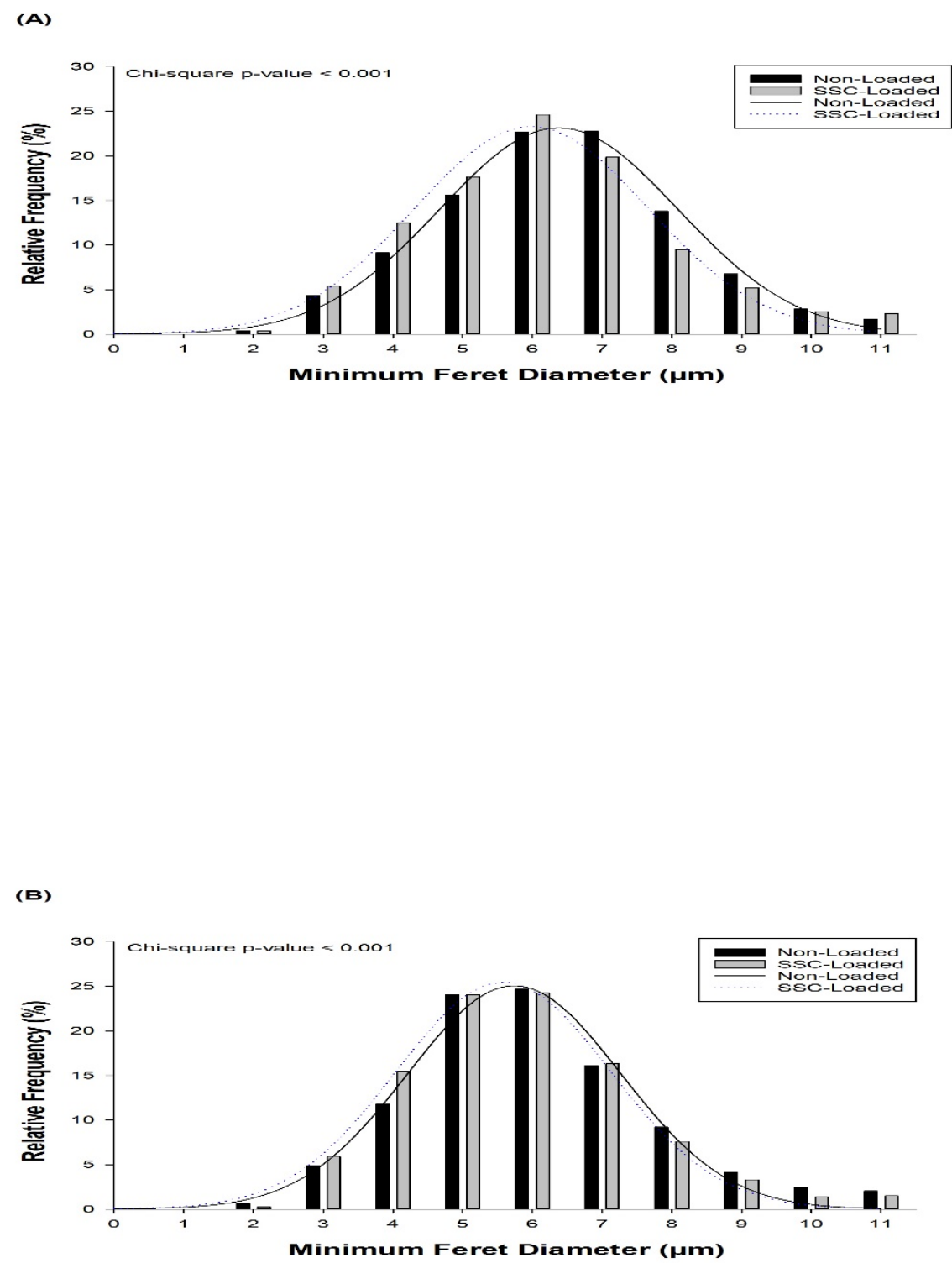

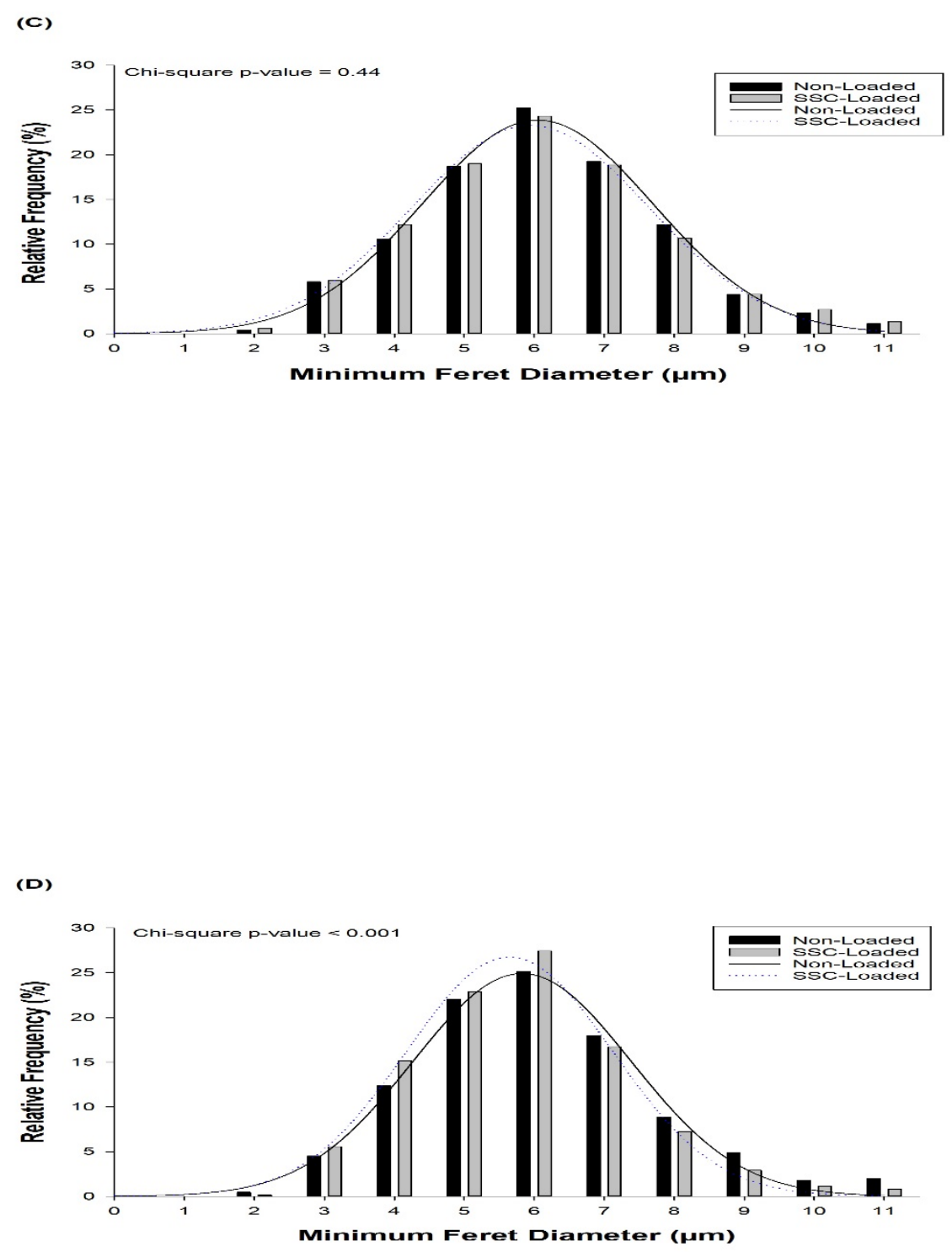


\section{FIGURE 9}
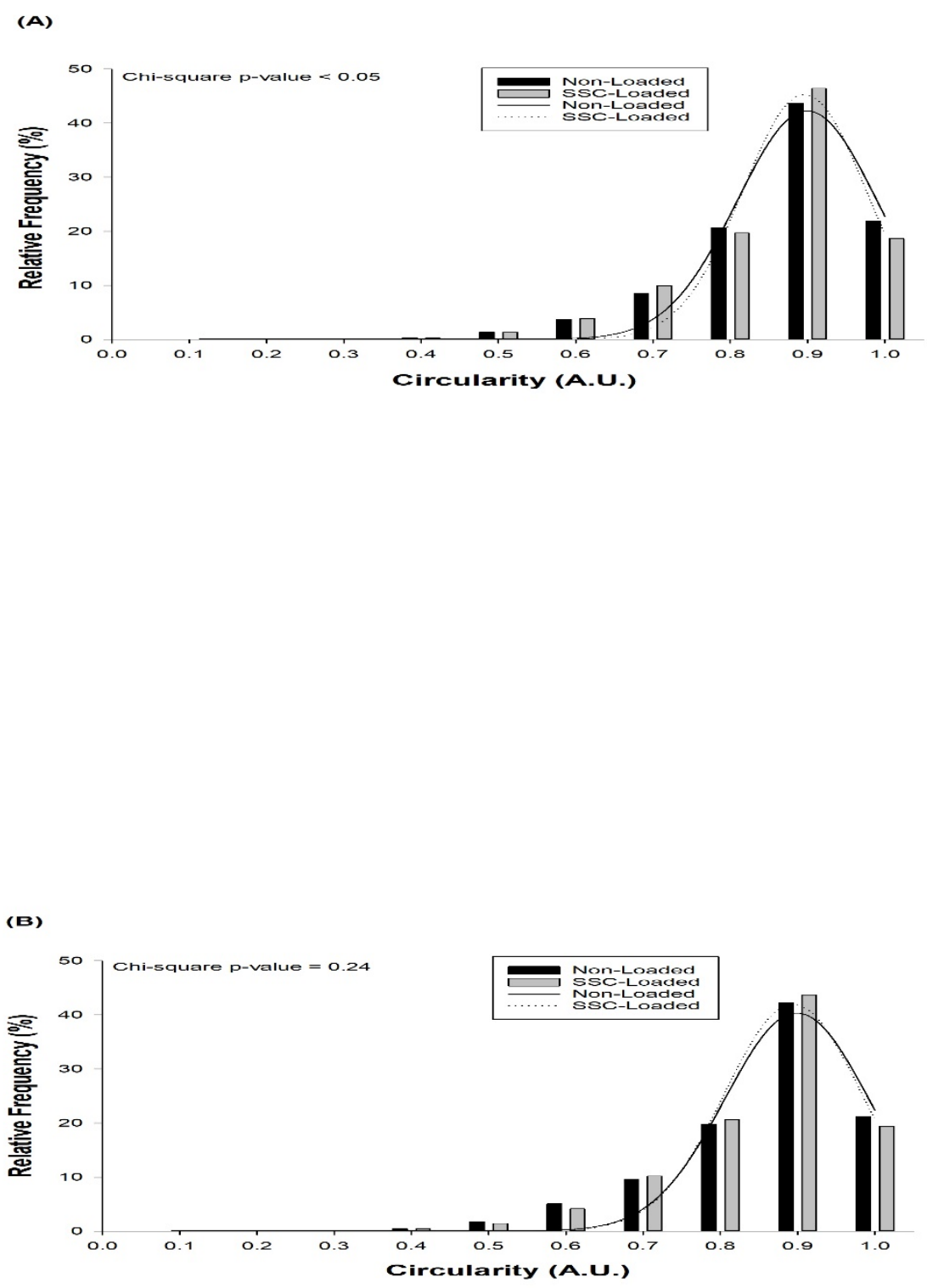

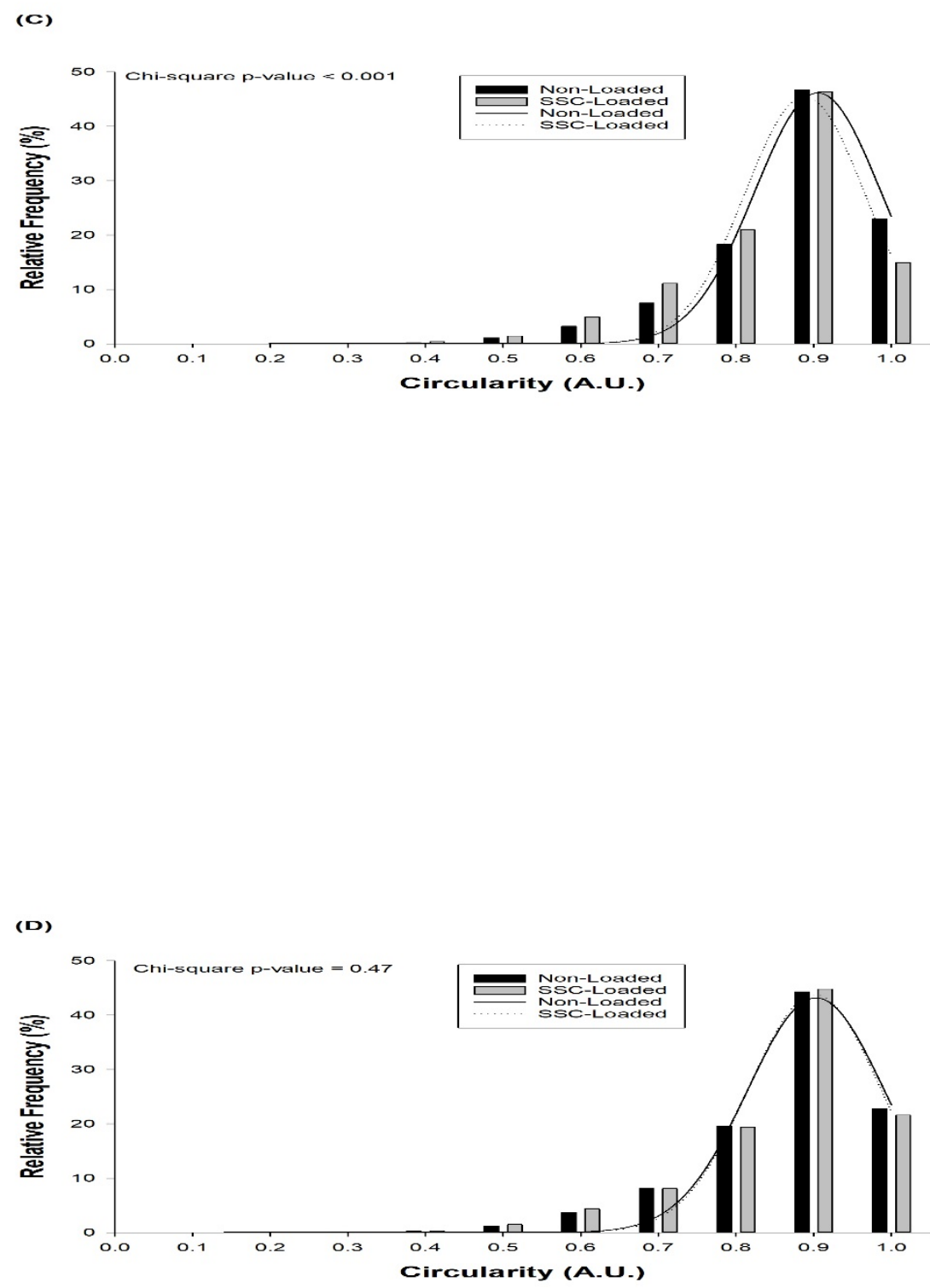


\section{FIGURE 10}
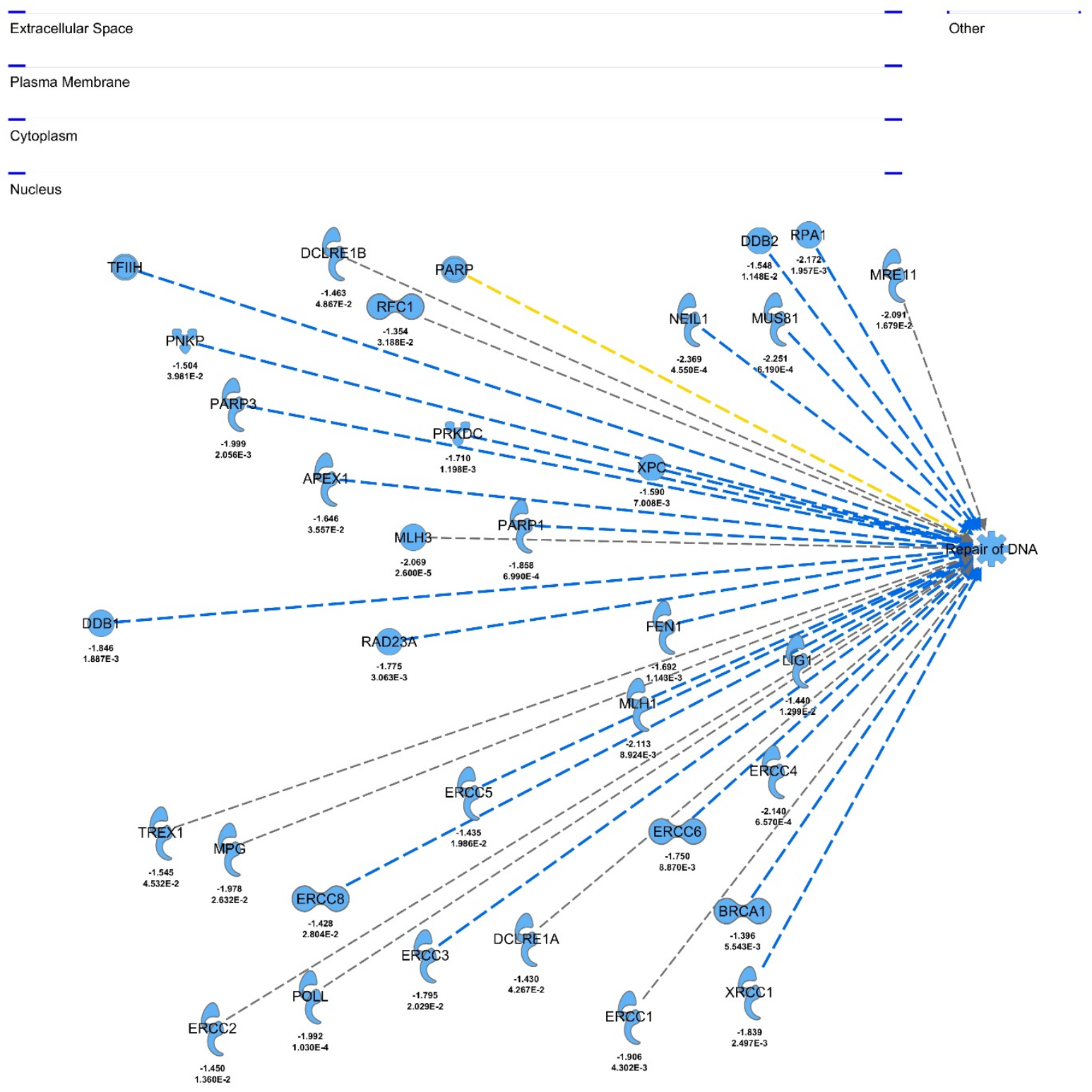
Plasma Membrane

Cytoplasm

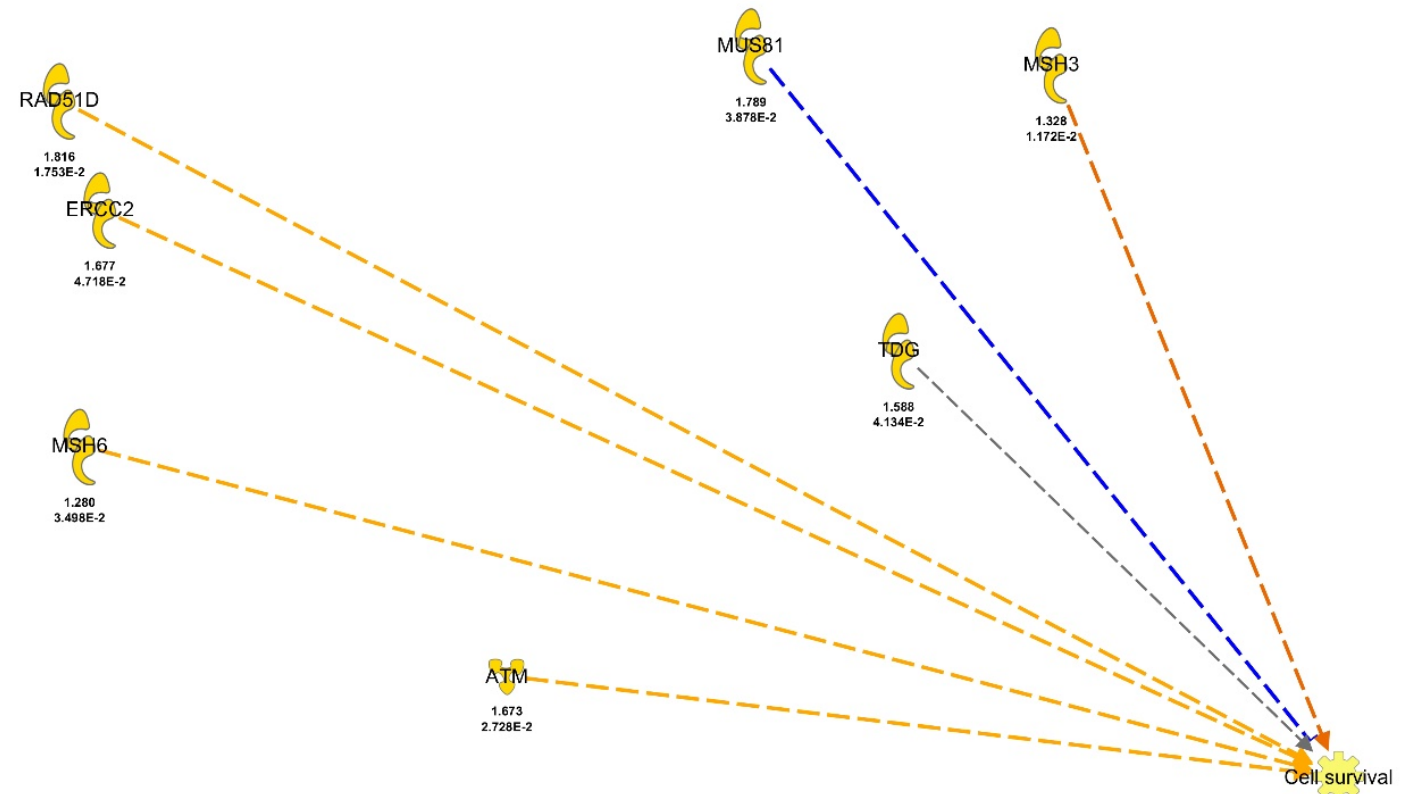




\section{FIGURE 11}

Adaptation (e.g.. Reduced Exercise Frequency)

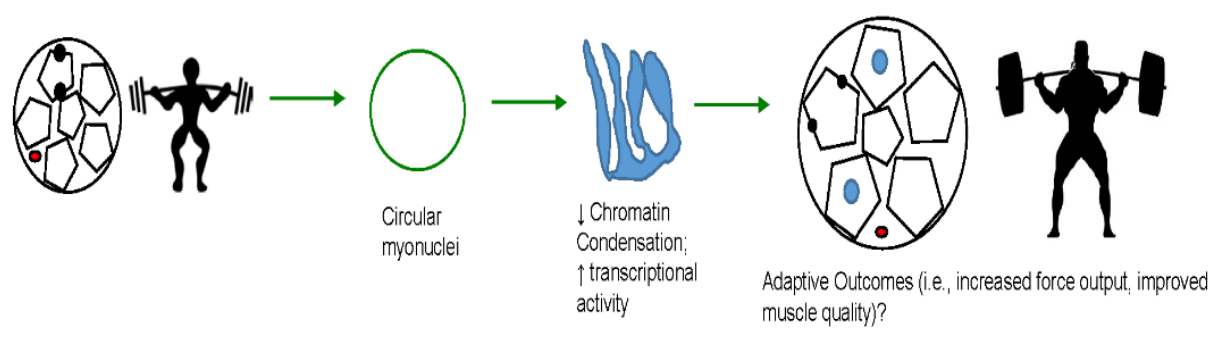

Maladaptation (e.g.. Higher Exercise Frequency)

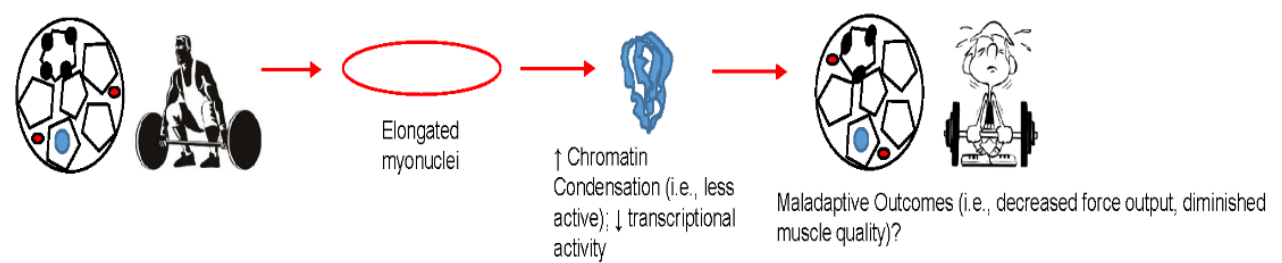




\section{CHAPTER VI}

THE THERAPEUTIC EFFECTS OF A RESISTANCE-TYPE EXERCISE TRAINING-RETRAINING PARADIGM ON TRANSCRIPTION FACTOR REGULATION AND MUSCLE ADAPTATION WITH AGING

Authors: Marshall A. Naimo ${ }^{1,2}$, Erik P. Rader ${ }^{1}$, James Ensey ${ }^{1}$, Michael L. Kashon ${ }^{1}$, Brent A. Baker ${ }^{1}$

Affiliations: ${ }^{1}$ Centers for Disease Control and Prevention, National Institute for Occupational Safety and Health, Health Effects Laboratory Division, Morgantown, West Virginia, USA; ${ }^{2}$ West Virginia University, School of Medicine, Division of Exercise Physiology, Morgantown, West Virginia, USA

Running Head: Cellular Muscle Memory Responses to Stretch-Shortening Contraction Training

Keywords: Stretch-shortening contractions, muscle memory, DNA methylation, epigenomics, Fisher 344 x Brown Norway, aging 


\section{ABSTRACT}

Previous data from our laboratory indicates that an altered adaptive phenotype following a validated, stretchshortening contraction (SSC) resistance-type exercise training (RTET) protocol occurs prior to complete biological development of the rodent - at six months of age in the adult rat. Interestingly, this is earlier than what we postulated and the exact explanations for this finding are unknown. The notion of muscle memory in the human performance literature have previously been conveyed, and more recent studies have started to establish a cellular basis for its existence following exercise training. However, the precise molecular underpinnings of this muscle memory are unknown. Therefore, the purpose was to determine whether exposure to high-intensity SSC RTET at a young age has positive effects on muscle adaptation as well as transcription factors (TFs) methylation and pathway gene expression with the progression into adulthood in skeletal muscle upon re-exposure to the same exercise stimulus. Methods: Dorsiflexor muscles of Fischer 344 x Brown Norway rats were exposed to a high-intensity SSC RTET stimulus on an in vivo dynamometer using a trainingretraining (TRT) paradigm, three days/week for one month, at three months and then again at six months of age; another group underwent the same training protocol with the exception that they were only trained at six months of age and thus were previously naïve prior to the exercise stimulus. Gene expression and DNA methylation of the tibialis anterior (TA) muscles were quantified via Profiler and Methylation Arrays (Qiagen ${ }^{\circledR}$ ). Results: TRT led to significant increases in both static (isometric) and dynamic (SSC) peak force as well as TA normalized muscle mass relative to naïve 6 month olds $(\mathrm{p}<0.05)$, thus augmenting the trainability of skeletal muscle into adulthood. The TRT group had 17 significantly $(\mathrm{p}<0.05)$ differentially expressed genes (SDEGs) in the TFs pathway, including MYF5 and ATF3; the 6-month trained only group had just 3 SDEGs. This response was accompanied

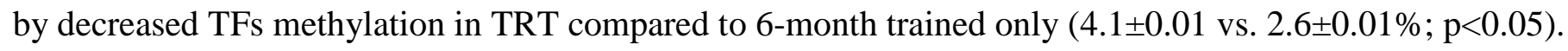

Conclusions: The augmented performance and muscle mass benefits at adulthood following an initial bout of SSC RTET at a younger age are supported by decreased TFs methylation and subsequent increased gene expression. These results advocate RTET at an earlier age as a means to preserve skeletal muscle function and plasticity as one ages through an epigenetic/epigenomic muscle memory mechanism. 


\section{Publications Disclaimers:}

"The findings and conclusions in this report are those of the author(s) and do not necessarily represent the official position of the National Institute for Occupational Safety and Health, Centers for Disease Control and Prevention." 


\section{INTRODUCTION}

One of the most defining features of skeletal muscle is its remarkable plasticity to adapt to various forms of stimuli. Skeletal muscle is dynamically regulated by changes in physical demands and environmental challenges (6). Epigenetics, literally meaning “above genetics," is defined as changes in gene expression as a result of structural modifications which are potentially reversible and occur independent of alterations to the actual genetic code itself $(8,38)$. Each cell type in the body has some form of a distinct epigenetic regulatory network referred to as the epigenome (38). Epigenomic processes are delineated as reversible marks that modulate chromatin structure, and comprise a macromolecular structure that contains unmodified as well as chemically modified nucleic acids and amino acids found in the nucleus (5).

Given the various mechanisms implicated in epigenetic/epigenomic control of cells, DNA methylation, the process of attaching a methyl group to the 5' position of a cytosine within DNA, is believed to be the most influential form of epigenomic regulation $(9,16)$. Transcriptional repression happens as a consequence of the attachment of methyl groups to promoter region of genes, which subsequently recruits methyl cytosine-guanine base pairing $(\mathrm{CpG})$ binding domain proteins. These $\mathrm{CpG}$ binding domain proteins modulate gene expression by acting as a blockade and subsequently keeping DNA in a closed state (i.e., heterochromatic), thereby leaving transcription factors (TFs) and RNA polymerase unable to access the DNA $(7,26,38)$. Although exceptions exist, DNA methylation located at promoter or enhancer regions of DNA almost exclusively attenuates gene expression. Therefore, while excluding other variables, higher levels of methylation generally amplifies transcriptional repression.

Aging is a biological process that is generally associated with an altered phenotype, with emerging evidence to suggest these changes are connected to epigenetic/epigenomic changes (51). In fact, scientific data indicates that the epigenome continuously maturates over the course of a person's lifespan and changes that occur with aging appear progressively due to a person's interactions with their environment (51). Although other factors also account for age-associated modifications in pathway signaling, a large portion of the changes can be linked to epigenomic mechanisms. Thus, epigenetic/epigenomic modifications of gene expression due to various genetic and environmental factors subsequently drives age-dependent patterns of gene expression. Interestingly, in post-mitotic tissues such as skeletal muscle, evidence suggests that DNA methylation is particularly actively involved due to an individual's interactions with their surrounding environment (16, 26, 38, $51)$.

For the previous two decades, our laboratory has established an in vivo rodent dynamometer model of stretch-shortening contraction (SSC) resistance-type exercise training (RTET) in order to explore the biomechanical, biochemical, and physiological effects of various skeletal muscle training paradigms (12, 34). Having a model that employs these type of movements is physiologically relevant because SSCs are inherent in 
activities of daily living as well as in the vast majority of exercise movements (e.g., walking, sprinting, jumping, resistance training, plyometrics, etc.) unlike sequestered isometric, concentric, or eccentric only movements $(36,54)$. The responses to RTET using this physiological model has been previously shown to range from adaptation, characterized by enhanced skeletal muscle performance along with increased muscle mass, to maladaptation, defined as an absence or diminishment of skeletal muscle performance and no improvements in muscle mass $(11,30,36,37)$. With respect to chronologically advancing age, previous data from our laboratory indicates that an altered adaptive phenotype following this validated, SSC RTET protocol occurs prior to complete biological development of the rodent - six months of age in the adult rat, and we believe this may point toward the onset of a loss in homeostatic control leading to an age-specific biological departure from the SSC-training adaptive response (35). Interestingly, this is much earlier than hypothesized and the exact explanations for this finding are unknown. Therefore, investigating if and how an initial, chronic bout of SSC RTET exposure affects the altered adaptive response observed in the adult rodent is crucial (11, 37).

Excitingly, recent research has shown exercise can modulate the amount of DNA methylation present in skeletal muscle. For instance, acute exposures to varying intensities of aerobic exercise have altered methylation levels as well as expression of genes related to muscle metabolism, mitochondrial biogenesis, and muscle growth in both human and rodent skeletal muscle tissue (3, 23, 25, 27). In regards to chronic exercise, past findings from others suggest that alterations in methylation are associated with changes in expression of genes related to muscle adaptation $(23,26,29,31,32)$; collectively, these studies demonstrated that a physiological stimulus with well-known health benefits (i.e., exercise) leads to epigenomic modifications in the form of DNA methylation, and these changes are associated with changes in gene expression as well as performance.

In general, the term muscle memory has been applied in the strength and conditioning, sport, and exercise domains as a general term describing the capability of skeletal muscle to respond more quickly to an applied stimulus that someone has been exposed to previously, making it more responsive and capable in its ability to rebuild muscle mass and strength in spite of periods of inactivity $(20,46)$. There is scientific evidence supporting this phenomenon in the human performance literature (22, 42, 49, 50). For example, a well-designed study performed by Taffe and colleagues (50) was carried out in which 13 men and women performed 24 weeks of resistance training, followed by 24 weeks of detraining, and then had a subsequent 12 weeks of retraining. The results of this study demonstrated that resuming resistance training following a detraining period significantly attenuated intramuscular fat content, improved muscle mass, and led to increased strength in older healthy adults compared to baseline and following the detraining period, while being similar to levels seen during the initial 24 week training period. 
Historically, the term muscle memory has been applied primarily in the context of motor learning and neurological adaptations to training $(15,40)$. However, a recent, very eloquent review article by Sharples et al. (46) endorsed the idea of a cellular based "skeletal muscle memory" in the context of epigenomic regulation by defining it as "the capacity of skeletal muscle to respond differently to environmental stimuli in an adaptive or maladaptive manner if the stimuli have been previously encountered.” In other words, the authors suggested that in this context muscle memory could refer to the ability of the tissue to retain prior environmental stimuli, such as adaptation to exercise training, which would change the responsivity of the muscle when that exact stimulus is reintroduced at a later time period. Under these circumstances, skeletal muscle could have the ability to retain cellular/molecular information after encountering a previous adaptive environmental stimulus in a manner that "primes" the tissue so that when it is re-exposed to that same stimulus it can bring about subsequent adaptation (i.e., "muscle epi-memory”) in an efficient manner (46). For instance, if the environment of the muscle is anabolic, leading to positive epigenomic adaptations in growth synthesis and muscle remodeling pathways, skeletal muscle cells may retain these modifications and subsequently influence the regulation of gene activity in a positive manner that would allow the muscle to be more likely to respond favorably to this same stimulus at later life by maintaining or perhaps even increasing muscle mass across the lifespan of the organism (46).

Research by this same group of investigators from the review by Sharples et al. (46) have begun to unravel the basis of a cellular form of muscle memory within the context of a training-retraining (TRT) paradigm in the human literature. The first study to provide scientific evidence for the existence of a cellular epigenetic/epigenomic muscle memory in skeletal muscle in response to RTET was by Seaborne et al. (43), who attempted to characterize a TRT paradigm by means of genome-wide methylation analysis in human skeletal muscle using previously untrained young male adults. For this study, participants completed an acute bout of resistance exercise, followed by seven weeks (three days per week) of RTET, had a subsequent seven week detraining period, and finally resumed a seven week (three days per week) RTET program. The main finding of the study was that there were 18,816 hypomethylated CpG sites following the retraining period, higher than at any other time point measured (i.e., baseline, acute, and the initial loading phases). Of particular note, the results showed significant hypomethylation within the PI3K-AKT pathway, a well-known regulator of muscle adaptation in response to RTET, when comparisons were made between baseline versus initial loading, as well as in the detrained versus retrained state. Other studies have come out with similar findings in support of the previous study mentioned $(28,41,53)$, thus providing reinforcing the idea that skeletal muscle is indeed able to retain epigenomic information from an exercise training stimulus in earlier life that can subsequently be reapplied upon introducing the same stimulus at a later age. 
Despite the therapeutic potential and supporting evidence for exercise, its application in positively influencing the total health of an individual across the lifespan is still not fully resolved. Moreover, even with the suggested underlying basis for the involvement of epigenetic/epigenomic regulation in the wherewithal of skeletal muscle to adapt to exercise, no studies have determined whether or not alterations in DNA methylation that occur as a result of exposure to an adaptive or maladaptive exercise stimulus using an in vivo physiological animal model of RTET can have a lasting influence on the epigenomic responsivity and adaptability of skeletal muscle upon reintroduction to the same stimulus at later life. Based on the therapeutic efficacy suggested by high-intensity SSC RTET for counteracting age-related declines in muscle mass and function, investigating the TRT paradigm could have important implications for furthering our understanding of muscle adaptation. In addition, showing beneficial effects in later life from SSC RTET at an earlier age has enormous therapeutic ramifications in preventing and/or decreasing the susceptibility to diseases directly associated with aging, such as sarcopenia, ultimately resulting in increased lifespan and healthspan (i.e., quality of life) in our increasingly growing aging population.

Therefore, the purpose of this study was to use a validated and reliable in vivo rodent model of highintensity SSC RTET in order to investigate the therapeutic efficacy of a RTET TRT paradigm in training at an earlier age on the prevention and/or attenuation of impairments of skeletal muscle during the later stages of life. Our hypothesis was that training resulting in adaptation at three months of age would lead to enhanced adaptation at six months of age upon reintroduction to the same exact high-intensity SSC RTET stimulus relative to naïve six month old rodents not receiving this previous bout of exercise training. Furthermore, these positive effects in adaptive outcomes (e.g., muscle performance, muscle quality) would be complemented by a molecular and DNA methylation signature in TFs signaling that would provide underlying support for the existence of a cellular form of muscle memory that subsequently helps aid in allowing adaptation to occur.

\section{MATERIALS AND METHODS}

\section{Animals}

Young (3 mo.) and adult (6 mo.) male Fischer x Brown Norway (F344xBN) rats were obtained from the National Institute of Aging colony and housed in an Association for Assessment and Accreditation of Laboratory Animal Care International-accredited animal quarters. All animal procedures were approved by the Animal Care and Use Committee at the National Institute for Occupational Safety and Health in Morgantown, WV and were in compliance with the NIH guide for the care and use of animals in research.

\section{RTET Paradigm}

F344xBN male rats were randomly assigned to a high-intensity SSC RTET protocol in one of the 
following two groups (Figure 1): 1) Young rats that underwent a TRT paradigm in which they were SSC-loaded three days per week for one month at three months of age, subsequently detrained for two months by simply staying in their cages without exercising, and finally were retrained again by undergoing a second one month SSC-loading bout at six months of age $(n=5) ; 2)$ adult rats SSC-loaded three days per week for one month only at six months of age, and therefore did not have a prior bout of high-intensity SSC RTET and were previously naïve to the exercise stimulus (6-month exercise control; $n=5)$. Six months of age was chosen because previous work from our laboratory demonstrated this was the age at which an altered adaptive phenotype following a validated, high-intensity SSC RTET protocol started to occur, thus may represent the potential starting point at which there is an age-specific biological departure from the SSC-training adaptive response (35). The loading paradigms were identical with the exception of the presence or absence of an initial one month bout of highintensity SSC RTET at three months of age. Training commenced on Mondays, Wednesdays, and Fridays, with 48 hours rest between training sessions. The training protocol was based on a previously validated, highintensity SSC RTET protocol $(11,12,37)$. Briefly, rats were anesthetized with isoflurane gas and placed in dorsal recumbency on a heated table with the left knee secured in flexion at $90^{\circ}$ and the left foot secured in a load cell. Platinum electrodes were then placed subcutaneously for activation of the dorsiflexor muscles. Muscle stimulation parameters (4-V magnitude, 0.2-ms pulse duration, and 120-Hz frequency) were based on previous studies of this model that found these settings to be optimal for producing supramaximal force outputs of the tibialis anterior (TA) muscle in male rats $(11,12)$. Training consisted of 80 total SSCs per session, which was comprised of eight sets of 10 repetitions, with two minute rest intervals between sets. For every SSC, the muscles were maximally activated, with the ankle angle set to $90^{\circ}$ flexion for $100 \mathrm{~ms}$, rotated out to $140^{\circ}$ flexion at a movement velocity of $60^{\circ}$ s, then returned back to $90^{\circ}$ at $60^{\circ} / \mathrm{s}$. Using this protocol, age-specific performance adaptation has been shown to occur along with a concomitant absence of overt muscle inflammation and degeneration in the days and weeks following training $(1,2,11,37)$. Static and dynamic muscle performance was assessed prior to the beginning of each training session by measurement of peak force, which was recorded and quantified using MatLab software (Version 9.3; MathWorks ${ }^{\circledR}$, Natick, MA, USA).

\section{INSERT FIGURE 1 HERE}

Seventy two hours after the final SSC-loading protocol, rats were euthanized and the TA muscle was surgically removed, weighed, and recorded in grams (i.e., wet-weight). Seventy two hours was chosen because this is considered beyond the acute effects of the first several hours post-training, but within the timeline where any overt signs of muscle degeneration as a consequence to the last training session would occur (37). Muscle quality (MQ) was derived from both static/dynamic performance and muscle mass values by taking the peak 
force and dividing by the normalized (i.e., muscle mass relative to tibia length) muscle mass values. The midbelly of the TA was coated with tissue freezing media (Tissue-Tek, 4583 O.C.T Compound; Sakura Finetek, Torrance, CA, USA) and frozen in isopentane cooled to the temperature of liquid nitrogen and stored at $-80^{\circ} \mathrm{C}$ prior to conducting quantitative morphology, gene expression, and protein analysis.

\section{Gene Expression Analysis}

Muscle tissue stored at $-80^{\circ} \mathrm{C}$ was retrieved and a $50 \mathrm{mg}$ portion was homogenized using a MiniBeadbeater 8 (Biospec Products, Bartlesville, OK, USA) with $1 \mathrm{ml}$ TRIzol and $1.0 \mathrm{~mm}$ zirconia beads (Catalog \#22079110zx; Biospec). RNA was subsequently isolated using the RNAqueous ${ }^{\mathrm{TM}}$ Phenol-free total RNA Isolation Kit (Cat\# AM1912; Life Technologies, Carlsbad, CA, USA) following kit instructions. Next, cDNA was synthesized using the RT ${ }^{2}$ First Strand Kit (Catalog\# 330401; Qiagen, Valencia, CA, USA). Samples were then analyzed using the Rat Skeletal Muscle Transcription Factors RT² Profiler PCR Array (Catalog\# PARN075Z; Qiagen) and $\mathrm{RT}^{2}$ SYBR Green Mastermix (Catalog\# 330523; Qiagen) per manufacturer’s instructions with use of an ABI PRISM 7500 instrument (Applied Biosystems, Foster City, CA, USA). The RT ${ }^{2}$ Profiler PCR Array was able to simultaneously assay 84 genes related to the TFs signaling pathway (Table 1). Fold changes and $P$ values were determined from comparisons of $\Delta \mathrm{C}_{\mathrm{t}}$ values between SSC-loaded and non-loaded control (NLC) muscles (i.e., the contralateral limb not subjected to RTET) and analyzed through the GeneGlobe Data Analysis Center (Qiagen). Five housekeeping genes [Actin beta (ACTB), beta-2 microglobulin (B2M), hypoxanthine phosphoribosyltransferase 1 (HPRT1), lactate dehydrogenase 1 (LDHA), and ribosomal protein, large, P1 (RPLP1)] were included on the array. The gene ACTB was chosen as the reference gene for normalization of values generated through GeneGlobe for the TFs array because it displayed the most stable results. Bioinformatic analysis was performed using IPA (Ingenuity Systems, www.ingenuity.com), which allowed for functional annotation of differentially expressed genes. All IPA biological functions except cancer specific, reproductive system, and psychological functions were evaluated.

\section{INSERT TABLE 1 HERE}

\section{DNA Methylation Analysis}

Total DNA was isolated using the DNeasy ${ }^{\circledR}$ Blood \& Tissue Kit (Qiagen) according to kit instructions. DNA extracts were subsequently prepared for methylation analysis following restriction digestions using the EpiTect Methyl II DNA Restriction Kit provided by Qiagen. Digested DNA samples were analyzed using the Rat Stem Cell Transcription Factors EpiTect Methyl II Signature PCR Array (catalog no. EARN-511ZA; Qiagen), which profiled the promoter-specific methylation status of a panel of 22 genes related to this pathway 
(Table 2). After digestion, the remaining DNA in each individual enzyme was quantified using quantitative real-time polymerase chain reaction (qRT-PCR). The qRT-PCR arrays were performed in an ABI PRISM 7500 instrument (Applied Biosystems). In a similar manner to previous studies (24, 30, 56), data was analyzed using the EpiTect Methyl II PCR Array system, which provided an integrated Microsoft Excel-based template that automatically performed all $\Delta \mathrm{Ct}$ based calculations from raw $\mathrm{Ct}$ values in order to quantify the percentage of DNA that was methylated for each gene on the array. The method employed by the Epitect Methyl II PCR System was based on the detection of remaining input DNA following cleavage with a methylation-sensitive and/or methylation-dependent restriction enzymes, which digested unmethylated and methylated DNA, respectively $(33,56)$. The relative fractions of methylated and unmethylated DNA for each gene of interest on the arrays were determined by comparing the amount in each digest with a "mock digest” (i.e., no enzymes added) using the $\Delta \mathrm{Ct}$ method (56).

\section{INSERT TABLE 2 HERE}

\section{Total RNA, DNA, and Protein}

RNA was isolated following the protocol outlined in the previous section on gene expression. Total DNA was isolated using the DNeasy ${ }^{\circledR}$ Blood \& Tissue Kit (Qiagen) according to kit instructions. Both RNA and DNA concentrations were obtained using a spectrophotometer (ND-1000; NanoDrop Technologies Inc., Wilmington, DE, USA). Cytoplasmic protein isolation extracts were obtained from the TA muscles using a method previously described by Siu et al. (48). Total protein contents of the cytoplasmic protein extracts were quantified and reported as protein concentration ( $\mu$ g protein/mg tissue - [protein]) in triplicate using the Pierce ${ }^{\mathrm{TM}}$ BCA Protein Assay Kit (Thermo Scientific). Additionally, values for the [RNA] to [DNA] ratio (i.e., assumed to be a surrogate measure of global transcriptional efficiency) and the [protein] to [RNA] ratio (i.e., assumed to be a surrogate measure of global translational efficiency) were analyzed (39).

\section{Statistical Analysis}

Peak force (means and percent difference), muscle mass (absolute and relative), MQ, DNA methylation, muscle RNA, muscle DNA, muscle protein, muscle transcriptional efficiency, and muscle translational efficiency following the one month exposure at six months of age for both groups were all analyzed using a mixed model analysis of variance (ANOVA) with the variable animal identification as a random factor to account for repeated measures (JMP version 13; SAS Institute, Inc., Cary, NC, USA). The peak force for the TRT group during the initial one month bout of SSC RTET at three months of age was analyzed using a one way mixed model ANOVA; the percent change for isometric and SSC peak force in the TRT group for this 
same training period was analyzed using a one-sample t-test in order to examine whether the data were different from a distribution that had a mean of zero (JMP version 13). The TFs PCR array data was analyzed using unpaired student’s t-test in the GeneGlobe Data Analysis Center (Qiagen) online platform. All data are expressed as means \pm SE, with $p<0.05$ being considered statistically significant.

\section{RESULTS}

\section{Dorsiflexor Muscle Performance, Muscle Mass, and Muscle Quality}

Muscle performance measured via isometric and SSC peak force performed prior to each training session for rodents in the TRT group exposed to the initial one month bout of high-intensity SSC RTET is shown in Figure 2. In response to this initial SSC-loading, TRT rodents displayed adaptation to the initial exercise stimulus as indicated by significant increases in both peak isometric and peak dynamic SSC force following one month of exercise. Additionally, the percent change for peak isometric (15.4 $\pm 3.4 \%)$ and peak dynamic SSC force (17.8 $\pm 5.3 \%)$ output were both found to be significantly different $(\mathrm{p}<0.05)$ from a distribution with a mean of zero when a two-tailed one-sample t-test was performed. Collectively, these results demonstrated that this initial bout of SSC-loading was effective in promoting adaptation and corroborates with previous research in our laboratory showing that one month of training enhances muscle performance in young three month old rats $(11,30,35,37)$.

\section{INSERT FIGURE 2 HERE}

Following two months of detraining in which these same rodents did not exercise, they were retrained following the same exercise stimulus for one month at six months of age and were compared to age-matched counterparts that underwent the same exercise stimulus as the TRT group but did not receive this initial one month training bout and therefore were naïve to this exposure prior to the SSC RTET protocol; the muscle performance responses to this training bout are shown in Figure 3. In response to dorsiflexion SSC-loading, isometric and SSC peak force was unchanged in the 6-month trained group; meanwhile, TRT rats adapted to SSC-training as indicated by significantly higher isometric and SSC peak force following training, which was also significantly higher compared to the final peak force in the 6-month trained only group (p<0.05) (Figure 3a and Figure 3c). When looking at relative changes in peak force, there were no significant differences between groups in isometric performance between the 6-month trained only group versus TRT (6.2 $\pm 6.3 \%$ vs.

14.6 $\pm 3.9 \%$; Figure 3b), but there was a significant difference in relative SSC performance between the two groups (-0.2 $\pm 5.2 \%$ vs. $12.5 \pm 1.7 \%, \mathrm{p}<0.05$; Figure $3 \mathrm{~d})$. 


\section{INSERT FIGURE 3 HERE}

Muscle mass data is shown in Figure 4. Both groups exhibited significantly increased muscle mass following one month of high-intensity SSC training, both in terms of absolute as well as normalized (i.e., muscle mass relative to tibia length) values in the SSC-loaded limb relative to NLC muscle ( $<<0.05)$ (Figure 4a and 4b). However, TRT also had significantly higher muscle mass in comparison to the 6-month trained only group for both absolute and normalized muscle mass values $(\mathrm{p}<0.05)$. Finally, while static (i.e., isometric) $\mathrm{MQ}$ was not different between groups ( $\mathrm{p}>0.05$ ), dynamic (i.e., SSC) MQ in the TRT group exhibited a trend ( $p=0.09)$ for being higher than the 6-month trained only group $(0.94 \pm 0.04 \mathrm{~N} / \mathrm{mg}$ per mm vs. $0.84 \pm 0.03 \mathrm{~N} / \mathrm{mg}$ per mm).

\section{INSERT FIGURE 4 HERE}

\section{Transcription Factors DNA Methylation, Gene Expression, and Bioinformatics Analysis}

The stem cell TFs pathway methylation profile from the EpiTect Methyl II PCR array is shown in Figure 5. When looking collectively at the entire pathway, there was no effect of age at baseline in the percentage of DNA methylation between young and old NLC, nor was there any effect of training on methylation levels in either group ( $>>0.05)$. However, following one month of high-intensity SSC RTET, there were significantly lower levels of methylation in the SSC-loaded limb of TRT compared to the 6-month trained only group $(\mathrm{p}<0.05)$. On an individual gene level, no genes were found that either had significantly higher levels of methylation with concomitant downregulation of gene expression nor significantly lower levels of methylation with a simultaneous upregulation of gene expression from the pathway array.

\section{INSERT FIGURE 5 HERE}

The significantly differentially expressed genes (SDEGs) in Rat Skeletal Muscle Transcription Factors RT $^{2}$ Profiler PCR Array are shown in Table 3 and Figure 6. The SDEGs were defined as having a fold change value of being up- or down-regulated by 1.1 fold or more along with a p-value less than 0.05 . The TRT group had 17 SDEGs compared to just three in the 6-month trained only group in the TFs signaling pathway; all but two of the genes in TRT were downregulated, while all three SDEGs were upregulated in 6-month trained only. Notably, the two genes upregulated in TRT were activating transcription factor 3 (ATF3) and myogenic factor 5 (MYF5), two genes that have previously been implicated in skeletal muscle to be highly involved in the stress and/or hypertrophic response to exercise $(17,18,21)$. 
INSERT TABLE 3 HERE

INSERT FIGURE 6 HERE

Bioinformatics analysis using IPA is shown in Table 4; the top canonical pathways in IPA revealed that both groups had similar physiological system and development function categories related to development and organ/tissue morphology. However, apparent differences between TRT and the 6-month only group were also revealed in the IPA analyses. For instance, the top canonical pathways in the 6-month only group were mainly associated with cell cycle regulation and stem cell pluripotency, such as wingless-related integration site $(\mathrm{WNT}) / \beta$-catenin signaling, S-phase entry, and G1/S checkpoint regulation, whereas the TRT group had canonical pathway signaling mainly related to growth and developmental regulation, such as WNT/Ca+ pathway, PI3K signaling, glucocorticoid receptor signaling, and protein kinase A signaling. In addition, the 6month only group had just one top network, which was related to “organismal injury.” Because they only had one network, we were unable to perform a top-merged network functions analysis. When analyzing the top processes based upon the one available top network included "tissue fibrosis" and "gene silencing” ( $<<0.0001$ for all). In comparison, the TRT group had three top networks, all of which were related to growth and tissue remodeling (e.g., gene expression, organ morphology). Importantly, after the three networks were merged in order to generate top merged-network functions, the top five processes in the merged analysis based on p-value in the TRT group included "transcription of DNA," "activation of DNA endogenous promoter," "transcription of RNA," "transcription,” and “transactivation of RNA” ( $<<0.0001$ for all).

\section{INSERT TABLE 4 HERE}

\section{Muscle RNA, DNA and Protein}

Figure 7 displays skeletal muscle concentrations for DNA, RNA, and protein. There were no age or treatment effects for any group on muscle [DNA] nor [RNA] (p>0.05) (Figure 7a-7b). For muscle [protein], the 6-month only group displayed a significant attenuation following one month of high-intensity SSC RTET compared to NLC muscle ( $\mathrm{p}<0.05)$; additionally, the trained limb of the TRT group had significantly higher muscle [protein] in comparison to the trained limb of the 6-month only group $(\mathrm{p}<0.001)$ (Figure 7c). There were no significant effects of training nor treatment $(\mathrm{p}>0.05)$ on the surrogate muscle translational efficiency index for either group (data not shown). 


\section{DISCUSSION}

The main findings from this study are: 1) There is a distinct molecular signature following high-intensity SSC RTET utilizing a TRT paradigm that accompanies adaptive outcomes, and these responses appear to be connected to the TFs signaling pathway, with a distinct pattern of enhanced regulation of specific molecules; 2) the positive adaptive effects from a bout of RTET at an earlier age resulted in augmented positive adaptive outcomes during adulthood, including enhanced muscle performance, muscle mass, and muscle protein; 3) DNA methylation results were in agreement with previous findings demonstrating an effect of training at earlier life on causing decreased methylation when the stimulus was reintroduced at later life, confirming this is a highly influential epigenomic process involved in the underlying muscle memory response to exercise in aged skeletal muscle; and 4) this work advances previous findings from our laboratory demonstrating the therapeutic efficacy of our in vivo physiological rodent dynamometer model of SSC RTET aiding in the adaptability of aged muscle, and also complements, as well as builds upon, the TRT paradigm that had been previously shown in the human literature.

To determine if muscles are capable of adapting to high-intensity RTET at a later period of time following a previous initial training bout, we exposed muscles of young rats at three months of age to 80 SSCs, three days per week (72 hours between training bouts) for one month and had them detrain for two months before re-exposing them to the same exercise stimulus for one month during adulthood at 6 months of age, then directly compared them to a separate group that only trained at 6 months of age. Similar to the response to 80 SSCs, three days per week exposure that our previous work has shown to augment adaptive outcomes following training, $(85,86,88,89)$, the TRT exposure paradigm resulted in positive adaptations reflected by increases in static and dynamic skeletal muscle performance, as well as muscle mass, upon reintroduction to the same exercise stimulus at six months of age. This result is particularly noteworthy because our laboratory had previously demonstrated this was the age in which an altered adaptive phenotype occurred following this same exact high-intensity SSC RTET protocol, which we believed may represent the point of onset that a loss in homeostatic control leading to a biological departure from the SSC-training adaptive response occurs (35). Our results shown in the 6-month trained only group confirm our previous work, as they demonstrated limited increases in skeletal muscle mass concomitant with no improvements in isometric nor dynamic muscle performance following one month of training. In contrast, six month old rats that had received a previous bout of this adaptive exercise stimulus at three months of age not only had augmented improvements in both skeletal muscle performance and muscle mass gains compared to contralateral NLC muscle, but were also significantly greater compared to the SSC-loaded limb of rats that only trained at six months of age. This implies that adult rodent muscles are robust in their ability to adapt to parameters of the biomechanical loading signature in a high-intensity SSC RTET paradigm only following a previously encountered bout of the same exercise at earlier 
life, hence lending supportive evidence to previous literature showing an existence of muscle memory, but for the first time using an in vivo, validated mechanical loading animal model of high-intensity RTET.

Our results clearly showed that adult rats who followed the TRT paradigm and received a prior adaptive exercise exposure at three months of age were superior over rats that trained only at six months of age as indicated by the ability to undergo adaptation via increases in skeletal muscle performance and improved muscle mass, along with a trend for greater MQ. Indeed, with appreciation for the current molecular data described herein (i.e., profiler array gene expression, IPA, and muscle protein), as well as that of skeletal muscle performance and muscle mass values which are complementary to other investigations in humans using a TRT paradigm (22, 41, 43, 49, 50), this study has provided evidence that high-intensity RTET prescribed in the appropriate manner at an earlier age can be greatly beneficial for adult skeletal muscle that has aged when the same exercise stimulus is reintroduced at later life, which can be particularly beneficial for those that have to be sedentary for an extended period of time due to injury and/or disease.

Long-term adaptations to RTET occur as a result of an accumulative effect of repeated, acute increases in gene expression for exercise-induced pathways of skeletal muscle growth, stress, homeostasis, and remodeling that follow each individual exercise stimulus $(21,28)$. However, the present data is suggestive at a molecular level that skeletal muscle cells retain an ability to more aptly adapt to a high-intensity SSC RTET paradigm only after encountering a previous adaptive bout of RTET at an earlier time point compared to naïve animals at the same age, and this is accompanied by a distinct epigenomic profile and gene expression signature. For instance, adult rats following the TRT paradigm had significantly less DNA methylation in the stem cell TFs pathway compared to the six month trained only group. Interestingly, although the TRT group adapted as reflected by increased skeletal muscle performance, muscle mass, and muscle protein, there were differences in the responses underlying adaptation in comparison to the group that trained just at six months of age which only partially adapted. Specifically, there was primarily a downregulation in TFs gene expression in the SSC-loaded limb of the TRT group. The downregulation of TFs was unanticipated; however, there is evidence to indicate that individuals who adapt to resistance training results in an increase in ribosomal biogenesis that serves as a key underlying process regulating training-induced muscle adaptation (49). Moreover, Rader and colleagues (36) showed a downregulation of genes related to myogenesis and muscle energetics in young C57BL/6 mice after one month of high-intensity SSC RTET (36). Therefore, it is plausible that in response to RTET training that is properly prescribed to promote adaptation at a younger age, individuals respond upon reintroduction to the same exercise stimulus later on in life by becoming highly efficient at the transcriptional level (partially as a result of decreased methylation) in that they require less TF signaling at each subsequent "new" baseline, as well as at the translational level since protein synthesis is more proficient. Alternatively, given that older individuals’ systemic milieu is desensitized at onset, appropriate, age-specific 
RTET must initially “re-establish/restore” a “younger” baseline, which was manifested in the six month trained only group in the TFs pathway as there were only three SDEGs but they were all upregulated; the specific mechanisms accounting for these differences should be the subject of future investigations.

Interestingly, the two genes significantly upregulated in the TRT group, ATF3 and MYF5, have previously been implicated in the adaptive growth and cellular stress response to SSC RTET. ATF3 has been demonstrated to increase in response to stress signals and is generally thought to be involved in the cellular stress response by regulating the expression of inflammation-related genes in order to maintain homeostasis (17). Moreover, its expression has previously been shown to increase following exercise, including RTET; for instance, ATF3 was found to modulate chemokine and cytokine expression in mouse skeletal muscle following endurance running, thereby aiding in the molecular adaptations to exercise training (17). Moreover, ATF3 was shown to regulate the expression of chemokines both in vitro using C2C12 myotubes following electrical stimulation and in vivo in mouse skeletal muscle following eccentric downhill running (18). MYF5 is part of a family of TFs related to myogenesis, defined as the process of forming new muscle tissue, either through repair to injury or remodeling in response to exercise training. Myogenesis is a tightly orchestrated process controlled by a family of proteins called the myogenic regulatory factors, which includes MYF5. Satellite cells are one of the key contributors for postnatal skeletal muscle growth during myogenesis; MYF5 and other myogenic regulatory factors are transcriptional and epigenetic regulators of myogenesis that act to control satellite cell fate and thereby facilitate muscle repair/remodeling (55). The majority of satellite cells express MYF5 and are committed to the muscle lineage, while a small subset of satellite cells that do not contain MYF5 are considered satellite "stem" cells whose primary purpose is to maintain the overall satellite cell pool of for future use (55). Therefore, the present results indicate that in a TRT paradigm that despite an overall downregulation of TFs, there is a selective upregulation in specific molecules crucial for growth and homeostasis that are subsequently aiding to promote adaptation at an age previously shown to have a decreased adaptive capacity.

Previous work has found that chronic exercise training leads to alterations in DNA methylation of genes important in the regulation of skeletal muscle. Nitert et al. (32) examined human skeletal muscle from adult males following six months of aerobic exercise; the main findings of the study were that six months of endurance exercise resulted in significant reductions in promoter DNA methylation of genes with known functions in both muscle and type 2 diabetes mellitus, including thyroid adenoma associated (THADA), myocyte enhancer factor 2A (MEF2A), runt related transcription factor 1 (RUNX1), and NADH dehydrogenase [ubiquinone] 1 subunit C2 (NDUFC2). Additionally, the amount of gene expression of THADA, MEF2A, RUNX1, and NDUFC2 were all negatively correlated with higher levels of DNA methylation. Kanzleiter and colleagues (23) had nine-week old male mice perform five days of endurance treadmill exercise training for four weeks. An initial bisulfite sequencing analysis found that compared to sedentary controls, exercised mice 
had 2,762 genes that displayed differentially methylated CpGs in their promoter regions. Subsequent evaluation of gene expression data revealed approximately 200 out of 2,762 genes with negative correlations between CpG methylation status and gene expression, with most of these genes being related to muscle growth, differentiation, and metabolism. Nguyen et al. (31) used a male mice model of severe dyslipidemia as a consequence to a deficiency of low-density lipoprotein receptor and had them go through three months of endurance exercise using a voluntary wheel. The investigators found that one of the most important antioxidant enzymes, glutathione peroxidase 1 had significant alterations in gene expression along with significantly decreased DNA methylation following the end of training.

Finally, Lindholm and colleagues (29) conducted a three month one-legged exercise training study in young adult humans using the non-exercise leg as an inter-individual control. Training was comprised of onelegged knee extension endurance training on a cycle four times per week followed by two unilateral knee extension performance tests implemented on a modified cycle prior to and following the end of the three month training period. The authors described that the main findings were that endurance training induced significant changes in methylation (primarily at the enhancer regions) at approximately 5,000 sites across the genome along with significant differential expression of roughly 4,000 genes. Collectively, the authors concluded that a physiological stimulus with well-known health benefits (i.e., exercise) induced epigenomic modifications in the form of DNA methylation, and that these changes were associated with alterations in gene expression as well as performance (29). Therefore, our results of decreased methylation in the stem cell TFs pathway in the SSCloaded limb of the TRT group in comparison to the six month trained only group are consistent with past findings showing alterations in DNA methylation status following adaptive exercise with respect to chronic exercise training .

However, the interpretation of these results are limited by the fact that on an individual gene level, there were no genes found that had either significantly higher levels of methylation with concomitant downregulation of gene expression nor significantly lower levels of methylation with simultaneous upregulation of gene expression from the pathway array. The main reason for this finding were that unfortunately only a handful of genes (11 out of the 22 total genes) that were on the stem cell TFs methylation array were also on the profiler pathway gene expression array. Out of the minority of genes that were on both arrays, only three of them were either SDEGs or showed a trend for having increased or decreased expression, which were FBJ osteosarcoma oncogene (FOS), myelocytomatosis oncogene (MYC), and GATA binding protein 2 (GATA2). As stated previously, none of these three genes were found to have significantly decreased methylation in the SSC-loaded relative to NLC muscle following one month of high-intensity SSC RTET, indicating that changes in expression may be controlled through other post-translational modifications and/or other epigenetic/epigenomic mechanisms, such as acetylation. However, from an overall pathway and signaling cascade perspective, our 
results for TF gene expression and DNA methylation showed that an overall decline in methylation along with the selective upregulation in a small subset of genes important in the growth and regulation of skeletal muscle are playing an important role in the adaptive responses (i.e., significantly improved muscle performance as well as increases in muscle mass and muscle protein) that occurred in adulthood as a result of a previous one month exposure to high-intensity SSC RTET at a younger age.

In general, the term muscle memory has been limited in its application to only describe changes within the context of motor learning and/or neurological based adaptation to training. However, as mentioned previously, a recently published review paper by Sharples et al. (46) promoted the existence of muscle memory through epigenetic/epigenomic regulation in response to an adaptive or maladaptive stimulus from a previously encountered bout of exercise. Since environmental stimuli and stressors modify changes in gene expression, epigenetics/epigenomics, with DNA methylation being a prime candidate mechanism, are highly likely to form the underlying foundation for skeletal muscle memory $(38,46)$. Recent evidence has emerged giving credence to the existence of a cellular form of muscle memory. This was first reported by Bruusgaard et al. (4), in which the researchers employed a synergistic ablation (SA) model where the TA muscles of mice were surgically removed from one leg, thus overloading the remaining extensor digitorum longus muscles as they moved around their cages; the authors then used live in vivo imaging in order to track and quantify myonuclei over an overload period followed by surgical denervation. Two weeks of overload with SA resulted in a near even gain in both myonuclei and skeletal muscle CSA (37\% vs. 35\%, respectively). When surgical denervation was introduced following the initial overload period, skeletal muscle CSA significantly and rapidly declined by approximately $40 \%$ as a result; however, the total number of myonuclei held steady with a very slight, nonsignificant decrease in number despite the loss of muscle CSA during the denervation period. These results indicate that the addition of nuclei seems to be long lasting and persistent even through a period where the skeletal muscle was denervated. The authors proposed a model on the relationship between myonuclei number and skeletal muscle hypertrophy in which an increase in myonuclei number precedes muscle mass gains, with a subsequent detraining period leading to muscle atrophy but little to no loss of myonuclei. Subsequently, a previous exposure to skeletal muscle hypertrophy (e.g., SA, mechanical overload, etc.), would bring about more rapid gains in muscle mass to reach previous levels in comparison to those without this previous experience, thus forming the underlying basis for a cellular muscle memory. As a follow up to this study by Egner et al. (15), mice administered testosterone for 14 days showed significantly robust increases in myonuclei number as well as skeletal muscle mass. The authors were also able to illustrate that not only were these myonuclei retained following the removal of the drug, despite skeletal muscle mass going back down to baseline levels, but that a subset of mice re-exposed to a subsequent physical loading stimulus exhibited significantly increased muscle mass relative to control mice. The authors concluded that although skeletal muscle hypertrophy can be 
reversed as a consequence of an atrophic stimulus (e.g., inactivity), a previous anabolic event leaves a lasting imprint on the skeletal muscle fiber in the form of increased myonuclei and suggested that because myonuclei increase as a result of RTET, it may benefit younger individuals to perform this type of exercise modality in order to gain benefits later at advanced age (15).

However, a very recent study by Dungan et al. (14) trained adult female mice for eight weeks using a progressive weighted wheel running model and found results in direct contrast to this previous study. Specifically, the authors demonstrated that following the cessation of training, the plantaris muscle was in an untrained state as reflected by decreased skeletal muscle mass and, more importantly, had less myonuclei following detraining, which is in direct disagreement with the previous studies mentioned $(4,15)$. As stated by the authors, one of the biggest factors for the conflicted results could be the model; these authors used a volitional, non-surgical, physiological model of exercise training, which is fully reversible due to the noninvasiveness compared to the SA model. However, differences also exist as well between all the studies that confound the interpretation of the results, which included the use of different strains of mice and a lack of reported ages by Bruusgaard's and Egner's groups $(4,15)$. Most prominently, all of these studies did not incorporate a true detraining phase followed by a retraining period to simulate past work done in human exercise studies previously mentioned (22, 42, 49, 50), which limits the translation of the results for application towards the concept of skeletal muscle memory within the context of exercise training and a TRT protocol.

As mentioned previously, recent studies have begun to elucidate the existence of a cellular form of skeletal muscle memory within the context of TRT paradigm in the human literature. Turner and colleagues (53) performed a complex analysis using bioinformatics as a follow up to examine whether or not large scale alterations in gene expression as a result of changes in DNA methylation following RTET were present in the transcriptome. In order to do this, they took publicly available transcriptome data from previous acute and chronic resistance exercise studies, combined them and subsequently directly compared these findings with their previous results of genome-wide DNA methylation in human skeletal muscle following acute resistance exercise as well as the chronic TRT paradigm from the Seaborne et al. study mentioned earlier (43). Using Venn diagram analysis, their results revealed 2,018 upregulated and 430 downregulated genes; nearly 600 out of the 2,018 genes upregulated also had decreased methylation, while approximately 100 out of the 430 genes had increased methylation following training. Collectively, through subsequent bioinformatics using the Kyoto Encyclopedia of Genes and Genomes pathway analysis tool, the combined methylation and transcriptome data implicated important roles of genes that were regulated through DNA methylation with well-known processes involved in the adaptive response to RTET, such as extracellular matrix remodeling, mechanotransduction, and transforming growth factor beta (TGF- $\beta$ ) signaling. Another very recent, and complementary, study was conducted by Sailani et al. (41) that examined the effects of lifelong physical activity (defined as having 
exercised on average three or more times per week throughout the majority of their lives) in older, healthy aged men compared to sedentary controls on global DNA methylation status. The authors discovered a distinct DNA methylation profile in the lifelong physically active group in comparison to sedentary age-matched counterparts in which over 700 genes had significantly lower methylation at the promoter region, including genes involved in a variety of critical skeletal muscle regulatory processes such as glycogen metabolism, glycolysis, muscular contraction, myogenesis, and oxidative stress. Moreover, these results complement on an epigenomic level previous research showing the beneficial effects of lifelong physical activity on morphological and physiological outcomes in aged skeletal muscle (10, 44, 45, 47, 52, 57).

Finally, it should be noted that to our knowledge, prior to the current work described herein, there has only been one study to date that looked at the effects of a true TRT paradigm in an animal model of RTET. Lee and colleagues (28) used female Sprague-Dawley rats and had them perform volitional weight-loaded ladder climbing exercise two times per week for eight weeks. Rats were randomly assigned to one of four different experimental groups: a non-exercised control group; a "training” group that performed RTET for eight weeks at 36-44 weeks of age; a "pre-training/detraining” group that trained for eight weeks at 8-16 weeks of age; and a “retraining” group that exercised at 8-16 weeks of age, and then again at 36-44 weeks of age following a 20 week deloading phase. The results demonstrated a significantly higher number of myonuclei in all trained groups compared to control, with no differences amongst the three other three groups. However, retraining resulted in significantly higher relative muscle mass compared to the training group. Additionally, it was found that retraining resulted in enhanced mitochondrial biogenesis as mitochondrial content and DNA copy number were significantly enhanced compared to all other groups. Similar results were also found for expression in genes important for mitochondrial function, including PGC-1 $\alpha$, mitofusin-2, mitochondrial fission 1 , and dynamin-related protein 1. Following separate complementary experiments using myonuclear-enriched myotubes, the authors indicated that the beneficial effects seen in mitochondria were due to an increased number of myonuclei that resulted with training. Collectively, the authors concluded that an increased number of myonuclei from RTET is a potential underlying cause of enhanced skeletal muscle hypertrophy and mitochondrial biogenesis, and that exposing muscles to RTET at a younger age could potentially have benefits in improving the metabolic phenotype of skeletal muscle at later life (28). While we believe the results from the present study provide valuable information about the TRT paradigm of high-intensity RTET aiding in the adaptability of adult skeletal muscle as a result of previous bout of exercise at a young age, our study was limited to two age groups, which consisted of three month old and six month old rodents. Given the past findings by our laboratory and others demonstrating an impairment in adaptation to RTET at old age (11, 13, 19, 35), it is important to investigate whether or not the adaptive benefits of the TRT paradigm shown in the present study are applicable at older ages. 
In conclusion, adult skeletal muscle demonstrates an impairment in its ability to adapt properly to highintensity SSC RTET; however, when previously exposing adult rodents to this type of exercise at a younger age previously shown to stimulate adaptation (Figure 2), adult muscle was capable of going through the putative adaptive process as reflected by an augmentation of skeletal muscle performance as well as enhanced muscle mass. Moreover, we provide key insights into the molecular underpinnings of a TRT paradigm in the regulation of skeletal muscle to appropriately prescribed RTET by showing for the first time evidence involving modified TFs pathway methylation and gene expression, which we believe helps shape downstream events that ultimately enables the muscle to undergo adaptation. Therefore, the findings in the present study supports the concept that an appropriately prescribed exercise prescription at a young age positively impacts and/or categorically attenuates aged-associated negative alterations in skeletal muscle outcomes during adulthood, which helps restore the systemic microenvironment in a favorable manner, eventually leading to muscle adaptation. Future studies should focus on further characterizing both the current TRT paradigm at more advanced ages as well as how various molecular, epigenetic/epigenomic, and morphological variables are influenced by other components of the exercise prescription (e.g., repetition number, velocity, exercise selection, etc.) in order to fully characterize what entails an "optimal” RTET TRT exercise program that is necessary for muscle adaptation to occur throughout the aging process. 


\section{REFERENCES}

1. Baker BA, Hollander MS, Kashon ML, and Cutlip RG. Effects of glutathione depletion and age on skeletal muscle performance and morphology following chronic stretch-shortening contraction exposure. Eur $J$ Appl Physiol 108: 619-630, 2010.

2. Baker BA, Hollander MS, Mercer RR, Kashon ML, and Cutlip RG. Adaptive stretch-shortening contractions: diminished regenerative capacity with aging. Appl Physiol Nutr Metab 33: 1181-1191, 2008.

\section{Barres R, Yan J, Egan B, Treebak JT, Rasmussen M, Fritz T, Caidahl K, Krook A, O'Gorman}

DJ, and Zierath JR. Acute exercise remodels promoter methylation in human skeletal muscle. Cell Metab 15: 405-411, 2012.

4. Bruusgaard JC, Johansen IB, Egner IM, Rana ZA, and Gundersen K. Myonuclei acquired by overload exercise precede hypertrophy and are not lost on detraining. Proc Natl Acad Sci U S A 107: 1511115116, 2010.

5. Bultmann S, and Stricker SH. Entering the post-epigenomic age: back to epigenetics. Open Biol 8: 2018.

6. Caldow MK, Thomas EE, Dale MJ, Tomkinson GR, Buckley JD, and Cameron-Smith D. Early myogenic responses to acute exercise before and after resistance training in young men. Physiol Rep 3: 2015.

7. Cedar H, and Bergman Y. Linking DNA methylation and histone modification: patterns and paradigms. Nat Rev Genet 10: 295-304, 2009.

8. Cheedipudi S, Puri D, Saleh A, Gala HP, Rumman M, Pillai MS, Sreenivas P, Arora R, Sellathurai J, Schroder HD, Mishra RK, and Dhawan J. A fine balance: epigenetic control of cellular quiescence by the tumor suppressor PRDM2/RIZ at a bivalent domain in the cyclin a gene. Nucleic Acids Res 43: 6236-6256, 2015.

9. Clark SJ, Statham A, Stirzaker C, Molloy PL, and Frommer M. DNA methylation: bisulphite modification and analysis. Nat Protoc 1: 2353-2364, 2006.

10. Crane JD, Macneil LG, and Tarnopolsky MA. Long-term aerobic exercise is associated with greater muscle strength throughout the life span. J Gerontol A Biol Sci Med Sci 68: 631-638, 2013. 
11. Cutlip RG, Baker BA, Geronilla KB, Mercer RR, Kashon ML, Miller GR, Murlasits Z, and Alway

SE. Chronic exposure to stretch-shortening contractions results in skeletal muscle adaptation in young rats and maladaptation in old rats. Appl Physiol Nutr Metab 31: 573-587, 2006.

12. Cutlip RG, Stauber WT, Willison RH, McIntosh TA, and Means KH. Dynamometer for rat plantar flexor muscles in vivo. Med Biol Eng Comput 35: 540-543, 1997.

13. Degens H, and Alway SE. Skeletal muscle function and hypertrophy are diminished in old age. Muscle Nerve 27: 339-347, 2003.

14. Dungan CM, Murach KA, Frick KK, Jones SR, Crow SE, Englund DA, Vechetti IJ, Jr., Figueiredo VC, Levitan BM, Satin J, McCarthy JJ, and Peterson CA. Elevated myonuclear density during skeletal muscle hypertrophy in response to training is reversed during detraining. Am J Physiol Cell Physiol 2019.

15. Egner IM, Bruusgaard JC, Eftestol E, and Gundersen K. A cellular memory mechanism aids overload hypertrophy in muscle long after an episodic exposure to anabolic steroids. J Physiol 591: 6221-6230, 2013.

16. Eilertsen KJ, Power RA, Harkins LL, and Misica P. Targeting cellular memory to reprogram the epigenome, restore potential, and improve somatic cell nuclear transfer. Anim Reprod Sci 98: 129-146, 2007.

17. Fernandez-Verdejo R, Vanwynsberghe AM, Essaghir A, Demoulin JB, Hai T, Deldicque L, and Francaux M. Activating transcription factor 3 attenuates chemokine and cytokine expression in mouse skeletal muscle after exercise and facilitates molecular adaptation to endurance training. FASEB J 31: 840-851, 2017.

18. Fernandez-Verdejo R, Vanwynsberghe AM, Hai T, Deldicque L, and Francaux M. Activating transcription factor 3 regulates chemokine expression in contracting C2C12 myotubes and in mouse skeletal muscle after eccentric exercise. Biochem Biophys Res Commun 492: 249-254, 2017.

19. Greig CA, Gray C, Rankin D, Young A, Mann V, Noble B, and Atherton PJ. Blunting of adaptive responses to resistance exercise training in women over 75y. Exp Gerontol 46: 884-890, 2011.

20. Gundersen K. Muscle memory and a new cellular model for muscle atrophy and hypertrophy. $J$ Exp Biol 219: 235-242, 2016. 
21. Harridge SD. Plasticity of human skeletal muscle: gene expression to in vivo function. Exp Physiol 92: 783-797, 2007.

22. Henwood TR, and Taaffe DR. Detraining and retraining in older adults following long-term muscle power or muscle strength specific training. J Gerontol A Biol Sci Med Sci 63: 751-758, 2008.

\section{Kanzleiter T, Jahnert M, Schulze G, Selbig J, Hallahan N, Schwenk RW, and Schurmann A.}

Exercise training alters DNA methylation patterns in genes related to muscle growth and differentiation in mice. Am J Physiol Endocrinol Metab 308: E912-920, 2015.

24. Karatzas PS, Mantzaris GJ, Safioleas M, and Gazouli M. DNA methylation profile of genes involved in inflammation and autoimmunity in inflammatory bowel disease. Medicine (Baltimore) 93: e309, 2014.

25. King-Himmelreich TS, Schramm S, Wolters MC, Schmetzer J, Moser CV, Knothe C, Resch E, Peil J, Geisslinger G, and Niederberger E. The impact of endurance exercise on global and AMPK genespecific DNA methylation. Biochem Biophys Res Commun 474: 284-290, 2016.

26. Laker RC, and Ryall JG. DNA Methylation in skeletal muscle stem cell specification, proliferation, and differentiation. Stem Cells Int 2016: 5725927, 2016.

27. Lane SC, Camera DM, Lassiter DG, Areta JL, Bird SR, Yeo WK, Jeacocke NA, Krook A, Zierath JR, Burke LM, and Hawley JA. Effects of sleeping with reduced carbohydrate availability on acute training responses. J Appl Physiol (1985) 119: 643-655, 2015.

28. Lee H, Kim K, Kim B, Shin J, Rajan S, Wu J, Chen X, Brown MD, Lee S, and Park JY. A cellular mechanism of muscle memory facilitates mitochondrial remodelling following resistance training. $J$ Physiol 596: 4413-4426, 2018.

\section{Lindholm ME, Marabita F, Gomez-Cabrero D, Rundqvist H, Ekstrom TJ, Tegner J, and}

Sundberg CJ. An integrative analysis reveals coordinated reprogramming of the epigenome and the transcriptome in human skeletal muscle after training. Epigenetics 9: 1557-1569, 2014.

30. Naimo MA, Rader EP, Ensey J, Kashon ML, and Baker BA. Reduced frequency of resistance-type exercise training promotes adaptation of the aged skeletal muscle microenvironment. J Appl Physiol (1985) 2019. 
31. Nguyen A, Duquette N, Mamarbachi M, and Thorin E. Epigenetic regulatory effect of exercise on glutathione peroxidase 1 expression in the skeletal muscle of severely dyslipidemic mice. PLoS One 11: e0151526, 2016.

\section{Nitert MD, Dayeh T, Volkov P, Elgzyri T, Hall E, Nilsson E, Yang BT, Lang S, Parikh H,} Wessman Y, Weishaupt H, Attema J, Abels M, Wierup N, Almgren P, Jansson PA, Ronn T, Hansson O, Eriksson KF, Groop L, and Ling C. Impact of an exercise intervention on DNA methylation in skeletal muscle from first-degree relatives of patients with type 2 diabetes. Diabetes 61: 3322-3332, 2012.

33. Ordway JM, Bedell JA, Citek RW, Nunberg A, Garrido A, Kendall R, Stevens JR, Cao D, Doerge RW, Korshunova Y, Holemon H, McPherson JD, Lakey N, Leon J, Martienssen RA, and Jeddeloh JA. Comprehensive DNA methylation profiling in a human cancer genome identifies novel epigenetic targets. Carcinogenesis 27: 2409-2423, 2006.

34. Rader EP, and Baker BA. Inflammaging and the age-specific responsiveness to stretch-shortening contractions. Exerc Sport Sci Rev 45: 195-200, 2017.

35. Rader EP, Layner K, Triscuit AM, Chetlin RD, Ensey J, and Baker BA. Age-dependent muscle adaptation after chronic stretch-shortening contractions in rats. Aging Dis 7: 1-13, 2016.

36. Rader EP, Naimo MA, Ensey J, and Baker BA. High-intensity stretch-shortening contraction training modifies responsivity of skeletal muscle in old male rats. Exp Gerontol 104: 118-126, 2018.

\section{Rader EP, Naimo MA, Layner KN, Triscuit AM, Chetlin RD, Ensey J, and Baker BA.}

Enhancement of skeletal muscle in aged rats following high-intensity stretch-shortening contraction training. Rejuvenation Res 20: 93-102, 2017.

38. Rasmussen M, Zierath JR, and Barres R. Dynamic epigenetic responses to muscle contraction. Drug Discov Today 19: 1010-1014, 2014.

39. Roberts MD, Kerksick CM, Dalbo VJ, Hassell SE, Tucker PS, and Brown R. Molecular attributes of human skeletal muscle at rest and after unaccustomed exercise: an age comparison. J Strength Cond Res 24: 1161-1168, 2010.

40. Rutherford OM, and Jones DA. The role of learning and coordination in strength training. Eur J Appl Physiol Occup Physiol 55: 100-105, 1986. 


\section{Sailani MR, Halling JF, Moller HD, Lee H, Plomgaard P, Pilegaard H, Snyder MP, and}

Regenberg B. Lifelong physical activity is associated with promoter hypomethylation of genes involved in metabolism, myogenesis, contractile properties and oxidative stress resistance in aged human skeletal muscle. Sci Rep 9: 3272, 2019.

42. Sakugawa RL, Moura BM, Orssatto L, Bezerra ES, Cadore EL, and Diefenthaeler F. Effects of resistance training, detraining, and retraining on strength and functional capacity in elderly. Aging Clin Exp Res 31: 31-39, 2019.

43. Seaborne RA, Strauss J, Cocks M, Shepherd S, O'Brien TD, van Someren KA, Bell PG, Murgatroyd C, Morton JP, Stewart CE, and Sharples AP. Human skeletal muscle possesses an epigenetic memory of hypertrophy. Sci Rep 8: 1898, 2018.

44. Seals DR, Justice JN, and LaRocca TJ. Physiological geroscience: targeting function to increase healthspan and achieve optimal longevity. J Physiol 594: 2001-2024, 2016.

45. Seals DR, and Melov S. Translational geroscience: emphasizing function to achieve optimal longevity. Aging (Albany NY) 6: 718-730, 2014.

46. Sharples AP, Stewart CE, and Seaborne RA. Does skeletal muscle have an 'epi'-memory? The role of epigenetics in nutritional programming, metabolic disease, aging and exercise. Aging Cell 15: 603-616, 2016.

47. Shibata S, Fujimoto N, Hastings JL, Carrick-Ranson G, Bhella PS, Hearon CM, Jr., and Levine BD. The effect of lifelong exercise frequency on arterial stiffness. J Physiol 596: 2783-2795, 2018.

48. Siu PM, Bryner RW, Martyn JK, and Alway SE. Apoptotic adaptations from exercise training in skeletal and cardiac muscles. FASEB J 18: 1150-1152, 2004.

49. Staron RS, Leonardi MJ, Karapondo DL, Malicky ES, Falkel JE, Hagerman FC, and Hikida RS. Strength and skeletal muscle adaptations in heavy-resistance-trained women after detraining and retraining. $J$ Appl Physiol (1985) 70: 631-640, 1991.

50. Taaffe DR, Henwood TR, Nalls MA, Walker DG, Lang TF, and Harris TB. Alterations in muscle attenuation following detraining and retraining in resistance-trained older adults. Gerontology 55: 217-223, 2009. 
51. Tan Q, Christiansen L, von Bornemann Hjelmborg J, and Christensen K. Twin methodology in epigenetic studies. J Exp Biol 218: 134-139, 2015.

52. Tucker LA. Physical activity and telomere length in U.S. men and women: an NHANES investigation. Prev Med 100: 145-151, 2017.

53. Turner DC, Seaborne RA, and Sharples AP. Comparative transcriptome and methylome analysis in human skeletal muscle anabolism, hypertrophy and epigenetic memory. Sci Rep 9: 4251, 2019.

54. Vaczi M, Nagy SA, Koszegi T, Ambrus M, Bogner P, Perlaki G, Orsi G, Toth K, and Hortobagyi T. Mechanical, hormonal, and hypertrophic adaptations to 10 weeks of eccentric and stretch-shortening cycle exercise training in old males. Exp Gerontol 58: 69-77, 2014.

55. Wang YX, and Rudnicki MA. Satellite cells, the engines of muscle repair. Nat Rev Mol Cell Biol 13: 127-133, 2011.

56. Wichnieski C, Maheshwari K, Souza LC, Nieves F, Tartari T, Garlet GP, Carneiro E, Letra A, and Silva RM. DNA methylation profiles of immune response-related genes in apical periodontitis. Int Endod $J$ 52: 5-12, 2019.

57. Zampieri S, Pietrangelo L, Loefler S, Fruhmann H, Vogelauer M, Burggraf S, Pond A, GrimStieger M, Cvecka J, Sedliak M, Tirpakova V, Mayr W, Sarabon N, Rossini K, Barberi L, De Rossi M, Romanello V, Boncompagni S, Musaro A, Sandri M, Protasi F, Carraro U, and Kern H. Lifelong physical exercise delays age-associated skeletal muscle decline. J Gerontol A Biol Sci Med Sci 70: 163-173, 2015. 
Table 1. RT ${ }^{2}$ Profiler Transcription Factors PCR Array.

$\begin{array}{ccccccccccccccc} & 1 & 2 & 3 & 4 & 5 & 6 & 7 & 8 & 9 & 10 & 11 & 12 \\ \text { A } & \text { Ar } & \text { Arnt } & \text { Atf1 } & \text { Atf2 } & \text { Atf3 } & \text { Atf4 } & \text { Cebpa } & \text { Cebpb } & \text { Cebpg } & \text { Clasrp } & \text { Creb1 } & \text { Crebbp } \\ \text { B } & \text { Ctnnb1 } & \text { Dr1 } & \text { E2f1 } & \text { E2f6 } & \text { Egr1 } & \text { Esr1 } & \text { Ets1 } & \text { Ets2 } & \text { Fos } & \text { Foxa2 } & \text { Foxg1 } & \text { Gata1 } \\ \text { C } & \text { Gata2 } & \text { Gata3 } & \text { Gtf2b } & \text { Gtf2f1 } & \text { Hand1 } & \text { Hand2 } & \text { Hdac1 } & \text { Hif1a } & \text { Hnf1a } & \text { Hnf4a } & \text { Hoxa5 } & \text { Hsf1 } \\ \text { D } & \text { Id1 } & \text { Irf1 } & \text { Jun } & \text { Junb } & \text { Jund } & \text { Kcnh8 } & \text { Max } & \text { Mef2a } & \text { Mef2b } & \text { Mef2c } & \text { Myc } & \text { Myf5 } \\ \text { E } & \text { Myod1 } & \text { Nanos2 } & \text { Nfat5 } & \text { Nfatc2 } & \text { Nfatc3 } & \text { Nfatc4 } & \text { Nfkb1 } & \text { Nfyb } & \text { Nr3c1 } & \text { Pax6 } & \text { Pou2af1 } & \text { Ppara } \\ \text { F } & \text { Pparg } & \text { Rb1 } & \text { Rel } & \text { Rela } & \text { RGD1560225 } & \text { Smad1 } & \text { Smad4 } & \text { Smad5 } & \text { Smad9 } & \text { Sp1 } & \text { Sp3 } & \text { Stat1 } \\ \text { G } & \text { Stat2 } & \text { Stat3 } & \text { Stat4 } & \text { Stat5a } & \text { Stat5b } & \text { Stat6 } & \text { Tbp } & \text { Tcf7l2 } & \text { Tcfap2a } & \text { Tgif1 } & \text { Tp53 } & \text { Yy1 } \\ \text { H } & \text { Actb } & \text { B2m } & \text { Hprt1 } & \text { Ldha } & \text { Rplp1 } & \text { RGDC } & \text { RTC } & \text { RTC } & \text { RTC } & \text { PPC } & \text { PPC } & \text { PPC }\end{array}$


Table 2. Details of the Rat Stem Cell Transcription Factors DNA Methylation PCR Array. All information on gene function(s) was obtained from Entrez Gene Summary and/or UniProtKB/Swiss-Prot.

\begin{tabular}{|c|c|c|}
\hline Genes & Symbol & Description \\
\hline $\begin{array}{l}\text { Cell Differentiation } \\
\text { Genes/Developmental } \\
\text { Regulators }\end{array}$ & Gata2 & GATA binding protein 2 \\
\hline
\end{tabular}

Jun

Klf4

Neurod1

Neurog1

Notch2

Pax6
Jun oncogene

Kruppel-like factor 4 (gut)

Neurogenic differentiation 1

Neurogenin 1

Notch homolog 2 (Drosophila)
Gene Function

Involved in stem cell maintenance with key roles in hematopoietic development; transcriptional activator regulates endothelin-1 gene expression in endothelial cells

Interacts directly with specific target DNA sequences to regulate gene expression; promotes activity of Nr5a1 when phosphorylated by Hipk3, leading to increased steroidogenic gene expression upon cAMP signaling pathway stimulation Plays important role in maintaining embryonic stem cells, and in preventing their differentiation; thought to control the G1-to-S transition of the cell cycle following DNA damage by mediating p53 (contributes to its down-regulation)

Forms heterodimers with other bHLH proteins and activates transcription of genes that contain a specific DNA sequence called the E-box; regulates expression of the insulin gene; contributes to the regulation of several cell differentiation pathways; associates with chromatin to enhancer regulatory elements in genes encoding key transcriptional regulators of neurogenesis

Involved in the initiation of neuronal differentiation; activates transcription by binding to the Ebox; associates with chromatin to enhancer regulatory elements in genes encoding key regulators of neurogenesis

The Notch signaling network is an evolutionarily conserved intracellular signaling pathway that plays a key role in development; functions as a receptor for membrane-bound ligands to regulate cell-fate determination; affects the implementation of differentiation, proliferation, and apoptotic programs; involved in bone remodeling and homeostasis; may play a role in vascular, renal and hepatic development

Activity is key in the development of neural tissues; important functions in the development of the eye, nose, central nervous 
Pparg

Runx1

Runx2

Smad2

Sp1

Chromatin Modification Genes

Hdac1
Peroxisome proliferator-activated receptor gamma

Runt-related transcription factor 1

Runt-related transcription factor 2

SMAD family member 2

Sp1 transcription factor

Histone deacetylase 1 system, and pancreas; required for the differentiation of pancreatic islet alpha cells

Nuclear receptor that binds peroxisome proliferators such as hypolipidemic drugs and the betaoxidation pathway of fatty acids; regulator of adipocyte differentiation and glucose homeostasis; acts as a critical regulator of gut homeostasis by suppressing NF-kappa-Bmediated proinflammatory responses; plays a key role in the regulation of cardiovascular circadian rhythms

Forms the heterodimeric complex core-binding factor with Cbfb; essential for development of normal hematopoiesis; controls the suppressive function of regulatory T-cells by associating with Foxp3

Can bind DNA both as a monomer or, with more affinity, as a subunit of a heterodimeric complex; involved in osteoblastic differentiation and skeletal morphogenesis

Intracellular signal transducer and transcriptional modulator activated by TGF- $\beta$ and active type 1 receptor kinases; mediates the signal of TGF- $\beta$ and regulates multiple cellular processes, such as cell proliferation, apoptosis, and differentiation; phosphorylation induces association with SMAD family member 4 , which is important for the translocation of this protein into the nucleus, where it binds to target promoters and forms a transcription repressor complex with other cofactors

Can activate or repress transcription in response to physiological and pathological stimuli; involved in many cellular processes, including cell differentiation, cell growth, apoptosis, immune responses, response to DNA damage, and chromatin remodeling; posttranslational modifications such as phosphorylation, acetylation, glycosylation, and proteolytic processing significantly affect the activity of this protein, which can be an activator or repressor Responsible for the deacetylation of lysine residues on the Nterminal part of the core histones (H2A, H2B, H3, and H4); histone deacetylation gives a tag for 
Sox2

Environmental \& Intracellular Fos Stimuli Responses
SRY (sex determining region Y)box 2

FBJ osteosarcoma oncogene

Hypoxia-inducible factor 1, alpha subunit (basic helix-loop-helix transcription factor)

Signal transducer and activator of transcription 1 epigenetic repression and plays an important role in transcriptional regulation, cell cycle progression and developmental events; upon calcium stimulation, Hdac1 is released from the complex and Crebbp is recruited, which facilitates transcriptional activation; interacts with retinoblastoma tumor-associated protein and is a key element in the control of cell proliferation and differentiation; required for transcriptional repression of circadian target genes

Forms a trimeric complex with Oct4 on DNA and controls the expression of a number of genes involved in embryonic development; involved in the determination of cell fate; critical for early embryogenesis and for embryonic stem cell pluripotency; may function as a switch in neuronal development; keeps neural cells undifferentiated by counteracting the activity of proneural proteins and suppresses neuronal differentiation

Nuclear phosphoprotein which can dimerize with proteins of the Jun family, thereby forming the transcription factor complex AP1 ; been implicated as regulators of cell proliferation, differentiation, and transformation; has a critical function in regulating the development of cells destined to form and maintain the skeleton; in some cases, expression of Fos has been associated with apoptotic cell death

Alpha subunit of hypoxiainducible factor-1 (HIF-1). HIF-1 functions as a master regulator of the cellular and systemic homeostatic response to hypoxia by activating transcription of many genes involved in energy metabolism, angiogenesis, apoptosis, and other genes whose protein products increase oxygen delivery or facilitate metabolic adaptation to hypoxia ligands including interferonalpha, interferon-gamma, EGF, PDGF and IL6; mediates the expression of a variety of genes, which is thought to be important for cell viability in response to different cell stimuli and pathogens; signal transducer and transcription activator that mediates cellular responses to 
Other Stem Cell Maintenance \& Differentiation Genes
Foxa2 (Hnf3b)

Lin28

Myc

Nfya

Pcna
Forkhead box A2

Lin-28 homolog (C. elegans)

Myelocytomatosis oncogene

Nuclear transcription factor-Y alpha

Proliferating cell nuclear antigen interferons, along with various cytokines and growth factors

Involved in embryonic

development, establishment of tissue-specific gene expression and regulation of gene expression in differentiated tissues; regulates the expression of genes important for glucose sensing in pancreatic beta-cells and glucose homeostasis; involved in the regulation of fat metabolism; is thought to act as a 'pioneer' factor opening compacted chromatin for other proteins through interactions with nucleosomal core histones and thereby replacing linker histones at target enhancer and/or promoter sites

Acts as a posttranscriptional regulator of genes involved in developmental timing and selfrenewal in embryonic stem cells; prevents the terminal processing of the LET7 family of microRNAs, which are major regulators of cellular growth and differentiation; 'translational enhancer' that drives specific mRNAs to polysomes and increases the efficiency of protein synthesis

Activates the transcription of growth-related genes; binds to the VEGFA promoter, promoting VEGFA production and subsequent sprouting angiogenesis; a proto-oncogene that plays a role in cell cycle progression, apoptosis and cellular transformation; forms a heterodimer with the related transcription factor MAX One subunit of a trimeric complex, forming a highly conserved transcription factor that binds to CCAAT motifs in the promoter regions in a variety of genes; subunit $\mathrm{A}$ associates with a tight dimer composed of the $\mathrm{B}$ and $C$ subunits, resulting in a trimer that binds to DNA with high specificity and affinity Found in the nucleus and is an auxiliary protein of DNA polymerase delta that is involved in the control of eukaryotic DNA replication by increasing the polymerase's processing ability during elongation of the leading strand; induces a robust stimulatory effect on Apex2 activities; plays a key role in the DNA damage response by being conveniently positioned at the 
replication fork to coordinate DNA replication with DNA repair and DNA damage tolerance pathways; acts as a loading platform to recruit DNA damage response proteins that allows completion of DNA replication after damage and promotes postreplication repair 
Table 3. Significantly differentially expressed genes (SDEGs) relevant to the transcription factors pathway for the tibialis anterior muscle following dorsiflexion SSC-loading relative to non-loaded control muscles.

\begin{tabular}{|c|c|c|c|c|c|c|c|}
\hline & & & & \multicolumn{2}{|c|}{ 6-Month Trained } & \multicolumn{2}{|c|}{ Trained-Retrained } \\
\hline Genes & Symbol & Description & RefSeq \# & $\mathrm{FC}$ & $P$ value & FC & $P$ value \\
\hline $\begin{array}{l}\text { Androgen Receptor } \\
\text { Signaling }\end{array}$ & $\mathrm{Rb} 1$ & Retinoblastoma 1 & NM_017045 & $\downarrow 1.00$ & 0.6744 & $\downarrow 1.38$ & 0.0751 \\
\hline \multirow[t]{4}{*}{$\begin{array}{l}\text { Growth Factor } \\
\text { Signaling }\end{array}$} & Nfatc2 & $\begin{array}{l}\text { Nuclear factor of activated } \\
\text { T-cells, cytoplasmic, } \\
\text { calcineurin-dependent } 2\end{array}$ & NM_001107805 & $\downarrow 1.09$ & 0.7771 & $\downarrow 2.16$ & 0.0171 \\
\hline & Nfatc3 & $\begin{array}{l}\text { Nuclear factor of activated } \\
\text { T-cells, cytoplasmic, } \\
\text { calcineurin-dependent } 3\end{array}$ & NM_001108447 & $\downarrow 1.13$ & 0.8924 & $\downarrow 1.71$ & 0.0019 \\
\hline & Stat5a & $\begin{array}{l}\text { Signal transducer and } \\
\text { activator of transcription } \\
5 \mathrm{~A}\end{array}$ & NM_017064 & $\uparrow 1.07$ & 0.7099 & $\downarrow 1.86$ & 0.0231 \\
\hline & Fos & $\begin{array}{l}\text { FBJ osteosarcoma } \\
\text { oncogene }\end{array}$ & NM_022197 & $\uparrow 1.16$ & 0.8158 & $\uparrow 2.00$ & 0.0653 \\
\hline \multirow[t]{4}{*}{ TGF- $\beta /$ BMP Signaling } & Id1 & $\begin{array}{l}\text { Inhibitor of DNA binding } \\
1\end{array}$ & NM_012797 & $\downarrow 1.36$ & 0.3123 & $\downarrow 2.05$ & 0.0266 \\
\hline & Smad4 & SMAD family member 4 & NM_019275 & $\downarrow 1.01$ & 0.6983 & $\downarrow 1.50$ & 0.0272 \\
\hline & Smad5 & SMAD family member 5 & NM_021692 & $\downarrow 1.04$ & 0.9133 & $\downarrow 1.63$ & 0.0712 \\
\hline & Smad9 & SMAD family member 9 & NM_138872 & $\uparrow 1.12$ & 0.4611 & $\downarrow 1.52$ & 0.0765 \\
\hline \multirow[t]{3}{*}{ JAK/STAT Signaling } & Crebbp & CREB binding protein & NM_133381 & $\downarrow 1.22$ & 0.5588 & $\downarrow 1.72$ & 0.0430 \\
\hline & Мус & $\begin{array}{l}\text { Myelocytomatosis } \\
\text { oncogene }\end{array}$ & NM_012603 & $\uparrow 2.40$ & 0.0413 & $\downarrow 1.16$ & 0.7048 \\
\hline & Stat6 & $\begin{array}{l}\text { Signal transducer and } \\
\text { activator of transcription } 6\end{array}$ & NM_001044250 & $\uparrow 1.79$ & 0.0057 & $\downarrow 1.69$ & 0.1762 \\
\hline \multirow[t]{2}{*}{ WNT Signaling } & Tcf7l2 & $\begin{array}{l}\text { Transcription factor 7-like } \\
2 \text { (T-cell specific, HMG- } \\
\text { box) }\end{array}$ & NM_001191052 & $\uparrow 1.40$ & 0.0242 & $\downarrow 1.01$ & 0.7871 \\
\hline & Tp53 & Tumor protein p53 & NM_030989 & $\uparrow 1.45$ & 0.2495 & $\downarrow 1.89$ & 0.0004 \\
\hline \multirow[t]{2}{*}{ Immune Signaling } & Nr3c1 & $\begin{array}{l}\text { Nuclear receptor } \\
\text { subfamily } 3 \text {, group C, } \\
\text { member } 1\end{array}$ & NM_012576 & $\downarrow 1.22$ & 0.4982 & $\downarrow 1.69$ & 0.0262 \\
\hline & Rela & $\begin{array}{l}\text { V-rel reticuloendotheliosis } \\
\text { viral oncogene homolog A } \\
\text { (avian) }\end{array}$ & NM_199267 & $\downarrow 1.32$ & 0.6469 & $\downarrow 1.73$ & 0.0396 \\
\hline MAP Kinase Signaling & Atf2 & $\begin{array}{l}\text { Activating transcription } \\
\text { factor } 2\end{array}$ & NM_031018 & $\downarrow 1.09$ & 0.9127 & $\downarrow 1.38$ & 0.0453 \\
\hline
\end{tabular}




\begin{tabular}{|c|c|c|c|c|c|c|c|}
\hline & Atf4 & $\begin{array}{l}\text { Activating transcription } \\
\text { factor } 4 \text { (tax-responsive } \\
\text { enhancer element B67) }\end{array}$ & NM_024403 & $\downarrow 1.45$ & 0.5569 & $\downarrow 1.77$ & 0.0156 \\
\hline & Max & MYC associated factor $\mathrm{X}$ & NM_022210 & $\downarrow 1.55$ & 0.2354 & $\downarrow 1.67$ & 0.0613 \\
\hline \multirow[t]{8}{*}{$\begin{array}{l}\text { Other Transcription } \\
\text { Factors }\end{array}$} & Atf3 & $\begin{array}{l}\text { Activating transcription } \\
\text { factor } 3\end{array}$ & NM_012912 & $\uparrow 3.99$ & 0.0655 & $\uparrow 7.19$ & 0.0379 \\
\hline & Cebpg & $\begin{array}{l}\text { CCAAT/enhancer binding } \\
\text { protein (C/EBP), gamma }\end{array}$ & NM_012831 & $\downarrow 1.24$ & 0.5540 & $\downarrow 2.12$ & 0.0076 \\
\hline & Ets2 & $\begin{array}{l}\text { V-ets erythroblastosis } \\
\text { virus E26 oncogene } \\
\text { homolog } 2 \text { (avian) }\end{array}$ & NM_001107107 & $\downarrow 1.46$ & 0.3860 & $\downarrow 2.36$ & 0.0431 \\
\hline & Myf5 & Myogenic factor 5 & NM_001106783 & $\uparrow 1.69$ & 0.1480 & $\uparrow 1.40$ & 0.0008 \\
\hline & Myod1 & $\begin{array}{l}\text { Myogenic differentiation } \\
1\end{array}$ & NM_176079 & $\downarrow 1.38$ & 0.3853 & $\downarrow 2.36$ & 0.0068 \\
\hline & Nfyb & $\begin{array}{l}\text { Nuclear transcription } \\
\text { factor-Y beta }\end{array}$ & NM_031553 & $\uparrow 1.24$ & 0.5941 & $\downarrow 1.78$ & 0.0091 \\
\hline & Gata2 & GATA binding protein 2 & NM_033442 & $\uparrow 1.12$ & 0.4407 & -1.94 & 0.0583 \\
\hline & Junb & Jun B proto-oncogene & NM_021836 & $\uparrow 1.84$ & 0.0517 & $\downarrow 1.18$ & 0.9450 \\
\hline
\end{tabular}

Out of 84 mRNA transcripts in the transcription factors pathway array, a total of 20 were classified as SDEGs in one or more experimental groups, while another 7 had a trend for being an SDEG. All SDEGs that surpassed a 1.1-fold change with a $P$ value $<0.05$ have bold text. Italicized text $=$ trend $(0.05<P<0.10)$ for increased/decreased expression. Sample sizes were $N=5$ to 6 per group. FC $=$ fold change. 
Table 4. Ingenuity Pathway Analysis (IPA) of gene expression results from the Transcription Factors RT ${ }^{2}$ Profiler Pathway Array for the tibialis anterior muscle following dorsiflexion SSC-loading relative to nonloaded control muscles; the top 5 results from each category were included in the table.

\begin{tabular}{|c|c|c|c|c|c|c|}
\hline Condition & Analyses & \multicolumn{5}{|c|}{ Highest - - } \\
\hline \multirow{3}{*}{$\begin{array}{c}\text { 6-Month } \\
\text { Trained } \\
\text { (SSC-loaded } 3 \\
\text { days per week) }\end{array}$} & $\begin{array}{c}\text { Top Canonical } \\
\text { Pathways (p-value; z- } \\
\text { score) }\end{array}$ & $\begin{array}{l}\text { Mouse Embryonic } \\
\text { Stem Cell } \\
\text { Pluripotency } \\
\text { (7.93E-05) } \\
\end{array}$ & $\begin{array}{l}\text { Wnt/ } \beta \text {-catenin } \\
\text { Signaling } \\
(1.87 \mathrm{E}-04)\end{array}$ & $\begin{array}{c}\text { Estrogen- } \\
\text { mediated S-phase } \\
\text { Entry } \\
(3.60 \mathrm{E}-03) \\
\end{array}$ & $\begin{array}{c}\text { Cell Cycle: G1/S } \\
\text { Checkpoint } \\
\text { Regulation } \\
(9.26 E-03) \\
\end{array}$ & $\begin{array}{l}\text { Thrombopoietin } \\
\text { Signaling } \\
(9.81 \mathrm{E}-03)\end{array}$ \\
\hline & $\begin{array}{l}\text { Top Networks } \\
\text { (Score) }\end{array}$ & $\begin{array}{c}\text { Organismal Injury } \\
\text { and } \\
\text { Abnormalities, } \\
\text { Reproductive } \\
\text { System Disease, } \\
\text { Connective Tissue } \\
\text { Disorders } \\
\text { (9) }\end{array}$ & N/A & N/A & N/A & N/A \\
\hline & $\begin{array}{c}\text { Physiological System } \\
\text { Development and } \\
\text { Function } \\
\text { (p-value range) }\end{array}$ & $\begin{array}{c}\text { Organ } \\
\text { Morphology } \\
(3.74 \mathrm{E}-03- \\
\text { 3.67E-07) }\end{array}$ & $\begin{array}{c}\text { Digestive System } \\
\text { Development and } \\
\text { Function } \\
(2.77 \mathrm{E}-03- \\
\text { 3.05E-06) }\end{array}$ & $\begin{array}{c}\text { Organismal } \\
\text { Development } \\
(3.60 \mathrm{E}-03- \\
3.05 \mathrm{E}-06)\end{array}$ & $\begin{array}{c}\text { Tissue } \\
\text { Morphology } \\
(3.74 \mathrm{E}-03-8.52 \mathrm{E}- \\
06)\end{array}$ & $\begin{array}{c}\text { Organ Development } \\
(3.32 \mathrm{E}-03-2.82 \mathrm{E}- \\
05)\end{array}$ \\
\hline
\end{tabular}

\begin{tabular}{|c|c|c|c|c|c|c|}
\hline Condition & Analyses & \multicolumn{5}{|c|}{ 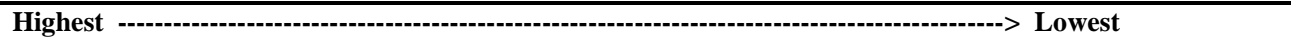 } \\
\hline \multirow{3}{*}{$\begin{array}{c}\text { Trained- } \\
\text { Retrained } \\
\text { (SSC-loaded } 3 \\
\text { days per week) }\end{array}$} & $\begin{array}{c}\text { Top Canonical } \\
\text { Pathways } \\
\text { (p-value; z-score) }\end{array}$ & $\begin{array}{c}\text { Wnt/Ca+ Pathway } \\
\text { (5.74E-12) }\end{array}$ & $\begin{array}{l}\text { PI3K Signaling in B } \\
\text { Lymphocytes } \\
\text { (6.43E-10) }\end{array}$ & $\begin{array}{c}\text { Glucocorticoid } \\
\text { Receptor Signaling } \\
\text { (4.16E-09) }\end{array}$ & $\begin{array}{c}\text { B Cell Receptor } \\
\text { Signaling } \\
(5.46 \mathrm{E}-09)\end{array}$ & $\begin{array}{c}\text { Protein Kinase A } \\
\text { Signaling } \\
(1.18 E-08)\end{array}$ \\
\hline & $\begin{array}{l}\text { Top Networks } \\
\text { (Score) }\end{array}$ & $\begin{array}{c}\text { Gene Expression, } \\
\text { Cell Cycle, } \\
\text { Respiratory } \\
\text { Disease (22) }\end{array}$ & $\begin{array}{c}\text { Organ } \\
\text { Morphology, } \\
\text { Tissue } \\
\text { Development, } \\
\text { Cell Death and } \\
\text { Survival } \\
\text { (11) }\end{array}$ & $\begin{array}{l}\text { Cell-Mediated } \\
\text { Immune } \\
\text { Response, } \\
\text { Cellular } \\
\text { Development, } \\
\text { Cellular Function } \\
\text { \& Maintenance } \\
\text { (6) }\end{array}$ & N/A & N/A \\
\hline & $\begin{array}{c}\text { Physiological System } \\
\text { Development and } \\
\text { Function } \\
\text { (p-value range) } \\
\text { (\# molecules) }\end{array}$ & $\begin{array}{c}\text { Organismal } \\
\text { Development } \\
(2.01 \mathrm{E}-04- \\
1.08 \mathrm{E}-13)\end{array}$ & $\begin{array}{c}\text { Lymphoid Tissue } \\
\text { Structure and } \\
\text { Development } \\
\text { (1.87E-04- } \\
\text { 4.51E-11) }\end{array}$ & $\begin{array}{c}\text { Tissue } \\
\text { Morphology } \\
(1.87 \mathrm{E}-04- \\
4.51 \mathrm{E}-11)\end{array}$ & $\begin{array}{c}\text { Organismal } \\
\text { Survival (1.59E-04 } \\
-7.34 \mathrm{E}-11)\end{array}$ & $\begin{array}{c}\text { Embryonic } \\
\text { Development } \\
(2.01 \mathrm{E}-04-1.19 \mathrm{E}- \\
10)\end{array}$ \\
\hline
\end{tabular}

The top canonical pathways and top networks were color highlighted based upon the percentage of genes associated with the pathway array that were up- or downregulated. All IPA functions with the exception of cancer specific, reproductive system, psychological functions, and cell lines were considered for this table. 
Sample sizes were $N=5$ per group. N/A = Not available; SSC = stretch-shortening contraction; Wnt = wingless-related integration site; PI3K = phosphoinositide-3-kinase. 


\section{FIGURE LEGENDS}

Figure 1. Conceptual flow chart showing our experimental design and setup. F344xBN male rats were randomly assigned to a high-intensity SSC resistance-type exercise training protocol for one month in one of the following two groups: 1) Young rats at three months of age that underwent a training-retraining paradigm in which they were SSC-loaded three days per week, were subsequently detrained for two months by simply staying in their cages without exercising, and finally were retrained again by undergoing a second SSC loading bout at six months of age $(n=5) ; 2)$ adult rats at six months of age that were SSC-loaded three days per week for one month who did not have a prior bout of high-intensity SSC resistance-type exercise training and therefore were previously naïve to the exercise stimulus $(n=5) .72$ hours post-exercise TA muscle tissue was harvested for subsequent molecular and biochemical analyses. Six months of age was chosen because previous work from our laboratory demonstrated that this was the age at which an altered adaptive phenotype following a validated, SSC resistance-type exercise training protocol started to occur, thus we believed this to be the potential starting point at which there is an age-specific biological departure from the SSC-training adaptive response. F344xBN = Fisher 344 x Brown Norway; SSC = stretch-shortening contraction; TA = tibialis anterior.

Figure 2. An initial one month bout of high-intensity SSC resistance-type exercise training in rodents following a training-retraining paradigm results in adaptation in young male rats through improved muscle performance. During each training session, TA isometric and dynamic muscle performance was assessed prior to the first training set. The maximum force was measured and recorded for each group; A) dorsiflexion static performance in terms of isometric peak force performed prior to commencement of the first training set; B) dorsiflexion dynamic performance in terms of SSC peak force performed prior to commencement of the first training set. For each group, the peak force performed was averaged based on recorded values during the first week (initial) and last week of training (final). Sample sizes were $N=5$ per group. ${ }^{*}$ Different from initial value, $\mathrm{p}<0.05$. Data presented as means \pm SEM. SSC $=$ stretch-shortening contraction; TA = tibialis anterior.

Figure 3. A training-retraining paradigm of SSC resistance-type exercise training improves muscle performance of adult male rats. During each training session, TA isometric and dynamic muscle performance was assessed prior to the first training set. The maximum force was measured and recorded for each group; A) dorsiflexion static performance in terms of isometric peak force performed prior to commencement of the first training set; B) relative changes in isometric peak force; C) dorsiflexion dynamic performance in terms of SSC peak force performed prior to commencement of the first training set; D) relative changes in SSC peak force. For each group, the peak force performed was averaged based on recorded values during the first week (initial) and last week of training (final). Sample sizes were $N=5$ per group. ${ }^{*}$ Different from initial within group; 
${ }^{\#}$ Different from final value of the 6-month trained only group, $\mathrm{p}<0.05$. Data presented as means \pm SEM. SSC $=$ stretch-shortening contraction; $\mathrm{TA}=$ tibialis anterior.

Figure 4. A training-retraining paradigm of SSC resistance-type exercise training improves muscle mass of adult male rats. Seventy two hours after the final SSC-loading protocol, rats were euthanized and the TA muscle was surgically removed, weighed, and recorded in grams (i.e., wet-weight). The wet-weight was measured and recorded for each group; A) absolute muscle mass that was recorded immediately following removal; B) TA muscle mass normalized by tibia length. Sample sizes were $\mathrm{N}=5$ per group. ${ }^{*}$ Different from NLC muscle within group; \# Different from SSC-loaded muscle of the 6-month trained only group, $\mathrm{p}<0.05$. Data presented as means \pm SEM. SSC = stretch-shortening contraction; TA = tibialis anterior; NLC = non-loaded control.

Figure 5. A training-retraining paradigm of SSC resistance-type exercise training alters DNA methylation in the TA skeletal muscle of adult male rats. Epitect Methyl II PCR array looking at the promoter-specific methylation status of genes implicated as important regulators of the transcription factors pathway were calculated as a percentage for each gene and then collectively combined to quantify the overall DNA methylation status of the entire pathway for both groups. Analyses were done comparing SSC-loaded TA muscle following one month of high-intensity SSC RTET with age-matched NLC muscle. Sample sizes were $N$ $=4$ to 5 per group. ${ }^{~}$ Different from SSC-loaded limb of 6-month trained, $\mathrm{p}<0.05$. Data presented as means \pm SEM. SSC = stretch-shortening contraction; TA = tibialis anterior; NLC = non-loaded control.

Figure 6. A training-retraining paradigm of SSC resistance-type exercise training increases transcription factors signaling in the TA skeletal muscle of adult male rats. The volcano plot graphs of the transcription factors PCR array. These graphs show the log2 of the fold change for each gene's expression between SSC-loaded and NLC muscle for each group. Fold changes and $P$ values were determined from comparisons of $\Delta \mathrm{C}_{\mathrm{t}}$ values between SSC-loaded and NLC TA muscles analyzed through the GeneGlobe Data Analysis Center (Qiagen). A) Adult rats trained only at 6 months of age; B) young rats trained at 3 months of age, detrained, and subsequently retrained at 6 months of age. Sample sizes were $N=5$ per group. The vertical solid line indicates fold changes of 0 . Vertical dotted lines indicate that the fold change in gene expression threshold is 1.1. The horizontal solid line indicates that the P-value of the $t$ test threshold is 0.05 . Genes that were significantly differentially expressed are labeled and colored according to whether or not they were up- or downregulated. SSC = stretchshortening contraction; TA = tibialis anterior; NLC = non-loaded control. 
Figure 7. A training-retraining paradigm of SSC resistance-type exercise training enhances muscle protein content in aged rats. Comparison of the molecular attributes between 6-month trained only and trained-retrained rodent skeletal muscle for A) muscle RNA; B) muscle DNA; C) muscle protein. Both RNA and DNA concentrations were obtained using a spectrophotometer (ND-1000; NanoDrop Technologies Inc., Wilmington, DE, USA). Total protein contents of the cytoplasmic protein extracts were quantified and reported as protein concentration ( $\mu$ g protein/mg tissue - [protein]) between groups. * Different from NLC muscle within group; "Different from SSC-loaded muscle of the 6-month trained only group, $\mathrm{p}<0.05$. Data presented as means \pm SEM. SSC = stretch-shortening contraction; TA = tibialis anterior; NLC = non-loaded control. 


\section{FIGURE 1}

\begin{tabular}{|c|c|c|c|c|}
\hline & & & & $\begin{array}{l}\text { Quantify \& } \\
\text { characterize } \\
\text { Gene } \\
\text { Expression }\end{array}$ \\
\hline \multirow[t]{2}{*}{$\begin{array}{l}\text { Initial adaptive SSC } \\
\text { loading at } 3 \text { months } \\
\text { of age for } 1 \text { month } \\
\text { ( } 8 \text { sets of } 10 \mathrm{SSCs} 3 \\
\text { days/wk) }\end{array}$} & 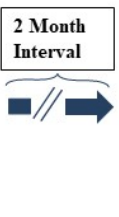 & \begin{tabular}{|l} 
Second SSC \\
loading bout at 6 \\
months of age for 1 \\
month (8 sets of 10 \\
SSCs 3 days/wk) \\
\end{tabular} & $\Longrightarrow \begin{array}{l}\begin{array}{l}\text { Soft-tissue } \\
\text { collected 72 } \\
\text { hours } \\
\text { following last } \\
\text { exposure }\end{array} \\
\end{array}$ & $\begin{array}{l}\text { Quantify \& } \\
\text { characterize } \\
\text { DNA } \\
\text { Methylation }\end{array}$ \\
\hline & \multicolumn{3}{|c|}{$\begin{array}{l}\text { Static \& } \\
\text { Dynamic } \\
\text { Performance } \\
\text { Measurements }\end{array}$} & $\begin{array}{l}\text { Quantify \& } \\
\text { characterize } \\
\text { Protein } \\
\text { Response } \\
\end{array}$ \\
\hline
\end{tabular}


FIGURE 2

(A)

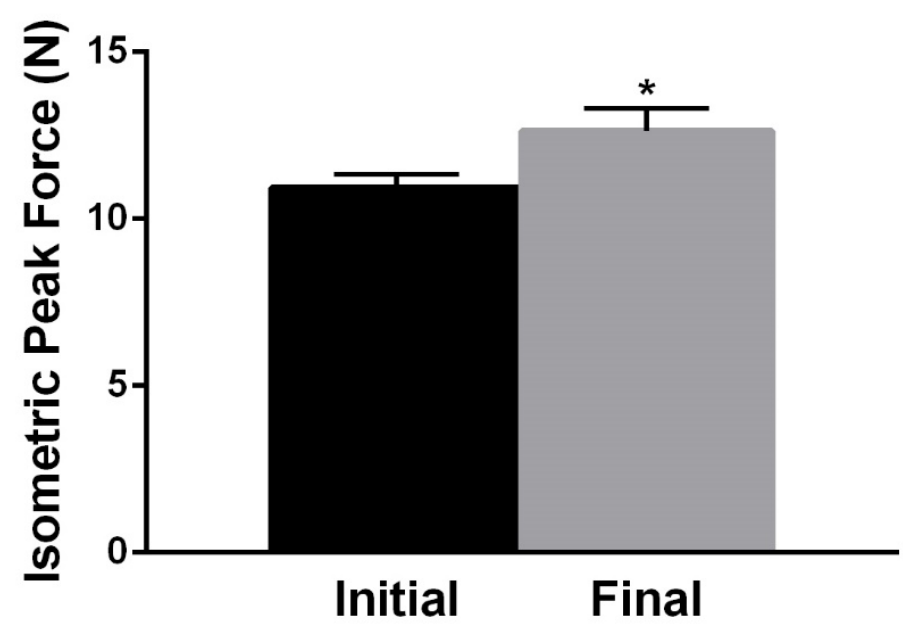

- Initial

Final

(B)

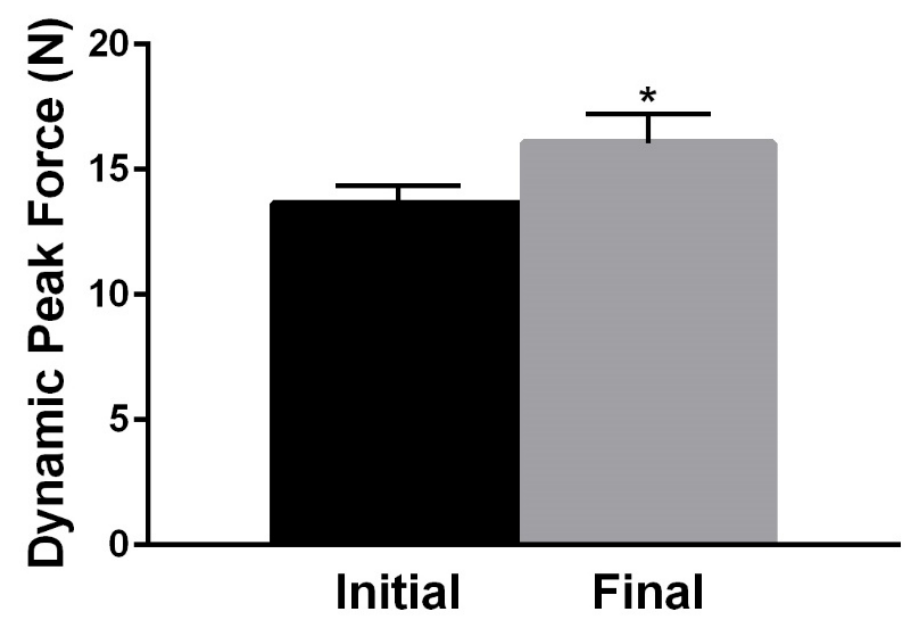


FIGURE 3

(A)

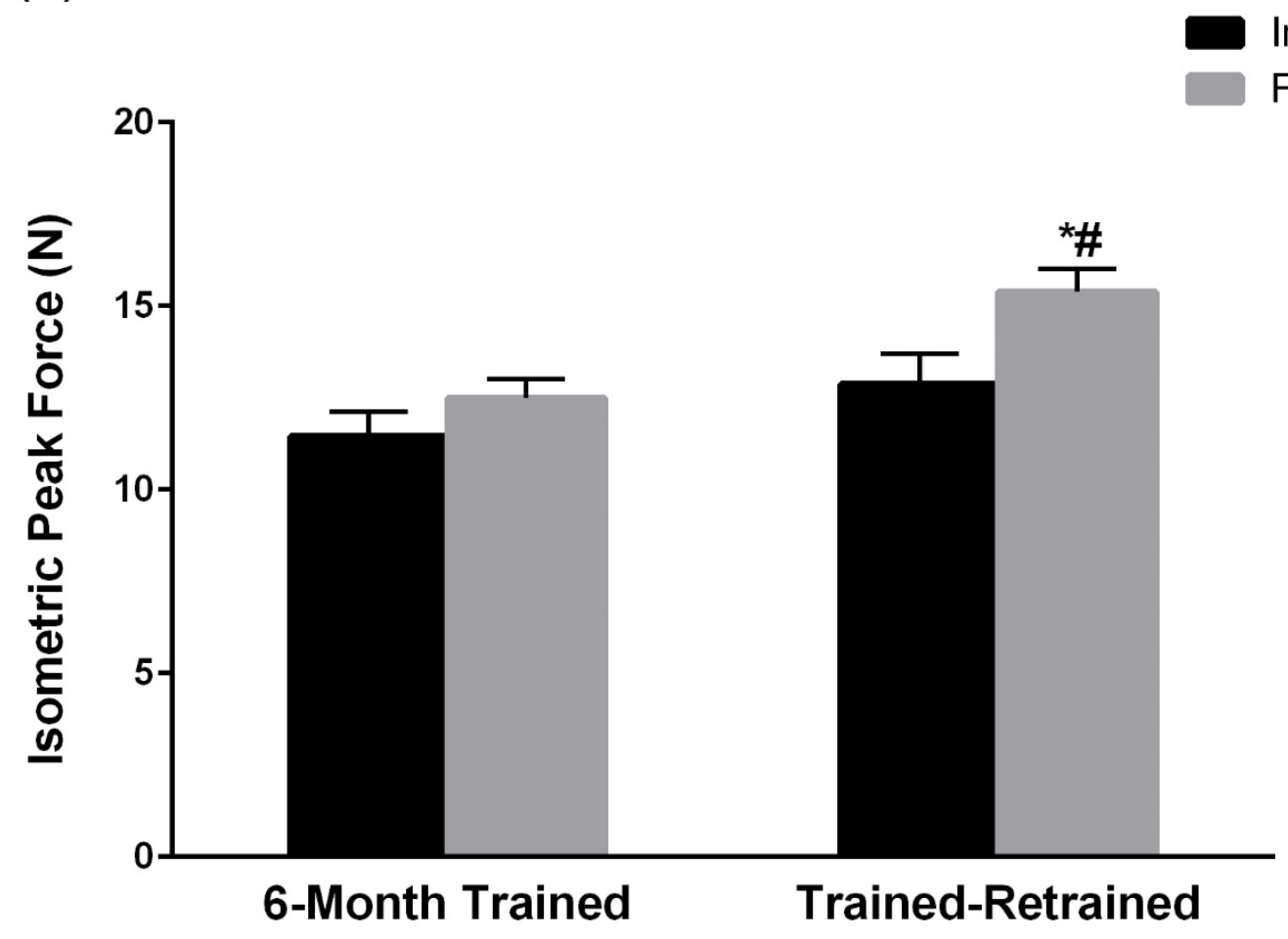

(B)

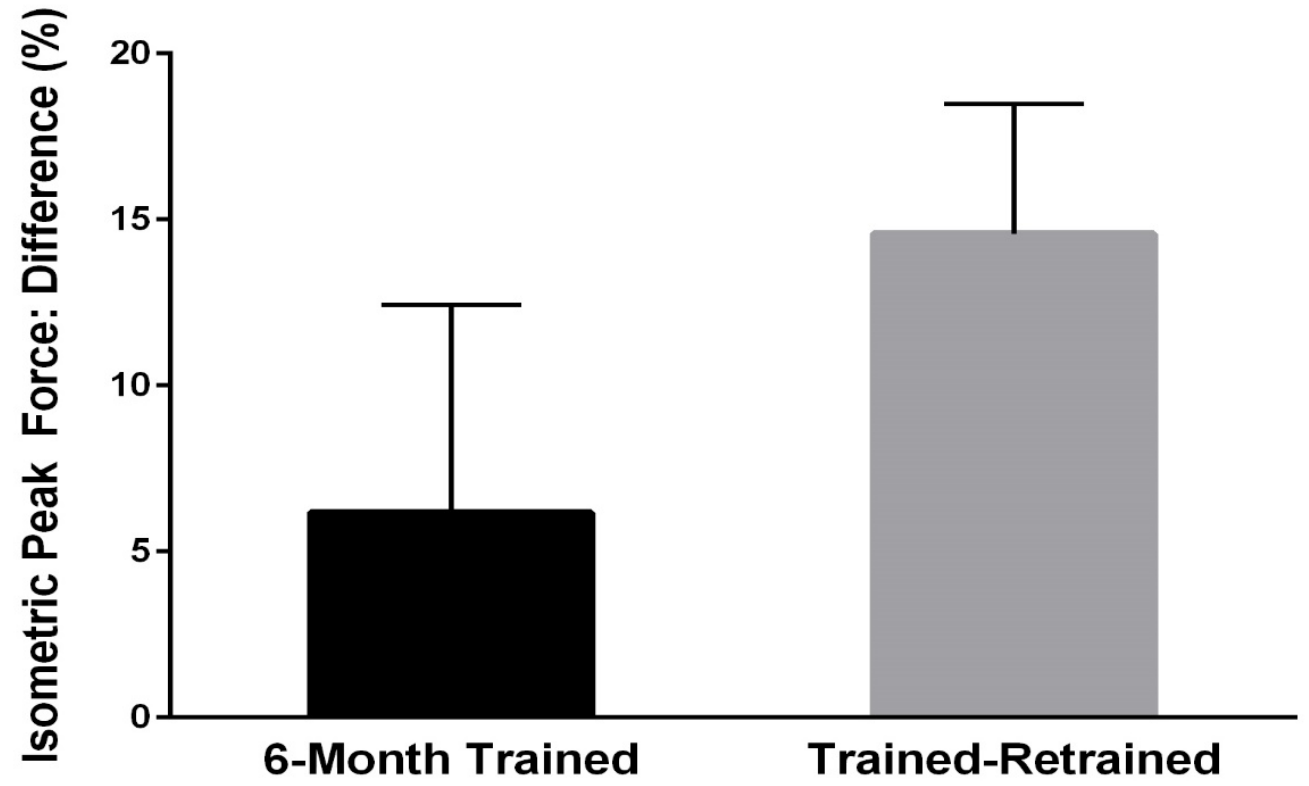


(C)

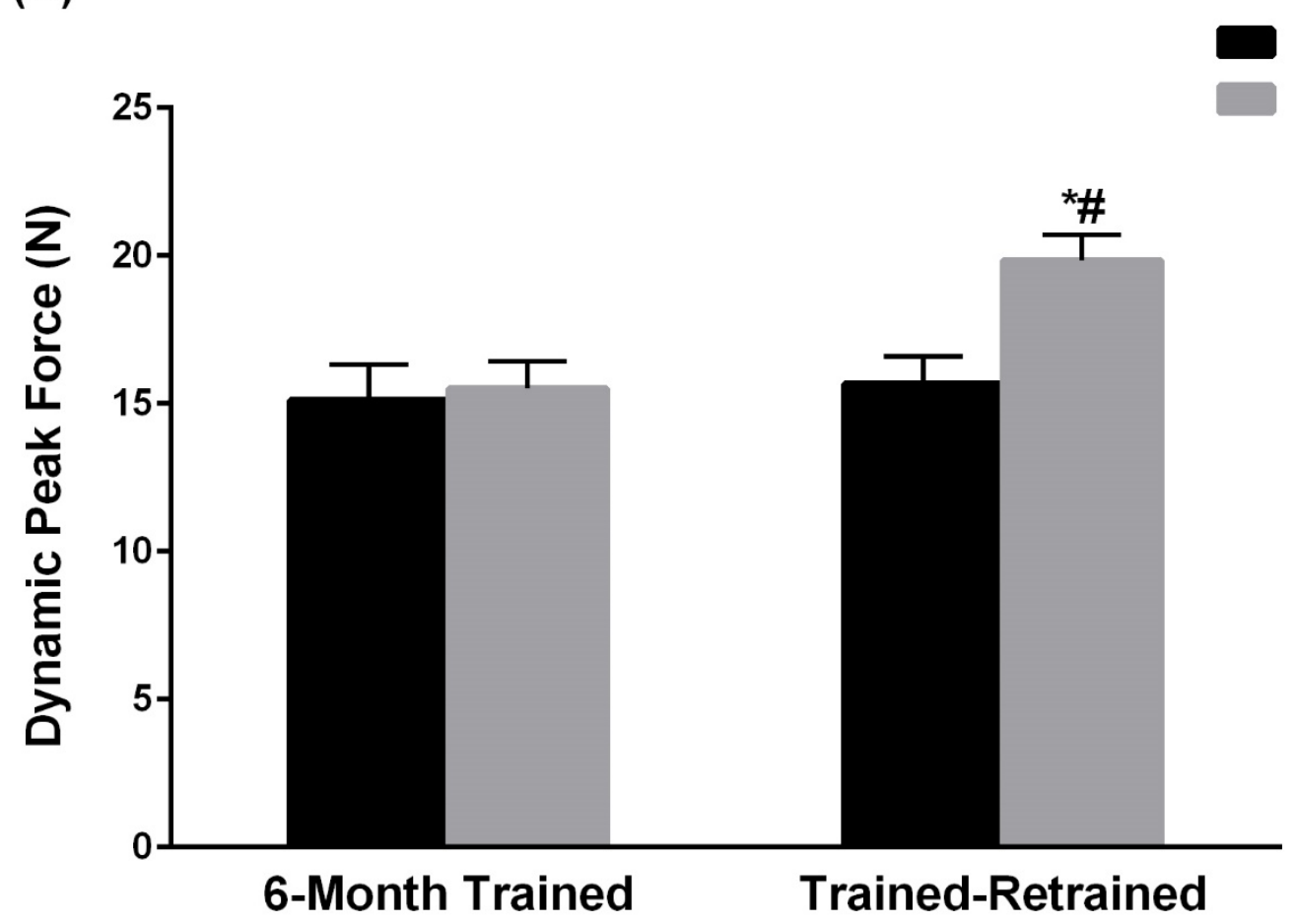

(D)

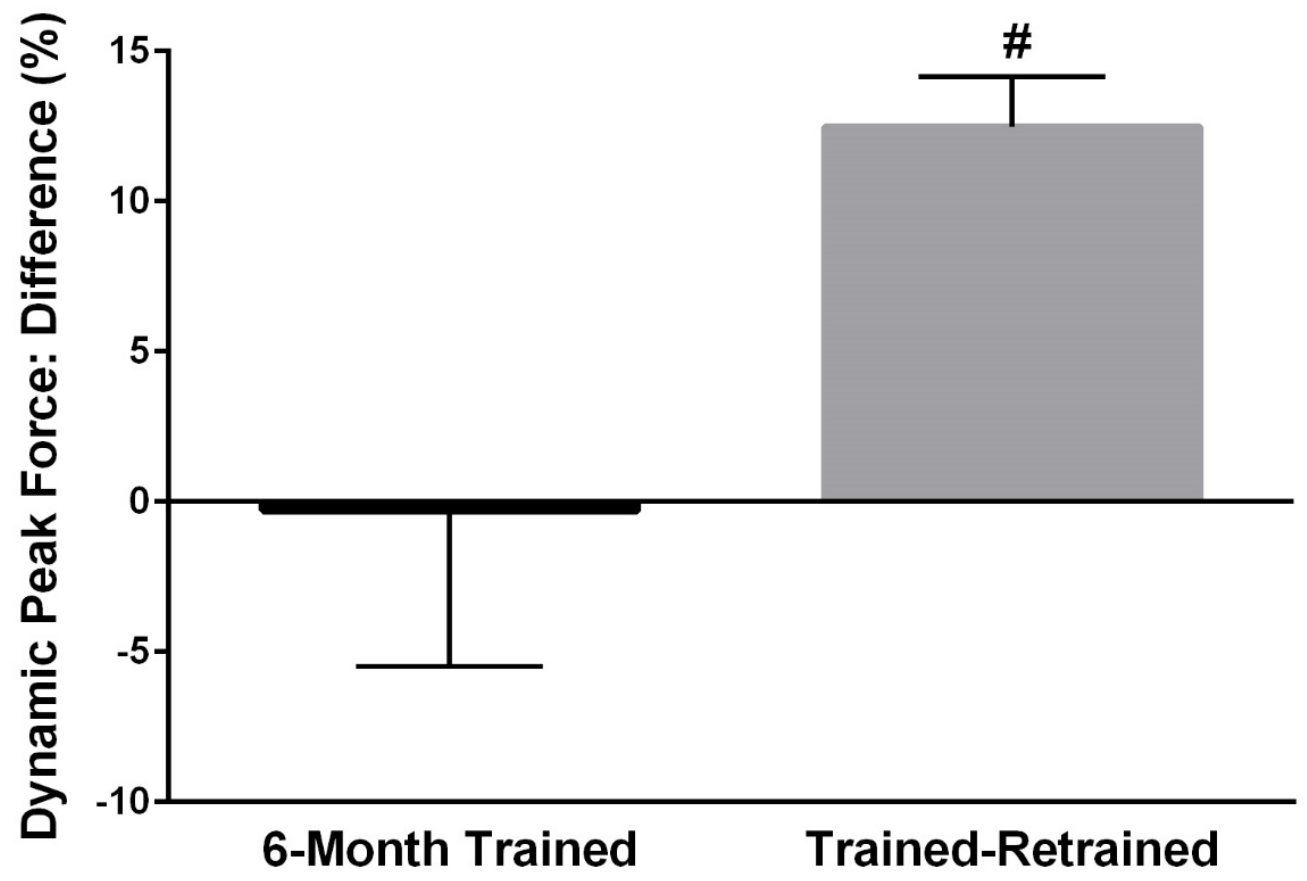


FIGURE 4

(A)

Non-Loaded

SSC-Loaded

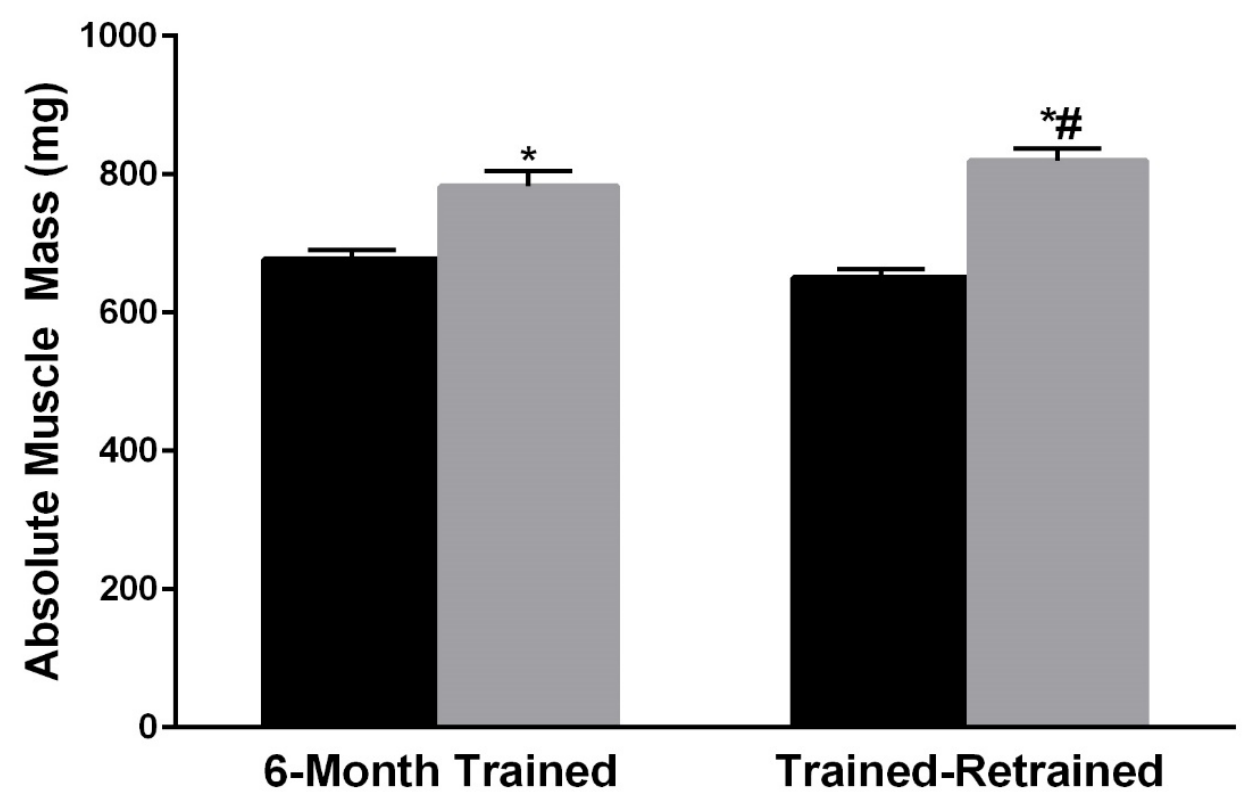

(B)

Non-Loaded

SSC-Loaded

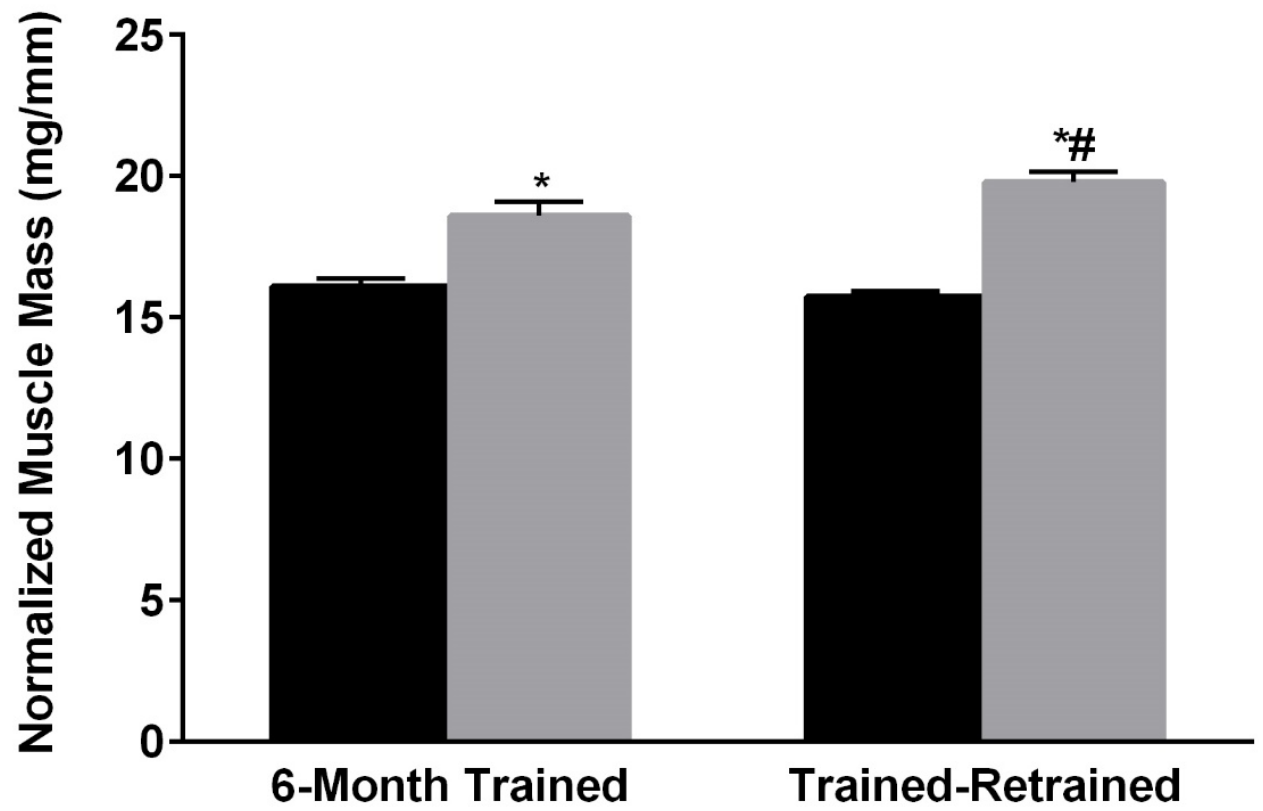


FIGURE 5

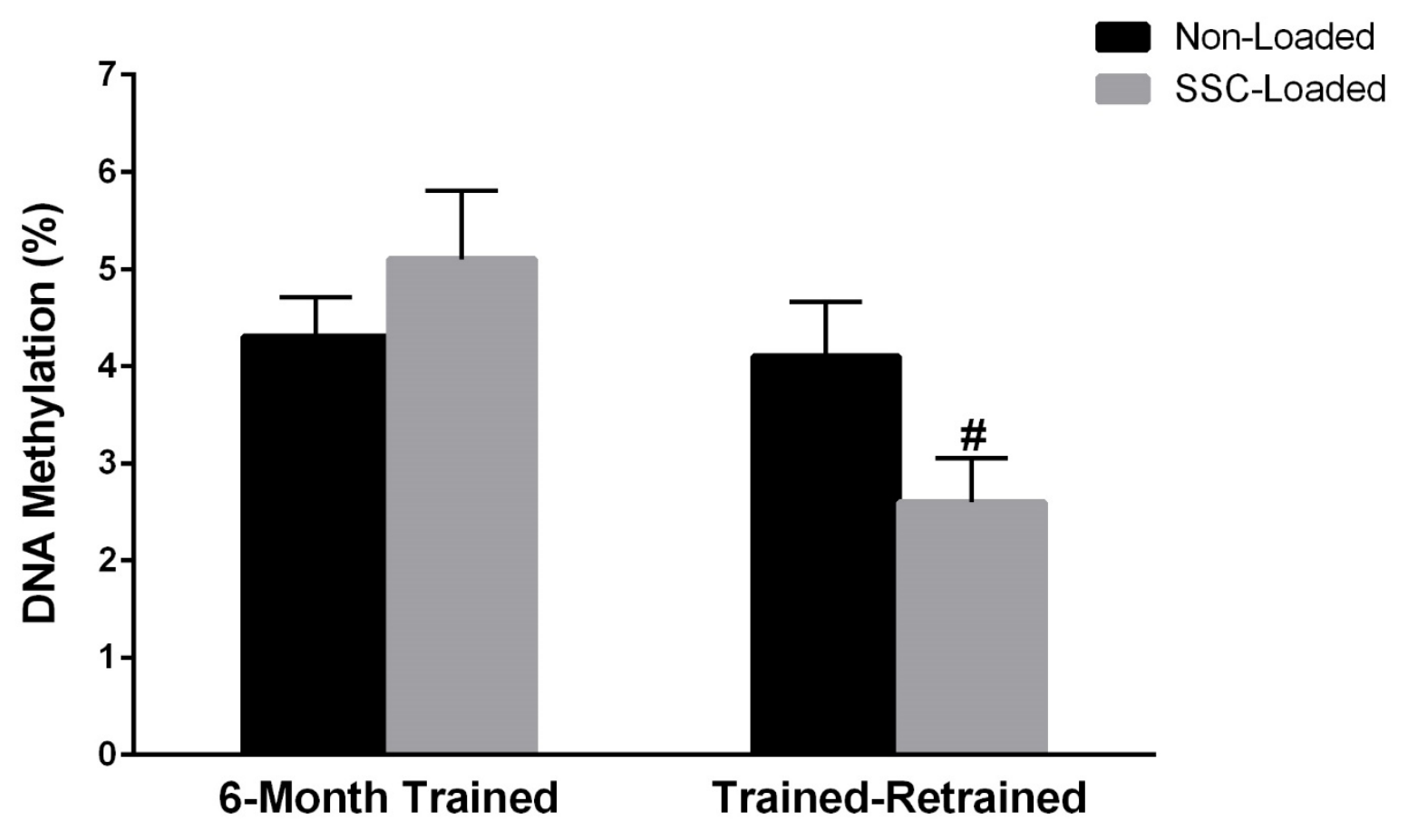




\section{FIGURE 6}

(A)

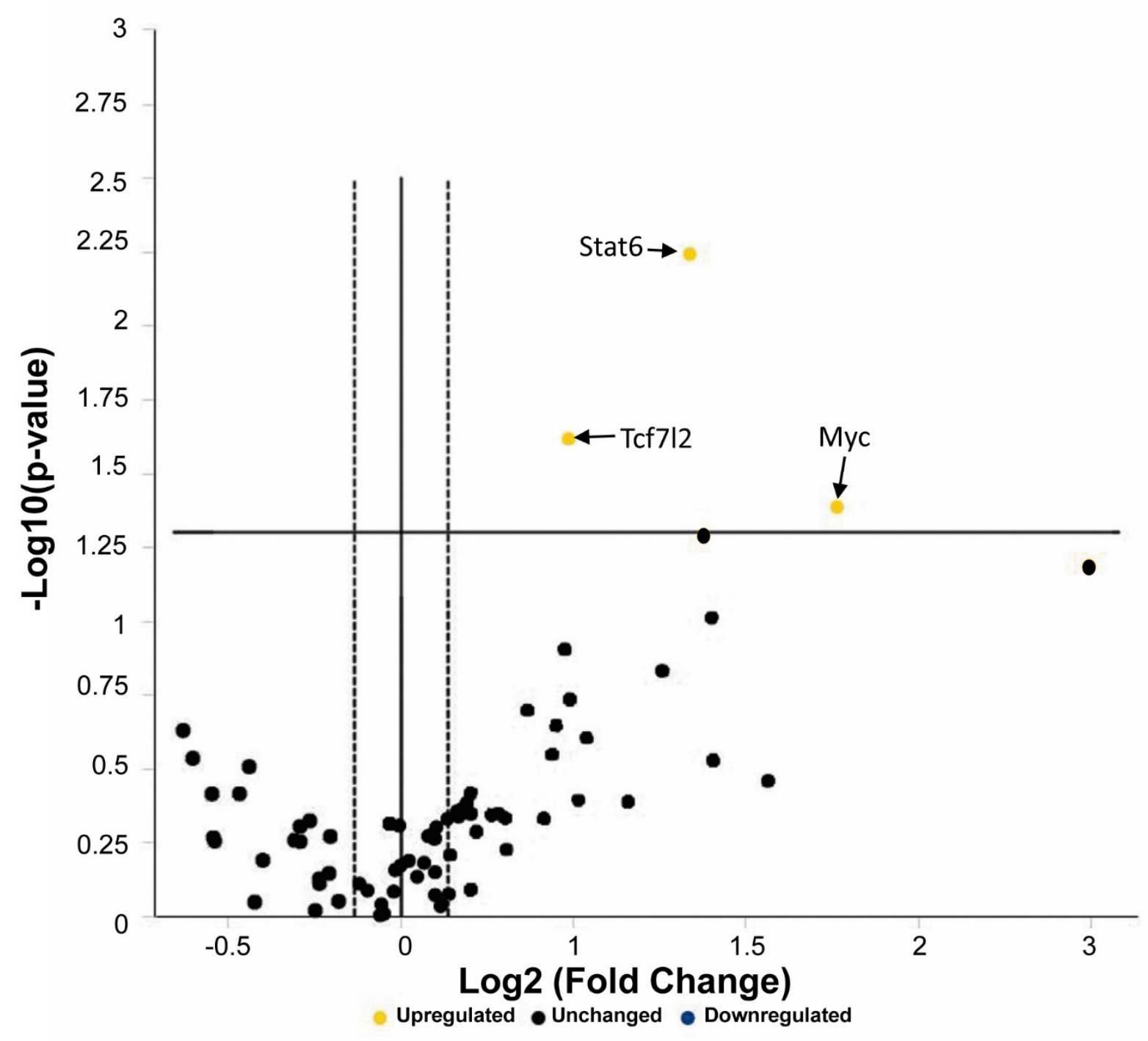


(B)

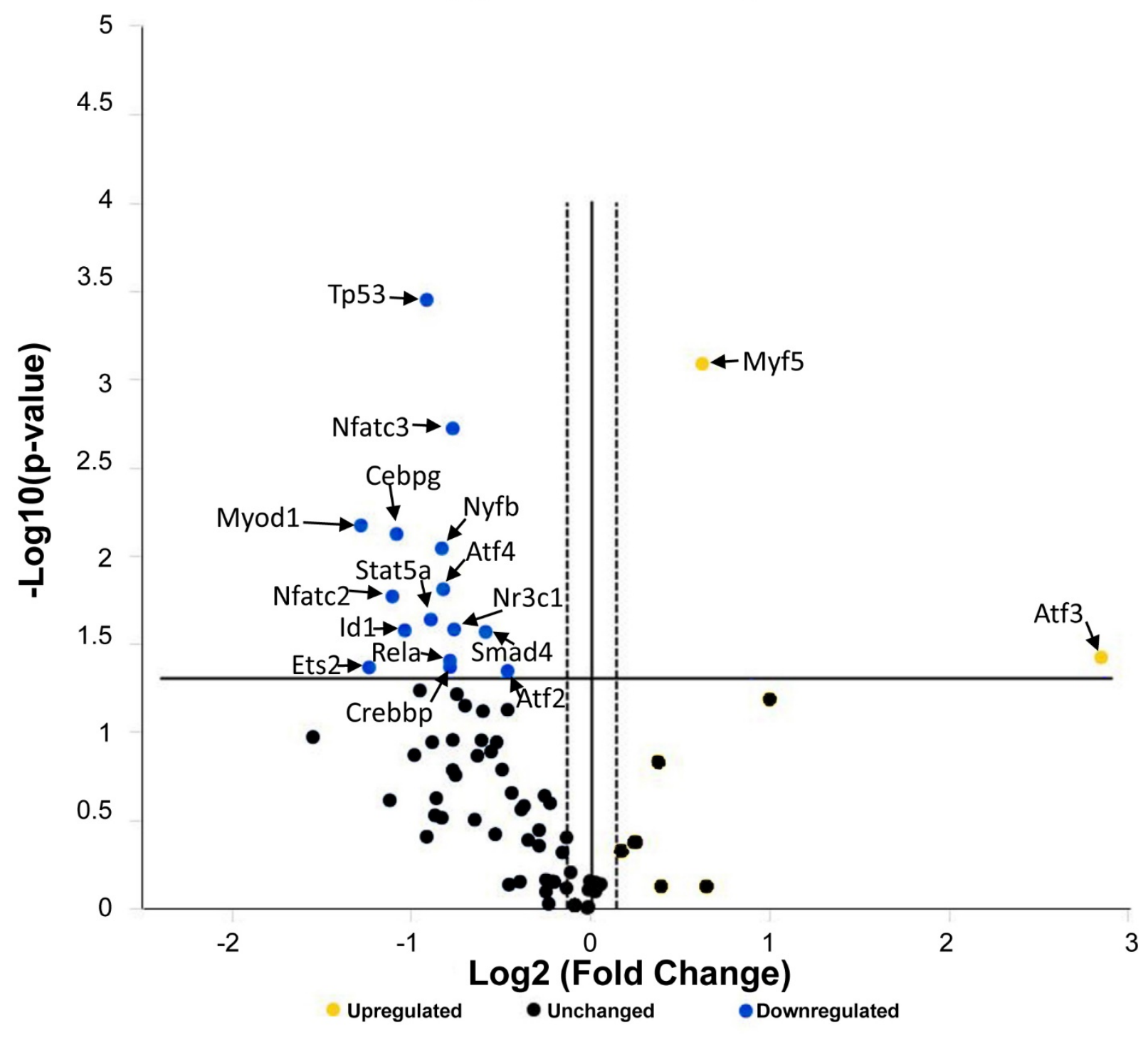


FIGURE 7

(A)

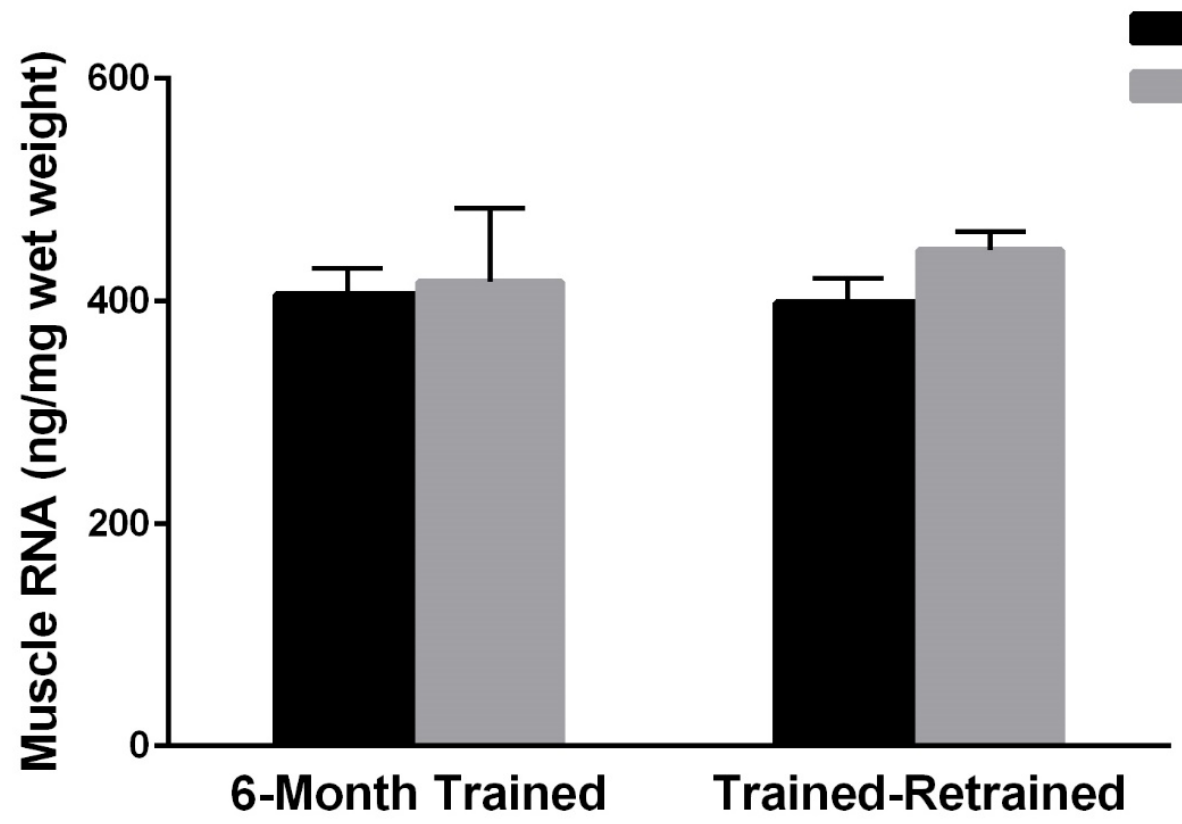

(B)

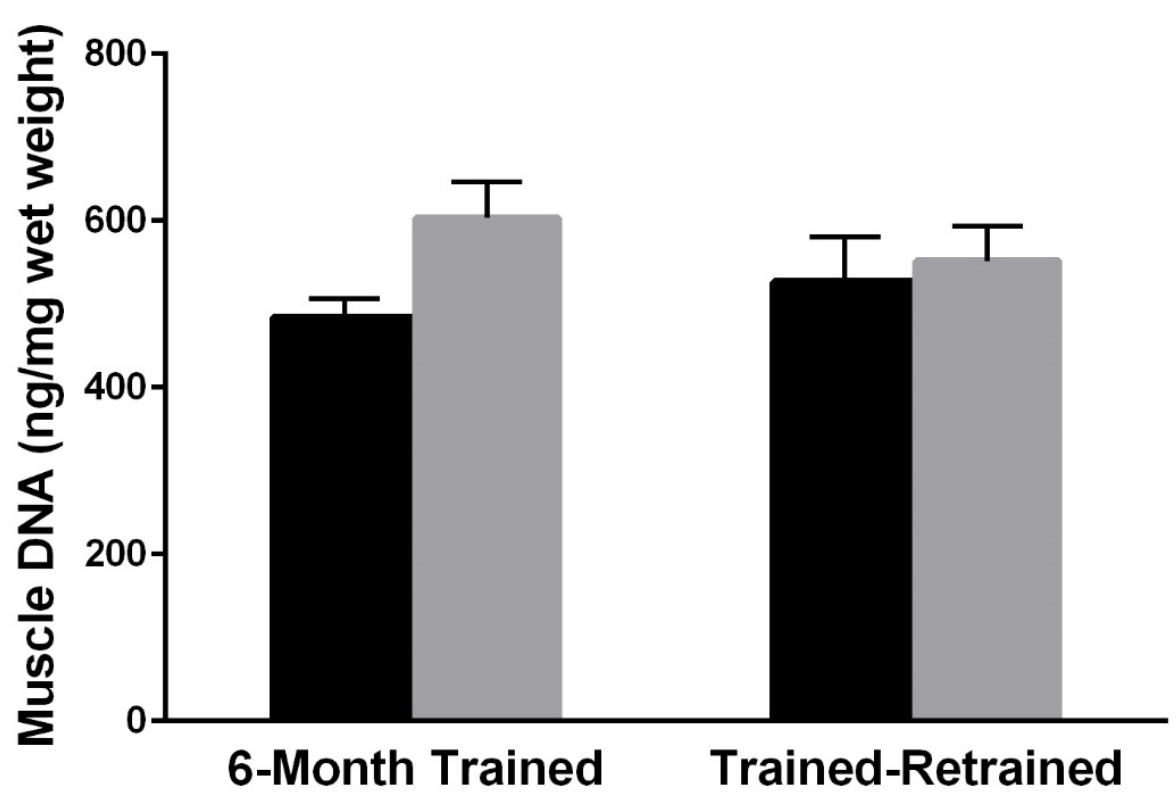


(C)

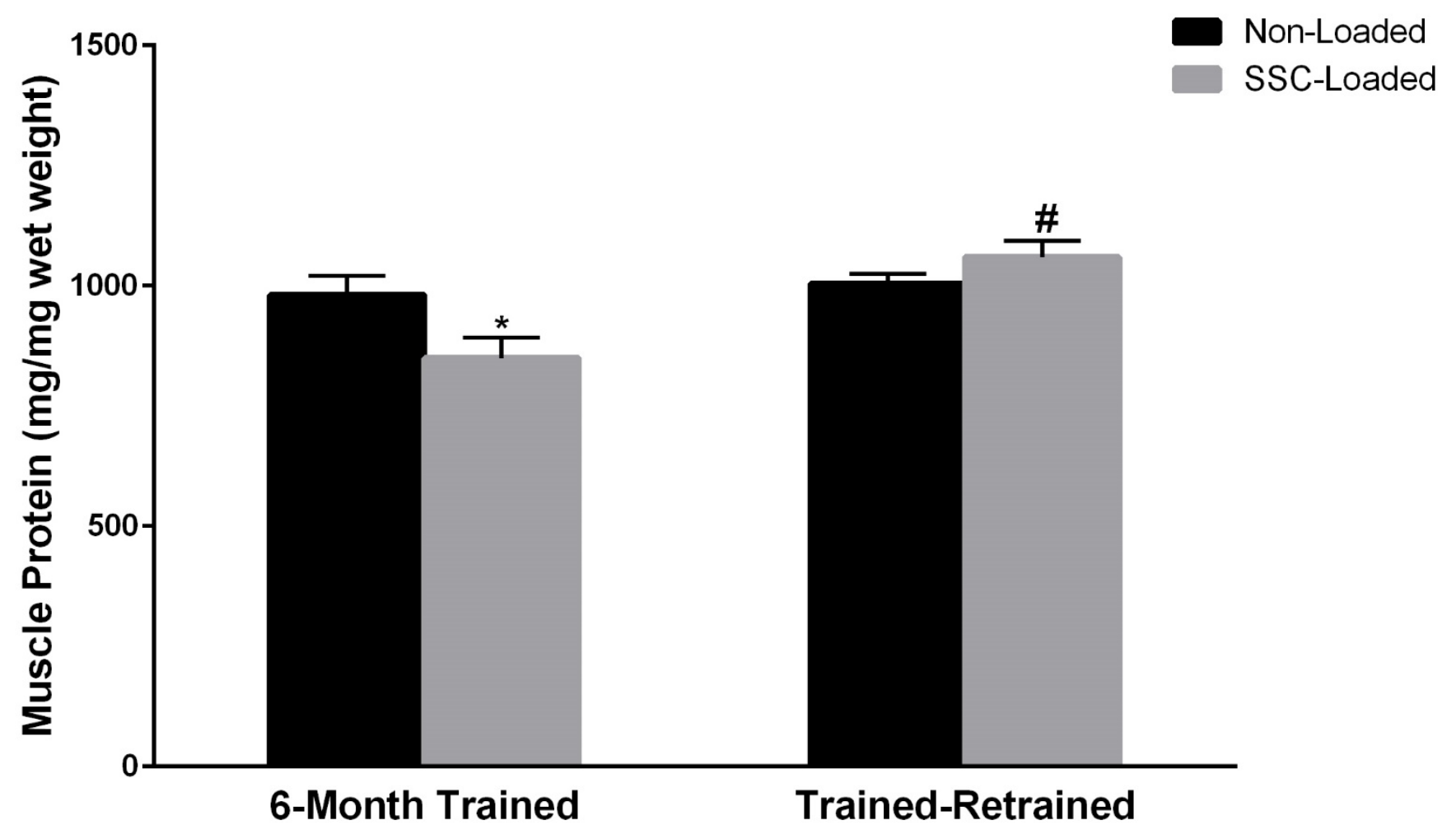




\section{CHAPTER VII}

\section{DISCUSSION}

The aims and experiments outlined in this dissertation were the first attempt at characterizing the effects of a reduced frequency of high-intensity stretch-shortening contraction (SSC) resistance-type exercise training (RTET) using a valid and reliable in vivo rodent dynamometer model in order to fill in the gaps in our knowledge about the underlying molecular signaling pathways and epigenomic processes (i.e., DNA methylation) modulating aged-related muscle adaptation as well as the therapeutic efficacy of a high-intensity SSC RTET training-retraining (TRT) paradigm, and whether or not being exposed to an adaptive exercise stimulus at an earlier age is able to prevent and/or attenuate age-related impairments of skeletal muscle during the later stages of life. The hypotheses for this research were that older animals that underwent high-intensity SSC RTET at a reduced frequency would respond in an appropriate manner (i.e., adaptation) and more closely resemble young rats in terms of the molecular signaling pathway and DNA methylation responses compared to age-matched counterparts exposed to a higher frequency of training. Moreover, aged skeletal muscle that underwent high-intensity SSC RTET would undergo maladaptation when exposed to an inappropriate exercise prescription (i.e., higher frequency), and this would occur as a consequence to a dysregulated molecular signaling response that would be seen in distinct pathways crucial in muscle growth, homeostasis, and remodeling; we further hypothesized that these potential age-related impairments in signaling events at the transcriptional level would occur as a consequence to increased levels of DNA methylation. Finally, it was hypothesized that training rodents at a younger relative age (i.e., three months of age) using high-intensity SSC RTET would positively influence the ability of skeletal muscle to adapt upon reintroduction to the same paradigm at a later age previously shown to have the inability to go through the full adaptive response (i.e., 6 months of age), and this would be attributed in part to an attenuated DNA methylation and enhanced gene expression response in the transcription factors pathway.

Sarcopenia, traditionally defined as the age-related decline in skeletal muscle mass (11) but more recently has become a more all-encompassing term in which declines in strength and muscle quality (MQ) are not only included but are considered more important features than the actual loss of muscle mass itself (5), results in a loss of functional capacity; which, subsequently increases the risk of disease, disability, frailty, and all-cause mortality. Exercise is well-known for its benefits in improving health and attenuating or preventing many chronic diseases, while being arguably more efficacious than any other lifestyle, pharmacological, or nutritional supplement intervention (33). For the previous two decades, our laboratory has utilized an in vivo rodent dynamometer model that employs the use of SSCs in order to explore the underlying mechanics of muscle adaptation to various parameters of the exercise prescription and have shown that the responses to 
RTET using this physiological model range from adaptation, characterized as leading to increases in muscle mass and performance, to maladaptation, a term used to describe the absence of muscle mass gains and diminished performance $(1,6,22,24)$. Collectively, the results from this dissertation provide important information regarding the understanding of the underlying biomechanical performance, physiological, molecular, and environmental factors influencing the capability of aged skeletal muscle to undergo adaptation in response to high-intensity SSC RTET in response to an appropriate exercise stimulus of reduced frequency training, which has important implications in the attenuation and/or reversal of sarcopenia. Additionally, the results demonstrate that a TRT paradigm is an effective therapeutic tool that can be utilized for attenuating and/or preventing the functional and physiological decrements of skeletal muscle that typically occur during the later stages of life by training at an earlier age before the onset of age-associated declines in skeletal muscle size and function. Despite the therapeutic efficacy of RTET, a fundamental basis for evidence-based exercise prescription was still vastly unknown because the integrated molecular, cellular and physiological pathways involved in exercise-induced muscle adaptation had not been fully characterized. Additionally, it was unclear whether or not manipulating the frequency or other variables of exercise could be used to change the underlying responsivity of signaling pathways important in the regulation of skeletal muscle, particularly in a high-intensity SSC in vivo animal model of RTET; this was the focus of chapters three through five of the present dissertation research.

The purpose of the third chapter of this dissertation was to determine whether decreasing the frequency of training, and thereby increasing recovery time between training sessions, would be sufficient to induce an adaptive response. Specifically, the hypothesis tested was that decreasing the frequency of training would improve adaptive outcomes in aged skeletal muscle for old rats. In order to attempt to answer this question, muscle mass (normalized to tibia length), isometric force, MQ (i.e. isometric force divided by normalized muscle mass), dynamic peak force, negative work, and positive work were all calculated and quantified. Furthermore, quantitative morphology for tissue percentage of normal muscle fibers, degenerative muscle fibers, non-cellular interstitium, and cellular interstitium was assessed by standardized stereological methods. Lipid peroxidation was also quantified as an indicator of oxidative stress because of findings in previous reports indicating oxidative stress as a factor in SSC-induced adaptation/maladaptation (28, 29). The results showed that modulating the exercise prescription by reducing the training frequency from three to days per week not only resulted in adaptation as indicated by positive augmentations in muscle performance and muscle mass, but also resulted in decreased oxidative stress (via levels of lipid peroxidation) and enhanced MQ in older rodents to levels indistinguishable to younger rats that adapted. These findings demonstrated that age-appropriate highintensity SSC-training has the potential to reduce lipid peroxidation levels, offset sarcopenia via enhanced MQ, and promote healthy aging. Collectively, this chapter established for the first time that modifying the exercise 
prescription by reducing the frequency of RTET attenuates age-dependent maladaptation and restores MQ and levels of oxidative stress to that of a younger phenotype.

The purpose of the fourth chapter of this dissertation was to define and extend the previous findings from chapter three by implementing an integrated systems biology approach using representative PI3K-AKT pathway gene expression and bioinformatics via ingenuity pathway analysis (IPA), along with various biochemical, morphological, and physiological muscle measures. Our hypothesis was that following highintensity SSC RTET young rats would respond at the performance, physiological and molecular level in an adaptive manner regardless of whether or not training frequency was reduced, and that old rats trained at a reduced frequency would also undergo adaptation while having a molecular growth and remodeling signaling response that would be similar to the younger rats. In contrast, old rats trained at a higher frequency would have a blunted response indicative of maladaptation.

The main findings from this chapter were: 1) There was a distinct molecular signature following RTET utilizing SSCs in old rats that accompanied adaptive outcomes, and that these responses appear to be connected to the PI3K-AKT signaling pathway, with SDEGs involved in skeletal muscle growth and remodeling that were present in both young rats as well as old rats trained at a reduced frequency; 2) the effects on gene expression in old rats trained at a reduced frequency had a positive influence on downstream outcomes including central nucleation and increased MND; and 3) this work advanced previous research from our laboratory demonstrating the efficacy of a reduced-frequency exercise paradigm aiding the adaptability of aged skeletal muscle in response to high-intensity SSC RTET, as reflected by positive changes in muscle performance and muscle mass. Overall, old rats trained at a reduced frequency of two days per week was clearly superior over old rats trained at a higher frequency of three days per week as indicated by increased skeletal muscle performance and enhanced muscle fiber area and MND similar to younger rodents. Moreover, the results from this chapter showed that appropriate, age-specific RTET in old rats trained at a reduced frequency of exercise led to increased expression of PI3K-AKT genes as reflected by the $\mathrm{RT}^{2}$ Profiler $^{\mathrm{TM}}$ Arrays and bioinformatics analysis via IPA, whereas a dysregulated response occurred in old rats trained at a higher frequency. These results indicate that when prescribed in a proper manner, high-intensity SSC RTET can directly modify PI3K-AKT pathway gene activity within the skeletal muscle, ultimately leading to adaptation. Therefore, reducing the frequency of RTET in a manner in which increased recovery time between sessions allows for skeletal muscle to initiate muscle repair and remodeling processes provides an ideal environment to help restore and/or promote improved muscle performance, physiology as well as positive changes in the skeletal muscle microenvironment, which ultimately helps enrich soft-tissue quality and functional outcomes with advanced age. Collectively, this chapter has provided key insights into the growth and remodeling signaling process in skeletal muscle in response to appropriately prescribed, age-specific RTET involving PI3K-AKT pathway expression, which 
partially underlies training-induced skeletal muscle adaptation with aging, and supports the notion that reducing the frequency of training can abolish aged-associated negative signaling events in key pathways involved in the growth and remodeling of muscle, as well as revitalize the systemic environment in a favorable manner that results in adaptation.

The purpose of the fifth chapter of this dissertation was to determine the effects of aging and a reduced training frequency on the activation of molecular signaling pathways associated with the maintenance and adaptation of skeletal muscle (i.e., apoptosis, DNA repair) following one month of high-intensity SSC RTET in old rats. Moreover, we sought to investigate whether or not DNA methylation was playing an active role in the molecular signaling events underlying the ability of skeletal muscle to go through adaptation following highintensity SSC RTET, and whether or not a reduced frequency of training would modulate the DNA methylation status of aged muscle. Our hypothesis for this research was that older animals exposed to a reduced frequency of high-intensity SSC RTET would respond in an appropriate manner (i.e., adaptation) and would more closely resemble young rats in terms of the underlying molecular signaling pathway, DNA methylation, and redox environment events compared to age-matched counterparts exposed to a higher training frequency that induced maladaptation. In addition, we postulated that the ability of aged muscle to adapt to high-intensity SSC RTET would be compromised when exposed to an inappropriate (i.e., maladaptive) stimulus as a consequence of an impaired molecular signaling response, and that this would be noticeable in distinct pathways important in the maintenance of muscle homeostasis and tissue remodeling; we further hypothesized that alterations in gene activity would occur as a consequence to modifications in levels of DNA methylation.

The main findings from this study were that there was a distinct molecular signature following RTET utilizing SSCs in old rats that accompanied adaptive and maladaptive outcomes, which seems to be associated with a distinct pattern of SDEGs involved in the skeletal muscle stress response and muscle homeostasis (i.e., apoptosis and DNA repair pathways) that was present in both young groups and old rats trained at a reduced frequency. Furthermore, these effects on gene expression in old rodents trained at a reduced frequency had a positive impact on downstream outcomes, including modifications in myonuclei size and morphology. Additionally, our hypothesis of DNA methylation being a highly influential epigenomic factor involved in the regulation of the aged muscle stress response to exercise seems to have been in part supported by our findings of decreased methylation in the apoptosis pathway, although it does not appear be the primary regulator of control in the DNA repair pathway. Finally, this work advanced previous research from our laboratory demonstrating the efficacy of a high-intensity SSC RTET reduced frequency paradigm assisting in the adaptability of aged muscle as reflected by positive changes in muscle performance and MQ.

Our results demonstrated that old rats trained at a reduced frequency of exercise were clearly superior compared to old rats trained at a higher frequency in terms of the adaptive response to SSC-training, given the 
increases in muscle performance and improvements in MQ shown that were more similar to younger rodents. Additionally, the present data revealed that old rats trained at a reduced frequency were able to undergo adaptation concomitant with enhanced activation of the apoptosis and DNA repair pathways. Given the current molecular data reported in this chapter (i.e., profiler array gene expression, IPA, cell death ELISA, myonuclei size and myonuclei morphology) along with the skeletal muscle performance and muscle mass data that corroborates with other studies in humans $(3,15-18,21,35)$, our results indicate that high-intensity SSC RTET prescribed in the appropriate manner (e.g., a reduced training frequency) can be highly beneficial for aged muscle, particularly in individuals who respond unfavorably to higher frequencies of exercise. Of note, this chapter contains, to our knowledge, the first data showing age and training specific alterations in DNA methylation of the apoptosis pathway following high-intensity SSC RTET. Specifically, rats that maladapted to a higher frequency of training had higher levels of methylation along with decreased gene expression of the apoptosis pathway. More importantly, modifying the exposure in old rodents from three to two days per week resulted in an improved molecular environment as indicated by lower levels of DNA methylation along with enhanced gene expression of the apoptosis pathway. Additionally, we found that old rats exposed to a higher frequency of training had significantly higher cell death in comparison to old NLC following training, whereas cell death was attenuated as a result of training in old rats trained at a reduced frequency. Collectively, these findings, along with the well-known functions of various apoptotic molecules as essential regulators of a host of events relative to development, homeostasis and “stimulus-driven events” within skeletal muscle (8), supports our contention that DNA methylation may be a mechanism that is a key component leading to a dysregulated apoptotic signaling response in old maladaptive muscle exercised at a higher frequency, which ultimately has a negative impact on its adaptability in response to inappropriately prescribed high-intensity SSC RTET. In skeletal muscle exposed to an appropriate exercise prescription of a reduced frequency of high-intensity SSC RTET that promotes adaptation, the data shows that increased apoptosis signaling seems to be an underlying mechanism contributing to the adaptive process.

In regards to the DNA repair pathway, the present chapter demonstrates that appropriate, age-specific RTET for old rats trained at a reduced frequency results in an enhanced expression of DNA repair genes, whereas an irregular response occurs in old rats that maladapted to a higher frequency of training as indicated by a downregulation of numerous genes in this pathway. This is an important point because these observations suggest that appropriately prescribed high-intensity SSC RTET at a reduced frequency can directly modify local DNA repair activity within skeletal muscle, ultimately leading to an adaptive or maladaptive fate. Despite these differential patterns of gene expression shown, and against our hypothesis, levels of DNA methylation in this pathway did not corroborate accordingly to these responses; old rats trained at a higher frequency of exercise actually had the least amount of methylation, yet had a robust downregulation of DNA repair gene expression 
following training. While further investigations will have to be undertaken to confirm our results, these results suggest that methylation is not the primary epigenetic/epigenomic regulator of DNA repair pathway gene expression, and perhaps other regulatory mechanisms (i.e. chromatin morphology) may provide an active role in the control of the DNA repair pathway gene expression and/or another epigenomic process, such as acetylation, is more prominently involved.

Despite a large body of previous work investigating the number and functions of myonuclei in skeletal muscle in response to both aging and/or exercise training, there is a paucity of literature regarding specific modifications on myonuclei size and morphology. Therefore, possibly the most fascinating component of this chapter is that for the first time training-induced alterations in myonuclei size and morphology in response to a resistance exercise training paradigm are reported. Specifically, the frequency distributions for myonuclei size and morphology in young rats as well as old rats trained at a reduced frequency showed that the myonuclei minimum feret decreased in these groups, whereas there were no effects in old rats trained at a higher frequency that maladapted. Additionally, decreases in circularity (i.e., more elongated) of myonuclei were only noted in old rats trained at a higher frequency, whereas young rats and old rats trained at a reduced frequency had no alterations in circularity, thus were able to maintain a more circular shape following RTET. Interestingly, previous work showed there is an association between cell shape and changes in nuclei morphology along with cellular proliferation in endothelial cells in which as cells became more elongated, this led to decreases in nuclear volume, which subsequently caused the nuclei to elongate and became less circular; this elongation then resulted in increased chromatin condensation and eventual decreases in cell proliferation. (38). Our results showing elongation of myonuclei in old rats that maladapted in comparison to old rats trained at a reduced frequency that adapted are directly in line with these previous findings. Collectively, our data supports the hypothesis that alterations in myonuclei morphology following exposure to either an appropriate (i.e., adaptation to a reduced frequency) or inappropriate (i.e., maladaptation to a higher frequency) high-intensity SSC RTET stimulus are directly altering the myonuclei size and morphology, which subsequently affects the molecular signaling events and ultimately the global biological response (see Figure 11 of chapter five). Overall, this chapter has provided critical insights into the regulation of skeletal muscle to appropriately prescribed, age-specific RTET involving apoptosis and DNA repair pathway expression, which partially underlies the adaptive training response with advanced age by supporting the concept that appropriate exercise prescription (e.g., reduced frequency of training) reverses and/or attenuates aged-associated negative alterations in key pathways involved in the stress response and remodeling of skeletal muscle, helps rejuvenate the systemic environment in order to maintain muscle homeostasis, and eventually leads to muscle adaptation.

The purpose of the sixth chapter of this dissertation was to investigate the impact of a high-intensity SSC RTET TRT paradigm in terms of how training at a young age affects skeletal muscle form and function 
during adulthood. Our hypothesis was that adaptive high-intensity SSC RTET at three months of age would result in adaptation at six months of age upon reintroduction to the same exercise stimulus in comparison to naïve six month old rodents not receiving this previous bout of exercise training. Furthermore, we believe these positive effects in adaptive outcomes (e.g., skeletal muscle performance, muscle mass, and MQ) in a TRT paradigm would be complemented by a molecular and epigenomic pattern in TFs signaling that would provide underlying support for the existence of a cellular muscle memory that subsequently would allow adaptation to occur. The main findings from this study were: 1) A distinct molecular signature following high-intensity RTET utilizing SSCs in a TRT paradigm of training exists that underlies adaptive outcomes, and these responses appear to be connected to the TFs signaling pathway; 2) these effects from an high-intensity bout of SSC RTET at an earlier age resulted in an augmentation of positive adaptive outcomes in adulthood upon reintroduction to the same exercise stimulus, including skeletal muscle performance, muscle mass, and muscle protein; 3) our results were consistent with previous findings by others showing that training leads to decreased DNA methylation when the same adaptive stimulus was reintroduced at later life, thus confirming that methylation appears to be a highly influential epigenomic factor involved in the underlying muscle memory response to exercise in aged skeletal muscle; and 4) this work advances previous research from our laboratory demonstrating the efficacy of our validated, in vivo, physiological rodent dynamometer model of high-intensity SSC RTET as an aid to prompt skeletal muscle to undergo adaptation, and builds upon the TRT paradigm that has been reported in recent human literature.

Despite the suggested underlying basis for the involvement of epigenetic/epigenomic regulation in the ability of skeletal muscle to adapt to exercise, no research prior to this chapter had determined whether or not alterations in DNA methylation that occur as a result of exposure to an exercise stimulus using an in vivo physiological animal model of high-intensity SSC RTET could have a lasting or permanent influence on the epigenetic/epigenomic responsivity as well as the adaptability of skeletal muscle upon reintroduction to the same stimulus at a later period of life. Thus, the results of this chapter have important implications for furthering our understanding of muscle adaptation in the context of a TRT paradigm, and the present results showing beneficial effects in later life from SSC RTET at an earlier age have enormous therapeutic ramifications in decreasing and/or preventing against the susceptibility to diseases directly associated with aging, such as sarcopenia, which ultimately can help improve healthspan in the aging population.

To determine if muscles were capable of adapting to high-intensity SSC RTET at a later period of time following a previous training bout, we exposed muscles of young rats at three months of age to 80 SSCs, three days per week, (72 hours between training bouts) for one month, then had them detrain for two months before re-exposing them to the same exercise stimulus that they did previously for one month during adulthood at six months of age, then directly compared them to a separate group that only trained at six months of age. Similar 
to the response to 80 SSCs, three days per week exposure shown from our previous work to augment adaptive outcomes following training $(6,22)$, the TRT exposure paradigm enhanced adaptive outcomes which was shown through improved static and dynamic muscle performance, as well as increased muscle mass and a trend for higher $\mathrm{MQ}$, upon reintroduction to the same exercise protocol at six months of age. This data is particularly noteworthy because our laboratory had previously demonstrated that six months of age was where an altered adaptive phenotype started to occur following this precise high-intensity SSC RTET protocol (25). The six month trained only group confirmed this previous work, as they had increases in muscle mass but no improvements in isometric or dynamic SSC performance following one month of training, whereas six month old rats that had received a previous bout of this adaptive exercise stimulus at three months of age not only had augmented improvements in both muscle performance and muscle mass gains compared to NLC control muscle, but was also significantly greater compared to the SSC-loaded limb of rats that only trained at six months of age. This substantiates our hypothesis that adult skeletal muscle is robust in its ability to undergo adaptation in response to a high-intensity SSC RTET paradigm after previously encountering the same bout of exercise at earlier life, supporting previous studies showing an existence of muscle memory (30, 32), but for the first time using a validated, in vivo, physiological mechanical loading model.

Taken together, this chapter provides key insights into the molecular and physiological responses of skeletal muscle using a TRT paradigm of appropriately prescribed high-intensity SSC RTET. Part of this response is due to selective changes in the TFs pathway gene expression, which partially underlies training adaptation with aging. Specifically, the current data is suggestive that the TRT paradigm is accompanied by a distinct epigenetic/epigenomic signature; adult rats in the TRT group had significantly decreased methylation of the stem cell TFs pathway. Interestingly, there were differences in the responses underlying adaptation in the TRT group compared to rats that trained only at six months of age that partially adapted. Decreased expression of the majority of SDEGs in the TRT group on the TFs pathway array was found, which went against what we had hypothesized; however, despite this overall downregulation, it was notable that the two genes that were significantly upregulated in the TRT group, ATF3 and MYF5, have been previously implicated to be highly involved in the adaptive growth and cellular stress response to RTET $(12,13,39)$. Therefore, while there is an overall downregulation of TFs in the TRT paradigm, there is an upregulation in select molecules critical for growth and homeostasis that are subsequently assisting in the process of allowing muscle adaptation to occur at an age previously shown to have an impaired adaptive capacity. Additionally, our results of decreased DNA methylation in the stem cell TFs pathway in the SSC-loaded limb of the TRT group in comparison to the six month trained only group are in line with past findings showing alterations in methylation status following adaptive exercise with respect to chronic exercise training (19, 20, 23). Overall, from a global perspective for gene expression and DNA methylation, our results show there is an overall decrease in stem cell TF methylation 
along with a discriminatory upregulation of a small subset of genes important in the growth and regulation of skeletal muscle. Overall, these signaling events are ultimately playing an important role in the TRT group being able to undergo adaptation (i.e., significantly improved skeletal muscle performance as well as increases in muscle mass and muscle protein) into adulthood as a product of their previous exposure to high-intensity SSC RTET at a younger age.

To summarize, adult skeletal muscle demonstrates an impairment in its ability to adapt properly to highintensity SSC RTET shown to promote adaptation in younger rodents; however, when exposing rodents to this same exercise stimulus at a younger age that is proven to promote adaptation, aged skeletal muscle is capable of adapting through augmented muscle performance and improved muscle mass. Moreover, for the first time, we provide evidence that an appropriately prescribed high-intensity SSC RTET TRT exercise paradigm induces positive modifications in TFs pathway gene expression and methylation, which we believe helps shape downstream events that ultimately enables skeletal muscle to undergo adaptation. Therefore, the findings in the present study support the concept that an appropriate exercise prescription at a young age reverses and/or attenuates aged-associated negative outcomes during adulthood and rejuvenates the systemic environment in a favorable manner that leads to muscle adaptation.

Finally, Appendix A, while not a direct component of the dissertation research, was nonetheless very complementary to the experiments and results outlined in chapters three through five of this dissertation. For this appendix, we used a secondary analysis of data from the National Health and Nutrition Examination Survey (NHANES) in order to examine the statistical associations between sufficient levels of resistance training and MQ. For this research, we obtained publicly available data from the NHANES database for two different sampling periods from 1999-2002 who were above the age of 55. For this study, individuals filled out a physical activity questionnaire and were asked about their participation in resistance training based activities from the past 30 days; based on their responses, these individuals were placed in to one of two groups: 1) “insufficient” if they performed resistance training less than two times per week; and 2) “sufficient” if they performed resistance training three or more times per week. MQ was calculated based on muscle strength data of the knee extensors that was obtained by NHANES staff on an isokinetic dynamometer, along with leg lean muscle mass that was obtained using dual-energy absorptiometry. Collectively, the results from this appendix are the first published secondary analysis of NHANES data that has attempted to characterize the statistical associations between sufficient levels of resistance training based on the frequency of resistance exercise with levels of MQ in older adults. The main findings were that in an NHANES population-based cohort of humans over the age of 55 years old that the frequency of resistance training is associated with greater MQ in select age groups, as there were statistically significant associations in those aged 55-64 and 55-79 years of age, but not in individuals aged 65 and older, as well as in those stratified in to a $65-79$ group as well as $\geq 80$ years of age. Collectively, our current 
NHANES results from Appendix A, in combination with our previous data from chapters three through five of this dissertation, suggests that the adaptive capacity of skeletal muscle in response to high-intensity SSC RTET is age-specific and dependent upon how components of the exercise prescription are structured and that with age, individuals are increasingly sensitive to the parameters of training presented to them and become more susceptible to the untoward effects of an inappropriate exercise prescription. Therefore, appropriately modified exercise prescription is very likely to be a critical component enabling improved MQ.

\section{FUTURE DIRECTIONS}

While the results from the present dissertation provide valuable information about the molecular and epigenomic underpinnings to muscle adaptation with aging in response to high-intensity SSC RTET, in an aged versus young state, there are several key areas currently unknown and need to be resolved in future studies. First, there are immediate experiments that can be performed in order to complement and advance the findings of the current dissertation work; to accomplish this, immunohistochemistry will be performed in order to visualize and quantify targets of interest that we have already examined biochemically either at an mRNA level or at a protein level. Specifically, we would like to take frozen TA muscle sections and stain using an antiDNA/RNA damage antibody (Abcam), an anti-5-methylcytosine antibody (Calbiochem), and terminal deoxynucleotidyl transferase dUTP nick end labeling (TUNEL) using a flurometric TUNEL detection kit (Roche).

The anti-DNA/RNA damage antibody binds with high affinity to 8-OHdG as well as other derivatives such as 8-oxo-7,8-dihydroguanine and 8-oxo-7,8-dihydroguanosine; we will use this label to provide visual evidence along with subsequent quantification via stereological analyses in order to complement and/or confirm the ELISA 8-OHdG results presented in the fifth chapter of this dissertation. It will be particularly interesting to analyze how much colocalization exists with DNA/RNA damage at the myonuclei using DAPI staining and whether or not aged rats trained at a higher frequency that maladapt have greater amounts of DNA/RNA damage positive myonuclei in comparison to aged rats trained at a lower frequency that underwent adaptation. The TUNEL assay detects DNA breaks formed following fragmentation in response to damage incurred to the DNA and, subsequently, apoptosis, and we will quantify the number of TUNEL positive myonuclei using frozen TA sections in order to supplement the cell death ELISA data presented from the fifth chapter of this dissertation. Specifically, we will be looking to see if old rats exposed to a higher frequency of exercise have greater amounts of TUNEL positive myonuclei present in comparison to old rats trained at a reduced frequency of exercise. Finally, the anti-5-methylcytosine antibody will provide visual evidence along with subsequent stereological quantification to detect its presence in the skeletal muscle tissue in order to confirm the pathway promotor-specific methylation profiles examined in the fifth and sixth chapters of this dissertation. Moreover, 
we would again like to see whether or not there is colocalization between the target antibody with myonuclei and whether or not differences exist between aged rats undergoing adaptation versus maladaptation as well as in the rodents who exercised following the TRT paradigm versus six month trained only animals. Based on the work presented in this dissertation, we would expect there to be higher amounts of myonuclei with colocalized 5-methylcytosine in rats that have undergone maladaptation following a higher frequency of exercise in chapters three through five of the dissertation along with six month old trained only rats in comparison to those following a TRT paradigm from chapter six.

Another experiment to follow up the work presented in this dissertation in the near future will be to use the Simple Western platform system (ProteinSimple) to quantify protein expression for various targets of interest. The Simple Western is a recently developed technique which utilizes a capillary nanoimmunoassay system that has overcome some of the logistical and methodology constraints of traditional Western Blots by utilizing capillaries that allow for separation, capture, immobilization, and detection of proteins via chemiluminescence in a significantly shorter amount of time (36). Using this technique, we would like to target individual SDEGs from the gene pathway arrays that have been implicated previously in the regulation of skeletal muscle in response to high-intensity SSC RTET in order to confirm and/or extend the findings that were shown at the transcriptional level. Specifically, given the data presented in this manuscript, we would like to examine the following molecules: ITGB1, FOS, and JUN based on the findings seen in the fourth chapter of this dissertation; TRAF3, TWEAK, and TDG from the fifth chapter of this dissertation; MYF5 and ATF3 from the sixth chapter of this dissertation. Moreover, because we looked at the PI3K-AKT pathway in the fourth chapter of the dissertation, it would be interesting to examine AKT along with other molecules involved in the hypertrophic response to resistance exercise, such as paired box 7 and the myogenic regulatory factors MYOD, myogenin, and myogenic factor 6.

In regards to new research questions, there are several different areas to consider as part of future research to the present work outlined in this dissertation manuscript. First, all of the work characterizing the molecular and epigenetic/epigenomic regulation of skeletal muscle in response to a reduced frequency of RTET should be extended and include more age groups in order to characterize the exercise induced adaptive response across the entire aging spectrum. Our laboratory and others previously showed that rat skeletal muscle in adulthood (6-9 months of age) and advanced adulthood (26-29 months of age) have significantly less muscle mass and/or muscle performance, which indicates that the susceptibility to milder forms of maladaptation can occur at ages earlier than previously thought $(7,25)$. Another variable that could be examined in future studies is the fiber type distribution. Previously, our laboratory was able to show that one month of high-intensity SSC RTET for three days per week induced a noticeable shift in the fiber type distribution in younger rodents as indicated by significant changes from type IIb to IIx fibers, whereas this response was absent in old rats that 
maladapted to the same three day per week exercise protocol (25). Moreover, it was found that the younger rats had a significantly enhanced ability to recover from fatigue compared to older rodents. These results suggest that shifts in fiber type distribution could be playing a role in the adaptive or maladaptive response to highintensity SSC RTET. Thus, a future research question to look at is what the fiber type distribution would look like in aged skeletal muscle following a reduced frequency of high-intensity SSC RTET shown in the present work to promote adaptation; based on the current data contained herein, we would speculate that aged muscle exposed to a reduced frequency would have a fiber type distribution shift that more closely looks like younger rats that adapted to the three day per week loading paradigm.

Further investigating and more fully characterizing the stress response involvement in high-intensity exercise following the modification of variables involving the RTET prescription with aging are warranted in future research as well, with the findings shown in chapter five of this dissertation providing an underlying foundation to build upon. For instance, given our results shown from IPA, it would be interesting to more closely examine the TWEAK/FN14 response to high-intensity SSC RTET to examine age and training-specific alterations in this signaling axis underlying adaptation or maladaptation following training. Furthermore, given the results seen of an altered DNA damage and repair response occurring in aged skeletal muscle as a result of inappropriately prescribed higher frequency exercise, it would be interesting to examine the precise factors that are accounting for this blunted response. For instance, since our results from the fifth chapter of the dissertation seem to indicate that methylation of the DNA repair pathway is not playing an important role in its regulation, examining other regulatory mechanisms such as acetylation or microRNA regulation of both individual molecules and globally from an overall pathway perspective could provide key insights in to the regulation of the DNA repair response underlying skeletal muscle adaptation in response to high-intensity SSC RTET.

Another important topic in future research is to more fully characterize how various molecular, epigenetic/epigenomic, and morphological outcomes to high-intensity SSC RTET are influenced by other components of the exercise prescription besides frequency (e.g., repetition number, velocity, exercise selection, intensity, etc.) in order to resolve in full what comprises the most optimal exercise program that will aid the ability of aged skeletal muscle to undergo adaptation. For instance, a recent study from our laboratory demonstrated that when the repetition and contraction type were modulated, young rats had performance and muscle mass gains regardless of whether they were exposed to four high-intensity isometric contractions (four sets of one repetition) or 40 SSCs (four sets of 10 repetitions) for three days per week. Only old rats exposed to the 40 SSCs protocol showed any sign of adaptation, as they were able to have gains in muscle mass and had reductions of TNF- $\alpha$ to levels similar to younger rats (26). However, in contrast to data shown in older rats trained at a reduced frequency in the third chapter of this dissertation work, there were no improvements in performance nor any changes in MQ in old rats exposed to this moderate SSC protocol, demonstrating a partial 
but incomplete adaptive response. Overall, these findings, along with the results shown from the third through fifth chapters of this dissertation, as well as Appendix A, demonstrates the increased sensitivity and complexities of what comprises an appropriate exercise prescription as one advances in age that promotes adaptation to high-intensity SSC RTET. Therefore, there is a critical need for additional, well-designed, randomized controlled trials in older adults focusing on characterizing those parameters (mode, frequency, intensity, etc.) that result in positive training-induced modifications in skeletal muscle until a consensus can be reached as to what encompasses the optimal RTET prescription that gives the best chance for aged muscle to respond favorably by undergoing adaptation as reflected by changes molecularly, physiologically, and at the performance level.

Because the experiments outlined and conducted in the sixth chapter of the current dissertation work was an initial exploratory analysis, there are several other experiments that could be done to build upon the current work. First, it would be beneficial to have slides stained for hematoxylin \& eosin in order to do stereological analyses and quantify various parameters of the morphological response in the same manner that we have previously reported $(2,27)$, which would include calculating the percentages of normal/degenerative fibers, cellular interstitium (consisting of all possible infiltrating cells such including inflammatory cells, endothelial cells, and fibroblasts) and non-cellular interstitium (indicative of swelling/edema). Moreover, we would also measure the total number of myonuclei as well as the percentage of CNFs present in rats trained at three months of age and then re-exposed to a second one month bout of high-intensity RTET at six months of age versus those only trained at six months old. Because of the conflict of results in studies explained previously in chapters two and six of the dissertation $(4,9,10)$, it would be interesting to see whether or not a higher myonuclei count and/or CNF response persists in rats following the TRT paradigm in comparison to those trained only at six months of age. In addition to stereological analysis, based on the results seen in the methylation of stem cell TFs pathway outlined in chapter six, an interesting follow-up would be to characterize specific histone marks that may be affected by a TRT paradigm that is resulting in reduced methylation. Based on previous research, we would potentially look to see if rats following the TRT paradigm have decreased trimethylation of lysines nine and 27 of histone H3 (H3K9me 2/3 and H3K27me3) or at lysine 20 of histone four (H4K20me3) since these are associated with a suppressed gene expression response (34). Alternatively or in combination, we could also look at the regulation of acetylation between groups by looking at the activity of histone acetyltransferases and the histone deacetylases to examine whether or not they are acting in concert with the DNA methylation responses contributing to a cellular skeletal muscle memory in rats following the TRT paradigm. Finally, given the results shown for minimum feret diameter and circularity of myonuclei outlined in the fifth chapter of this dissertation, staining slides for 4-HNE, $\beta$-dystroglycan, and DAPI and following it with a subsequent tracing analysis using ImageJ would be very useful for looking at modifications in myonuclear 
size and morphology. These characteristics have yet to be reported in rats following a TRT paradigm and at adulthood in animals that trained only at six months of age; we speculate that rats following a TRT protocol would have decreased minimum feret diameter and maintain circularity in a manner similar to the results shown in chapter five of the dissertation for young and old rats trained at a reduced frequency that underwent adaptation.

In regards to the applicability towards the TRT paradigm outlined in the sixth chapter of this dissertation, a previous study from Rader et al. (25) notably showed that six month old male rats who underwent one month of high-intensity SSC RTET had significantly enhanced fatigue recovery, as values for peak force, negative work, and positive work improved by $11 \%$ to $15 \%$, whereas these values were unchanged in three month old rats exposed to the same protocol. Moreover, it was found this improved fatigue recovery was accompanied by significant decreases in type IIb fibers along with concomitant increases in type IIx and type IIa fibers. Young three month old rats had no improvements in fatigue recovery, but as stated earlier, they had a shift in fiber type from type IIb to IIx; in contrast to the six month old rats, there were no changes in type IIa fibers. Given these previous results, it would be very intriguing to see the fiber type distribution and fatigue recovery curves of rats at six months of age following a TRT paradigm in comparison to their age-matched counterparts not receiving this previous bout of exercise at earlier life. We hypothesize that rats following a TRT paradigm of being exposed to an earlier adaptive bout of exercise training would lead to an augmented ability to recover from fatigue along with an even more pronounced shift in the fiber distribution phenotype (i.e., increased type IIa and/or type IIx) compared to rats trained only at six months of age.

Given the past findings by our laboratory and others demonstrating an impairment in adaptation to RTET at old age $(6,7,14,25)$, it is important to investigate whether or not the adaptive benefits of the TRT paradigm shown in the present study are applicable at older ages. Specifically, future studies should focus on further characterizing both the current TRT paradigm at even more advanced ages as well as how these molecular, epigenetic/epigenomic, and morphological variables are influenced by other components of the exercise prescription (e.g., repetition number, velocity, exercise selection, etc.) in order to fully define what comprises an optimal high-intensity SSC RTET TRT exercise paradigm necessary for muscle adaptation to occur as one ages. For instance, we reported in chapters three through five of this dissertation that 30 month old rats exposed to a reduced frequency of high-intensity SSC RTET (i.e., two days per week) promotes adaptation, whereas age-matched counterparts exposed to a higher frequency of this same exercise protocol (i.e., three days per week) induces maladaptation; thus, it would be intriguing to investigate a TRT paradigm in which 30 month old rats are trained for one month two times per week at 30 months of age, then retrained for a second one month bout of two day per week high-intensity SSC RTET at 33 months of age and see if they respond as favorably as the six month old rats exposed to a TRT paradigm from the sixth chapter of this dissertation. 
Another potential experimental design could be to have three month old rats perform one month of highintensity SSC RTET for three days per week, have them go through an extended detraining phase until they reach 30 months of age, and then reintroduce them to the same exercise stimulus and see if a muscle memory persists following this extended period of inactivity. The answers to these questions have important ramifications in the clinical efficacy of how exposure to high-intensity resistance exercise at a younger age is able to improve adaptive outcomes at a later time point following long periods of inactivity.

\section{CONCLUSIONS}

In conclusion, despite the fact that aged skeletal muscle has deficiencies in the ability to respond favorably to RTET, evidence from the third through fifth chapters of this dissertation provides clear evidence that the tissue still retains the capacity to undergo adaptation, both physiologically as well molecularly in the microenvironment, as long as the stimulus is prescribed in the appropriate manner. While acknowledging that it is not the sole component of the biomechanical loading signature involved in the biological response to exercise, the findings presented in this document supports the notion that in a high-intensity SSC in vivo animal physiological model of RTET, a reduced frequency of training is a highly influential factor that can be utilized in the titration of the exercise prescription in order to make aged skeletal muscle adapt properly, whereas an inappropriately prescribed exercise prescription using a higher frequency of training can lead to aged muscle undergoing maladaptation. Not only has this been established through traditional adaptive parameters such as skeletal muscle performance, muscle mass, and MQ, but also at the cellular level through integrated physiological pathways involved in exercise-induced muscle growth, homeostasis, and remodeling, as well as at the biochemical level through various protein measures.

In regards to various mechanisms that could potentially be influencing the molecular signaling responsivity to high-intensity SSC RTET that leads to an adaptive or maladaptive fate, a host of literature that was previously outlined in the first and second chapters of this dissertation points to the epigenetic/epigenomic regulation of gene expression as having a clearly defined role in both the overall aging process as well as in response to acute or chronic exercise, particularly DNA methylation. The results from the fifth chapter of this dissertation demonstrating decreased DNA methylation along with a concomitant increase in expression of the apoptosis pathway that accompanies adaptation to a reduced frequency of exercise confirms the fact that this epigenetic/epigenomic mechanism is a highly influential factor regulating the molecular signaling responses to high-intensity SSC RTET, and ultimately has an impact on the capability of aged skeletal muscle to undergo adaptation. Overall, the present results from chapters three through five of this dissertation demonstrate that aging is accompanied by an increased sensitivity to training frequency and approaches aimed at modifying the exercise prescription in a manner that reduces global levels of DNA methylation can be undertaken in order to 
help enhance gene expression and subsequently help rejuvenate the growth, remodeling, and stress response potential of skeletal muscle in order to attenuate and/or prevent negative age-related negative clinical maladies, such as sarcopenia; the data presented herein specifically implicates a reduced frequency of high-intensity SSC RTET as an effective therapeutic means for achieving these beneficial molecular, physiological, and functional outcomes in aged skeletal muscle.

While the term muscle memory, referring to the capability of a person to more quickly or superiorly respond to a specific exercise stimulus that has previously been encountered in comparison to a naïve one (e.g., improvement in muscle mass, strength, etc.), has typically been viewed to rely almost exclusively on the motor learning and neurological adaptations to exercise training, the results shown from the sixth chapter of this dissertation gives credence to more recent evidence establishing a cellular mechanism for the existence of muscle memory through epigenomic regulation by means of DNA methylation. Specifically, in response to an adaptive exercise stimulus at a younger age, rats following a TRT paradigm were shown to have less TFs pathway DNA methylation upon reintroduction to the same exercise stimulus during adulthood compared to adult rats not given this previous encounter of high-intensity SSC RTET. Given the decreased DNA methylation along with selective upregulation of transcripts important in the growth and homeostatic regulation of skeletal muscle, the results presented in this chapter supports previous work of methylation being an important underlying epigenomic regulator comprising a cellular form of muscle memory that occurs in response to an adaptive exercise stimulus, which has a lasting influence on the overall responsivity and adaptability of skeletal muscle upon reintroduction to the same stimulus at later life. This was indicated by the fact that rats trained at a young age had significantly enhanced skeletal muscle performance and muscle mass upon retraining in adulthood (i.e., TRT) compared to age-matched counterparts not receiving this previous bout of exercise. In summary, based on the therapeutic potential suggested by high-intensity SSC RTET for counteracting and/or attenuating age-related declines in skeletal muscle mass and function due to its ability to maximize motor unit recruitment; and, subsequently the number as well as the type of muscle fibers capable of being activated during training (i.e., type II) $(15,24,25,31,37)$, investigating the various parameters of the exercise prescription both in general adaptability to training as well as within the context of a TRT paradigm has been shown to have critical implications for aged skeletal muscle to have the ability to go through adaptation. Overall, the results presented from this entire dissertation work have demonstrated significant beneficial therapeutic effects from high-intensity SSC RTET and have substantial clinical relevance in preventing and/or decreasing the susceptibility to diseases directly associated with aging, such as sarcopenia, ultimately resulting in improved healthspan and perhaps contributing to increased lifespan in a rapidly and increasingly growing global aging population. 


\section{REFERENCES}

1. Baker BA, Hollander MS, Mercer RR, Kashon ML, and Cutlip RG. Adaptive stretch-shortening contractions: diminished regenerative capacity with aging. Appl Physiol Nutr Metab 33: 1181-1191, 2008.

2. Baker BA, Mercer RR, Geronilla KB, Kashon ML, Miller GR, and Cutlip RG. Stereological analysis of muscle morphology following exposure to repetitive stretch-shortening cycles in a rat model. Appl Physiol Nutr Metab 31: 167-179, 2006.

3. Borde R, Hortobagyi T, and Granacher U. Dose-response relationships of resistance training in healthy old adults: a systematic review and meta-analysis. Sports Med 45: 1693-1720, 2015.

4. Bruusgaard JC, Johansen IB, Egner IM, Rana ZA, and Gundersen K. Myonuclei acquired by overload exercise precede hypertrophy and are not lost on detraining. Proc Natl Acad Sci U S A 107: 1511115116, 2010.

5. Cruz-Jentoft AJ, Bahat G, Bauer J, Boirie Y, Bruyere O, Cederholm T, Cooper C, Landi F, Rolland Y, Sayer AA, Schneider SM, Sieber CC, Topinkova E, Vandewoude M, Visser M, Zamboni M, Writing Group for the European Working Group on Sarcopenia in Older P, and the Extended Group for E. Sarcopenia: revised European consensus on definition and diagnosis. Age Ageing 48: 16-31, 2019.

6. Cutlip RG, Baker BA, Geronilla KB, Mercer RR, Kashon ML, Miller GR, Murlasits Z, and Alway SE. Chronic exposure to stretch-shortening contractions results in skeletal muscle adaptation in young rats and maladaptation in old rats. Appl Physiol Nutr Metab 31: 573-587, 2006.

7. Degens H, and Alway SE. Skeletal muscle function and hypertrophy are diminished in old age. Muscle Nerve 27: 339-347, 2003.

8. Dostert C, Grusdat M, Letellier E, and Brenner D. The TNF family of ligands and receptors: communication modules in the immune system and beyond. Physiol Rev 99: 115-160, 2019.

9. Dungan CM, Murach KA, Frick KK, Jones SR, Crow SE, Englund DA, Vechetti IJ, Jr., Figueiredo VC, Levitan BM, Satin J, McCarthy JJ, and Peterson CA. Elevated myonuclear density during skeletal muscle hypertrophy in response to training is reversed during detraining. Am J Physiol Cell Physiol 2019. 
10. Egner IM, Bruusgaard JC, Eftestol E, and Gundersen K. A cellular memory mechanism aids overload hypertrophy in muscle long after an episodic exposure to anabolic steroids. J Physiol 591: 6221-6230, 2013.

11. Evans WJ. What is sarcopenia? J Gerontol A Biol Sci Med Sci 50 Spec No: 5-8, 1995.

12. Fernandez-Verdejo R, Vanwynsberghe AM, Essaghir A, Demoulin JB, Hai T, Deldicque L, and Francaux M. Activating transcription factor 3 attenuates chemokine and cytokine expression in mouse skeletal muscle after exercise and facilitates molecular adaptation to endurance training. FASEB J 31: 840-851, 2017.

13. Fernandez-Verdejo R, Vanwynsberghe AM, Hai T, Deldicque L, and Francaux M. Activating transcription factor 3 regulates chemokine expression in contracting C2C12 myotubes and in mouse skeletal muscle after eccentric exercise. Biochem Biophys Res Commun 492: 249-254, 2017.

14. Greig CA, Gray C, Rankin D, Young A, Mann V, Noble B, and Atherton PJ. Blunting of adaptive responses to resistance exercise training in women over 75y. Exp Gerontol 46: 884-890, 2011.

\section{Hagerman FC, Walsh SJ, Staron RS, Hikida RS, Gilders RM, Murray TF, Toma K, and Ragg}

KE. Effects of high-intensity resistance training on untrained older men. I. Strength, cardiovascular, and metabolic responses. J Gerontol A Biol Sci Med Sci 55: B336-346, 2000.

16. Hakkinen K, Kraemer WJ, Newton RU, and Alen M. Changes in electromyographic activity, muscle fibre and force production characteristics during heavy resistance/power strength training in middle-aged and older men and women. Acta Physiol Scand 171: 51-62, 2001.

17. Hakkinen K, Pakarinen A, Kraemer WJ, Hakkinen A, Valkeinen H, and Alen M. Selective muscle hypertrophy, changes in EMG and force, and serum hormones during strength training in older women. $J$ Appl Physiol (1985) 91: 569-580, 2001.

18. Hikida RS, Staron RS, Hagerman FC, Walsh S, Kaiser E, Shell S, and Hervey S. Effects of highintensity resistance training on untrained older men. II. Muscle fiber characteristics and nucleo-cytoplasmic relationships. J Gerontol A Biol Sci Med Sci 55: B347-354, 2000.

19. Kanzleiter T, Jahnert M, Schulze G, Selbig J, Hallahan N, Schwenk RW, and Schurmann A. Exercise training alters DNA methylation patterns in genes related to muscle growth and differentiation in mice. Am J Physiol Endocrinol Metab 308: E912-920, 2015. 
20. Lindholm ME, Marabita F, Gomez-Cabrero D, Rundqvist H, Ekstrom TJ, Tegner J, and Sundberg CJ. An integrative analysis reveals coordinated reprogramming of the epigenome and the transcriptome in human skeletal muscle after training. Epigenetics 9: 1557-1569, 2014.

21. Melov S, Tarnopolsky MA, Beckman K, Felkey K, and Hubbard A. Resistance exercise reverses aging in human skeletal muscle. PLoS One 2: e465, 2007.

22. Naimo MA, Rader EP, Ensey J, Kashon ML, and Baker BA. Reduced frequency of resistance-type exercise training promotes adaptation of the aged skeletal muscle microenvironment. J Appl Physiol (1985) 2019.

23. Nitert MD, Dayeh T, Volkov P, Elgzyri T, Hall E, Nilsson E, Yang BT, Lang S, Parikh H, Wessman Y, Weishaupt H, Attema J, Abels M, Wierup N, Almgren P, Jansson PA, Ronn T, Hansson O, Eriksson KF, Groop L, and Ling C. Impact of an exercise intervention on DNA methylation in skeletal muscle from first-degree relatives of patients with type 2 diabetes. Diabetes 61: 3322-3332, 2012.

24. Rader EP, and Baker BA. Inflammaging and the age-specific responsiveness to stretch-shortening contractions. Exerc Sport Sci Rev 45: 195-200, 2017.

25. Rader EP, Layner K, Triscuit AM, Chetlin RD, Ensey J, and Baker BA. Age-dependent muscle adaptation after chronic stretch-shortening contractions in rats. Aging Dis 7: 1-13, 2016.

26. Rader EP, Naimo MA, Ensey J, and Baker BA. High-intensity stretch-shortening contraction training modifies responsivity of skeletal muscle in old male rats. Exp Gerontol 104: 118-126, 2018.

27. Rader EP, Naimo MA, Layner KN, Triscuit AM, Chetlin RD, Ensey J, and Baker BA.

Enhancement of skeletal muscle in aged rats following high-intensity stretch-shortening contraction training. Rejuvenation Res 20: 93-102, 2017.

28. Ryan MJ, Dudash HJ, Docherty M, Geronilla KB, Baker BA, Haff GG, Cutlip RG, and Alway SE. Aging-dependent regulation of antioxidant enzymes and redox status in chronically loaded rat dorsiflexor muscles. J Gerontol A Biol Sci Med Sci 63: 1015-1026, 2008.

29. Ryan MJ, Dudash HJ, Docherty M, Geronilla KB, Baker BA, Haff GG, Cutlip RG, and Alway SE. Vitamin E and C supplementation reduces oxidative stress, improves antioxidant enzymes and positive muscle work in chronically loaded muscles of aged rats. Exp Gerontol 45: 882-895, 2010. 


\section{Sailani MR, Halling JF, Moller HD, Lee H, Plomgaard P, Pilegaard H, Snyder MP, and}

Regenberg B. Lifelong physical activity is associated with promoter hypomethylation of genes involved in metabolism, myogenesis, contractile properties and oxidative stress resistance in aged human skeletal muscle. Sci Rep 9: 3272, 2019.

31. Schoenfeld BJ, Wilson JM, Lowery RP, and Krieger JW. Muscular adaptations in low- versus highload resistance training: a meta-analysis. Eur J Sport Sci 16: 1-10, 2016.

\section{Seaborne RA, Strauss J, Cocks M, Shepherd S, O'Brien TD, van Someren KA, Bell PG,} Murgatroyd C, Morton JP, Stewart CE, and Sharples AP. Human skeletal muscle possesses an epigenetic memory of hypertrophy. Sci Rep 8: 1898, 2018.

33. Seals DR, and Melov S. Translational geroscience: emphasizing function to achieve optimal longevity. Aging (Albany NY) 6: 718-730, 2014.

34. Sharples AP, Stewart CE, and Seaborne RA. Does skeletal muscle have an 'epi'-memory? The role of epigenetics in nutritional programming, metabolic disease, aging and exercise. Aging Cell 15: 603-616, 2016.

\section{Stec MJ, Thalacker-Mercer A, Mayhew DL, Kelly NA, Tuggle SC, Merritt EK, Brown CJ,} Windham ST, Dell'Italia LJ, Bickel CS, Roberts BM, Vaughn KM, Isakova-Donahue I, Many GM, and Bamman MM. Randomized, four-arm, dose-response clinical trial to optimize resistance exercise training for older adults with age-related muscle atrophy. Exp Gerontol 99: 98-109, 2017.

36. Tobias IS, Lazauskas KK, Arevalo JA, Bagley JR, Brown LE, and Galpin AJ. Fiber type-specific analysis of AMPK isoforms in human skeletal muscle: advancement in methods via capillary nanoimmunoassay. J Appl Physiol (1985) 124: 840-849, 2018.

\section{Vaczi M, Nagy SA, Koszegi T, Ambrus M, Bogner P, Perlaki G, Orsi G, Toth K, and Hortobagyi} T. Mechanical, hormonal, and hypertrophic adaptations to 10 weeks of eccentric and stretch-shortening cycle exercise training in old males. Exp Gerontol 58: 69-77, 2014.

38. Versaevel M, Grevesse T, and Gabriele S. Spatial coordination between cell and nuclear shape within micropatterned endothelial cells. Nat Commun 3: 671, 2012.

39. Wang YX, and Rudnicki MA. Satellite cells, the engines of muscle repair. Nat Rev Mol Cell Biol 13: 127-133, 2011. 


\title{
APPENDIX A \\ RESISTANCE TRAINING FREQUENCY CONFERS GREATER MUSCLE QUALITY IN AGED INDIVIDUALS: A BRIEF NHANES REPORT
}

Running Head: Resistance Training Frequency and Muscle Quality Response

\author{
Marshall A. Naimo ${ }^{1,2}$
}

Ja K. Gu${ }^{1}$

Christa Lilly ${ }^{3}$

George A. Kelley ${ }^{3}$

${ }^{1}$ Centers for Disease Control and Prevention, National Institute for Occupational Safety and Health, Health Effects Laboratory Division, Morgantown, West Virginia, USA

${ }^{2}$ West Virginia University, School of Medicine, Division of Exercise Physiology, Morgantown, West Virginia, USA

${ }^{3}$ West Virginia University, School of Public Health, Department of Biostatistics, Morgantown, West Virginia, USA 
Corresponding Author:

Brent A. Baker

1095 Willowdale Road, MS-L3027

Morgantown, WV 26505-2888

bwb3@cdc.gov

Office: 304-285-5968 Fax: 304-285-6126

Marshall A. Naimo, MS, 1095 Willowdale Road, MS-L3014, Morgantown, WV, 26505-2888

Ja K. (Jack) Gu, MSPH, 1095 Willowdale Road, MS-L4050, Morgantown, WV, 26505-2888

Dr. Christa Lilly, School of Public Health, Department of Biostatistics, WV, 26506-9190

Dr. George A. Kelley, FACSM, School of Public Health, Department of Biostatistics, WV, 26506-9190 


\section{Publications Disclaimers:}

"The findings and conclusions in this report are those of the authors and do not necessarily represent the official position of the National Institute for Occupational Safety and Health, Centers for Disease Control and Prevention.” 


\section{ABSTRACT}

Background: Sarcopenia, the age-related decline in skeletal muscle mass, results in a loss of strength and functional capacity, which subsequently increases the risk of disease, disability frailty, and all-cause mortality. Skeletal muscle quality (MQ), i.e., strength per unit muscle mass, is the ability of muscle to perform its functions, and evidence indicates it is a more influential variable underlying age-related declines in muscle function than losses in muscle mass. Resistance training (RT) is known for enhancing skeletal MQ, improving health span, and reducing mortality. However, to the best of our knowledge, no studies have examined the relationship between RT frequency and MQ in an aged population. Thus, this study was designed to test the hypothesis that greater MQ in older individuals is associated with RT frequency. Methods: Utilizing data from 2,391 older adults in the National Health and Nutrition Survey (NHANES; 1999-2002), a secondary analysis of data was performed to see if an association existed between RT frequency and MQ in persons aged 55 years and older. Data were analyzed using analysis of covariance (ANCOVA) with three different models. Individuals were stratified into two groups based on how many days per week they performed RT: Insufficient (i.e., < two days per week) or sufficient ( $\geq$ two days per week). Muscle quality was calculated by taking the average peak force (Newtons) obtained from an isokinetic dynamometer and dividing it by leg lean mass, excluding bone mineral content (grams), obtained from dual-energy X-ray absorptiometry. The alpha level was set at $<0.05$. Results: For persons aged 55 and over, a statistically significant association was found between sufficient RT and greater MQ in both unadjusted as well as adjusted models that accounted for various demographic, behavioral, and clinical characteristics ( $\mathrm{p}<0.05$ for all). However, when limited to those 65 and older, no statistically significant associations were observed between sufficient RT and greater MQ (p>0.05 for all). When partitioned according to those 55 to 64 years of age and those 55 to 79 years, a statistically significant association was again observed ( $<<0.05$ for all). No statistically significant associations were observed for individuals 65-79 years of age or those $\geq 80$ years of age ( $\mathrm{p}>0.05$ for all). Conclusions: Sufficient amounts of RT are associated with greater MQ in selected older individuals. A need exists for future randomized controlled trials that examine the dose-response relationship between resistance training and MQ in older adults.

Keywords: Aging; sarcopenia; muscle composition; exercise prescription; cohort study 


\section{INTRODUCTION}

Sarcopenia, defined as the age-related loss of skeletal muscle mass and muscle performance (6), is a major health problem associated with a number of adverse outcomes. These include, but are not limited to, disability, frailty, poor quality of life and premature all-cause mortality $(3,10,14)$. This issue is especially important given the aging of our population worldwide. For example, by 2050, it is estimated that at least $25 \%$ of the population will be over the age of 65 , with prevalence rates as high as $40 \%$ in certain regions (20). In the United States, an estimated 20\% of the labor force is over the age of 55 but by 2020, that percentage is projected to increase to $25 \%$ (1). Not surprisingly, sarcopenia is associated with significant healthcare and socio-economic costs (11). Given the former, it is critical to examine interventions aimed at preventing and/or attenuating the negative effects of sarcopenia.

Aging is associated with the progressive decline of physiological and biomechanical functions that potentially leads to a loss of functional capacity. With advanced age, there are reductions in physical activity, specifically load-dependent movements that contribute to declines in motor function, metabolic quality of skeletal muscle, and a loss of muscle mass (26). In particular, muscle quality (MQ), defined as muscle strength or power per unit muscle mass, has been demonstrated by our laboratory as well as others to be a particularly sensitive indicator of age-related negative outcomes, and perhaps more functionally important than the actual amount of muscle mass for describing alterations in muscle performance as a consequence of sarcopenia (10, 17). In fact, several recent studies support the notion that a linear relationship exists between MQ status and overall mortality $(5,6,22)$.

Regular physical activity, including resistance training, has provided the most convincing evidence to date regarding the preservation of function with aging (21). Several studies have demonstrated the benefits of regularly-structured physical exercise as an effective, therapeutic approach to mitigate sarcopenic outcomes with aging $(8,18,24,27)$. Evidence suggests that high-intensity resistance training (RT) is the most potent intervention for attenuating the deleterious effects of sarcopenia, thus making it a potentially effective form of “physiotherapy” specifically directed at mitigating age-related decrements in muscle function (7). Along those lines, multiple studies have indicated that RT is associated with decreased overall mortality $(3,14,15,22)$.

Despite the increasing evidence highlighting RT for improving MQ as well as functional and healthrelated outcomes, to the best of our knowledge, no studies have examined the relationship between sufficient levels of RT and MQ in a nationally representative cohort of aged individuals over an extended period of time. Therefore, the purpose of this study was to examine the association between RT and MQ in aged individuals. We hypothesized that those persons who engaged in sufficient levels of RT activities, defined as two or more times per week based upon previous evidence in older individuals $(4,9,18,23)$, would be associated with greater MQ. 


\section{METHODS}

\section{Study Population and Design}

This study was conducted using data from the National Health and Nutrition Examination Survey (NHANES), an ongoing program of studies conducted by the Centers for Disease Control and Prevention's (CDC) National Center for Health Statistics. The purpose of NHANES is to measure the lifestyle, health, and nutrition behavior of a nationally representative sample of U.S. citizens (12). Specific to this project, NHANES data for calculating MQ, i.e., muscle performance and muscle mass data, were available for a four-year period and two data collection cycles spanning the years 1999-2002. For this study, only individuals that were 55 years of age and older were included in the data that was available for the 1999-2000 and 2001-2002 cycles.

Of 21,004 individuals that had data reported from the two NHANES cycles, a total of 3,737 were above the age of 55. After excluding those persons who did not have complete data and/or did not participate in one or both of the muscle RT and muscle mass measurements $(n=1,346)$, a final sample of 2,391 (1,220 males and 1,171 females) was used for the present study. Written consent was previously obtained from each NHANES participant and approved by the National Center for Health Statistics Research Ethics Review Board under protocol \#98-12.

\section{Measurement Methods}

The exposure variable (independent variable) was identified in NHANES as physical activity in which subjects were asked about their participation in RT-based activities performed in the past 30 days that was specifically designed to strengthen muscles (e.g., lifting weights, push-ups, sit-ups, etc.), and how frequently they did these activities. No advice was given by the NHANES staff on how to appropriately define RT performance; participants simply reported whether they engaged in any muscle RT activities and how many days per week they participated in RT. For the current study, frequency of training per week was dichotomized as either insufficient (less than two days per week) or sufficient (two or more days per week).

Based on the available data in NHANES, MQ was calculated from muscle strength, measured by the average peak force in Newtons using a knee dynamometer, as well as leg muscle mass, (i.e., leg lean mass using dual-energy X-ray absorptiometry (DXA) (model QDR-4500A, Hologic, Inc., Bedford, MA, USA). Covariates included gender, age, race/ethnicity, educational attainment, annual household income, pregnancy (yes/no), number of alcoholic drinks, smoking status, blood pressure, glucose readings, cardiovascular disease status, and leg \% fat. Body composition (lean body mass, fat mass, total mass) was measured using DXA following the manufacturer's acquisition procedures. Dual-energy X-ray absorptiometry scans were administered to eligible survey participants in the NHANES mobile examination centers. The scan for each survey participant was reviewed and analyzed by the University of California, San Francisco, Department of Radiology, using standard 
radiologic techniques and study-specific protocols developed for NHANES. Soft tissue measures included leg fat mass (grams), leg lean mass including bone mineral content (grams), leg lean mass excluding bone mineral content (grams), and percent (\%) body fat obtained for the legs. An isokinetic Kin Com MP dynamometer (Chattanooga Group, Inc., Chattanooga, TN, USA) was used to evaluate the strength of the knee extensors and flexors. Average peak force (Newtons) of the quadriceps and hamstrings was assessed at 60 degrees per second. Muscle quality was calculated by taking the measured average peak force in Newtons and dividing it by leg lean mass in grams, excluding bone mineral content.

\section{Statistical Analysis}

SAS SUDAAN 11.0.1 statistical software (Research Triangle Institute, Research Triangle Park, NC, USA) was used for all data analysis. Appropriate sample weights were used in calculating statistically reliable estimates in all analyses given that NHANES data were obtained using a complex, multistage sampling design that involves stratification, clustering, and oversampling of specific population subgroups. Standard errors were estimated using Taylor series linearization. Associations for various cohort characteristics with sufficient RT and MQ were assessed using chi-squared tests for categorical variables and analysis of variance (ANOVA) as

well as ordinary least squares (OLS) regression for continuous variables. The association between sufficient RT and MQ was calculated by an analysis of covariance (ANCOVA). Four different models were produced: 1) unadjusted estimates; 2) adjustments for age, gender, and race/ethnicity; 3) adjustment for model 2 covariates (age, gender, and race/ethnicity) as well as alcohol use, smoking status, and cardiovascular disease; 4) adjustment for model 2 and 3 covariates as well as \% leg fat. Sensitivity analysis for older ages were conducted based on previously used classifications for age, with 65+ defined as elderly (19). All reported p-values were two-sided, and alpha was set to 0.05 .

\section{RESULTS}

A total of 2,391 participants were included in the analyses (1,220 males, 1,171 females). Table 1 shows the association between selected study participant characteristics with muscle strength and MQ. Statistically significant associations were found between muscle strength and education level, annual household income, alcohol intake, smoking status and leg fat \%. Statistically significant associations were also found between MQ and age, race/ethnicity, education level, annual household income, alcohol intake, cardiovascular disease risk, and leg fat \%.

INSERT TABLE 1 HERE

When the analysis was performed using the entire study population, i.e., those individuals 55 years of age or older, there was a statistically significant association between sufficient RT of two or more sessions per 
week and increased MQ in models 1-3, with a trend for a statistically significant association in Model 4 $(p=0.053)$ (Table 2). When results were categorized according to participants 55 to 64 years of age a statistically significant association between sufficient RT and increased MQ for models 1 and 2 and a trend for statistical significance ( $\mathrm{p}=0.06$ ) for model 3 was observed (Table 3). However, no statistically significant association was observed for model 4. For those 55 to 79 years of age, a statistically significant association was observed for models 1-3 but not model 4 (Table 3). Results for those 65 years of age and older are shown in Table 4 . As can be seen, no statistically significant associations were observed between RT and increased MQ for any of the models ( $p>0.05$ for all). Similar findings were observed when the analysis was limited to those 65 to 79 years of age as well as those $\geq 80$ years of age (Table 5 ).

\section{INSERT TABLES 2-5 HERE}

\section{DISCUSSION}

To the best of our knowledge, this is the first secondary analysis of NHANES data that has attempted to characterize the association between sufficient levels of RT and greater MQ in older adults. The results of our study suggest that frequency of RT and increased MQ is associated with selected age groups. For example, statistically significant associations and statistical trends were found between sufficient frequency of RT and MQ in those 55 years of age and older as well as when stratified according to those 55 to 64 and 55 to 79 years of age. However, when limited to those 65 years of age and older as well as stratification according to 65 to 79 and $\geq 80$ year age groups, no statistically significant associations were observed between RT and MQ. Given the results and acknowledging the limitation that these activities were unsupervised/without instruction, this suggests that as one gets older, specific, appropriate exercise prescription may be a critical component for enabling skeletal muscle to undergo adaptation and achieve the beneficial effects of RT.

While muscle mass has historically been the primary focus of RT and other therapeutic options for improving muscle function in aging individuals, recent efforts support the notion that MQ is a more precise indicator of age-related negative health outcomes and perhaps more functionally relevant than the overall amount of muscle mass present for describing alterations in muscle performance as a consequence of sarcopenia $(10,17)$. In fact, muscle strength seems to decline at a faster rate than muscle mass, suggesting that decreases in MQ is the result of reduced functional capacity (2). Sarcopenia has many negative consequences, including, but not limited to, declines in mobility, increased risk of disability, increased susceptibility to musculoskeletal disorders, a higher prevalence of frailty, and premature all-cause mortality $(2,3,13,14)$. Recent research suggests that various measures of MQ are predictors of longevity and/or mortality in older adults (5, 22). Resistance training appears to be the primary therapeutic measure for improving MQ and overall muscle 
function, and subsequently counteracting and/or attenuating the negative consequences of sarcopenia (5, 10, 22). The results of the present study suggest that such an association exists in selected adult humans 55 years of age and older.

The lack of a statistically significant association in those 65 years of age and older may be the result of one or more of the following factors. First, the variable of sufficient versus insufficient RT activities was based on a self-reported questionnaire administered by NHANES staff without any oversight or supervision as to any of the parameters of their exercise program. In a recent meta-analysis conducted by Lacroix et al. (16), they reported that supervised resistance training induced larger effects on muscle strength and power compared to unsupervised resistance training. In addition, our laboratory has previously shown in rodents that while younger skeletal muscle displays a robust ability to adapt to multiple RT paradigms, aged muscle exhibits an inability to adapt properly to a non-specific, generalized exercise program; yet, by titrating the parameters of the exercise prescription (e.g., frequency, intensity) for aged muscle, there is an observed restoration of lipid peroxidation levels and MQ to a younger phenotype $(8,18)$. Furthermore, recent findings from our laboratory also suggest that altering the exercise prescription in aged muscle has an effect on muscle performance, mass and overall quality, findings which were supported by concomitant alterations in growth and remodeling signaling pathways in skeletal muscle that are underlying these performance-related changes of muscle (unpublished results). Therefore, the results of the present study in combination with previous data suggest that as individual's age, they become more susceptible to untoward effects of the specific exercise stimulus presented to them, and thus, appropriately modified exercise prescription may be a critical component underlying improvements in MQ. When prescribed in the proper manner, RT appears to be capable of promoting muscle adaptation, i.e., improvements in muscle mass and performance, in older individuals. However, inappropriately applied RT programs may result in a lack of significant muscle adaptation.

While the results of the present study are important, there were potential limitations that should be considered when interpreting the results. First, because the data was self-reported via answers to a questionnaire, there is an inherent self-report bias that is inherent with this type of analysis (e.g., social desirability, etc.) that may have led to some inaccurate answers. In addition, based on the nature of this study and results, we were unable to control any parameters of the exercise prescription, including intensity and the types of exercise performed. However, the fact that the results of the present study showed an association between enhanced MQ with a sufficient frequency of training suggests that these associations may be stronger if the exercise prescription was supervised given the recent findings that supervised exercise leads to larger effects in measures of muscle strength and power in older adults (16).

In conclusion, our findings in an NHANES population-based cohort of adult humans 55 years of age and older suggest that frequency of RT is associated with greater MQ in selected age groups. Given that exercise, 
particularly RT, is the most accessible, efficacious intervention to maximize skeletal muscle size and strength in the treatment of sarcopenia, a need exists for additional, well-designed, randomized controlled trials in older adults focusing on characterizing those parameters (mode, frequency, intensity, etc.) that augment adaptive responses in skeletal muscle before any degree of certainty can be reached regarding RT and MQ in older adults. 


\section{ACKNOWLEDGEMENTS}

The authors certify that they comply with the ethical guidelines for authorship and publishing of the Journal of Cachexia, Sarcopenia and Muscle (25).

\section{FUNDING}

This manuscript was supported by internal NIOSH funds. This study was conducted using data from the National Health and Nutrition Examination Survey (NHANES), an ongoing program of studies conducted by the Centers for Disease Control and Prevention’s (CDC) National Center for Health Statistics.

\section{CONFLICT of INTEREST}

None declared. 


\section{REFERENCES}

1. Baker BA. An old problem: Aging and skeletal muscle strain injury. J Sport Rehabil 26: 180-188, 2017.

2. Barbat-Artigas S, Rolland Y, Zamboni M, and Aubertin-Leheudre M. How to assess functional status: a new muscle quality index. J Nutr Health Aging 16: 67-77, 2012.

3. Beaudart C, Zaaria M, Pasleau F, Reginster JY, and Bruyere O. Health outcomes of sarcopenia: A systematic review and meta-analysis. PLoS One 12: e0169548, 2017.

4. Borde R, Hortobagyi T, and Granacher U. Dose-response relationships of resistance training in healthy old adults: a systematic review and meta-analysis. Sports Med 45: 1693-1720, 2015.

5. Brown JC, Harhay MO, and Harhay MN. The muscle quality index and mortality among males and females. Ann Epidemiol 26: 648-653, 2016.

6. Brown JC, Harhay MO, and Harhay MN. Sarcopenia and mortality among a population-based sample of community-dwelling older adults. J Cachexia Sarcopenia Muscle 7: 290-298, 2016.

7. Cholewa J, Guimaraes-Ferreira L, da Silva Teixeira T, Naimo MA, Zhi X, de Sa RB, Lodetti A, Cardozo MQ, and Zanchi NE. Basic models modeling resistance training: an update for basic scientists interested in study skeletal muscle hypertrophy. J Cell Physiol 229: 1148-1156, 2014.

8. Cutlip RG, Baker BA, Geronilla KB, Mercer RR, Kashon ML, Miller GR, Murlasits Z, and Alway SE. Chronic exposure to stretch-shortening contractions results in skeletal muscle adaptation in young rats and maladaptation in old rats. Appl Physiol Nutr Metab 31: 573-587, 2006.

9. Fisher JP, Steele J, Gentil P, Giessing J, and Westcott WL. A minimal dose approach to resistance training for the older adult; the prophylactic for aging. Exp Gerontol 99: 80-86, 2017.

10. Fragala MS, Kenny AM, and Kuchel GA. Muscle quality in aging: a multi-dimensional approach to muscle functioning with applications for treatment. Sports Med 45: 641-658, 2015.

11. Harper S. Economic and social implications of aging societies. Science 346: 587-591, 2014.

12. https://www.cdc.gov/nchs/nhanes/about_nhanes.htm. About the National Health and Examination Survey. 
13. Keevil VL, and Romero-Ortuno R. Ageing well: a review of sarcopenia and frailty. Proc Nutr Soc 74: 337-347, 2015.

14. Kelley GA, and Kelley KS. Is sarcopenia associated with an increased risk of all-cause mortality and functional disability? Exp Gerontol 96: 100-103, 2017.

\section{Kraschnewski JL, Sciamanna CN, Poger JM, Rovniak LS, Lehman EB, Cooper AB, Ballentine} NH, and Ciccolo JT. Is strength training associated with mortality benefits? A 15-year cohort study of US older adults. Prev Med 87: 121-127, 2016.

16. Lacroix A, Hortobagyi T, Beurskens R, and Granacher U. Effects of supervised vs. unsupervised training programs on balance and muscle strength in older adults: A systematic review and meta-analysis. Sports Med 47: 2341-2361, 2017.

17. McGregor RA, Cameron-Smith D, and Poppitt SD. It is not just muscle mass: a review of muscle quality, composition and metabolism during ageing as determinants of muscle function and mobility in later life. Longev Healthspan 3: 9, 2014.

\section{Rader EP, Naimo MA, Layner KN, Triscuit AM, Chetlin RD, Ensey J, and Baker BA.}

Enhancement of skeletal muscle in aged rats following high-intensity stretch-shortening contraction training. Rejuvenation Res 20: 93-102, 2017.

19. Sabharwal S, Wilson H, Reilly P, and Gupte CM. Heterogeneity of the definition of elderly age in current orthopaedic research. Springerplus 4: 516, 2015.

20. Seals DR, Justice JN, and LaRocca TJ. Physiological geroscience: targeting function to increase healthspan and achieve optimal longevity. J Physiol 594: 2001-2024, 2016.

21. Seals DR, and Melov S. Translational geroscience: emphasizing function to achieve optimal longevity. Aging (Albany NY) 6: 718-730, 2014.

22. Srikanthan $\mathbf{P}$, and Karlamangla AS. Muscle mass index as a predictor of longevity in older adults. Am J Med 127: 547-553, 2014.

23. Stec MJ, Thalacker-Mercer A, Mayhew DL, Kelly NA, Tuggle SC, Merritt EK, Brown CJ, Windham ST, Dell'Italia LJ, Bickel CS, Roberts BM, Vaughn KM, Isakova-Donahue I, Many GM, and 
Bamman MM. Randomized, four-arm, dose-response clinical trial to optimize resistance exercise training for older adults with age-related muscle atrophy. Exp Gerontol 99: 98-109, 2017.

24. Tucker LA. Physical activity and telomere length in U.S. men and women: an NHANES investigation. Prev Med 100: 145-151, 2017.

25. von Haehling S, Morley JE, Coats AJS, and Anker SD. Ethical guidelines for publishing in the journal of cachexia, sarcopenia and muscle: update 2017. J Cachexia Sarcopenia Muscle 8: 1081-1083, 2017.

26. Welle S. Cellular and molecular basis of age-related sarcopenia. Can J Appl Physiol 27: 19-41, 2002.

27. Yarasheski KE. Managing sarcopenia with progressive resistance exercise training. J Nutr Health Aging 6: 349-356, 2002. 
Table 1. Association between participant characteristics, muscle strength and muscle quality in those $\geq 55$ years of age.

\begin{tabular}{|c|c|c|c|c|c|c|}
\hline & \multirow{2}{*}{$\begin{array}{l}\text { Sampled } \\
(\mathrm{n}=2,391)\end{array}$} & \multicolumn{3}{|c|}{ Muscle Strength } & \multicolumn{2}{|c|}{ Muscle Quality } \\
\hline & & $\begin{array}{l}\text { Insufficient } \\
\text { Percent }^{\mathrm{a}}\end{array}$ & $\begin{array}{l}\text { Sufficient } \\
\text { Percent }^{\mathrm{a}}\end{array}$ & P-value ${ }^{b}$ & $\operatorname{Mean}^{\mathrm{a}}(95 \% \mathrm{CI})$ & P-value ${ }^{c}$ \\
\hline \multicolumn{7}{|l|}{ Age (years) } \\
\hline $55-64$ & 932 & 45.6 & 52.7 & \multirow{3}{*}{0.1952} & $19.60(19.09-20.10)$ & \multirow{3}{*}{$<0.0001 *$} \\
\hline $65-74$ & 858 & 33.7 & 29.7 & & $18.12(17.61-18.64)$ & \\
\hline $75+$ & 601 & 20.7 & 17.6 & & $16.02(15.36-16.69)$ & \\
\hline \multicolumn{7}{|l|}{ Gender } \\
\hline Male & 1,220 & 46.2 & 51.4 & \multirow[t]{2}{*}{0.1697} & $18.25(17.73-18.77)$ & \multirow[t]{2}{*}{0.3934} \\
\hline Female & 1,171 & 53.8 & 48.6 & & $18.50(17.95-19.06)$ & \\
\hline \multicolumn{7}{|l|}{ Race/ethnicity } \\
\hline White & 1,397 & 81.8 & 85.7 & \multirow{3}{*}{0.1714} & 18.49 (17.98-18.99) & \multirow{3}{*}{$0.0002 *$} \\
\hline Black & 393 & 7.7 & 6.5 & & $16.80(16.23-17.38)$ & \\
\hline Hispanic/others & 601 & 10.5 & 7.8 & & 18.70 (17.77-19.63) & \\
\hline \multicolumn{7}{|l|}{ Education } \\
\hline$<12^{\text {th }}$ years & 906 & 27.6 & 17.3 & \multirow{4}{*}{$<0.0001^{*}$} & $17.81(17.23-18.38)$ & \multirow{4}{*}{$0.0084 *$} \\
\hline High S Grad. & 543 & 27.6 & 15.0 & & $18.43(17.65-19.21)$ & \\
\hline Some college & 507 & 23.8 & 29.1 & & 18.44 (17.95-18.93) & \\
\hline College+ & 432 & 21.0 & 38.6 & & 18.93 (18.17-19.69) & \\
\hline \multicolumn{7}{|l|}{ Income (thousands) } \\
\hline $0-25$ & 801 & 33.7 & 18.2 & \multirow{4}{*}{ 0.0011* } & $17.22(16.55-17.89)$ & \multirow{4}{*}{ 0.0001* } \\
\hline $25-45$ & 525 & 25.8 & 22.1 & & $18.24(17.52-18.95)$ & \\
\hline $45-75$ & 387 & 21.9 & 25.3 & & $18.91(18.17-19.65)$ & \\
\hline $75^{+}$ & 326 & 18.7 & 34.3 & & 19.23 (18.54-19.93) & \\
\hline \multicolumn{7}{|l|}{ Alcoholic intake } \\
\hline No drink & 610 & 29.9 & 16.9 & \multirow{3}{*}{ 0.0038* } & $17.70(17.11-18.28)$ & \multirow{3}{*}{$0.0032 *$} \\
\hline 1 drink/day & 706 & 36.7 & 49.0 & & $18.61(18.25-18.98)$ & \\
\hline 2 drinks/day & 644 & 33.4 & 34.1 & & $18.83(18.17-19.48)$ & \\
\hline \multicolumn{7}{|l|}{ Smoking status } \\
\hline Current & 321 & 15.3 & 5.5 & \multirow{3}{*}{ 0.0002* } & 18.99 (18.30-19.69) & \multirow{3}{*}{0.0560} \\
\hline Former & 961 & 39.2 & 46.7 & & $18.40(17.77-19.03)$ & \\
\hline Never & 1,104 & 45.6 & 47.8 & & $18.20(17.63-18.78)$ & \\
\hline \multicolumn{7}{|l|}{ Blood pressure } \\
\hline No controlled & 1,901 & 79.2 & 75.7 & \multirow[t]{2}{*}{0.3694} & 18.33 (17.85-18.81) & \multirow[t]{2}{*}{0.3422} \\
\hline Controlled & 457 & 20.8 & 24.3 & & $18.76(17.90-19.61)$ & \\
\hline Glucose & & & & 0.7252 & & 0.1708 \\
\hline
\end{tabular}




\begin{tabular}{|c|c|c|c|c|c|c|}
\hline & \multirow{3}{*}{$\begin{array}{l}\text { Sampled } \\
(n=2,391)\end{array}$} & \multicolumn{3}{|c|}{ Muscle Strength } & \multicolumn{2}{|c|}{ Muscle Quality } \\
\hline & & Insufficient & Sufficient & & & \\
\hline & & Percent $^{\mathrm{a}}$ & Percent $^{\mathrm{a}}$ & P-value ${ }^{b}$ & $\operatorname{Mean}^{\mathrm{a}}(95 \% \mathrm{CI})$ & P-value ${ }^{c}$ \\
\hline No controlled & 191 & 12.7 & 11.5 & & 17.58 (16.34-18.82) & \\
\hline Controlled & 997 & 87.3 & 88.5 & & $18.36(17.90-18.82)$ & \\
\hline \multicolumn{7}{|l|}{ CVD } \\
\hline Yes & 235 & 9.5 & 7.4 & 0.2404 & $17.24(16.51-17.97)$ & $0.0017^{*}$ \\
\hline No & 2,156 & 90.5 & 92.6 & & 18.50 (18.04-18.97) & \\
\hline \multicolumn{7}{|l|}{ Leg fat $\%$} \\
\hline$<30 \%$ & 798 & 29.5 & 38.6 & \multirow{3}{*}{$0.0367 *$} & $18.78(18.17-19.40)$ & \multirow{3}{*}{$0.0460 *$} \\
\hline $30-<40 \%$ & 659 & 26.6 & 26.1 & & 17.95 (17.36-18.55) & \\
\hline$\geq 40 \%$ & 935 & 43.9 & 35.4 & & 18.37 (17.80-18.93) & \\
\hline
\end{tabular}

Notes: a , percent means weighted; ${ }^{b}$, derived from chi-square tests; ${ }^{c}$, derived from ANOVA or regression; CI, confidence intervals; CVD, cardiovascular disease; *, statistically significant $(\mathrm{P}<0.05)$. 
Table 2. Association between frequency of resistance training and muscle quality in those $\geq 55$ years of age $(n=2,391)$.

\begin{tabular}{|c|c|c|c|}
\hline & $\begin{array}{l}\text { Insufficient }^{\mathrm{a}} \\
\quad(\mathrm{n}=2,075)\end{array}$ & $\begin{array}{l}\text { Sufficient }^{b} \\
\quad(n=316)\end{array}$ & P-value ${ }^{c}$ \\
\hline Muscle Quality & Mean (95\% CI) & Mean (95\% CI) & \\
\hline Model $1^{\mathrm{d}}$ & $18.22(17.75-18.68)$ & 19.43 (18.68-20.18) & $0.0032 *$ \\
\hline Model $2^{\mathrm{e}}$ & 18.25 (17.82-18.67) & 19.25 (18.60-19.90) & $0.0050 *$ \\
\hline Model $3^{\mathrm{f}}$ & $18.31(17.92-18.70)$ & $19.16(18.45-19.86)$ & $0.0175^{*}$ \\
\hline Model $4^{\mathrm{g}}$ & 18.34 (17.97-18.71) & 18.99 (18.30-19.68) & $0.0533 * *$ \\
\hline
\end{tabular}

Notes: ${ }^{\mathrm{a}}$ Insufficient, $<2$ times per week, ${ }^{\mathrm{b}}$, Sufficient, $\geq 2$ times per week; ${ }^{\mathrm{c}}$, $\mathrm{P}$-value derived from

ANCOVA; d, unadjusted; e, adjusted for age, gender, race/ethnicity; f, adjusted for age, gender,

race/ethnicity, alcohol, smoking status, and cardiovascular disease; g. adjusted for age, gender,

race/ethnicity, alcohol, smoking status, cardiovascular disease, and \% leg fat; $\mathrm{CI}$, confidence intervals;

*, statistically significant $(\mathrm{P}<0.05)$; **, trend for statistical significance $(\mathrm{P}<0.10)$. 
Table 3. Association between frequency of resistance training and muscle quality partitioned by age 55.

\begin{tabular}{|c|c|c|c|c|c|}
\hline \multicolumn{3}{|c|}{ 55-64 years } & \multicolumn{3}{|c|}{ 55-79 years } \\
\hline $\begin{array}{c}\text { Insufficient }^{\mathrm{a}} \\
\qquad(\mathrm{n}=801)\end{array}$ & $\begin{array}{l}\text { Sufficient }^{\mathrm{b}} \\
(\mathrm{n}=131)\end{array}$ & P-value ${ }^{c}$ & $\begin{array}{c}\text { Insufficient }^{\mathrm{a}} \\
(\mathrm{n}=1,768)\end{array}$ & $\begin{array}{c}\text { Sufficient }^{\mathrm{b}} \\
(\mathrm{n}=268)\end{array}$ & $\mathrm{P}$-value ${ }^{\mathrm{a}}$ \\
\hline
\end{tabular}

\begin{tabular}{|c|c|c|c|c|c|c|}
\hline $\begin{array}{l}\text { Muscle } \\
\text { Quality }\end{array}$ & Mean $(95 \%$ CI) & Mean (95\% CI) & & Mean $(95 \%$ CI) & Mean $(95 \%$ CI) & \\
\hline Model $1^{\mathrm{d}}$ & $\begin{array}{c}19.39(18.88- \\
19.89)\end{array}$ & $\begin{array}{c}20.74(19.70- \\
21.77)\end{array}$ & 0.0128* & $\begin{array}{c}18.48(18.03- \\
19.01)\end{array}$ & $\begin{array}{c}19.76(19.01- \\
20.51)\end{array}$ & 0.0014* \\
\hline Model $2^{\mathrm{e}}$ & $\begin{array}{c}19.41(18.93- \\
19.89)\end{array}$ & $\begin{array}{c}20.62(19.64- \\
21.60)\end{array}$ & $0.0198 *$ & $\begin{array}{c}18.51(18.10- \\
18.93)\end{array}$ & $\begin{array}{c}19.57(18.88- \\
20.25)\end{array}$ & $0.0043^{*}$ \\
\hline Model $3^{f}$ & $\begin{array}{c}19.47 \text { (18.99- } \\
19.96)\end{array}$ & $\begin{array}{c}20.58(19.50- \\
21.67)\end{array}$ & $0.0593 * *$ & $\begin{array}{c}18.59 \text { (18.19- } \\
18.99)\end{array}$ & $\begin{array}{c}19.41(18.64- \\
20.17)\end{array}$ & $0.0329 *$ \\
\hline Model $4^{\mathrm{g}}$ & $\begin{array}{c}19.54(19.09- \\
20.00)\end{array}$ & $\begin{array}{c}20.23(19.26- \\
21.20)\end{array}$ & 0.1724 & $\begin{array}{c}18.62(18.24- \\
19.00)\end{array}$ & $\begin{array}{c}19.21(18.45- \\
19.97)\end{array}$ & 0.1143 \\
\hline
\end{tabular}

Notes: a, Insufficient, $<2$ times per week, ${ }^{\text {b, }}$ Sufficient, $\geq 2$ times per week; ${ }^{c,}$ P-value derived from

ANCOVA; ${ }^{\mathrm{d}}$ unadjusted; ${ }^{\mathrm{e}}$, adjusted for age, gender, race/ethnicity; ${ }^{\mathrm{f}}$, adjusted for age, gender, race/ethnicity, alcohol, smoking status, and cardiovascular disease; g. adjusted for age, gender, race/ethnicity, alcohol, smoking status, cardiovascular disease, and \% leg fat; CI, confidence intervals;

*, statistically significant (P-value $<0.05)$; **, trend for statistical significance $(\mathrm{P}<0.10)$. 
Table 4. Association between frequency of resistance training and muscle quality in participants $\geq 65$ of age, $(n=1,459)$.

\begin{tabular}{cccc} 
& $\begin{array}{c}\text { Insufficient }^{\mathrm{a}} \\
(\mathrm{n}=1,274)\end{array}$ & $\begin{array}{c}\text { Sufficient }^{\mathrm{b}} \\
(\mathrm{n}=185)\end{array}$ & P-value $^{\mathrm{c}}$ \\
\hline Muscle Quality & Mean $(95 \% \mathrm{CI})$ & Mean $(95 \% \mathrm{CI})$ & \\
\hline Model 1 $^{\mathrm{d}}$ & $17.24(16.69-17.79)$ & $17.97(17.03-18.91)$ & 0.1723 \\
Model 2 $^{\mathrm{e}}$ & $17.23(16.71-17.76)$ & $17.99(17.14-18.85)$ & 0.1257 \\
Model 3 $^{\mathrm{f}}$ & $17.21(16.69-17.72)$ & $17.78(16.94-18.62)$ & 0.2039 \\
Model 4 $^{\mathrm{g}}$ & $17.22(16.71-17.72)$ & $17.73(16.87-18.60)$ & 0.2635 \\
\hline
\end{tabular}

Notes: ${ }^{\text {a, }}$ Insufficient, $<2$ times per week, ${ }^{\text {b, }}$ Sufficient, $\geq 2$ times per week; ${ }^{c}$, $\mathrm{P}$-value derived from

ANCOVA; ${ }^{\mathrm{d}}$ unadjusted; ${ }^{\mathrm{e}}$ adjusted for age, gender, race/ethnicity; $\mathrm{f}$, adjusted for age, gender, race/ethnicity, alcohol, smoking status, and cardiovascular disease; ' ${ }^{\text {. }}$ adjusted for age, gender, race/ethnicity, alcohol, smoking status, cardiovascular disease, and \% leg fat; $\mathrm{CI}$, confidence intervals. 
Table 5. Association between frequency of resistance training and muscle quality categorized by age.

\begin{tabular}{|c|c|c|c|c|c|c|}
\hline & \multicolumn{2}{|c|}{65 to 79 years } & \multicolumn{3}{|c|}{$\geq 80$ years } & \multirow[b]{2}{*}{$\begin{array}{c}\mathrm{P}- \\
\text { value }^{\mathrm{C}}\end{array}$} \\
\hline & $\begin{array}{c}\text { Insufficient }^{\mathrm{a}} \\
\quad(\mathrm{n}=967)\end{array}$ & $\begin{array}{l}\text { Sufficient }^{\mathrm{b}} \\
\quad(\mathrm{n}=137)\end{array}$ & $\begin{array}{c}\text { P- } \\
\text { value }^{\mathrm{a}}\end{array}$ & $\begin{array}{l}\text { Insufficient }^{\mathrm{a}} \\
\quad(\mathrm{n}=307)\end{array}$ & $\begin{array}{l}\text { Sufficient }^{\mathrm{b}} \\
\qquad(\mathrm{n}=48)\end{array}$ & \\
\hline \multicolumn{7}{|l|}{ Muscle } \\
\hline Quality & Mean (95\% CI) & Mean $(95 \%$ CI) & & Mean $(95 \%$ CI) & Mean (95\% CI) & \\
\hline Model $1^{\mathrm{d}}$ & $\begin{array}{c}17.56(17.02- \\
18.11)\end{array}$ & $\begin{array}{c}18.39(17.42- \\
19.37)\end{array}$ & 0.1256 & $\begin{array}{c}15.74(14.84- \\
16.63)\end{array}$ & $\begin{array}{c}16.29(15.20- \\
17.38)\end{array}$ & 0.3909 \\
\hline Model $2^{\mathrm{e}}$ & $\begin{array}{c}17.56(17.05- \\
18.08)\end{array}$ & $\begin{array}{c}18.39(17.47- \\
19.32)\end{array}$ & 0.1118 & $\begin{array}{c}15.80(14.98- \\
16.61)\end{array}$ & $\begin{array}{c}15.93(14.79- \\
17.06)\end{array}$ & 0.8377 \\
\hline Model $3^{f}$ & $\begin{array}{c}17.57(17.04- \\
18.10)\end{array}$ & $\begin{array}{c}18.04(17.03- \\
19.05)\end{array}$ & 0.3626 & $\begin{array}{c}15.50(14.75- \\
16.25)\end{array}$ & $\begin{array}{c}16.17(14.94- \\
17.39)\end{array}$ & 0.3709 \\
\hline Model $4^{\mathrm{g}}$ & $\begin{array}{c}17.58(17.06- \\
18.10)\end{array}$ & $\begin{array}{c}17.97(16.92- \\
19.02)\end{array}$ & 0.4634 & $\begin{array}{c}15.49(14.72- \\
16.25)\end{array}$ & $\begin{array}{c}16.27(15.06- \\
17.48)\end{array}$ & 0.2906 \\
\hline
\end{tabular}

Notes: a Insufficient, $<2$ times per week, ${ }^{\text {b, }}$ Sufficient, $\geq 2$ times per week; c, P-value derived from ANCOVA; ${ }^{\text {d, }}$ unadjusted; e, adjusted for age, gender, race/ethnicity; ${ }^{\mathrm{f}}$, adjusted for age, gender, race/ethnicity, alcohol, smoking status, and cardiovascular disease; ${ }^{\text {g. }}$ adjusted for age, gender, race/ethnicity, alcohol, smoking status, cardiovascular disease, and \% leg fat; CI, confidence intervals. 


\title{
BIOGRAPHICAL SKETCH
}

\author{
MARSHALL A. NAIMO
}

Marshall received his Bachelor of Science degree in Exercise Science at The Florida State University in Tallahassee, Florida during the Fall 2008 semester. Also at The Florida State University under the advisement of Dr. Lynn Panton, he obtained his Master of Science degree in Exercise Physiology during the Spring 2011 semester; his thesis work eventually became a first author publication in a peer-reviewed journal in 2013. While collecting his research, Marshall taught courses in Functional Anatomy and Physiology Laboratory, Exercise Testing and Prescription Laboratory, and Nutrition and Sports. After graduation, Marshall spent two years teaching classes and conducting research studies at the University of Tampa. Teaching duties included both lecture and/or laboratory courses in Human Anatomy and Physiology, Biokinetics and Conditioning, and Exercise Physiology. Collectively, his research conducted during this time in the areas of sports nutrition and human performance in response to resistance exercise training and/or high intensity interval training resulted in being an author on nine publications, including one as first author. After enrolling in the Biomedical Sciences Doctor of Philosophy program at West Virginia University starting in the Fall 2013 semester, Marshall eventually joined the Systems Mechanophysiology and Aging Research Team at CDC/NIOSH under the mentorship of Dr. Brent A. Baker starting in 2014, and spent the next several years of his research characterizing various underlying molecular, physiological, and biomechanical characteristics of young and aged skeletal muscle following contraction-induced injury or high-intensity resistance-type exercise training paradigms. His contributions from his dissertation work as well as other internally funded projects have led to being an author on six additional publications (two as first author) along with another two first author publications that will be submitted shortly after graduation. Upon successful defense of his dissertation in the Summer 2019 semester, Marshall’s immediate plans are remaining at CDC/NIOSH in a full-time postdoctoral research capacity with plans of developing a rodent model of volitional ladder climbing in hopes of further characterizing at the molecular, physiological, and performance levels various components of the exercise prescription with the purpose of more fully developing clinically relevant optimal training programs aimed at restoring and/or augmenting the ability of aged skeletal muscle to undergo adaptation. 